\title{
RECOMENDACIONES \\ DE USO ADECUADO \\ DE PRUEBAS Y \\ SUPLEMENTOS DE \\ VITAMINA D EN \\ POBLACIÓN GENERAL
}

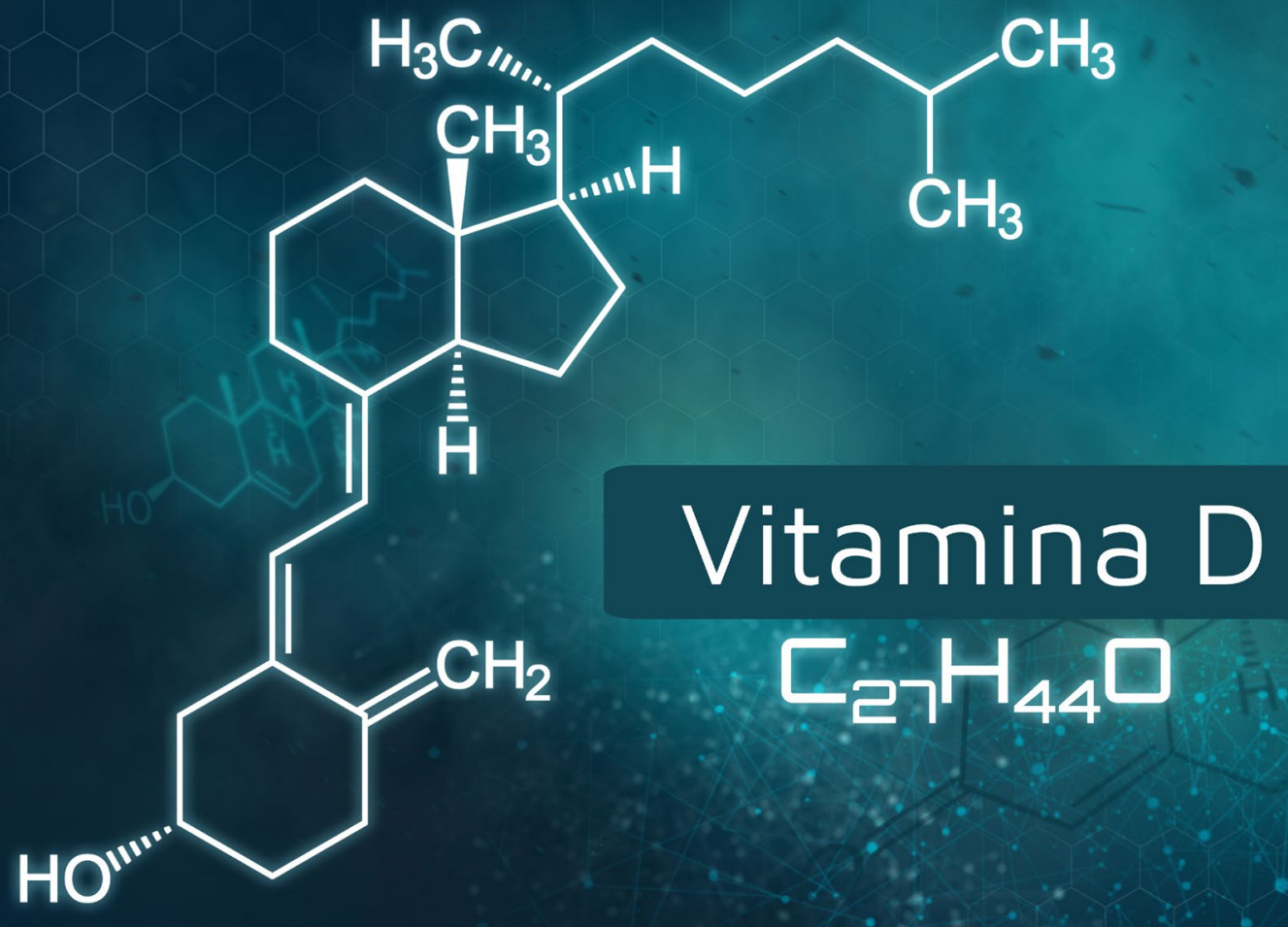


El contenido del presente informe es responsabilidad exclusiva de la Unidad de Asesoramiento Científicotécnico, Avalia-t.

Este documento puede ser reproducido total o parcialmente, por cualquier medio, siempre que se cite explícitamente su procedencia.

Fecha de edición: 2021

Edita: Ministerio de Sanidad

Agencia Gallega para la Gestión del Conocimiento en Salud (ACIS),

Unidad de Asesoramiento Científico-técnico, avalia-t

NIPO: 133-21-095-3

Depósito legal: C 1667-2021

Contacto: Avalia_t.Acis@sergas.es

Maquetación: Tórculo Comunicación Gráfica, S. A 
Este documento ha sido realizado por la Unidad de Asesoramiento Científico-técnico, Avalia-t de la Agencia Gallega para la Gestión del Conocimiento en Salud (ACIS) en el marco de la financiación del Ministerio de Sanidad para el desarrollo de las actividades del Plan anual de trabajo de la Red Española de Agencias de Evaluación de Tecnologías Sanitarias y Prestaciones del Sistema Nacional de Salud.

Para citar este documento:

Grupo de trabajo del OPBE del uso adecuado de pruebas y suplementos de vitamina D en población general. Recomendaciones de uso adecuado de pruebas y suplementos de vitamina D en población general. Madrid: Ministerio de Sanidad; Santiago de Compostela: Agencia Gallega para la Gestión del Conocimiento en Salud (ACIS), Unidad de Asesoramiento Científico-técnico, Avalia-t; 2021.

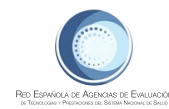





\section{Índice}

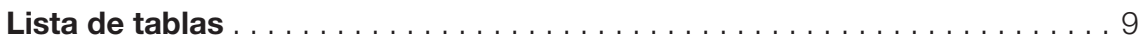

Lista de figuras $\ldots \ldots \ldots \ldots \ldots \ldots \ldots \ldots \ldots \ldots \ldots$

Autoría y colaboraciones $\ldots \ldots \ldots \ldots \ldots \ldots \ldots \ldots \ldots \ldots \ldots \ldots$

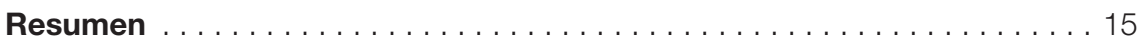

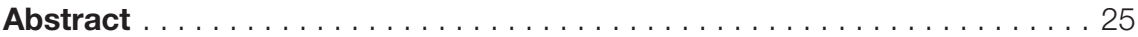

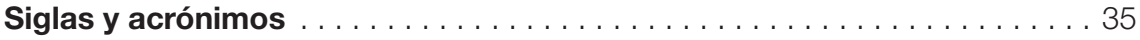

Glosario de términos. . . . . . . . . . . . . . . . . . . . . . . . 37

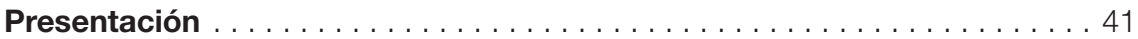

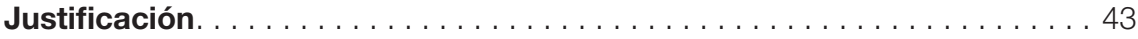

1 Alcance y objetivos . . . . . . . . . . . . . . . . 45

1.1 Alcance. . . . . . . . . . . . . . . . . . . . . 45

1.2 Objetivos........................... 45

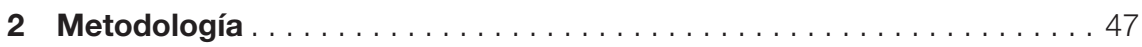

2.1 Constitución del grupo de trabajo . . . . . . . . . . . . . 47

2.2 Formulario de preguntas clínicas y estrategia de respuesta . . . . . . . . . 48

2.3 Búsqueda, evaluación y síntesis de la evidencia . . . . . . . . . . . . . 49

2.3.1 Búsqueda. . . . . . . . . . . . . . . . . . . . . 49

2.3 .2 Evaluación . . . . . . . . . . . . . . . . . . . . . . . . . 50

2.3 .3 Síntesis y recomendaciones . . . . . . . . . . . . . . . . . . . . . . . 50

2.4 Identificación y desarrollo de indicadores de evaluación . . . . . . . . . . . 51

2.5 Redacción del documento . . . . . . . . . . . . . . . . . . . 51

2.6 Revisión externa . . . . . . . . . . . . . . . . . . . . . . . 51 


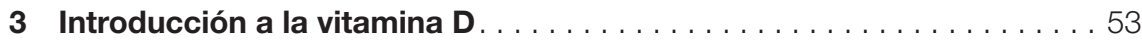

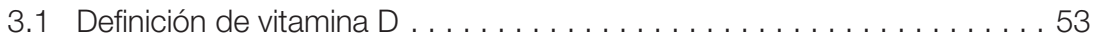

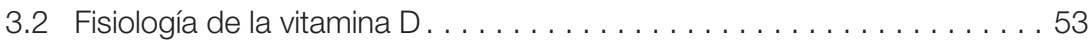

3.3 Fuentes de vitamina D. . . . . . . . . . . . . . . . . . . . . . . . 54

3.4 Niveles óptimos de vitamina $D \ldots \ldots \ldots \ldots \ldots \ldots \ldots \ldots \ldots \ldots \ldots \ldots \ldots \ldots$

3.5 Prevalencia y carga de la enfermedad. . . . . . . . . . . . . . . 59

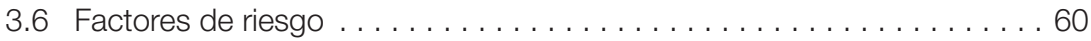

3.7 Intervenciones y tratamiento . . . . . . . . . . . . . . . . . . . 61

3.8 Efectos adversos del tratamiento con vitamina $\mathrm{D} \ldots \ldots \ldots \ldots \ldots \ldots \ldots$

3.9 Fármacos con vitamina D comercializados en España . . . . . . . . . . . . . 64

4 Revisión de la evidencia científica para las recomendaciones de uso adecuado de pruebas y suplementos de vitamina $D$ en

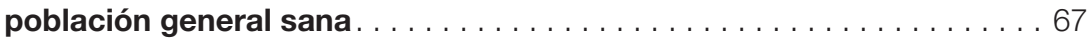

4.1 Delphi modificado. Concentración óptima de vitamina $\mathrm{D}$ en población sana . . . . . . . . . . . . . . . . . . . . . . . . . . . . . . 67

4.2 Revisión de la evidencia científica . . . . . . . . . . . . . . . . . . . 70

4.3 Pregunta 1: ¿Cuál es la asociación entre los niveles séricos de 25(OH)D y los resultados en salud? Resultados en: mortalidad, cáncer, fracturas, caídas, enfermedad cardiovascular, diabetes, depresión, funcionamiento cognitivo, estado funcional, etc. . . . . . . . 70

4.3.1 Resultados de los niveles séricos de 25(OH)D en salud . . . . . . . 72

4.4 Pregunta 2: ¿Cuál es la efectividad de la vitamina $D$, en variables duras/finales, incluyendo enfermedades cardiovasculares, obesidad, cáncer, sistema inmunitario, fractura osteoporótica, insuficiencia renal y/o la calcificación de los tejidos blandos? . . . . . . . . . . . . . . . 93

4.4.1 Resultados de la efectividad del suplemento de vitamina D en salud .............................. 94

\section{Recomendaciones de uso adecuado de la vitamina D en}

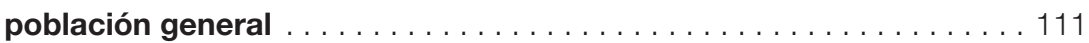

5.1 Consideraciones previas . . . . . . . . . . . . . . . . . . . 111

5.2 Recomendaciones de uso en población general sana. . . . . . . . . . . . . 112

5.2.1 Recomendaciones sobre cuantificación de vitamina D en población general sana. . . . . . . . . . . . . . . . . . . . . . . . 112

5.2.2 Recomendaciones de suplementación con vitamina $D$ en población general sana. . . . . . . . . . . . . . . . . . 112 
6. Aplicabilidad del OPBE

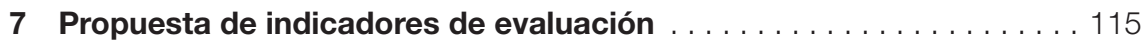

8 Actualización del OPBE. . . . . . . . . . . . . . . . . . . . . . 119

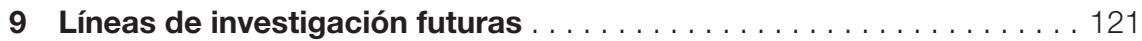

Referencias bibliográficas $\ldots \ldots \ldots \ldots \ldots \ldots \ldots \ldots \ldots \ldots \ldots \ldots$

Anexos . . . . . . . . . . . . . . . . . . . . . . . . . . . . . . . . . . . . 147

Anexo 1. Declaración de conflictos de interés . . . . . . . . . . . . . . . . . 147

Anexo 2. Preguntas a responder. . . . . . . . . . . . . . . . . 148

Anexo 3. Estrategia de búsqueda . . . . . . . . . . . . . . . . . . . . . . . . 149

Anexo 3.1 Pregunta 1: Niveles de vitamina D y resultados en salud . . . . . . 149

Anexo 3.2. Pregunta 2: Efectividad de vitamina D y resultados en salud . . . . 154

Anexo 4. Análisis de sesgos de estudios incluidos . . . . . . . . . . . . . . . 157

Anexo 4.1. Pregunta 1: Niveles de vitamina D y resultados en salud . . . . . . 157

Anexo 4.2. Pregunta 2: Efectividad de vitamina D y resultados en salud . . . . 165

Anexo 5. Evaluación y características de los estudios incluidos. . . . . . . . . 176

Anexo 5.1. Pregunta 1: Niveles de 25(OH)D y resultados en salud . . . . . . . 176

Anexo 5.2. Pregunta 2: Efectividad vitamina D en resultados en salud . . . . 232

Anexo 6. Fármacos comercializados en España que contienen vitamina D . . 355 



\section{Lista de tablas}

Tabla 1 Alimentos ricos en vitamina D . . . . . . . . . . . . . . 55

Tabla 2 Factores de riesgo para niveles no óptimos de vitamina D . . . . . . . . 61

Tabla 3 Recomendaciones de la Agencia Española de Medicamentos y productos Sanitarios para la hipercalcemia . . . . . . . . . . . 64

Tabla 4 Resultados ronda 1 Delphi. . . . . . . . . . . . . . . . 68

Tabla 5 Resultados Ronda 2 Delphi . . . . . . . . . . . . . . . . . . 69

Tabla 6 Concentración de vitamina D. . . . . . . . . . . . . . . . . 70

Tabla 7 Desarrollo pregunta PICO, pregunta $1 \ldots \ldots \ldots \ldots \ldots \ldots \ldots$

Tabla 8 Criterios de inclusión y exclusión de estudios, pregunta $1 \ldots \ldots$. . . 71

Tabla 9 Bases de datos electrónicas de la literatura biomédica, pregunta 1 . . 72

Tabla 10 Desarrollo pregunta PICO, pregunta $2 \ldots \ldots \ldots \ldots \ldots . \ldots 9$

Tabla 11 Criterios de inclusión y exclusión de estudios, pregunta $2 \ldots . . . . .99$

Tabla 12 Bases de datos electrónicas de la literatura biomédica, pregunta 2 . . 94

Tabla 13 Grados de cumplimiento y clasificación . . . . . . . . . . . . . . . . 115

Tabla 14 Indicadores propuestos . . . . . . . . . . . . . . . 116

Tabla 15 Fichas de los indicadores propuestos . . . . . . . . . . . . . . 116

\section{Lista de figuras}

Figura 1 Vías de síntesis de vitamina $\mathrm{D} \ldots \ldots \ldots \ldots \ldots \ldots \ldots \ldots$

Figura 2 Gráfico de riesgo de sesgos de estudios observacionales.

Pregunta 1: Niveles de vitamina $D$ y resultados en salud. . . . . . . . 160

Figura 3 Gráfico de riesgos de sesgos de ECAs. Pregunta 2: Efectividad de vitamina $D$ y resultados en salud. . . . . . . . . . . . . 171 



\section{Autoría y colaboraciones}

\section{Grupo de elaboración (por orden alfabético)}

Silvia Sandra Abelleira Ramos. Médica de Familia, Centro de Salud O Saviñao, Lugo.

María Carmen Blas Fraga. FEA de laboratorio de Análisis Clínicos del Hospital Lucus Augusti Lugo.

Rosendo Bugarín González. Médico de Familia. Centro Salud Monforte de Lemos, Lugo.

Rosa Burgo López. FEA Servicio de Endocrinología Hospital Público de Monforte de Lemos, Lugo.

Carmen Casal Llorente. Farmacéutica. Servicio de Uso Racional de Medicamento y Atención Farmacéutica. Subdirección Xeral de FarmaciaDirección Xeral de Asistencia Sanitaria.

Jesús Calviño Varela. Jefe de Servicio de Nefrología del Hospital Lucus Augusti Lugo.

Manuel Castro Pazos. Médico de Familia. Centro Salud Monte Porreiro, Pontevedra.

José Antonio Díaz Peromingo. FEA del Servicio de Medicina Interna del Hospital Clínico Universitario Santiago de Compostela, A Coruña.

María José Faraldo Vallés. Jefa de Servicio de la Unidad de Asesoramiento Científico-técnico, avalia-t. Agencia Gallega para el Conocimiento en Salud (ACIS).

Begoña Graña Suárez.FEA del Área de Oncología del Hospital Universitario de A Coruña.

María del Carmen Maceira Rozas. Farmacéutica. Unidad de Asesoramiento Científico-técnico, avalia-t. Agencia Gallega para el Conocimiento en Salud (ACIS).

Ana Belén Mariño Prol. Farmacéutica de Atención Primaria. Centro Salud Lalín, Pontevedra.

Xoan Miguéns Váquez. Jefe Servicio de Rehabilitación del Complexo Hospitalario Universitario de Ourense.

Susana Romero Yuste. Jefa de Servicio de Reumatología del Complexo Hospitalario Universitario de Pontevedra. 
Leonor Varela Lema. Profesora Ayudante Doctora. Área de Medicina Preventiva y Salud Pública. Universidad de Santiago de Compostela (USC).

\section{Coordinación}

Área clínica

Silvia Sandra Abelleira Ramos. Médica de Familia, Centro de salud O Saviñao. Lugo.

Área metodológica

María del Carmen Maceira Rozas. Farmacéutica. Unidad de Asesoramiento Científico-técnico, Avalia-t. Agencia Gallega para el Conocimiento en Salud (ACIS)

\section{Revisión externa (por orden alfabético)}

Pilar Aguado Acín. Médico Adjunto del Servicio de Reumatología. Coordinadora Unidad Metabólica Ósea. Hospital Universitario La Paz, Madrid. En representación de la Sociedad Española de Reumatología (SER).

José Carlos Bastida Calvo. Médico de Familia. Centro de Salud de Marín, Pontevedra. En representación de la Sociedad Española de Médicos Generales y de Familia (SEMG).

Dolores Cambeiro Pais. Farmacéutica de Atención Primaria. Centro de Salud de Teis, Vigo. En representación de la Sociedad Española de Farmacéuticos de Atención Primaria (SEFAP).

Isidoro Cano Rodríguez. Licenciado especialista en Endocrinología y Nutrición del Complejo Asistencial Universitario de León (CAULE). En representación de Sociedad Española de Endocrinología y Nutrición (SEEN).

Cristina Carbonell Abella. Médico de Familia. Centro de Salud Vía Roma, Barcelona. En representación de la Sociedad Española de Médicos de Atención Primaria (SEMERGEN).

Santos Castañeda Sanz. Servicio de Reumatología. Hospital de la Princesa, Madrid. En representación de la Sociedad Española de Reumatología (SER). 
Federico Cuesta Triana. Servicio de Geriatría. Hospital Clínico San Carlos, Madrid. En representación de la Sociedad Española de Geriatría y Gerontología (SEGG).

Jesús Alberto García Vadillo. Jefe de Sección de Reumatología del Hospital Universitario La Princesa, Madrid. A propuesta de Ministerio de Sanidad.

M Luisa González Casaus. FEA de Análisis Clínicos del Hospital Central de la Defensa Gómez Ulla, Madrid. En representación de la Sociedad Española de Nefrología (SEN).

Luis Ángel León Mateos. Servicio de Oncología Médica \& Grupo de Oncología Médica Traslacional. Hospital Clínico Universitario \& Instituto de Investigación Sanitaria de Santiago de Compostela (IDIS). En representación de la Sociedad Española de Oncología Médica (SEOM).

Isabel Lorenzo Lorenzo. Especialista en Oncología Médica. Complejo Hospitalario Universitario de Vigo. En representación de la Sociedad Española de Oncología Médica (SEOM).

Guillermo Martínez Díaz-Guerra. Jefe de sección de la Unidad de Endocrinología y Nutrición del Hospital Universitario 12 de Octubre, Madrid. En representación de la Sociedad Española de Investigación Ósea y Metabolismo Mineral (SEIOM).

María Medina Abellán. Médico de Familia y Comunitaria. Centro de Salud Totana Sur, Murcia. En representación de la Sociedad Española de Medicina de Familia y Comunitaria (SEMFYC).

Ricardo Mencía Barrio. Jefe de Unidad de Cadera, Servicio de Cirugía Ortopédica y Traumatología del Complejo Asistencial Universitario de León. Vocal de asuntos profesionales de la SECOT. En representación de la Sociedad Española de Cirugía Ortopédica y Traumatología (SECOT).

José Luis Pérez Castrillón. Servicio de Medicina Interna. Hospital Río Hortega. Catedrático de Medicina de la Universidad de Valladolid. En representación de la Sociedad Española de Medicina Interna (SEMI).

Álvaro Pérez Martín. Médico de familia. Centro de Salud Isabel II, Santander. Miembro de grupo de trabajo nacional de enfermedades reumatológicas. En representación de la Sociedad Española de Medicina de Familia y Comunitaria (SEMFYC).

Manuel Portela Romero. Subdirector de Prestación Farmacéutica, Formación, Docencia, Investigación e Innovación. Área sanitaria de Santiago de Compostela e Barbanza. En representación de la Sociedad Española de Médicos de Atención Primaria (SEMERGEN). 
Jimena Ramón García. Farmacéutica de Atención Primaria. Dirección Asistencial Sur, Sistema Madrileño de Salud. En representación de la Sociedad Española de Farmacéuticos de Atención Primaria (SEFAP).

\section{Sociedades Científicas Colaboradoras (por orden alfabético)}

Sociedad Española de Cirugía Ortopédica y Traumatología (SECOT)

Sociedad Española de Endocrinología y Nutrición (SEEN)

Sociedad Española de Farmacéuticos de Atención Primaria (SEFAP)

Sociedad Española de Geriatría y Gerontología (SEGG)

Sociedad Española de Investigación Ósea y Metabolismo Mineral (SEIOM)

Sociedad Española de Medicina de Familia y Comunitaria (SEMFYC)

Sociedad Española de Medicina Interna (SEMI)

Sociedad Española de Médicos de Atención Primaria (SEMERGEN)

Sociedad Española de Médicos Generales y de Familia (SEMG)

Sociedad Española de Nefrología (SEN)

Sociedad Española de Oncología Médica (SEOM)

Sociedad Española de Reumatología (SER)

\section{Documentación}

Beatriz Casal Acción. Documentalista. Unidad de Asesoramiento Científicotécnico, Avalia-t. Agencia Gallega para el Conocimiento en Salud (ACIS).

\section{Declaración de intereses}

Todos los autores, colaboradores y revisores externos de este OPBE han firmado un documento en el que se han hecho explícitas las relaciones que puedan ser origen potencial de conflictos de interés. 


\section{Resumen}

\section{Antecedentes}

En los últimos años, en los países de rentas altas, han aumentado exponencialmente tanto el número de determinaciones de vitamina D (VitD) como el número de suplementos recomendados. Esto ha sido motivado por la suposición de la existencia de un vínculo entre niveles no óptimos de vitamina D y diferentes resultados adversos en salud. Sin embargo, no está demostrado que la detección de valores no óptimos de VitD y la suplementación puedan tener efectos beneficiosos sobre la salud en población general.

\section{Introducción}

La VitD es un nutriente esencial necesario para la homeostasis ósea. Existen dos formas de VitD: Colecalciferol (VitD3) y Ergocalciferol (VitD2). La primera (VitD3) se forma principalmente en la piel después de la exposición al sol, de ahí se obtiene casi el 90 \% (1), además de la ingesta de algunos alimentos. La segunda (VitD2) se obtiene solo por la dieta y/o los suplementos alimenticios $(1,2)$. Una vez en el organismo, la VitD debe sufrir dos hidroxilaciones para tener una forma biológicamente activa. La capacidad de absorción de la piel se ve modificada a lo largo de los años y el aprovechamiento de la VitD contenida en los alimentos, depende de la capacidad de absorción del individuo alterado por distintas patologías (3).

Para la medición de niveles de VitD existe un consenso universal que defiende la medición en sangre de la forma inactiva, el 25(OH)D como marcador del nivel de VitD en el organismo $(2,4)$. Para la cuantificación existen distintos métodos como cromatográficos como HPL y LCMS-MS o inmunoquímicos como radioinmunoensayo, enzimoinmunoensayo o quimioinmunoensayo. De ellos el LCMS-MS (calibrada frente al SMR 2972) es considerada la técnica patrón de oro para la cuantificación de $25(\mathrm{OH}) \mathrm{D}$ $(3,5)$.

A día de hoy, todavía no existe un consenso en las sociedades científicas y profesionales sanitarios sobre los valores óptimos de la VitD. Esto provoca que cuando se toman valores umbrales de $25(\mathrm{OH}) \mathrm{D}$ mayores de $30 \mathrm{ng} / \mathrm{ml}$, más del $88 \%$ de la población presenta niveles bajos de VitD; sin embargo, 
cuando el umbral aplicado es de $20 \mathrm{ng} / \mathrm{ml}$, la cifra de la población con niveles bajos, disminuye al $37 \%$ y al $7 \%$ si nos referimos a valores menores de $10 \mathrm{ng} / \mathrm{ml}$. Cuando se analiza por grupos de edad, entre el 80-100\% de los mayores de 65 años presentan valores de 25(OH)D por debajo del $20 \mathrm{ng} / \mathrm{ml}$. Estos valores a nivel mundial se pueden extrapolar a España $(2,6,7)$.

En población general y especialmente en ancianos, la causa más frecuente de niveles no óptimos de VitD, es el estilo de vida sedentario y la institucionalización, que provocan falta de exposición al sol. Otros factores determinantes son la estación del año en la cual se hace la determinación, la latitud donde se vive, los factores climáticos como la nubosidad, el uso de protectores solares o la pigmentación de la piel. Además de enfermedades que provocan mala absorción o el consumo de determinados fármacos (8-11).

\section{Objetivo}

Determinar las pautas de uso adecuado de la cuantificación sérica de la VitD y su suplementación, en población general adulta sana.

\section{Metodología}

Se realizó una revisión sistemática de la literatura científica existente y se analizaron los resultados de la revisión por un grupo de expertos. Para la constitución del grupo de trabajo multidisciplinar se contactó con profesionales clínicos de diferentes especialidades relevantes para el tema, entre ellas reumatología, nefrología, medicina de familia, farmacia de atención primaria, laboratorio de análisis clínicos, medicina interna, endocrinología, rehabilitación y oncología de distintos centros sanitarios.

Fuentes de datos. La búsqueda bibliográfica se realizó de forma escalonada. La primera fase se centró en la búsqueda de guías de práctica clínica (GPC) y revisiones sistemáticas (RS), publicadas hasta mayo de 2018. Se buscó en bases de datos específicas como National Guideline Clearinghouse, Guidelines International Network (GIN), TRIPDatabase, Cochrane Library y bases de datos generales como PubMed y Embase, entre otras. Se incluyeron palabras claves como: vitamin D deficienc, adult*, general population, screening, 25-hydroxyvitamin, etc. La segunda fase consistió en una búsqueda específica para cada una de las preguntas formuladas, que se realizó en bases de datos generales (Cochrane Library, CRD Databases, Medline y Embase) utilizando, para cada una de las preguntas, palabras claves específicas. Esta búsqueda abarcó hasta 2019. 
Formulación de preguntas clínicas. Después de una lectura crítica de GPC y RS, el grupo priorizó dos de las 10 preguntas formuladas, para responder en este documento.

- Pregunta 1: ¿Cuál es la asociación entre los niveles séricos de 25(OH)D y los resultados en salud incluyendo mortalidad, cáncer, fracturas, caídas, enfermedad cardiovascular, diabetes, depresión, funcionamiento cognitivo, estado funcional, etc.?

- Pregunta 2: ¿Cuál es la efectividad de la vitamina $\mathrm{D}$, en variables duras/finales, incluyendo enfermedades cardiovasculares, obesidad, cáncer, sistema inmunitario, fractura osteoporótica, insuficiencia renal y/o la calcificación de los tejidos blandos?

El análisis y la selección de los estudios se realizó por al menos dos revisoras independientes. La evaluación del riesgo de sesgo de los estudios se realizó mediante distintos instrumentos:AGREE-II (para GPC),AMSTAR-2 (para RS), la herramienta de la Colaboración Cochrane (para ECAs), la herramienta ACROBAT-NRSI resumida (para estudios observacionales) y ROBINS-1 (estudios de intervenciones no aleatorios). La evaluación de la calidad de la evidencia se realizó siguiendo la metodología GRADE (Grade of Recomendations, Assessment, Development and Evaluation).

El grupo analizó los resultados de la revisión y realizó las recomendaciones de uso adecuado por consenso a través de discusiones realizadas presencialmente y online con los integrantes del grupo multidisciplinar. Para llegar a un consenso sobre la definición de niveles óptimos de 25(OH)D total en suero o su deficiencia se realizó un Delphi modificado que incluyó dos rondas de votación remota y una reunión de mesa redonda.

Además, el grupo identificó indicadores de evaluación realizados también por consenso del grupo. El borrador del documento pasó por una revisión externa de expertos propuestos por las principales sociedades científicas.

\section{Resultados de la revisión de la literatura científica}

En la búsqueda general de revisiones y guías se localizó un documento realizado por la Task Force (4) y publicado en 2014 que se adoptó para la contestación de las dos preguntas como punto de partida. 
Pregunta 1: ¿Cuál es la asociación entre los niveles séricos de 25(OH)D y los resultados en salud incluyendo mortalidad, cáncer, fracturas, caídas, enfermedad cardiovascular, diabetes, depresión, funcionamiento cognitivo, estado funcional, etc.?

La Task Force (4) incluía en esta pregunta estudios prospectivos de cohortes, caso-control anidados o revisiones sistemáticas que examinaban la asociación entre los valores de 25(OH)D y los resultados en salud. La actualización de este documento para la pregunta 1 identificó 862 referencias, de las cuales se incluyeron 40 , más 3 procedentes de la búsqueda manual.

Los estudios localizados presentaron calidad media/baja, dado que eran todos caso-control o cohortes con alto riesgo de sesgo. También se localizaron algunas RS, pero solo incluían estudios de caso-control o cohorte, también con alto nivel de sesgo. Este tipo de diseños no se vale para establecer una relación causal entre el nivel de VitD y la patología encontrada.

De forma general, la evidencia encontrada muestra que valores bajos de $25(\mathrm{OH}) \mathrm{D}$ en suero no reflejan asociación con resultados adversos de salud en población general, a nivel de enfermedades cardiovasculares, cáncer en distintas localizaciones (próstata, mama, ovario, páncreas, otros), vértigo posicional paroxístico benigno, enfermedades del sistema circulatorio, enfermedades oculares (oclusión de la vena retiniana central, el glaucoma primario de ángulo abierto o la degeneración macular tardía asociada a la edad) o alergias, entre otras. En otras patologías no se ha podido establecer una conclusión clara debido a los resultados contradictorios de los estudios o a las limitaciones metodológicas de estos, no pudiendo establecer relaciones causales, como en las caídas, deterioro cognitivo, depresión, o dismenorrea, entre otras.

A continuación, se muestran el resumen elaborado por el grupo de trabajo en base a la evidencia encontrada en la relación de la VitD con distintos riesgos.

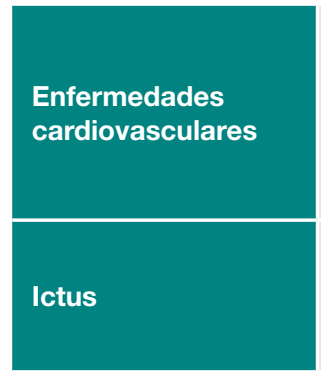

En población general, los resultados son discordantes en los estudios incluidos en la revisión de partida. Sin embargo, las publicaciones posteriores presentan la misma orientación, no encontrando asociación significativa entre los valores bajos de $25(\mathrm{OH}) \mathrm{D}$ y los riesgos cardiovasculares. (Calidad de la evidencia MUY BAJA) (4, 12-14).

En población general, los estudios de la revisión de partida y los posteriores van todos en la misma línea, apuntando a que valores bajos de 25(OH)D se asocian con aumento de riesgo de ictus. (Calidad de la evidencia MUY BAJA) $(4,15)$. 


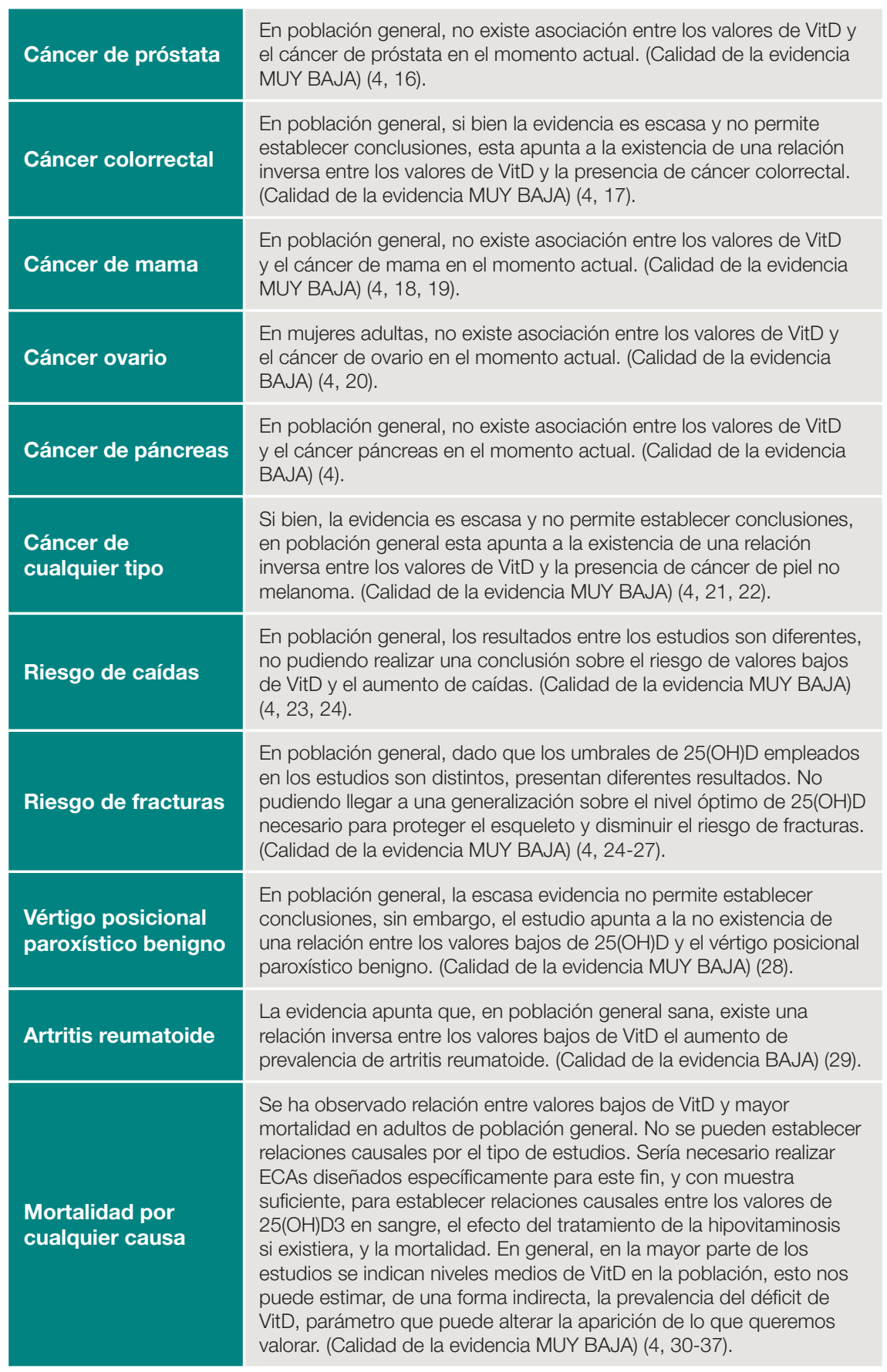




\begin{tabular}{|c|c|}
\hline Sistema circulatorio & $\begin{array}{l}\text { En población general, los estudios disponibles analizados no permiten } \\
\text { establecer una relación entre los valores de VitD y la incidencia de } \\
\text { enfermedad tromboembólica. (Calidad de la evidencia BAJA) }(38,39) \text {. }\end{array}$ \\
\hline $\begin{array}{l}\text { Riesgo de deterioro } \\
\text { cognitivo }\end{array}$ & $\begin{array}{l}\text { En población general, los resultados de los estudios presentan } \\
\text { diferencias entre ellos. Estudios que muestran asociación entre los } \\
\text { valores bajos de } 25(\mathrm{OH}) \mathrm{D} \text { y aumento de riesgo de déficit cognitivo, } \\
\text { y estudios que no encuentran ninguna asociación. (Calidad de la } \\
\text { evidencia BAJA) }(4,40-43) \text {. }\end{array}$ \\
\hline $\begin{array}{l}\text { Enfermedades } \\
\text { oculares }\end{array}$ & $\begin{array}{l}\text { En población general, no se puede establecer una asociación causal } \\
\text { entre el bajo nivel de } 25(\mathrm{OH}) \mathrm{D} \text { y la oclusión de la vena central de la } \\
\text { retina (OVRC) ni con el glaucoma primario de ángulo abierto. Por } \\
\text { otro lado, las concentraciones de } 25(\mathrm{OH}) \mathrm{D} \text { por debajo de } 50 \mathrm{nmol} / \mathrm{L} \\
\text { se podrían asociar con la degeneración macular tardía asociada a la } \\
\text { edad. (Calidad de la evidencia MUY BAJA) (44-46). }\end{array}$ \\
\hline Enfermedad renal & $\begin{array}{l}\text { Al margen de la asociación entre valores de vitamina D, } \\
\text { hiperparatiroidismo y proteinuria, en población general, la evidencia de } \\
\text { que el déficit de vitamina D contribuya significativamente al desarrollo } \\
\text { de enfermedad renal es escasa para establecer conclusiones. No } \\
\text { obstante, esta apunta a la existencia de una relación inversa entre } \\
\text { niveles de vitamina D y enfermedad renal. (Calidad de la evidencia } \\
\text { MUY BAJA) }(47,48) \text {. }\end{array}$ \\
\hline $\begin{array}{l}\text { Riesgo de } \\
\text { depresión }\end{array}$ & $\begin{array}{l}\text { En población general, los estudios muestran diferentes resultados que } \\
\text { no permiten establecer una conclusión general sobre el problema. } \\
\text { (Calidad de la evidencia BAJA) }(4,49) \text {. }\end{array}$ \\
\hline Tuberculosis & $\begin{array}{l}\text { La VitD tiene efectos importantes en las células del sistema inmune. } \\
\text { Los macrófagos y los linfocitos T y B expresan el receptor de la VitD. } \\
\text { En población general, hay pocos estudios sobre valores plasmáticos } \\
\text { de VitD y la posible asociación a tuberculosis. (Calidad de la evidencia } \\
\text { MUY BAJA) (50). }\end{array}$ \\
\hline Dismenorrea & $\begin{array}{l}\text { En mujeres sanas, aunque la evidencia es escasa y no permite } \\
\text { establecer conclusiones, esta apunta a la existencia de una relación } \\
\text { inversa entre los valores bajos de VitD y la dismenorrea primaria. } \\
\text { (Calidad de la evidencia MUY BAJA) (51). }\end{array}$ \\
\hline Riesgo de alergias & $\begin{array}{l}\text { En población general, no se puede establecer una asociación causal } \\
\text { entre el nivel 25(OH)D y la rinitis alérgica. (Calidad de la evidencia MUY } \\
\text { BAJA) (52). }\end{array}$ \\
\hline $\begin{array}{l}\text { Enfermedad de } \\
\text { Crohn }\end{array}$ & $\begin{array}{l}\text { La revisión sistemática concluye que los resultados sugieren una } \\
\text { asociación entre los valores bajos de VitD y la enfermedad de Crohn. } \\
\text { Sin embargo, hay que tener en cuenta que la alimentación de sujetos } \\
\text { con enfermedad de Crohn es deficiente, restrictiva y selectiva lo que } \\
\text { no permite establecer una relación causal. (Calidad de la evidencia } \\
\text { MODERADA) (53). }\end{array}$ \\
\hline $\begin{array}{l}\text { Fragilidad en el } \\
\text { adulto mayor }\end{array}$ & $\begin{array}{l}\text { En población general sana, los estudios observacionales sugieren una } \\
\text { asociación entre estatus deficiente de VitD y la debilidad muscular en } \\
\text { personas mayores. (Calidad de la evidencia BAJA) (54). }\end{array}$ \\
\hline
\end{tabular}


Pregunta 2: ¿Cuál es la efectividad de la vitamina $D$, en variables duras/ finales, incluyendo enfermedades cardiovasculares, obesidad, cáncer, sistema inmunitario, fractura osteoporótica, insuficiencia renal y/o la calcificación de los tejidos blandos?

Para esta pregunta la Task Force (4) incluía ensayos clínicos aleatorizados (ECAs) que examinaban la efectividad de la VitD en resultados de salud. La actualización del documento de la Task Force (4) para esta pregunta identificó 1739 referencias, de las cuales se incluyeron 66 (55 ECAs y 11 RS).

La evidencia encontrada sobre los suplementos de VitD en población general asintomática tanto con déficit o nivel bajo de VitD como sin déficit de VitD, no muestra beneficios de la suplementación en la incidencia del cáncer, enfermedades cardiovasculares, riesgo de fracturas, alivio del dolor, riesgo de infecciones o procesos inflamatorios, entre otras.

La revisión también aportó resultados contradictorios o insuficientes sobre el beneficio de la suplementación en la deficiencia de VitD asintomática en otros resultados de salud como la función pulmonar, la función cognitiva o la función tiroidea.

A continuación, se muestran el resumen elaborado por el grupo de trabajo en base a la evidencia encontrada en la relación de la VitD con distintas patologías.

\begin{tabular}{|c|c|}
\hline Riesgo de cáncer & $\begin{array}{l}\text { En población general, los estudios, de forma general, no } \\
\text { mostraron que exista una menor incidencia de cáncer al } \\
\text { administrar suplementos de VitD. (Calidad de la evidencia ALTA) } \\
(4,55-58) \text {. }\end{array}$ \\
\hline $\begin{array}{l}\text { Enfermedad } \\
\text { cardiovascular }\end{array}$ & $\begin{array}{l}\text { En población general, los resultados obtenidos en los estudios } \\
\text { no avalan la indicación de tratamiento con VitD para modificar } \\
\text { la incidencia de enfermedades cardiovasculares en población } \\
\text { general. (Calidad de la evidencia MUY BAJA) }(4,58-69) \text {. }\end{array}$ \\
\hline Fracturas y caídas & $\begin{array}{l}\text { Las publicaciones revelaron que los suplementos de VitD no } \\
\text { muestran una reducción significativa en el riesgo de caídas o } \\
\text { fracturas en la población general adulta. (Calidad de la evidencia } \\
\text { ALTA) (4, 70-73). } \\
\text { Evidencia Complementaria: } \\
\text { - Metabolismo óseo (variable subrogada) } \\
\text { Con los datos actuales los suplementos de VitD en mujeres } \\
\text { mayores de } 50 \text { años no modifican los marcadores de } \\
\text { remodelado óseo. (Calidad de la evidencia MODERADA) (4, } \\
74-77) \text {. }\end{array}$ \\
\hline
\end{tabular}




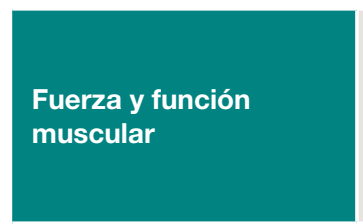

Mortalidad

Dolor

Infecciones

Procesos inflamatorios

\section{Niveles de \\ testosterona en \\ varones y en el \\ antígeno prostático \\ específico}

\section{Tromboembolismo} venoso

Función pulmonar

Función renal

Función cognitiva

Función tiroidea

Diabetes tipo 2
Existe una gran heterogeneidad en los estudios. Aunque la evidencia es mixta, en general, sugiere una falta de efecto de la suplementación de VitD sobre la función y fuerza muscular, tanto en sujetos con déficit de VitD como sin él. El mayor número de estudios se han realizado en mujeres postmenopáusicas. (Calidad de la evidencia BAJA) (4, 78-85).

No hay evidencias que sostengan que la administración de VitD disminuya la mortalidad en la población general. (Calidad de la evidencia MODERADA) $(4,86)$.

Hasta el momento no existe un beneficio claro sobre la suplementación de VitD en el dolor crónico en adultos. (Calidad de la evidencia ALTA) $(87,88)$.

Las últimas publicaciones de mayor calidad muestran que no existe evidencia suficiente para respaldar la administración de VitD para reducción en el riesgo de infecciones del tracto superior en población general. (Calidad de la evidencia MODERADA) (89-94).

En población general sana, los estudios muestran que los suplementos de VitD no modifican los valores de biomarcadores inflamatorios. (Calidad de la evidencia MUY BAJA) (95-98).

El pequeño número de ECAs localizados no ha demostrado un efecto de los suplementos de VitD sobre la concentración de testosterona o el nivel de PSA en varones. (Calidad de la evidencia MUY BAJA) $(82,99,100)$.

El ECA localizado no encuentra un efecto de los suplementos de VitD sobre la reducción de tromboembolismo venoso en mujeres postmenopáusicas. (Calidad de la evidencia MUY BAJA) (101).

Hasta el momento, en población general sana, no existe evidencia suficiente para extraer conclusiones. (Calidad de la evidencia MUY BAJA) $(67,102)$.

Hasta el momento, no existe evidencia suficiente para extraer conclusiones. Al margen de su relación con el metabolismo mineral, los resultados muestran en general que no existe relación entre el suplemento de VitD y la función renal en población general sana. (Calidad de la evidencia MUY BAJA) (103-105).

En población general sana, los estudios muestran resultados mixtos. Hasta el momento con el bajo número de estudios y el pequeño seguimiento no se puede realizar una conclusión general. (Calidad de la evidencia MUY BAJA) (106-108).

No existe evidencia suficiente para extraer conclusiones. Calidad de la evidencia MUY BAJA) (109).

En población general, los resultados son mixtos. Con los estudios disponibles no se puede realizar una conclusión general. (Calidad de la evidencia MODERADA) (4, 110-120).

En población general sana, no existen datos suficientes para establecer la relación entre los suplementos de VitD y la mejora en la calidad de vida. Los estudios se restringen a mujeres postmenopáusicas. (Calidad de la evidencia BAJA) $(121,122)$. 


\section{Recomendaciones}

A partir de la evidencia revisada, el grupo de trabajo ha emitido las siguientes recomendaciones relativas al uso adecuado de la determinación de niveles de VitD y a la indicación de suplementación en población general sana.

\section{Pregunta 1: Sobre cuantificación de VitD en población general sana}

- En adultos sanos asintomáticos y sin factores de riesgo para el déficit de VitD no es necesaria la determinación de valores de VitD. A efectos de esta recomendación, se consideran tanto los factores de riesgo extrínsecos (por ejemplo, ingesta inadecuada o hiperpigmentación cutánea); como intrínsecos (por ejemplo, la edad avanzada o la malabsorción: gastrectomía, enfermedades intestinales o cirrosis biliar primaria).

Pregunta 2: Sobre suplementación con VitD en población general sana

- En adultos sanos asintomáticos la suplementación farmacológica, hasta el momento, no ha demostrado beneficios, independientemente de los niveles de VitD.

- Podría considerarse la suplementación en población institucionalizada mayor de 70 años, sin necesidad de determinación.

\section{Indicadores}

Por parte del grupo elaborador se han propuesto tres indicadores, uno de estructura y dos de procesos. EL indicador de estructura trata de conocer qué número de laboratorios presentan protocolos actualizados para la petición de determinación de VitD. Los indicadores de proceso tratan por un lado de determinar el número de determinaciones solicitadas de VitD y por el otro el gasto farmacéutico en suplementos con VitD en población general sana. 



\section{Abstract}

\section{Background}

In recent years, in high-income countries, vitamin $\mathrm{D}$ screenings and supplementation have increased exponentially. This has been motivated by the assumption that suboptimal vitamin D (VitD) levels are associated to various adverse health outcomes. However, important uncertainties exist regarding the benefits of screening for suboptimal VitD values and supplementation in the general population.

\section{Introduction}

VitD is an essential nutrient necessary for bone homeostasis. There are two forms of VitD: Cholecalciferol (VitD3) and Ergocalciferol (VitD2). The first (VitD3) is mainly generated in the skin after exposure to the sun, from which almost $90 \%$ is obtained (1); remaining VitD3 is obtained from food sources. The second (VitD2) is obtained only through diet and/or food supplements $(1,2)$. Once in the body, VitD must undergo two hydroxylations to obtain a biologically active form. The absorption capacity of the skin is modified over the years and the use of VitD contained in food depends on the absorption capacity of the individual, which is altered by various pathologies (3).

There is a general consensus that the inactive form, 25(OH)D, should be measured in blood as a marker of the VitD levels in the body $(2,4)$. For quantification there are different methods such as chromatographic methods like HPL and LCMS-MS or immunochemical methods like radioimmunoassay, enzyme immunoassay or chemoimmunoassay. Of these, LCMS-MS (calibrated against SMR 2972) is considered the gold standard technique for the quantification of $25(\mathrm{OH}) \mathrm{D}(3,5)$.

However, to date, there is no consensus among scientific societies and health professionals on optimal VitD values. The use of different cut-off values are concerning because they can lead to very different percentages of people been considered to have low VitD levels and being supplemented with VitD. Using threshold values of 25(OH)D greater than $30 \mathrm{ng} / \mathrm{ml}$, more than $88 \%$ of the population would be valued to have low levels of VitD; however, when the threshold applied is $20 \mathrm{ng} / \mathrm{ml}$, this number decreases to $37 \%$, and to $7 \%$ for values lower than $10 \mathrm{ng} / \mathrm{ml}$. When analysed by age 
group, between $80-100 \%$ of people over 65 years of age have $25(\mathrm{OH}) \mathrm{D}$ values below $20 \mathrm{ng} / \mathrm{ml}$. These worldwide values can be extrapolated to Spain $(2,6,7)$.

In the general population and especially in the elderly, the most frequent cause of suboptimal VitD levels is a sedentary lifestyle and institutionalisation, which lead to a lack of sun exposure. Other determining factors are the season of the year in which the assessment is made, the latitude where one lives, climatic factors such as cloudiness, the use of sunscreens or skin pigmentation, and diseases that cause malabsorption or the consumption of certain medicines (8-11).

\section{Aim}

To develop guidelines for the appropriate use of serum VitD quantification and supplementation in the general healthy adult population.

\section{Methods}

A systematic review (SR) of the existing scientific literature was carried out and the results of the review were analysed by a multidisciplinary group of experts. This expert working group was composed by clinical professionals from different specialties relevant to the topic, including rheumatology, nephrology, family medicine, primary care pharmacy, clinical analysis laboratory, internal medicine, endocrinology, rehabilitation and oncology from different health centres. This expert group was responsible for formulating and prioritizing the research questions approached in the guideline.

Data sources. The literature search was conducted in a stepwise manner. The first phase focused on the search for clinical practice guidelines (CPG) and SR, published up to May 2018. We searched specific databases such as the National Guideline Clearinghouse, Guidelines International Network (GIN), TRIPDatabase, Cochrane Library and general databases such as PubMed and Embase, among others. Key words included were: vitamin D deficiency, adult*, general population, screening, 25-hydroxyvitamin, etc. The second phase consisted of a specific search for each of the research questions, which was carried out in general databases (Cochrane Library, CRD Databases, Medline and Embase) using specific keywords for each of the questions. This search covered the period up to 2019. 
Selection of key research questions. After a critical reading of CPG and SR, the group prioritised two of the 10 questions initially formulated, to answer in this document.

- Question 1: What is the association between serum 25(OH)D levels and health outcomes including mortality, cancer, fractures, falls, cardiovascular disease, diabetes, depression, cognitive functioning, functional status, etc.?

- Question 2: What is the effectiveness of vitamin D on hard/end variables including cardiovascular disease, obesity, cancer, immune system, osteoporotic fracture, renal failure and/or soft tissue calcification?

The analysis and selection of studies was performed by at least two independent reviewers. Risk of bias assessment of the studies was performed using different instruments: AGREE-II (for CPGs), AMSTAR-2 (for SRs), the Cochrane Collaboration tool (for RCTs) and ROBINS-1 (studies of non-randomised interventions). The quality of the evidence was assessed using the GRADE (Grade of Recommendations, Assessment, Development and Evaluation) methodology.

The multidisciplinary expert group analysed the results of the review and achieved consensus recommendations through face-to-face and online discussions. To reach consensus on the definition of optimal serum total 25(OH)D levels or deficiency, a modified Delphi was conducted including two rounds of remote voting and a roundtable meeting.

In addition, the group developed evaluation indicators that were also established consensus. The draft document underwent an external review by experts proposed by the main scientific societies.

\section{Results of the review of the scientific literature}

The general search for reviews and guidelines identified a SR published in 2014 by the Task Force (4), which was adopted to answer the two questions as a starting point. 
Question 1: What is the association between serum levels of 25(OH)D and health outcomes including mortality, cancer, fractures, falls, cardiovascular disease, diabetes, depression, cognitive functioning, functional status, etc.?

The Task Force (4) included prospective cohort, nested case-control or systematic review studies examining the association between $25(\mathrm{OH}) \mathrm{D}$ values and health outcomes. The update of this paper for question 1 identified 862 references, of which 40 were included, plus 3 from the manual search.

The studies located were of medium/low quality, as they were all case-control or cohort studies with a high risk of bias. Some SR were also located, but they only included case-control or cohort studies, also with a high level of bias. This type of design is not suitable for establishing a causal relationship between the level of VitD and the pathology found.

In general, the evidence found shows no association between low serum $25(\mathrm{OH}) \mathrm{D}$ values and risk of adverse health outcomes in the general population, including cardiovascular diseases, cancer in different locations (prostate, breast, ovarian, pancreatic, others), benign paroxysmal positional vertigo, circulatory system diseases, eye diseases (central retinal vein occlusion, primary open angle glaucoma or late age-related macular degeneration, etc.) and other diseases (e.g., central retinal vein occlusion, primary open angle glaucoma or late age-related macular degeneration, etc.) or allergies, among others. In other pathologies, it has not been possible to establish a clear conclusion due to the contradictory results of the studies or their methodological limitations, and causal relationships cannot be established, such as in falls, cognitive impairment, depression, or dysmenorrhoea, among others.

The following is a summary of the evidence-based working group's findings on the relationship between VitD and various risks.

\begin{tabular}{l|l}
$\begin{array}{l}\text { The results of the studies included in the initial review were inconsistent } \\
\text { Cardiovascular } \\
\text { diseases }\end{array}$ & $\begin{array}{l}\text { regarding the risk in the general population. Subsequent publications } \\
\text { present similar findings, showing no significant association between low } \\
25(\mathrm{OH}) \mathrm{D} \text { values and cardiovascular risks. (VERY LOW quality of evidence) } \\
(4,12-14) .\end{array}$ \\
\hline Stroke & $\begin{array}{l}\text { Both baseline and subsequent studies suggested that low 25(OH)D values } \\
\text { are associated with an increased risk of stroke in general population. (VERY } \\
\text { LOW quality of evidence) (4, 15). }\end{array}$ \\
\hline Prostate cancer & $\begin{array}{l}\text { No association between VitD values and prostate cancer has been found at } \\
\text { the present time in the general population (VERY LOW quality of evidence) } \\
\text { (4, 16). }\end{array}$
\end{tabular}




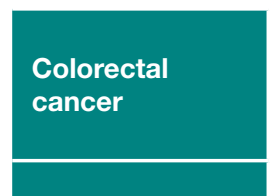

Breast cancer

Ovarian cancer

Pancreatic

cancer

(n)

Any type of

cancer

the

Risk of falls

Risk of

fractures

\begin{tabular}{l} 
Benign \\
paroxysmal \\
positional \\
vertigo \\
\hline
\end{tabular}

Rheumatoid

arthritis

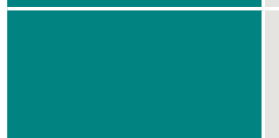

All-cause

mortality



Circulatory

system

Risk of

cognitive

impairment
Although the evidence is scarce and does not allow for conclusions to be drawn, it points to an inverse relationship between VitD values and the presence of colorectal cancer in the general population. (VERY LOW quality of evidence) $(4,17)$.

In the general population, no association has been found between VitD values and breast cancer at the present time. (VERY LOW quality of evidence.) $(4,18,19)$.

In adult women, no association has been found between VitD values and ovarian cancer at the present time. (LOW quality of evidence) $(4,20)$.

In the general population, no association has been found between VitD values and pancreatic cancer at the present time. (LOW quality of evidence) (4).

Although the evidence is scarce and does not allow conclusions to be drawn regarding the general population, it points to the existence of an inverse relationship between VitD values and the presence of nonmelanoma skin cancer. (VERY LOW quality of evidence) $(4,21,22)$.

In the general population, results between the studies are inconsistent, and no conclusion can be drawn about the potential risk of low VitD values and increased falls. (VERY LOW quality of evidence.) $(4,23,24)$.

In the general population, it is not possible to generalise about the optimum level of 25(OH)D needed to protect the skeleton and reduce the risk of fractures given the different results attending to the $25(\mathrm{OH})$ thresholds used in included studies. (VERY LOW quality of evidence) $(4,24-27)$.

Although the evidence is scarce and does not allow for conclusions to be drawn regarding the risk in the general population, it points to the non-existence of a relationship between low 25(OH)D values and benign paroxysmal positional vertigo. (VERY LOW quality of evidence) (28).

The evidence suggests that, in a healthy general population, there is an inverse relationship between low VitD values and increased prevalence of rheumatoid arthritis. (LOW quality of Evidence) (29).

A relationship has been observed between low VitD values and increased mortality in adults in the general population. Causal relationships cannot be established because of the low quality of the evidence. RCTs specifically designed for this purpose, and with a sufficient sample size, would be necessary to establish causal relationships between blood 25(OH)D3 values, the effect of treatment of hypovitaminosis, if any, and mortality. In general, most studies indicate average VitD levels in the population, which can indirectly estimate the prevalence of VitD deficiency, a parameter that may alter the occurrence of what we want to assess (VERY LOW quality of evidence) (4, 30-37).

In the general population, the available studies reviewed do not allow for establishing a relationship between VitD values and the incidence of thromboembolic disease. (LOW quality of evidence) $(38,39)$.

In the general population, results are inconsistent. Studies showing an association between low 25(OH)D values and increased risk of cognitive deficits, and studies finding no association have been found. (LOW quality of evidence) (4, 40-43). 


\begin{tabular}{|c|c|}
\hline Eye diseases & $\begin{array}{l}\text { In the general population, a causal association cannot be established } \\
\text { between low } 25(\mathrm{OH}) \mathrm{D} \text { and central retinal vein occlusion (CRVO) and } \\
\text { primary open-angle glaucoma. Furthermore, 25(OH)D concentrations below } \\
50 \text { nmol/L could be associated with late age-related macular degeneration. } \\
\text { (VERY LOW Quality of Evidence) (44-46). }\end{array}$ \\
\hline Kidney disease & $\begin{array}{l}\text { Apart from the association between vitamin D levels, hyperparathyroidism } \\
\text { and proteinuria in the general population, the evidence that vitamin D } \\
\text { deficiency contributes significantly to the development of kidney disease is } \\
\text { too weak to draw any conclusions. However, it does point to the existence } \\
\text { of an inverse relationship between vitamin D levels and kidney disease. } \\
\text { (VERY LOW quality of evidence) }(47,48) \text {. }\end{array}$ \\
\hline $\begin{array}{l}\text { Risk of } \\
\text { depression }\end{array}$ & $\begin{array}{l}\text { Studies in general population show different results, making it impossible } \\
\text { to arrive at a general conclusion on the problem. (LOW quality of evidence) } \\
(4,49) \text {. }\end{array}$ \\
\hline Tuberculosis & $\begin{array}{l}\text { VitD is noted to have important effects on cells of the immune system. } \\
\text { Macrophages, T and B lymphocytes express the VitD receptor. In the } \\
\text { general population, there are few studies on plasma VitD values and the } \\
\text { possible association with tuberculosis. (VERY LOW quality of evidence) (50). }\end{array}$ \\
\hline Dysmenorrhoea & $\begin{array}{l}\text { In healthy women, although the evidence is scarce and does not allow } \\
\text { conclusions to be drawn, it points to the existence of an inverse relationship } \\
\text { between low VitD values and primary dysmenorrhoea (VERY LOW quality of } \\
\text { evidence) (51). }\end{array}$ \\
\hline Risk of allergies & $\begin{array}{l}\text { In the general population, a causal association between } 25(\mathrm{OH}) \mathrm{D} \text { level and } \\
\text { allergic rhinitis cannot be established. (VERY LOW quality of evidence) (52). }\end{array}$ \\
\hline Crohn's disease & $\begin{array}{l}\text { The systematic review concludes that the results suggest an association } \\
\text { between low VitD values and Crohn's disease. However, it must be taken } \\
\text { into account that the diet of subjects with Crohn's disease is deficient, } \\
\text { restrictive and selective, which does not allow a causal relationship to be } \\
\text { established. (MODERATE quality of evidence) (53). }\end{array}$ \\
\hline $\begin{array}{l}\text { Frailty in the } \\
\text { elderly }\end{array}$ & $\begin{array}{l}\text { In the general healthy population, observational studies suggest an } \\
\text { association between deficient VitD status and muscle weakness in older } \\
\text { people. (LOW quality of evidence) (54). }\end{array}$ \\
\hline
\end{tabular}

\section{Question 2: What is the effectiveness of vitamin D on hard/end variables including cardiovascular disease, obesity, cancer, immune system, osteoporotic fracture, renal failure and/or soft tissue calcification?}

For this question the Task Force (4) included randomised clinical trials (RCTs) examining the effectiveness of VitD on health outcomes. The Task Force (4) document update for this question identified 1739 references, of which 66 (55 RCTs and 11 SRs) were included).

The evidence found on VitD supplementation in the general asymptomatic population, both VitD deficient or low VitD deficient and non VitD deficient, shows no benefit of supplementation on cancer 
incidence, cardiovascular disease, fracture risk, pain relief, risk of infections or inflammatory processes, among others.

The review also provided conflicting or insufficient results on the benefit of supplementation in asymptomatic VitD deficiency on other health outcomes such as lung function, cognitive function or thyroid function.

The following is a summary drawn up by the working group based on the evidence found on the relationship between VitD and different pathologies.

\begin{tabular}{|c|c|}
\hline Risk of cancer & $\begin{array}{l}\text { In the general population, the studies generally did not show a } \\
\text { lower incidence of cancer with VitD supplementation. (HIGH quality } \\
\text { of evidence) }(4,55-58) \text {. }\end{array}$ \\
\hline $\begin{array}{l}\text { Cardiovascular } \\
\text { disease }\end{array}$ & $\begin{array}{l}\text { In the general population, the results obtained in the studies do } \\
\text { not support the indication of treatment with VitD to modify the } \\
\text { incidence of cardiovascular disease in the general population. } \\
\text { (VERY LOW quality of evidence) }(4,58-69) \text {. }\end{array}$ \\
\hline Fractures and falls & $\begin{array}{l}\text { The literature found that VitD supplementation does not show a } \\
\text { significant reduction in the risk of falls or fractures in the general } \\
\text { adult population. (HIGH quality of evidence) }(4,70-73) \text {. } \\
\text { Complementary evidence: } \\
\text { - Bone mineral density (surrogate variable): } \\
\text { Based on current data, VitD supplementation in women over } \\
50 \text { years of age does not modify bone remodelling markers. } \\
\text { (MODERATE quality of evidence) }(4,74-77) \text {. }\end{array}$ \\
\hline $\begin{array}{l}\text { Strength and muscle } \\
\text { function }\end{array}$ & $\begin{array}{l}\text { The studies vary significantly. Although the evidence is mixed, in } \\
\text { general, it suggests a lack of effect of VitD supplementation on } \\
\text { muscle function and strength in both VitD-deficient and non- } \\
\text { VitD-deficient subjects. The largest number of studies have been } \\
\text { conducted with postmenopausal women. (LOW quality of evidence) } \\
(4,78-85) \text {. }\end{array}$ \\
\hline Mortality & $\begin{array}{l}\text { There is no evidence to support that VitD administration decreases } \\
\text { mortality in the general population. (MODERATE quality of evidence) } \\
(4,86) \text {. }\end{array}$ \\
\hline Pain & $\begin{array}{l}\text { So far there is no clear benefit of VitD supplementation in chronic } \\
\text { pain in adults. (HIGH quality of evidence) }(87,88) \text {. }\end{array}$ \\
\hline Infections & $\begin{array}{l}\text { The latest and highest quality publications show that there is } \\
\text { insufficient evidence to support the administration of VitD for } \\
\text { reduction in the risk of upper tract infections in the general } \\
\text { population. (MODERATE quality of evidence) (89-94). }\end{array}$ \\
\hline $\begin{array}{l}\text { Inflammatory } \\
\text { processes }\end{array}$ & $\begin{array}{l}\text { In a healthy general population, studies show that VitD } \\
\text { supplementation does not change inflammatory biomarker values. } \\
\text { (VERY LOW quality of evidence) (95-98). }\end{array}$ \\
\hline
\end{tabular}


Male testosterone

levels and prostate

specific antigen (PSA)

levels

Venous

thromboembolism

Pulmonary function

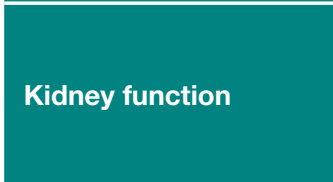

Cognitive function

Thyroid function

Type 2 diabetes

Quality of life
The small number of localised RCTs have not demonstrated an effect of VitD supplementation on testosterone concentration or PSA level in men. (VERY LOW quality of evidence) $(82,99,100)$.

Localised RCT finds no effect of VitD supplementation on the reduction of venous thromboembolism in postmenopausal women. (VERY LOW quality of evidence) (101).

So far, in a healthy general population, there is insufficient evidence to draw any conclusions. (VERY LOW quality of evidence.) (67, 102).

So far, there is insufficient evidence to draw conclusions. Apart from its relationship with mineral metabolism, the results show in general that there is no relationship between VitD supplementation and kidney function in a healthy general population. (VERY LOW quality of evidence) (103-105).

In the general healthy population, studies show mixed results. So far, due to the low number of studies and the small follow-up, no general conclusion can be drawn. (VERY LOW quality of evidence) (106-108).

There is insufficient evidence to draw conclusions. (VERY LOW quality of evidence) (109).

In the general population, the results are mixed. No overall conclusion can be drawn from the available studies. (MODERATE quality of evidence) $(4,110-120)$.

In the general healthy population, there are insufficient data to establish the relationship between VitD supplementation and improved quality of life. Studies are restricted to postmenopausal women (LOW quality of evidence) $(121,122)$.

\section{Recommendations}

Based on the evidence reviewed, the working group has issued the following recommendations regarding the appropriate use of VitD level determination and indication for supplementation in a healthy general population.

\section{Question 1: On VitD quantification in a healthy general population}

- In asymptomatic healthy adults without risk factors for VitD deficiency, determination of VitD values is not necessary. For the purpose of this recommendation, both extrinsic risk factors (e.g. inadequate intake or skin hyperpigmentation) and intrinsic risk factors (e.g. advanced age or malabsorption: gastrectomy, intestinal diseases or primary biliary cirrhosis) are considered. 
Question 2: On VitD supplementation in a healthy general population

- In asymptomatic healthy adults, pharmacological supplementation has so far shown no benefit, irrespective of VitD levels.

- Supplementation could be considered in the institutionalised population over 70 years of age, without the need for determination.

\section{Indicators}

Three indicators have been proposed by the development group, one structure and two process indicators. The structure indicator aims to determine the number of laboratories with updated protocols for VitD determination requests. The process indicators aim, on the one hand, to determine the number of VitD determinations requested and, on the other hand, the pharmaceutical expenditure on VitD supplements in the general healthy population. 



\section{Siglas y acrónimos}

ACROBAT-NRSI: A Cochrane Risk of Bias Assessment Tool for Non-Randomized Studies of Interventions

AHRQ: Agency for Healthcare Research and Quality

AMSTAR: Assessing the Methodological Quality of Systematic Reviews

CCAA: Comunidades Autónomas

CIE-10: Clasificación Internacional de Enfermedades

CRD: Centre for Reviews and Dissemination

DMAE: Degeneración Macular Asociada a la Edad

ECAs: Ensayos Clínicos Aleatorios

EE. UU.: Estados Unidos

GPC: Guía de práctica clínica

GRADE: Grade of Recomendations, Assessment, Development and Evaluation

GPAA: Glaucoma primario de ángulo abierto

IARC: International Agency for Research on Cancer

IMC: Índice de masa corporal

IHE: Institute of Health Economics

IOM: Institute of Medicine

IOF: International Osteoporosis Foundation

MeSH: Medical Subject Headings

OPBE: Otros productos basados en la evidencia

PICO: Patient, Intervention, Comparator, Outcome

PTH: Hormona paratiroidea

RS: Revisión sistemática

ROBINS I: Risk of bias tools

SEIOMM: Sociedad Española de Investigación Ósea y del Metabolismo Mineral

SNS: Sistema Nacional de Salud

TVP: Tromboembolismo venoso o pulmonar

USPSTF: US Preventive Service Task Force 



\section{Glosario de términos}

1a-hidroxicolecalciferol: análogo sintético de 1,25-dihidroxivitamina D.

1,25-dihidroxivitamina D (1,25 (OH)2D): principal metabolito activo de la vitamina $\mathrm{D}$ en el cuerpo. Se produce en el riñón a partir de 25-hidroxivitamina D.

25-hidroxivitamina D (25(OH)D): metabolito de la vitamina D producido en el hígado a partir de la vitamina D. Circula en la sangre y es un marcador de exposición a la vitamina $\mathrm{D}$, lo que refleja el suministro de vitamina D de la síntesis cutánea y la dieta.

Alfacalcidol: otro nombre para 1a-hidroxicolecalciferol.

Biomarcador: indicador bioquímico, fisiológico u otro que se mide objetivamente para evaluar los efectos de los procesos biológicos normales, el progreso de una enfermedad, condición o la respuesta a una intervención.

Calcidiol: otro nombre para 25-hidroxivitamina D.

Calcifediol: otro nombre para 25-hidroxivitamina D.

Calcitonina: una hormona peptídica, producida por la glándula tiroides, implicada en la regulación de la homeostasis del calcio.

Calcitriol: otro nombre para 1,25-dihidroxivitamina D.

Colecalciferol: otro nombre para la vitamina D3.

Densidad mineral ósea (DMO): es una unidad esquelética $\left(\mathrm{g} / \mathrm{cm}^{3}\right)$. Cuando se mide mediante técnicas de rayos $\mathrm{X}$ de energía simple o doble, representa la masa de mineral óseo medida dentro de un área escaneada $\left(\mathrm{g} / \mathrm{cm}^{2}\right)$ y no es una medición de densidad real.

Ergocalciferol: otro nombre para la vitamina D2.

Factor de crecimiento de fibroblastos 23 (FGF23): es una proteína sintetizada en el osteocito que inhibe la reabsorción proximal de fósforo $(\mathrm{P})$, promueve la disminución de los niveles de 1,25 (OH)2 VitD3 (calcitriol) al inhibir la $a-1$ hidroxilasa renal y bloquea la síntesis/liberación de paratohormona (PTH) intacta (PTHi) 2. 
Fenotipo: características físicas y/o bioquímicas observables de la expresión de un gen en un conjunto particular de condiciones ambientales.

Fortificación: adición de nutrientes a los alimentos durante el proceso de fabricación.

Genoma: secuencia completa de ADN de un organismo.

Genotipo: constitución genética de un individuo a diferencia de sus características expresadas.

Grupos de riesgo: grupos de población que tienen un riesgo más alto que la población general de tener niveles deficientes de vitamina D.

Heterogéneo: variado en contenido, compuesto de diferentes partes.

Hipercalciuria: calcio elevado en la orina. Definido como excreción urinaria de calcio > 250 mg/día en mujeres y 275-300 mg/día en hombres.

Hiperparatiroidismo: trastorno en el cual las glándulas paratiroides del cuello producen demasiada hormona paratiroidea (PTH).

Homeostasis: proceso por el cual los sistemas internos del cuerpo mantienen un equilibrio a pesar de las condiciones externas.

Homogéneo: formado por partes que son todas similares o que consisten en una sola sustancia.

Hormona paratiroidea (PTH): hormona secretada por las glándulas paratiroides. Participa en la regulación del homeostasis del calcio.

Índice de masa corporal (IMC): medición de la obesidad propuesta por la Organización Mundial de la Salud. Está correlacionado con el grado de adiposidad. Se obtiene dividiendo el peso (en $\mathrm{kg}$ ) por la talla $\left(\mathrm{en} \mathrm{m}^{2}\right)$.

Linfocitos (B y T): las células $\mathrm{T}$ (linfocitos $\mathrm{T}$ ) son responsables de la inmunidad celular interactuando con diferentes células del sistema inmunitario mediante la producción de citoquinas y otros mediadores. Los linfocitos B son los responsables de la inmunidad humoral mediada por anticuerpos.

Malabsorción: trastorno de la digestión causado por una inadecuada absorción de los nutrientes en el tracto gastrointestinal. Puede originar diarrea, gases, anemia y pérdida de peso. 
Metabolitos: cualquier sustancia producida durante los diversos metabolismos (digestión u otros procesos químicos corporales). También se puede referir al producto que queda después de la descomposición (metabolismo) de un fármaco por parte del cuerpo.

Obesidad: acúmulo excesivo de grasa corporal que se define con un valor de índice de masa corporal o IMC (peso/talla ${ }^{2}$ ) mayor o igual a $30 \mathrm{~kg} / \mathrm{m}^{2}$. Es un factor de riesgo para enfermedades cardíacas, diabetes e hipertensión arterial.

Osteoide: porción orgánica sin mineralizar de la matriz ósea que se forma con anterioridad a la maduración del tejido óseo.

Osteomalacia: trastorno esquelético que se desarrolla como resultado de la deficiencia de vitamina D y los estados hipofosfatémicos. Causa dolor severo en los huesos y músculos y debilidad muscular. Causado por un deterioro en la fase de mineralización de la remodelación ósea, lo que resulta en una proporción más baja de mineral óseo a osteoide de lo normal. El daño renal o hepático, que interfiere con el metabolismo de la vitamina $\mathrm{D}$, también puede causar osteomalacia.

Osteoporosis: trastorno esquelético progresivo caracterizado por una reducción de la resistencia ósea debido a la pérdida de masa ósea y al deterioro de la microarquitectura del hueso, donde la proporción de mineral óseo a osteoide es normal. Conduce a una mayor fragilidad ósea y riesgo de fractura.

Polimorfismo genético: variación natural en un gen, secuencia de ADN o cromosoma.

Radiación ultravioleta: es parte del espectro electromagnético emitido por el sol con longitudes de onda de alrededor de 100-400 nm. Las subregiones del espectro de la radiación ultravioleta se han definido de acuerdo con la transmisión en el tejido humano y los posibles efectos sobre la salud: UVA (315-400 nm), UVB (280-315 nm) y UVC (100-280 nm).

Valores dietéticos de referencia: los valores de referencia dietéticos reflejan la cantidad de energía y nutrientes que necesitan las personas sanas según su edad y género, y demandas asociadas con situaciones específicas.

Veganos: personas que siguen una dieta solo de productos vegetales, evitan alimentos, bebidas y artículos no alimentarios que contengan productos animales. 
Vida media: tiempo requerido para que la concentración de una sustancia en el cuerpo disminuya a la mitad.

Vitamina D: en sentido estricto, la denominada vitamina D es una hormona con capacidad de regulación del equilibrio calcio-fósforo en el organismo, de la que hoy en día se conocen además otras acciones extraesqueléticas. Se obtiene tras una exposición a la radiación ultravioleta o en menor medida de los alimentos, especialmente en el pescado, la leche y los lácteos. La deficiencia de vitamina $D$ puede provocar raquitismo en niños y osteomalacia en adultos, también se asocia con osteoporosis y el riesgo subsiguiente de fracturas (fragilidad ósea).

Vitamina D2: también conocida como ergocalciferol. Formado en hongos y levaduras por exposición a UVB del esteroide, ergosterol.

Vitamina D3: también conocida como colecalciferol. Sintetizado en la piel de los humanos a partir del 7-deshidrocolesterol por la acción de la radiación solar UVB. 


\section{Presentación}

Las normas de buena práctica clínica son un conjunto de condiciones que debe cumplir el profesional para asegurar que está realizando una correcta asistencia sanitaria. Las guías de práctica clínica (GPC) y los productos basados en la evidencia (OPBE) pretenden ser una ayuda para alcanzar una adecuada práctica clínica, asegurar a los profesionales que están realizando una buena asistencia sanitaria, y ayudarlos en la toma de decisiones.

Uno de los principales papeles de la vitamina $\mathrm{D}$ en el organismo es aumentar la absorción de calcio y fósforo para mineralizar el esqueleto. El déficit de vitamina $\mathrm{D}$ puede traer consigo niveles de calcio en sangre bajos y provocar secundariamente niveles elevados de hormona paratiroidea (PTH), ocasionando un recambio óseo acelerado que se puede traducir clínicamente en osteoporosis u osteomalacia, si se trata de un déficit de larga duración o gravedad. A día de hoy, faltan estudios específicos bien diseñados que confirmen su relevancia y la del tratamiento de su déficit a nivel clínico en acciones extraesqueléticas.

Para la elaboración de este OPBE se contó con la participación de un equipo multidisciplinar de profesionales que trabajan en diferentes sectores de la sanidad. Es un proyecto en el marco de la financiación del Ministerio de Sanidad, Consumo y Bienestar Social junto con GuíaSalud para el desarrollo de las actividades del Plan anual de trabajo de la Red Española de Agencias de Evaluación de Tecnologías Sanitarias y Prestaciones del Sistema Nacional de Salud. 



\section{Justificación}

Desde finales de los años 90, a partir del conocimiento de las acciones extraesqueléticas de la vitamina $\mathrm{D}$, surge un creciente interés por determinar los posibles efectos adversos para la salud y la supervivencia, de tener un nivel insuficiente de dicha hormona y los efectos beneficiosos que la suplementación de ésta pudiera aportar.

La distinta interpretación de sus efectos beneficiosos y de los potenciales riesgos ha llevado a distintas recomendaciones por parte de las sociedades científicas, lo que generó una controversia sin una respuesta clara todavía. A día de hoy, no existe un consenso respecto a la indicación de a quién realizar la determinación de niveles séricos de vitamina $\mathrm{D}$ en población sana, sobre los puntos de corte que se deben utilizar para distinguir los estados de deficiencia y de normalidad de vitamina $\mathrm{D}$, o como proceder en el manejo clínico de los pacientes con deficiencia.

Por otra parte, el cribado de vitamina D de forma indiscriminada, y su tratamiento con pautas no rigurosas en cuanto a dosis, frecuencia y duración, ha provocado, además de la aparición de efectos secundarios, una carga económica para el sistema sanitario cuya justificación, está sin determinar.

En 2016 la Sociedad Española de Medicina de Laboratorio dentro del proyecto Compromiso por la Calidad de las Sociedades Científicas en España coordinado por el Ministerio de Sanidad, Consumo y Bienestar Social, Guía Salud y la Sociedad Española de Medicina Interna recomendaron "no realizar cribado poblacional de déficit de vitamina D, mediante la concentración en suero de 25(OH)D (calcidiol)". Países como Francia, limitaron, ya en 2013, las indicaciones de las mediciones de 25(OH) $\mathrm{D}$, debido a que las determinaciones se habían multiplicado por diez en menos de diez años. Así, en Francia el reembolso de las mediciones séricas de 25(OH)D en suero se restringió a los casos de raquitismo/osteomalacia, adultos mayores con caídas recurrentes, control del trasplante renal en adultos y tratamiento quirúrgico de la obesidad en adultos.

El proyecto de OPBE surge a petición de la Dirección General de Cartera Básica de Servicios del SNS y Farmacia a propuesta de la Unidad de Asesoramiento Científico-técnico, Avalia-t. 



\section{Alcance y objetivos}

\subsection{Alcance}

\section{Población diana}

Población general sana, definida en este proyecto como personas mayores de 18 años, que no presentan síntomas de déficit de vitamina $\mathrm{D}$ y/o patologías susceptibles de déficit de vitamina $\mathrm{D}$ como el raquitismo o la osteomalacia.

Quedan excluidas las personas que padezcan enfermedades crónicas grave, mal controladas o con enfermedad terminal y las mujeres gestantes por tener necesidades terapéuticas y nutricionales diferentes.

\section{Niveles asistenciales}

Atención Primaria y de Atención Hospitalaria del Sistema Nacional de Salud.

\section{Proceso asistencial}

Este documento se centra en la determinación analítica de los niveles séricos de 25-hidroxivitamina D [25(OH)D] y la prescripción de suplementación con vitamina D.

\section{Profesionales a quienes va dirigido}

Profesionales del SNS que prestan asistencia sanitaria y toman decisiones de práctica clínica, así como sociedades científicas y gestores sanitarios.

\subsection{Objetivos}

El objetivo general del proyecto es realizar un OPBE donde se establezcan las pautas de uso adecuado de la determinación sérica de la vitamina $\mathrm{D}$ y suplementación, en población general adulta sana, mediante la realización de una revisión sistemática de la literatura científica existente y el consenso de expertos. 



\section{Metodología}

Para la realización de este OPBE, elaborado mediante revisión sistemática (RS) de la literatura y consenso de expertos, se siguió la metodología desarrollada por GuíaSalud para la elaboración de guías y protocolos, recogida en los manuales: "Manual Metodológico para la Elaboración de Guías de Práctica Clínica (GPC) en el Sistema Nacional de Salud" (123) y la "Guía Metodológica para la Elaboración de Protocolos Basados en la Evidencia" (124), ambos pueden ser consultados en la página Web de la Biblioteca de GPC del SNS, GuíaSalud.

En este apartado se describen las principales etapas del proceso de elaboración, que responden a la necesidad de que las recomendaciones que se recojan en él se basen en la mejor evidencia científica disponible.

\subsection{Constitución del grupo de trabajo}

Para la elaboración del OPBE, el primer paso fue formar los distintos grupos que colaboraron en la elaboración:

- Grupo coordinador: integrado por una médica de atención primaria como coordinadora clínica con formación y experiencia en el tema de estudio y una técnica de la Unidad de Asesoramiento Científicotécnico, Avalia-t como coordinadora metodóloga.

Sus funciones fueron: coordinar el proyecto y dar soporte al grupo de trabajo, establecer el alcance y objetivos del OPBE, formular las preguntas, establecer el grupo de expertos para el desarrollo del OPBE y coordinarlos, elaborar el cronograma, planificar las reuniones y supervisar los resultados de la revisión sistemática de la evidencia científica.

- Grupo elaborador: en él están representados todos los grupos relevantes para el tema a tratar, profesionales clínicos entre ellos analistas clínicos, reumatólogos, nefrólogos, médicos de familia, farmacéuticos de atención primaria, internistas, endocrinólogos, rehabilitadores y oncólogos de distintos centros sanitarios que abarcaran todo el territorio de la Comunidad Autónoma (CCAA) gallega y metodólogos y documentalista pertenecientes a Unidad de Asesoramiento Científico-técnico, Avalia-t. 
Las funciones de este grupo, entre otras, fueron: revisar el alcance y objetivos del OPBE planteados por el grupo coordinador, realizar la revisión sistemática y elaborar el documento.

- Grupo de revisores externos: formado por expertos en el tema y en su mayoría propuestos por Sociedades Científicas implicadas en el tema a tratar.

Su función principal es revisar el borrador del documento final.

Todos los participantes cubrieron y firmaron una declaración de conflictos de intereses antes del inicio de la elaboración del OPBE (Anexo 1).

\subsection{Formulario de preguntas clínicas y estrategia de respuesta}

Una vez delimitado el alcance y objetivos del OPBE, el siguiente paso fue concretar un listado completo de preguntas clínicas que desarrollaran el tema que aborda el OPBE. Con este listado se consiguió un mapa de preguntas, cuyas respuestas constituyen el contenido del documento.

Se formularon 12 preguntas que fueron reorganizadas en 10, de las cuales el grupo elaborador priorizó 2 para su respuesta en este OPBE (Anexo 2). El resto de preguntas se incluyeron en el apartado de Líneas de investigación futuras (Apartado IX).

La metodología para contestar las preguntas está basada en tres puntos:

- En primer lugar, se realizó una búsqueda general de GPC y RS para responder a las preguntas clínicas. Se valoró la calidad de las mismas, realizándose una síntesis de la información y procediendo a seleccionar las de mayor nivel de evidencia, como se especifica en el punto 3.3.2 de este apartado.

Se adoptaron como válidas, aquellas recomendaciones o conclusiones de las GPC y RS, que contestaban la pregunta planteada y alcanzaban un nivel alto de evidencia, de forma que no planteaban dudas que pudieran ser invalidadas por nuevas referencias.

- En segundo lugar, cuando las GPC o RS solo contestaban parcialmente las preguntas clínicas planteadas y/o cuando la nueva 
evidencia podría modificar las recomendaciones o conclusiones existentes se realizó una nueva búsqueda para actualizar la pregunta, teniendo como base de partida la fecha de búsqueda de las GPC o RS utilizadas en esa pregunta.

\subsection{Búsqueda, evaluación y síntesis de la evidencia}

\subsubsection{Búsqueda}

La búsqueda de literatura científica se llevó a cabo en dos tiempos, siguiendo el esquema anteriormente desarrollado para la estrategia de respuesta de las preguntas:

- Una primera búsqueda para localizar RS y GPCs sobre la medición de vitamina D en adultos. Esta búsqueda se realizó sin límite temporal hasta mayo 2018.

Una vez realizada la búsqueda, se procedió a la lectura de los resúmenes de los artículos recuperados, y se seleccionaron aquellos que podrían servir como punto de partida para la realización del OPBE.

Posteriormente, y definidas las preguntas a contestar en el documento, se realizó una búsqueda (2018) específica para cada una de las preguntas que no estaban contestadas con la evidencia encontrada en GPC o RS. Estas búsquedas tenían límite temporal en función de la documentación encontrada. En 2019 se realizó una actualización de esta búsqueda.

- Se realizó también una búsqueda general en Internet, y se revisaron páginas de diferentes organizaciones nacionales e internacionales y de sociedades científicas, etc. Por último, se procedió a la revisión de las referencias bibliográficas recogidas en los artículos incluidos.

Las estrategias de las búsquedas bibliográficas se recogen en el anexo 3 de este documento, y están disponibles en la página web de Avalia-t (ACIS) (https://acis.sergas.es/).

Una vez realizadas las búsquedas bibliográficas, se procedió a la lectura de los resúmenes de los artículos recuperados, y se seleccionaron aquellos que contestaban las preguntas formuladas en el OPBE. 
La selección de los estudios a incluir se llevó a cabo por dos o más revisores de forma independiente según de la pregunta.

\subsubsection{Evaluación}

La evaluación del riesgo de sesgo y calidad de los estudios seleccionados se realizó mediante el instrumento AGREE II para las GPC y la herramienta AMSTAR-2 en el caso de RS.

En la actualización de las preguntas se usó la herramienta de la Colaboración Cochrane para la evaluación de riesgo de sesgo de ECAs y la herramienta ROBINS-I para los estudios de intervenciones no aleatorios. Todos ellos recogidos en el anexo 4 y 5 (125).

El análisis se realizó de forma independiente por lo menos por dos evaluadores, que valoraron cada uno de los ítems de los instrumentos utilizados.

Se decidió elegir como guías base, aquellas que superasen al menos la puntuación de $40 \%$ en el dominio de rigor en la elaboración y de 4 sobre 7 en la calidad global de la guía. Con respecto a las revisiones, todas aquellas que presentasen un valor de AMSTAR-2 de calidad alta.

Finalmente, la evaluación de la calidad de la evidencia se realizó siguiendo la metodología GRADE (Grade of Recomendations, Assessment, Development and Evaluation) (126). Cuando no existía información que permitiera confirmar un nivel de evidencia sobre un aspecto determinado, o se tratara de aspectos organizativos o logísticos, la recomendación se estableció por consenso, basado en la experiencia del grupo de elaboración del OPBE.

\subsubsection{Síntesis y recomendaciones}

Para la extracción de los datos aportados en los artículos se empleó un formulario diseñado específicamente para tal fin. Dicha extracción, así como su posterior análisis, se llevó a cabo por, al menos, dos revisores de forma independiente.

La información se sintetizó en las correspondientes tablas guías donde se recogen la referencia, la recomendación de las GPC, la conclusión de las RS y los hallazgos de los distintos estudios, además del grado y nivel de evidencia en el que está basada/o (Anexos 4 y 5). 


\subsection{Identificación y desarrollo de indicadores de evaluación}

La monitorización y/o auditoría se realizará mediante unos indicadores para registrar la adherencia o la implementación de las recomendaciones del OPBE, y así mejorar su uso.

Los indicadores identificados por el grupo elaborador fueron derivados de las actividades o procedimientos establecidos en el OPBE y están basados en la evidencia científica y en el consenso de expertos. Estos indicadores nos permiten saber si se están consiguiendo los objetivos previstos. Se han agrupado según la clasificación de Donabedian (127), en tres categorías estructurales, de procesos o de resultados.

\subsection{Redacción del documento}

Después de examinar la evidencia de cada una de las preguntas se realizó un borrador del documento que se envió al grupo de trabajo para su revisión, tanto de contenidos, como de estructura y referencias incluidas. El grupo elaborador aportó diferentes sugerencias que fueron incluidas en el borrador tras consensuarlas en una reunión.

Una vez el grupo elaborador ratificó el documento este se envió a revisión externa.

\subsection{Revisión externa}

Una vez finalizado el documento y antes de su publicación, se sometió a un proceso de revisión externa por parte de expertos propuestos por las sociedades científicas implicadas en el tema.

Las aportaciones de los revisores externos fueron en primer lugar valoradas por el grupo coordinador y en caso de dudas planteadas al grupo elaborador donde se decidió sobre su inclusión en el documento. 



\section{Introducción a la vitamina D}

\subsection{Definición de vitamina D}

El término de vitamina D (VitD) es usado para referirse a un grupo de compuestos liposolubles que están involucrados en el sistema endocrino que regula la homeostasis mineral, protege la integridad del esqueleto y modula el crecimiento y la diferenciación celular en una amplia variedad de tejidos, desempeñando, por tanto, un papel importante en el metabolismo del calcio, fósforo y hormonal, así como en funciones reguladoras celulares, y por todo ello es muy importante que sus valores sean óptimos $(4,128)$.

\subsection{Fisiología de la vitamina D}

Hay dos formas de VitD: colecalciferol o VitD3 y ergocalciferol o VitD2. La primera se forma principalmente en la piel después de la exposición al sol (radiación UV), aunque también se encuentra en algunos alimentos de origen animal o vegetal y la segunda, VitD2 se obtiene por la dieta, con la ingesta de alimentos de origen vegetal $(1,2)$.

Independientemente de la forma en la que el organismo obtiene la VitD, ésta debe sufrir dos hidroxilaciones para tener una forma biológicamente activa.

La primera, se lleva a cabo en el hígado, transformando el colecalciferol en 25-hidroxicolecalciferol (calcifediol o calcidiol), y la segunda, que le confiere realmente capacidad biológica activa, se lleva a cabo fundamentalmente a nivel renal, en el interior la célula epitelial del túbulo proximal, convirtiéndose en 1,25 dihidroxicolecalciferol (calcitriol) gracias a la acción de la enzima 1a-hidroxilasa. Para acceder al interior de esta célula, el $25(\mathrm{OH}) \mathrm{D}$, unido a su proteína transportadora (DBP), debe ser filtrado en el glomérulo renal, y unirse a un receptor (denominado megalina) que permita este paso (129).

En los pacientes con insuficiencia renal, cuyo filtrado glomerular está disminuido, y en aquellos que presentan albuminuria, este proceso se encuentra limitado, en el primer caso, por la disminución de la cantidad de complejo 25(OH)D-DBP filtrado que puede acceder al receptor megalina, y en el segundo caso, porque este tiene también afinidad por la albúmina, 
quedando ocupados por esta, los receptores necesarios para el acceso al interior de la célula para el complejo 25(OH)D-DBP.

La presencia de la enzima 1 $\alpha$-hidroxilasa se ha encontrado en un gran número de tejidos, no sólo a nivel renal, lo que sugirió a los investigadores que sus funciones no estaban limitadas a su actuación endocrina a nivel musculoesquelético, y fue punto de partida para estudios posteriores sobre sus acciones extraesqueléticas y su interés en la salud en general.

En la siguiente figura (figura 1) podemos ver las vías de síntesis de la VitD.

Figura 1. Vías de síntesis de vitamina D

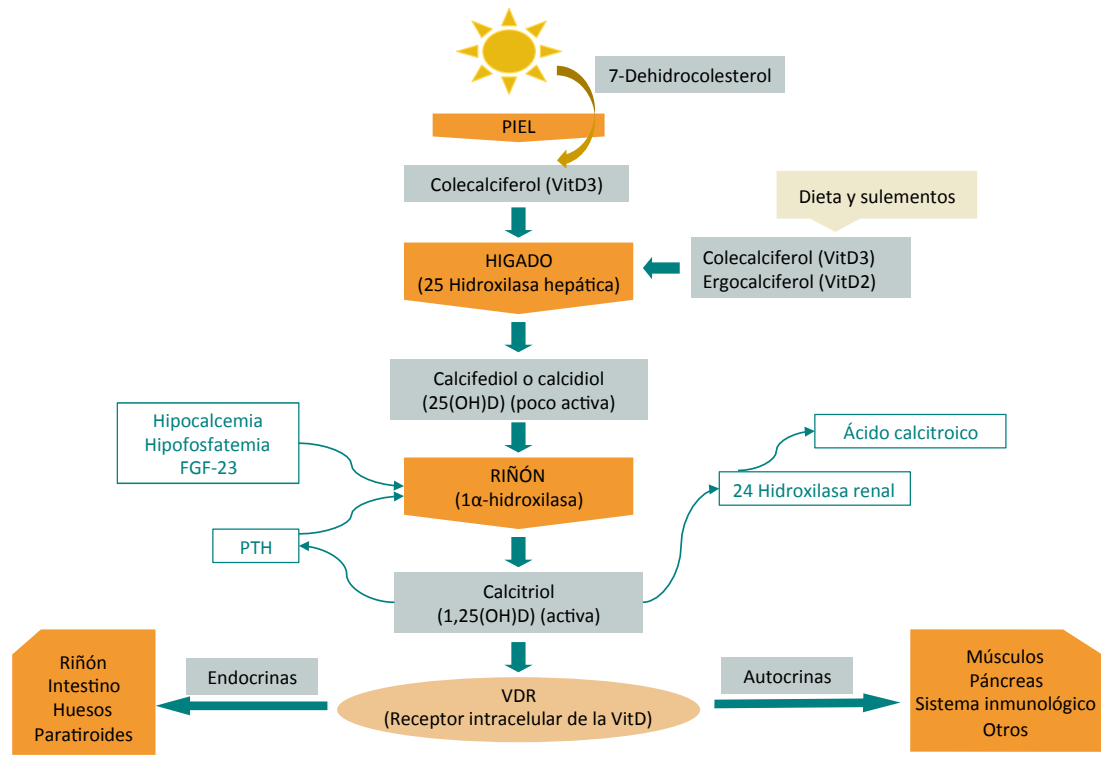

Fuente: elaboración propia

\subsection{Fuentes de vitamina D}

La VitD se obtiene de dos formas, la mayor parte (cerca del $90 \%$ ) se sintetiza en la piel por la acción de la radiación ultravioleta B (UVB) de la luz solar $(1,130)$, y en segundo lugar, a través de la dieta, por la toma de alimentos fortificados o ricos en VitD de forma natural $(1,2,11)$.

La producción de VitD en la piel está condicionada no sólo por la incidencia de la radiación ultravioleta B (UVB), que varía según la latitud 
en la que se viva, la estación del año, la hora y la duración del día, la capa de nubes, la contaminación, el contenido de melanina en la piel y la protección solar, sino también por la capacidad de los queratinocitos de la piel de convertir el 7-dehidrocolesterol en colecalciferol endógeno, capacidad que está alterada por los cambios de la epidermis producidos por la edad.

Muy pocos alimentos en la naturaleza contienen VitD. Los pescados grasos (salmón, atún y caballa) y aceites de hígado de pescado se encuentran entre las mejores fuentes. Pequeñas cantidades de colecalciferol y su metabolito $25(\mathrm{OH}) \mathrm{D}_{3}$ se hallan en el hígado de vacuno, el queso y yemas de huevo. Algunos hongos proporcionan $\mathrm{VitD}_{2}$ en cantidades variables, aunque esta forma es menos activa que colecalciferol de origen animal.

En la tabla 1 podemos ver la cantidad de VitD por 100 gr de alimento.

\section{Tabla 1. Alimentos ricos en vitamina D}

\begin{tabular}{|l|l|}
\hline Alimento & $\begin{array}{l}\text { Ul de VitD: } \\
=\mathbf{0} .025 \text { mcg colecalciferol } \\
=1 \mathbf{~ m c g ~ d e ~ V i t D ~ D ~ = ~ 4 0 ~ U I ~ V i t D ~}\end{array}$ \\
\hline Salmón asado o cocido & $360 \mathrm{UI} / 100 \mathrm{~g}$ \\
\hline Caballa cocida & $345 \mathrm{UI} / 100 \mathrm{~g}$ \\
\hline Sardinas enlatadas con aceite & $500 \mathrm{UI} / 100 \mathrm{~g}$ \\
\hline Atún enlatado en aceite & $232 \mathrm{UI} / 100 \mathrm{~g}$ \\
\hline Anguila cocida & $200 \mathrm{UI} / 100 \mathrm{~g}$ \\
\hline Huevo entero & $20 \mathrm{UI}$ \\
\hline Hígado vaca cocido & $15 \mathrm{UI} / 100 \mathrm{~g}$ \\
\hline Aceite hígado bacalao & $9067 \mathrm{UI} / 100 \mathrm{~g}$ \\
\hline Gambas & $152 \mathrm{UI} / 100 \mathrm{~g}$ \\
\hline Yogur (entero, desnatado, semidesnatado) & $89 \mathrm{UI} / 100 \mathrm{~g}$ \\
\hline
\end{tabular}

Fuente: Modificado de Base de datos española de composición de alimentos (131).

En los suplementos y alimentos enriquecidos, la VitD está disponible en dos formas, $\mathrm{D}_{2} \mathrm{y}_{3}$. En muchos países, alimentos como la leche, el yogurt, la margarina, las pastas, los cereales o la bollería y el pan están fortificados con VitD. En EE.UU. y Canadá la leche fortificada normalmente proporciona 100 UI por taza (132).

El aprovechamiento de la VitD contenida en los alimentos, depende de la capacidad de absorción del individuo. Así, en pacientes con enfermedades 
intestinales o hepáticas que provoquen malabsorción, o que reciban tratamiento con fármacos que provoquen secuestro de sales biliares en el intestino (colestiramina), dicho aprovechamiento puede estar muy limitado. Problemas como la intolerancia a la lactosa pueden implicar una reducción del consumo de lácteos, y con ello, una reducción en la ingesta de VitD, al ser estos, los alimentos más comúnmente fortificados. Personas obesas por secuestro en tejido adiposo y la causa del déficit en las personas que toman fármacos que afectan al metabolismo de la VitD por ser grupos con riesgo de déficit de VitD.

Pero no solo problemas de salud modifican la absorción de la VitD, la capacidad de la piel para sintetizar VitD va reduciéndose con los años, así personas de 70 años pueden tener reducida la capacidad al $30 \%$. Otros factores que afectan a la producción cutánea de VitD son la cantidad de melanina en la piel, la latitud geográfica, hora del día y estación del año (3).

La Comisión Autonómica Central de Farmacia y Terapéutica de Galicia (133) publicó en 2018 una serie de recomendaciones para el uso de la VitD en la población general basada en la revisión de la literatura, donde concluía que la principal fuente de VitD es la síntesis cutánea tras una exposición solar de entre 10 y 15 minutos al día en brazos y piernas (suficiente sólo en los meses de verano) y consumo de alimentos ricos en VitD.

Se ha observado, que los niveles de VitD, si bien mejoran al final del verano en la población que se expone al sol, siguen estando por debajo de los niveles óptimos en un $2.7 \%$ de los sujetos del estudio, considerando niveles óptimos $\geq 30 \mathrm{ng} / \mathrm{ml}$ de 25(OH)D en sangre venosa (134).

\subsection{Niveles óptimos de vitamina D}

Se pueden establecer tres criterios para definir la concentración óptima de 25(OH)D en el suero (135):

- Supresión máxima de la hormona paratiroidea (PTH): a medida que caen los valores de $25(\mathrm{OH}) \mathrm{D}$ la absorción intestinal de calcio disminuye, lo que conduce a un descenso de la concentración de calcio sérico. Esta reducción del calcio sérico es uno de los estímulos para el aumento de las concentraciones séricas de PTH que a su vez induce la conversión de 25(OH)D en 1,25-dihidroxiVitamina D y el mantenimiento de la absorción de calcio. 
- La concentración de 25(OH)D estimada para suprimir la PTH varía. En algunos informes se agrupan en el rango de 27.5 a $30 \mathrm{ng} / \mathrm{ml}$ (67.5 a $75 \mathrm{nmol} / \mathrm{L})$. Sin embargo, otros expertos apoyan la tesis de que la supresión de la PTH por 25(OH)D es continua a lo largo de un amplio rango de concentraciones de VitD y los valores superiores a $20 \mathrm{ng} / \mathrm{ml}(50 \mathrm{nmol} / \mathrm{L})$ son adecuados para suprimir la $\mathrm{PTH}$, asumiendo una función renal normal.

- Absorción intestinal de calcio adecuada: cuando la concentración de 25(OH)D se reducen, no hay suficiente sustrato para su conversión a 1,25 - dihidroxivitamina $\mathrm{D}$, incluso con altas concentraciones de PTH, y la absorción intestinal de calcio disminuye. El nivel de $25(\mathrm{OH}) \mathrm{D}$ en el que ocurre esto se estima en aproximadamente $4.4 \mathrm{ng} / \mathrm{ml}(11 \mathrm{nmol} / \mathrm{L})$. Por lo tanto, las concentraciones séricas de $25(\mathrm{OH}) \mathrm{D}$ por debajo de $4.4 \mathrm{ng} / \mathrm{ml}(11 \mathrm{nmol} / \mathrm{L})$ se han asociado con una disminución de la eficiencia de absorción de calcio.

- Salud ósea: el riesgo de fracturas viene determinado por la densidad mineral ósea (DMO) y por alteraciones en la microarquitectura. La concentración óptima de 25(OH)D también se puede definir a través un criterio clínico, como el nivel óptimo para la prevención de fracturas. En algunos ensayos, la suplementación con VitD para alcanzar concentraciones de $25(\mathrm{OH}) \mathrm{D}$ de 28 a $40 \mathrm{ng} / \mathrm{ml}(70$ a $99 \mathrm{nmol} / \mathrm{L}$ ) redujo el riesgo de fractura.

Existe un consenso universal que defiende la medición en sangre de la forma inactiva, el 25(OH)D como marcador del nivel de VitD en el organismo $(2,4)$. Se llega a este consenso, debido a la mayor vida media, concentración y estabilidad de este metabolito inactivo en suero.

Los métodos para la cuantificación de 25(OH)D pueden ser cromatográficos como HPL y LCMS-MS o inmunoquímicos como radioinmunoensayo, enzimoinmunoensayo o quimioinmunoensayo (el más usado en los laboratorios clínicos). Existe buena correlación entre ambos métodos, siendo LCMS-MS (calibrada frente al SMR 2972) la técnica patrón de oro para la cuantificación de $25(\mathrm{OH}) \mathrm{D}$. Los métodos inmunoquímicos miden la $25(\mathrm{OH}) \mathrm{D}$ total, mientras que los cromatográficos $25(\mathrm{OH}) \mathrm{D} 2$ y 25(OH)D3 por separado $(3,5)$.

Los valores de 25(OH)D a partir de los que se diagnostica y gradúa la deficiencia de VitD, han variado a lo largo de los años. En la actualidad, no existe un consenso general sobre las concentraciones óptimas, pero sí un convenio de mínimos, que indican la necesidad de obtener niveles séricos 
de $25(\mathrm{OH}) \mathrm{D}$ superiores a $20 \mathrm{ng} / \mathrm{ml}(50 \mathrm{nmol} / \mathrm{L})(136,137)$, y que los valores menores de $10 \mathrm{ng} / \mathrm{ml}(25 \mathrm{nmol} / \mathrm{L})$ deben tratar de evitarse en todas las edades (138).

La Sociedad Española de Investigación Ósea y del Metabolismo Mineral (SEIOMM) y Sociedades afines establecieron en 2011 (1) la valoración de niveles de $25(\mathrm{OH}) \mathrm{D}$ séricos considerando valores suficientes de VitD mayores de $30 \mathrm{ng} / \mathrm{ml}$ (>75 nmol/L), valores insuficientes entre 20-30 ng/ml (50-75 nmol/L) y niveles deficientes menores de $20 \mathrm{ng} / \mathrm{ml}(<50 \mathrm{nmol} / \mathrm{L})$.

Ese mismo año el Institute Of Medicine (IOM) define después de una revisión extensa y completa de la literatura y de una evaluación de los indicadores de salud (como: la salud ósea y esquelética, enfermedades crónicas extraesqueléticas, cáncer, enfermedad cardiovascular, diabetes, caídas o trastornos autoinmunes, etc.) que la salud ósea era el único factor que cumplía con los criterios para su uso como "indicador" ya que establecía causalidad. El comité sugirió que la concentración 25(OH)D igual a 16 ng/ml (40 nmol/L) satisfacen las necesidades de aproximadamente la mitad de la población y valores de $20 \mathrm{ng} / \mathrm{ml}$ (50 nmol/L) cubrirían los requerimientos del $97.5 \%$ de la población general (139).

Por su parte, la International Osteoporosis Foundation (IOF) establecía como cifra umbral óptima un valor de $30 \mathrm{ng} / \mathrm{ml}(4,128)$.

La Comisión Autonómica Central de Farmacia y Terapéutica de Galicia (133), considera que valores séricos de 25(OH)D entre 30-50 ng/ml (75-125 nmol/L) son valores óptimos para asegurar los beneficios a nivel óseo y mantener un nivel de seguridad. Una reciente publicación de enero de 2019 en Reino Unido titulada Guidelines on the diagnosis and management of vitamin $D$ deficiency in children and adults fijaba los valores de $25(\mathrm{OH})$ $\mathrm{D}$ para laboratorio en deficiencia $<12 \mathrm{ng} / \mathrm{ml}(30 \mathrm{nmol} / \mathrm{L})$, insuficiencia $12-20 \mathrm{ng} / \mathrm{ml}$ (30-50 nmol/L y adecuada > $30 \mathrm{ng} / \mathrm{ml}(50 \mathrm{nmol} / \mathrm{L})(140)$.

La recomendación realizada en el documento de consenso del 2017 de la Sociedad Española de Endocrinología y Nutrición sugería mantener concentraciones séricas de 25(OH)D entre 30-50 ng/ml (75-125 nmol/L) para conseguir los beneficios de salud que aporta la VitD. Esta recomendación presentaba una fuerza de recomendación débil y un nivel de evidencia muy bajo (7). 


\subsection{Prevalencia y carga de la enfermedad}

Según la CIE-10 (Clasificación Internacional de Enfermedades), el déficit de VitD se encuadran dentro del capítulo IV de enfermedades endocrinas, nutricionales y metabólicas, con los códigos E-55 (141).

La prevalencia de niveles óptimos de VitD varía según el modelo de definición de umbral que vimos en el apartado anterior. Así, con valores estrictos de 25(OH)D (>30 ng/ml) más del $88 \%$ de la población presentaría niveles bajos de VitD. Cuando aplicamos umbrales de $20 \mathrm{ng} / \mathrm{ml}$, la cifra de la población con niveles bajos, disminuye al $37 \%$ y al $7 \%$ cuando nos referimos a valores menores de $10 \mathrm{ng} / \mathrm{ml}(7)$.

La SEIOMM en 2011 publicaba un trabajo titulado" Documento de posición sobre las necesidades y valores óptimos de VitD" (1), en el cual los autores se preguntaban si son adecuados los valores de VitD en la población española. Se tomaba como base óptima de niveles de VitD valores entre $30-75 \mathrm{ng} / \mathrm{ml}$ y déficit valores inferiores a $20 \mathrm{ng} / \mathrm{ml}$. Con estas referencias consideraban que la mayoría de la población española no alcanzaba los niveles óptimos de VitD, presentando déficit un $30 \%$ de jóvenes y un $87 \%$ de ancianos institucionalizados y teniendo valores intermedios 50-70\% las edades intermedias (adultos y postmenopáusicas) y ancianos no institucionalizados.

La realidad es que la situación de hipovitaminosis D subclínica es muy prevalente en la población general. De hecho, los países mediterráneos privilegiados de sol, son los que muestran los niveles más bajos de calcidiol sérico de Europa por malinterpretar que las fuentes solares son suficientes (142).

Una revisión del 2013, encontraba a nivel mundial que el $88 \%$ de las muestras analizadas presentaban valores séricos menores de $30 \mathrm{ng} / \mathrm{ml}$ $(75 \mathrm{nmol} / \mathrm{L})$, el $37 \%$ menores de $20 \mathrm{ng} / \mathrm{ml}(50 \mathrm{nmol} / \mathrm{L})$ y un $7 \%$ menores de $10 \mathrm{ng} / \mathrm{ml}$ (25 nmol/L) (2). En España, estudios publicados de distintas regiones muestran valores similares $(2,6)$.

El documento de consenso de la Sociedad Española de Endocrinología y Nutrición (7) realizado en 2017 por un grupo de expertos para realizar una serie de recomendaciones de VitD para la población general mostraba situaciones similares a las del resto del mundo, así individuos mayores de 65 años presentan valores de $25(\mathrm{OH}) \mathrm{D}$ por debajo del $20 \mathrm{ng} / \mathrm{ml}$ en el $80-100 \%$ de la población y en menores de 56 años el porcentaje se sitúa en torno al $40 \%$. 


\subsection{Factores de riesgo}

La causa más frecuente de niveles no óptimos de VitD, en gran parte de la población y especialmente en ancianos, es la falta de exposición al sol debido al estilo de vida sedentario y a la institucionalización. También es importante considerar otros factores como $(8,9)$ :

- la estación del año, ya que se estima que los valores de 25(OH)D séricos descienden cerca de un $20 \%$ en invierno,

- la latitud donde se vive, áreas por encima de $40^{\circ}$ (hemisferio norte) presentan mayor déficit, dado que la exposición a la UV es más baja,

- factores climáticos, como la nubosidad o la contaminación que limita la síntesis de VitD

- el tanto por cien de la superficie corporal expuesta (ropa, vestimenta por motivos culturales ...),

- uso de protectores solares

- pigmentación de la piel, es uno de los factores importantes en el nivel de VitD en el organismo, ya que afecta al tiempo que es necesario exponerse al sol para alcanzar unos valores óptimos de VitD, en este caso la población de piel oscura necesita más tiempo solar para alcanzar los mismos valores de VitD que población de piel clara.

Otras causas de déficit de VitD son las enfermedades que provocan mala absorción (enfermedad celíaca...), la resección intestinal secundaria a enfermedad, o a cirugía bariátrica por obesidad $(10,11)$, y el consumo de fármacos (anticonvulsivantes, inmunosupresores, antirretrovirales...).

En la tabla 2 podemos ver los diferentes mecanismos patógenos y causas de carencia de VitD clasificados por mecanismo extrínseco o intrínseco. 
Tabla 2: Factores de riesgo para niveles no óptimos de vitamina D

\section{Extrínseca}

- Pigmentación de la piel

- Uso de protectores solares

- Ingesta inadecuada

- Escasa exposición a la luz solar:

- Institucionalización o reclusión

- Prendas/vestimentas de ocultación

\section{Intrínsecas}

- Edad avanzada (> 65 años)

- Ancianos con historial de caídas o fracturas por fragilidad

- Malabsorción:

- Gastrectomía

- Enfermedades intestinales

- Cirrosis biliar primaria

- Insuficiencia pancreática

- Tratamiento con colestiramina

- Colestasis crónica

- Incremento del catabolismo de la VitD

- Rifampicina,

- Anticonvulsivantes,

- Terapia antiretroviral

- Dosis altas de glucocorticoides

- Tuberculostáticos

- Hiperparatiroidismo

- Deficiencia de la 1a-hidroxilación renal

- Insuficiencia renal crónica

- Raquitismo dependiente de VitD tipo I

- Hipoparatiroidismo

- Pérdida renal de 25(OH)D

- Síndrome nefrótico

- Enfermedad ósea de Paget

- Enfermedades granulomatosas crónicas

- Deficiencia de 25-hidroxilación hepática

- Raquitismo dependiente de VitD tipo II

- Obesidad

- Mujeres embarazadas y en lactancia

- Etc.

Fuente: modificado de los estudios $(7,140)$.

\subsection{Intervenciones y tratamiento}

De forma general no se recomienda el cribado poblacional sobre el déficit de VitD. Hasta el momento, no hay evidencia de los beneficios del cribado universal del déficit de VitD en población general asintomática y sin factores de riesgo. Distintas publicaciones a nivel internacional y nacional coinciden en que solo debe realizarse la determinación de los valores de $25(\mathrm{OH}) \mathrm{D}$ en personas de alto riesgo $(4,133)$, como afectados por alteraciones del metabolismo óseo (osteoporosis, osteomalacia, raquitismo, etc.), obesos, sujetos con malabsorción, y antes de cirugía bariátrica entre otros. 
La Sociedad Española de Endocrinología y Nutrición recomienda la determinación de déficit de VitD exclusivamente en individuos con factores de riesgo de deficiencia de VitD. Esta es una recomendación fuerte con nivel de evidencia bajo, basada en la opinión de un grupo de expertos. A su vez se recomienda no realizar el cribado en población sin factores de riesgo, también recomendación fuerte con nivel de evidencia bajo (7).

La Sociedad Española de Medicina del laboratorio (SEQC) realiza una serie de recomendaciones para la valoración bioquímica del estatus de vitD en 2019 a través del posicionamiento de la Comisión de Hormonas de la SEQC. Este comité determino tres recomendaciones (143):

- No se recomienda el cribado en población general adulta sin factores de riesgo de déficit de VitD.

- Está ampliamente aceptado que es recomendable medir la concentración de VitD en pacientes sintomáticos y pacientes con enfermedades crónicas que pueden afectar a la absorción y el metabolismo de la VitD y

- No existe acuerdo unánime respecto a la necesidad de medir la concentración de $25(\mathrm{OH}) \mathrm{D}$ en población con riesgo de presentar déficit de vitamina $\mathrm{D}$, pero sin enfermedades crónicas que afectan a la absorción o metabolismo de la VitD.

La deficiencia de VitD se puede tratar con una mayor ingesta dietética (alimentos ricos en VitD o fortificados), una mayor exposición a la luz UV según la época del año y con tratamiento farmacológico.

Al igual que sucede para definir los umbrales de déficit de VitD, el tipo de intervención y tratamiento también están sujetos a controversia. Existen recomendaciones diferentes de distintos organismos y sociedades sobre el principio activo y la posología para normalizar los niveles de VitD.

Así, la IOM (139) sugería para alcanzar concentraciones mayores de $20 \mathrm{ng} / \mathrm{ml}$ en población sana dosis de hasta $600 \mathrm{UI} /$ día para adultos entre 18 y 70 años y 800 IU/día para mayores de 70 años, sin embargo, la Sociedad de Endocrinología de EE.UU. consideraba que se debían alcanzar valores superiores a $30 \mathrm{ng} / \mathrm{ml}$, para lo que era necesaria una ingesta de entre 15002000 UI/día $(4,128)$.

En España, la SEIOMM publicaba en 2011 que existen dudas sobre el tratamiento en monoterapia del calcio y la VitD; no así, cuando se administran conjuntamente que parecen tener cierta eficacia en la prevención de fracturas 
no vertebrales. Esto es más claro en personas deficitarias de estos elementos, como lo ancianos institucionalizados. En pacientes con osteoporosis, hay consenso universal para que reciban calcio y VitD junto al tratamiento de la enfermedad. El aporte recomendado se sitúa entre 1000- $1200 \mathrm{mg} / \mathrm{día}$ de calcio y 800 UI/día de VitD. A su vez, también la Sociedad Española de Endocrinología y Nutrición (7) sugería una ingesta diaria de 600 UI de VitD en menores de 70 años y de 800 UI para mayores de 70 años. La recomendación era débil y el nivel de evidencia bajo.

La Comisión Autonómica Central de Farmacia y Terapéutica de Galicia en 2018 (133), determinó, como la IOM que para el tratamiento del déficit de VitD la dosis necesaria dependía de la causa y la severidad, considerando adecuada una ingesta diaria de 600 UI en menores de 70 años y de 800 UI en mayores de 70 años en aquellos pacientes de los cuales se desconoce su nivel de 25(OH)D.

La guía reciente publicada en Reino Unido (140) recomienda que personas mayores de 65 años y aquellas que tienen poca o ninguna exposición al sol deben tomar un suplemento diario que contenga $400 \mathrm{UI}$ (10 mcg) de VitD.

La dosis necesaria de VitD no solo depende de la causa sino también de la severidad del déficit, de la capacidad de absorción del individuo y de otros determinantes genéticos. Así, en sujetos obesos (por "secuestro" de la VitD en el tejido graso), con malabsorción o aquellos que reciben tratamiento con fármacos que puede interferir en la absorción y metabolismo de la VitD, pueden ser necesario administrar dosis 2 o 3 veces mayores que otra población general (128).

Existen además muchas y diferentes formas de presentación de la VitD (cápsulas, gotas, soluciones orales), distintas dosis que van desde 200 a 100000 UI y distintos patrones o regímenes de administración, pudiendo ser diario, semanal o mensual. El régimen anual de toma única, está desaconsejado al haberse asociado con mayor número de caídas y fracturas en ancianos. Podemos encontrarla, además, asociada a otros medicamentos como calcio o bifosfonatos.

\subsection{Efectos adversos del tratamiento con vitamina D}

Los efectos adversos por suplementación son infrecuentes. Aun así, la toxicidad puede ser causada por un exceso de ingesta oral a través de suplementación. El principal efecto secundario de la sobredosificación 
de VitD es la hipercalcemia, que puede producir desde anorexia, náuseas, vómitos, polidipsia, debilidad muscular, litiasis renal, arritmias cardíacas, cefalea, depresión, confusión y coma $(8,144)$.

La Agencia Española de Medicamentos y productos sanitarios (AEMPS) publicó en marzo de 2019 una nota informativa sobre los casos graves de hipercalcemia por sobredosificación de VitD tanto en pacientes pediátricos como adultos (145). La AEMPS concreta que los casos notificados de hipercalcemia están relacionados con la sobredosificación con preparados de colecalciferol en niños y calcifediol en adultos y que en ambos casos están relacionados con errores en la prescripción, como en la dispensación o en la administración del medicamento. Por ello la AEMPS hace recomendaciones (ver tabla 3) tanto a nivel de médicos prescriptores como de farmacéuticos dispensadores.

\section{Tabla 3. Recomendaciones de la Agencia Española de Medicamentos y productos Sanitarios para la hipercalcemia}

\section{Médicos}

- Seleccionar la presentación del medicamento adecuada para cada situación

- Asegurarse de que en la receta aparecen anotados con claridad tanto la presentación, como la dosis por toma y frecuencia de administración. Confirmar que se está administrando correctamente el producto en las visitas médicas sucesivas.

- En adultos: explicar con claridad a los pacientes la pauta de administración del medicamento prescripto, haciendo especial hincapié en la frecuencia de administración del mismo, asegurándose de que el paciente la comprende.

- Explicar los riesgos derivados de la sobredosis de VitD e instruir acerca de los síntomas que sugieran su existencia.

\section{Farmacéuticos}

- Comprobar que la prescripción y la frecuencia de administración son adecuados para la situación, antes de la dispensación.

- En el momento de la dispensación, revisar con los pacientes que conocen con exactitud la pauta posológica prescrita.

Fuente: modificado de (133).

\subsection{Fármacos con vitamina D comercializados en España}

En España no se dispone de VitD2 (ergocalciferol) por vía oral, por ello tanto para la prevención como para el tratamiento se usa la VitD3 (colecalciferol). 
Los derivados hidroxilados en posición 25(OH)D (calcifediol) solo están autorizados para la indicación de tratamiento y no de prevención (128).

En nuestro país, existen distintas especialidades farmacéuticas que contienen VitD. Se presentan tanto como principio activo único o asociado a otros elementos como el calcio, el ácido alendrónico o distintas vitaminas y minerales. Pueden encontrarse en el mercado en diferentes presentaciones como cápsulas, gotas, soluciones, etc.

En el anexo 6 podemos ver los distintos fármacos comercializados en España que contiene VitD, en él se recogen tanto el nombre comercial, como el principio activo, la forma farmacéutica y la dosis (actualizado a junio 2020). 



\section{Revisión de la evidencia científica para las recomendaciones de uso adecuado de pruebas y suplementos de vitamina D en población general sana}

Como se reflejó en el apartado de Alcance y objetivos, se ha definido como población general sana, aquella población mayor de 18 años con o sin factores de riesgo de déficit VitD, que no presenta síntomas ni patologías que pudieran manifestarse por su déficit. Además, este OPBE tampoco se ocupa de las personas con enfermedades crónicas graves o mal controladas, personas con enfermedad terminal o mujeres embarazadas, al necesitar todos ellos terapias nutricionales diferentes.

Antes de desarrollar las preguntas y dado que existe una gran controversia para establecer la concentración óptima de VitD, se desarrolló un consenso basado en la opinión de expertos del grupo de trabajo mediante un método Delphi modificado.

\subsection{Delphi modificado. Concentración óptima de vitamina $\mathrm{D}$ en población sana}

Para el desarrollo de las definiciones de consenso relacionadas con la concentración óptima de la VitD, se formó un panel con los doce profesionales multidisciplinares, que participaban en el grupo de trabajo del OPBE.

El proceso Delphi modificado incluyó dos rondas de votación remota y una reunión de mesa redonda donde se presentaron y discutieron los resultados. La pregunta planteada en el Delphi fue la siguiente: ¿Cuáles son los umbrales estándares de $25(\mathrm{OH}) \mathrm{D}$ para definir el nivel óptimo y déficit de VitD en población general adulta?

Para la primera ronda, el nivel de acuerdo con cada una de las cinco declaraciones se calificó en una escala Likert de cinco puntos: totalmente de acuerdo, de acuerdo, neutral, en desacuerdo y totalmente en desacuerdo (ordenado entre el 1 y el 5, siendo el 5 totalmente de acuerdo y el 1 totalmente en desacuerdo). En la primera ronda se analiza el coeficiente de variación 
(CV \%) y la mediana para obtener los ítems del segundo cuestionario. Solo se consideraron para la segunda ronda aquellas opciones que obtuvieron un valor de mediana $\geq 3$ y a su vez un CV bajo (26-50\%) o muy bajo ( $\leq 25 \%)$.

Los resultados en la primera ronda fueron los reflejados en la tabla siguiente (tabla 4).

Tabla 4. Resultados ronda 1 Delphi

\begin{tabular}{|c|c|c|c|c|c|}
\hline & Opción 1 & Opción 2 & Opción 3 & Opción 4 & Opción 5 \\
\hline Óptima & $\begin{array}{c}\geq 30 \mathrm{ng} / \mathrm{ml} \\
(\geq 75 \mathrm{nmol} / \mathrm{L})\end{array}$ & $\begin{array}{c}\geq 20 \mathrm{ng} / \mathrm{ml} \\
(\geq 50 \mathrm{nmol} / \mathrm{L})\end{array}$ & $\begin{array}{l}\text { Entre } 30-50 \mathrm{ng} / \mathrm{ml} \\
(75-125 \mathrm{nmol} / \mathrm{L})\end{array}$ & $\begin{array}{c}>20 \mathrm{ng} / \mathrm{ml} \\
(>50 \mathrm{nmol} / \mathrm{L})\end{array}$ & $\begin{array}{l}\text { Entre } 30-76 \mathrm{ng} / \mathrm{ml} \\
(75-190 \mathrm{nmol} / \mathrm{L})\end{array}$ \\
\hline Insuficiencia & $\begin{array}{l}\text { Entre } 20-29 \mathrm{ng} / \mathrm{ml} \\
(50-74 \mathrm{nmol} / \mathrm{L})\end{array}$ & & $\begin{array}{l}\text { Entre } 20-29 \mathrm{ng} / \mathrm{ml} \\
(50-74 \mathrm{nmol} / \mathrm{L})\end{array}$ & $\begin{array}{l}\text { Entre } 12-20 \mathrm{ng} / \mathrm{ml} \\
(30-50 \mathrm{nmol} / \mathrm{L})\end{array}$ & $\begin{array}{l}\text { Entre } 21-29 \mathrm{ng} / \mathrm{ml} \\
(49-74 \mathrm{nmol} / \mathrm{L})\end{array}$ \\
\hline Deficiencia & $\begin{array}{c}<20 \mathrm{ng} / \mathrm{ml} \\
(<50 \mathrm{nmol} / \mathrm{L})\end{array}$ & $\begin{array}{c}<20 \mathrm{ng} / \mathrm{ml} \\
(<50 \mathrm{nmol} / \mathrm{L})\end{array}$ & $\begin{array}{c}<20 \mathrm{ng} / \mathrm{ml} \\
(<50 \mathrm{nmol} / \mathrm{L})\end{array}$ & $\begin{array}{c}\leq 12 \mathrm{ng} / \mathrm{ml} \\
(\leq 30 \mathrm{nmol} / \mathrm{L})\end{array}$ & $\begin{array}{l}<20 \mathrm{ng} / \mathrm{ml} \\
(<50 \mathrm{nmol} / \mathrm{L})\end{array}$ \\
\hline Participante 1 & 3 & 2 & 5 & 1 & 4 \\
\hline Participante 2 & 5 & 2 & 3 & 1 & 4 \\
\hline Participante 3 & 4 & 3 & 5 & 1 & 2 \\
\hline Participantes 4 & 3 & 1 & 4 & 2 & 5 \\
\hline Participantes 5 & 5 & 1 & 4 & 2 & 3 \\
\hline Participantes 6 & 5 & 3 & 4 & 1 & 2 \\
\hline Participantes 7 & 3 & 5 & 2 & 4 & 1 \\
\hline Participantes 8 & 3 & 2 & 4 & 1 & 5 \\
\hline Participantes 9 & 2 & 4 & 3 & 5 & 1 \\
\hline Participantes 10 & 2 & 4 & 3 & 5 & 1 \\
\hline Participantes 11 & 3 & 1 & 4 & 2 & 5 \\
\hline Participantes 12 & 5 & 2 & 4 & 1 & 3 \\
\hline $\begin{array}{l}\text { Totalmente de acuerdo } \\
\text { (puntuación 5) }\end{array}$ & 4 & 1 & 2 & 2 & 3 \\
\hline De acuerdo (puntuación 4) & 1 & 2 & 6 & 1 & 2 \\
\hline Neutral (puntuación 3) & 5 & 2 & 3 & 0 & 2 \\
\hline $\begin{array}{l}\text { En desacuerdo } \\
\text { (puntuación 2) }\end{array}$ & 2 & 4 & 1 & 3 & 2 \\
\hline $\begin{array}{l}\text { Totalmente en desacuerdo } \\
\text { (puntuación 1) }\end{array}$ & 0 & 3 & 0 & 6 & 3 \\
\hline Mediana & 3 & 2 & 4 & 1.5 & 3 \\
\hline $\begin{array}{l}\text { Coeficiente Variación } \\
(\mathrm{CV} \%)\end{array}$ & & $53 \%$ & & $73 \%$ & $53 \%$ \\
\hline
\end{tabular}

Fuente: elaboración propia con datos del Delphi realizado 
La segunda ronda se completó por el mismo número de participantes, pasando a esta ronda dos alternativas, la opción 1 y la opción 3. Los resultados se reflejan en la tabla siguiente.

Tabla 5. Resultados Ronda 2 Delphi

\begin{tabular}{|c|c|c|}
\hline & Opción 1 & Opción 3 \\
\hline Óptima & $\begin{array}{c}\geq 30 \mathrm{ng} / \mathrm{ml} \\
(75 \mathrm{nmol} / \mathrm{L})\end{array}$ & $\begin{array}{l}\text { Entre } 30-50 \mathrm{ng} / \mathrm{ml} \\
(75-125 \mathrm{nmol} / \mathrm{L})\end{array}$ \\
\hline Insuficiencia & $\begin{array}{l}\text { Entre } 20-29 \mathrm{ng} / \mathrm{ml} \\
(50-74 \mathrm{nmol} / \mathrm{L})\end{array}$ & $\begin{array}{l}\text { Entre } 20-29 \mathrm{ng} / \mathrm{ml} \\
(50-74 \mathrm{nmol} / \mathrm{L})\end{array}$ \\
\hline Deficiencia & $\begin{array}{l}<20 \mathrm{ng} / \mathrm{ml} \\
(50 \mathrm{nmol} / \mathrm{L})\end{array}$ & $\begin{array}{l}<20 \mathrm{ng} / \mathrm{ml} \\
(50 \mathrm{nmol} / \mathrm{L})\end{array}$ \\
\hline Participante 1 & 1 & 2 \\
\hline Participante 2 & 1 & 2 \\
\hline Participante 3 & 1 & 2 \\
\hline Participante 4 & 1 & 2 \\
\hline Participante 5 & 2 & 1 \\
\hline Participante 6 & 1 & 2 \\
\hline Participante 7 & 2 & 1 \\
\hline Participante 8 & 2 & 1 \\
\hline Participante 9 & 1 & 2 \\
\hline Participante 10 & 1 & 2 \\
\hline Participante 11 & 1 & 2 \\
\hline Participante 12 & 1 & 2 \\
\hline TOTALMENTE DE ACUERDO (valor 2) & 3 & \\
\hline TOTALMENTE EN DESACUERDO (valor 1) & 9 & 3 \\
\hline \multicolumn{3}{|c|}{ El $70 \%$ de 12 participantes es 8.4 . La opción 3 ha tenido 9 votos superando el $70 \%$} \\
\hline
\end{tabular}

Fuente: elaboración propia con datos del Delphi realizado

En esta ronda se consideró que existía consenso cuando la pregunta obtuvo una puntuación de por lo menos el $70 \%$ de los participantes, obteniendo la opción 3 nueve votos representando el $75 \%$ de los votos y siendo por tanto la opción elegida.

El panel determinó para la pregunta ¿cuáles son los umbrales estándares de $25(\mathrm{OH}) \mathrm{D}$ para definir el nivel óptimo y el de déficit de vitamina $\mathrm{D}$ en población general adulta? El panel determinó los siguientes valores (tabla 6): 
Tabla 6. Concentración de vitamina D

\begin{tabular}{|l|l|}
\hline Óptima & $30-50 \mathrm{ng} / \mathrm{ml}(75-125 \mathrm{nmol} / \mathrm{L})$ \\
\hline Insuficiencia & Entre $20-29.9 \mathrm{ng} / \mathrm{ml}(50-74.9 \mathrm{nmol} / \mathrm{L})$ \\
\hline Deficiencia & $<20 \mathrm{ng} / \mathrm{ml}(<50 \mathrm{nmol} / \mathrm{L})$ \\
\hline
\end{tabular}

Fuente: elaboración propia con datos del Delphi realizado

\subsection{Revisión de la evidencia científica}

Las preguntas clínicas a responder en este documento, se contestarán en base a la mejor evidencia encontrada, de acuerdo con la secuencia de actividades planteadas en la metodología.

De forma general, se adoptó para la contestación de las dos preguntas como punto de partida un documento realizado por la Task Force (4) y publicado en 2014. Este fue localizado en la búsqueda general de revisiones y guías y teniendo como propósito general realizar una revisión sistemática para desarrollar una serie de recomendaciones sobre el déficit de VitD. En el instrumento AMSTAR-2 (146), para la evaluación de la calidad metodológica de la revisión sistemática, presentaba una valoración alta.

Cada una de las preguntas fue elaborada de forma independiente y se realizó una búsqueda bibliográfica, para cada una de ellas, limitada en el tiempo en función de la fecha de la referencia del documento de partida, año 2014.

No se realizaron preguntas de novo.

\subsection{Pregunta 1: ¿Cuál es la asociación entre los niveles séricos de 25(OH)D y los resultados en salud? Resultados en: mortalidad, cáncer, fracturas, caídas, enfermedad cardiovascular, diabetes, depresión, funcionamiento cognitivo, estado funcional, etc.}

Se ha formulado una pregunta de investigación en formato PICO (Patient, Intervention, Comparator, Outcome) que podemos ver en la tabla 7. 
Tabla 7. Desarrollo pregunta PICO, pregunta 1

\begin{tabular}{|l|l|}
\hline Población diana & Población general adulta \\
\hline Intervención & Niveles séricos de $25(\mathrm{OH})$ \\
\hline Comparación & Déficit niveles $25(\mathrm{OH}) \mathrm{D}$ \\
\hline Resultados & $\begin{array}{l}\text { Resultados en salud: mortalidad, cáncer, fracturas, caídas, enfermedad } \\
\text { cardiovascular, diabetes, depresión, funcionamiento cognitivo, estado funcional, } \\
\text { etc. }\end{array}$ \\
\hline
\end{tabular}

Fuente: elaboración propia.

Para la contestación de esta pregunta, como comentamos anteriormente, se tomó como punto de partida un documento realizado por la Task Force (4) y publicado en 2014.

La revisión realizaba la pregunta dentro de un marco contextual. Se incluyeron estudios prospectivos de cohortes, caso-control anidados o revisiones sistemáticas que examinaban la asociación entre los valores de $25(\mathrm{OH}) \mathrm{D}$ y los resultados en salud, encontrando estudios sobre mortalidad, cáncer, fracturas, caídas, enfermedades cardiovasculares, diabetes, función cognitiva y estado funcional.

La búsqueda bibliográfica realizada en septiembre de 2018 sobre la actualización del documento de partida (4) y su actualización en 2019, arrojó, tras eliminar duplicados, un total de 862 referencias de estudios. Se seleccionaron 47 para su lectura a texto completo. De ellos se han seleccionado 40 que cumplieron los criterios de inclusión vistos en la tabla 8. En la tabla 9 se pueden ver los resultados según la fuente consultada.

\section{Tabla 8. Criterios de inclusión y exclusión de estudios, pregunta 1}

\begin{tabular}{|l|l|}
\hline Aspecto considerado & Criterios de inclusión/exclusión \\
\hline Diseño del estudio & $\begin{array}{l}\text { Inclusión: RS, ECAs, estudios de casos-controles y cohortes con } \\
\text { grupo de comparación posteriores al } 2014 . \\
\text { Exclusión: estudios con menor nivel de evidencia de los incluidos } \\
\text { en documento de partida }\end{array}$ \\
\hline Tamaño muestral & No se establecieron limitaciones sobre el tamaño muestral \\
\hline Variables de resultado & $\begin{array}{l}\text { Datos relativos a la salud en términos de: mortalidad, cáncer, } \\
\text { fracturas, caídas, enfermedad cardiovascular, diabetes, depresión, } \\
\text { funcionamiento cognitivo, estado funcional, etc. }\end{array}$ \\
\hline Idioma & $\begin{array}{l}\text { Inclusión: castellano, inglés, francés, portugués e italiano. } \\
\text { Exclusión: otros idiomas. }\end{array}$ \\
\hline Límite temporal & Inclusión: a partir del año 2014. \\
\hline
\end{tabular}

Fuente: elaboración propia. 
Tabla 9. Bases de datos electrónicas de la literatura biomédica, pregunta 1

\begin{tabular}{|l|c|}
\hline Bases de datos & Resultados \\
\hline Cochrane Library Trials (Wiley) & 392 \\
\hline Pubmed (Medline) & 463 \\
\hline Embase (Ovid) & 35 \\
\hline CRD database & 12 \\
\hline Total & 862 \\
\hline Seleccionados lectura texto completo & 47 \\
\hline Incluidos & 40 \\
\hline
\end{tabular}

Fuente: elaboración propia.

\subsubsection{Resultados de los niveles séricos de 25(OH)D en salud}

Además de la revisión de partida resumimos 43 estudios, 40 procedentes de la búsqueda y tres que se encontraron de forma manual o en búsquedas posteriores. De los 43 estudios, 37 eran artículos primarios y 6 eran revisiones. La evidencia nueva se centró principalmente en mortalidad, cáncer, fracturas o enfermedades cardiovasculares. Los hallazgos fueron inconsistentes entre los estudios. Los métodos utilizados para analizar el suero 25(OH)D en los trabajos era diferente en muchos de ellos, así como el valor umbral utilizado para definir el nivel óptimo de VitD. Además, los hallazgos variaron según el grupo de edad y el estado de salud de los participantes, el estado basal de VitD y el período de seguimiento.

Otra de las limitaciones de estos estudios, que aumenta el riesgo de sesgo, es que no fueron, en su mayoría, específicamente diseñados para evaluar el nivel de VitD, la mayoría de la población procedía de publicaciones diseñadas con otros objetivos. Además, el tamaño muestral con frecuencia carecía de poder suficiente para los resultados esperados y variaban ampliamente en calidad metodológica y en los métodos utilizados para medir el estado de VitD.

Todos los estudios localizados presentan calidad media/baja, dado que son todos caso-control o cohortes con alto riesgo de sesgo. Aunque para algunas patologías se localizaron revisiones sistemáticas, éstas solo incluían estudios de caso-control o cohorte, también con alto nivel de sesgo. Con este tipo de estudios no se puede establecer la relación causal entre el nivel de VitD y la patología encontrada. 
Hay que reseñar que la ausencia de ECAs impide la obtención de conclusiones definitivas y la consiguiente realización de recomendaciones con alto nivel de evidencia científica con respecto a los niveles de VitD y las distintas patologías.

\section{Vitamina D y enfermedades cardiovasculares}

La revisión de partida llevada a cabo por la Task Force (4) incluye para contextualizar las enfermedades cardiovasculares y su relación con la VitD, revisiones y meta-análisis que concluyen que valores $<24 \mathrm{ng} / \mathrm{ml}$ podrían estar asociados con un aumento de riesgo de enfermedad cardiovascular. La asociación para valores $>25$ ng/mg no está clara y esta asociación puede estar relacionada con la raza o etnia.

Posterior a la revisión de partida se localizaron tres estudios nuevos (12-14). Los tres son de cohortes como parte de análisis poblacionales, publicados dos de ellos en $2014(12,14)$ y otro en 2015 (13). Dos fueron realizados en Europa $(13,14)$ y otro en EE.UU. (12). La calidad de la evidencia es muy baja, en los tres casos el nivel de sesgo es serio dado que la población no había sido reclutada para el objetivo del estudio y solo presentan una medida de $25(\mathrm{OH}) \mathrm{D}$, entre otras limitaciones.

Dos de los trabajos realizan el análisis solo en hombres $(12,14)$ ( $\mathrm{n}=3019$ y 3731 respectivamente) y ambos en mayores de 60 años, mientras el estudio de Vitezona et al, 2015 (13) ( $n=3395)$ incluía ambos sexos y mayores de 55 años.

Ninguno encuentra una asociación significativa entre bajas concentraciones séricas de $25(\mathrm{OH}) \mathrm{D}$ y resultados clínicos cardiovasculares, incluso realizando análisis por distintos modelos de ajustes de covariables y utilizando umbrales distintos para el déficit de VitD.

\section{Resumen: Enfermedades cardiovasculares}

En población general, los resultados son discordantes en los estudios incluidos en la revisión de partida. Sin embargo, las publicaciones posteriores presentan la misma orientación, no encontrando asociación significativa entre los valores bajos de 25(OH)D y los riesgos cardiovasculares.

Calidad de la evidencia MUY BAJA $\oplus$ $\odot 0$

\section{Ictus}

La revisión de partida (4) incluye un meta-análisis de siete estudios prospectivos (147), que mostró un aumento de riesgo de ictus para valores 
$<12 \mathrm{ng} / \mathrm{ml}$ frente a $<19 \mathrm{ng} / \mathrm{ml}$. La asociación puede variar dependiendo de la raza/etnia.

Posteriormente se recuperó un estudio de casos y controles anidado (15) de calidad baja y riesgo de sesgo serio. Se publicó en el 2016 en EE.UU., y la población son adultos blancos y afroamericanos con edad media de 45 años ( $\mathrm{n}=1547,610$ casos y 937 controles). Encontró que los valores de deficiencia de $25(\mathrm{OH}) \mathrm{D}$ se asocian con alto riesgo de ictus independientemente de la raza y del ajuste a factores de confusión. Sin embargo, los resultados hay que tomarlos con cautela ya que el trabajo presenta limitaciones importantes. En primer lugar, solo realiza una medida de concentración de 25(OH)D no puede extrapolarse a otras etnias, no se obtienen mediciones directas de VitD y no ajusta los resultados a la actividad física, además de obtener las características basales mediante entrevista telefónica, con el consiguiente sesgo de memoria y presentar diferencias significativas entre el grupo control y casos a nivel de sexo, raza, Índice de masa corporal (IMC), presión arterial, tabaquismo, diabetes, etc.

No se identificaron ECAs para el informe actual que evaluaran los efectos de la VitD en los resultados clínicos de la enfermedad cardiovascular.

\section{Resumen: Ictus}

En población general, los estudios de la revisión de partida y los posteriores van todos en la misma línea, apuntando a que valores bajos de $25(\mathrm{OH}) \mathrm{D}$ se asocian con aumento de riesgo de ictus.

Calidad de la evidencia MUY BAJA $\oplus \circ 0 \bigcirc$

\section{Vitamina D y cáncer}

La Task Force (4) incluyó distintos estudios para el cáncer de distintas localizaciones. En la búsqueda posterior también se hallaron estudios referentes a distintas localizaciones de cáncer como colorrectal, mama, próstata, etc., que desarrollamos a continuación.

\section{Cáncer de próstata}

La Task Force (4) incluye en el 2014 cuatro revisiones sistemáticas/ metanálisis de estudios prospectivos (148-152), no encontrando asociación en ninguno de ellos.

La búsqueda posterior arrojó un nuevo estudio anidado de casos y controles (16) con riesgo de sesgo serio. Evalúa la asociación de las concentraciones basales séricas de 25(OH)D y el riesgo de cáncer de próstata 
en hombres mayores de 55 años ( $\mathrm{n}=1695$ casos y $\mathrm{n}=1682$ controles). Los autores sugieren una posible asociación protectora entre el nivel de 25(OH)D y el riesgo de cáncer, y no encuentran diferencias por edad o IMC. Sin embargo, hay que destacar el alto riesgo de sesgo al no estar la población reclutada específicamente para este análisis. Además, los autores solo realizan una determinación de la concentración de $25(\mathrm{OH}) \mathrm{D}$ sin tener en cuenta los cambios que se pueden producir a lo largo del tiempo y no realizan análisis por subgrupos de población en base al momento del año en el que se realiza la extracción de la sangre para la medición de VitD.

La tendencia se observó cuando los participantes se dividieron por cuartiles de concentración de $25(\mathrm{OH}) \mathrm{D}$, pero no se realizó una evaluación por categorías de suficiencia de VitD.

No se identificaron ECAs elegibles.

\section{Resumen: Cáncer de próstata}

En población general, no existe asociación entre los valores de VitD y el cáncer de próstata en el momento actual.

Calidad de la evidencia MUY BAJA $\oplus$ MOO

\section{Cáncer colorrectal}

En la RS (4) de partida se incluyeron:

- Una revisión sistemática y metanálisis de la US Preventive Service Task Force (USPSTF) realizada en el 2011 (149), que incluía cuatro estudios de casos y controles anidados de buena calidad y dos revisiones sistemáticas $2014(148,151)$, todos con resultados en la misma dirección: una asociación inversa entre el nivel de VitD y el riesgo de cáncer colorrectal.

- Y una revisión llevada a cabo por el grupo de la IARC en 2009 (152), que concluyó que la relación dosis-respuesta era bastante lineal hasta valores de $35-40 \mathrm{ng} / \mathrm{ml}$. Pero con resultados inconsistentes.

En la búsqueda posterior no se identificaron nuevos ECAs, solo se recuperó un estudio de casos y controles anidado (17) publicado en el 2015 en EE. UU., y cuya población es solo mujeres $(\mathrm{n}=274$ casos y $\mathrm{n}=274$ controles). El trabajo encuentra tendencias de aumento de la incidencia de cáncer colorrectal y cáncer de colon con concentraciones decrecientes de $25(\mathrm{OH}) \mathrm{D}$ y no en el cáncer rectal. La asociación es significativa en valores de $25(\mathrm{OH}) \mathrm{D}>29 \mathrm{ng} / \mathrm{ml}$ experimentando mayor reducción de la incidencia 
del cáncer colorrectal. La mortalidad presenta significación $(\mathrm{p}=0.048)$ en el análisis sin ajustes a factores de confusión y asociación límite cuando se ajusta $(p=0.051)$. Sin embargo, como en las publicaciones referenciadas para otras patologías, los resultados hay que tomarlos con cautela ya que presenta serias limitaciones, en primer lugar, se trata de un estudio retrospectivo, que solo realiza una medida de concentración de $25(\mathrm{OH}) \mathrm{D}$ y no ajusta los resultados a la exposición al sol o al consumo de suplementos, entre otras variables. EL riesgo de sesgo del estudio es serio.

Resumen: Cáncer colorrectal

En población general, si bien la evidencia es escasa y no permite establecer conclusiones, esta apunta a la existencia de una relación inversa entre los valores de VitD y la presencia de cáncer colorrectal.

Calidad de la evidencia MUY BAJA $\oplus \circ \circ \bigcirc$

\section{Cáncer de mama}

La revisión (4) de partida incluye en esta localización cuatro metanálisis, una revisión de la USPSTF de estudios prospectivos $(148-151,153)$ y un estudio anidado de caso-control (154), todos ellos concluyen que los datos son consistentes en cuanto a la falta de asociación con los valores de $25(\mathrm{OH})$ D y el riesgo de cáncer de mama.

En la búsqueda posterior se identificaron dos nuevos estudios observacionales $(18,19)$ que evalúan la asociación entre $25(\mathrm{OH}) \mathrm{D}$ y cáncer de mama. Ambos con diseño de casos y controles, uno anidado a estudio sobre estilo de vida y otro de base hospitalaria. Jamshidinaeini et al, (18) presentan 270 mujeres (135 en cada grupo) mayores de 50 años que muestran una asociación inversa entre los valores de $25(\mathrm{OH}) \mathrm{D}$ y el riesgo de cáncer a nivel del total de mujeres, tanto en el modelo no ajustado $(\mathrm{p}=0.002)$, como en el ajustado ( $\mathrm{p}=0.001)$; sin embargo, la asociación no se encuentra cuando el análisis se realiza solo en mujeres postmenopáusicas $(\mathrm{p}=0.096)$, pero si en premenopáusicas $(\mathrm{p}=0.007)$ ambas en el modelo sin ajustes. El estudio de Kim et al, (19) realizado en mujeres postmenopáusicas entre 45-75 años ( $\mathrm{n}=707$ por grupo), señalan una asociación inversa entre el nivel de VitD y el riesgo de cáncer cuando los valores son menores de $20 \mathrm{ng} / \mathrm{ml}$.

Los estudios muestran un aumento en el riesgo de cáncer de mama con concentraciones decrecientes de 25(OH)D, pero en ambos casos los estudios presentan riesgo de sesgo, Jamshidinaeini et al,(18) crítico y Kim et al, (19) moderado. Como en los casos anteriores la población no había sido reclutada para el objetivo del análisis, solo se realiza una determinación del nivel sérico de VitD y, además, presentan en ambos casos sesgo de selección 
y no ajustan por variables importantes como actividad física, consumo de alcohol o uso de anticonceptivos. En el caso de Jamshidinaeini et al, (18) presenta también sesgo de memoria al informar de variables confusoras a través de una encuesta postal.

No se identificaron ECAs.

\section{Resumen: Cáncer de mama}

En población general, no existe asociación entre los valores de VitD y el cáncer de mama en el momento actual.

Calidad de la evidencia MUY BAJA $\oplus$ MO०

\section{Cáncer de ovario}

EL documento de la Task Force (4) hacía referencia a dos revisiones sistemáticas del $2014(148,151)$ que incluían distintas localizaciones tumorales entre ellas la de ovario, concluyendo que no se encontraba asociación entre el nivel de VitD y los tumores.

Para el informe actual se localizó un estudio de casos y controles de base poblacional, realizado en 2016 en EE.UU., en mujeres afroamericanas (20) ( $n=490$ casos y $n=656$ controles). El trabajo evalúa la asociación de las concentraciones séricas de $25(\mathrm{OH}) \mathrm{D}$ por ingesta de VitD por dieta o sol y cáncer de ovario. Los autores no encuentran una asociación significativa entre el nivel de VitD y el riesgo de cáncer de ovario. Presenta un riego de sesgo moderado, como los estudios anteriores, por falta de datos sobre el uso de protección solar o vestimenta y solo una toma de niveles de VitD.

No se identificaron ECAs relevantes.

\section{Resumen: Cáncer ovario}

En mujeres adultas, no existe asociación entre los valores de VitD y el cáncer de ovario en el momento actual.

Calidad de la evidencia BAJA $\oplus$ BO०

\section{Cáncer de páncreas}

La revisión (4) de partida incluyó:

- Dos metanálisis de estudios prospectivos $(150,152)$, que no encontraron una asociación significativa, aunque algunos estudios de forma individual, observaron una asociación en forma de U. 
- Dos revisiones $(148,151)$ que presentaban datos contradictorios

La búsqueda posterior no encontró publicaciones sobre el tema.

\section{Resumen: Cáncer de páncreas}

En población general, no existe asociación entre los valores de VitD y el cáncer páncreas en el momento actual.

Calidad de la evidencia BAJA $\oplus \oplus \circ 0$

\section{Cáncer de cualquier tipo}

La revisión de la Task Force (4) incluyó dos revisiones sistemáticas $(148,151)$ para cánceres en distintas localizaciones. No se observó asociación entre el nivel de VitD y cánceres de esófago, gástricos, endometrio, vejiga, riñón o linfoma no Hodgkin.

En la búsqueda posterior se localizaron dos publicaciones nuevas (21, 22). Las dos son de estudios de cohortes retrospectivos basados en datos de distintos trabajos. Uno de ellos, realizado en 2016 (21), solo incluye mujeres mayores de $55(\mathrm{n}=2304)$ y excluye el cáncer de piel, encontrando una asociación inversa entre los niveles séricos de $25(\mathrm{OH}) \mathrm{D}$ y el aumento de la incidencia del cáncer de manera general y una asociación protectora con valores $25(\mathrm{OH}) \mathrm{D} \geq 40 \mathrm{ng} / \mathrm{ml}$. EL otro (22), publicado en 2014 y realizado en Dinamarca sobre la base de estudios poblacionales, que incluye tanto hombres como mujeres $(n=12204)$, no muestra asociación significativa entre el estado sérico de la VitD y la incidencia de cáncer total, tanto en modelos ajustados como en no ajustado a factores de confusión. El análisis por tipo de cáncer muestra que solo el cáncer colorrectal $(\mathrm{p}=0.05)$ y el cáncer de piel no melanoma ( $\mathrm{p}=0.003)$ en modelos no ajustados señalan asociación entre el nivel sérico de VitD y el riesgo de cáncer.

En ambos casos el riesgo de sesgo de los estudios es serio, dado que son estudios retrospectivo y parte de la evidencia procede de análisis secundarios. No se localizaron ECAs.

\section{Resumen: Cáncer de cualquier tipo}

Si bien, la evidencia es escasa y no permite establecer conclusiones, en población general esta apunta a la existencia de una relación inversa entre los valores de VitD y la presencia de cáncer de piel no melanoma.

Calidad de la evidencia MUY BAJA $\oplus \circ \bigcirc \bigcirc$ 
Vitamina D y la salud ósea

Los resultados informados en esta sección se basan en los estudios que abordan problemas de fracturas, caídas y artritis.

\section{Caídas}

La Task Force (4) incluyó para este apartado una revisión paraguas (revisión de revisiones) realizada en el 2014 (107 revisiones sistemáticas y 74 meta-análisis de estudios observacionales) (151), y estableció que la evidencia era inconsistente y que no se podían extraer conclusiones respecto a la asociación entre valores bajos de $25(\mathrm{OH}) \mathrm{D}$ y riesgo de caídas, por el contrario, la evidencia sugiere que niveles altos están asociados a mayor riesgo.

Posteriormente, solo se identificaron dos publicaciones de 2017 (23, 24) que evalúa el nivel sérico de VitD y las caídas. Duval et al (23) presenta diseño de caso-control anidado, en sujetos ancianos mayores de 75 años que sufren caídas $(n=72)$ frente a controles sanos sin caídas $(n=144)$. Los autores encuentran una asociación significativa $(\mathrm{p}=0.014)$ entre el déficit de VitD y el riego de caídas en pacientes geriátricos, cuando se ajusta por factores de confusión.

El estudio de Rothenbacher et al (24) es un estudio de cohortes prospectivo de base poblacional en población con media de edad de 75.6 años y un total de 1506 participantes donde se estudia la asociación entre la función renal y el nivel de $25(\mathrm{OH}) \mathrm{D}$ y se evalua su rol en el riesgo de caídas. Los autores concluyen que la función renal podría explicar parte de la deficiencia de VitD, y los valores de VitD se asocian de forma inversa a los niveles de caída en pacientes con valores de calcio por encima de la media.

En ambos estudios el riesgo de sesgo es serio, dado que la población proviene de otros estudios, mientras la publicación de un estudio (23) presenta elevada fragilidad, en el otro (24) no se ajusta por fragilidad, no existe información sobre la dieta o por el tiempo en exterior ya que es población de residencias comunitarias. En el caso de Duval et al (23) el tamaño muestral es pequeño, restando potencia a los resultados.

No se encontraron ECAs para el estudio de caídas.

Resumen: Riesgo de caídas

En población general, los resultados entre los estudios son diferentes, no pudiendo realizar una conclusión sobre el riesgo de valores bajos de VitD y el aumento de caídas.

Calidad de la evidencia MUY BAJA $\oplus \circ 0 \bigcirc$ 


\section{Fracturas}

La revisión de la Task Force (4) incluye en este apartado, la misma revisión paraguas que para el estudio de las caídas (151), y concluye que la evidencia es sugestiva solo para fracturas no vertebrales y que no se pueden extraer conclusiones respecto a otras fracturas. Habitualmente, los estudios prospectivos que muestran una asociación, refieren mayor riesgo para valores $<20 \mathrm{ng} / \mathrm{ml}$. Basado en estos estudios, y en los niveles óptimos para suprimir la hormona paratiroidea y maximizar la absorción de $\mathrm{Ca}$, los expertos generalmente están de acuerdo respecto a que los valores $<20 \mathrm{ng} / \mathrm{ml}$ no son óptimos para mantener la salud ósea, aunque no hay un acuerdo general respecto a si los niveles deberían ser $>20 \mathrm{ng} / \mathrm{ml}$ para proteger el sistema esquelético.

En este apartado se han recuperado cuatro estudios, dos de cohortes de base poblacional, Julian et al, 2016 (25) y Rothenbacher et al, 2014 (24), y dos de caso-control, Maier et al, 2015 (26) y Williams et al, 2018 (27). Los cuatro incluyen población de ambos sexos y evalúan la asociación entre las concentraciones séricas de $25(\mathrm{OH}) \mathrm{D}$ y distintas fracturas metatarsianas (27), vertebrales (26), y fracturas en cualquier localización (25), y el último, tiene como objetivo analizar la asociación entre la función renal y la 25(OH) D y evaluar la relación de ambos factores, con el riesgo de caídas y fracturas óseas. Todos los estudios presentan riesgo de sesgo serio, excepto Julian et al (25) que presenta riesgo moderado.

Las cuatro publicaciones muestran resultados diferentes en cuanto a la asociación entre el nivel sérico de VitD y el riesgo de fractura. Julian et al, (25) señala un riesgo menor de fracturas en sujetos de edad entre 40-79 años ( $\mathrm{n}=14$ 624, de ellos 1183 con fractura) y valores séricos de 25(OH)D entre $50-70 \mathrm{nmol} / \mathrm{L}$ frente a sujetos con niveles menores de $30 \mathrm{nmol} / \mathrm{L}$. La incidencia de riesgo de fractura aumenta con los años de seguimiento y no depende del modelo de análisis y las covariables incluidas en ellos. Maier et al, (26) en un estudio de casos y controles con 638 sujetos (246 casos y 392 controles) que evalúan las fracturas vertebrales, encuentran que el $89 \%$ de sujetos con este tipo de fracturas presenta valores muy bajos de VitD frente al $60 \%$ en el grupo control $(p=0.036)$. No se señalan diferencias entre los grupos por edad, pero si por otras covariables como obesidad, consumo de tabaco y diabetes. Williams et al, (27) ( $\mathrm{n}=99$ sujetos, 71 casos y 28 controles) estudian la prevalencia de las fracturas metatarsianas de baja energía frente a pacientes con esguinces de tobillo y su asociación con el nivel bajo de VitD. Los grupos presentan diferencias estadísticamente significativas entre ellos a nivel basal (edad, sexo, consumo nicotina y estación de extracción). No se encuentra asociación significativa entre la fractura o esguince y el nivel de VitD. 
Rothernbacher et al (24) ( $\mathrm{n}=1385)$, evalúan el efecto de la $25(\mathrm{OH})$ $\mathrm{D}$ en la función renal y el riesgo de caídas y fracturas, la muestra presenta una edad media de 75.6 años. No encuentran asociación entre el nivel de VitD y fracturas.

De forma resumida podemos decir que, pacientes con fracturas metatarsianas no presenta niveles más bajos de 25(OH)D que sujetos con esguinces. Sin embargo, el estudio que asocia actividad física y edad con el riesgo de fracturas, señala una asociación inversa entre el riesgo de fractura y el nivel de 25(OH)D.

Los resultados están limitados por el riesgo de sesgo, debido a la realización de solo una toma de niveles séricos de VitD, entre otras.

No se encontraron ECAs para el estudio de fracturas.

\section{Resumen: Riesgo de fracturas}

En población general, dado que los umbrales de 25(OH)D empleados en los estudios son distintos, presentan diferentes resultados. No pudiendo llegar a una generalización sobre el nivel óptimo de 25(OH)D necesario para proteger el esqueleto y disminuir el riesgo de fracturas.

Calidad de la evidencia MUY BAJA $\oplus \circ 0 \bigcirc$

\section{Vértigo posicional paroxístico benigno (VPPB)}

Para evaluar el efecto de la VitD y la osteoporosis en la etiología del VPPB se incluyó solo una publicación de 2017 (28) de caso-control con base hospitalaria. Se desarrolla en Turquía, con un número reducido de sujetos ( $\mathrm{n}=156,78$ casos y 78 controles) de ambos sexos. No hay diferencias significativas entre los grupos a nivel basal y no se encuentran diferencias entre el nivel de VitD y el VPPB por edad, pero si en el análisis por sexo. Las mujeres premenopáusicas presentan diferencias a nivel de VitD $(\mathrm{p}=0.016)$ y prevalencia de déficit ( $\mathrm{p}=0.007$ ), pero no en mujeres postmenopáusicas o en hombres. Los autores sugieren que la osteoporosis y la deficiencia de VitD no son factores determinantes para el riesgo de vértigo posicional paroxístico. Presenta un nivel de sesgo serio, hay que tener en cuenta que sujetos de entorno hospitalario pueden no ser extrapolables a población general.

\section{Resumen: Vértigo posicional paroxístico benigno}

En población general, la escasa evidencia no permite establecer conclusiones, sin embargo, el estudio apunta a la no existencia de una relación entre los valores bajos de 25(OH)D y el vértigo posicional paroxístico benigno.

Calidad de la evidencia MUY BAJA $\oplus \circ 0 \bigcirc$ 


\section{Artritis reumatoide}

Se localizó una RS con metanálisis realizada por Lee et al 2016 (29) en el estudio de la artritis reumatoide. La RS presenta una calidad metodológica alta, medida con el instrumento de criterios del AMSTAR-2 (146), incluye 14 estudios de caso control con un total de 1143 casos y 963 controles. El objetivo es determinar si el nivel sérico de $25(\mathrm{OH}) \mathrm{D}$ está disminuido en pacientes con artritis frente a controles sanos. El metanálisis encuentra una relación significativa entre los valores VitD y artritis $(p=0.017)$ y la prevalencia del déficit de VitD y artritis $(\mathrm{p}=0.023)$, en ambos casos en comparación con controles sanos.

Sin embargo, aunque la revisión es de buena calidad, presenta limitaciones, ya que los trabajos incluidos eran heterogéneos entre si y el análisis de factores de confusión era deficitario.

\section{Resumen: Artritis reumatoide}

La evidencia apunta que, en población general sana, existe una relación inversa entre los valores bajos de VitD el aumento de prevalencia de artritis reumatoide.

Calidad de la evidencia BAJA $\oplus \oplus \circ \bigcirc$

\section{Vitamina D y mortalidad}

La revisión de partida (4) incluye para contextualizar la mortalidad:

- Una revisión paraguas, antes mencionada (151), que concluye que no existía evidencia suficiente para extraer conclusiones entre la asociación de los niveles de VitD y la mortalidad.

- Una revisión de la AHRQ del 2009, que incluyó cuatro estudios de cohortes (155), donde el estudio de mayor calidad mostró una tendencia significativa de disminución del riesgo de muerte con mayores concentraciones de $25(\mathrm{OH}) \mathrm{D}$, aunque sugirió una asociación en forma de U; los otros no encontraron una asociación significativa.

- Un metanálisis del 2012 con 11 estudios prospectivos de cohortes (156), que concluyó que existía una disminución no lineal del riesgo de muerte, observando que los valores de 30-35 ng/ml eran los que estaban más relacionados con la disminución de la mortalidad.

- Tres estudios posteriores al 2012 (157-159) que concluyeron que tanto los valores altos como los bajos estaban relacionados con 
aumentos en el riesgo de mortalidad, con valores óptimos entre $20-40 \mathrm{ng} / \mathrm{ml}$.

- Y dos revisiones del 2014 que incluían 31 y 73 estudios, respectivamente $(148,160)$ y donde ambas concluían que únicamente los valores bajos estaban asociados a un aumento en el riesgo.

Posteriormente a la revisión de partida se identificaron siete estudios observacionales (30-32, 34-37), derivados en su mayoría de análisis poblacionales, que evalúan la asociación de concentraciones bajas de $25(\mathrm{OH}) \mathrm{D}$ y el riego de mortalidad por todas las causas o por mortalidad específica. Cuatro de ellos son prospectivos $(30-32,35)$ y tres retrospectivos $(34,36,37)$. Tres de los trabajos se realizan en mujeres $(30,34,37)$ y los otros en población general adulta. Los resultados son muy variados.

Buchebner et al (30) realizado en mujeres mayores de 75 años con o sin fracturas $(\mathrm{n}=1011)$, que se evalúan repitiendo las determinaciones de VitD cada 5 años, hasta los 90 años de edad. Se observa que aquellas mujeres que tenían valores bajos de VitD tiene mayor mortalidad, esta relación significativa se produce a los 10 años de seguimiento, pero no a los 5 años. En el mismo estudio, las mujeres que tuvieron fracturas, tenían menor mortalidad general, lo que sugiere que la relación entre los valores de VitD y la mortalidad en general, es independiente del estado de salud, fragilidad y la mortalidad relacionada con las fracturas. Riesgo de sesgo alto.

Daraghmeh et al $(31),(\mathrm{n}=10$ 517) muestran una asociación significativa entre valores bajos de VitD y mortalidad cardiovascular y los niveles de 25(OH)D entre 32-160 ng/ml cuando no se ajusta por variables de confusión, pero no cuando está ajustada. No ocurría lo mismo con la mortalidad por todas las causas, donde la asociación es significativa con o sin ajustar por factores. Riesgo de sesgo alto.

Otros tres trabajos (34-36) $(\mathrm{n}=7937$ total $)$ encuentran asociación entre los valores bajos de $25(\mathrm{OH}) \mathrm{D}$ y riesgo de mortalidad tanto en población general adulta, como en mujeres de mediana edad y, tanto en mortalidad por todas las causas $(\mathrm{p}>0.05)$ como específica por enfermedad cardiovascular $(p<0.01)$ o ictus $(p>0.01)$. Samefors et al, (35) señalan que en pacientes mayores de 65 años, institucionalizados durante al menos un año, se ha observado que no hay diferencias entre sexos, y que existe un aumento del riesgo de mortalidad significativo con el aumento de edad $(\mathrm{p}>0.01)$ y por la falta de actividad física $(\mathrm{p}=0.001)$. Wong et al, (37) realizado en ancianas $(\mathrm{n}=1188)$ para determinar la asociación entre las concentraciones séricas de VitD y la mortalidad específica por cáncer, encuentran que concentraciones 
bajas de $25(\mathrm{OH}) \mathrm{D}$, se asocian tanto en análisis ajustados como no ajustados a factores de confusión, con un aumento significativo de mortalidad por cáncer.

Sin embargo, Formiga et al (32), un estudio realizado en España en una población de 312 individuos mayores de 85 años que viven en la comunidad, con una prevalencia de hipovitaminosis del $52 \%$, no encuentran asociación entre mortalidad por enfermedad cardiovascular y déficit de VitD.

Todos los estudios localizados son observacionales, lo cual permite hacer una posible asociación, pero no establecer ninguna relación causal. Cuatro de los estudios presentan un riesgo de sesgo serio $(31,32,34,35)$ y tres un riesgo moderado $(30,36,37)$. En general, se hacen pocas mediciones del nivel sérico de VitD, mayoritariamente una medida o máximo tres; además, muchos de los datos sobre mortalidad proceden de registros que pueden tener falta de datos al utilizar diferentes fuentes y no constar, o no tener estandarizadas las causas de muerte y muchos de los trabajos con baja participación $(32,35)$, etc. Otra limitación es la no generalización de los resultados debido a que en su mayoría son poblaciones con características especiales. Hay que tener en cuenta en el análisis que, al tener diseño observacional, no pueden realizar conclusiones sobre causalidad.

También se localizó una RS con metanálisis, Gaksch et al, 2017 (33), que incluía 8 estudios de cohortes realizados en el norte de Europa sobre mortalidad $(\mathrm{n}=26916)$. Los resultados muestran que los sujetos con valores de $25(\mathrm{OH}) \mathrm{D}$ en el extremo inferior $(25-30 \mathrm{nmol} / \mathrm{L})$ presentan riesgo significativamente mayor de mortalidad por cualquier causa en un seguimiento a 10.5 años. Este riesgo aumenta a medida que disminuye el nivel de $25(\mathrm{OH}) \mathrm{D}$, mientras que en la mayor parte de la población con valores entre $50-125 \mathrm{nmol} / \mathrm{L}$ el riesgo no difiere significativamente. Sin embargo, no se pudo establecer una asociación consistente entre los valores de VitD y la mortalidad. Cuando el análisis es ajustado a mortalidad por causas cardiovasculares o cáncer $(\mathrm{n}=24325)$ se observa un riesgo significativo de mortalidad en enfermedades cardiovasculares al disminuir el nivel de $25(\mathrm{OH}) \mathrm{D}$, pero no se encuentra asociación de riesgo de muerte por cáncer y niveles bajos de $25(\mathrm{OH}) \mathrm{D}$. La revisión presenta una calidad metodológica críticamente baja, medida con el instrumento de criterios del AMSTAR-2 (146), al presentar más de una debilidad crítica y una serie de limitaciones, ya que, los resultados no son extrapolables a población general.

En la búsqueda actual no se localizó ningún ECAs que abordara la mortalidad. 
Resume: Mortalidad por cualquier causa

Se ha observado relación entre valores bajos de VitD y mayor mortalidad en adultos de población general.

No se pueden establecer relaciones causales por el tipo de estudios. Sería necesario realizar ECAs diseñados específicamente para este fin, y con muestra suficiente, para establecer relaciones causales entre los valores de 25(OH)D3 en sangre, el efecto del tratamiento de la hipovitaminosis si existiera, y la mortalidad.

En general, en la mayor parte de los estudios se indican niveles medios de VitD en la población, esto nos puede estimar, de una forma indirecta, la prevalencia del déficit de VitD, parámetro que puede alterar la aparición de lo que queremos valorar.

Calidad de la evidencia MUY BAJA $\oplus$ MO०

\section{Vitamina $D$ y el sistema circulatorio}

Para el informe actual, no identificamos nuevos ECAs que abordan la relación de las concentraciones séricas de $25(\mathrm{OH}) \mathrm{D}$ y enfermedades del sistema circulatorio. Se localizaron dos estudios $(38,39)$, ambos con el objetivo de analizar si el riesgo de enfermedad tromboembólica (ETV) está relacionado con el nivel sérico de 25(OH)D. Folsom et al, 2014 (39) hacen un análisis retrospectivo de un estudio de cohorte poblacional, donde 537 sujetos entre 45-64 años presentan ETV de una población de 12 752. Los autores realizan tres modelos de análisis por covariables, y en ninguno de ellos encuentran una asociación entre el nivel bajo de VitD y el riesgo de sufrir una ETV. Por su parte Andro et al, 2016 (38), realiza un estudio de caso-control en sujetos mayores de 75 años con ETV o embolia pulmonar, con 340 sujetos en cada uno de los grupos y en el que los autores tampoco encuentran asociación significativa entre los valores de $25(\mathrm{OH}) \mathrm{D}$ y el riesgo de ETV.

Ambos presentan un riesgo de sesgo moderado, presentan limitaciones al mostrar solo una medida del nivel de VitD.

Resumen: Sistema circulatorio

En población general, los estudios disponibles analizados no permiten establecer una relación entre los valores de VitD y la incidencia de enfermedad tromboembólica.

Calidad de la evidencia BAJA $\oplus \oplus \circ \bigcirc$

\section{Vitamina D y deterioro cognitivo}

La revisión de partida (4) identificó dentro del deterioro cognitivo dos revisiones sistemáticas $(148,151)$, que sugieren una asociación entre los valores altos de $25(\mathrm{OH}) \mathrm{D}$ y la disminución cognitiva. 
En el informe actual, identificamos tres estudios de cohortes retrospectivos que evalúan la asociación entre las concentraciones séricas de suero $25(\mathrm{OH}) \mathrm{D}$ y el riesgo de deterioro cognitivo (demencia y Alzheimer) (41-43). También se localizó una RS con metanálisis publicada en 2017 por Goodwill et al (40), cuyo objetivo era investigar si el nivel bajo de VitD interviene en la función cognitiva de adultos sanos, para poder realizar una intervención preventiva.

Los autores de la RS (40) encuentran una relación inversa entre el déficit de VitD y la función cognitiva o deterioro cognitivo, sin embargo, los mismos autores concluyen que la mayoría de los estudios incluidos presentan seguimientos a cinco años cuando deberían ser a diez años por lo que puede ser una causa inversa. Los estudios realizados por Littlejohns et al, 2014 y Moon et al, 2015 (41, 42), ( $n=1658$ y 412 respectivamente), en población anciana de ambos sexos muestran de forma significativa que los niveles bajos de 25(OH)D aumentan el riesgo de demencia por todas las causas y enfermedad de Alzhéimer, así como una asociación significativa con la progresión de la enfermedad, tanto en modelos de análisis ajustados como no. Sin embargo, Olsson et al, en 2017 (43), en una muestra de 1182 sujetos, también ancianos pero solo hombres no encuentran esta asociación.

Las limitaciones de los tres estudios son similares, con falta de mediciones repetidas de concentración de VitD y falta de ajuste con variables de confusión. Los tres estudios presentan un riesgo de sesgo serio. Por su parte, la RS (40) presenta una calidad metodológica alta, medida con el instrumento de criterios del AMSTAR-2 (146).

No se han localizado ECAs.

\section{Resumen: Riesgo de deterioro cognitivo}

En población general, los resultados de los estudios presentan diferencias entre ellos. Estudios que muestran asociación entre los valores bajos de $25(\mathrm{OH}) \mathrm{D}$ y aumento de riesgo de déficit cognitivo, y estudios que no encuentran ninguna asociación.

Calidad de la evidencia BAJA $\oplus \oplus \circ 0$

\section{Vitamina D y enfermedades oculares}

Se incluyen dos estudios, ambos de casos y controles $(45,46)$ con riesgo de sesgo serio. Ambos de pequeño tamaño muestral limitando la potencia de los resultados, además de no ajustar por factores de confusión. También se incluye una RS con metanálisis (44), que presenta una calidad metodológica críticamente baja, medida con el instrumento de criterios del AMSTAR-2 (146), al presentar más de una debilidad crítica. El objetivo fue 
revisar sistemáticamente y evaluar cuantitativamente la asociación de la concentración de $25(\mathrm{OH}) \mathrm{D}$ circulante con el desarrollo de la degeneración macular asociada a la edad (DMAE).

La RS con metanálisis realizada por Annweiler et al 2016 (44), que incluyó 11 estudios observacionales (10 estudios transversales y 1 estudio de cohorte) realizados en el hemisferio norte, concluye que las altas concentraciones de $25(\mathrm{OH}) \mathrm{D}$ podrían ser protectoras contra la DMAE, y que las concentraciones de $25(\mathrm{OH}) \mathrm{D}$ por debajo de $50 \mathrm{nmol} / \mathrm{L}$ se asocian con la DMAE tardía. La asociación con $25(\mathrm{OH}) \mathrm{D}<25 \mathrm{nmol} / \mathrm{L}$ no alcanzó significación estadística. Cabe destacar que las concentraciones más bajas de 25(OH)D y la DMAE son dos hallazgos frecuentes en adultos mayores. Lanzan la hipótesis de que las concentraciones más bajas de VitD, pueden contribuir a la DMAE; sin embargo, se necesitan más estudios prospectivos de observación e intervención bien diseñados, para una mejor comprensión de la participación de la VitD en la aparición y progresión de la misma. Presenta una serie de limitaciones por la heterogeneidad y tamaño relativamente pequeño de los estudios, duración de la deficiencia de VitD, así como la heterogeneidad en la evaluación de los resultados, lo que hace tener baja potencia estadística.

Epstein et al, 2017 (45) ( $\mathrm{n}=216,72$ casos y 144 controles), plantean la hipótesis de que los pacientes con oclusión de la vena central de la retina tienen mayor deficiencia de VitD, en comparación con controles sanos. No encontraron diferencias significativas de valores de VitD entre ambos grupos.

Goncalves et al, 2015 (46) ( $\mathrm{n}=314,150$ casos y 164 controles), analizan si pacientes con glaucoma primario de ángulo abierto (GPAA) presentan menor concentración de nivel sérico de VitD frente a controles sanos. Los resultados muestran que una insuficiencia en VitD, se asocia directamente con el diagnóstico de GPAA ( $\mathrm{p}=0.034)$, pero no con la severidad del proceso. Los mismos autores reflejan que, aunque los hallazgos cumplían los criterios de causalidad, no pudieron responder a los problemas de temporalidad ni especificidad.

No se identificaron ECAs elegibles.

\section{Resumen: Enfermedades oculares}

En población general, no se puede establecer una asociación causal entre el bajo nivel de $25(\mathrm{OH})$ D y la oclusión de la vena central de la retina (OVRC) ni con el glaucoma primario de ángulo abierto.

Por otro lado, las concentraciones de 25(OH)D por debajo de $50 \mathrm{nmol} / \mathrm{L}$ se podrían asociar con la degeneración macular tardía asociada a la edad.

Calidad de la evidencia MUY BAJA $\oplus \circ \circ \bigcirc$ 
Vitamina D y enfermedad renal

En una búsqueda específica para enfermedad renal posterior a la actualización y sugerida por el grupo de autores de este OPBE, se identificaron dos estudios $(47,48)$.

Por un lado, Izumaru et al, 2014 (47), cuyo objetivo es determinar si la deficiencia de VitD se asocia con un mayor riesgo de insuficiencia renal. Población $\geq$ a 40 años ( $\mathrm{n}=2417,988$ hombres y 1429 mujeres) que presenta un seguimiento de 5 años. El estudio es una cohorte prospectiva en población japonesa que participa en una encuesta de salud sobre enfermedad cardiovascular. Los autores encuentran una relación significativa entre el nivel de suero de 1,25(OH)D2 más bajo y un mayor riesgo para el desarrollo de la etapa 3-5 de la enfermedad renal crónica.

La otra publicación seleccionada, realizada por Sahin et al 2014 (48), es un estudio de casos y controles con reclutamiento prospectivo $(n=403$ sujetos). De ellos, 74 presentaban nefropatía inducida por contraste que constituyen los casos y 329 sin nefropatía por contraste, los controles. Está publicado en Turquía y presenta como objetivo investigar si existe relación entre el déficit de VitD y la nefropatía inducida por contraste en pacientes sometidos a angiografía coronaria. Los autores señalan una asociación del déficit de VitD con una mayor incidencia de la nefropatía por contraste. Los mismos autores concluyen con la necesidad de realizar más estudios para confirmar el grado de la asociación.

Ambos estudios presentan riesgo de sesgo crítico, al presentar solo una determinación de VitD, análisis de poblaciones secundarias y pocos pacientes incluidos, lo que disminuye el poder estadístico.

No se localizaron ECAs.

\section{Resumen: Enfermedad renal}

Al margen de la asociación entre valores de vitamina $\mathrm{D}$, hiperparatiroidismo y proteinuria, en población general, la evidencia de que el déficit de vitamina D contribuya significativamente al desarrollo de enfermedad renal es escasa para establecer conclusiones. No obstante, esta apunta a la existencia de una relación inversa entre niveles de vitamina D y enfermedad renal. Calidad de la evidencia MUY BAJA $\oplus$ MOO

Vitamina D y otros eventos

\section{Depresión}

La Task Force (4) incluía los siguientes estudios para la depresión: 
- Dos revisiones sistemáticas $(148,151)$, que concluyen que la evidencia sugiere una disminución del riesgo de depresión y desórdenes del estado anímico con concentraciones altas de 25(OH)D.

- Dos estudios $(161,162)$ que sitúan los valores óptimos entre $21-30 \mathrm{ng} / \mathrm{ml}$.

En la búsqueda posterior solo se incluye un estudio anidado de cohortes (49) con riesgo de sesgo moderado. En este caso se evalúa si los valores séricos de $25(\mathrm{OH}) \mathrm{D}$ y $1,25(\mathrm{OH}) \mathrm{D}$ pueden predecir la remisión y el curso de la depresión, en pacientes entre 60 y 93 años, diagnosticados de depresión $(\mathrm{n}=378)$. No encuentran asociación significativa entre los valores de $25(\mathrm{OH}) \mathrm{D}$ o los de $1,25(\mathrm{OH}) \mathrm{D}$, ajustados o no ajustados a covariables, aunque refieren una tendencia menor de remisión de la depresión en sujetos con niveles muy bajos de VitD. El trabajo presenta poca potencia, y no está estratificado y ajustado por factores de confusión como la actividad física, la exposición al sol o la ingesta de VitD.

No se identificaron ECAs elegibles.

\section{Resumen: Riesgo de depresión}

En población general, los estudios muestran diferentes resultados que no permiten establecer una conclusión general sobre el problema.

Calidad de la evidencia BAJA $\oplus \oplus \circ 0$

\section{Tuberculosis}

Se localizó una publicación de 2017 de caso-control (50) ( $\mathrm{n}=805$ casos y n $=16049$ controles), con diseño transversal basado en los resultados de una encuesta poblacional de salud. El objetivo es investigar la prevalencia del déficit de VitD en sujetos con antecedentes de tuberculosis, frente a sujetos sin antecedentes. No se encuentran diferencias de prevalencia de déficit de VitD entre los dos grupos y en ambos grupos el tamaño de la población con déficit era alto. El análisis de las características basales entre los grupos daba diferencias estadísticamente significativas a nivel de sexo, edad, ocupación, hábito de fumar, IMC o precepción del estado de salud; además, dentro de cada uno de los grupos también había diferencias entre población con déficit y sin déficit de VitD a nivel de las mismas variables.

El estudio presenta riesgo de sesgo serio y no se han localizaron ECAs. 
Resumen: Tuberculosis

La VitD tiene efectos importantes en las células del sistema inmune. Los macrófagos y los linfocitos T y B expresan el receptor de la VitD. En población general, hay pocos estudios sobre valores plasmáticos de VitD y la posible asociación a tuberculosis.

Calidad de la evidencia MUY BAJA $\oplus$ MOO

\section{Dismenorrea}

Se recuperó un estudio reciente (2018) de casos y controles con reclutamiento prospectivo $(n=184$ casos y 184 controles $)$ (51). Está publicado en Turquía y presenta como objetivo, investigar el posible papel del déficit de VitD, en la dismenorrea primaria en mujeres entre 18 y 25 años en comparación con mujeres sanas. Los autores señalan una correlación significativa y positiva entre los valores bajos de $25(\mathrm{OH}) \mathrm{D}$ y la dismenorrea primaria. Las limitaciones del trabajo hacen difícil su extrapolación, ya que solo se realiza una única toma de muestras de VitD y no se tienen en cuenta las variaciones estacionales. Además, los grupos presentan diferencias estadísticamente significativas $(\mathrm{p}=0.001)$ en cuanto a las características basales, así el grupo de casos muestra mayor nivel de mujeres con depresión, irritabilidad, fatiga, dolor de cabeza y cambios en el apetito, entre otras variables. Pero también existían diferencias significativas dentro del grupo de casos entre las mujeres con déficit y no déficit de VitD.

El estudio presenta riesgo de sesgo serio. No se localizaron ECAs.

\section{Resumen: Dismenorrea}

En mujeres sanas, aunque la evidencia es escasa y no permite establecer conclusiones, esta apunta a la existencia de una relación inversa entre los valores bajos de VitD y la dismenorrea primaria.

Calidad de la evidencia MUY BAJA $\oplus \circ 0 \bigcirc$

\section{Alergias}

En la actualización solo se identificó una publicación, Vatankhah $\mathrm{V}$ et al, 2016 (52), que determina el nivel medio de VitD en pacientes con rinitis alérgica frente a grupo control ( $n=54$ casos, $n=54$ controles $)$. El estudio es un caso-control en población iraní que acude al servicio de otorrinolaringología. Los autores no encuentran una relación entre tener rinitis y presentar un nivel bajo de VitD en el total de los sujetos $(\mathrm{n}=0.88)$; sin embargo, cuando se analiza por sexo, solo las mujeres presentan diferencias en niveles de $25(\mathrm{OH}) \mathrm{D}$ entre grupos $(\mathrm{p}=0.001)$. 
El estudio presenta riesgo de sesgo serio, al no ajusta por distintas variables de confusión importantes, el tamaño muestral es pequeño y se realiza solo una medida del nivel sérico de 25(OH)D.

No se localizan ECAs.

Resumen: Riesgo de alergias

En población general, no se puede establecer una asociación causal entre el nivel 25(OH)D y la rinitis alérgica.

Calidad de la evidencia MUY BAJA $\oplus \circ 0 \bigcirc$

\section{Enfermedad de Crohn}

Se localizó una RS con metanálisis (53), que presenta una calidad metodológica críticamente baja, medida con el instrumento de criterios del AMSTAR-2 (146), al presentar más de una debilidad crítica. El objetivo era realizar un metanálisis que evaluara la $25(\mathrm{OH}) \mathrm{D}$ y la $1,25(\mathrm{OH}) \mathrm{D}$ en pacientes con enfermedad de Crohn en comparación con controles sanos. La revisión incluye un total de 58 estudios en cuatro metanálisis:

- Uno sobre la diferencia media de los valores de 25(OH)D en pacientes con enfermedad de Crohn $\left(\mathrm{n}^{\circ}\right.$ estudios $\left.=27\right)$ en comparación con controles sanos $(\mathrm{n}=25)$

- Otro realizando el mismo cálculo, pero con los valores de $1,25(\mathrm{OH})_{2} \mathrm{D}_{3}$ en pacientes con enfermedad de Crohn $(\mathrm{n}=7)$ en comparación con sanos $(\mathrm{n}=8)$

- Un metanálisis sobre la prevalencia de deficiencia de VitD $(n=34)$

- Y por último uno sobre el coeficiente de correlación entre la gravedad de la enfermedad de Crohn y el nivel de VitD $(n=6)$.

Los autores concluyen que los pacientes con enfermedad de Crohn presentan valores más bajos en comparación con controles sanos y muestran una correlación entre las concentraciones de VitD y la gravedad de la enfermedad.

Debe considerarse, una vez más, las limitaciones del trabajo, por su diseño (estudios observacionales), y la falta de datos, al no poder realizar un análisis de subgrupos por temporada o actividad de la enfermedad.

En la búsqueda actual no se localizó ningún ECAs. 
Resumen: Enfermedad de Crohn

La revisión sistemática concluye que los resultados sugieren una asociación entre los valores bajos de VitD y la enfermedad de Crohn. Sin embargo, hay que tener en cuenta que la alimentación de sujetos con enfermedad de Crohn es deficiente, restrictiva y selectiva lo que no permite establecer una relación causal.

Calidad de la evidencia MODERADA $\oplus \oplus \oplus \mathrm{O}$

\section{Fragilidad en el adulto mayor}

Posterior a la revisión de partida se localizó una revisión sistemática realizada por Zhou et al, 2016 (54) que presenta como objetivo efectuar una estimación de la relación entre el nivel bajo de VitD y la fragilidad (pérdida de peso, debilidad, agotamiento, etc.) en personas mayores. Incluye un total de siete estudios de cohortes prospectivos con un total de 17815 sujetos, de los cuales 9518 pertenecían a seis estudios que incluían población mayor o igual a 65 años y los otros 8297 sujetos restantes pertenecientes al otro estudio que incluía población entre 50 y 74 años. La revisión señala una asociación significativa entre bajas concentraciones séricas de $25(\mathrm{OH})$ $\mathrm{D}$ en grupos definidos por escala o criterios de Freid, pero no por índice o indicadores de fragilidad. No se identificaron ECAs, ni en la revisión ni en nuestro informe.

La revisión presenta varias limitaciones, como el uso de distintas definiciones de fragilidad y el número insuficiente de estudios para poder realizar un análisis por subgrupos, además presentan distintos umbrales para la definición de déficit de VitD y la asociación es compleja debido a que los sujetos tienen más problemas para exponerse al sol.

No se encontraron ECAs posteriores. La revisión presenta una calidad metodológica críticamente baja, medida con el instrumento de criterios del AMSTAR-2 (146), al presentar más de una debilidad crítica.

\section{Resumen: Fragilidad en el adulto mayor}

En población general sana, los estudios observacionales sugieren una asociación entre estatus deficiente de VitD y la debilidad muscular en personas mayores.

Calidad de la evidencia BAJA $\oplus \oplus \circ \bigcirc$ 


\subsection{Pregunta 2: ¿Cuál es la efectividad de la vitamina $D$, en variables duras/finales, incluyendo enfermedades cardiovasculares, obesidad, cáncer, sistema inmunitario, fractura osteoporótica, insuficiencia renal y/o la calcificación de los tejidos blandos?}

Se ha formulado una pregunta de investigación en formato PICO (Patient, Intervention, Comparator, Outcome) que podemos ver en la tabla 10.

\section{Tabla 10. Desarrollo pregunta PICO, pregunta 2}

\begin{tabular}{l|l}
\hline $\begin{array}{l}\text { Población } \\
\text { diana }\end{array}$ & Población general adulta sana \\
\hline Intervención & Suplemento de vitamina D \\
\hline Comparación & No suplemento de vitamina D \\
\hline Resultados & $\begin{array}{l}\text { Resultados en salud: mortalidad, cáncer, fracturas, caídas, enfermedad } \\
\text { cardiovascular, diabetes, depresión, funcionamiento cognitivo, estado } \\
\text { funcional, etc. }\end{array}$ \\
\hline
\end{tabular}

Fuente: elaboración propia.

Como para la primera pregunta, se tomó como punto de partida el documento realizado por la Task Force (7) y publicada en 2014.

Para esta pregunta se incluyeron ECAs que examinaban la efectividad de la VitD en resultados de salud, encontrando trabajos sobre mortalidad, fracturas, caídas, diabetes, función psicosocial, discapacidad y funcionamiento físico.

La búsqueda bibliográfica realizada en diciembre de 2018 sobre la actualización del documento de partida (4) y la posterior actualización en 2019, arrojó, tras eliminar duplicados, un total de 1739 referencias. Se seleccionaron 102 para su lectura a texto completo. De ellos se han incluido 66 (55 ECAs y $11 \mathrm{RS}$ ) que cumplieron los criterios de inclusión vistos en la tabla 11. En la tabla 12 se pueden ver los resultados de la búsqueda según la fuente consultada. 
Tabla 11. Criterios de inclusión y exclusión de estudios, pregunta 2

\begin{tabular}{|l|l|}
\hline Aspecto considerado & Criterios de inclusión/exclusión \\
\hline Diseño & $\begin{array}{l}\text { Inclusión: ECAs, posteriores al } 2014 . \\
\text { Exclusión: estudios con menor nivel de evidencia de los incluidos en } \\
\text { documento de partida }\end{array}$ \\
\hline Tamaño muestral & No se establecieron limitaciones sobre el tamaño muestral \\
\hline Variables de resultado & $\begin{array}{l}\text { Datos relativos a la salud en términos de: mortalidad, cáncer, } \\
\text { fracturas, caídas, enfermedad cardiovascular, diabetes, depresión, } \\
\text { funcionamiento cognitivo, estado funcional, etc. }\end{array}$ \\
\hline Idioma & $\begin{array}{l}\text { Inclusión: castellano, inglés, francés, portugués e italiano. } \\
\text { Exclusión: otros idiomas. }\end{array}$ \\
\hline Límite temporal & Inclusión: 2014. \\
\hline
\end{tabular}

Fuente: elaboración propia.

Tabla 12. Bases de datos electrónicas de la literatura biomédica, pregunta 2

\begin{tabular}{|l|c|}
\hline Bases de datos & Resultados \\
\hline Cochrane Library Trials (Wiley) & 785 \\
\hline Pubmed (Medline) & 859 \\
\hline Embase (Ovid) & 476 \\
\hline CRD database & 47 \\
\hline Total & 1739 \\
\hline Seleccionados lectura texto completo & 102 \\
\hline Incluidos & 68 \\
\hline
\end{tabular}

Fuente: elaboración propia.

\subsubsection{Resultados de la efectividad del suplemento de vitamina D en salud}

Resumimos los 57 ECAs y las 11 revisiones sistemáticas y metanálisis de ECAs posteriores a la revisión de partida. La evidencia disponible se centró, principalmente en enfermedades cardiovasculares, fragilidad y riesgo de diabetes.

Vitamina $D$ y riesgo de cáncer

La revisión de partida (4) presenta en este apartado dos ECAs $(163,164)$. Los datos limitados de los estudios, muestran que no se observaban asociaciones generales con el tratamiento con VitD en cánceres de mama y colorrectal. 
Para este apartado se incluyeron una revisión sistemática (56) y 3 ECAs $(55,57,58)$, posteriores a la revisión de partida, que investigan la asociación entre los suplementos de VitD y los eventos o riesgos de cáncer.

Con respecto a la revisión sistemática de ECAs con metanálisis, realizada por Bjelakovic et al 2014 (56) presenta una calidad metodológica alta, medida con el instrumento de criterios del AMSTAR-2 (146). Incluye 18 ECAs (16 ECAs con riesgo de sesgo medio y 2 ECAs con riesgo bajo) y un total de 50623 sujetos adultos con o sin déficit de VitD. El objetivo es analizar los efectos beneficiosos o perjudiciales de la suplementación de la VitD en la prevención del cáncer. Los autores no encuentran diferencias de beneficio o riesgo respecto a cáncer, pero si una disminución de mortalidad tanto por cáncer como por cualquier causa. Sin embargo, hay que tener en cuenta el riesgo de sesgo que presentan la mayoría de los estudios incluidos y el no alcance del tamaño requerido para el análisis.

Los tres ECAs encontrados $(55,57,58)$ presentan distinto riego de sesgo, dos estudios con bajo riesgo $(57,58)$ y uno con riesgo de sesgo alto (55). El riesgo de sesgo se halló según la "Herramienta y criterios de evaluación de riesgo de sesgo para ECAs de la Colaboración Cochrane" (165).

Todos los estudios, se realizan en población adulta, dos incluyen ambos sexos $(55,58)$ y otro solo mujeres postmenopáusicas sanas $(57)$. El tamaño muestral es variado, con 25871 en el estudio de Manson et al 2019 (58) y sobre los 2000 sujetos en los otros dos estudios. Todos administran el suplemento de forma diaria con dosis entre 1000 y 2000 UI/día, además los tres suplementan con calcio. El tiempo de seguimiento se sitúa entre los tres y cinco años. Los resultados son similares en los tres estudios: Manson et al 2019 (58) no encuentra reducción de incidencia de cáncer, ni reducción de mortalidad total o por cáncer, a diferencia de la revisión de Bjelakovic et al 2014 (56). Lappe et al 2017 (57) tampoco encuentra reducción de riesgo de cáncer y Baron et al (55) tampoco halla que el suplemento de VitD disminuya el riesgo de aparición de adenomas colorrectales en población que ya los ha tenido.

En población general, los estudios, de forma general, no mostraron que exista una menor incidencia de cáncer al administrar suplementos de VitD.

Calidad de la evidencia ALTA $\oplus \oplus \oplus \oplus$ 
Vitamina D y enfermedad cardiovascular

La revisión de partida (4) no hacía comentarios sobre este apartado. Posteriormente se localizaron tres RS $(62,66,68)$ y 9 ECAs $(58-61,63-65$, $67,69)$, que investigan la asociación entre las concentraciones séricas de $25(\mathrm{OH}) \mathrm{D}$ y el riesgos o los eventos cardiovasculares.

Con respecto a las revisiones sistemáticas de ECAs con metanálisis, la calidad metodológica se midió mediante el instrumento de criterios del AMSTAR-2 (146). Dos presentaban calidad metodológica baja $(62,68)$, con mayor número de estudios incluidos (30 y 8 respectivamente) y otra de calidad críticamente baja (66), al presentar una o más de una debilidad no crítica, con un total de siete estudios incluidos.

Dos de las revisiones $(62,68)$ informaron que no hubo efecto significativo sobre la presión arterial (PA) diastólica (PAD) o sistólica (PAS) en sujetos con o sin déficit de VitD. Sin embargo, la revisión de Shu et al 2018 (66), encontraba que pacientes con deficiencia de VitD y una suplementación suficiente podían reducir la PA periférica, mientras no influía en la PA central.

Los nueve ECAs encontrados $(58-61,63-65,67,69)$ presentan distinta calidad metodológica, con seis estudios de bajo riesgo de sesgo $(58,60,61$, 63-65), uno con riesgo de sesgo medio (67) y dos con riesgo de sesgo alto (59, 69). Como en el caso anterior y en el análisis futuro los riesgos de sesgos de ECAs se halló según la "Herramienta y criterios de evaluación de riesgo de sesgo para ECAs de la Colaboración Cochrane" (165).

Todos los estudios, excepto dos, se realizan en población que incluía ambos sexos. El rango de sujetos era muy variado desde 25871 a 40 personas, siendo muy heterogéneo (déficit o no de VitD, sobrepeso, prediabetes, hipertensos, raza, etc.), los estudios no solo son heterogéneos en la población incluida, sino también en otras características como la dosis administrada (alta o baja), régimen de dosis (diario, semanal o mensual) o el tiempo de seguimiento, que va desde los dos a los 60 meses. Aun así, siete de los nueve ECAs, concluyen que el uso de suplementos de VitD no modifica la incidencia o el riesgo de ECV. Solo dos estudios $(59,69)$, ambos con el menor número de sujetos incluidos 40 y 41 respectivamente, en sujetos con déficit de VitD, y con riesgo de sesgo alto, encontraban una disminución significativa del riesgo de eventos cardiovasculares, en el caso de Abdulricha et al 2018 (59), y un beneficio en la rigidez arterial, aunque no en la disminución de la PA en el caso de Zaleski et al 2015 (69). 
Resumen: Enfermedad cardiovascular

En población general, los resultados obtenidos en los estudios no avalan la indicación de tratamiento con VitD para modificar la incidencia de enfermedades cardiovasculares en población general.

Calidad de la evidencia MUY BAJA $\oplus \circ 0 \bigcirc$

Vitamina D y la salud ósea

\section{Fracturas y caídas}

En este apartado la RS de la Task Force (4) incluyó los siguientes estudios:

- Fracturas: cuatro ECAs de calidad moderada (166-169) y un estudio de casos y controles anidado (170). Los autores concluyen que ninguno de los estudios mostró una reducción significativa en el riesgo de fracturas cuando se aplicaron dosis de $\mathrm{VitD}_{3}$ de $400 \mathrm{a}$ 800 IU por día desde dos meses a siete años, con o sin calcio. No se observó riesgo para ninguno de los subgrupos evaluados.

- Caídas: incluyeron siete ECAs de calidad moderada (166, 168, 169, 171-173), donde evaluaron el efecto de dosis de 800 IU/día (con o sin calcio) durante dos a 36 meses o el efecto de dosis de 400-1000 IU/día (con o sin calcio). El RR global obtenido en los ECAs que aplicaron dosis más altas, con o sin calcio, no fue significativo, aunque los resultados fueron muy heterogéneos variando entre reducciones del $22 \%$ hasta aumentos del $2 \%$. Sin embargo, se observó una disminución significativa en el riesgo de caídas por individuo en los cinco ECAs que aplicaron dosis más bajas.

En la búsqueda posterior se incluyeron 3 revisiones sistemáticas (70,72, 73) y 1 ECA (71), que analizan la asociación entre el suplemento de VitD y el riesgo de caída o fractura. La calidad metodológica de las revisiones según el instrumento AMSTAR-2, es alta en dos de ellas $(70,72)$ y una puntuación baja en la revisión realizada por Kahwati et al 2018 (73) al presentar una debilidad crítica. El ECA localizado elaborado por Cangussu et al 2016 (71) presenta bajo riesgo de sesgo según la herramienta de la Colaboración Cochrane de evaluación del riesgo de sesgo de ECAs (165).

Dos de las revisiones fueron elaboradas para la organización de la US Prevencive Services Task Force, con distinto objetivo. Guirguis-Blake et al (72) para la prevención de caídas y Kahwati et al 2017 (73) en la de 
fracturas, ambos en población general adulta que vive en la comunidad. La otra revisión incluida está elaborada por Bolland et al 2018 (70) que analiza además de las caídas y las fracturas, la DMO.

La RS realizada por Bolland et al 2018 (6) incluye un total de 81 ECAs (fracturas 42 ECAs, caídas 37 ECAs y DMO 41 ECAs) con un total de 53537 sujetos. La revisión analiza sujetos con o sin déficit de vitamina D que reciben suplemento de VitD vs sujetos no tratados o tratados con dosis bajas $(<800 \mathrm{UI} /$ día). Los estudios presentan distinta dosis y formas de suplementación de VitD administrada, así como distinto período de seguimiento, población, etc. De forma general los estudios se realizaban en mujeres de población general con monoterapia. El análisis agrupado de la suplementación de VitD no aporta un efecto beneficioso tanto en el riesgo total de fracturas, como de caídas. Tampoco se encontraron diferencias significativas en la DMO en las distintas localizaciones analizadas. Los autores concluyen que hoy día no son necesarios nuevos estudios y que no se justifica el uso de suplemento de VitD para mejorar la salud musculoesquelético en población general.

La RS para evaluar el riesgo de distintas intervenciones en la prevención de caídas, elaborada por Guirguis-Blake et al (72), en el caso de la suplementación con VitD, incluye siete ECAs con 7531 sujetos 65 años o más, de la población general. Los estudios incluidos muestran resultados mixtos, sin embargo, cuando se realiza el análisis agrupado no se encuentra asociación significativa entre el suplemento de VitD y las caídas en cualquiera de los análisis, tanto en población general como en población que ya ha sufrido al menos una caída. A raíz de esta revisión, la organización de la US Prevencive Services Task Force, en una publicación posterior (174) no recomienda administrar suplementos de VitD para prevenir las caídas en adultos mayores de 65 años que viven en comunidad (grado de recomendación D).

En el caso de la revisión realizada por Kahwati et al 2018 (73) para evaluar el efecto del suplemento de VitD, sola o con calcio para la prevención de fracturas, los autores incluyeron un total de once ECAs con 51419 sujetos de población adulta mayor de 50 años, sin déficit de VitD y que viven en la comunidad. Como en los estudios incluidos en las revisiones anteriores, estos presentan distinta dosis, seguimiento, etc. En este caso, diez de los once ECAs no encuentran diferencias en la incidencia de las fracturas entre los grupos con suplemento de VitD vs placebo. Como en el caso de la revisión de Guirguis-Blake et al (72), a raíz de esta revisión la organización de la $U S$ Prevencive Services Task Force, en una publicación posterior (175) muestra una serie de recomendaciones por consenso: 
- La evidencia actual es insuficiente para evaluar el balance riesgo/ beneficio de los suplementos de VitD y calcio, solos o combinados en la prevención de fracturas primarias en hombres asintomáticos y mujeres premenopáusicas (recomendación por consenso)

- La evidencia actual es insuficiente para evaluar el balance riesgo/ beneficio de la suplementación diaria con dosis mayores 400 UI de VitD y $1000 \mathrm{mg}$ de calcio para la prevención primaria de fracturas en mujeres postmenopáusicas (recomendación por consenso)

- No se recomienda la suplementación diaria con 400 UI de VitD o menos y $1000 \mathrm{mg}$ de calcio o menos para la prevención primaria de fracturas en mujeres postmenopáusicas que viven en comunidad.

Por otro lado, el ECA (71) localizado, cuyo objetivo es evaluar las caídas o el control del equilibrio postural, como efecto de la suplementación de VitD vs placebo en 160 mujeres postmenopáusicas, con dosis de 1000 UI/día y un seguimiento de 9 meses, encuentra que la suplementación con VitD en mujeres con antecedentes de caídas es un factor protector de las caídas y mejora el equilibrio postural. Sin embargo, aunque el riesgo de sesgo es bajo, el ECA presenta distintas limitaciones como el sesgo del recuerdo, ya que las caídas se contabilizan al final del estudio mediante entrevista personal.

\section{Resumen: Fracturas y caídas}

Las publicaciones revelaron que los suplementos de VitD no muestran una reducción significativa en el riesgo de caídas o fracturas en la población general adulta.

Calidad de la evidencia ALTA $\oplus \oplus \oplus \oplus$

\section{Evidencia complementaria}

La pregunta 2 solo hace referencia a variables duras/finales, sin embargo, se consideró la inclusión del metabolismo óseo (variable subrogada), como información adicional a la salud ósea.

\section{- Metabolismo óseo}

Posterior a la revisión de Task Force (4), se han localizado cuatro ECAs (74-77) que cumplen los criterios de inclusión. Están publicados entre 2014 y 2018, tres de ellos con bajo riesgo de sesgo (74-76) y uno, el realizado por Trautvetter et al (77), que es el más antiguo, el de menor tamaño, menor seguimiento y el único que incluye ambos sexos, con riesgo poco claro o dudoso (riesgo medio). El riesgo de sesgo se calculó con la "Herramienta 
y criterios de evaluación de riesgo de sesgo para ECAs de la Colaboración Cochrane" (165).

Para la evaluación de la salud ósea los estudios consideran, dos de ellos $(74,76)$ el índice de densidad mineral ósea (DMO) y los otros dos $(75$, 77) el efecto en marcadores de recambio óseo. Tres de los estudios (74-76) se realizan en mujeres mayores de 50 años y solo el estudio de Trautvetter et al (77), como comentamos anteriormente, incluía ambos sexos. El rango de sujetos incluidos es variado y se encuentra entre 60 y 260 personas. Las dosis administradas de VitD también varía entre los estudios, aunque todos realizan una administración diaria, algunos son de baja y otros de alta dosis $\mathrm{y}$ todos frente a placebo. El rango de seguimiento va desde las ocho semanas a los tres años.

Los resultados de estos estudios, que consideran el efecto de la administración de suplementos de VitD en los índices de salud ósea, no encuentran una asociación significativa. Solamente, Nahas-Neto et al (75), donde las mujeres habían sido reclutadas para otro trabajo (análisis secundario), hallan que podría producirse una reducción en los marcadores de recambio óseo en el grupo con suplemento de VitD.

\section{Resumen: Metabolismo óseo}

Con los datos actuales los suplementos de VitD en mujeres mayores de 50 años no modifican los marcadores de remodelado óseo.

Calidad de la evidencia MODERADA $\oplus \oplus \oplus \circ$

\section{Vitamina D y su efecto en la fuerza y función muscular}

La Task Force en su RS (4) incluyó en este apartado un ECA de calidad moderada (176) que examina la administración de 16 dosis semanales de 8400 IU, sin observar mejoras en el funcionamiento físico.

En la actualización se incluyeron ocho ECAs (78-85) que muestran distinto riesgo de sesgo, así cinco presentan bajo riesgo de sesgo $(78,81,82$, $84,85)$, dos riesgo de sesgo medio $(80,83)$ y uno alto riesgo $(79)$.

Cinco $(78-80,83,85)$ de los estudios se realizan solo en mujeres postmenopáusicas, de los otros tres, uno en varones jóvenes (82), otro en minorías étnicas (81) y el llevado a cabo por Vaes et al 2018 (84) en población general ( $\geq 65$ años) y con nivel prefrágil o frágil según los criterios de fragilidad de Fried. El rango de sujetos incluidos en los estudios varía entre 78 y 461, siendo heterogéneos a nivel de déficit o no de VitD, dosis administrada (alta o baja), régimen (diario, semanal o quincenal) o el 
tiempo de seguimiento que va desde las seis semanas a los dos años. Aun así, de los ocho ECAs, siete (79-85) concluyen que el uso de suplementos de VitD no modifica la función, la fuerza muscular o el rendimiento físico, independientemente del aumento del nivel de $[25(\mathrm{OH}) \mathrm{D}]$ alcanzado. Por tanto, no apoyan el uso de suplementos de VitD para incrementar la fuerza muscular.

Solo el estudio realizado por Cangussu et al 2015 (78) en Brasil en 160 mujeres postmenopáusicas y nueve meses de seguimiento encontraba que la suplementación es un protector frente a la sarcopenia, aumentando la fuerza muscular. Este estudio presenta la limitación de no haber realizado análisis comparado, solo se evaluaron diferencias, con respecto a nivel basal de VitD en sangre, de la fuerza muscular en los grupos.

\section{Resumen: Fuerza y función muscular}

Existe una gran heterogeneidad en los estudios. Aunque la evidencia es mixta, en general, sugiere una falta de efecto de la suplementación de VitD sobre la función y fuerza muscular, tanto en sujetos con déficit de VitD como sin él. El mayor número de estudios se han realizado en mujeres postmenopáusicas.

Calidad de la evidencia BAJA $\oplus \oplus \circ \bigcirc$

\section{Vitamina D y su efecto sobre la mortalidad}

La Task Force (4) incluye en el 2014 un ECA de buena calidad (177), distintos ECAs de calidad moderada $(166,167,176,178-183)$ y un estudio de casos y controles anidado (184). Ninguna de las publicaciones mostró una reducción significativa en la mortalidad en población general con el tratamiento de $\mathrm{VitD}_{3}$ (dosis de $400 \mathrm{IU} /$ día a $40000 \mathrm{IU}$ por semana, con o sin calcio). Las estimaciones en algunos de los ensayos fueron muy imprecisas. En el análisis agregado el tratamiento con $\mathrm{VitD}_{3}$, solo o con $\mathrm{Ca}$, se asoció a una disminución en el riesgo versus placebo. En el análisis por subgrupos, el riesgo solo fue significativo para personas mayores de 70 años institucionalizadas.

Posterior a la revisión de partida se localizó una RS de Cochrane publicada por Bjelakovic et al 2014 (86) que evalúa el impacto sobre la mortalidad de la suplementación de VitD.

La revisión (86) incluye ECAs y realiza metanálisis. La calidad metodológica se midió mediante el instrumento de criterios del AMSTAR-2 (146) presentando un valor metodológico alto, sin embargo, los ECAs incluidos presentaban altas tasas de abandono, lo que aumenta el riesgo de sesgo de los estudios. Incluye un total de 56 ECAs con 95286 adultos sanos o con enfermedad en fase estable y con o sin déficit de VitD. Los resultados 
revelan que el suplemento de VitD3 podría conllevar una disminución de la mortalidad en personas mayores, mientras la VitD2 o las formas activas no tuvieron efectos sobre ella. Los propios autores de la revisión, recomiendan tomar los resultados con cautela, debido a la gran cantidad de abandonos que se producen en los ECAs incluidos, que pueden alterar los resultados.

\section{Resumen: Mortalidad}

No hay evidencias que sostengan que la administración de VitD disminuya la mortalidad en la población general.

Calidad de la evidencia MODERADA $\oplus \oplus \oplus \mathrm{O}$

Vitamina D y su efecto en el dolor

La Task Force (4) no incluye estudios sobre este apartado. Se localizaron una revisión sistemática Cochrane (88) y un ECA (87), que investigan la asociación entre los suplementos de VitD y la disminución del dolor.

La revisión sistemática de Straube et al 2015 (88), es una actualización de una RS Cochrane del año 2009. Incluye un total de diez ECAs, seis pertenecientes a la actualización y cuatro a la revisión de partida, con un total de 811 sujetos. La calidad metodológica se midió mediante el instrumento de criterios del AMSTAR-2 (146) presentando un valor medio. El objetivo es evaluar la eficacia y seguridad de la suplementación de VitD vs placebo en el dolor crónico. Debido a las limitaciones de los ECAs incluidos la evidencia debe considerarse incompleta, por ello, de momento, no existe evidencia de beneficio en el dolor crónico en adultos, con la suplementación con VitD.

El ECA realizado por Knutsen et al 2014 (87) presenta como objetivo evaluar si los suplementos de VitD reducirían el dolor musculoesquelético y de cabeza en población adulta sana específica de grupos étnicos minoritarios, que viven en Noruega. Presenta un bajo riesgo de sesgo según la herramienta de la Colaboración Cochrane de evaluación del riesgo de sesgo de ECAs (165). Incluye un total de 251 participantes, con un seguimiento de 16 semanas, observando que no existe reducción en la puntuación de las escalas de dolor

Resumen: Dolor

Hasta el momento no existe un beneficio claro sobre la suplementación de VitD en el dolor crónico en adultos.

Calidad de la evidencia ALTA $\oplus \oplus \oplus \oplus$ 
La Task Force (4) no incluye estudios sobre este apartado. Posteriormente a la revisión de partida se localizaron cinco ECAs que evalúan la asociación entre los suplementos de VitD y el efecto en el riesgo de infecciones.

Los cinco ECAs localizados $(89-91,93,94)$ presentan distinto riesgo de sesgo, tres con riesgo de sesgo medio (89-91), uno riesgo bajo (93) y otro con riesgo de sesgo alto (94).

Los estudios se realizan en poblaciones con distintas características, así, mientras dos de ellos $(89,91)$ se llevan a cabo en población mayor que vive en residencias de ancianos, otros dos realizan los estudios en población general sana $(93,94)$ y por último el estudio de Goodall et al 2014 (90) que incluye población joven (estudiantes $>18$ años). El rango de sujetos incluidos es variado y se encuentra entre 34 y 644 personas. En todos los estudios se administran altas dosis de VitD de forma semanal o mensual y todos realizan un seguimiento de un año, excepto el estudio de Simpson et al 2015 (93) donde es solo de cuatro meses.

Los resultados encontrados por los estudios apuntan en una misma dirección, los estudios revelan un efecto protector de la VitD frente las infecciones. Ginde et al 2017 (89) descubren una reducción de infecciones respiratorias agudas en sujetos con suplemento de VitD, sin embargo, también muestran un aumento de caídas, aunque no fracturas. Simpson et al 2015 (93) observan un efecto protector frente a las infecciones agudas en sujetos con déficit de VitD. Tran et al 2014 (94), por su parte comunican que dosis altas mensuales podrían reducir la necesidad de antibióticos en personas ancianas y Goodall et al (90) encuentran que la suplementación con VitD podría prevenir las infecciones del tracto respiratorio superior. El estudio llevado a cabo por Martineau et al 2015 (91) que analiza bolos altos intermitentes de VitD cada dos meses frente dosis diaria, hallan que los bolos intermitentes aumentan el riesgo de infecciones respiratorias.

Posterior a la búsqueda se localizó una revisión sistemática publicada en junio 2020 por la Scientific Advisory Committee on Nutrition (SACN) de Reino Unido. Cuyo objetivo es realizar una actualización del informe del 2016 sobre VitD y salud (92), solo en lo referente a las infecciones respiratorias agudas y VitD, y ver si cambia sus conclusiones anteriores. Los autores concluyen que, de forma general, la evidencia en este momento no respalda recomendar suplementos de VitD para prevenir las infecciones respiratorias agudas en la población general del Reino Unido. La revisión 
presenta una calidad metodológica alta, medida con el instrumento de criterios del AMSTAR-2 (146)

\section{Resumen: Infecciones}

Las últimas publicaciones de mayor calidad muestran que no existe evidencia suficiente para respaldar la administración de VitD para reducción en el riesgo de infecciones del tracto superior en población general.

Calidad de la evidencia MODERADA $\oplus \oplus \oplus \mathrm{O}$

\section{Vitamina D y su efecto en los procesos inflamatorios}

La Task Force (4) no incluye estudios sobre este apartado. En la búsqueda se localizaron un total de cuatro estudios que abordaban los procesos inflamatorios en relación a la VitD, una revisión sistemática (96) y 3 ECAs $(95,97,98)$.

La revisión sistemática de Jamka et al 2016 (96) incluye ECAs y realiza metanálisis. La calidad metodológica se midió mediante el instrumento de criterios del AMSTAR-2 (146) presentando un valor bajo, dado que había varías debilidades críticas. Incluye un total de trece ECAs con 1955 sujetos con sobrepeso u obesos. Los resultados del metanálisis no muestran un efecto beneficioso de la administración de suplementos de VitD en los valores de biomarcadores inflamatorios.

Los tres ECAs encontrados $(95,97,98)$ presentan distinta calidad metodológica, dos estudios con riesgo de sesgo alto $(95,97)$ y uno con riesgo de sesgo medio (poco claro o dudoso) (98).

Los estudios presentaban distinta población, sujetos prediabéticos y con déficit de VitD vs personas mayores vs adultos sanos con o sin déficit. También diferían en dosis y forma de administración (diaria vs semanal vs mensual), así como en el número de sujetos con un rango entre 46 y 615. Llama la atención el tiempo de seguimiento del estudio de Barker et al 2015 (95) con solo 35 días, es el único estudio que concluye que el suplemento de VitD podría aumentar los niveles de citocinas proinflamatorias en población con bajo nivel 25(OH)D. Los otros dos ECAs no encuentran una relación significativa entre el suplemento de VitD y los valores de biomarcadores inflamatorios.

Resumen: Procesos inflamatorios

En población general sana, los estudios muestran que los suplementos de VitD no modifican los valores de biomarcadores inflamatorios.

Calidad de la evidencia MUY BAJA $\oplus \circ \bigcirc \bigcirc$ 
Vitamina $D$ y efectos en los niveles de testosterona en varones y en el antígeno prostático específico (PSA)

La Task Force (4) no incluye estudios sobre este apartado. Se han localizado tres ECAs, dos que analizan los valores de testosterona en varones $(82,100)$ y uno los niveles de PSA (99).

Los ECAs $(82,100)$ que analizan los efectos de la suplementación con VitD (dosis semanal) vs placebo, en las concentraciones de testosterona en varones sanos jóvenes o de mediana edad con valores normales o déficit de $25(\mathrm{OH}) \mathrm{D}$, con un seguimiento entre seis semanas y tres meses, no encuentran efectos significativos de la suplementación con VitD. Ambos ECAs presentan riesgo de sesgo bajo según la herramienta de la Colaboración Cochrane de evaluación del riesgo de sesgo de ECAs (165).

El estudio de Chandler et al 2014 (99), se trata de un ECA con nivel de riesgo de sesgo medio (poco claro o dudoso). El estudio evaluó los beneficios de los suplementos de VitD en los valores de PSA en varones de raza negra sin cáncer de próstata. Con un seguimiento de tres meses y un total de 105 sujetos que recibieron dosis diarias de VitD de 1000, 2000 o 4000 UI, no se encontró una asociación significativa entre la suplementación de VitD y los niveles de PSA.

Resumen: Niveles de testosterona en varones y en el antígeno prostático específico

El pequeño número de ECAs localizados no ha demostrado un efecto de los suplementos de VitD sobre la concentración de testosterona o el nivel de PSA en varones.

Calidad de la evidencia MUY BAJA $\oplus$ MO०

Vitamina D y su efecto en el tromboembolismo venoso

La Task Force (4) no incluye estudios sobre este apartado. Se localizó solo un ECA para su lectura a texto completo. El estudio (101) evalúa si la suplementación con VitD vs placebo podría reducir el riesgo de tromboembolismo venoso (TEV). Blondon et al 2015 (101), se trata de un análisis secundario a otro ECA anterior que evaluaba el cáncer colorrectal y las fracturas de cadera (WHI CaD trial). El nivel de riesgo de sesgo en base al primer artículo es bajo. El estudio se desarrolla en 36282 mujeres posmenopáusicas con o sin déficit de VitD que participan en el nuevo análisis, donde se suplementan con VitD más calcio de forma diaria (1000 mg de $\mathrm{Ca}+400$ UI $\mathrm{D}_{3}$ ) vs placebo durante aproximadamente siete años, con un seguimiento postintervención medio de cinco años. No se halló 
una reducción significativa del riesgo de TEV y por tanto no respaldan el uso de suplementos de $\mathrm{Ca}+\mathrm{VitD}$ con este fin.

\section{Resumen: Tromboembolismo venoso}

El ECA localizado no encuentra un efecto de los suplementos de VitD sobre la reducción de tromboembolismo venoso en mujeres postmenopáusicas.

Calidad de la evidencia MUY BAJA $\oplus$ MO०

\section{Vitamina D y función pulmonar}

La Task Force (4) no incluye estudios sobre este apartado. En la búsqueda solo hubo un estudio que cumpliera los criterios de inclusión. El estudio de Sluyter et al (67) publicado en 2017 con riesgo de sesgo poco claro o dudoso según la "Herramienta y criterios de evaluación de riesgo de sesgo para ECAs de la Colaboración Cochrane" (165). El análisis de un subgrupo de sujetos adultos (442 participantes) procedentes de un ECA mayor (ViDA) (102), realizado en Nueva Zelanda, que reciben altas dosis de suplemento de VitD durante un año, no encuentran mejora en la función pulmonar a nivel general. Un análisis de subgrupo de pacientes fumadores (incluyen exfumadores) con déficit de $25(\mathrm{OH}) \mathrm{D}<50 \mathrm{nmol} / \mathrm{L}$ o con asma o EPOC sí muestran una mejoría en la función pulmonar con el suplemento de VitD. Estos resultados hay que tomarlos con cautela por el número reducido de pacientes incluidos.

\section{Resumen: Función pulmonar}

Hasta el momento, en población general sana, no existe evidencia suficiente para extraer conclusiones.

Calidad de la evidencia MUY BAJA $\oplus \circ \circ \bigcirc$

\section{Vitamina D y su efecto en la función renal}

La Task Force (4) no incluye estudios sobre este apartado. Se localizaron tres ECAs para su lectura a texto completo en la búsqueda posterior a la revisión de partida que investigan la asociación entre las concentraciones séricas de 25(OH)D y la enfermedad renal (103-105).

Los ECAs localizados (103-105) presentan los tres bajo riesgo de sesgo según la Herramienta de la Colaboración Cochrane de evaluación de riesgos de sesgos de ECAs (165).

Dos de los estudios $(104,105)$, incluyen ambos sexos y uno solo mujeres postmenopáusicas (103). El rango de sujetos se encuentra entre 
93 y 147, y todos están realizados en población con déficit de VitD. Los estudios presentan diferencias en otras características como, población sana vs población con sobrepeso u obesidad vs postmenopáusicas, seguimiento (rango 2 y 12 meses) y distintas dosis y régimen de aplicación (diarias vs semanal). EL objetivo es similar, ya que todos evalúan los efectos del suplemento de VitD en el metabolismo mineral a nivel renal, analizando la incidencia de hipercalcemia o hipercalciuria, factor de crecimiento fibroblastos 23 (FGF-23) o el efecto en el sistema renina-angiotensina. Con respecto a los resultados y las conclusiones de los autores, los tres estudios coinciden en la falta de diferencias significativas en los resultados entre sujetos suplementados con VitD vs no suplementados. McMullan et al (104) a nivel de actividad del sistema renina/angiotensina o la presión arterial, Nygaard et al (105) en factor de crecimiento fibroblastos (FGF23) y por último el estudio de Gallagher et al (103) a nivel de eventos de hipercalcemia o hipercalciuria.

La concentración baja de 25(OH)D y la suplementación con VitD no modifican los cambios de distintos metabolitos que pueden afectar a nivel del sistema renal.

\section{Resumen: Función renal}

Hasta el momento, no existe evidencia suficiente para extraer conclusiones. Al margen de su relación con el metabolismo mineral, los resultados muestran en general que no existe relación entre el suplemento de VitD y la función renal en población general sana.

Calidad de la evidencia MUY BAJA $\oplus \circ \circ \bigcirc$

Vitamina D y su efecto en la función cognitiva

La Task Force (4) no incluye estudios sobre este apartado. Se han localizado dos ECAs que analizan el efecto de la suplementación de VitD en la función cognitiva $(106,108)$.

Ambos estudios presentan un nivel de riesgo de sesgo bajo (106, 108) según los criterios de la herramienta de la Colaboración Cochrane de evaluación del riesgo de sesgo de ECAs (165).

Los dos ECAs $(106,108)$ analizan los efectos de la suplementación con VitD en la función cognitiva. Jorde et al 2019 (107) realiza la evaluación frente a placebo (dosis alta semanal) en 394 sujetos con bajos valores de VitD y con un seguimiento de cuatro meses, no encontrando diferencias significativas entre los grupos en la función cognitiva. Por su parte Pettersen et al 2017 (108), compara dosis altas vs bajas de VitD en 95 personas con o sin déficit de VitD y con un seguimiento similar al de Jorde et al (107) (4.5 
meses), encontrando en este caso que dosis altas de VitD presenta beneficios en la función cognitiva, específicamente en la memoria no verbal en sujetos con déficit de VitD.

\section{Resumen: Función cognitiva}

En población general sana, los estudios muestran resultados mixtos. Hasta el momento con el bajo número de estudios y el pequeño seguimiento no se puede realizar una conclusión general. Calidad de la evidencia MUY BAJA $\oplus \circ \circ \bigcirc$

\section{Vitamina D y su efecto en la función tiroidea}

La Task Force (4) no incluye estudios sobre este apartado. En la actualización de la búsqueda se localizó un ECA que evalúa si la suplementación con VitD vs placebo podría reducir los niveles de anticuerpos tiroideos. El estudio de Knutsen et al 2017 (109), se trata de un ECA con análisis secundario de otro ECA anterior, no diseñado para el este objetivo. El nivel de riesgo de sesgo en base al primer estudio es bajo. El estudio se desarrolla en minorías étnicas (250 sujetos) que viven en Noruega, a los que se suplementa con dosis diaria de VitD, baja (400 UI/día) o altas (1000 UI/día) y que presentan un seguimiento de dieciseis semanas. No se encontró un efecto significativo entre la suplementación de VitD3 en cualquiera de sus dosis y los valores de hormonas tiroideas.

\section{Resumen: Función tiroidea}

No existe evidencia suficiente para extraer conclusiones.

Calidad de la evidencia MUY BAJA $\oplus 000$

Vitamina $D$ y su efecto en el riesgo de diabetes tipo 2

La RS (4) de partida incluye para este apartado un ECA de calidad moderada (173) y un estudio de casos-controles anidado (185). Ninguno de los dos ECAs que evaluaron dosis de 400 IU a 1000 IU día, con o sin calcio, de uno a siete años, mostraron efectos significativos

En la búsqueda posterior se incluyeron once estudios que investigan la asociación entre las concentraciones séricas de $25(\mathrm{OH}) \mathrm{D}$ y sus efectos en el riesgo de la diabetes tipo 2. De ellos se incluyen una revisión sistemática (120) y diez ECAs (110-119), que evalúan en función de distintos análisis como: el estado glucémico, sensibilidad a la insulina o glucosa o el análisis de marcadores específicos, entre otros. 
Con respecto a la revisión sistemática de ECAs realizada por Zuk et al 2016 (120), la calidad metodológica es críticamente baja, con debilidades críticas, obtenida mediante el instrumento de criterios del AMSTAR-2 (146). La revisión incluye un total de catorce estudios y 1968 sujetos. Los autores concluyen que, de forma general, no hubo efecto significativo de la suplementación de VitD sobre marcadores glucémicos, en población con sobrepeso u obesidad.

De los diez ECAs incluidos, ocho presentan un riesgo de sesgo bajo $(110,112-115,117-119)$ y los otros dos riesgo de sesgo alto $(111,116)$.

Todos los estudios, se llevan a cabo en población que incluía ambos sexos, excepto el realizado Tepper et al (118) que incluyó solo población masculina. El rango de sujetos era muy variado desde 65 a 511, siendo muy heterogéneo (déficit o no de VitD, sobrepeso, prediabetes, inmigrantes, raza, etc.). Los estudios no solo son heterogéneos en la población incluida, sino también, en otras características como la dosis administrada (alta o baja), régimen de dosis (diario, semanal o mensual) o el tiempo de seguimiento que va desde los tres meses a los cinco años.

Aun así, seis (110-115) de los diez ECAs, concluyen que el uso de suplementos de VitD no mejora el metabolismo diabético y no presenta modificaciones en la resistencia a la insulina. Los otros cuatro ECAs (116119) obtienen mejoría de resistencia la insulina.

Resumen: Diabetes tipo 2

En población general, los resultados son mixtos. Con los estudios disponibles no se puede realizar una conclusión general.

Calidad de la evidencia MODERADA $\oplus \oplus \oplus \mathrm{O}$

\section{Vitamina D y su efecto en la calidad de vida}

La Task Force (4) no incluye estudios sobre este apartado. Se han localizado dos ECAs que analizan el efecto de la suplementación de VitD en la calidad de vida $(121,122)$.

Los estudios presentan un nivel de riesgo de sesgo bajo, según los criterios de la herramienta de la Colaboración Cochrane de evaluación del riesgo de sesgo de ECAs (165).

Los dos ECAs $(121,122)$ analizan los efectos de la suplementación con VitD en la calidad de vida, entre otras variables como el miedo a las caídas, el sueño, los síntomas depresivos o el bienestar mental. El análisis 
se realiza frente a placebo, en ambos casos, con administración diaria de VitD, aunque usando distintas dosis (800 UI y 2000 UI, Patil et al (121) y Mason et al (122), respectivamente). Los dos estudios se refieren a mujeres postmenopáusicas, y en el caso del estudio de Mason et al (122), las mujeres presentan sobrepeso. Incluyen un total de 627 mujeres con un seguimiento de uno o dos años, y en el estudio de Patil et al (121), además, realizan ejercicio físico. Ambos estudios, no encuentran diferencias significativas en la calidad de vida o el resto de valores analizados entre grupo con suplemento de VitD vs placebo.

Resumen: Calidad de vida

En población general sana, no existen datos suficientes para establecer la relación entre los suplementos de VitD y la mejora en la calidad de vida. Los estudios se restringen a mujeres postmenopáusicas.

Calidad de la evidencia BAJA $\oplus \circ \bigcirc$

\section{Otros resultados}

Los siguientes resultados solo se encuentran en la RS de partida (4) ya que no se han localizado nuevos estudios en la búsqueda posterior.

\section{Cribado}

No se encontraron estudios que examinen los beneficios y riesgos de realizar un cribado de VitD en población general.

\section{Funcionamiento psicosocial y discapacidad}

Las RS (4) localiza estudios sobre las siguientes patologías:

- Depresión y ansiedad: un ECA de buena calidad (186) que evaluó el efecto de $20000 \mathrm{IU} / \mathrm{semana}$ de $\mathrm{VitD}_{3}$ durante seis meses en personas sanas con nivel de $\mathrm{VitD}<22 \mathrm{ng} / \mathrm{ml}$, no mostrando ninguna mejoría en la depresión o ansiedad.

- Fibromialgia: un ECA de pequeño tamaño $(n=90)$ y calidad moderada (187). El estudio evaluó el efecto de 8 dosis semanales de 50000 IU mostrando el grupo tratado una mejora leve a corto plazo en la puntuación general del impacto de la fibromialgia, pero no mostraron mejora significativa en la mayoría de los síntomas musculoesqueléticos o en las actividades de la vida diaria. No influyó en la depresión o subescalas con la interferencia en el trabajo. 


\section{Recomendaciones de uso adecuado de la vitamina $>\mathrm{D}$ en población general}

\subsection{Consideraciones previas}

Actualment no existe consenso en la comunidad científica sobre la definición de deficiencia de VitD o el nivel óptimo de 25(OH)D total en suero. Esta falta de consenso hace difícil la determinación del problema, ya que el punto de corte utilizado condicionará la prevalencia de déficit de VitD en la población que se considere.

La evidencia disponible hasta el momento muestra que valores bajos de $25(\mathrm{OH}) \mathrm{D}$ en suero, no reflejan, asociación con resultados adversos de salud a nivel de enfermedades cardiovasculares, cáncer en distintas localizaciones (próstata, mama, ovario, páncreas, otros), vértigo posicional paroxístico benigno, enfermedades del sistema circulatorio, enfermedades oculares (oclusión de la vena retiniana central, el glaucoma primario de ángulo abierto o la degeneración macular tardía asociada a la edad) o alergias, entre otras.

En otras patologías no se ha podido establecer una conclusión clara debido a los resultados contradictorios de los estudios encontrados o a las limitaciones metodológicas de estudios incluidos, no pudiendo establecer relaciones causales, como en las caídas, deterioro cognitivo o depresión, dismenorrea, entre otras.

En cuanto a la evidencia encontrada sobre los suplementos de VitD en población general asintomática tanto con déficit como sin déficit de VitD, no muestra que la suplementación tenga beneficios para la incidencia del cáncer, enfermedades cardiovasculares, riesgo de fracturas, alivio del dolor, riesgo de infecciones o inflamaciones, entre otras.

La revisión también aportó resultados contradictorios o insuficientes sobre el beneficio de la suplementación en la deficiencia de VitD asintomática en otros resultados de salud como la función pulmonar, la función cognitiva o la función tiroidea. 


\subsection{Recomendaciones de uso en población general sana}

El grupo de trabajo considera que, en base al conocimiento actual, no está indicado, en general, cuantificar los niveles de VitD en población sin síntomas de déficit o factores de riesgo, ni suplementar valores $>20 \mathrm{ng} / \mathrm{ml}$.

Además, después de los 65 años, la prevalencia de deficiencia es muy alta por lo que la determinación rutinaria no estaría justificada. Según el documento de consenso desarrollado en 2016 por miembros del Grupo de Trabajo de Osteoporosis y Metabolismo Mineral de la SEEN, la insuficiencia/ deficiencia de VitD constituye una aparente epidemia a nivel mundial, con una situación similar en España donde entre el 80-100 \% de los individuos mayores de 65 años presentan concentraciones de 25[OH]D por debajo de $20 \mathrm{ng} / \mathrm{ml}(7)$.

\subsubsection{Recomendaciones sobre cuantificación de vitamina D en población general sana}

- En adultos sanos asintomáticos y sin factores de riesgo para el déficit de VitD no es necesaria la determinación de valores de VitD. A efectos de esta recomendación, se consideran tanto los factores de riesgo extrínsecos (por ejemplo, ingesta inadecuada o hiperpigmentación cutánea); como intrínsecos (por ejemplo, la edad avanzada o la malabsorción: gastrectomía, enfermedades intestinales ocirrosis biliar primaria).

\subsubsection{Recomendaciones de suplementación con vitamina D en población general sana}

- En adultos sanos asintomáticos la suplementación farmacológica, hasta el momento, no ha demostrado beneficios, independientemente de los niveles de VitD.

- Podría considerarse la suplementación en población institucionalizada mayor de 70 años, sin necesidad de determinación. 


\section{Aplicabilidad del OPBE}

Los OPBE son útiles para mejorar la calidad de la asistencia y los resultados en los pacientes. El gran reto en la actualidad es conseguir la adherencia de los profesionales a las recomendaciones de estos. Para ello es fundamental una estrategia de implementación dirigida a vencer las barreras existentes en el medio en el que se va a aplicar.

El plan para implantar las recomendaciones sobre el uso adecuado de vitamina $\mathrm{D}$ en población general incluye las siguientes intervenciones:

1. Necesidad de planificar actividades formativas dirigidas a los médicos de atención primaria y médicos especialistas de área que tengan relación con la medición, suplementación y valoración de los valores de la VitD, con el objetivo de difundir y consolidar el conocimiento de los niveles séricos de $25(\mathrm{OH}) \mathrm{D}$ y su relación con la salud.

2. Favorecer la difusión del OPBE entre los agentes implicados, administraciones sanitarias y colectivos profesionales. Destacando el envío de material informativo realizado con el objeto de favorecer su difusión.

3. Difusión en formato electrónico en las páginas web del Ministerio de Sanidad, de GuíaSalud, de la RedETS, de Avalia-t y de otras agencias de evaluación y sociedades implicadas en el proyecto.

4. Publicación en revistas científicas.

5. Evaluación de la efectividad de la implementación, estableciendo sistemas de apoyo a la decisión clínica, integrando las recomendaciones del OPBE y los indicadores en los programas informáticos utilizados en los Servicios sanitarios. 



\section{Propuesta de indicadores de evaluación}

Los indicadores son datos medidos que podemos utilizar como pauta para conocer y evaluar la calidad de distintas actividades. Es importante definir aquellos que permiten monitorizar la calidad del OPBE y su cumplimiento.

En este apartado se incluyen los propuestos por el grupo elaborador que permiten valorar diferentes aspectos de las recomendaciones de uso adecuado de pruebas y suplementos de vitamina $\mathrm{D}$ en población general. Estos se eligieron por cumplir características como la validez y la información que aportan.

EL objetivo de los indicadores propuestos es facilitar la implementación de las recomendaciones realizadas por el grupo de trabajo, ya que es importante conocer si se alcanzan los objetivos que se espera obtener con el cumplimiento del OPBE. Para cada uno de ellos se indica el criterio de calidad y se incluye una descripción operativa de los mismos, para garantizar la homogeneidad y la calidad de la información registrada.

El valor umbral se ha definido como un punto de corte de un determinado porcentaje. A continuación, proponemos aquí los siguientes grados de cumplimiento (tabla 13), proponiendo para el primer año un descenso de entre el 20-25\% en la determinación de VitD.

Tabla 13. Grados de cumplimiento y clasificación

\begin{tabular}{|c|c|}
\hline$\%$ de cumplimiento & Calificación \\
\hline$\geq 90 \%$ & Excelente \\
\hline $80-89 \%$ & Bueno \\
\hline $60-79 \%$ & Regular \\
\hline $40-59 \%$ & Pobre \\
\hline $0-39 \%$ & Muy pobre \\
\hline
\end{tabular}

Fuente: elaboración propia.

A continuación (tabla 14), se relacionan los indicadores propuestos. 
Tabla 14. Indicadores propuestos

\begin{tabular}{|l|l|l|l|}
\hline $\begin{array}{l}\text { Tipo de } \\
\text { indicador }\end{array}$ & Nombre del indicador & $\begin{array}{l}\text { Dimensión } \\
\text { de la calidad }\end{array}$ & $\begin{array}{l}\text { Nivel } \\
\text { asistencial }\end{array}$ \\
\hline Estructura & $\begin{array}{l}\text { Conocer a nivel del SNS los laboratorios con } \\
\text { protocolo actualizado*para la petición de } \\
\text { determinación de VitD. }\end{array}$ & $>90 \%$ & $1^{\dagger}$ y $2^{\ddagger}$ \\
\hline Proceso & $\begin{array}{l}\text { Número de determinaciones de VitD solicitados } \\
\text { en población general en AP y AH después de } \\
\text { la publicación del OPBE. }\end{array}$ & $80 \%-90 \%$ & 1 y 2 \\
\hline Proceso & $\begin{array}{l}\text { Gasto farmacéutico en suplementación con } \\
\text { VitD en población general sana después de la } \\
\text { publicación del OPBE. }\end{array}$ & $>90 \%$ & 1 y 2 \\
\hline
\end{tabular}

*Protocolo actualizado: protocolos desarrollados en los últimos años en base a la evidencia científica

†1: Atención primaria (AP),

¥2: Atención hospitalaria $(\mathrm{AH})$

Fuente: elaboración propia.

En la tabla 15, vemos las fichas descriptivas de los indicadores anteriormente propuestos.

\section{Tabla 15. Fichas de los indicadores propuestos}

\section{Laboratorios del SNS con protocolo actualizado para determinación de VitD}

$\mathrm{N}^{\circ}$ de laboratorios del SNS con protocolos específicos para la petición de determinación de VitD, solo a sujetos que tienen indicación

\section{Fórmula}

x 100

$\mathrm{N}^{\circ}$ total de laboratorios del SNS que realizan determinación de VitD

\begin{tabular}{|l|l|l|l|l|l|l|l|}
\hline $\begin{array}{l}\text { Inclusión y } \\
\text { exclusión }\end{array}$ & $\begin{array}{l}\text { El indicador incluye a todos los laboratorios de análisis que realicen } \\
\text { determinaciones de valores de VitD } \\
\text { Se excluyen aquellos laboratorios del SNS que no realicen determinaciones } \\
\text { de valores de VitD }\end{array}$ \\
\hline Fuente de datos & Gerencias de Áreas de Salud \\
\hline Tipo indicador & Estructura \\
\hline Ajuste de riesgos & No se contempla \\
\hline Umbral & $\geq 90 \%$ \\
\hline
\end{tabular}


Número de determinaciones de VitD solicitados en AP para población general sana

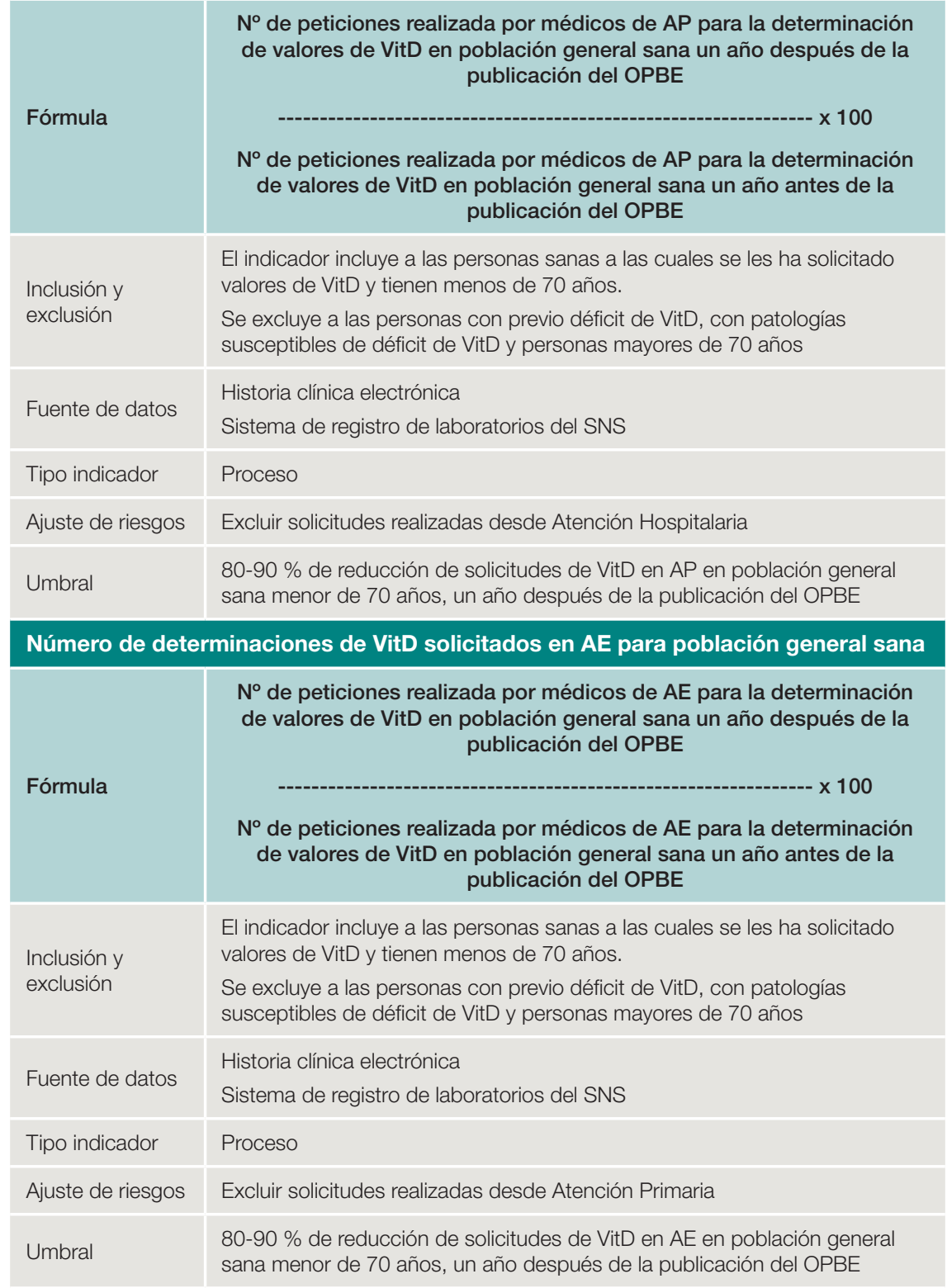


Gasto farmacéutico en suplementación con VitD en población general sana

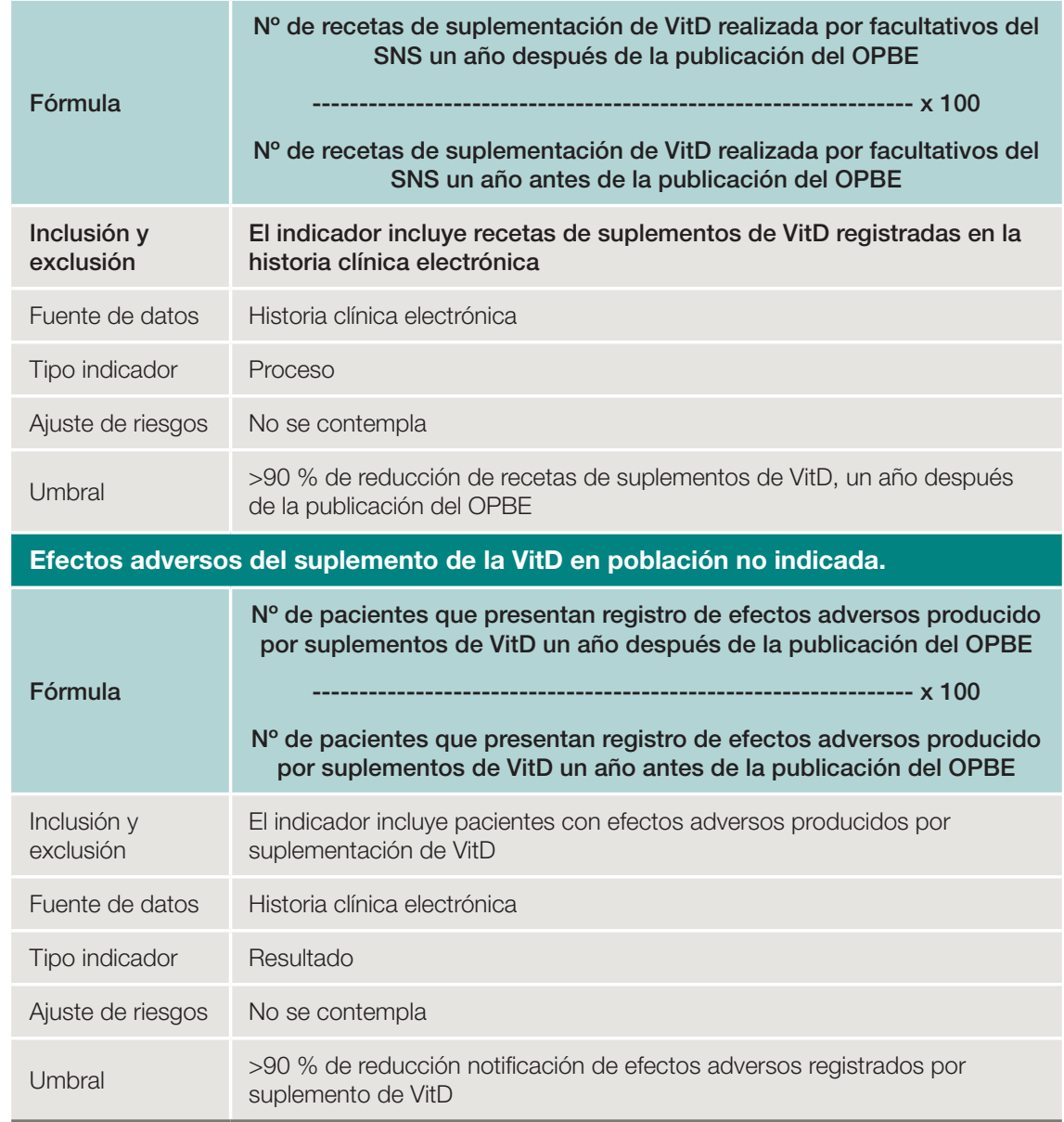

Fuente: elaboración propia. 


\section{Actualización del OPBE}

La actualización de las recomendaciones recogidas en el OPBE está prevista cada cinco años, sin que se descarte, en caso de ser necesario, una actualización anterior debida a nueva evidencia relevante.

Un año antes de su caducidad el equipo elaborador u otro, valorará la actualización teniendo en cuenta los siguientes criterios:

- Identificación y valoración de nuevas evidencias relevantes

- Opinión de los elaboradores del OPBE

- Percepción de los usuarios

- Análisis del contexto (incluido COVID-19) 



\section{Líneas de investigación futuras}

Los autores de este OPBE identificaron un gran número de incertidumbres en torno a:

- ¿Cuáles son los umbrales estándares de 25(OH)D para definir el déficit de vitamina D?

- ¿Cuáles son los grupos de riesgo de padecer déficit de vitamina D?

- ¿Cuál es la cantidad diaria recomendada de vitamina D en población general y en grupos de riesgo?

- ¿Cuál es la frecuencia adecuado para la monitorización de las concentraciones de vitamina D? (seguimiento del paciente, evitando las determinaciones en periodos de seguimiento muy corto y no justificado).

- ¿Cuáles son los efectos adversos del tratamiento con vitamina D en población general y en subgrupos de paciente?

- ¿Cuáles son los umbrales tóxicos de vitamina D?

- ¿Cuál es la relación coste efectividad del cribado de vitamina D en personas de alto riesgo (afectados por osteoporosis, sujetos con malabsorción, etc.)?

- ¿Cómo tratar a los pacientes sanos asintomáticos que llegan a la consulta con una determinación de VitD previa y que es deficiente?

Una prioridad particular son los ECAs rigurosos, a gran escala y aleatorizados para aumentar el nivel de evidencia sobre la VitD, tanto a nivel de resultados musculoesqueléticos como no esqueléticos, así como para identificar los puntos anteriormente expuestos.

Hay que destacar que una de las limitaciones encontrada para la extrapolación de recomendaciones es que los participantes, en su mayoría, no estaban reclutados para el estudio de la VitD y sus concentraciones séricas de $[25(\mathrm{OH}) \mathrm{D}]$ como nivel de déficit no está definida en todos los estudios de la misma forma. Por lo tanto, los ECAs futuros deben estratificar a los participantes, a priori, para incluir individuos con concentraciones séricas de $[25(\mathrm{OH}) \mathrm{D}]$ en lugar de confiar en el análisis de subgrupos secundarios. 



\section{Referencias bibliográficas}

1 Sociedad Española de Investigación Ósea y del Metabolismo Mineral (SEIOMM) y Sociedades afines, Gómez de Tejada Romero M, Sosa Henríquez M, Del Pino Montes J, Jódar Gimeno E, Quesada Gómez J, et al. Documento de posición sobre las necesidades y niveles óptimos de vitamina D. Rev Osteoporos Miner. 2011;3(1):53-4.

2 Navarro Valverde C, Quesada Gómez J. Deficiencia de vitamina D en España. ¿Realizadad o mito? Rev Osteoporos Metab Miner. 2014;6(Supli 1):S5-10.

3 Busturia Jimeno M. Vitamina D: visión desde el laboratorio. Rev Esp Endocrinol Pediatr. 2012;3(suppl):39-45.

4 LeBlanc E, Chou R, Zakher B, Daeges M, Pappas M. Screening for Vitamin D Deficiency: Systematic Review for the U.S. Preventive Services Task Force Recommendation. Rockville (MD): Agency for Healthcare Research and Quality; 2014. Informe $\mathrm{N}^{\circ}$.: Evidence Synthesis No. 119. [consultado 24 jul 2018]. Disponible en: https://www. ncbi.nlm.nih.gov/pubmedhealth/PMH0071094/.

5 Torrubia B, Alonso I, López-Ramiro E, Mahillo I, De la Piedra C. Comparación entre dos inmunoensayos automatizados por quimioluminiscencia para la cuantificación de $25(\mathrm{OH})$ vitamina D Rev Osteoporos Metab Miner. 2016;8(2):70-4.

6 Gómez de Tejada Romero M, Sosa Henríquez M, Del Pino Montes J, Jodar Gimeno E, Quesada Gómez J, Cancelo Hidalgo M, et al. Sociedad Española de Investigación Ósea y del Metabolismo Mineral (SEIOMM) y Sociedades afines. Documento de posición sobre las necesidades y niveles óptimos de vitamin D. Rev Osteoporos Metab Miner. 2011;3:53-64.

7 Varsavsky M, Rozas Moreno P, Becerra Fernández A, Luque Fernández I, Quesada Gómez JM, Ávila Rubio V, et al. Recomendaciones de vitamina D para la población general. Endocrinol Diabetes Nutr. 2017;64(51):7-14.

8 Nowson CA, McGrath JJ, Ebeling PR, Haikerwal A, Daly RM, Sanders KM, et al. Vitamin D and health in adults in Australia and New Zealand: 
a position statement. Med J Aust. 2012;196(11):686-7. PubMed PMID: 22708765 .

9 Smith H. Guideline on Diagnosis \& Management of Vitamin D Deficiency for Non-Specialists in Primary Care [Internet]. West Bromwich:Lancashire Medicines Management Group;2013. [consultado 27 mar 2019]. Disponible en: http://www.lancsmmg.nhs.uk/wp-content/ uploads/sites/3/2013/04/Diagnosis-and-Management-of-Vitamin-DDeficiency-for-Non-Specialists-in-Primary-Care-Version-21.pdf.

10 Keeney JT, Butterfield DA. Vitamin D deficiency and Alzheimer disease: Common links. Neurobiology of Disease. 2015;84:84-98.

11 Trincado P. Hipovitaminosis D. Rev Med Clin Condes. 2013;24(5):813-7.

12 Bajaj A, Stone KL, Peters K, Parimi N, Barrett-Connor E, Bauer D, et al. Circulating vitamin D, supplement use, and cardiovascular disease risk: the MrOS Sleep Study. Journal of Clinical Endocrinology \& Metabolism. 2014;99(9):3256-62. PubMed PMID: 24670083.

13 Vitezova A, Cartolano NS, Heeringa J, Zillikens MC, Hofman A, Franco $\mathrm{OH}$, et al. Vitamin D and the risk of atrial fibrillation - The Rotterdam study. PLoS ONE. 2015;10 (5)(e0125161).

14 Wannamethee SG, Welsh P, Papacosta O, Lennon L, Whincup PH, Sattar N. Elevated parathyroid hormone, but not vitamin D deficiency, is associated with increased risk of heart failure in older men with and without cardiovascular disease. Circulation: Heart Failure. 2014;7(5):732-9. PubMed PMID: 25104043.

15 Judd SE, Morgan CJ, Panwar B, Howard VJ, Wadley VG, Jenny NS, et al. Vitamin D deficiency and incident stroke risk in community-living black and white adults. International Journal of Stroke. 2016;11(1):93102. PubMed PMID: 26763025.

16 Schenk JM, Till CA, Tangen CM, Goodman PJ, Song X, Torkko KC, et al. Serum 25-hydroxyvitamin D concentrations and risk of prostate cancer: results from the Prostate Cancer Prevention Trial. Cancer Epidemiol Biomarkers Prev. 2014;23(8):1484-93. PubMed PMID: 25085836.

17 Chandler PD, Buring JE, Manson JE, Giovannucci EL, Moorthy MV, Zhang S, et al. Circulating vitamin D levels and risk of colorectal cancer 
in women. Cancer Prevention Research. 2015;8(8):675-82. PubMed PMID: 25813525.

18 Jamshidinaeini Y, Akbari ME, Abdollahi M, Ajami M, Davoodi SH. Vitamin D Status and Risk of Breast Cancer in Iranian Women: A Case-Control Study. Journal of the American College of Nutrition. 2016;35(7):639-46. PubMed PMID: 27331363.

19 Kim Y, Franke AA, Shvetsov YB, Wilkens LR, Cooney RV, Lurie G, et al. Plasma 25-hydroxyvitamin D3 is associated with decreased risk of postmenopausal breast cancer in whites: A nested case-control study in the multiethnic cohort study. BMC Cancer. 2014;14 (1) (no pagination) (29). PubMed PMID: 52967301.

20 Qin B, Moorman PG, Alberg AJ, Barnholtz-Sloan JS, Bondy M, Cote ML, et al. Dairy, calcium, vitamin D and ovarian cancer risk in AfricanAmerican women. Br J Cancer. 2016;115(9):1122-30. PubMed PMID: 27632371.

21 McDonnell SL, Baggerly C, French CB, Baggerly LL, Garland CF, Gorham ED, et al. Serum 25-Hydroxyvitamin D Concentrations $>/=40 \mathrm{ng} / \mathrm{ml}$ Are Associated with $>65 \%$ Lower Cancer Risk: Pooled Analysis of Randomized Trial and Prospective Cohort Study. PLoS One. 2016;11(4):e0152441. PubMed PMID: 27049526.

22 Skaaby T, Husemoen LL, Thuesen BH, Pisinger C, Jorgensen T, Roswall $\mathrm{N}$, et al. Prospective population-based study of the association between serum 25-hydroxyvitamin-D levels and the incidence of specific types of cancer. Cancer epidemiology, biomarkers \& prevention. 2014;23(7):1220-9. PubMed PMID: 24789846.

23 Duval GT, Pare PY, Gautier J, Walrand S, Dinomais M, Annweiler C. Vitamin D and the Mechanisms, Circumstances and Consequences of Falls in Older Adults: A Case-Control Study. J Nutr Health Aging. 2017;21(10):1307-13. PubMed PMID: 29188894.

24 Rothenbacher D, Klenk J, Denkinger MD, Herbolsheimer F, Nikolaus $\mathrm{T}$, Peter R, et al. Prospective evaluation of renal function, serum vitamin $\mathrm{D}$ level, and risk of fall and fracture in community-dwelling elderly subjects. Osteoporos Int. 2014;25(3):923-32. PubMed PMID: 24221451.

25 Julian C, Lentjes MA, Huybrechts I, Luben R, Wareham N, Moreno LA, et al. Fracture Risk in Relation to Serum 25-Hydroxyvitamin D and Physical Activity: Results from the EPIC-Norfolk Cohort Study. 
PLoS ONE [Revista en línea]. 2016;11(10):e0164160. PubMed PMID: 27749911.

26 Maier GS, Seeger JB, Horas K, Roth KE, Kurth AA, Maus U. The prevalence of vitamin $\mathrm{D}$ deficiency in patients with vertebral fragility fractures. Bone \& Joint Journal. 2015;97-B(1):89-93. PubMed PMID: 25568419.

27 Williams BR, Thomas AJ, Collier RC, Boffeli TJ, Anderson SA. Vitamin D Levels Do Not Predict Risk of Metatarsal Fractures. Foot \& Ankle Specialist. 2018;11(1):37-43. PubMed PMID: 28367641.

28 Karatas A, Acar Yuceant G, Yuce T, Haci C, Cebi IT, Salviz M. Association of Benign Paroxysmal Positional Vertigo with Osteoporosis and Vitamin D Deficiency: A Case Controlled Study. The Journal of International Advanced Otology. 2017;13(2):259-65. PubMed PMID: 28274898 .

29 Lee YH, Bae SC. Vitamin D level in rheumatoid arthritis and its correlation with the disease activity: a meta-analysis. Clin Exp Rheumatol. 2016;34(5):827-33. PubMed PMID: 27049238.

30 Buchebner D, McGuigan F, Gerdhem P, Ridderstrale M, Akesson K. Association Between Hypovitaminosis D in Elderly Women and Longand Short-Term Mortality-Results from the Osteoporotic Prospective Risk Assessment Cohort. J Am Geriatr Soc. 2016;64(5):990-7. PubMed PMID: 27225355.

31 Daraghmeh AH, Bertoia ML, Al-Qadi MO, Abdulbaki AM, Roberts MB, Eaton CB. Evidence for the vitamin D hypothesis: The NHANES III extended mortality follow-up. Atherosclerosis. 2016;255:96-101. PubMed PMID: 27855294.

32 Formiga F, Ferrer A, Megido MJ, Boix L, Contra A, Pujol R, et al. Low serum vitamin $\mathrm{D}$ is not associated with an increase in mortality in oldest old subjects: the Octabaix three-year follow-up study. Gerontology. 2014;60(1):10-5. PubMed PMID: 23689215.

33 Gaksch M, Jorde R, Grimnes G, Joakimsen R, Schirmer H, Wilsgaard $\mathrm{T}$, et al. Vitamin D and mortality: Individual participant data metaanalysis of standardized 25-hydroxyvitamin D in 26916 individuals from a European consortium. PLoS ONE. 2017;12(2):e0170791. PubMed PMID: 28207791. 
34 Leu Agelii M, Lehtinen-Jacks S, Zetterberg H, Sundh V, Bjorkelund C, Lissner L. Low vitamin D status in relation to cardiovascular disease and mortality in Swedish women - Effect of extended follow-up. Nutr Metab Cardiovasc Dis. 2017;27(12):1143-51. PubMed PMID: 29170060.

35 Samefors M, Ostgren CJ, Molstad S, Lannering C, Midlov P, Tengblad A. Vitamin D deficiency in elderly people in Swedish nursing homes is associated with increased mortality. Eur J Endocrinol.2014;170(5):66775. PubMed PMID: 24520134.

36 Sun YQ, Langhammer A, Skorpen F, Chen Y, Mai XM. Serum 25-hydroxyvitamin D level, chronic diseases and all-cause mortality in a population-based prospective cohort: the HUNT Study, Norway. BMJ Open. 2017;7(6):e017256. PubMed PMID: 28674149.

37 Wong G, Lim WH, Lewis J, Craig JC, Turner R, Zhu K, et al. Vitamin D and cancer mortality in elderly women. BMC Cancer. 2015;15:106. PubMed PMID: 25884436.

38 Andro M, Delluc A, Moineau MP, Tromeur C, Gouillou M, Lacut $\mathrm{K}$, et al. Serum levels of $25(\mathrm{OH}) \mathrm{D}$ are not associated with venous thromboembolism in the elderly population. A case-control study. Thrombosis \& Haemostasis. 2016;115(1):169-75. PubMed PMID: 26538494.

39 Folsom AR, Roetker NS, Rosamond WD, Heckbert SR, Basu S, Cushman M, et al. Serum 25-hydroxyvitamin D and risk of venous thromboembolism: the Atherosclerosis Risk in Communities (ARIC) Study. Journal of Thrombosis \& Haemostasis. 2014;12(9):1455-60. PubMed PMID: 25039645.

40 Goodwill AM, Szoeke C. A Systematic Review and Meta-Analysis of The Effect of Low Vitamin D on Cognition. J Am Geriatr Soc. 2017;65(10):2161-8. PubMed PMID: 28758188.

41 Littlejohns TJ, Henley WE, Lang IA, Annweiler C, Beauchet O, Chaves $\mathrm{PH}$, et al. Vitamin D and the risk of dementia and Alzheimer disease. Neurology. 2014;83(10):920-8.

42 Moon JH, Lim S, Han JW, Kim KM, Choi SH, Kim KW, et al. Serum 25-hydroxyvitamin D level and the risk of mild cognitive impairment and dementia: The Korean Longitudinal Study on Health and Aging (KLoSHA). Clin Endocrinol (Oxf). 2015;83(1):36-42. PubMed PMID: 602688523. 
43 Olsson E, Byberg L, Karlstrom B, Cederholm T, Melhus H, Sjogren P, et al. Vitamin D is not associated with incident dementia or cognitive impairment: an 18-y follow-up study in community-living old men. American Journal of Clinical Nutrition. 2017;105(4):936-43. PubMed PMID: 28202477.

44 Annweiler C, Drouet M, Duval GT, Pare PY, Leruez S, Dinomais M, et al. Circulating vitamin $\mathrm{D}$ concentration and age-related macular degeneration: Systematic review and meta-analysis. Maturitas. 2016;88:101-12. PubMed PMID: 27105707.

45 Epstein D, Kvanta A, Lindqvist PG. Vitamin D Deficiency in Patients with Central Retinal Vein Occlusion: a Case Control Study. Curr Eye Res. 2017;42(3):448-51. PubMed PMID: 27348601.

46 Goncalves A, Milea D, Gohier P, Jallet G, Leruez S, Baskaran M, et al. Serum vitamin D status is associated with the presence but not the severity of primary open angle glaucoma. Maturitas. 2015;81(4):470-4. PubMed PMID: 26059919.

47 Izumaru K, Ninomiya T, Nagata M, Usui T, Yoshida D, Yonemoto K, et al. Serum 1,25-dihydroxyvitamin $\mathrm{d}$ and the development of kidney dysfunction in a Japanese community. Circ J. 2014;78(3):732-7. PubMed PMID: 24351652.

48 Sahin I, Gungor B, Can MM, Avci, II, Guler GB, Okuyan E, et al. Lower blood vitamin D levels are associated with an increased incidence of contrast-induced nephropathy in patients undergoing coronary angiography. Can J Cardiol. 2014;30(4):428-33. PubMed PMID: 24680172.

49 van den Berg KS, Marijnissen RM, van den Brink RH, Naarding P, Comijs HC, Oude Voshaar RC. Vitamin D deficiency, depression course and mortality: Longitudinal results from the Netherlands Study on Depression in Older persons (NESDO). Journal of Psychosomatic Research. 2016;83:50-6. PubMed PMID: 27020077.

50 Joo MH, Han MA, Park SM, Shin HH. Vitamin D Deficiency among Adults with History of Pulmonary Tuberculosis in Korea Based on a Nationwide Survey. International Journal of Environmental Research \& Public Health [Revista en línea]. 2017;14(4):10. PubMed PMID: 28394278. 
51 Karacin O, Mutlu I, Kose M, Celik F, Kanat-Pektas M, Yilmazer M. Serum vitamin D concentrations in young Turkish women with primary dysmenorrhea: A randomized controlled study. Taiwan J Obstet Gynecol. 2018;57(1):58-63. PubMed PMID: 29458904.

52 Vatankhah $\mathrm{V}$, Lotfizadeh $\mathrm{M}$, Iranpoor $\mathrm{H}$, Jafari $\mathrm{F}$, Khazraei $\mathrm{H}$. Comparison vitamin D serum levels in allergic rhinitis patients with normal population. Revue francaise d'allergologie [Internet]. 2016; 56(7-8):[539-43 pp.]. Disponible en: http://cochranelibrary-wiley.com/o/ cochrane/clcentral/articles/547/CN-01290547/frame.html.

53 Sadeghian M, Saneei P, Siassi F, Esmaillzadeh A. Vitamin D status in relation to Crohn's disease: Meta-analysis of observational studies. Nutrition. 2016;32(5):505-14. PubMed PMID: 26837598.

54 Zhou J, Huang P, Liu P, Hao Q, Chen S, Dong B, et al. Association of vitamin D deficiency and frailty: A systematic review and meta-analysis. Maturitas. 2016;94:70-6. PubMed PMID: 27823748.

55 Baron JA, Barry EL, Mott LA, Rees JR, Sandler RS, Snover DC, et al. A Trial of Calcium and Vitamin D for the Prevention of Colorectal Adenomas. N Engl J Med. 2015;373(16):1519-30. PubMed PMID: 26465985.

56 Bjelakovic G, Gluud LL, Nikolova D, Whitfield K, Krstic G, Wetterslev J, et al. Vitamin D supplementation for prevention of cancer in adults. Cochrane Database Syst Rev. 2014 (6):CD007469. PubMed PMID: 24953955.

57 Lappe J, Watson P, Travers-Gustafson D, Recker R, Garland C, Gorham E, et al. Effect of Vitamin D and Calcium Supplementation on Cancer Incidence in Older Women: A Randomized Clinical Trial. JAMA. 2017;317(12):1234-43. PubMed PMID: 28350929.

58 Manson JE, Cook NR, Lee IM, Christen W, Bassuk SS, Mora S, et al. Vitamin D Supplements and Prevention of Cancer and Cardiovascular Disease. N Engl J Med. 2019;380(1):33-44. PubMed PMID: 30415629.

59 Abdulridha MK, Abdulaali AR, Arif IS. Modulation of endogenous angiotensin II, systolic, and diastolic blood pressure in hypovitaminosis D patients with cardiovascular risk after oral Vitamin D3 supplementation. Int J Res Pharm Sci. 2018;9(4):1382-92. 
60 Borgi L, McMullan C, Wohlhueter A, Curhan GC, Fisher ND, Forman JP. Effect of Vitamin D on Endothelial Function: A Randomized, DoubleBlind, Placebo-Controlled Trial. Am J Hypertens. 2017;30(2):124-9. PubMed PMID: 28077419.

61 Chacko SJ, Pauwaa S, Barengolts E, Ciubotaru I, Kansal MM. Vitamin D Attenuates Left Atrial Volume Changes in African American Males with Obesity and Prediabetes. Echocardiography. 2016;33(5):681-5. PubMed PMID: 26711179.

62 Golzarand M, Shab-Bidar S, Koochakpoor G, Speakman JR, Djafarian K. Effect of vitamin D3 supplementation on blood pressure in adults: An updated meta-analysis. Nutr Metab Cardiovasc Dis. 2016;26(8):66373. PubMed PMID: 27287826.

63 Kubiak J, Thorsby PM, Kamycheva E, Jorde R. Vitamin D supplementation does not improve CVD risk factors in vitamin D-insufficient subjects. Endocr Connect. 2018;7(6):840-9. PubMed PMID: 29764903.

64 Ramly M, Ming MF, Chinna K, Suboh S, Pendek R. Effect of vitamin D supplementation on cardiometabolic risks and health-related quality of life among urban premenopausal women in a tropical country--a randomized controlled trial. PLoS ONE [Internet].2014;9(10):[e110476 p.]. Disponible en: https://dx.doi.org/10.1371/journal.pone.0110476.

65 Scragg R, Stewart AW, Waayer D, Lawes CMM, Toop L, Sluyter J, et al. Effect of monthly high-dose vitamin D supplementation on cardiovascular disease in the vitamin D assessment study: A randomized clinical trial. JAMA Cardiology. 2017;2(6):608-16.

66 Shu L, Huang K. Effect of vitamin D supplementation on blood pressure parameters in patients with vitamin $\mathrm{D}$ deficiency: a systematic review and meta-analysis. J Am Soc Hypertens. 2018;12(7):488-96.

67 Sluyter JD, Camargo CA, Waayer D, Lawes CMM, Toop L, Khaw KT, et al. Effect of Monthly, High-Dose, Long-Term Vitamin D on Lung Function: A Randomized Controlled Trial. Nutrients. 2017;9(12):13. PubMed PMID: 29236049.

$68 \mathrm{Wu}$ L, Sun D. Effects of calcium plus vitamin D supplementation on blood pressure: a systematic review and meta-analysis of randomized controlled trials. J Hum Hypertens. 2017;31(9):547-54. PubMed PMID: 28230063. 
69 Zaleski A, Panza G, Swales H, Arora P, Newton-Cheh C, Wang T, et al. High-Dose versus Low-Dose Vitamin D Supplementation and Arterial Stiffness among Individuals with Prehypertension and Vitamin D Deficiency. Dis Markers. 2015:918968. PubMed PMID: 26451070.

70 Bolland MJ, Grey A, Avenell A. Effects of vitamin D supplementation on musculoskeletal health: a systematic review, meta-analysis, and trial sequential analysis. Lancet Diabetes Endocrinol. 2018;6(11):847-58. PubMed PMID: 30293909.

71 Cangussu LM, Nahas-Neto J, Orsatti CL, Poloni PF, Schmitt EB, Almeida-Filho B, et al. Effect of isolated vitamin D supplementation on the rate of falls and postural balance in postmenopausal women fallers: a randomized, double-blind, placebo-controlled trial. Menopause. 2016;23(3):267-74. PubMed PMID: 26554884.

72 Guirguis-Blake JM, Michael YL, Perdue LA, Coppola EL, Beil TL. Interventions to Prevent Falls in Older Adults: Updated Evidence Report and Systematic Review for the US Preventive Services Task Force. JAMA. 2018;319(16):1705-16. PubMed PMID: 29710140.

73 Kahwati L, Palmieri Weber R, Pan H, Gourlay M, LeBlanc E, CokerSchwimmer M, et al. Vitamin D, Calcium, or Combined Supplementation for the Primary Prevention of Fractures in Community-Dwelling Adults: An Evidence Review for the U.S. Preventive Services Task Force. Rockville (MD): Agency for Healthcare Research and Quality; 2018. Informe No.:Evidence Synthesis No. 160. [consultado 20 ene 2020]. Disponible en: https://www.ncbi.nlm.nih.gov/books/NBK525398/.

74 Aloia J, Fazzari M, Islam S, Mikhail M, Shieh A, Katumuluwa S, et al. Vitamin D Supplementation in Elderly Black Women Does Not Prevent Bone Loss: A Randomized Controlled Trial. J Bone Miner Res. 2018;33(11):1916-22. PubMed PMID: 29905969.

75 Nahas-Neto J, Cangussu LM, Orsatti CL, Bueloni-Dias FN, Poloni PF, Schmitt EB, et al. Effect of isolated vitamin D supplementation on bone turnover markers in younger postmenopausal women: a randomized, double-blind, placebo-controlled trial. Osteoporos Int. 2018;29(5):1125-33. PubMed PMID: 29450585.

76 Smith LM, Gallagher JC, Kaufmann M, Jones G. Effect of increasing doses of vitamin D on bone mineral density and serum N-terminal 
telopeptide in elderly women: a randomized controlled trial. J Intern Med. 2018;284(6):685-93. PubMed PMID: 30137647.

77 Trautvetter U, Neef N, Leiterer M, Kiehntopf M, Kratzsch J, Jahreis G. Effect of calcium phosphate and vitamin D(3) supplementation on bone remodelling and metabolism of calcium, phosphorus, magnesium and iron. Nutr J. 2014;13:6. PubMed PMID: 24438153.

78 Cangussu LM, Nahas-Neto J, Orsatti CL, Bueloni-Dias FN, Nahas EAP. Effect of vitamin D supplementation alone on muscle function in postmenopausal women: a randomized, double-blind, placebocontrolled clinical trial. Osteoporos Int. 2015;26(10):2413-21.

79 Gao LH, Zhu WJ, Liu YJ, Gu JM, Zhang ZL, Wang O, et al. Physical performance and life quality in postmenopausal women supplemented with Vitamin D: a two-year prospective study. Acta Pharmacol Sin. 2015;36(9):1065-73. PubMed PMID: 26279157.

80 Hansen K, Johnson R, Chambers K, Johnson M, Lemon C, Vo T, et al. Treatment of Vitamin D Insufficiency in Postmenopausal Women: A Randomized Clinical Trial. JAMA Intern Med. 2015;175(10):1612-21.

81 Knutsen KV, Madar AA, Lagerløv P, Brekke M, Raastad T, Stene LC, et al. Does vitamin D improve muscle strength in adults? A randomized, double-blind, placebo-controlled trial among ethnic minorities in Norway. Journal of clinical endocrinology and metabolism. 2014;99(1):194-202. PubMed PMID: CN-00979257.

82 Saha S, Goswami R, Ramakrishnan L, Vishnubhatla S, Mahtab S, Kar P, et al. Vitamin D and calcium supplementation, skeletal muscle strength and serum testosterone in young healthy adult males: Randomized control trial. Clin Endocrinol (Oxf). 2018;88(2):217-26. PubMed PMID: 29095521.

83 Suebthawinkul C, Panyakhamlerd K, Yotnuengnit P, Suwan A, Chaiyasit $\mathrm{N}$, Taechakraichana $\mathrm{N}$. The effect of vitamin D2 supplementation on muscle strength in early postmenopausal women: a randomized, double-blind, placebo-controlled trial. Climacteric. 2018;21(5):491-7. PubMed PMID: CN-01651817.

84 Vaes AMM,Tieland M, Toussaint N, Nilwik R, Verdijk LB, van Loon LJC, et al. Cholecalciferol or 25-Hydroxycholecalciferol Supplementation Does Not Affect Muscle Strength and Physical Performance in Prefrail 
and Frail Older Adults. J Nutr. 2018;148(5):712-20. PubMed PMID: 30053278 .

85 Wood AD, Secombes KR, Thies F, Aucott LS, Black AJ, Reid DM, et al. A parallel group double-blind RCT of vitamin D3 assessing physical function: is the biochemical response to treatment affected by overweight and obesity? Osteoporosis International. 2014;25(1):305-15. PubMed PMID: 23982800.

86 Bjelakovic G, Gluud LL, Nikolova D, Whitfield K, Wetterslev J, Simonetti RG, et al. Vitamin D supplementation for prevention of mortality in adults. Cochrane Database Syst Rev. 2014 (1):CD007470. PubMed PMID: 24414552.

87 Knutsen KV, Madar AA, Brekke M, Meyer HE, Natvig B, Mdala I, et al. Effect of vitamin D on musculoskeletal pain and headache: a randomized, double-blind, placebo-controlled trial among adult ethnic minorities in Norway. Pain. 2014;155(12):2591-8. PubMed PMID: 25261164.

88 Straube S, Derry S, Straube C, Moore RA. Vitamin D for the treatment of chronic painful conditions in adults. Cochrane Database Syst Rev. 2015 (5):CD007771. PubMed PMID: 25946084.

89 Ginde AA, Blatchford P, Breese K, Zarrabi L, Linnebur SA, Wallace JI, et al. High-Dose Monthly Vitamin D for Prevention of Acute Respiratory Infection in Older Long-Term Care Residents: A Randomized Clinical Trial. J Am Geriatr Soc. 2017;65(3):496-503. PubMed PMID: 27861708.

90 Goodall EC, Granados AC, Luinstra K, Pullenayegum E, Coleman BL, Loeb M, et al. Vitamin D3 and gargling for the prevention of upper respiratory tract infections: a randomized controlled trial. BMC Infect Dis. 2014;14:273. PubMed PMID: 24885201.

91 Martineau AR, Hanifa Y, Witt KD, Barnes NC, Hooper RL, Patel $\mathrm{M}$, et al. Double-blind randomised controlled trial of vitamin D3 supplementation for the prevention of acute respiratory infection in older adults and their carers (ViDiFlu). Thorax. 2015;70(10):953-60. PubMed PMID: 26063508.

92 Scientific Advisory Committee on Nutrition (SACN). Rapid review: Vitamin D and acute respiratory tract infections. London: SACN;2020 
93 Simpson S, van der Mei I, Stewart N, Blizzard L, Prudence Tettey \& Bruce Taylor. Weekly cholecalciferol supplementation results in significant reductions in infection risk among the vitamin $\mathrm{D}$ deficient: results from the CIPRIS pilot RCT. BMC Nutr. 2015; 1:7.

94 Tran B, Armstrong BK, Ebeling PR, English DR, Kimlin MG, van der Pols JC, et al. Effect of vitamin D supplementation on antibiotic use: a randomized controlled trial. American Journal of Clinical Nutrition. 2014;99(1):156-61. PubMed PMID: CN-00961035.

95 Barker T, Rogers VE, Levy M, Templeton J, Goldfine H, Schneider $\mathrm{ED}$, et al. Supplemental vitamin $\mathrm{D}$ increases serum cytokines in those with initially low 25-hydroxyvitamin D: a randomized, double blind, placebo-controlled study. Cytokine. 2015;71(2):132-8. PubMed PMID: 25461390 .

96 Jamka M, Wozniewicz M, Walkowiak J, Bogdanski P, Jeszka J, Stelmach-Mardas M. The effect of vitamin D supplementation on selected inflammatory biomarkers in obese and overweight subjects: a systematic review with meta-analysis. Eur J Nutr. 2016;55(6):2163-76. PubMed PMID: 26538075.

97 Sinha-Hikim I, Duran P, Shen R, Lee M, Friedman TC, Davidson MB. Effect of long term vitamin D supplementation on biomarkers of inflammation in Latino and African-American subjects with pre-diabetes and hypovitaminosis D. Horm Metab Res. 2015;47(4):280-3. PubMed PMID: 25011019.

98 Waterhouse M, Tran B, Ebeling PR, English DR, Lucas RM, Venn AJ, et al. Effect of vitamin D supplementation on selected inflammatory biomarkers in older adults: a secondary analysis of data from a randomised, placebo-controlled trial. Br J Nutr. 2015;114(5):693-9. PubMed PMID: 26206095.

99 Chandler PD, Giovannucci EL, Scott JB, Bennett GG, Ng K, Chan AT, et al. Null association between vitamin D and PSA levels among black men in a vitamin D supplementation trial. Cancer epidemiology, biomarkers \& prevention. 2014;23(9):1944-7. PubMed PMID: 24974387.

100 Lerchbaum E, Pilz S, Trummer C, Schwetz V, Pachernegg O, Heijboer AC, et al.Vitamin D and Testosterone in Healthy Men: A Randomized Controlled Trial. J Clin Endocrinol Metab. 2017;102(11):4292-302. PubMed PMID: 28938446. 
101 Blondon M, Rodabough RJ, Budrys N, Johnson KC, Berger JS, Shikany JM, et al. The effect of calcium plus vitamin D supplementation on the risk of venous thromboembolism: from the women's health initiative randomized controlled trial. Thromb Haemost. 2015;113(5):988-98. PubMed PMID: CN-01083920.

102 Scragg R, Waayer D, Stewart AW, Lawes CMM, Toop L, Murphy J, et al. The Vitamin D Assessment (ViDA) Study: design of a randomized controlled trial of vitamin D supplementation for the prevention of cardiovascular disease, acute respiratory infection, falls and non-vertebral fractures. J Steroid Biochem Mol Biol. 2016;164:318-25.

103 Gallagher JC, Smith LM, Yalamanchili V. Incidence of hypercalciuria and hypercalcemia during vitamin $\mathrm{D}$ and calcium supplementation in older women. Menopause. 2014;21(11):1173-80.

104 McMullan CJ, Borgi L, Curhan GC, Fisher N, Forman JP. The effect of vitamin $\mathrm{D}$ on renin-angiotensin system activation and blood pressure: a randomized control trial. Journal of hypertension. 2017;35(4):822-9. PubMed PMID: 28033130.

105 Nygaard B, Frandsen NE, Brandi L, Rasmussen K, Oestergaard OV, Oedum L, et al. Effects of high doses of cholecalciferol in normal subjects: A randomized double-blinded, placebo- Controlled trial. PLoS ONE. 2014;9 (8) (no pagination)(e102965).

106 Jódar Gimeno E. Recomendaciones sobre cómo administrar la vitamina D. Guías internacionales y nacionales. Rev Osteoporos Metab Miner. 2014;6(Supl 1):S19-22.

107 Jorde R, Kubiak J, Svartberg J, Fuskevag OM, Figenschau Y, Martinaityte I, et al. Vitamin D supplementation has no effect on cognitive performance after four months in mid-aged and older subjects. J Neurol Sci. 2019;396:165-71. PubMed PMID: 30472553.

108 Pettersen JA. Does high dose vitamin D supplementation enhance cognition?: a randomized trial in healthy adults. Experimental Gerontology. 2017;90:90-7. PubMed PMID: CN-01340582.

109 Knutsen KV, Madar AA, Brekke M, Meyer HE, Eggemoen AR, Mdala I, et al. Effect of vitamin D on thyroid autoimmunity: a randomized, double-blind, controlled trial among ethnic minorities. J Clin Endocrinol Metab. 2017;1(5):470-9. 
110 Barengolts E, Manickam B, Eisenberg Y,Akbar A, Kukreja S, Ciubotaru I. Effect of High-Dose Vitamin D Repletion on Glycemic Control in African-American Males with Prediabetes and Hypovitaminosis D. Endocrine Practice. 2015;21(6):604-12. PubMed PMID: 25716637.

111 Jorde R, Sollid ST, Svartberg J, Schirmer H, Joakimsen RM, Njolstad I, et al. Vitamin D 20,000 IU per Week for Five Years Does Not Prevent Progression From Prediabetes to Diabetes. J Clin Endocrinol Metab. 2016;101(4):1647-55. PubMed PMID: 26829443.

112 Mitchell DM, Leder BZ, Cagliero E, Mendoza N, Henao MP, Hayden DL, et al. Insulin secretion and sensitivity in healthy adults with low vitamin $\mathrm{D}$ are not affected by high-dose ergocalciferol administration: a randomized controlled trial. American Journal of Clinical Nutrition. 2015;102(2):385-92. PubMed PMID: 26156733.

113 Moreira-Lucas TS, Duncan AM, Rabasa-Lhoret R, Vieth R, Gibbs AL, Badawi A, et al. Effect of vitamin D supplementation on oral glucose tolerance in individuals with low vitamin $\mathrm{D}$ status and increased risk for developing type 2 diabetes (EVIDENCE): A double-blind, randomized, placebo-controlled clinical trial. Diabetes, Obesity \& Metabolism. 2017;19(1):133-41. PubMed PMID: 27717236.

114 Mousa A, Naderpoor N, de Courten MP, Teede H, Kellow N, Walker $\mathrm{K}$, et al. Vitamin D supplementation has no effect on insulin sensitivity or secretion in vitamin D-deficient, overweight or obese adults: a randomized placebo-controlled trial. American Journal of Clinical Nutrition. 2017;105(6):1372-81. PubMed PMID: 28490514.

115 Oosterwerff MM,Eekhoff EM, Van Schoor NM,Boeke AJ, Nanayakkara $\mathrm{P}$, Meijnen R, et al. Effect of moderate-dose vitamin D supplementation on insulin sensitivity in vitamin D-deficient non-Western immigrants in the Netherlands: a randomized placebo-controlled trial. American Journal of Clinical Nutrition. 2014;100(1):152-60. PubMed PMID: 24898240.

116 Osati S, Homayounfar R, Hajifaraji M. Metabolic effects of vitamin $\mathrm{D}$ supplementation in vitamin $\mathrm{D}$ deficient patients (a double-blind clinical trial). Diabetes \& Metabolic Syndrome. 2016;10(2 Suppl 1):S7S10. PubMed PMID: 27094871.

117 Sun X, Cao ZB, Tanisawa K, Ito T, Oshima S, Higuchi M. Vitamin $\mathrm{D}$ supplementation reduces insulin resistance in Japanese adults: a 
secondary analysis of a double-blind, randomized, placebo-controlled trial. Nutrition research (new york, NY). 2016;36(10):1121-9. PubMed PMID: $\mathrm{CN}-01248787$.

118 Tepper S, Shahar DR, Geva D, Ish-Shalom S. Differences in homeostatic model assessment (HOMA) values and insulin levels after vitamin D supplementation in healthy men: a double-blind randomized controlled trial. Diabetes, Obesity \& Metabolism. 2016;18(6):633-7. PubMed PMID: 26890031.

119 Zarrin R, Ayremlou P, Ghassemi F. The effect of vitamin D supplementation on the glycemic status and the percentage of body fat mass in adults with prediabetes: a randomized clinical trial. Iran Red Crescent Med J. 2017;19(3). PubMed PMID: CN-01366601.

120 Zuk A, Fitzpatrick T, Rosella LC. Effect of Vitamin D3 Supplementation on Inflammatory Markers and Glycemic Measures among Overweight or Obese Adults: A Systematic Review of Randomized Controlled Trials. PLoS ONE [Revista en línea]. 2016;11(4):e0154215. PubMed PMID: 27116227.

121 Patil R, Karinkanta S, Tokola K, Kannus P, Sievänen H, Uusi-Rasi K. Effects of Vitamin D and Exercise on the Wellbeing of Older Community-Dwelling Women: a Randomized Controlled Trial. Gerontology. 2016;62(4):401-8. PubMed PMID: CN-01165808.

122 Mason C, de Dieu Tapsoba J, Duggan C, Wang CY, Korde L, McTiernan A. Repletion of vitamin $\mathrm{D}$ associated with deterioration of sleep quality among postmenopausal women. Preventive Medicine. 2016;93:166-70.

123 Grupo de trabajo sobre Guías de Práctica Clínica. Elaboración de Guías del Práctica Clínica en el Sistema Nacional de Salud: Actualización del Manual Metodológico [Internet]. Madrid: Ministerio de Sanidad, Consumo y Bienestar Social; Zaragoza: Guía Salud. 2016. [consultado 27 jul 2018]. Disponible en: http://portal.guiasalud.es/emanuales/ elaboracion_2/Capitulos/completo.pdf.

124 Comet Cortés P, Salcedo Fernández F. Guía metodológica para la elaboración de protocolos basados en la evidencia [Internet]. Zaragoza: Instituto Aragonés de Ciencias de la Salud; Servicio Aragonés de Salud. 2009. [consultado 27 jul 2018]. Disponible en: http://www.ics.aragon.es/ awgc/contenido.detalle.do?idContenido $=1431 \&$ vienede $=$ null . 
125 Puñal-Riobóo J, Baños Álvarez E, Varela Lema L, Castillo Muñoz M, Atienza Merino G, Ubago Pérez R, et al. Guía para la elaboración y adaptación de informes rápidos de evaluación de tecnologías sanitarias. Santiago de Compostela: Agencia Gallega para la Gestión del Conocimiento en Salud. Unidad de Asesoramiento Científicotécnico, avalia-t; Madrid: Ministerio de Sanidad, Servicios Sociales e Igualdad; 2016. [consultado 27 jul 2018]. Disponible en: http://hdl. handle.net/20.500.11940/9007.

126 The GRADE working group. Grading of Recommendations of Assessment Development and Evaluations [Internet]. [consultado 27 jul 2018]. Disponible en: http://www.gradeworkinggroup.org/.

127 Donabedian A, Ann Arbor M. Explorations in Quality Assessment and Monitoring. The Definition of Quality and Approaches to Its Assessment. 1: Health administration press; 1980.

128 Aguilar del Rey F. Protocolo de tratamiento de la deficiencia de vitamina D. Med Clin (Barc). 2014;142(3):125-31.

129 Dusso A. El sistema hormonal de la vitamina D: lo que sabemos y lo que nos queda por saber. Nefrología Sup Ext. 2011;2(5):37-43.

130 Working Group on Vitamin D. Vitamin D and Health. London: Scientific Advisory Committee on Nutrition (SACN);2016. [consultado 24 jul 2020]. Disponible en: https://www.gov.uk/government/groups/ scientific-advisory-committee-on-nutrition.

131 BEDCA Base de Datos Española de Composición de Alimentos [Base de datos en línea]. Madrid:Agencia española de seguridad alimentaria y nutrición; 2006. [Consultado 27 jul 2020]. Listado de alimentos de la consulta, Vitamina D. Disponible en: https://www.bedca.net/bdpub/ index.php.

132 Alimentos con vitaminas. Alimentos para una dieta rica en vitamina. Alimentos ricos en vitamina D [Internet]. [consultado 13 may 2019]. Disponible en: https://alimentosvitaminas.com/alimentos-vitamina-d.

133 Comisión Autonómica Central de Farmacia e Terapéutica. Vitamina D: Recomendacións de uso na poboación xeral [Documento Interno]. 2018.

134 Fernández Moreno A, Donnay Candil S, Beamud Lagos M. Cambios estacionales en las concentraciones séricas de vitamina $\mathrm{D}$ en pacientes 
atendidos en un Centro de Salud. Rev Osteoporos Metab Miner. 2012;4(2):63-8.

135 Dawson-Hughes B. Vitamin D deficiency in adults: Definition, clinical manifestations, and treatment [Internet]. UpToDate; 2019 [consultado 20 may 2020]. Disponible en: https://www.uptodate.com/contents/ vitamin-d-deficiency-in-adults-definition-clinical-manifestations-andtreatment.

136 Holick MF. Vitamin D: Evolutionary, Physiological and Health Perspectives. Current Drug Targets. 2011;12(1):4-18.

137 Pludowski P, Karczmarewicz E, Bayer M, Carter GD, Chlebna-Sokol D, Czech-Kowalska J, et al. Practical guidelines for the supplementation of vitamin D and the treatment of deficits in Central Europe recommended vitamin $\mathrm{D}$ intakes in the general population and groups at risk of vitamin D deficiency. Endokrynol Pol. 2013;64(4):319-27.

138 Bouillon R. Comparative analysis of nutritional guidelines for vitamin D. Nat Rev Endocrinol. 2017;13:466-79.

139 Ross A, Manson J, Abrams S, Aloia J, Brannon P, Clinton S, et al. The 2011 report on dietary reference intakes for calcium and vitamin D from the Institute of Medicine: What clinicians need to know. J Clin Endocrinol Metab. 2011;96(1):53-8.

140 Cardiff \& Vale University Health Board. Diagnosis and Management of Vitamin D Deficiency in Children and Adults Guidelines [Internet]. Cardif: Cardiff and Vale UHB; 2016. [consultado 27 mar 2019]. Disponible en: http://www.cardiffandvaleuhb.wales.nhs.uk/sitesplus/ documents/1143/UHB205\%20V2\%20January\%2016.pdf.

141 Ministerio de Sanidad Servicios Sociales e Igualdad. Manual de codificación. CIE-10-ES Diagnósticos. Unidad Técnica de Codificación CIE-10-ES. Madrid: Ministerio de Sanidad, Servicios Sociales e Igualdad; 2016. [consultado 20 ene 2020]. Disponible en: https:// www.mscbs.gob.es/estadEstudios/estadisticas/normalizacion/CIE10/ UT_MANUAL_DIAG_2016_prov1.pdf.

142 van Schoor N, Lips P. Worldwide vitamin D status. Best Pract Res Clin Endocrinol Metab. 2011;25(4):671-80.

143 Urgell R, Alfayate R, Ferrer M, Granada E, Álvarez E, Berlanga G, et al. Recomendaciones para la valoración bioquímica del estatus 
de vitamina D. Posicionamiento de la Comisión de Hormonas de la Sociedad Española de Medicina de Laboratorio (SEQCML). Barcelona:Sociedad Española de Medicina de Laboratorio (SEQCML); 2019. Informe $\mathrm{N}^{\circ} .:$ Rec Vit D. Versión 1. A. [consultado 23 dic 2020]. Disponible en: https://seqc.es/docs/Comisiones/Hormonas/Rec\%20 Vit\%20D.pdf.

144 Vitamina D: evidencias y controversias. Infac. 2012;20(2):7-12.

145 Ministerio de Sanidad Consumo y Bienestar Social. Vitamina D: casos graves de hipercalcemia por sobredosificación en pacientes adultos y en pediatría [Internet]. Madrid: Ministerio de Sanidad, Consumo y Bienestar Social; 2019. [consultado 27 mar 2019]. Disponible en: https://www.aemps.gob.es/informa/notasInformativas/ medicamentosUsoHumano/seguridad/2019/NI_MUH_FV-2-2019vitamina-D.htm.

146 Shea BJ, Reeves BC, Wells G, Thuku M, Hamel C, Moran J, et al. AMSTAR 2: a critical appraisal tool for systematic reviews that include randomised or non-randomised studies of healthcare interventions, or both. BMJ. 2017;358:j4008. PubMed PMID: 28935701.

147 Sun Q, Pan A, Hu F, Manson J, Rexrode K. 25-Hydroxyvitamin D levels and the risk for stroke: a prospective study and meta-analysis. Stroke. 2012;43(6):1470-7.

148 Autier P, Boniol M, Pizot C, Mullie P. Vitamin D status and ill health: a systematic review. Lancet Diabetes Endocrinol. 2014;2(1):76-89.

149 Chung M, Lee J, Terasawa T, Lau J, Trikalinos T. Vitamin D with or without calcium supplementation for prevention of cancer and fractures: an updated meta-analysis for the U.S. Preventive Services Task Force. Ann Intern Med. 2011; 155(12):827-38.

150 Gandini S, Boniol M, Haukka J, Byrnes G, Cox B, Sneyd M, et al. Metaanalysis of observational studies of serum 25-hydroxyvitamin D levels and colorectal, breast and prostate cancer and colorectal adenoma. Int J Cancer. 2011;128(6):1414-24.

151 Theodoratou E, Tzoulaki I, Zgaga L, Ioannidis J. Vitamin D and multiple health outcomes: umbrella review of systematic reviews and meta-analyses of observational studies and randomised trials. BMJ. 2014;348:1-19. 
152 Yin L, Grandi N, Raum E, Haug U, Arndt V, Brenner H. Meta-analysis: longitudinal studies of serum vitamin $\mathrm{D}$ and colorectal cancer risk. Aliment Pharmacol Ther. 2009;30(2):113-25. PubMed PMID: 19392870.

153 Yin L, Grandi N, Raum E, Haug U, Arndt V, Brenner H. Metaanalysis: serum vitamin D and breast cancer risk. Eur J Cancer. 2010;46(12):2196-205.

154 Eliassen A, Spiegelman D, Hollis B, Horst R, Willett W, Hankinson S. Plasma 25-hydroxyvitamin D and risk of breast cancer in the Nurses' Health Study II. Breast Cancer Res. 2011;13(3):R50.

155 Chung M, Balk M, Brendel M, Ip S, Lau J, Lee J, et al. Vitamin D and Calcium: A Systematic Review of Health Outcomes. AHRQ Publication No. 09-E015. Rockville, MD: Agency for healthcare Research and Quality. 2009. [consultado 2019]. Disponible en: http:// www.ahrq.gov/downloads/pub/evidence/pdf/vitadcal/vitadcal.pdf.

156 Zittermann A, Iodice S, Pilz S, Grant WB, Bagnardi V, Gandini S. Vitamin D deficiency and mortality risk in the general population: a meta-analysis of prospective cohort studies. Am J Clin Nutr. 2012;95(1):91-100. PubMed PMID: 22170374.

157 Dror Y, Giveon SM, Hoshen M, Feldhamer I, Balicer RD, Feldman BS. Vitamin D levels for preventing acute coronary syndrome and mortality: evidence of a nonlinear association. J Clin Endocrinol Metab. 2013;98(5):2160-7. PubMed PMID: 23533239.

158 Durup D, Jorgensen HL, Christensen J, Schwarz P, Heegaard AM, Lind B. A reverse J-shaped association of all-cause mortality with serum 25-hydroxyvitamin D in general practice: the CopD study. J Clin Endocrinol Metab. 2012;97(8):2644-52. PubMed PMID: 22573406.

159 Sempos C, Durazo-Arvizu R, Dawson-Hughes B, Yetley E, Looker A, Schleicher R, et al. Is there a reverse J-shaped association between 25-hydroxyvitamin D and all-cause mortality? Results from the U.S. nationally representative NHANES. J Clin Endocrinol Metab. 2013;98(7):3001-9.

160 Chowdhury R, Kunutsor S, Vitezova A, Oliver-Williams C, Chowdhury S, Kiefte-de-Jong JC, et al. Vitamin D and risk of cause specific death: systematic review and meta-analysis of observational cohort and randomised intervention studies. BMJ. 2014;348:g1903. PubMed PMID: 24690623. 
161 Maddock J, Berry DJ, Geoffroy MC, Power C, Hypponen E. Vitamin $\mathrm{D}$ and common mental disorders in mid-life: cross-sectional and prospective findings. Clin Nutr. 2013;32(5):758-64. PubMed PMID: 23395104.

162 Milaneschi Y, Shardell M, Corsi AM, Vazzana R, Bandinelli S, Guralnik JM, et al. Serum 25-hydroxyvitamin D and depressive symptoms in older women and men. J Clin Endocrinol Metab. 2010;95(7):3225-33. PubMed PMID: 20444911.

163 Chlebowski RT, Johnson KC, Kooperberg C, Pettinger M, WactawskiWende J, Rohan T, et al. Calcium plus vitamin D supplementation and the risk of breast cancer. J Natl Cancer Inst. 2008;100(22):1581-91. PubMed PMID: 19001601.

164 Wactawski-Wende J, Kotchen JM, Anderson GL, Assaf AR, Brunner RL, O'Sullivan MJ, et al. Calcium plus vitamin D supplementation and the risk of colorectal cancer. N Engl J Med. 2006;354(7):684-96. PubMed PMID: 16481636.

165 Centro Cochrane Iberoamericano, trad. Manual cochrane de revisiones sistemáticas de intervenciones, versión 5.1.0 [Internet]. Barcelona: Centro Cochrane Iberoamericano; 2012 [actualizado mar 2011; consultado 18 jul 2020]. Disponible en: http:/www.cochrane.es/?q=es/ node/269.

166 Chapuy MC, Pamphile R, Paris E, Kempf C, Schlichting M, Arnaud S, et al. Combined calcium and vitamin D3 supplementation in elderly women: confirmation of reversal of secondary hyperparathyroidism and hip fracture risk: the Decalyos II study. Osteoporos Int. 2002;13(3):25764. PubMed PMID: 11991447.

167 Lips P, Graafmans W, Ooms M, al. e. Vitamin D supplementation and fracture incidence in elderly persons. A randomized, placebo-controlled clinical trial. Ann Intern Med. 1996;124(4):400-6.

168 Pfeifer M, Begerow B, Minne HW, Abrams C, Nachtigall D, Hansen C. Effects of a short-term vitamin D and calcium supplementation on body sway and secondary hyperparathyroidism in elderly women. J Bone Miner Res. 2000;15(6):1113-8. PubMed PMID: 10841179.

169 Pfeifer M, Begerow B, Minne HW, Suppan K, Fahrleitner-Pammer A, Dobnig H.Effects of a long-term vitamin D and calcium supplementation on falls and parameters of muscle function in community-dwelling 
older individuals. Osteoporos Int. 2009;20(2):315-22. PubMed PMID: 18629569.

170 Jackson RD, LaCroix AZ, Gass M, Wallace RB, Robbins J, Lewis CE, et al. Calcium plus vitamin D supplementation and the risk of fractures. N Engl J Med. 2006;354(7):669-83. PubMed PMID: 16481635.

171 Bischoff HA, Stahelin HB, Dick W, Akos R, Knecht M, Salis C, et al. Effects of vitamin D and calcium supplementation on falls: a randomized controlled trial. J Bone Miner Res. 2003;18(2):343-51. PubMed PMID: 12568412.

172 Kärkkäinen M, Tuppurainen M, Salovaara K, al. e. Does daily vitamin D $800 \mathrm{IU}$ and calcium $1000 \mathrm{mg}$ supplementation decrease the risk of falling in ambulatory women aged 65-71 years? A 3-year randomized population-based trial (OSTPRE-FPS). Maturitas. 2010;65(4):359-65.

173 Wood AD, Secombes KR, Thies F, Aucott L, Black AJ, Mavroeidi A, et al. Vitamin D3 supplementation has no effect on conventional cardiovascular risk factors: a parallel-group, double-blind, placebocontrolled RCT. J Clin Endocrinol Metab. 2012;97(10):3557-68. PubMed PMID: 22865902.

174 Kahwati LC, Weber RP, Pan H, Gourlay M, LeBlanc E, CokerSchwimmer M, et al.Vitamin D, Calcium, or Combined Supplementation for the Primary Prevention of Fractures in Community-Dwelling Adults: Evidence Report and Systematic Review for the US Preventive Services Task Force. JAMA. 2018;319(15):1600-12. PubMed PMID: 29677308.

175 Grossman DC, Curry SJ, Owens DK, Barry MJ, Caughey AB, Davidson $\mathrm{KW}$, et al. Interventions to Prevent Falls in Community-Dwelling Older Adults: US Preventive Services Task Force Recommendation Statement. JAMA. 2018;319(16):1696-704. PubMed PMID: 29710141.

176 Lips P, Binkley N, Pfeifer M, Recker R, Samanta S, Cohn DA, et al. Once-weekly dose of 8400 IU vitamin $\mathrm{D}$ (3) compared with placebo: effects on neuromuscular function and tolerability in older adults with vitamin D insufficiency. Am J Clin Nutr. 2010;91(4):985-91. PubMed PMID: 20130093.

177 Gallagher JC, Sai A, Templin T, 2nd, Smith L. Dose response to vitamin D supplementation in postmenopausal women: a randomized trial. Ann Intern Med. 2012;156(6):425-37. PubMed PMID: 22431675. 
178 Brazier M, Grados F, Kamel S, Mathieu M, Morel A, Maamer M, et al. Clinical and laboratory safety of one year's use of a combination calcium + vitamin D tablet in ambulatory elderly women with vitamin D insufficiency: results of a multicenter, randomized, doubleblind, placebo-controlled study. Clin Ther. 2005;27:1885-93.

179 Gallagher JC, Jindal PS, Smith LM. Vitamin D supplementation in young White and African American women. J Bone Miner Res. 2014;29(1):173-81. PubMed PMID: 23761326.

180 Gallagher JC, Peacock M, Yalamanchili V, Smith LM. Effects of vitamin D supplementation in older African American women. J Clin Endocrinol Metab. 2013;98(3):1137-46. PubMed PMID: 23386641.

181 Grimnes G, Figenschau Y, Almas B, Jorde R. Vitamin D, insulin secretion, sensitivity, and lipids: results from a case-control study and a randomized controlled trial using hyperglycemic clamp technique. Diabetes. 2011;60(11):2748-57. PubMed PMID: 21911741.

182 Kärkkäinen M, Tuppurainen M, Salovaara K, Sandini L, Rikkonen $\mathrm{T}$, Sirola J, et al. Effect of calcium and vitamin D supplementation on bone mineral density in women aged 65-71 years: a 3-year randomized population-based trial (OSTPRE-FPS). Osteoporos Int. 2010;21(12):2047-55. PubMed PMID: 20204604.

183 Krieg MA, Jacquet AF, Bremgartner M, Cuttelod S, Thiebaud D, Burckhardt P. Effect of supplementation with vitamin D3 and calcium on quantitative ultrasound of bone in elderly institutionalized women: a longitudinal study. Osteoporos Int. 1999;9(6):483-8. PubMed PMID: 10624454.

184 LaCroix AZ, Kotchen J, Anderson G, Brzyski R, Cauley JA, Cummings $\mathrm{SR}$, et al. Calcium plus vitamin D supplementation and mortality in postmenopausal women: the Women's Health Initiative calciumvitamin D randomized controlled trial. J Gerontol A Biol Sci Med Sci. 2009;64(5):559-67. PubMed PMID: 19221190.

185 De Boer I, Tinker L, Connelly S, al. e. Calcium plus vitamin D supplementation and the risk of incident diabetes in the Women's Health Initiative. Diabetes Care. 2008;31(4):701-7.

186 Kjaergaard M, Waterloo K, Wang CE, Almas B, Figenschau Y, Hutchinson MS, et al. Effect of vitamin D supplement on depression scores in people with low levels of serum 25-hydroxyvitamin D: nested 
case-control study and randomised clinical trial. Br J Psychiatry. 2012;201(5):360-8. PubMed PMID: 22790678.

187 Arvold DS, Odean MJ, Dornfeld MP, Regal RR, Arvold JG, Karwoski $\mathrm{GC}$, et al. Correlation of symptoms with vitamin D deficiency and symptom response to cholecalciferol treatment: a randomized controlled trial. Endocr Pract. 2009;15(3):203-12. PubMed PMID: 19364687. 



\section{Anexos}

\section{Anexo 1. Declaración de conflictos de interés}

Cada una de las personas participantes en la elaboración y revisión del OPBE ha realizado una declaración de intereses sobre la materia que se trata en el OPBE, sometida posteriormente a evaluación por parte del grupo coordinador.

En la siguiente tabla se muestra de forma resumida la declaración de intereses de cada uno de ellos. Las declaraciones de intereses completas se encuentran disponibles (a petición) para su consulta en avalia-t.

\begin{tabular}{|l|l|}
\hline Autor & Conflicto \\
\hline Silvia Sandra Abelleira Ramos & Sin conflicto \\
\hline María Carmen Blas Fraga & Sin conflicto \\
\hline Rosendo Bugarín González & Sin conflicto \\
\hline Rosa Burgo López & Sin conflicto \\
\hline Carmen Casal Llorente & Sin conflicto \\
\hline Jesús Calviño Varela & Sin conflicto \\
\hline Manuel Castro Pazos & Sin conflicto \\
\hline José Antonio Díaz Peromingo & Sin conflicto \\
\hline María José Faraldo Vallés & Sin conflicto \\
\hline Begoña Graña Suárez & Sin conflicto \\
\hline María del Carmen Maceira Rozas & Sin conflicto \\
\hline Ana Belén Mariño Prol & Sin conflicto \\
\hline Xoan Miguéns Váquez & Sin conflicto \\
\hline Susana Romero Yuste & Sin conflicto \\
\hline Leonor Varela Lema & Sin conflicto \\
\hline
\end{tabular}




\section{Anexo 2. Preguntas a responder}

Pregunta 1: ¿Cuál es la asociación entre los niveles séricos de 25(OH)D y los resultados en salud en población general sana? Resultados en: mortalidad, cáncer, fracturas, caídas, enfermedad cardiovascular, diabetes, depresión, funcionamiento cognitivo, estado funcional, etc.

Pregunta 2: ¿Cuál es la efectividad de la vitamina D, en variables duras/ finales, incluyendo enfermedades cardiovasculares, obesidad, cáncer, sistema inmunitario, osteoporosis, insuficiencia renal y la calcificación de los tejidos blandos, en población general sana? 


\section{Anexo 3. Estrategia de búsqueda}

\section{Anexo 3.1 Pregunta 1: Niveles de vitamina D y resultados en salud}

\section{Cochrane Library, Trials y Systematic Reviews (Wiley)}

\begin{tabular}{|c|c|c|}
\hline & Términos de búsqueda & ítems \\
\hline \#1 & 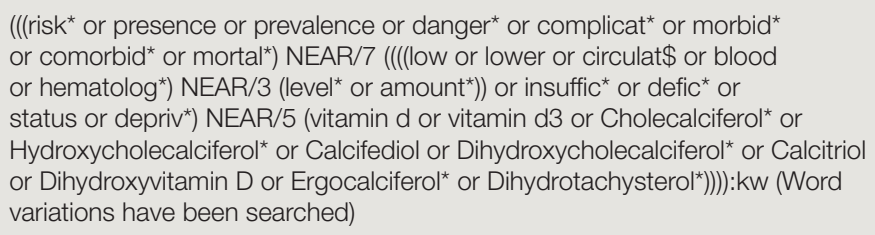 & 579 \\
\hline \#2 & $\begin{array}{l}\left(\left(\left(\text { risk }^{\star} \text { or presence or prevalence or danger }{ }^{\star} \text { or complicat }{ }^{\star} \text { or } \text { morbid }^{\star} \text { or }\right.\right.\right. \\
\left.\text { comorbid }{ }^{\star} \text { or mortal }{ }^{\star}\right) \text { NEAR7 (hypovitamin }{ }^{\star} \text { NEAR d))):kw (Word variations } \\
\text { have been searched) }\end{array}$ & 1 \\
\hline \#3 & \#1 OR \#2 with Cochrane Library publication date from Jan 2014 to present & 392 \\
\hline
\end{tabular}

\section{Crd Databases}

\begin{tabular}{|c|c|c|}
\hline & Términos de búsqueda & ítems \\
\hline 1 & 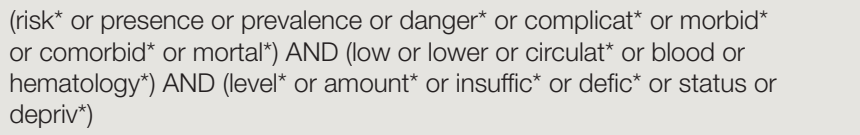 & 7839 \\
\hline 2 & 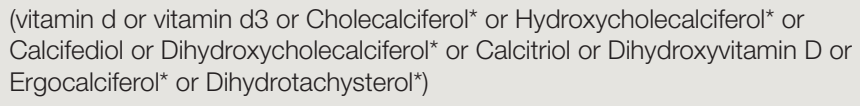 & 398 \\
\hline 3 & \#1 AND \#2 & 94 \\
\hline 4 & $\begin{array}{l}\text { (risk }^{\star} \text { or presence or prevalence or danger } \\
\text { comorbid }^{\star} \text { or } \text { mortal }^{\star} \text { ) AND (“hypovitamin } \text { d") }^{*}\end{array}$ & 0 \\
\hline 5 & \#3 OR \#4 & 94 \\
\hline 6 & * FROM 2014 TO 2018 & 14388 \\
\hline 7 & \#5 AND \#6 & 12 \\
\hline
\end{tabular}


Medline y preMedline (Ovid SP)

\begin{tabular}{|c|c|c|}
\hline & Términos de búsqueda & ítems \\
\hline 1 & exp vitamin $\mathrm{d} /$ & 54702 \\
\hline 2 & vitamin D deficiency/ & 13925 \\
\hline 3 & 1 or 2 & 57878 \\
\hline 4 & exp Mass Screening/ & 118595 \\
\hline 5 & Diagnostic Tests, Routine/ & 10345 \\
\hline 6 & 4 or 5 & 128303 \\
\hline 7 & 3 and 6 & 179 \\
\hline 8 & $\begin{array}{l}\text { ((risk\$ or presence or prevalence or danger } \$ \text { or complicat\$ or morbid } \$ \\
\text { or comorbid } \$ \text { or mortal\$) adj7 (((low or lower or circulat } \$ \text { or blood } \\
\text { or hematolog\$) adj3 (level\$ or amount\$)) or insuffic } \$ \text { or defic } \$ \text { or } \\
\text { status or depriv\$) adj5 (vitamin d or vitamin d3 or Cholecalciferol\$ or } \\
\text { Hydroxycholecalciferol\$ or Calcifediol or Dihydroxycholecalciferol\$ or } \\
\text { Calcitriol or Dihydroxyvitamin D or Ergocalciferol\$ or Dihydrotachysterol\$))). } \\
\text { mp. }\end{array}$ & 5119 \\
\hline 9 & $\begin{array}{l}\text { ((risk\$ or presence or prevalence or danger } \$ \text { or complicat } \$ \text { or morbid } \$ \\
\text { or comorbid } \$ \text { or mortal\$) adj7 ((((low or lower or circulat } \$ \text { or blood or } \\
\text { hematolog } \$ \text { ) adj3 (level\$ or amount } \$)) \text { or insuffic } \$ \text { or defic } \$ \text { or status or } \\
\text { depriv } \$) \text { adj5 (hypovitamin } \$ \text { adj d }))) \text {.mp. }\end{array}$ & 28 \\
\hline 10 & 8 or 9 & 5126 \\
\hline 11 & 3 and 10 & 3947 \\
\hline 12 & 7 or 11 & 4095 \\
\hline 13 & exp retrospective study/ & 721641 \\
\hline 14 & $\begin{array}{l}\text { ((Post adj facto adj design) or (retrospective adj design) or (retrospective adj } \\
\text { panel adj studies) or (retrospective adj panel adj study) or (retrospective adj } \\
\text { studies) or retrospective).ab,hw,ti. }\end{array}$ & 860742 \\
\hline 15 & 13 or 14 & 860742 \\
\hline 16 & Epidemiologic Studies/ & 7822 \\
\hline 17 & 12 and 16 & 5 \\
\hline 18 & $\begin{array}{l}\text { limit } 17 \text { to (controlled clinical trial or guideline or meta analysis or } \\
\text { randomized controlled trial) }\end{array}$ & 2 \\
\hline 19 & 17 or 18 & 5 \\
\hline 20 & exp "OUTCOME AND PROCESS ASSESSMENT (HEALTH CARE)"/ & 999474 \\
\hline 21 & 12 and 20 & 209 \\
\hline 22 & exp Vital Statistics/ & 859137 \\
\hline
\end{tabular}


Términos de búsqueda

ítems

\begin{tabular}{|c|c|c|}
\hline 23 & 12 and 22 & 1294 \\
\hline 24 & 19 or 21 or 23 & 1441 \\
\hline 25 & mo.fs. & 528028 \\
\hline 26 & pc.fs. & 1205140 \\
\hline 27 & 25 or 26 & 1689001 \\
\hline 28 & 12 and 27 & 826 \\
\hline 29 & 24 or 28 & 1999 \\
\hline 30 & $\begin{array}{l}\text { limit } 29 \text { to (yr="2014 -Current" and ("adult (19 to } 44 \text { years)" or "young adult } \\
\text { and adult (19-24 and 19-44)" or "middle age ( } 45 \text { to } 64 \text { years)" or "middle } \\
\text { aged ( } 45 \text { plus years)" or "all aged ( } 65 \text { and over)" or "aged ( } 80 \text { and over)")) }\end{array}$ & 540 \\
\hline 31 & 30 not 15 & 463 \\
\hline 1 & exp vitamin d/ & 54702 \\
\hline 2 & vitamin d deficiency/ & 13925 \\
\hline 3 & 1 or 2 & 57878 \\
\hline 4 & exp Mass Screening/ & 118595 \\
\hline 5 & Diagnostic Tests, Routine/ & 10345 \\
\hline 6 & 4 or 5 & 128303 \\
\hline 7 & 3 and 6 & 179 \\
\hline 8 & $\begin{array}{l}\text { ((risk\$ or presence or prevalence or danger } \$ \text { or complicat } \$ \text { or morbid } \$ \\
\text { or comorbid } \$ \text { or mortal\$) adj7 ((((low or lower or circulat\$ or blood } \\
\text { or hematolog\$) adj3 (level\$ or amount\$)) or insuffic } \$ \text { or defic } \$ \text { or } \\
\text { status or depriv\$) adj5 (vitamin d or vitamin d3 or Cholecalciferol\$ or } \\
\text { Hydroxycholecalciferol\$ or Calcifediol or Dihydroxycholecalciferol\$ or } \\
\text { Calcitriol or Dihydroxyvitamin D or Ergocalciferol\$ or Dihydrotachysterol\$ or } \\
\text { or 25-hydroxyvitamin* or "25(OH)D"))).mp. }\end{array}$ & 5119 \\
\hline 9 & $\begin{array}{l}\text { ((risk\$ or presence or prevalence or danger } \$ \text { or complicat } \$ \text { or morbid } \$ \\
\text { or comorbid } \$ \text { or mortal\$) adj7 ((((low or lower or circulat } \$ \text { or blood or } \\
\text { hematolog } \$ \text { ) adj3 (level\$ or amount } \$)) \text { or insuffic } \$ \text { or defic } \$ \text { or status or } \\
\text { depriv } \$ \text { ) adj5 (hypovitamin } \$ \text { adj d }))) . m p \text {. }\end{array}$ & 28 \\
\hline 10 & 8 or 9 & 5126 \\
\hline 11 & 3 and 10 & 3947 \\
\hline 12 & 7 or 11 & 4095 \\
\hline 13 & exp retrospective study/ & 721641 \\
\hline 14 & $\begin{array}{l}\text { ((Post adj facto adj design) or (retrospective adj design) or (retrospective adj } \\
\text { panel adj studies) or (retrospective adj panel adj study) or (retrospective adj } \\
\text { studies) or retrospective).ab,hw,ti. }\end{array}$ & 860742 \\
\hline
\end{tabular}


Términos de búsqueda

ítems

\begin{tabular}{|c|c|c|}
\hline 15 & 13 or 14 & 860742 \\
\hline 16 & Epidemiologic Studies/ & 7822 \\
\hline 17 & 12 and 16 & 5 \\
\hline 18 & $\begin{array}{l}\text { limit } 17 \text { to (controlled clinical trial or guideline or meta analysis or } \\
\text { randomized controlled trial) }\end{array}$ & 2 \\
\hline 19 & 17 or 18 & 5 \\
\hline 20 & exp "OUTCOME AND PROCESS ASSESSMENT (HEALTH CARE)"/ & 999474 \\
\hline 21 & 12 and 20 & 209 \\
\hline 22 & exp Vital Statistics/ & 859137 \\
\hline 23 & 12 and 22 & 1294 \\
\hline 24 & 19 or 21 or 23 & 1441 \\
\hline 25 & mo.fs. & 528028 \\
\hline 26 & pc.fs. & 1205140 \\
\hline 27 & 25 or 26 & 1689001 \\
\hline 28 & 12 and 27 & 826 \\
\hline 29 & 24 or 28 & 1999 \\
\hline 30 & $\begin{array}{l}\text { limit } 29 \text { to (yr="2014 -Current" and ("adult (19 to } 44 \text { years)" or "young adult } \\
\text { and adult (19-24 and 19-44)" or "middle age ( } 45 \text { to } 64 \text { years)" or "middle } \\
\text { aged ( } 45 \text { plus years)" or "all aged ( } 65 \text { and over)" or "aged ( } 80 \text { and over)")) }\end{array}$ & 540 \\
\hline 31 & 30 not 15 & 463 \\
\hline
\end{tabular}




\section{Embase (Ovid SP)}

\begin{tabular}{|c|c|c|}
\hline & Términos de búsqueda & ítems \\
\hline 1 & exp vitamin $\mathrm{d} /$ & 128212 \\
\hline 2 & vitamin d deficiency/ & 26072 \\
\hline 3 & 1 or 2 & 132051 \\
\hline 4 & exp Mass Screening/ & 216891 \\
\hline 5 & Diagnostic Tests, Routine/ & 74720 \\
\hline 6 & 4 or 5 & 288700 \\
\hline 7 & 3 and 6 & 1330 \\
\hline 8 & 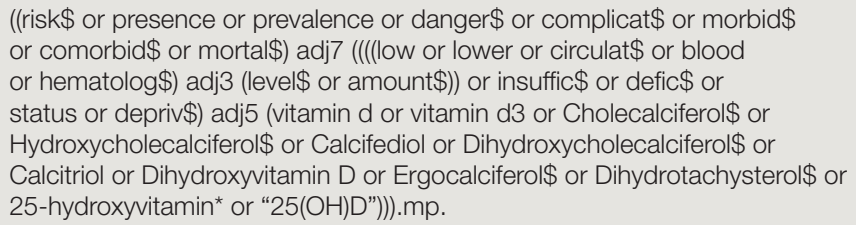 & 9326 \\
\hline 9 & $\begin{array}{l}\text { ((risk\$ or presence or prevalence or danger } \$ \text { or complicat } \$ \text { or morbid } \$ \\
\text { or comorbid } \$ \text { or mortal\$) adj7 ((((low or lower or circulat\$ or blood or } \\
\text { hematolog\$) adj3 (level\$ or amount\$)) or insuffic } \$ \text { or defic } \$ \text { or status or } \\
\text { depriv\$) adj5 (hypovitamin } \$ \text { adj d ))).mp. }\end{array}$ & 60 \\
\hline 10 & 8 or 9 & 9349 \\
\hline 11 & 3 and 10 & 9205 \\
\hline 12 & 7 or 11 & 10466 \\
\hline 13 & Epidemiologic Studies/ & 202038 \\
\hline 14 & exp "OUTCOME AND PROCESS ASSESSMENT (HEALTH CARE)"/ & 1470061 \\
\hline 15 & exp Vital Statistics/ & 3299 \\
\hline 16 & mo.fs. & 0 \\
\hline 17 & pc.fs. & 1070725 \\
\hline 18 & 13 or 14 or 15 or 16 or 17 & 2625169 \\
\hline 19 & 12 and 18 & 1618 \\
\hline 20 & 12 and 17 & 1001 \\
\hline 21 & $\begin{array}{l}\text { limit } 20 \text { to (embase and yr="2014 -Current" and (adult }<18 \text { to } 64 \text { years }>\text { or } \\
\text { aged }<65+\text { years }>\text { )) }\end{array}$ & 35 \\
\hline
\end{tabular}




\title{
Anexo 3.2. Pregunta 2: Efectividad de vitamina $D$ y resultados en salud
}

\author{
Cochrane Library, Trials y Systematic Reviews (Wiley)
}

\begin{tabular}{|c|c|c|}
\hline & Términos de búsqueda & ítems \\
\hline \#1 & $\begin{array}{l}\text { (((take or taking or takes or give or giving or prescri } \$ \text { or provid } \$ \text { or } \\
\text { oral\$ or parenteral\$ or diet\$ or food } \$ \text { or pill or pills or tablet\$) NEAR/5 } \\
\text { supplement } \$ \text { NEAR/5 (vitamin d or vitamin d3 or Cholecalciferol\$ or } \\
\text { Hydroxycholecalciferol\$ or Calcifediol or Dihydroxycholecalciferol\$ } \\
\text { or Calcitriol or Dihydroxyvitamin D or Ergocalciferol\$ or } \\
\text { Dihydrotachysterol\$))):ti,ab,kw (Se han buscado variaciones de la palabra) }\end{array}$ & 1079 \\
\hline \#2 & $\begin{array}{l}\text { ((supplement\$ NEAR/5 (((low or lower or circulat\$ or blood or serum } \\
\text { or hematolog\$) NEAR/3 (level\$ or amount } \$)) \text { or insuffic } \$ \text { or defic } \$ \text { or } \\
\text { status or depriv\$) NEAR/5 (vitamin d or vitamin d3 or Cholecalciferol\$ } \\
\text { or Hydroxycholecalciferol\$ or Calcifediol or Dihydroxycholecalciferol } \$ \text { or } \\
\text { Calcitriol or Dihydroxyvitamin D or Ergocalciferol } \$ \text { or Dihydrotachysterol\$))):ti } \\
\text {,ab,kw (Se han buscado variaciones de la palabra) }\end{array}$ & 895 \\
\hline \#3 & \#1 OR \#2 con año de publicación de 2014 hasta hoy, en Ensayos & 785 \\
\hline
\end{tabular}

\section{Crd Databases}

\begin{tabular}{|c|c|c|}
\hline & Términos de búsqueda & ítems \\
\hline 1 & 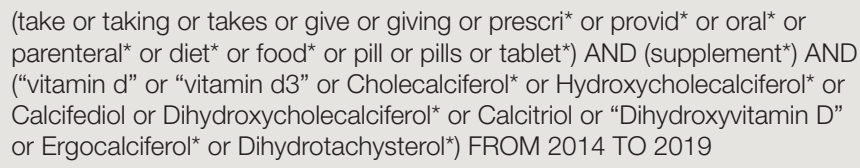 & 33 \\
\hline 2 & $\begin{array}{l}\text { (low or lower or circulat* or blood or serum or hematolog*) AND (level }{ }^{\star} \text { or } \\
\text { amount*) FROM } 2014 \text { TO } 2019\end{array}$ & 387 \\
\hline 3 & (insuffic* or defic* or status or depriv*) FROM 2014 TO 2019 & 978 \\
\hline 4 & \#2 OR \#3 & 1257 \\
\hline 5 & $\begin{array}{l}\text { ("vitamin d" or "vitamin d3" or Cholecalciferol* or Hydroxycholecalciferol* or } \\
\text { Calcifediol or Dihydroxycholecalciferol* or Calcitriol or "Dihydroxyvitamin D" } \\
\text { or Ergocalciferol* or Dihydrotachysterol*) FROM } 2014 \text { TO } 2019\end{array}$ & 68 \\
\hline 6 & \#4 AND \#5 & 31 \\
\hline 7 & \#1 OR \#6 & 47 \\
\hline
\end{tabular}




\section{Medline y preMedline (Ovid SP)}

\begin{tabular}{|c|c|c|}
\hline & Términos de búsqueda & ítems \\
\hline 1 & vitamin d deficiency/ & 14155 \\
\hline 2 & $\begin{array}{l}\text { ((take or taking or takes or give or giving or prescri } \$ \text { or provid } \$ \text { or oral } \$ \text { or } \\
\text { parenteral } \$ \text { or diet } \$ \text { or food } \$ \text { or pill or pills or tablet } \$ \text { ) adj5 supplement } \$ \text { adj5 } \\
\text { (vitamin d or vitamin } \mathrm{d} 3 \text { or Cholecalciferol } \$ \text { or Hydroxycholecalciferol } \$ \text { or } \\
\text { Calcifediol or Dihydroxycholecalciferol } \$ \text { or Calcitriol or Dihydroxyvitamin D or } \\
\text { Ergocalciferol } \$ \text { or Dihydrotachysterol } \$)) . m p \text {. }\end{array}$ & 2407 \\
\hline 3 & $\begin{array}{l}\text { (supplement } \$ \text { adj5 (((low or lower or circulat } \$ \text { or blood or serum } \\
\text { or hematolog\$) adj3 (level\$ or amount } \$) \text { ) or insuffic } \$ \text { or defic } \$ \text { or } \\
\text { status or depriv\$) adj5 (vitamin d or vitamin d3 or Cholecalciferol\$ or } \\
\text { Hydroxycholecalciferol\$ or Calcifediol or Dihydroxycholecalciferol\$ or } \\
\text { Calcitriol or Dihydroxyvitamin D or Ergocalciferol\$ or Dihydrotachysterol\$))). } \\
\text { mp. }\end{array}$ & 933 \\
\hline 4 & 2 or 3 & 3103 \\
\hline 5 & $\begin{array}{l}\text { exp Vitamin D/ad, ae, po, tu, to [Administration \& Dosage, Adverse Effects, } \\
\text { Poisoning, Therapeutic Use, Toxicity] }\end{array}$ & 21190 \\
\hline 6 & 4 or 5 & 22537 \\
\hline 7 & 1 and 6 & 4924 \\
\hline 8 & $\begin{array}{l}\text { limit } 7 \text { to (yr="2014 -Current" and ("adult (19 to } 44 \text { years)" or "young adult } \\
\text { and adult ( } 19-24 \text { and } 19-44) \text { " or "middle age ( } 45 \text { to } 64 \text { years)" or "middle } \\
\text { aged ( } 45 \text { plus years)" or "all aged ( } 65 \text { and over)" or "aged ( } 80 \text { and over)")) }\end{array}$ & 859 \\
\hline
\end{tabular}

\section{Embase (Ovid SP)}

\begin{tabular}{|l|l|l|}
\hline & Términos de búsqueda & ítems \\
\hline 1 & exp vitamin d/ & 128865 \\
\hline 2 & vitamin d deficiency/ & 26309 \\
\hline 3 & 1 or 2 & 132741 \\
\hline 4 & exp Mass Screening/ & 217853 \\
\hline 5 & Diagnostic Tests, Routine/ & 74731 \\
\hline 6 & 4 or 5 & 289665 \\
\hline 7 & $\begin{array}{l}3 \text { and } 6 \\
\text { ((take or taking or takes or give or giving or prescri\$ or provid\$ or oral\$ or } \\
\text { parenteral\$ or diet\$ or food\$ or pill or pills or tablet\$) adj5 supplement\$ adj5 } \\
\text { (vitamin d or vitamin d3 or Cholecalciferol\$ or Hydroxycholecalciferol\$ or } \\
\text { Calcifediol or Dihydroxycholecalciferol\$ or Calcitriol or Dihydroxyvitamin D or } \\
\text { Ergocalciferol\$ or Dihydrotachysterol\$)).mp. }\end{array}$ & 3090 \\
\hline 8
\end{tabular}


(supplement\$ adj5 ()((low or lower or circulat\$ or blood or serum or hematolog\$) adj3 (level\$ or amount\$)) or insuffic $\$$ or defic $\$$ or status or depriv $\$$ ) adj5 (vitamin d or vitamin d3 or Cholecalciferol $\$$ or Hydroxycholecalciferol\$ or Calcifediol or Dihydroxycholecalciferol\$ or Calcitriol or Dihydroxyvitamin D or Ergocalciferol\$ or Dihydrotachysterol\$))). $\mathrm{mp}$.

10 or 9

11 exp Vitamin D/ad, ae, po, tu, to [Administration \& Dosage, Adverse Effects, Poisoning, Therapeutic Use, Toxicity] aged $<65+$ years $>$ )) 


\title{
Anexo 4. Análisis de sesgos de estudios incluidos
}

\section{Anexo 4.1. Pregunta 1: Niveles de vitamina D y resultados en salud}

\author{
Anexo 4.1.1. Revisiones sistemáticas
}

Herramienta de valoración de la calidad metodológica de revisiones sistemáticas AMSTAR-II

\begin{tabular}{|c|c|c|c|c|c|c|c|c|}
\hline Estudio & 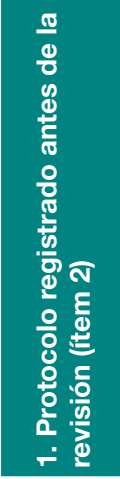 & 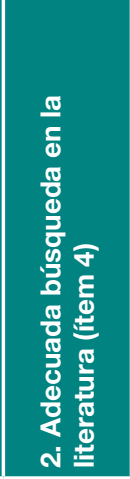 & 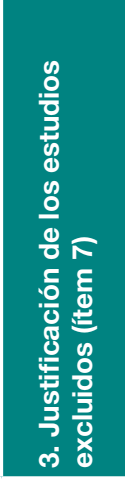 & 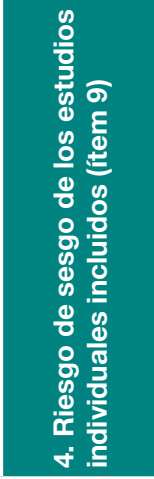 & 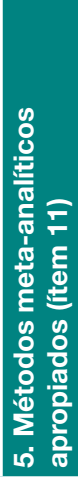 & 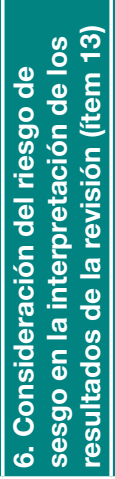 & 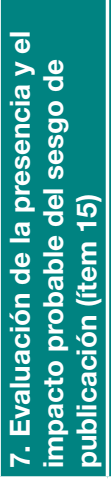 & 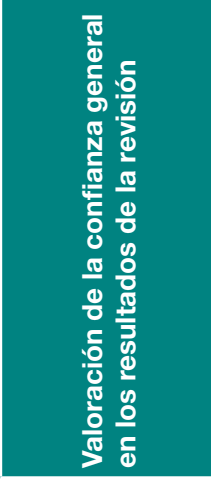 \\
\hline \multicolumn{9}{|l|}{ Salud ocular } \\
\hline Annweiler et al. 2016 (44) & No & No & Sí, parcial & $\begin{array}{l}\text { Solo incluye } \\
\text { EINA }\end{array}$ & No & Sí & No & Críticamente baja \\
\hline \multicolumn{9}{|l|}{ Deterioro cognitivo } \\
\hline Goodwill et al. 2017 (40) & Sí & Sí, parcial & Sí parcial & Sí & Sí & Sí & Sí & Alta \\
\hline \multicolumn{9}{|l|}{ Artritis } \\
\hline Lee et al. 2016 (29) & Sí & Sí, parcial & Sí, parcial & Sí, parcial & Sí & Sí & Sí & Alta \\
\hline \multicolumn{9}{|l|}{ Mortalidad } \\
\hline Gaksch et al. 2017 (33) & Sí, parcial & No & No & Sí, parcial & Sí & $\mathrm{Si}$ & No & Críticamente baja \\
\hline \multicolumn{9}{|l|}{ Fragilidad } \\
\hline Zhou et al. 2016 (54) & Si & Sí, parcial & No & Sí, parcial & Sí & $\mathrm{Si}$ & No & Críticamente baja \\
\hline \multicolumn{9}{|l|}{ Enfermedad Crohn } \\
\hline Sadeghian et al. 2016 (53) & No & Sí, parcial & No & $\mathrm{Si}$ & $\mathrm{Si}$ & $\mathrm{Si}$ & $\mathrm{Si}$ & Críticamente baja \\
\hline Confianza & \multicolumn{8}{|c|}{ Justificación } \\
\hline Alta & \multicolumn{8}{|c|}{$\begin{array}{l}\text { Ninguna debilidad crítica y hasta una no crítica: la RS proporciona un resumen exacto y } \\
\text { completo de los resultados de los estudios disponibles. }\end{array}$} \\
\hline Media & \multicolumn{8}{|c|}{$\begin{array}{l}\text { Ninguna debilidad crítica y más de una debilidad no crítica (aunque si son muchas podría } \\
\text { justificarse una baja confianza): la RS tiene debilidades, pero no hay defectos críticos, } \\
\text { pudiendo proporcionar un resumen preciso de los resultados de los estudios disponibles. }\end{array}$} \\
\hline Baja & \multicolumn{8}{|c|}{$\begin{array}{l}\text { Hasta una debilidad crítica, con o sin puntos débiles no críticos: la RS puede no } \\
\text { proporcionar un resumen exacto y completo de los estudios disponibles. }\end{array}$} \\
\hline Criticamente baja & \multicolumn{8}{|c|}{ Más de una debilidad crítica, con o sin debilidades no críticas: la RS no es fiable. } \\
\hline
\end{tabular}


Anexo 4.1.2. Estudios observacionales

Herramienta para evaluación de riesgo de sesgo en estudios no aleatorizados (observacionales), ROBINS-I.

\begin{tabular}{|c|c|c|c|c|c|c|c|c|}
\hline Estudio & 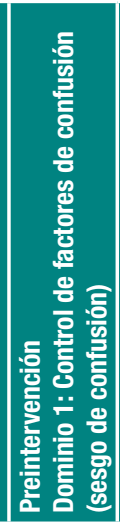 & 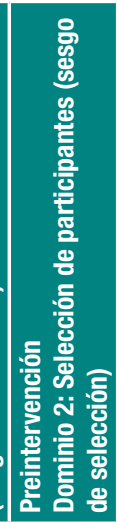 & 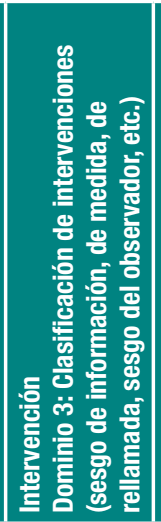 & 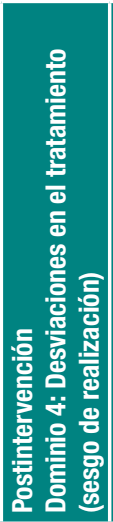 & 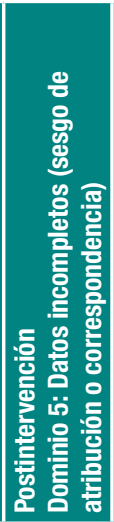 & 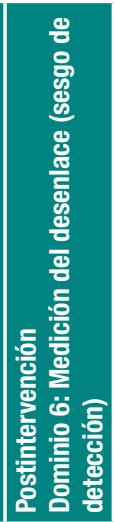 & 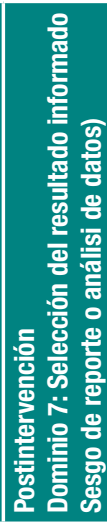 & 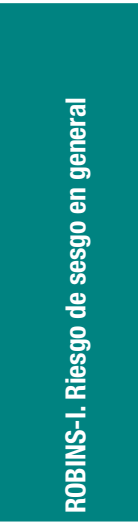 \\
\hline \multicolumn{9}{|l|}{ Cardiovascular } \\
\hline Bajaj et al 2014 (12) & & & & & & & & Serio \\
\hline Vitezona et al 2015 (13) & & & & & & & & Serio \\
\hline Wannamethee et al 2014 (14) & & & & & $\square$ & & & Serio \\
\hline \multicolumn{9}{|l|}{ Ictus } \\
\hline Judd et al 2016 (15) & & & & & $\square$ & & & Serio \\
\hline \multicolumn{9}{|l|}{ Deterioro cognitivo } \\
\hline Littlejohns et al 2014 (41) & & & & & E & & & Serio \\
\hline Moon et al 2015 (42) & & & & & 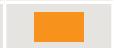 & & & Serio \\
\hline Olsson et al 2017 (43) & & . & 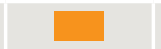 & & $\square$ & & 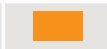 & Serio \\
\hline \multicolumn{9}{|l|}{ Circulatorio } \\
\hline Folsom et al 2014 (39) & & & & & & & & Moderado \\
\hline Andro et al 2016 (38) & & & & & 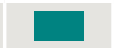 & & & Moderado \\
\hline \multicolumn{9}{|l|}{ Salud ósea } \\
\hline Karatas et al 2017 (28) & & & & & & & & Serio \\
\hline Duval et al 2017 (23) & & & & & $\square$ & & & Serio \\
\hline Julian et al 2016 (25) & & & & & 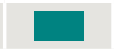 & & & Moderado \\
\hline Maier at al 2015 (26) & & & & & & & & Serio \\
\hline Rothenbacher et al 2014 (24) & & & & & & & & Seria \\
\hline Williams et al 2018 (27) & & & & & & & & Serio \\
\hline \multicolumn{9}{|l|}{ Cáncer } \\
\hline Chandler et al 2015 (17) & & & & & & & & Serio \\
\hline Jamshidinaeini et al 2016 (18) & & & 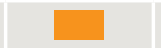 & & & & & Crítico \\
\hline McDonnell et al 2016 (21) & & 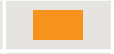 & 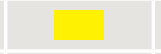 & & & & & Serio \\
\hline Schenk et al 2014 (16) & & & $\square$ & & $\square$ & & 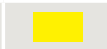 & Serio \\
\hline
\end{tabular}




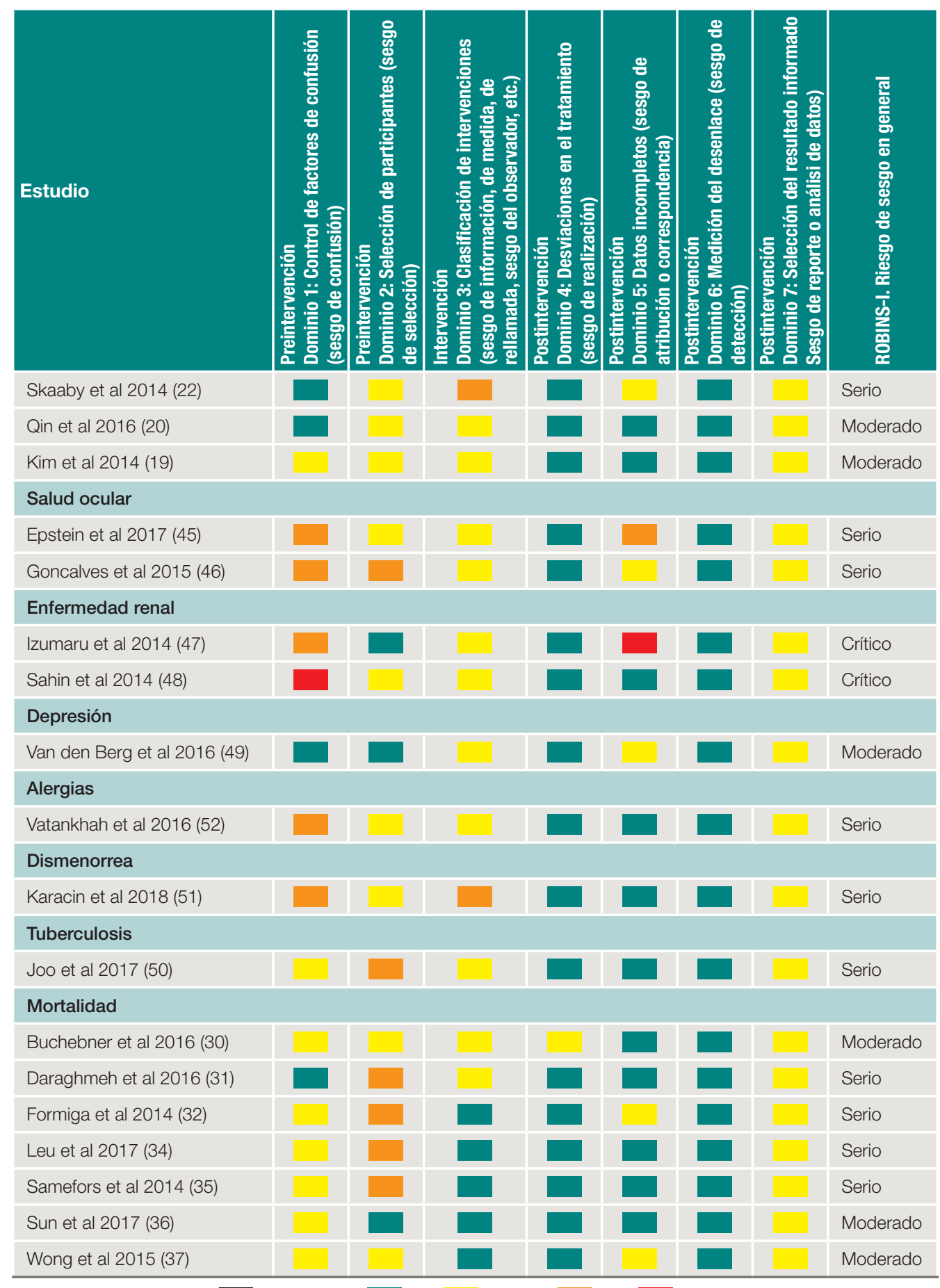

Evaluación de riesgo de sesgo: $\square$ Sin información; Bajo; Moderado; $\quad$ Serio; $\quad$ Crítico 


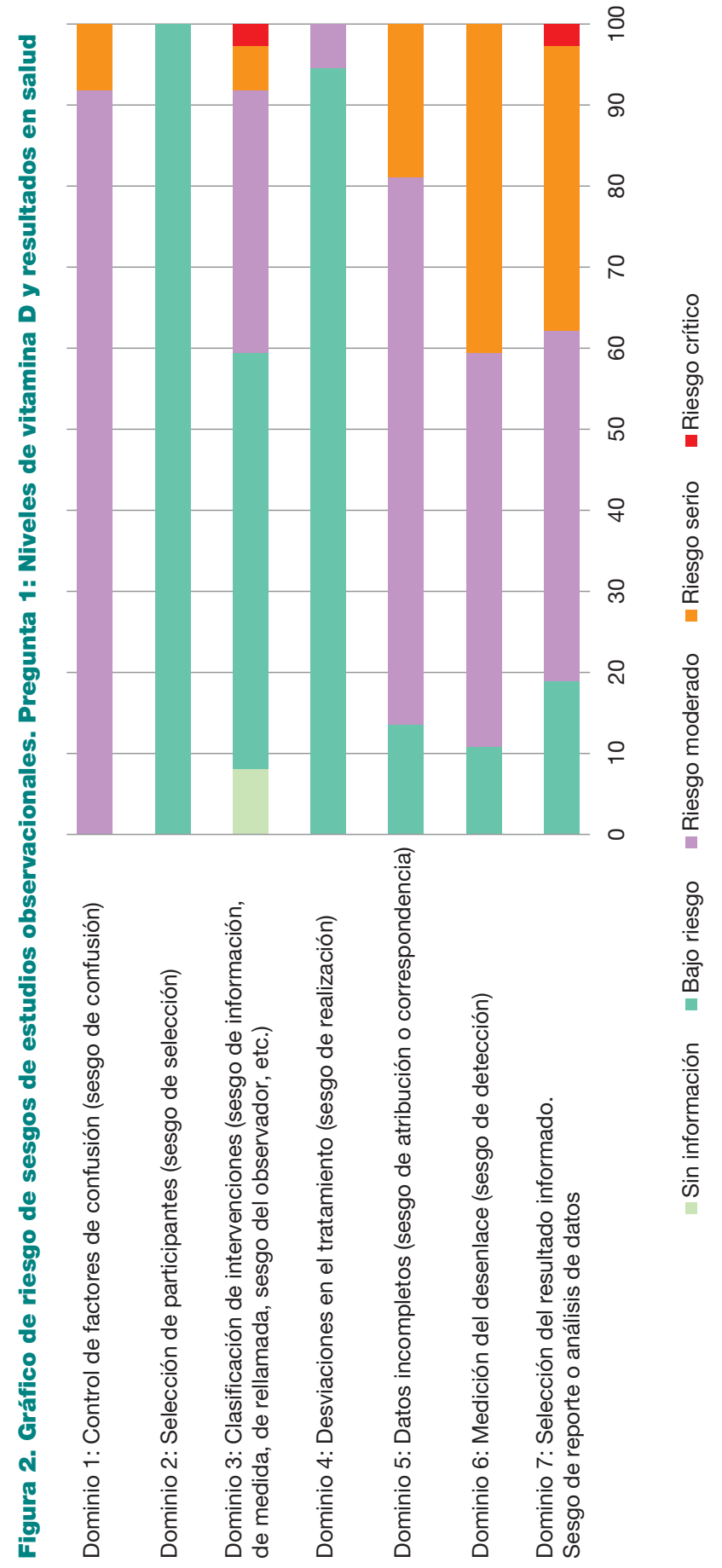




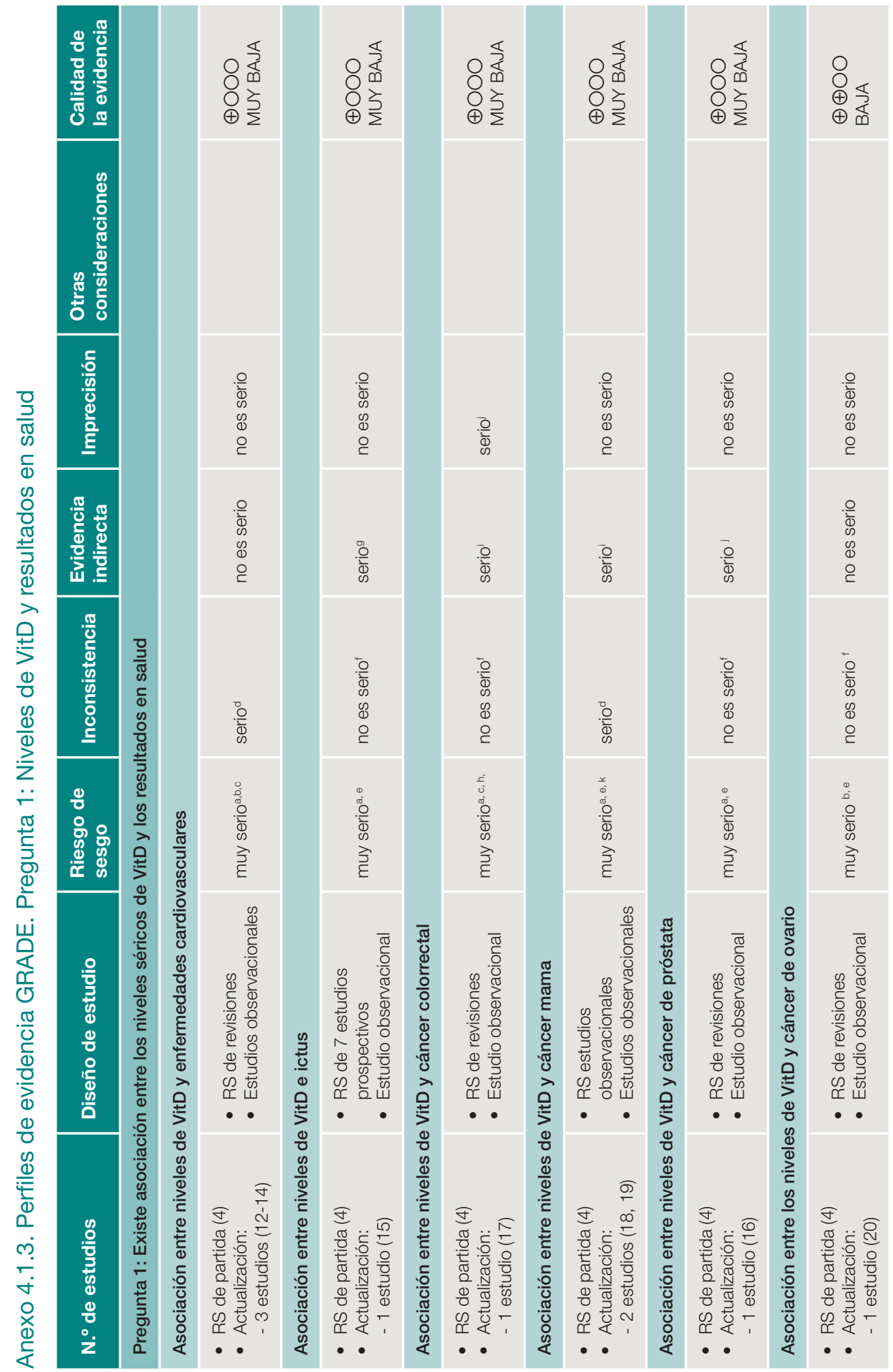




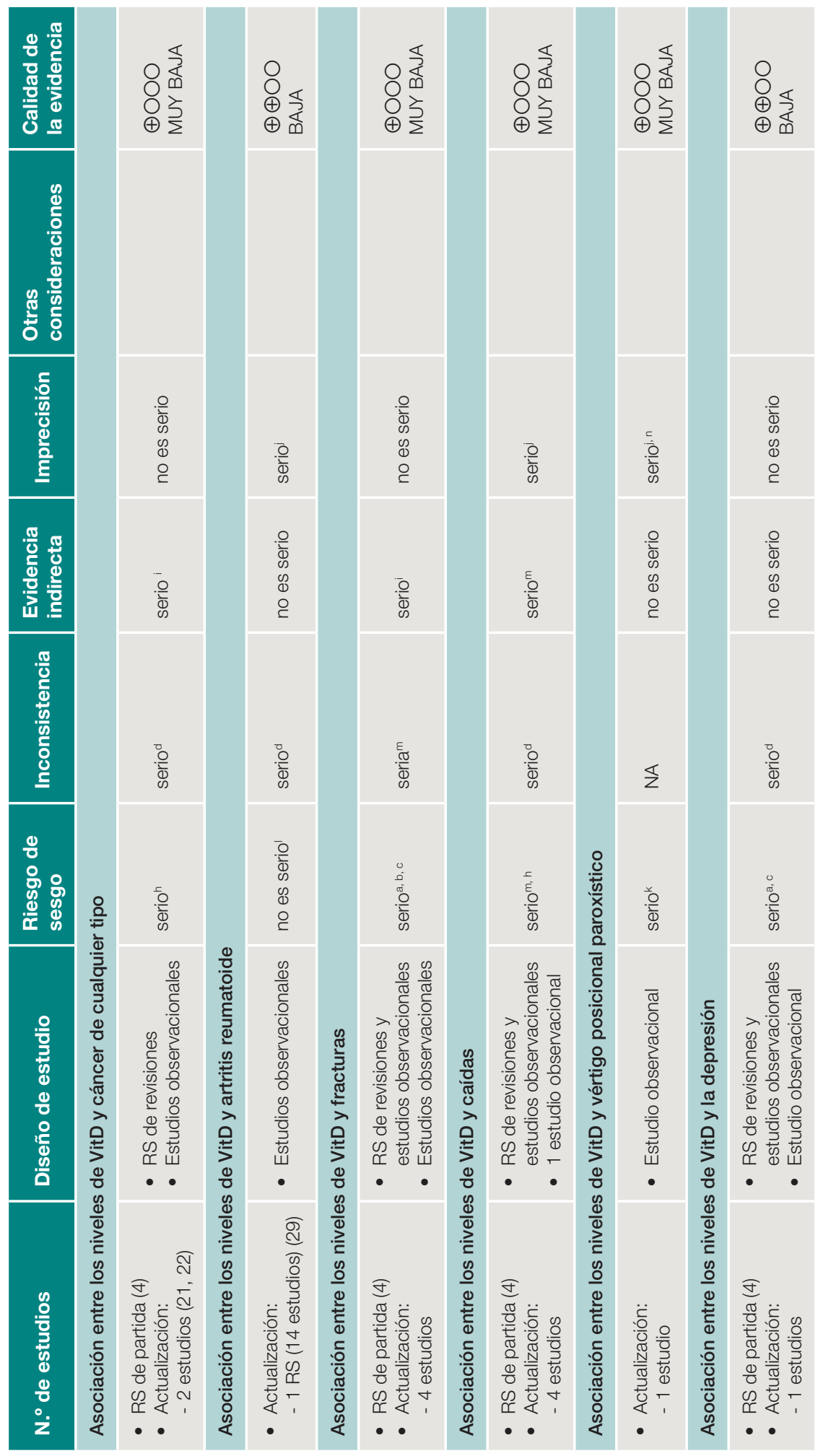




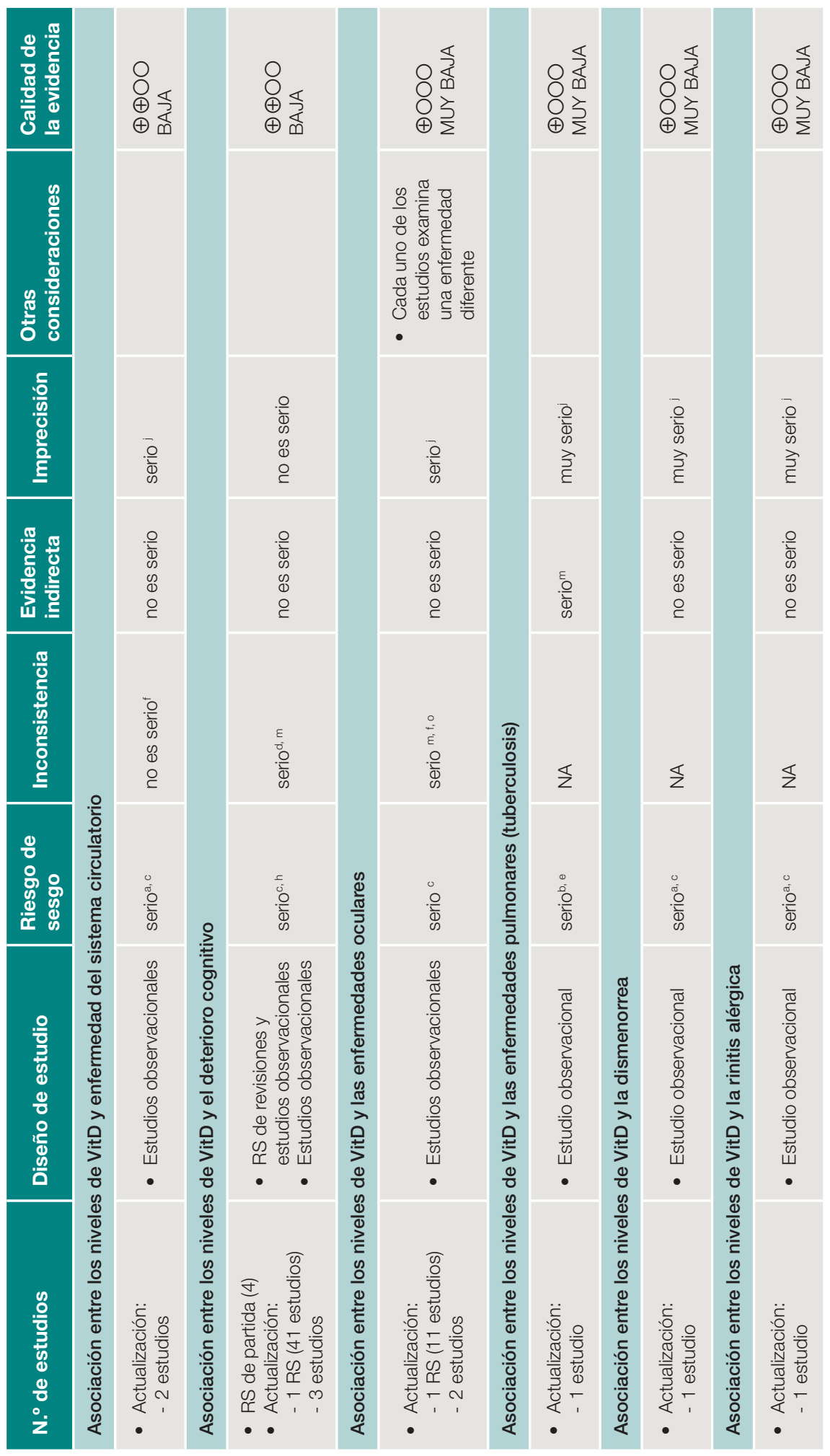




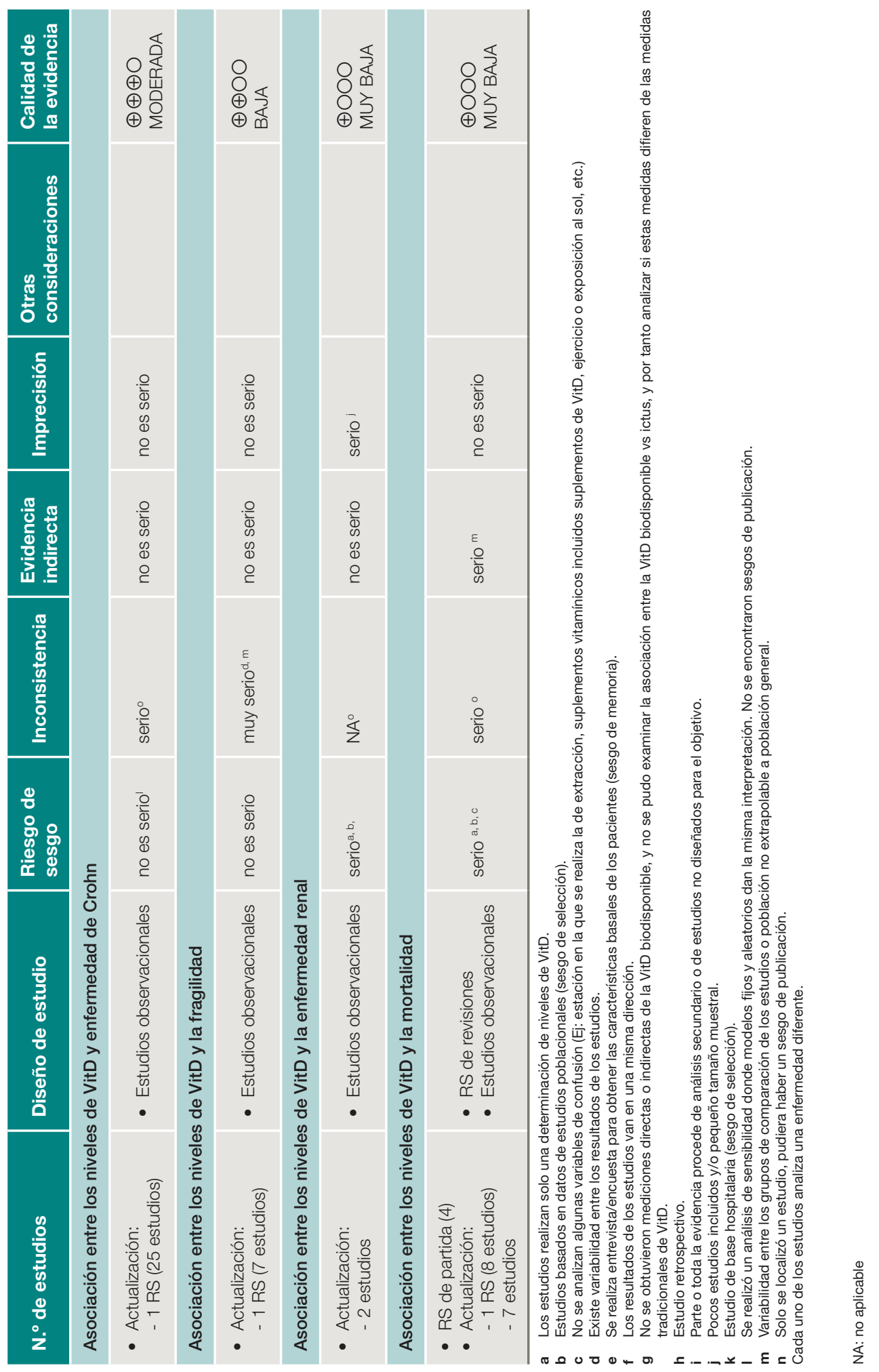




\section{Anexo 4.2. Pregunta 2: Efectividad de vitamina D y resultados en salud}

Anexo 4.2.1. Revisiones sistemáticas

Herramienta de valoración de la calidad metodológica de revisiones sistemáticas AMSTAR-II.

\begin{tabular}{|c|c|c|c|c|c|c|c|c|}
\hline Estudio & 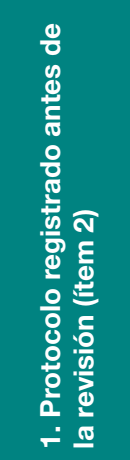 & 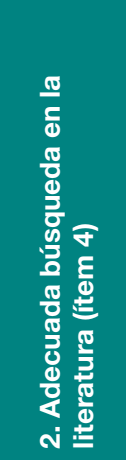 & 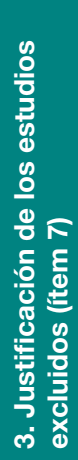 & 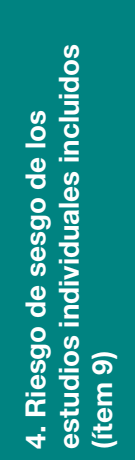 & 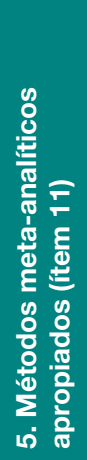 & 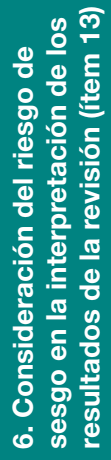 & 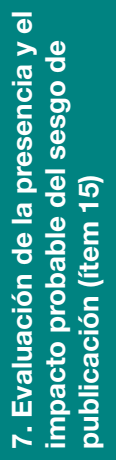 & 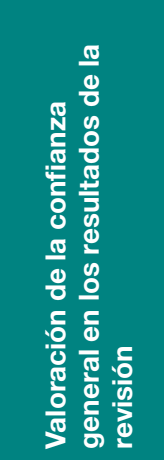 \\
\hline \multicolumn{9}{|c|}{ Fracturas y caídas } \\
\hline $\begin{array}{l}\text { Bolland et al. } \\
2018(70)\end{array}$ & Sí, parcial & Sí, parcial & Sí & Sí & Sí & Sí & Sí & Alta \\
\hline $\begin{array}{l}\text { Guirguis-Blake } \\
\text { et al. } 2018 \text { (72) }\end{array}$ & Sí & Sí, parcial & Sí & Sí, parcial & Sí & Sí & Sí & Alta \\
\hline $\begin{array}{l}\text { Kahwati et al. } \\
2018 \text { (174) }\end{array}$ & Sí & Sí & Sí & Sí, parcial & Sí & Sí & No & Baja \\
\hline \multicolumn{9}{|l|}{ Cáncer } \\
\hline $\begin{array}{l}\text { Bjelakovic et al. } \\
2014(56)\end{array}$ & Sí, parcial & Sí & Sí & Sí & Sí & Sí & Sí & Alta \\
\hline \multicolumn{9}{|l|}{ Diabetes tipo 2} \\
\hline $\begin{array}{l}\text { Zuk et al. } 2016 \\
(120)\end{array}$ & Sí, parcial & Sí, parcial & No & Sí & No & Sí & No & $\begin{array}{l}\text { Críticamente } \\
\text { baja }\end{array}$ \\
\hline \multicolumn{9}{|c|}{ Enfermedad cardiovascular } \\
\hline $\begin{array}{l}\text { SHU et al. } 2010 \\
(66)\end{array}$ & Sí & Sí, parcial & No & No & Sí & Sí & Sí & $\begin{array}{l}\text { Críticamente } \\
\text { baja }\end{array}$ \\
\hline $\begin{array}{l}\text { Wu et al. } 2017 \\
(68)\end{array}$ & Sí & Sí, parcial & No & Sí & Sí & Sí & Sí & Baja \\
\hline $\begin{array}{l}\text { Golzarand et al. } \\
2016(62)\end{array}$ & Sí, parcial & Sí, parcial & No & Sí & Sí & Sí & Sí & Baja \\
\hline
\end{tabular}




\begin{tabular}{|c|c|c|c|c|c|c|c|c|}
\hline Estudio & 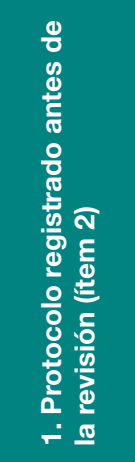 & 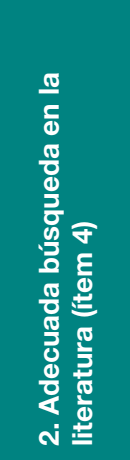 & 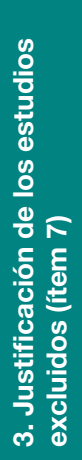 & 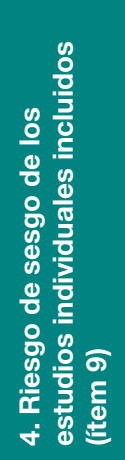 & 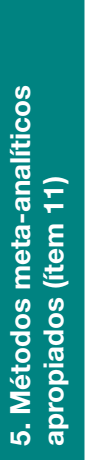 & 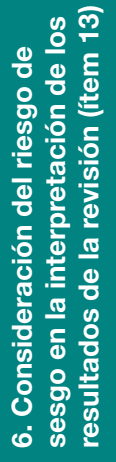 & 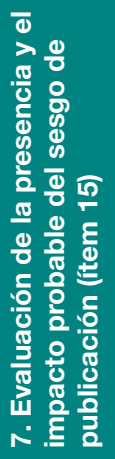 & 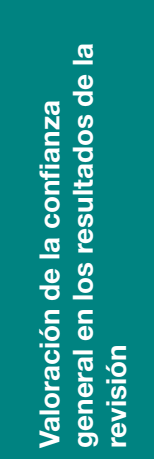 \\
\hline \multicolumn{9}{|c|}{ Procesos inflamatorios } \\
\hline $\begin{array}{l}\text { Jamka et al. } \\
2016(96)\end{array}$ & Sí, parcial & Sí, parcial & No & Sí, parcial & Sí & Sí & Sí & Baja \\
\hline \multicolumn{9}{|l|}{ Dolor } \\
\hline $\begin{array}{l}\text { Straube et al. } \\
2015 \text { (88) }\end{array}$ & Sí, parcial & Sí, parcial & Sí & Sí & Sí & Sí & Sí & Media \\
\hline \multicolumn{9}{|l|}{ Mortalidad } \\
\hline $\begin{array}{l}\text { Bjelakovic et al. } \\
2014 \text { (86) }\end{array}$ & Sí, parcial & Sí & Sí & Sí & Sí & Sí & Sí & Alta \\
\hline Confianza & \multicolumn{8}{|c|}{ Justificación } \\
\hline Alta & \multicolumn{8}{|c|}{$\begin{array}{l}\text { Ninguna debilidad crítica y hasta una no crítica: la RS proporciona un resumen exacto y } \\
\text { completo de los resultados de los estudios disponibles. }\end{array}$} \\
\hline Media & \multicolumn{8}{|c|}{$\begin{array}{l}\text { Ninguna debilidad crítica y más de una debilidad no crítica (aunque si son muchas podría } \\
\text { justificarse una baja confianza): la RS tiene debilidades, pero no hay defectos críticos, } \\
\text { pudiendo proporcionar un resumen preciso de los resultados de los estudios disponibles. }\end{array}$} \\
\hline Baja & \multicolumn{8}{|c|}{$\begin{array}{l}\text { Hasta una debilidad crítica, con o sin puntos débiles no críticos: la RS puede no proporcionar } \\
\text { un resumen exacto y completo de los estudios disponibles. }\end{array}$} \\
\hline $\begin{array}{l}\text { Criticamente } \\
\text { baja }\end{array}$ & \multicolumn{8}{|c|}{ Más de una debilidad crítica, con o sin debilidades no críticos: la RS no es confiable. } \\
\hline
\end{tabular}


Anexo 4.2.2. ECA

Herramienta y criterios de evaluación de riesgo de sesgo para ECAs de la Colaboración Cochrane.

\begin{tabular}{|c|c|c|c|c|c|c|c|c|}
\hline Estudio & 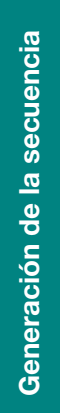 & 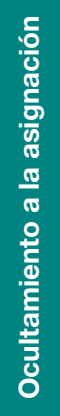 & 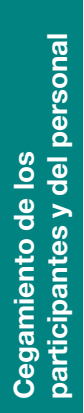 & 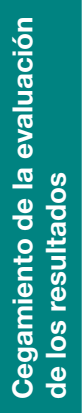 & 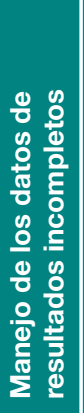 & 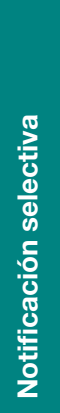 & 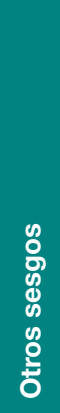 & 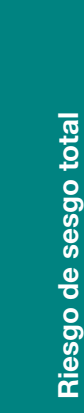 \\
\hline \multicolumn{9}{|l|}{ Salud ósea } \\
\hline Smith et al 2018 (76) & + & + & + & 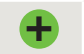 & + & + & + & Bajo \\
\hline Nahas-Neto et al 2018 (75) & + & + & + & + & + & + & + & Bajo \\
\hline Aloia et al 2018 (74) & + & $?$ & + & $?$ & + & + & + & Bajo \\
\hline Trautvetter et al 2014 (77) & + & $?$ & + & $?$ & + & $?$ & + & Medio \\
\hline \multicolumn{9}{|l|}{ Fracturas y caídas } \\
\hline Cangussu et al 2016 (71) & + & + & + & $?$ & + & + & + & Bajo \\
\hline \multicolumn{9}{|l|}{ Cáncer } \\
\hline Manson et al 2019 (58) & + & + & + & $?$ & + & + & + & Bajo \\
\hline Lappe et al 2017 (57) & + & $?$ & + & + & + & + & + & Bajo \\
\hline Baron et al 2015 (55) & $?$ & $?$ & + & + & $?$ & + & $?$ & Alto \\
\hline \multicolumn{9}{|l|}{ Diabetes tipo 2} \\
\hline Zarrin et al 2017 (119) & + & + & + & + & + & + & + & Bajo \\
\hline Mousa et al 2017 (114) & + & + & + & + & + & + & + & Bajo \\
\hline Moreira-Lucas et al 2017 (113) & + & + & + & $?$ & + & + & + & Bajo \\
\hline Tepper at al 2016 (118) & + & + & + & $?$ & + & + & + & Bajo \\
\hline Sun et al 2016 (117) & + & + & + & $?$ & + & + & + & Bajo \\
\hline Osati et al 2016 (116) & $?$ & $?$ & $?$ & $?$ & $?$ & + & $?$ & Alto \\
\hline Jorde et al 2016 (111) & + & $?$ & $?$ & $?$ & $?$ & + & + & Alto \\
\hline Michell et al 2015 (112) & + & + & + & + & + & + & + & Bajo \\
\hline Barengolts et al 2015 (110) & + & + & + & + & + & + & + & Bajo \\
\hline
\end{tabular}




\begin{tabular}{|c|c|c|c|c|c|c|c|c|}
\hline Estudio & 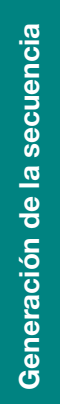 & 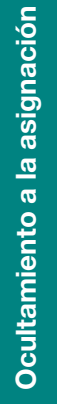 & 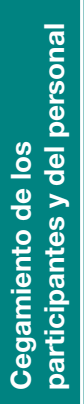 & 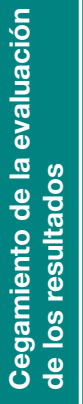 & 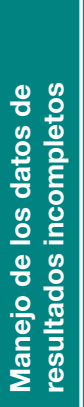 & 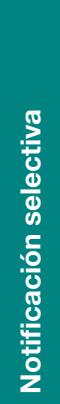 & 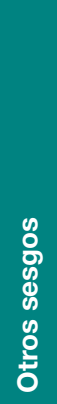 & 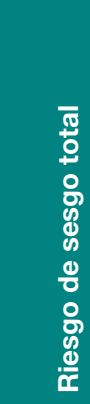 \\
\hline Oosterweff et al 2014 (115) & + & + & $\oplus$ & + & $\oplus$ & + & + & Bajo \\
\hline \multicolumn{9}{|l|}{ Enfermedad cardiovascular } \\
\hline Manson et al 2019 (58) & + & + & + & $?$ & + & + & + & Bajo \\
\hline Kubiak et al 2018 (63) & + & + & + & $?$ & + & $?$ & + & Bajo \\
\hline Abdulridha et al 2018 (59) & $?$ & - & - & - & + & $?$ & + & Alto \\
\hline Sluyter et al 2017 (67) & $?$ & $?$ & $?$ & $?$ & + & + & + & Medio \\
\hline Scragg et al 2017 (65) & + & + & + & $?$ & + & $?$ & $?$ & Bajo \\
\hline Borgi et al 2017 (60) & + & + & + & + & + & + & + & Bajo \\
\hline Chacko et al 2016 (61) & + & + & + & $?$ & + & + & $?$ & Bajo \\
\hline Zaleski et al 2015 (69) & $?$ & $?$ & $?$ & $?$ & + & $?$ & $?$ & Alto \\
\hline Ramly et al 2014 (64) & + & + & + & + & + & + & $?$ & Bajo \\
\hline \multicolumn{9}{|l|}{ Enfermedad renal } \\
\hline McMullan et al 2017 (104) & + & + & + & + & + & $?$ & + & Bajo \\
\hline Nygaard et al 2014 (105) & + & + & + & $?$ & + & + & + & Bajo \\
\hline Gallagher et al 2014 (103) & + & + & $\oplus$ & $\oplus$ & $\oplus$ & $?$ & + & Bajo \\
\hline \multicolumn{9}{|l|}{ Procesos infecciosos } \\
\hline Ginde et al 2017 (89) & + & + & + & + & + & + & $\theta$ & Medio \\
\hline Martineau et al 2015 (91) & + & + & + & + & 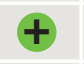 & + & - & Medio \\
\hline Simpson et al 2015 (93) & + & + & + & $\oplus$ & $?$ & + & + & Bajo \\
\hline Tran et al 2014 (94) & + & + & $\oplus$ & $?$ & $\oplus$ & $?$ & - & Alto \\
\hline Goodall et al 2014 (90) & + & + & + & + & + & + & - & Medio \\
\hline \multicolumn{9}{|l|}{ Calidad de vida } \\
\hline Mason et al 2016 (122) & + & $?$ & $?$ & $?$ & $?$ & + & + & Bajo \\
\hline Patil et al 2016 (121) & + & + & $?$ & $?$ & + & + & + & Bajo \\
\hline
\end{tabular}




\begin{tabular}{|c|c|c|c|c|c|c|c|c|}
\hline Estudio & 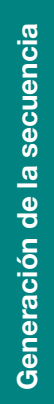 & 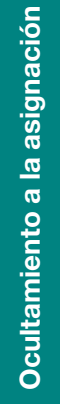 & 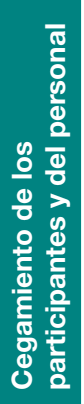 & 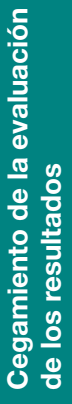 & 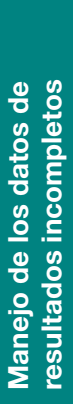 & 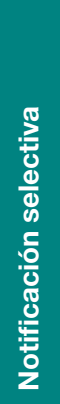 & 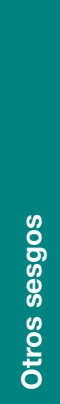 & 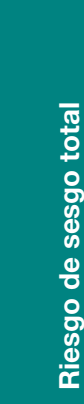 \\
\hline \multicolumn{9}{|l|}{ Función cognitiva } \\
\hline Jorde et al 2019 (107) & + & + & + & $?$ & + & + & + & Bajo \\
\hline Pettersen et al 2017 (108) & + & + & + & $?$ & + & $?$ & + & Bajo \\
\hline \multicolumn{9}{|l|}{ Función muscular } \\
\hline Vaes et al 2018 (84) & + & + & + & + & + & + & + & Bajo \\
\hline Suebthawinkul et al 2018 (83) & + & $?$ & $?$ & $?$ & + & + & + & Medio \\
\hline Saha et al 2018 (82) & + & + & + & + & + & $?$ & + & Bajo \\
\hline Hansen et al 2015 (80) & $?$ & + & + & $?$ & + & + & $?$ & Medio \\
\hline Gao et al 2015 (79) & - & $?$ & $\theta$ & $\theta$ & $?$ & $?$ & $?$ & Alto \\
\hline Cangussu et al 2015 (78) & + & + & + & + & $?$ & + & + & Bajo \\
\hline Wood et al 2014 (85) & + & + & + & + & + & + & + & Bajo \\
\hline Knutsen et al 2014 (81) & + & + & + & + & + & + & + & Bajo \\
\hline \multicolumn{9}{|l|}{ Función tiroidea } \\
\hline Knutsen et al 2017 (109) & + & + & + & + & + & $?$ & + & Bajo \\
\hline \multicolumn{9}{|l|}{ Procesos inflamatorios } \\
\hline Sinha-Hikim et al 2015 (97) & $?$ & $?$ & $?$ & $?$ & $?$ & $?$ & $?$ & Alto \\
\hline Waterhause et al 2015 (98) & + & $?$ & + & + & $?$ & $?$ & + & Medio \\
\hline Barker et al 2015 (95) & + & $?$ & $?$ & $?$ & + & + & $?$ & Alto \\
\hline \multicolumn{9}{|l|}{ Función pulmonar } \\
\hline Sluyte at al 2017 (67) & + & $?$ & + & $?$ & + & + & + & Bajo \\
\hline \multicolumn{9}{|l|}{ Dolor } \\
\hline Knutsen et al 2014 (87) & 4 & + & + & + & + & + & + & Bajo \\
\hline \multicolumn{9}{|l|}{ Circulatorio } \\
\hline Blondon et al 2015 (101) & + & + & + & + & + & $?$ & + & Bajo \\
\hline
\end{tabular}




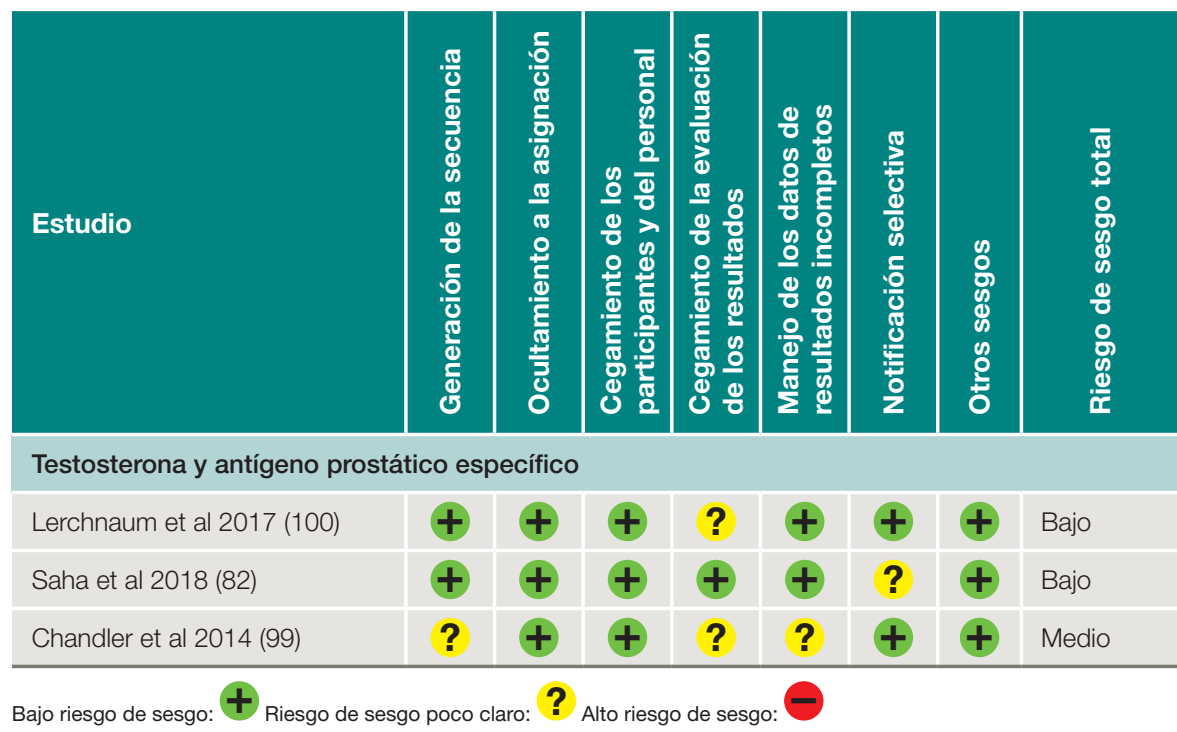




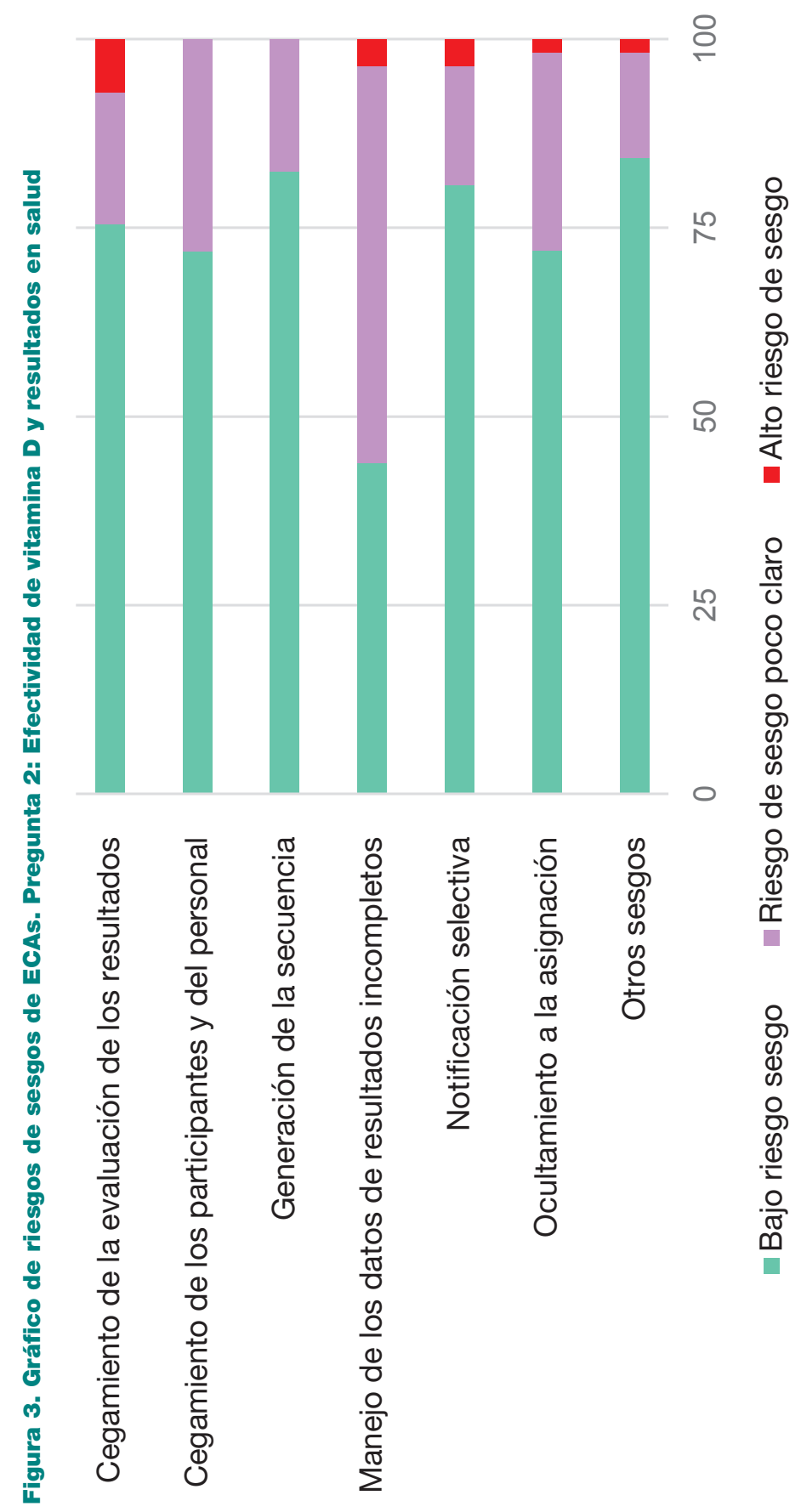




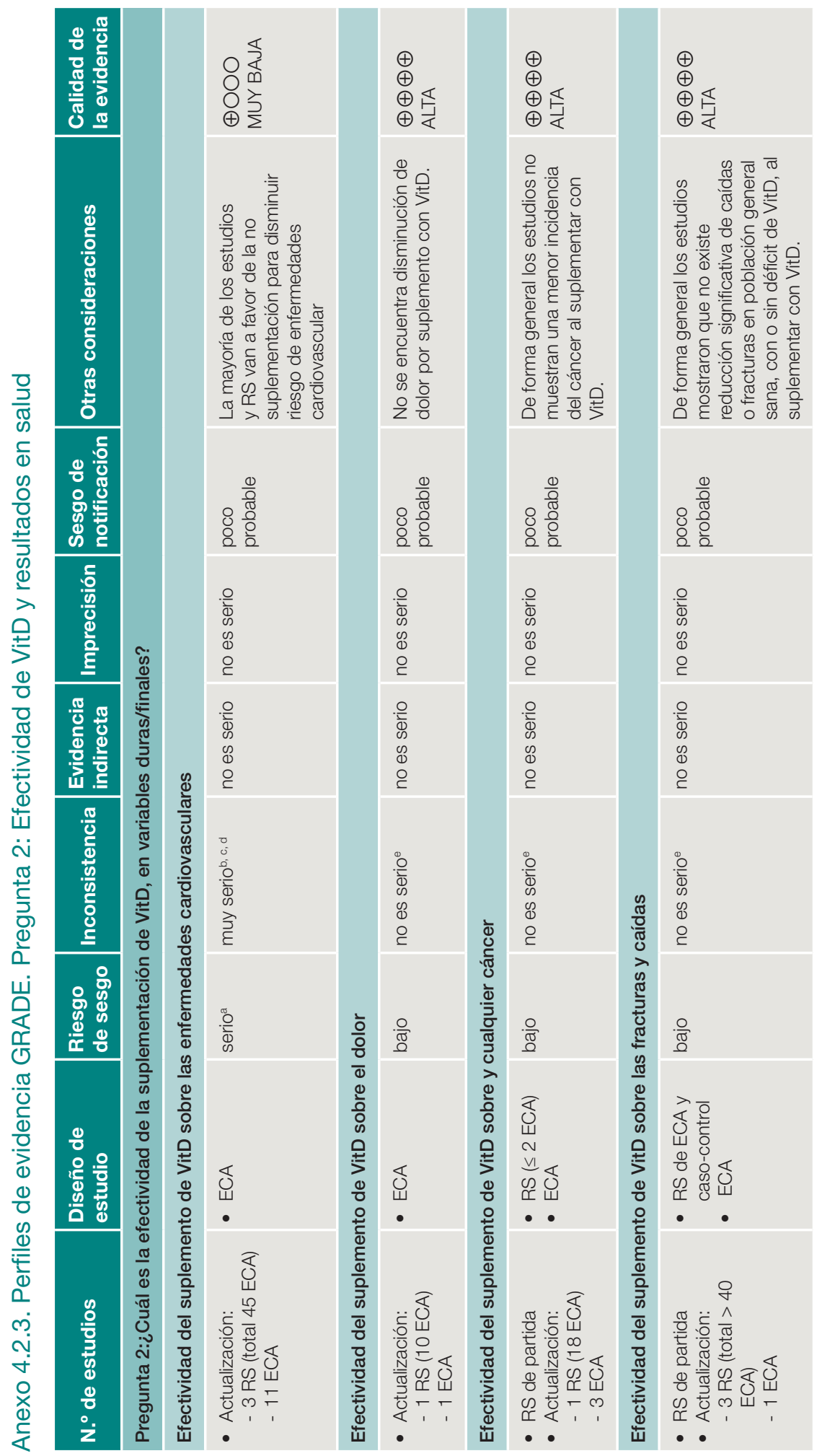




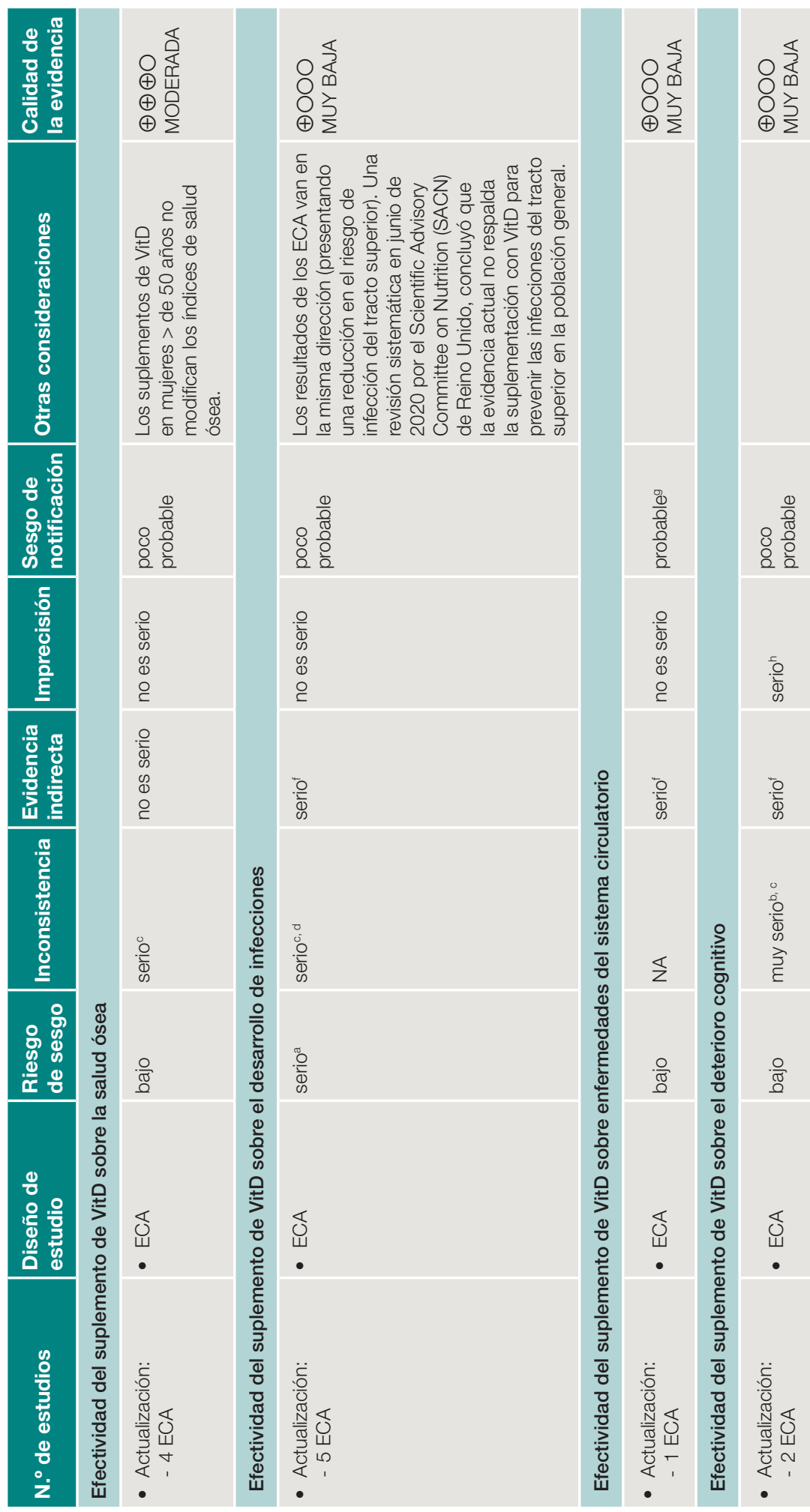




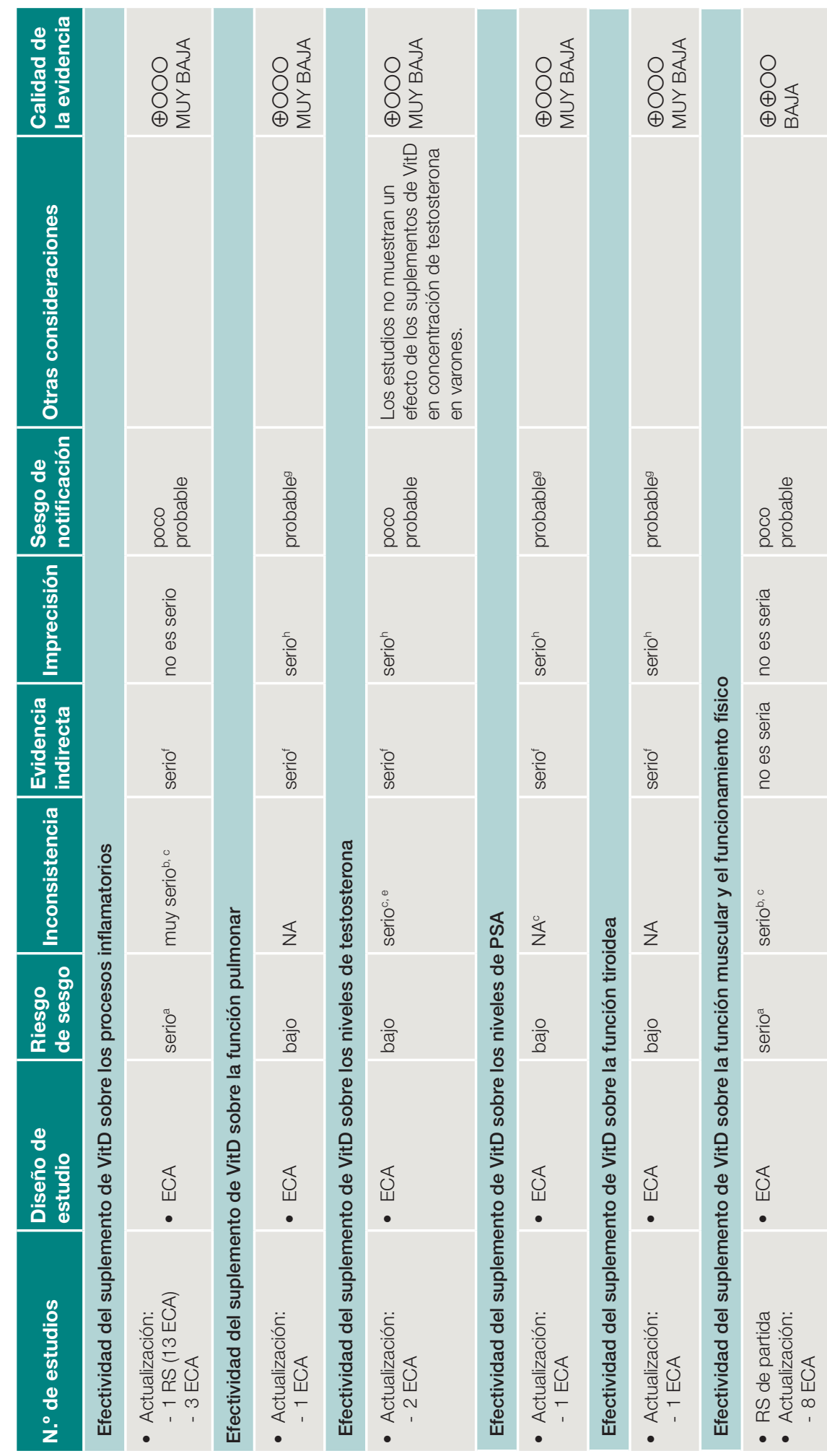




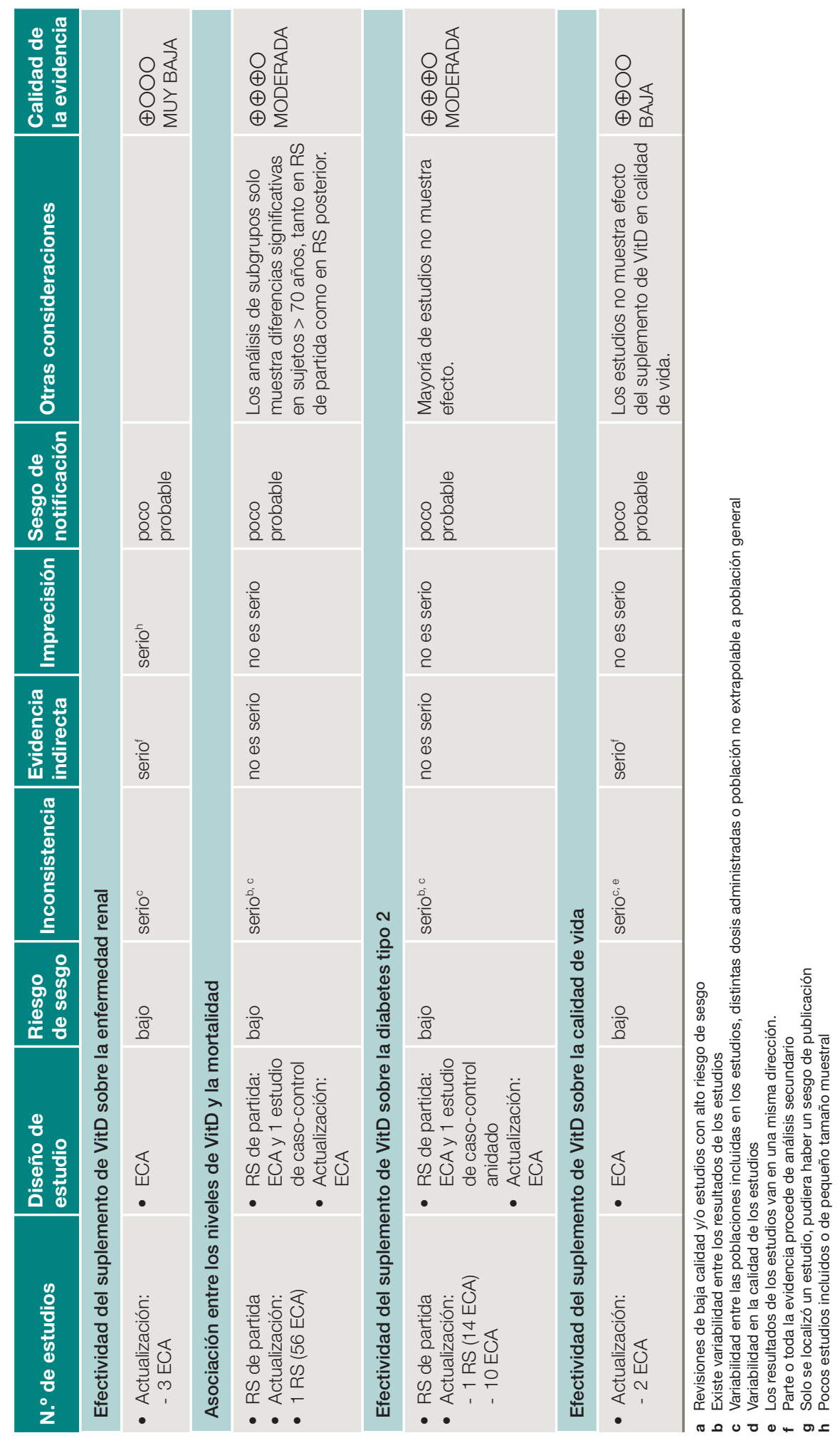


III

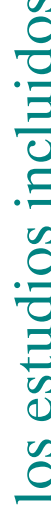

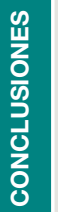

ช

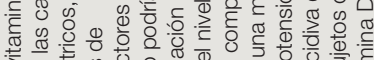

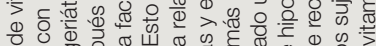

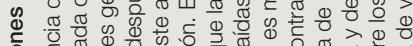

क

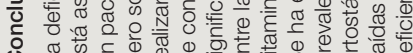
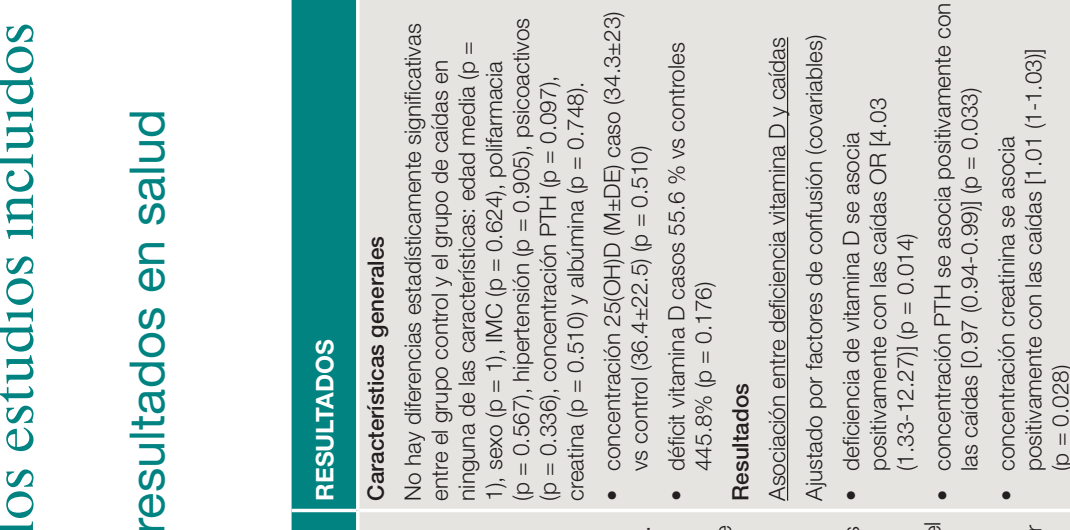

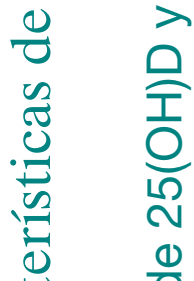

중

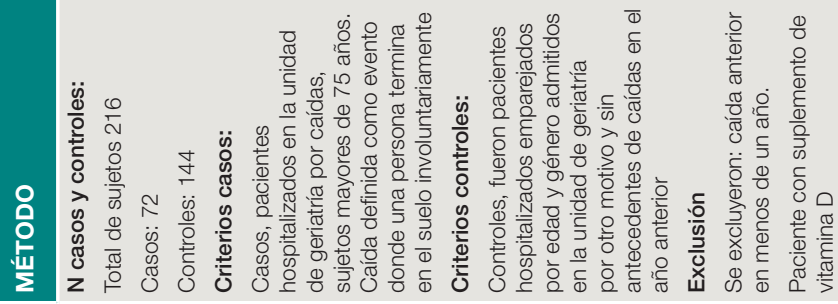

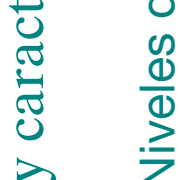

10

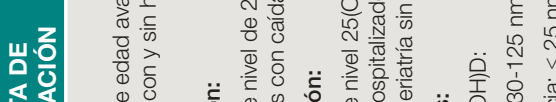

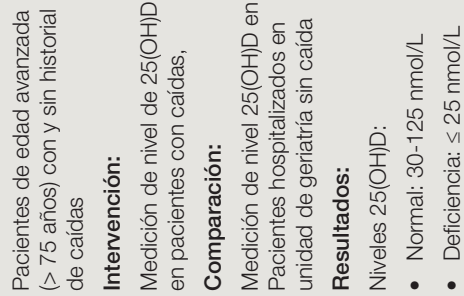

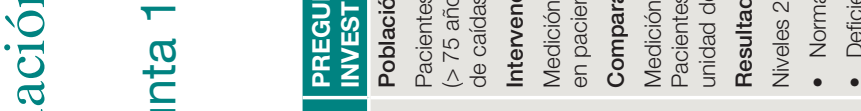

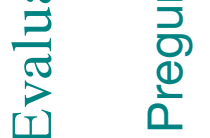

ఠ

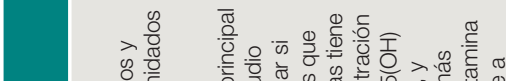

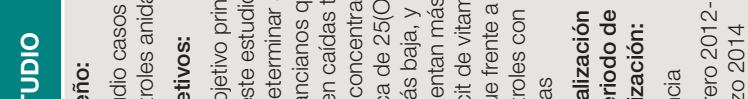

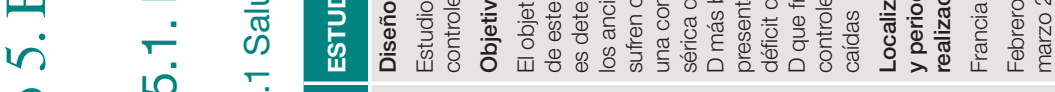

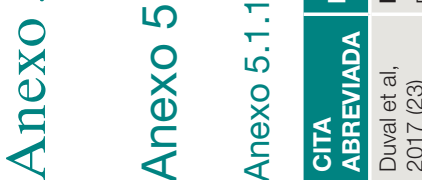




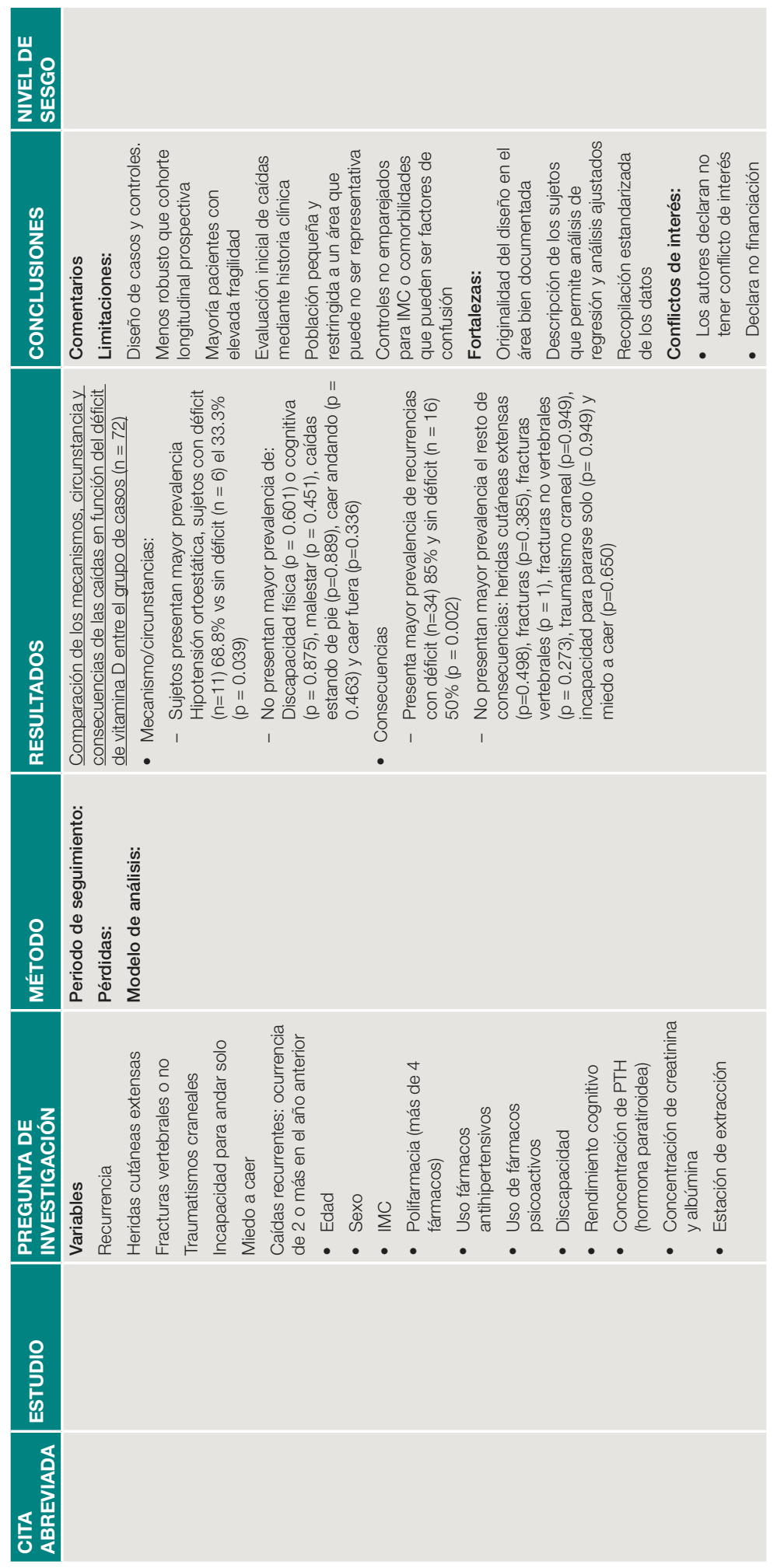




\section{WII}

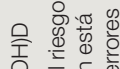

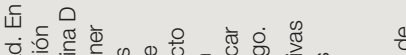

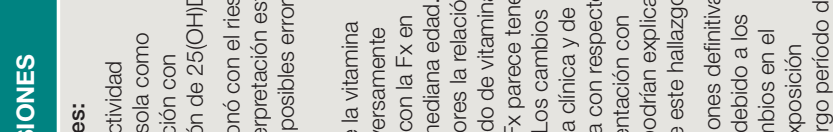

崖

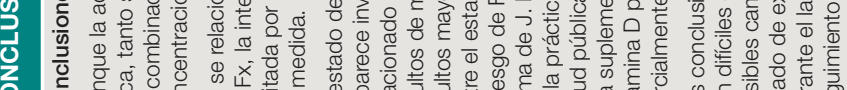

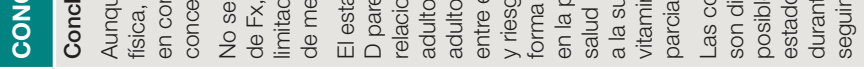

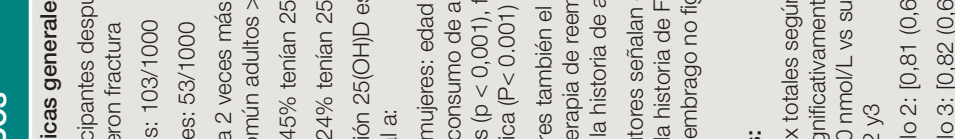

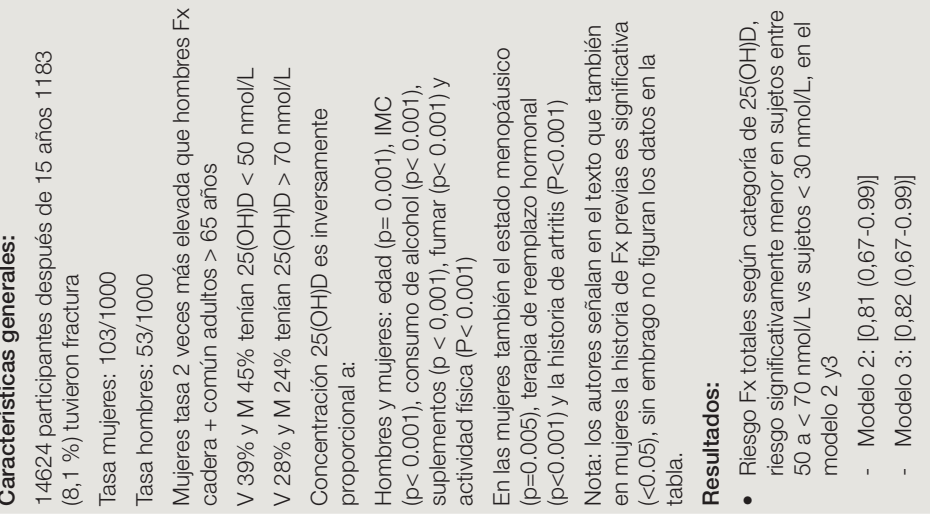

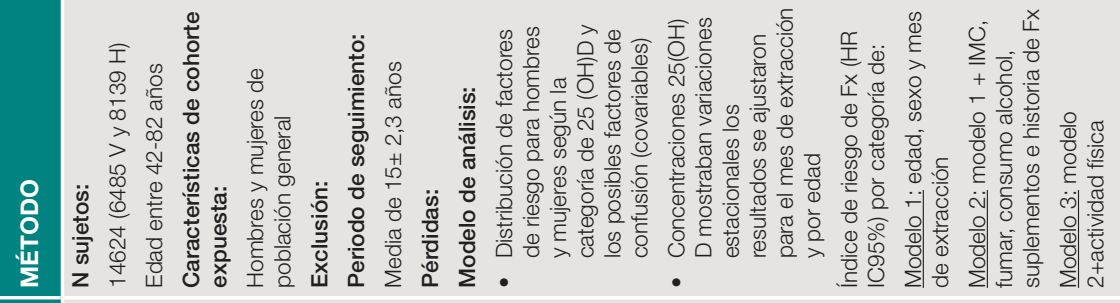

$\frac{\sqrt{0}}{\frac{0}{3}} \frac{10}{0} \quad \frac{0}{0}$

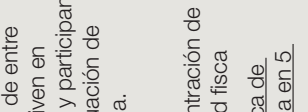

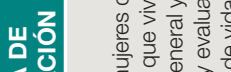

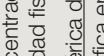

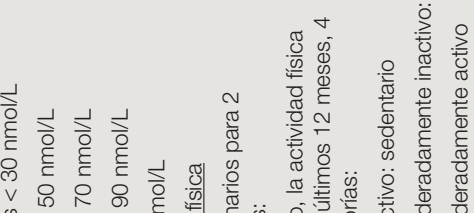

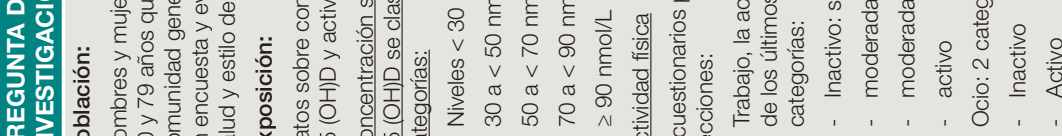

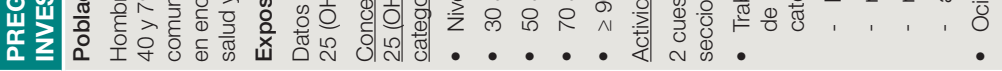

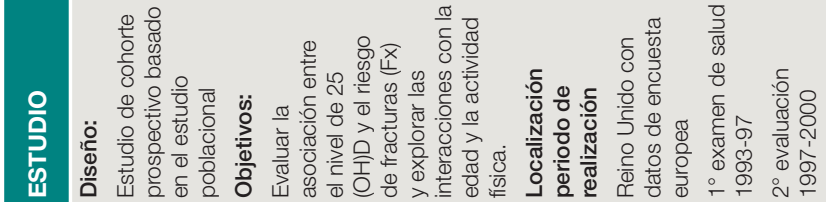

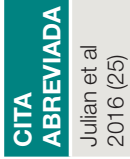




\section{㟧。}

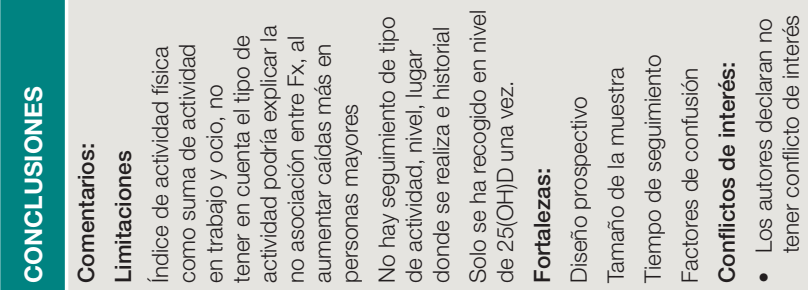

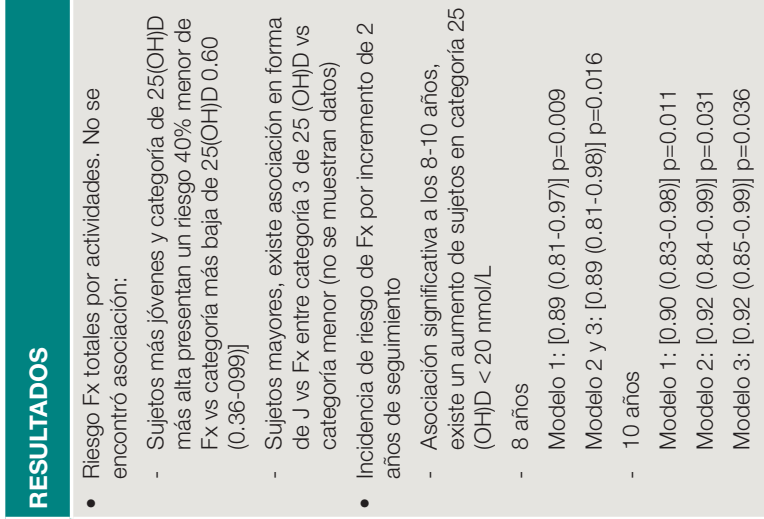

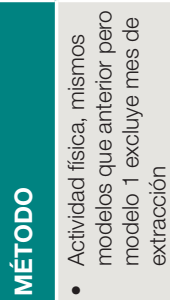

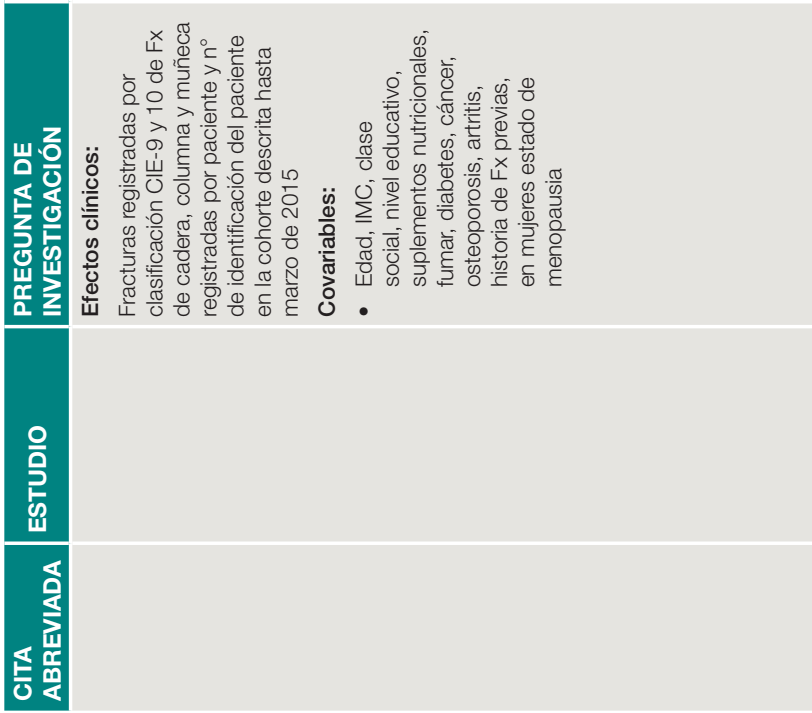




\section{III}

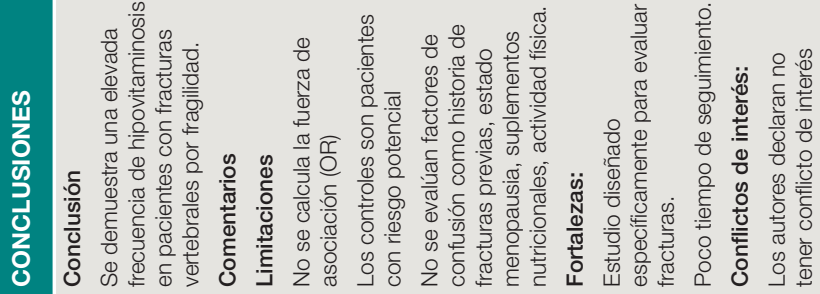

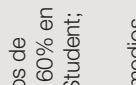

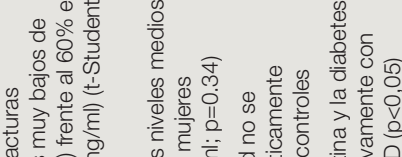

要它

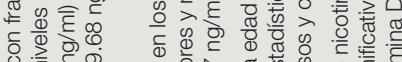

o

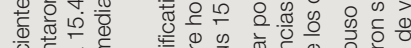

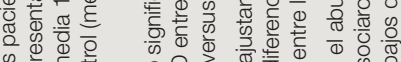

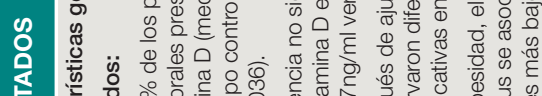

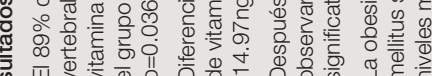

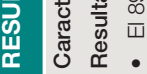

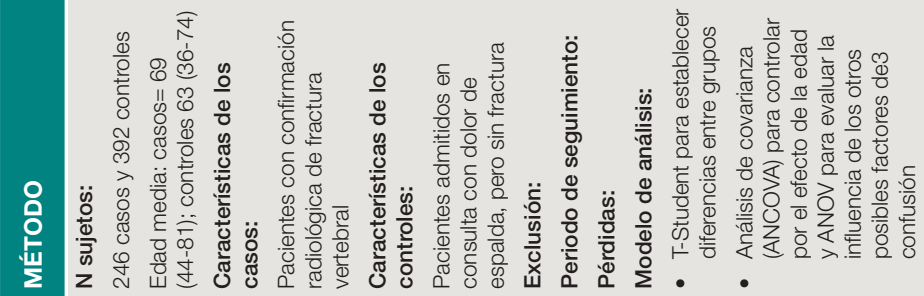

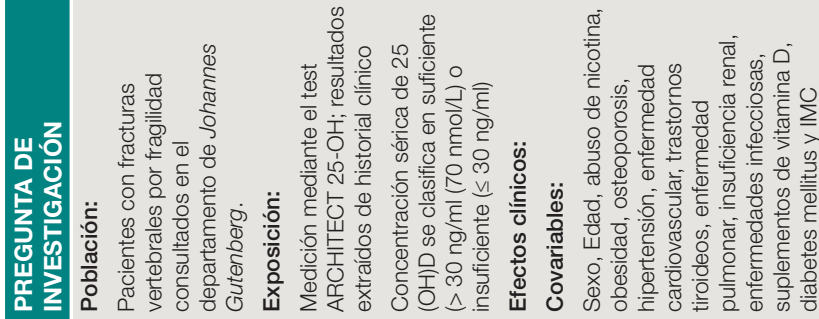

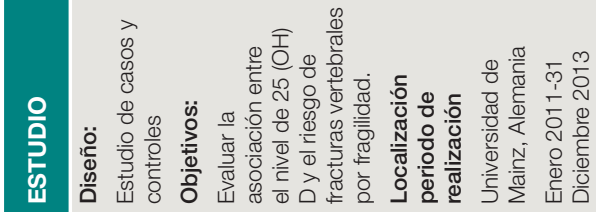

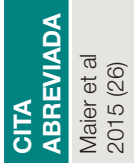




\section{III}

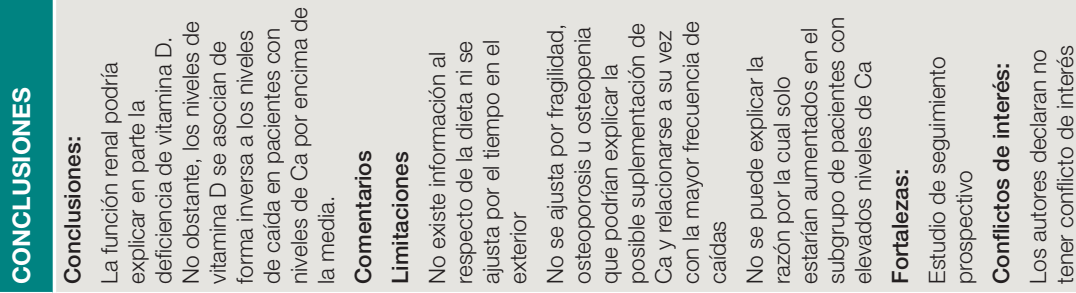

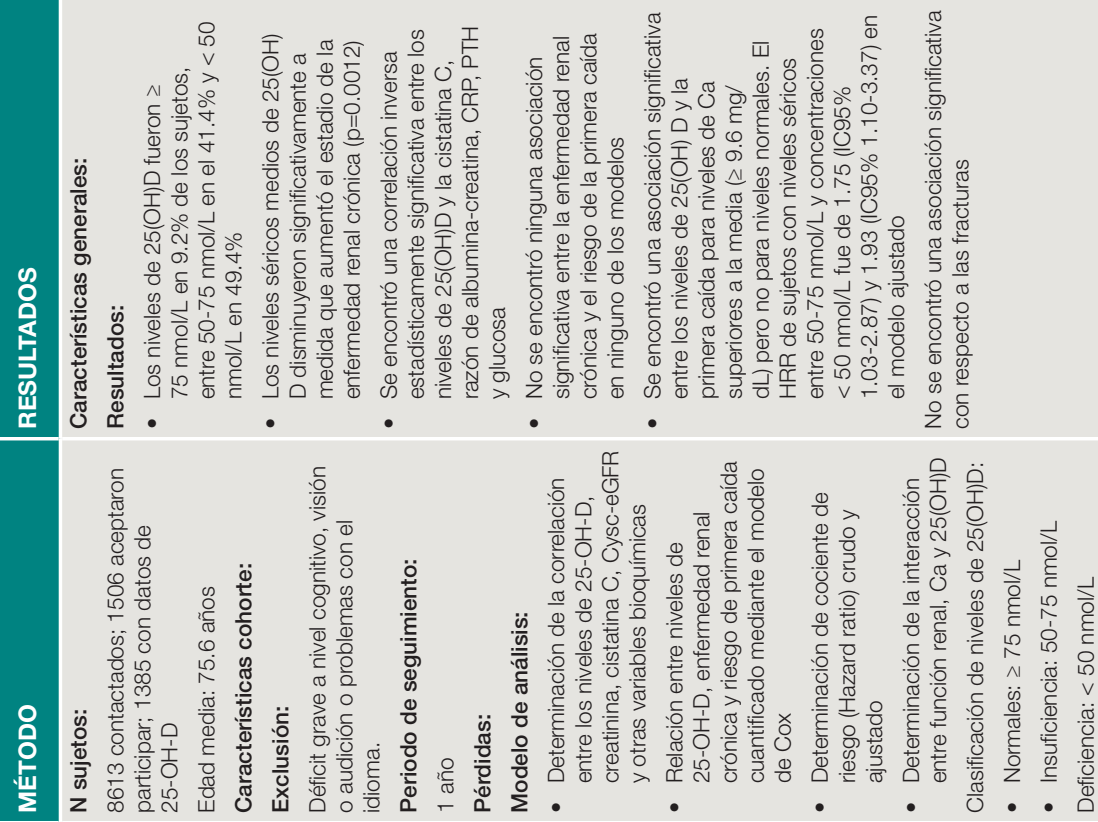

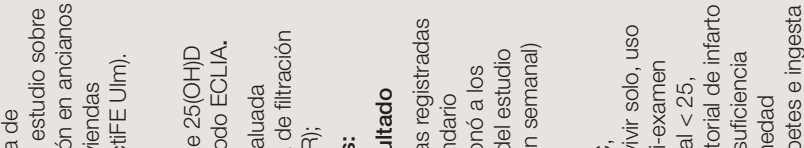

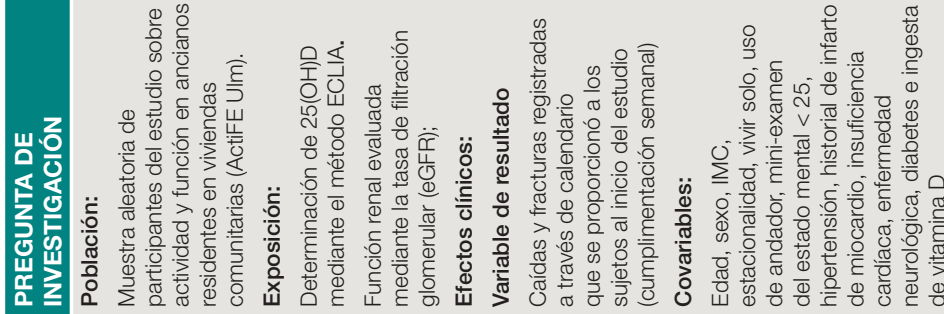

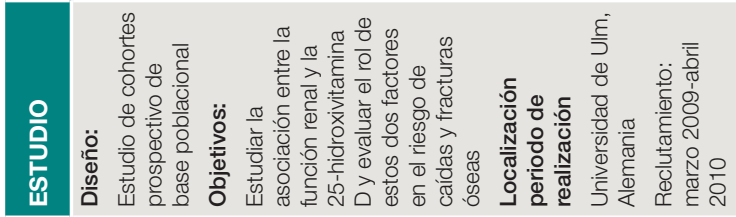

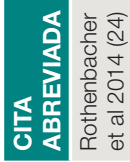




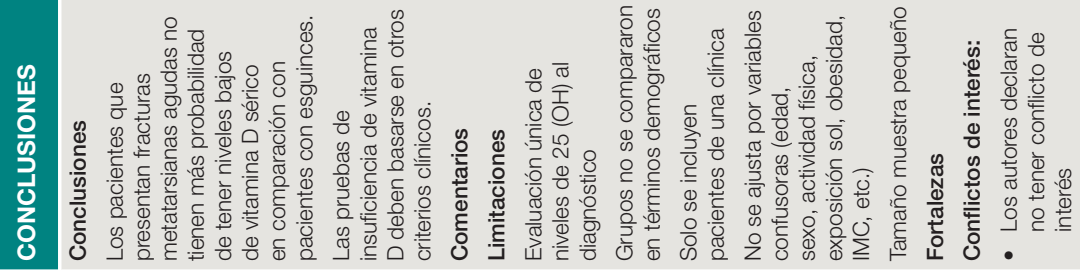

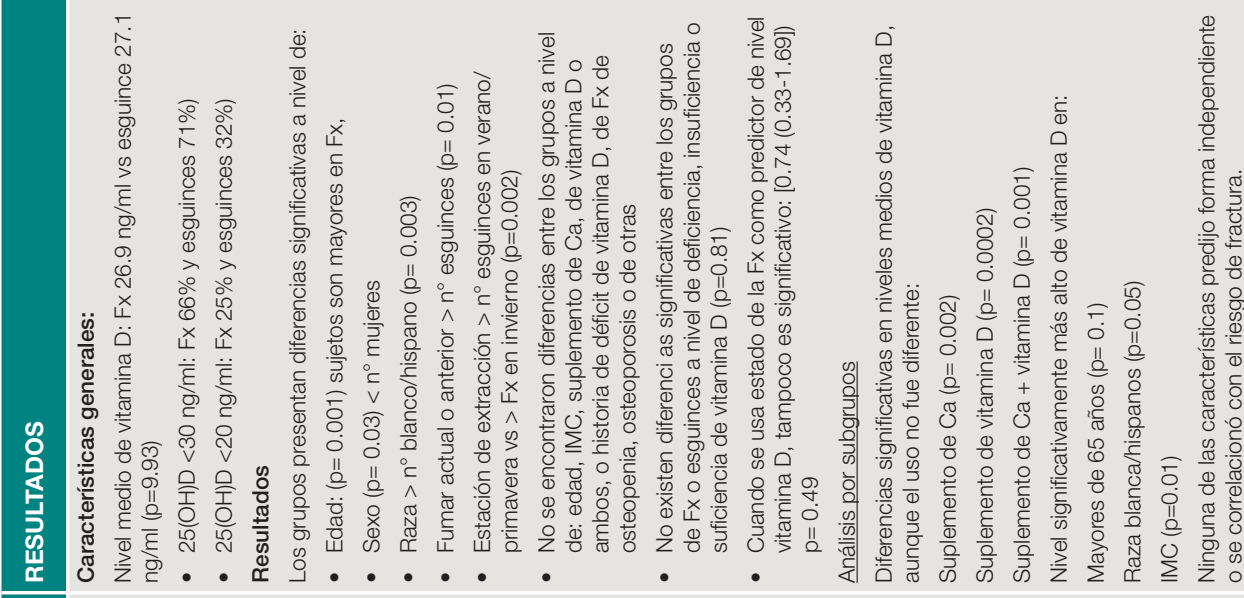

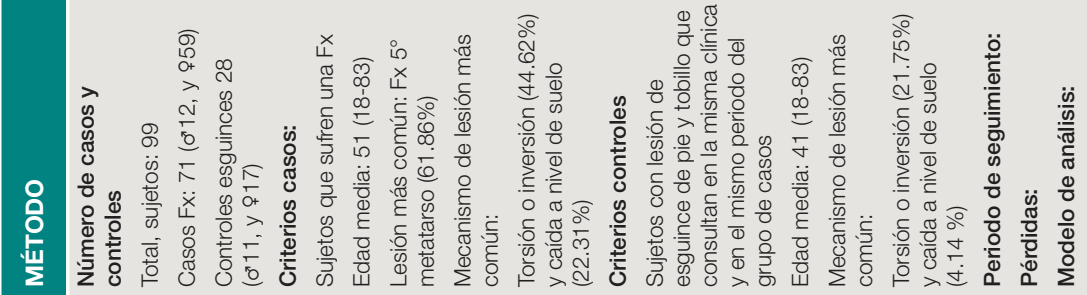

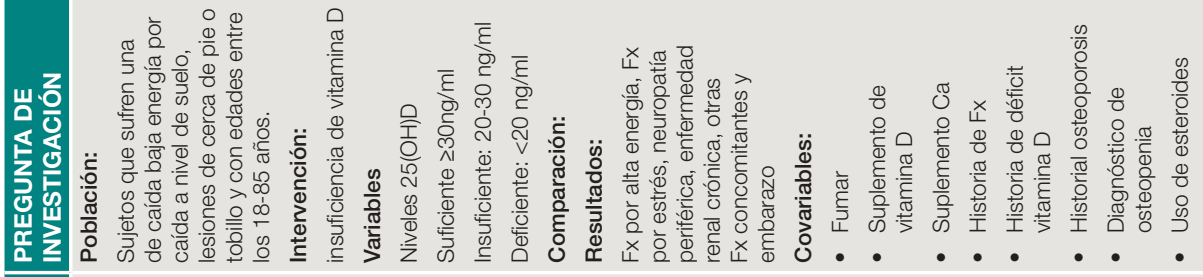

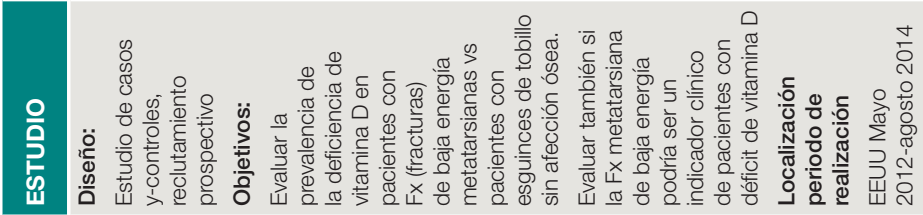

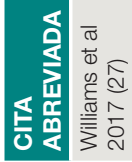


in

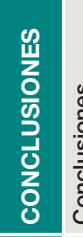

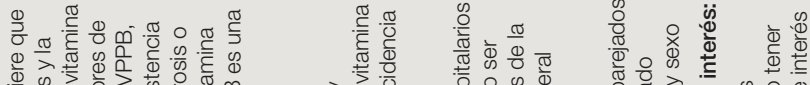

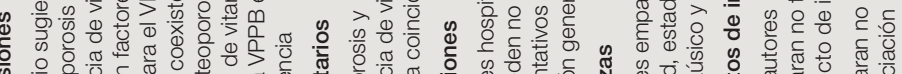

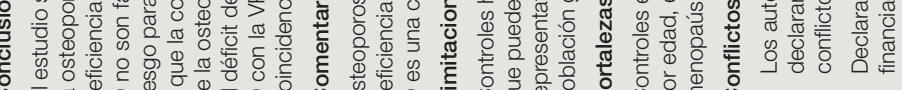
ब

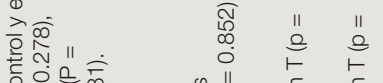

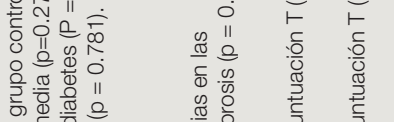

ब

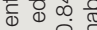

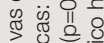

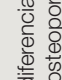

离

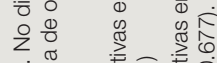

震

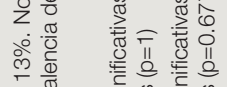

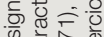

क्ष

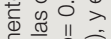

贻

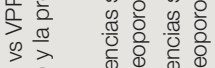

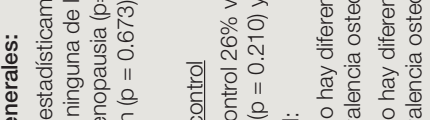

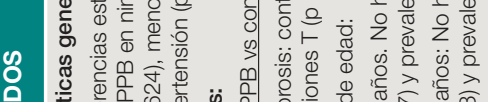

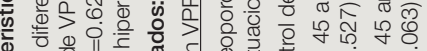

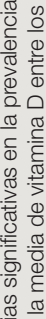

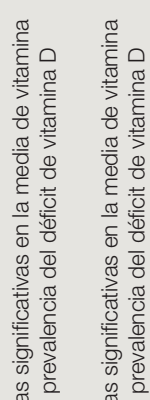

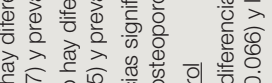

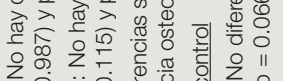

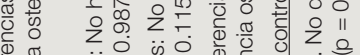

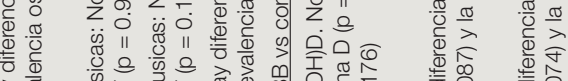

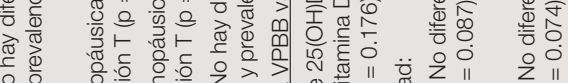

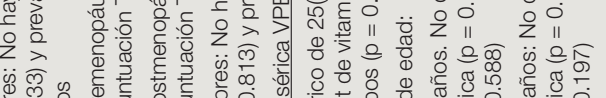

要

邹

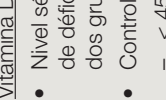

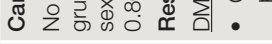

\section{is}

$\frac{\pi}{\frac{\pi}{0}}: \frac{\pi}{0}$

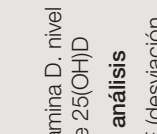

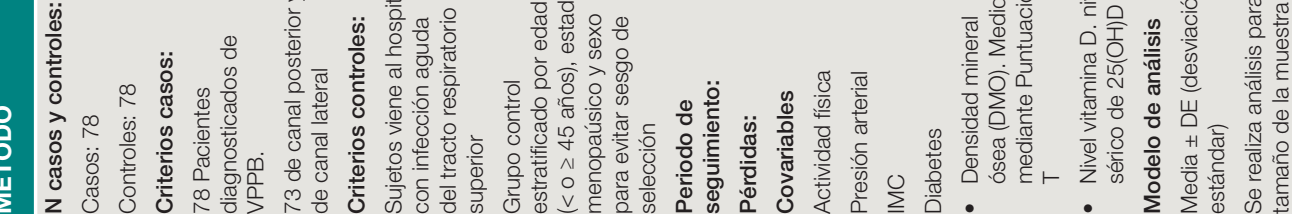

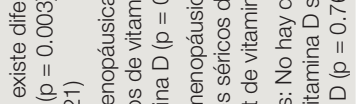

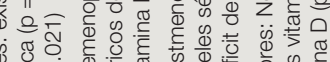

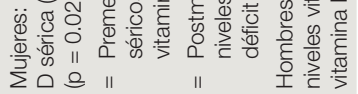

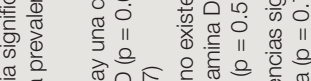

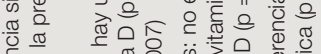
离文

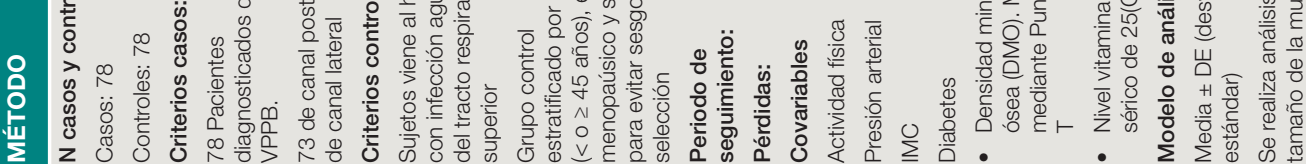

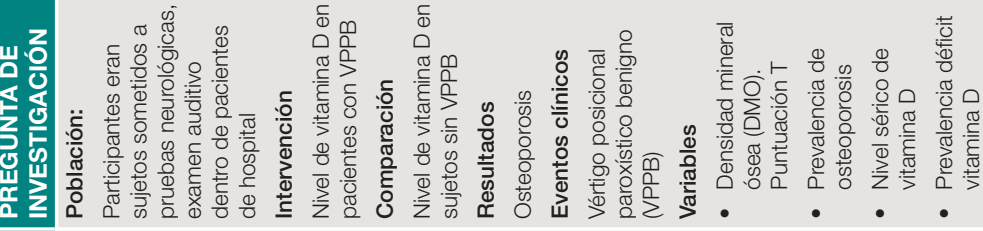

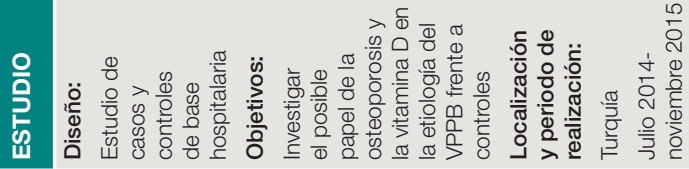




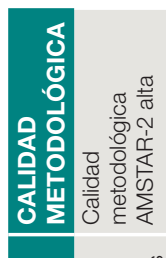

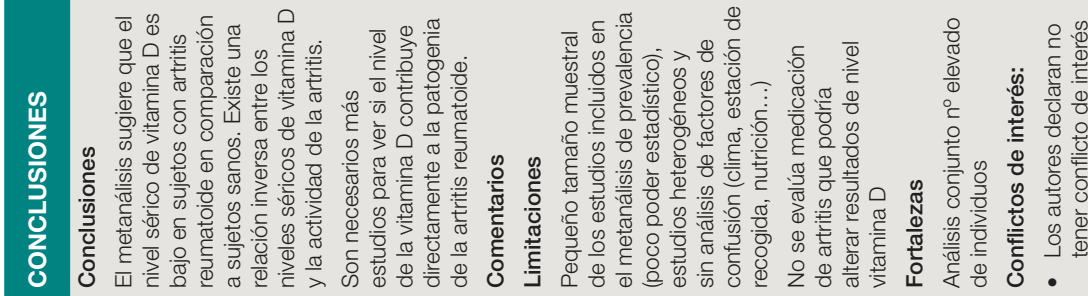

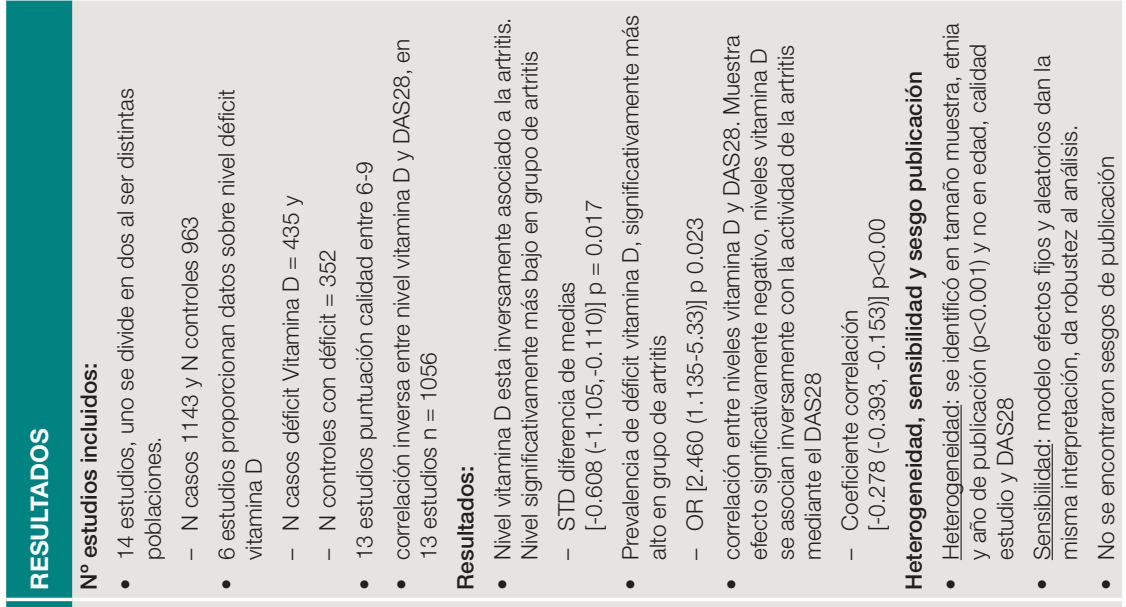

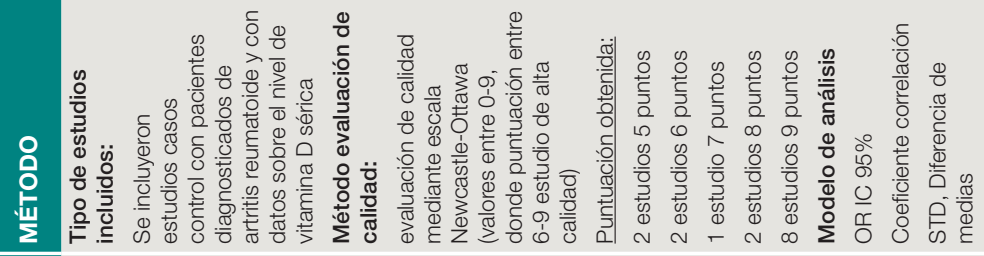

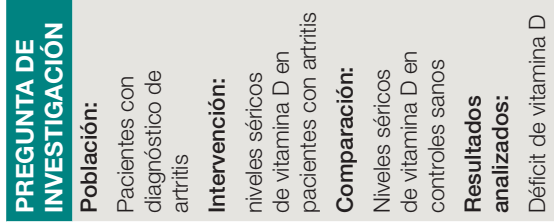

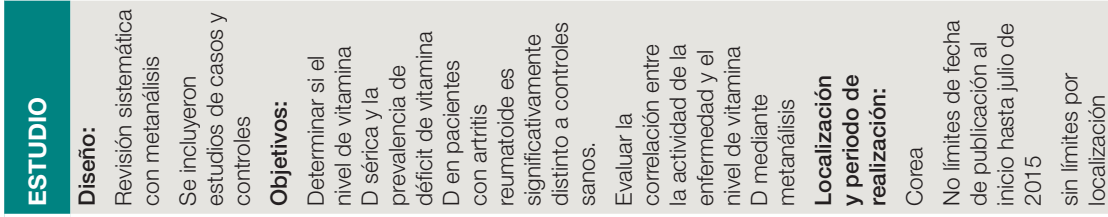

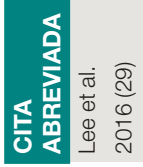


I

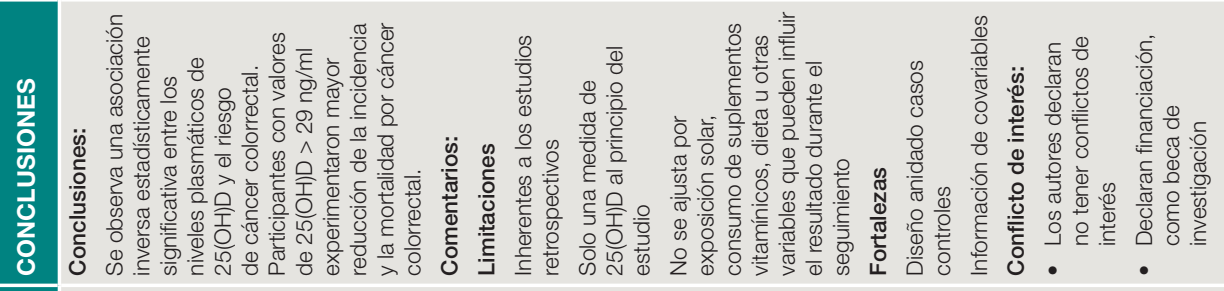

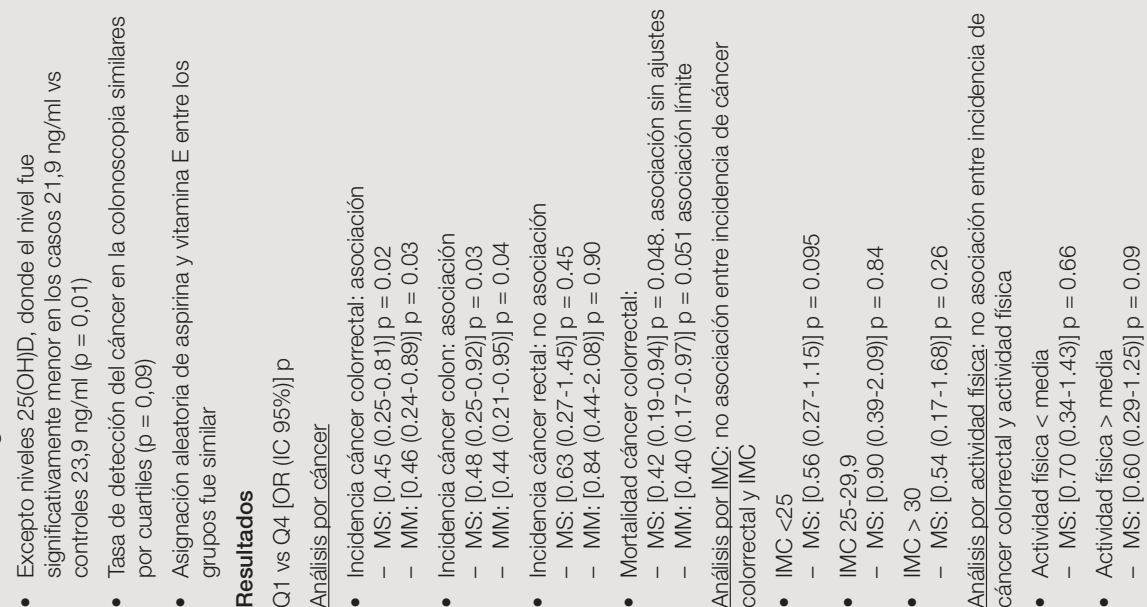

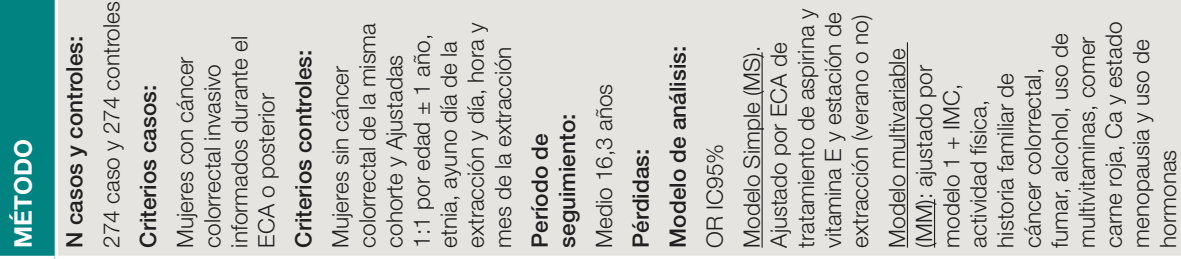

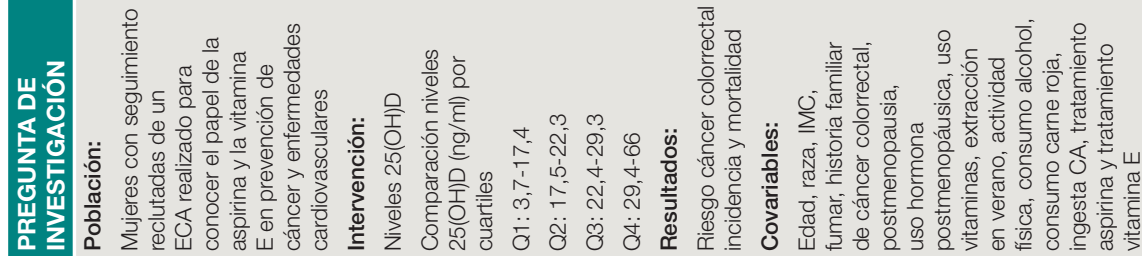

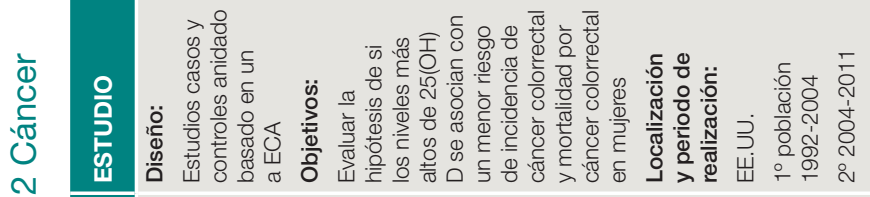

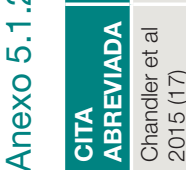




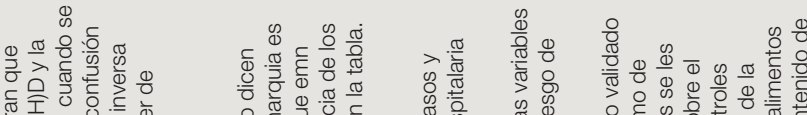

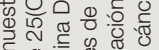

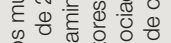

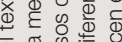

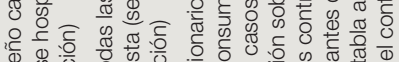

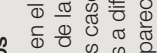

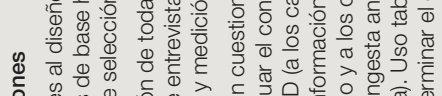

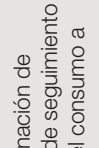

.ึำ

$\frac{\bar{\Phi}}{\Phi}$

is 0

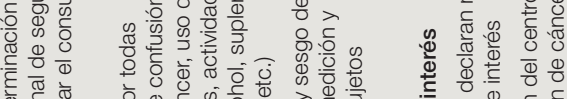

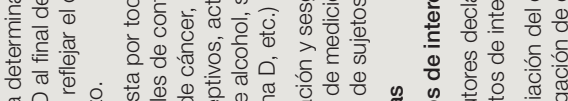

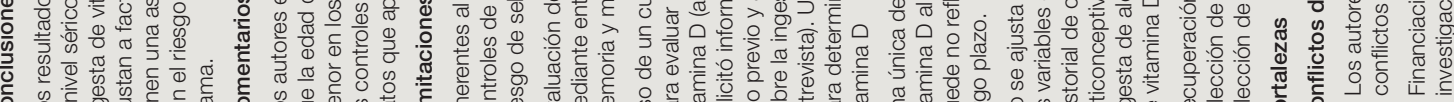

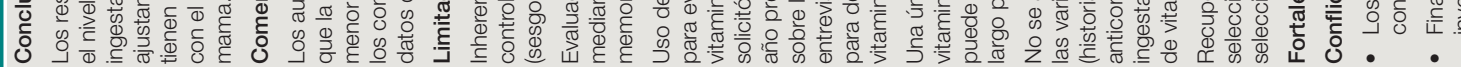

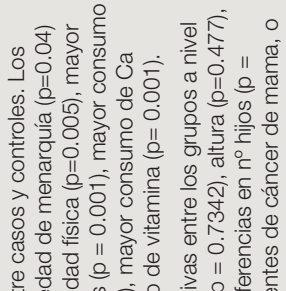

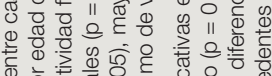

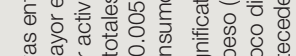

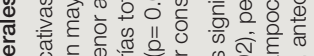

is

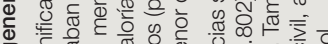

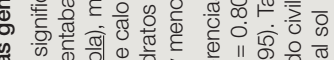

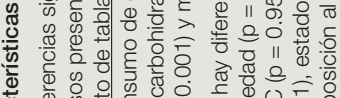

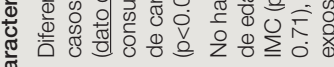

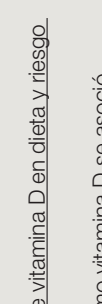

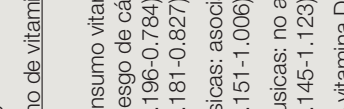

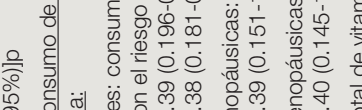

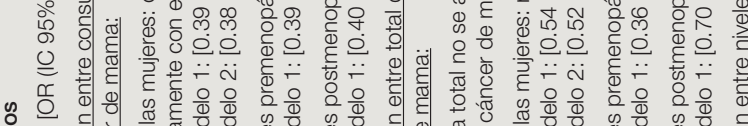

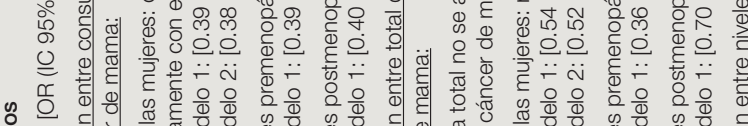

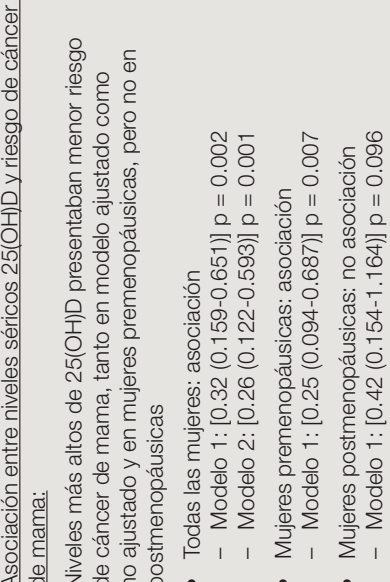

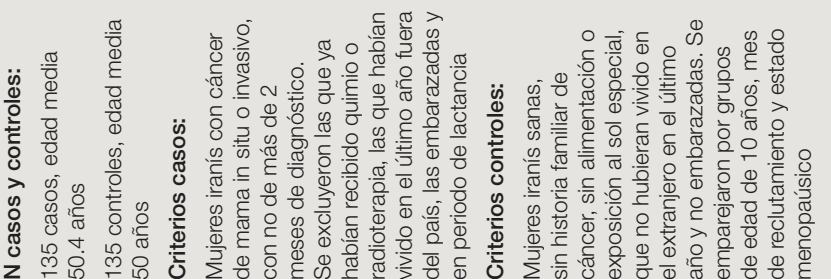

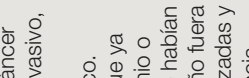

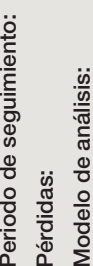

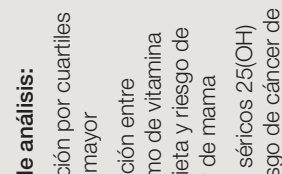

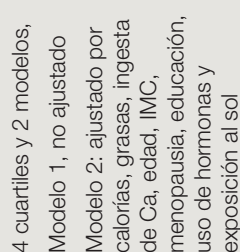

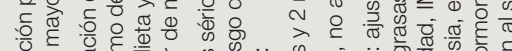

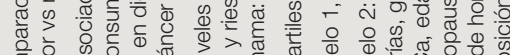

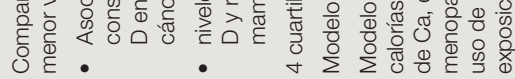

\section{ฮ $\bigcirc$}

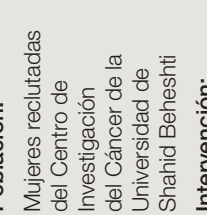

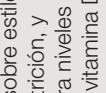

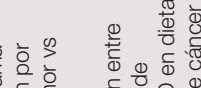

离

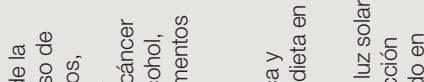

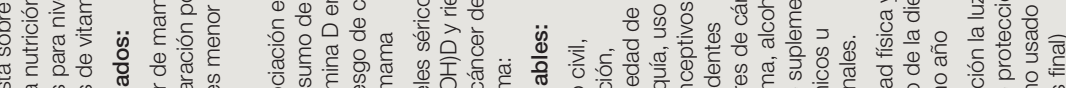

雪.

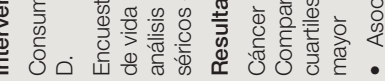

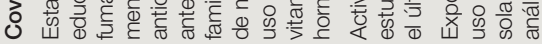

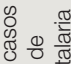
음
立 $>\frac{0}{8}$
हैं
ه

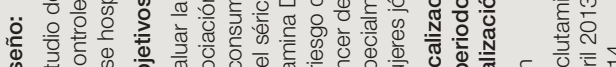

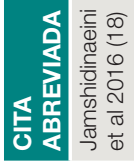




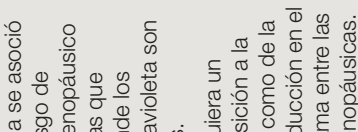

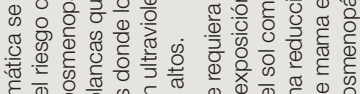

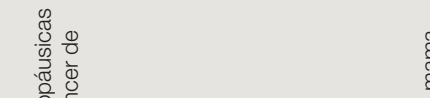

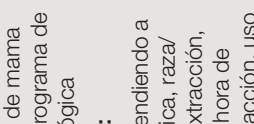

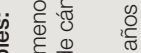

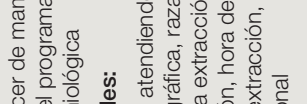

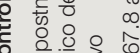

牙家

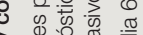

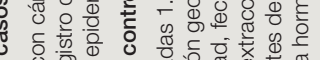

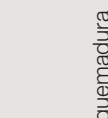

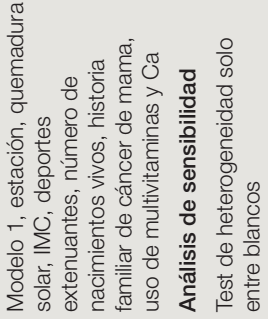

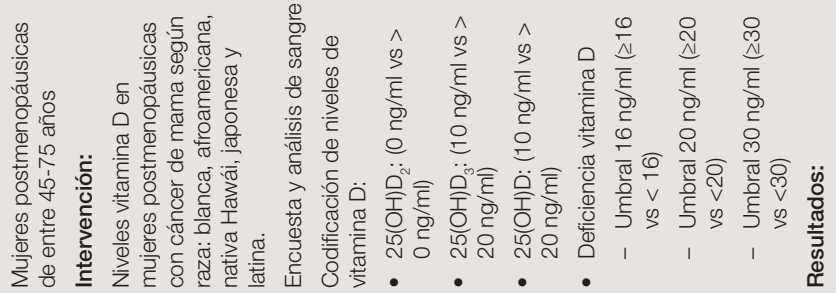

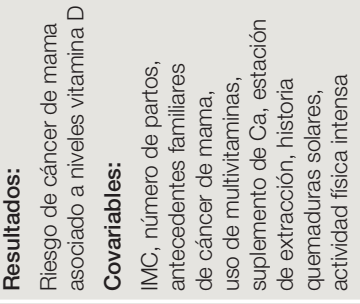

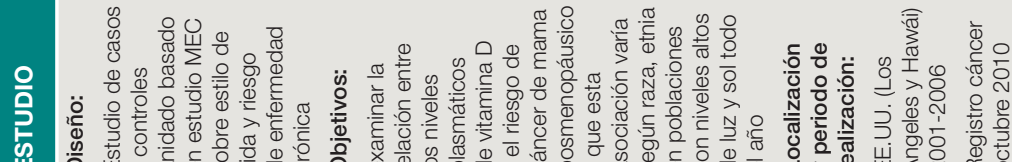

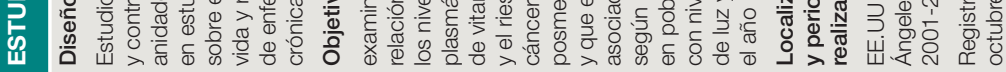




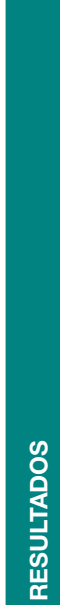

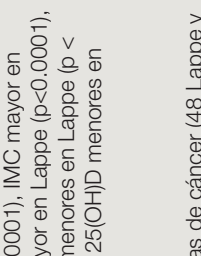

\section{จ}

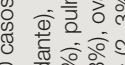

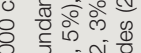

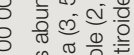

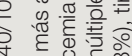

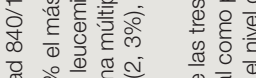

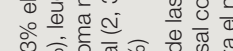

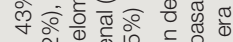
ㅎำ

is is

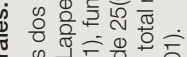

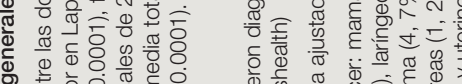

늘 है है है

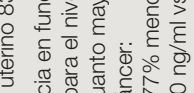

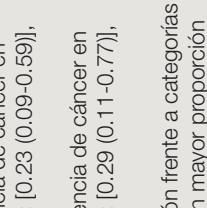

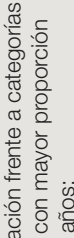

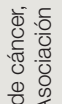

요

ठำ

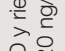

오ำ

क्ण 을 है

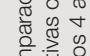

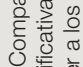

U. ऐ)

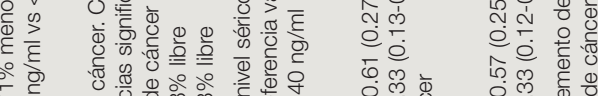

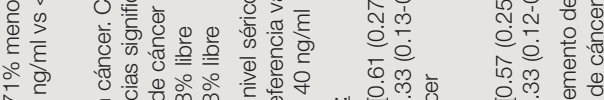

蛋

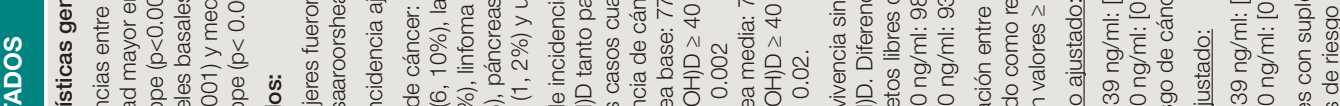

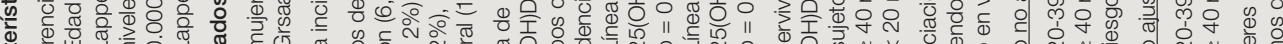

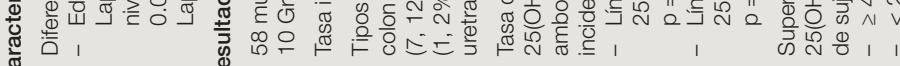
क ल

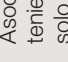

.

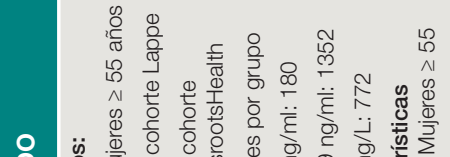

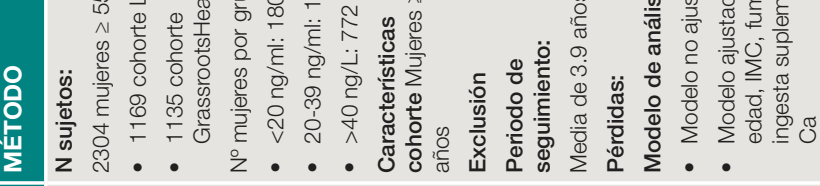

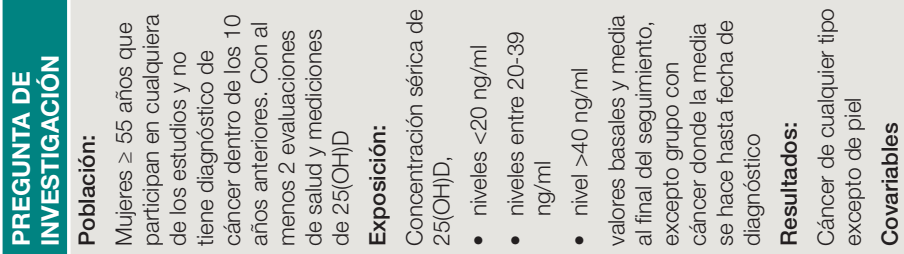

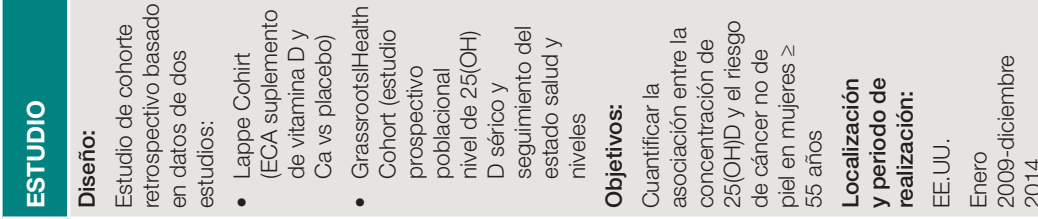

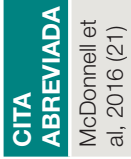




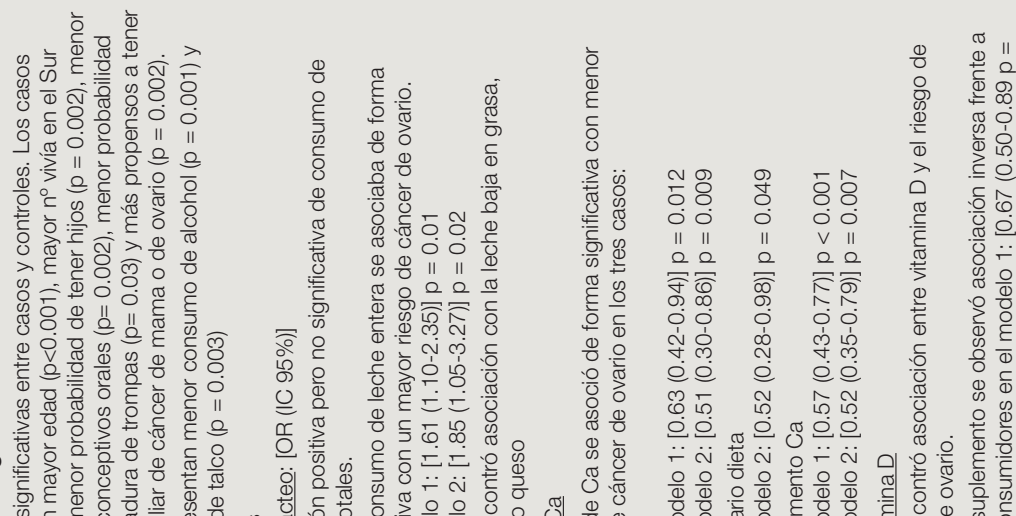

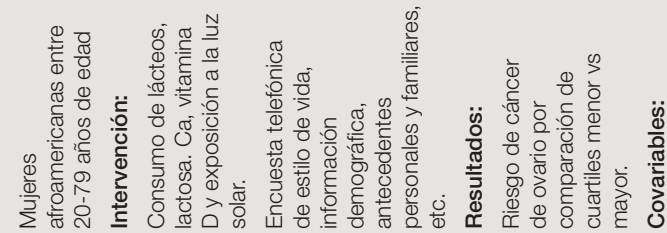

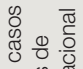




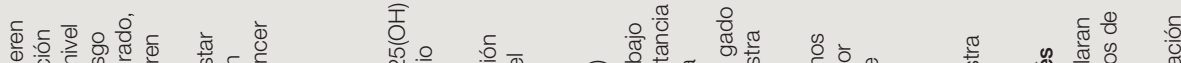

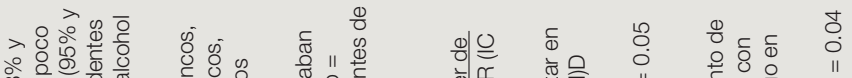

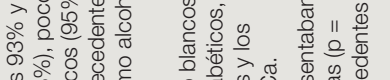

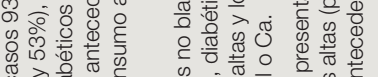

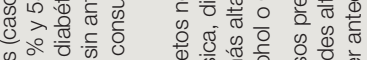

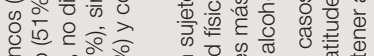

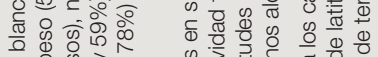

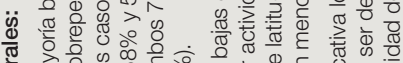

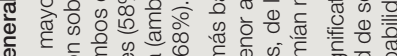

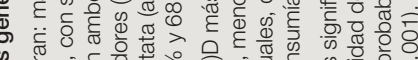

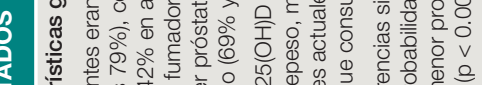

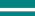

a.

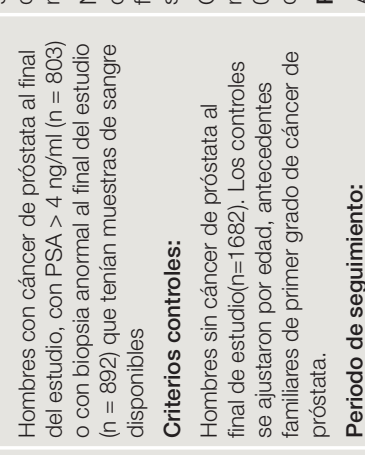

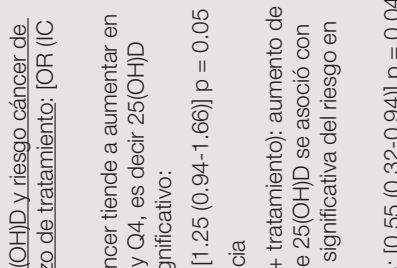

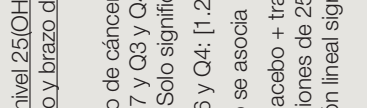

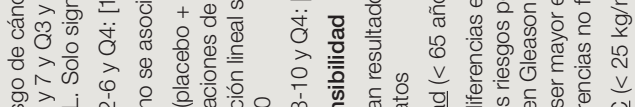




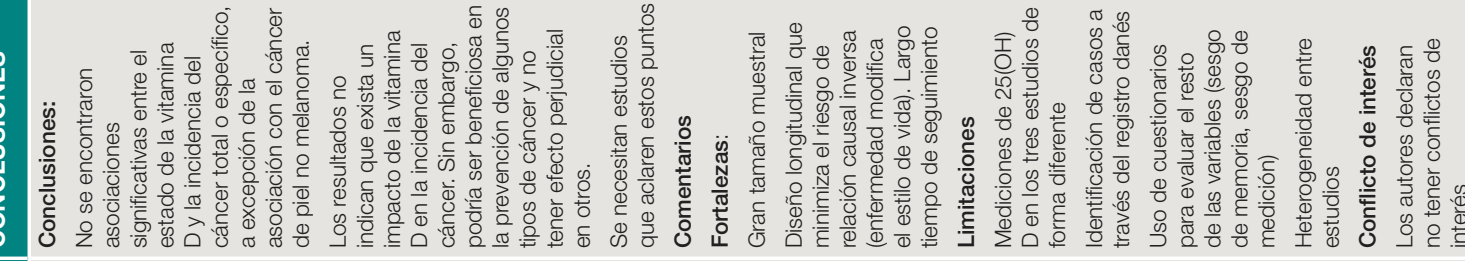

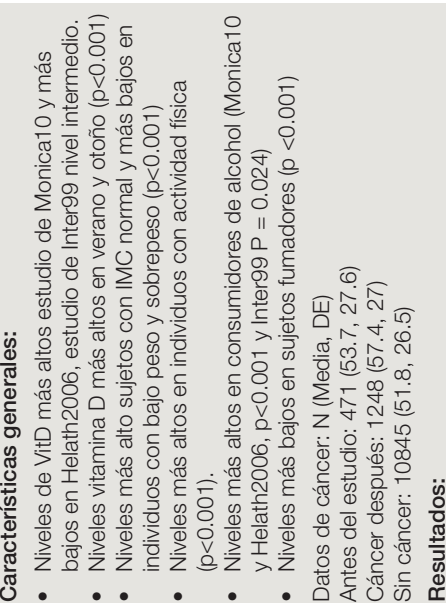

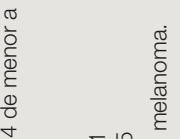

a

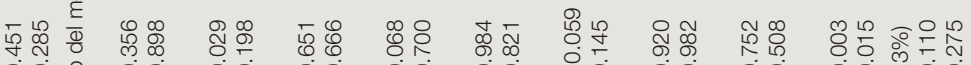

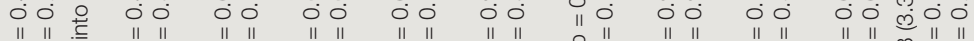

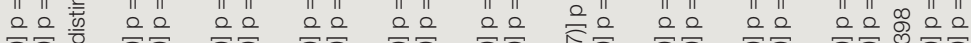

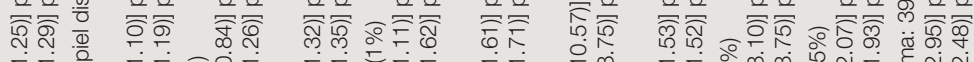

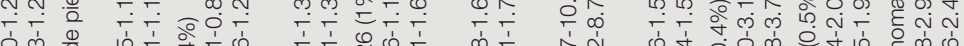

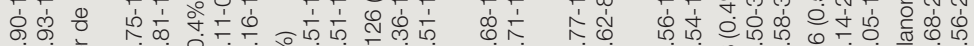

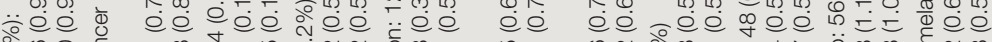

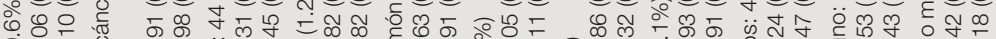

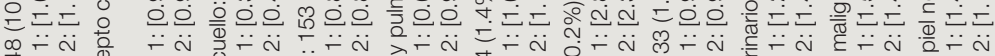

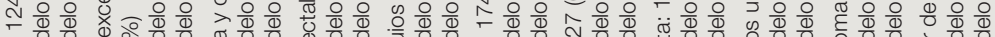

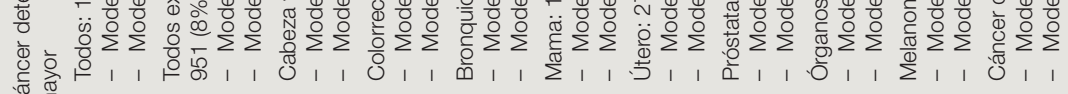

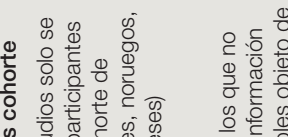

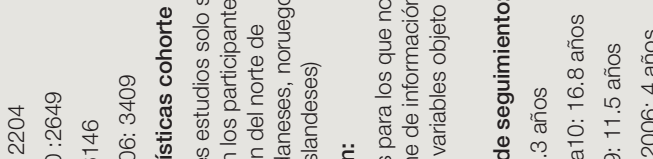

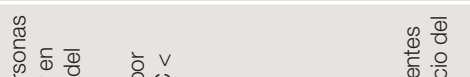

这

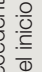

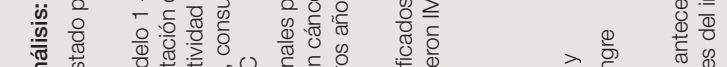

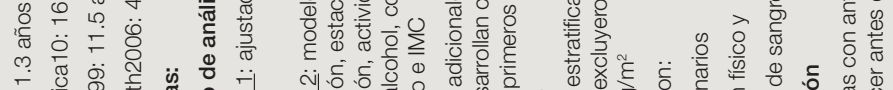

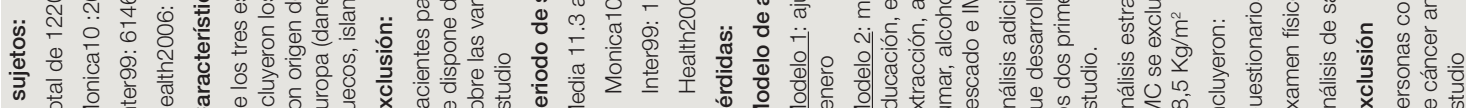

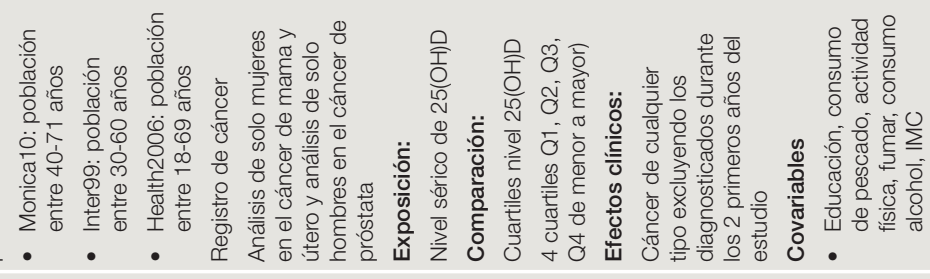

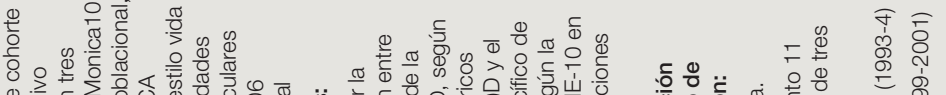



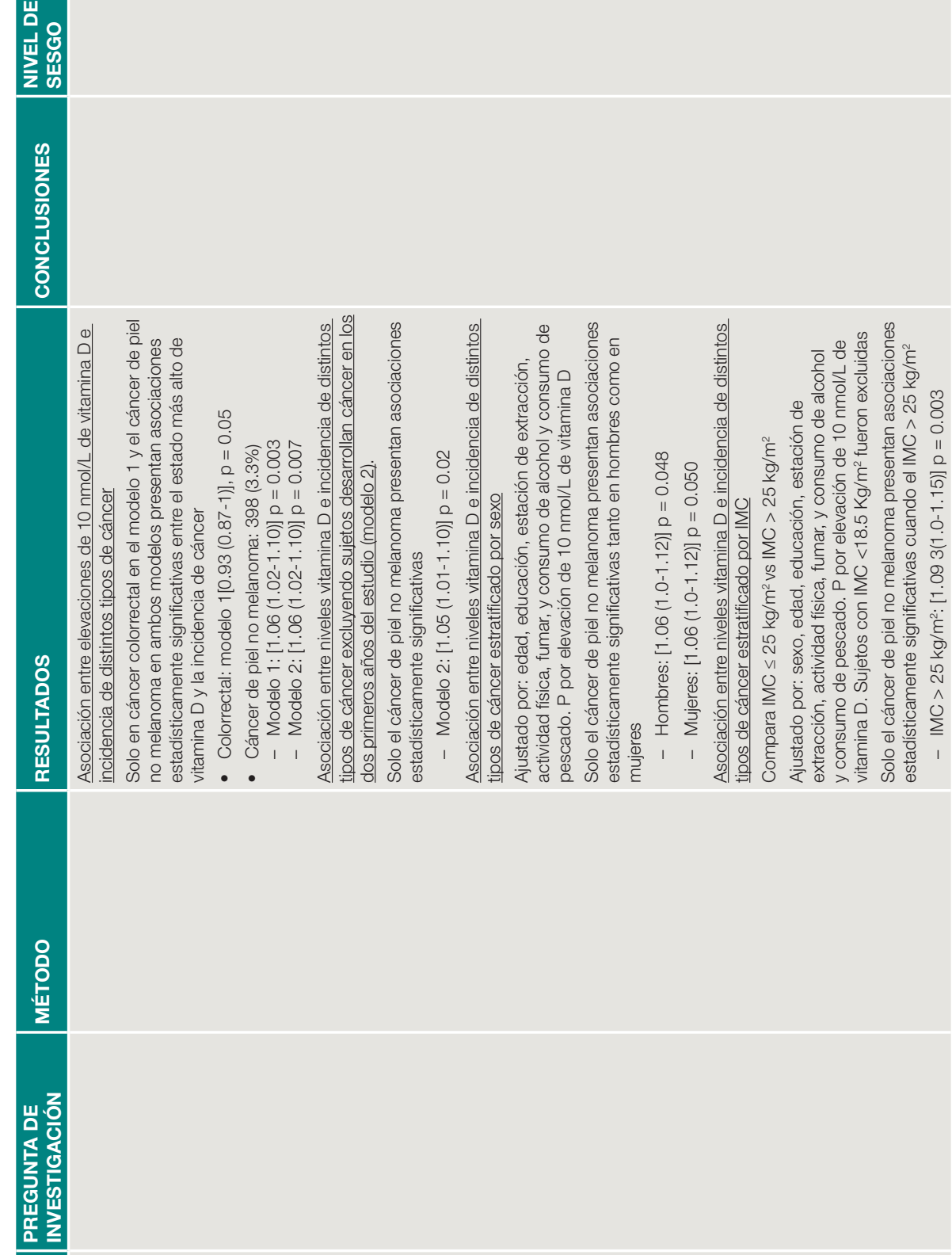

음 


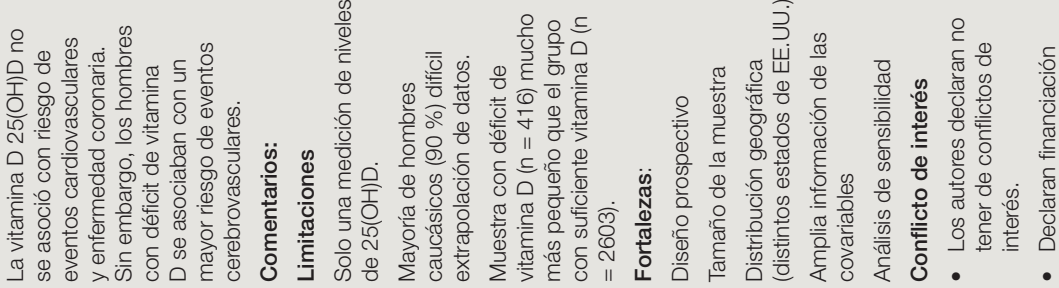

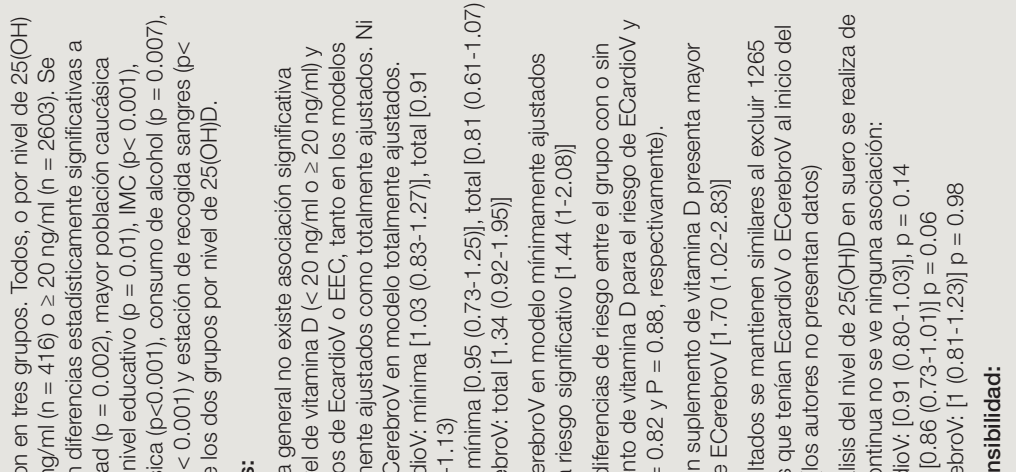

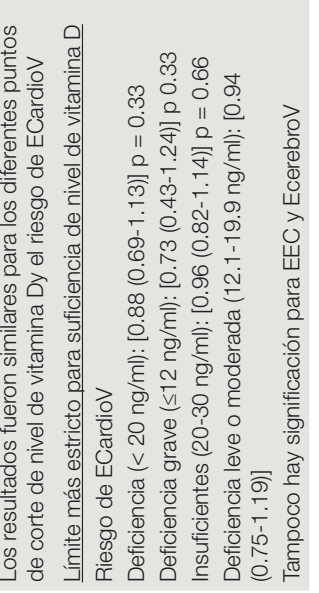

을

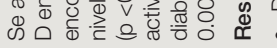

(1)

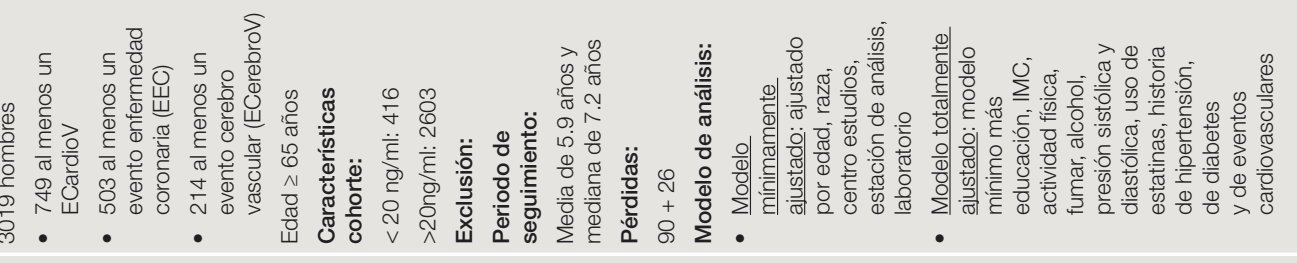

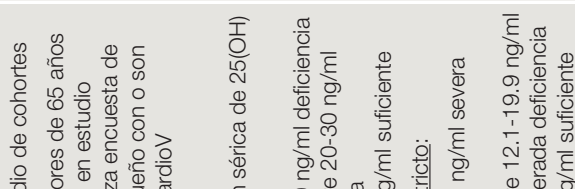
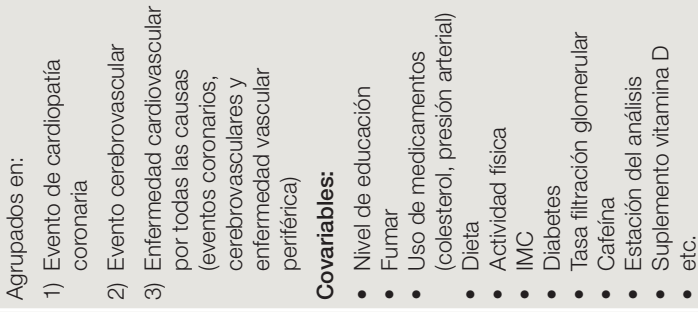

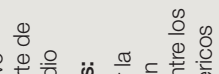

\section{里}

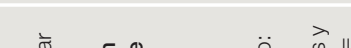

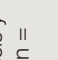

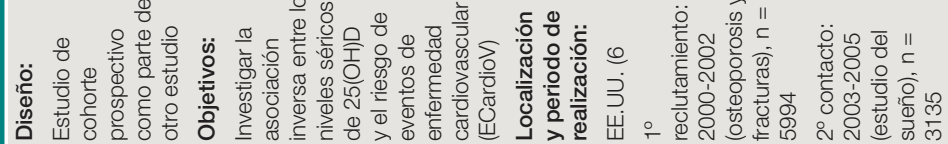


IIIIIIIIIIIIIUIIIII

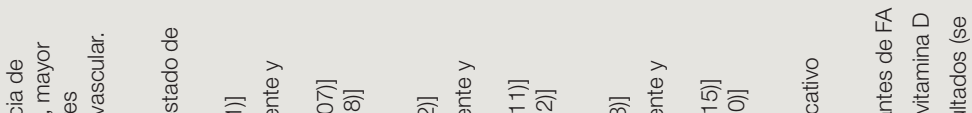

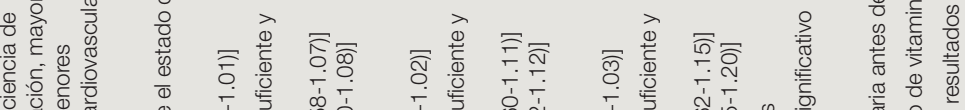

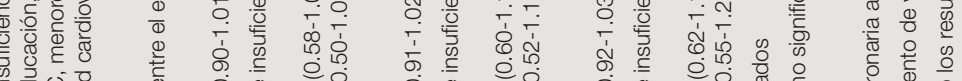

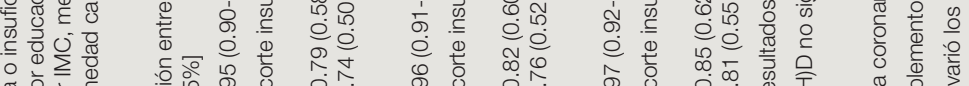

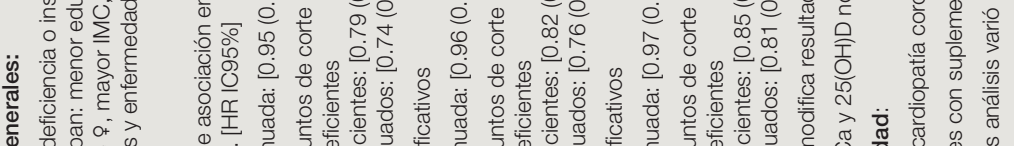

a

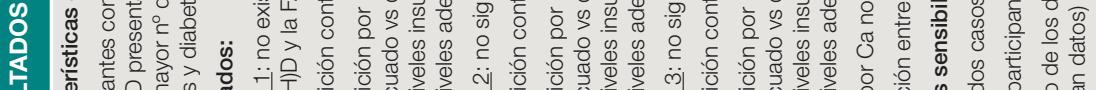

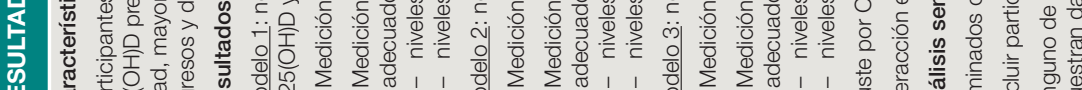

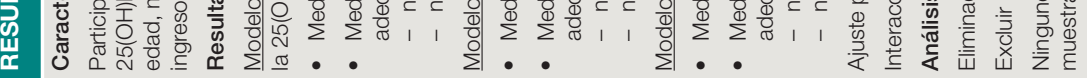

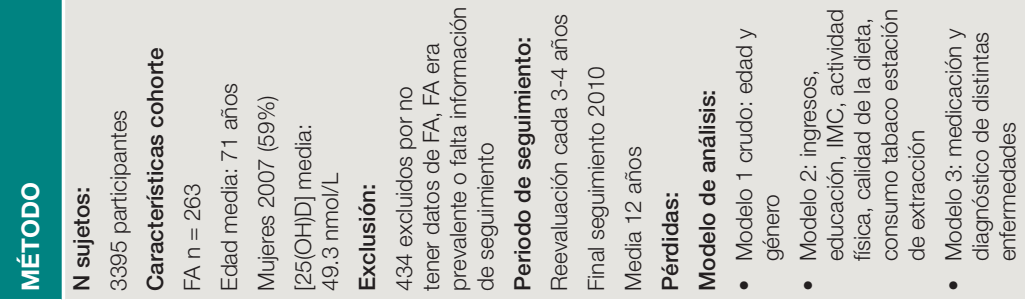

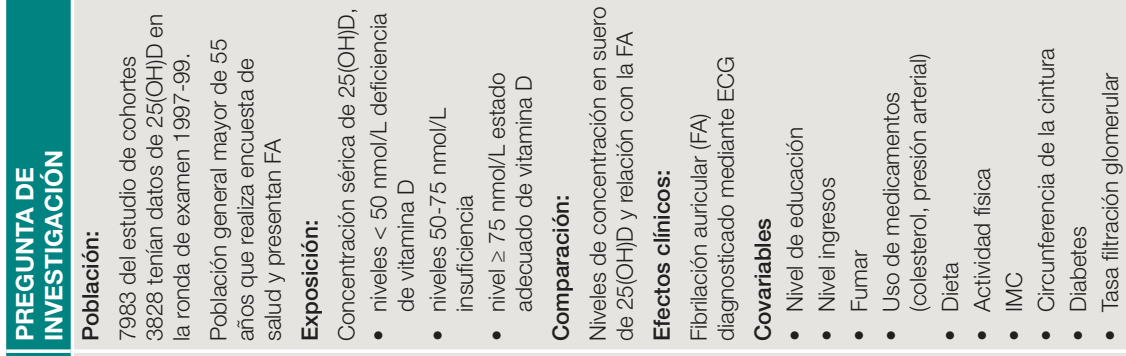

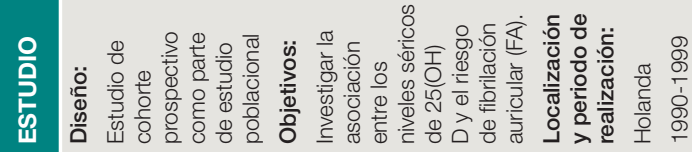

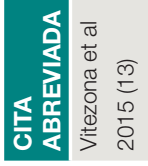




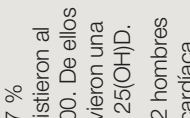

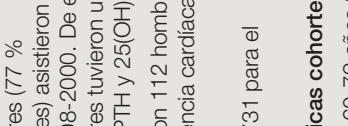

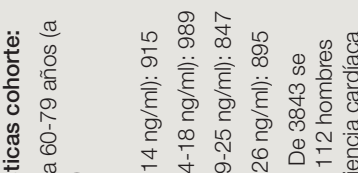




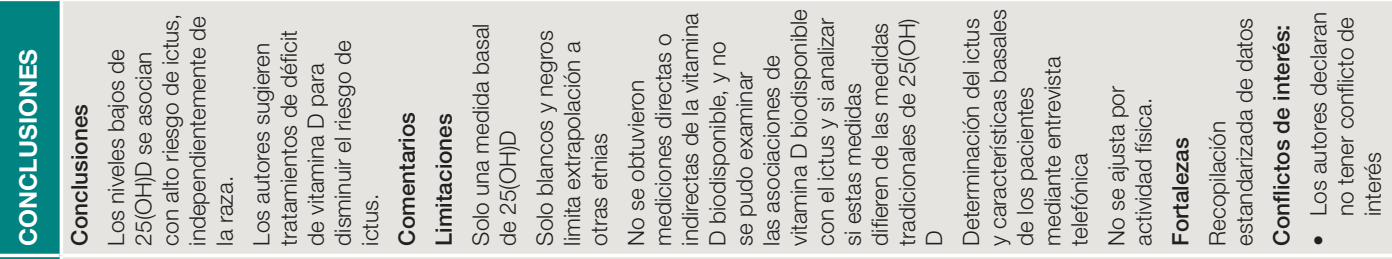

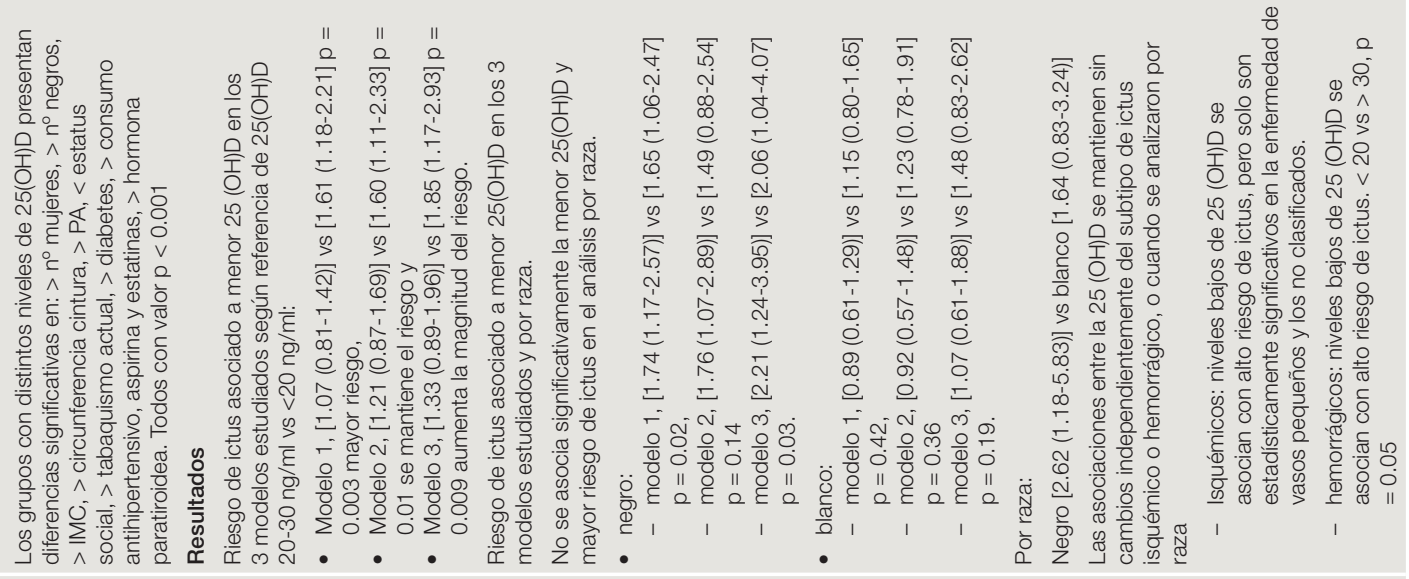

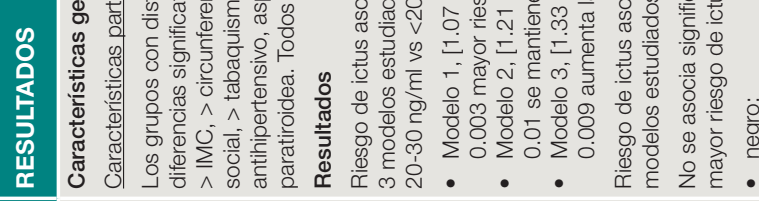

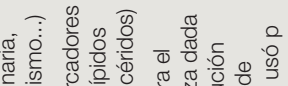

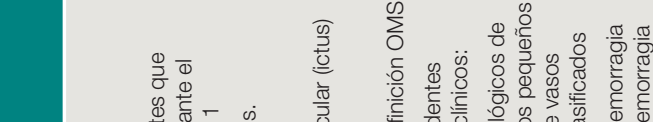

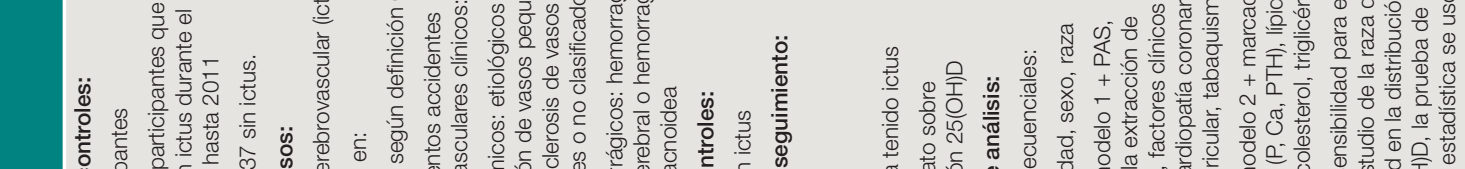

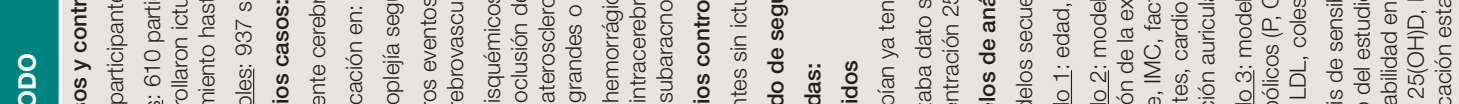

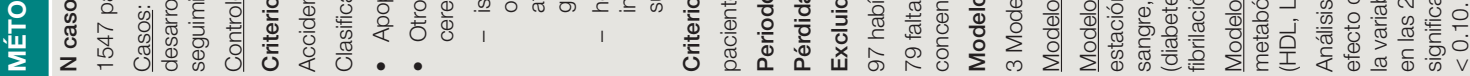

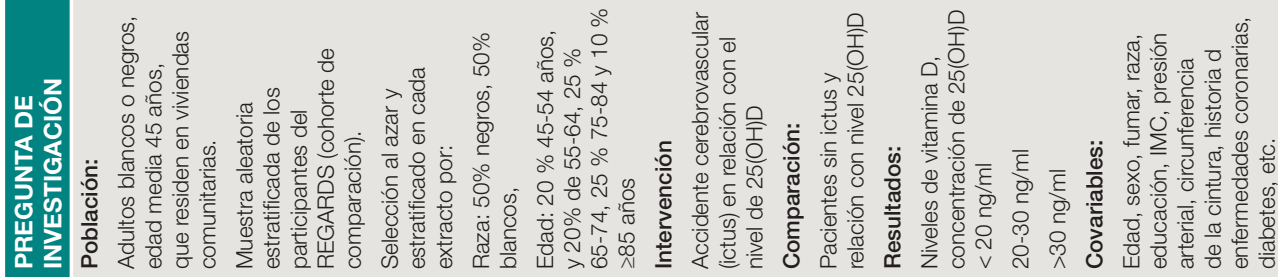

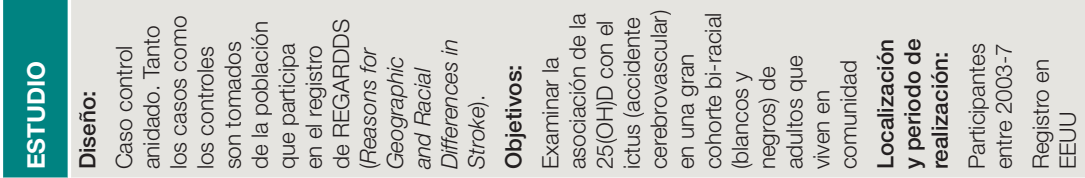

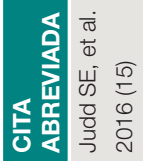




\section{IIII}

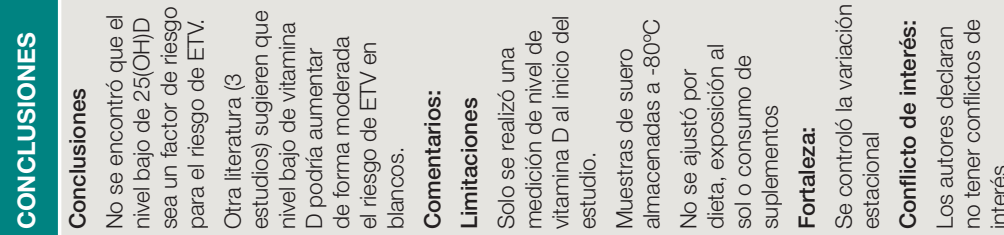

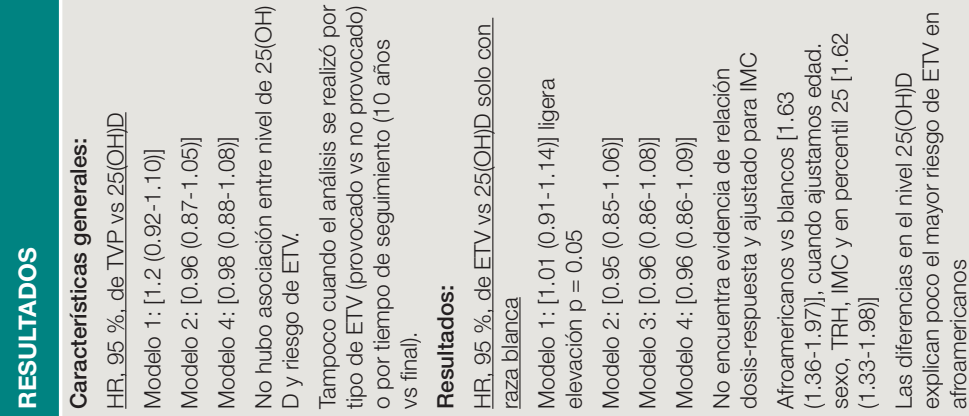

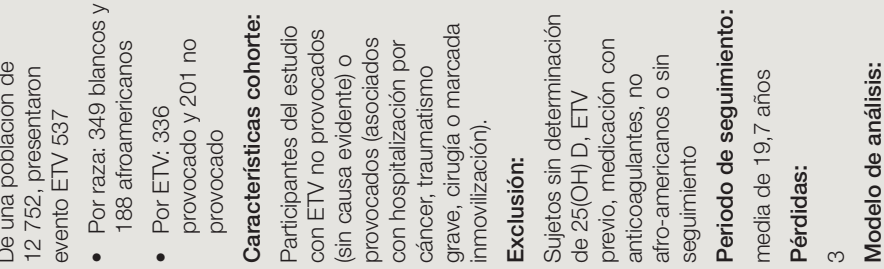

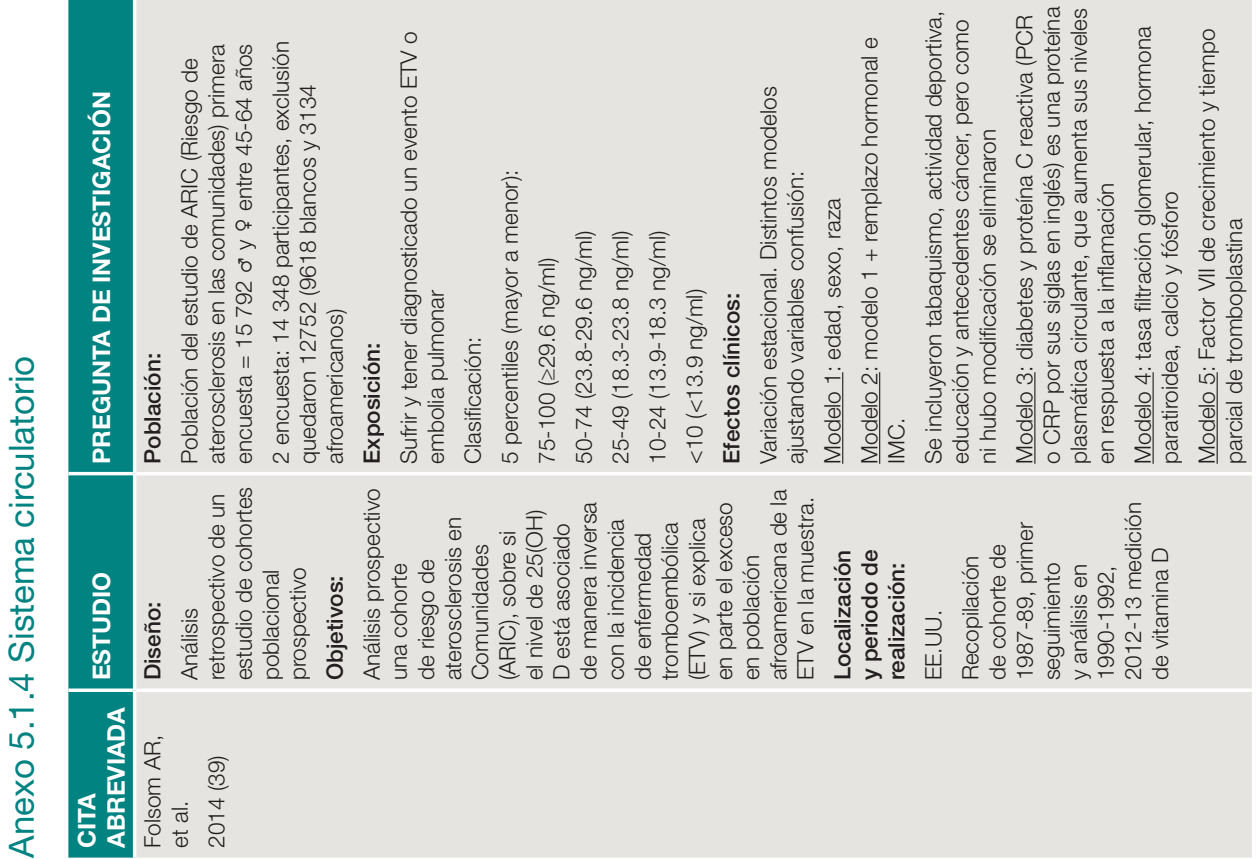




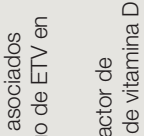

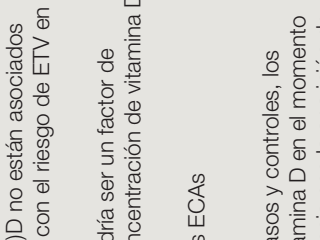

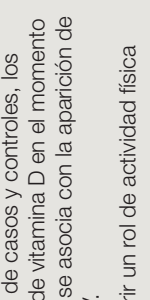

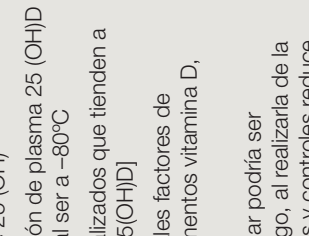

क)

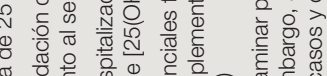

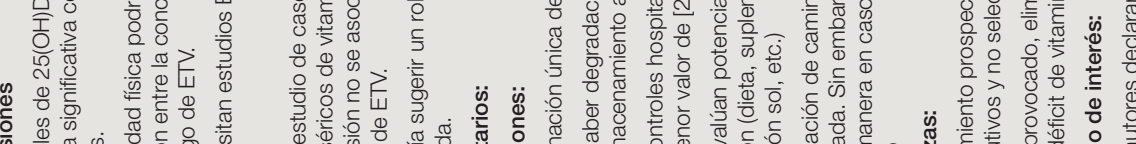

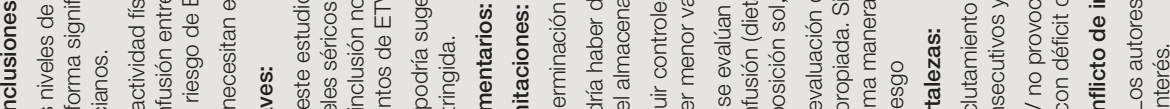

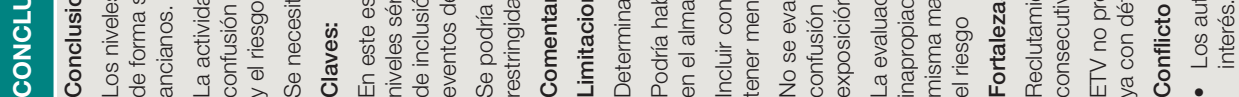




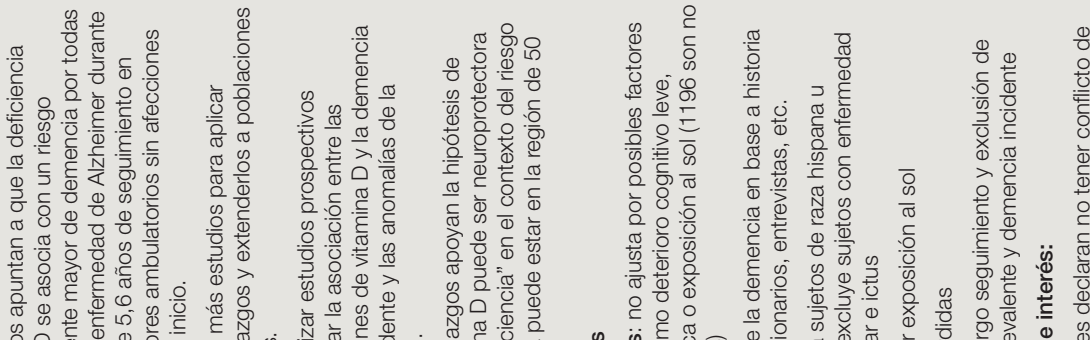

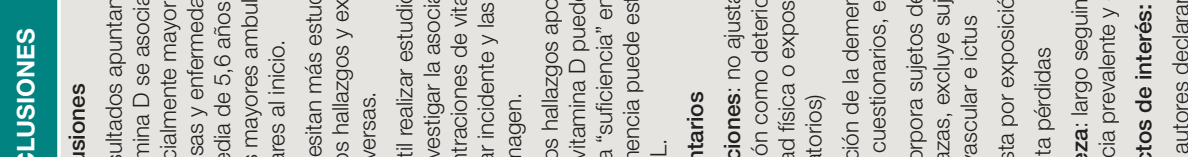

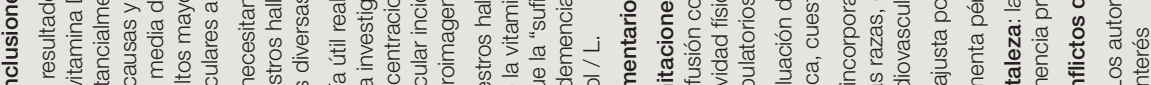

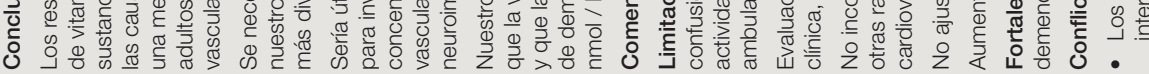

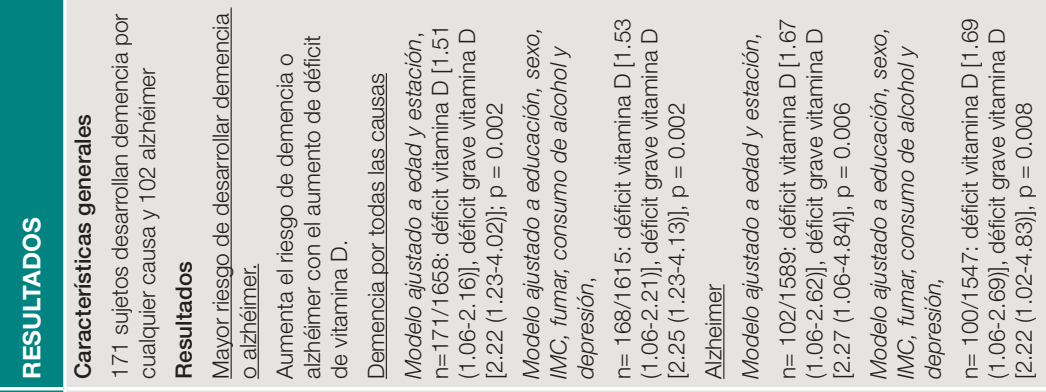

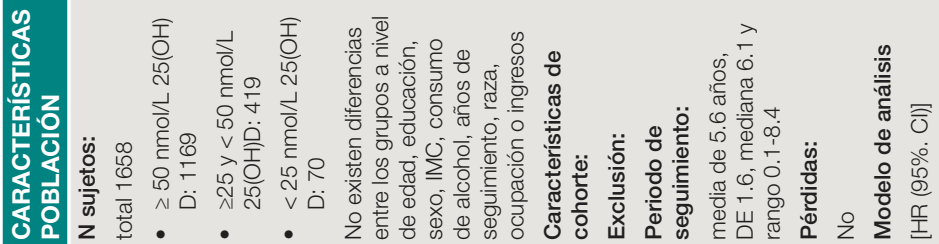

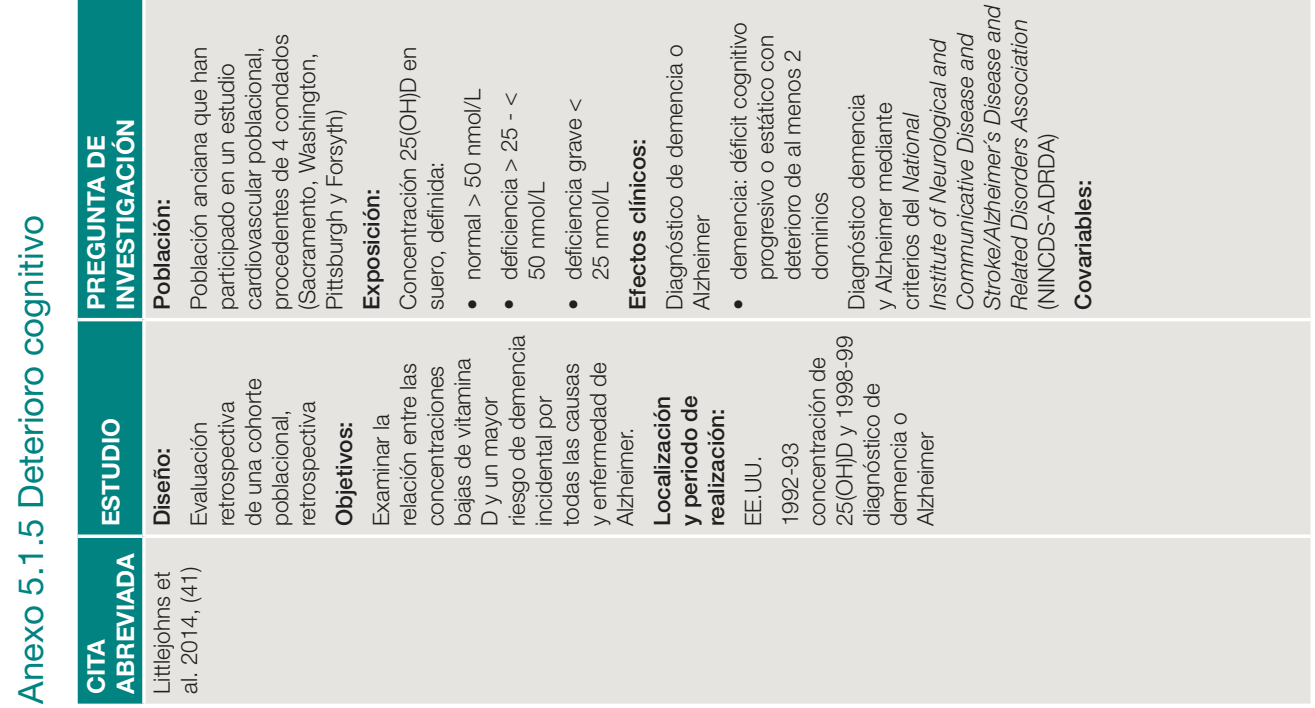


III

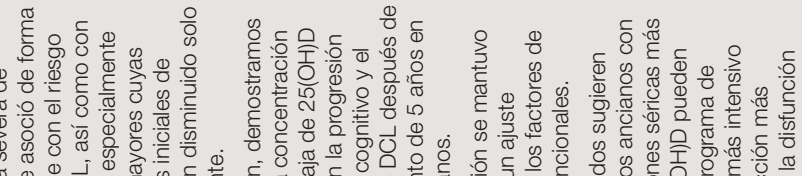

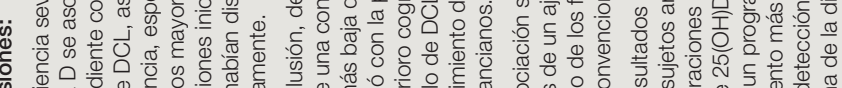

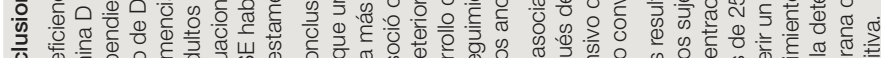

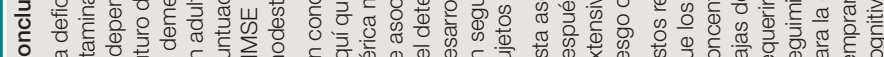

\section{ช}

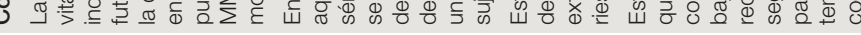
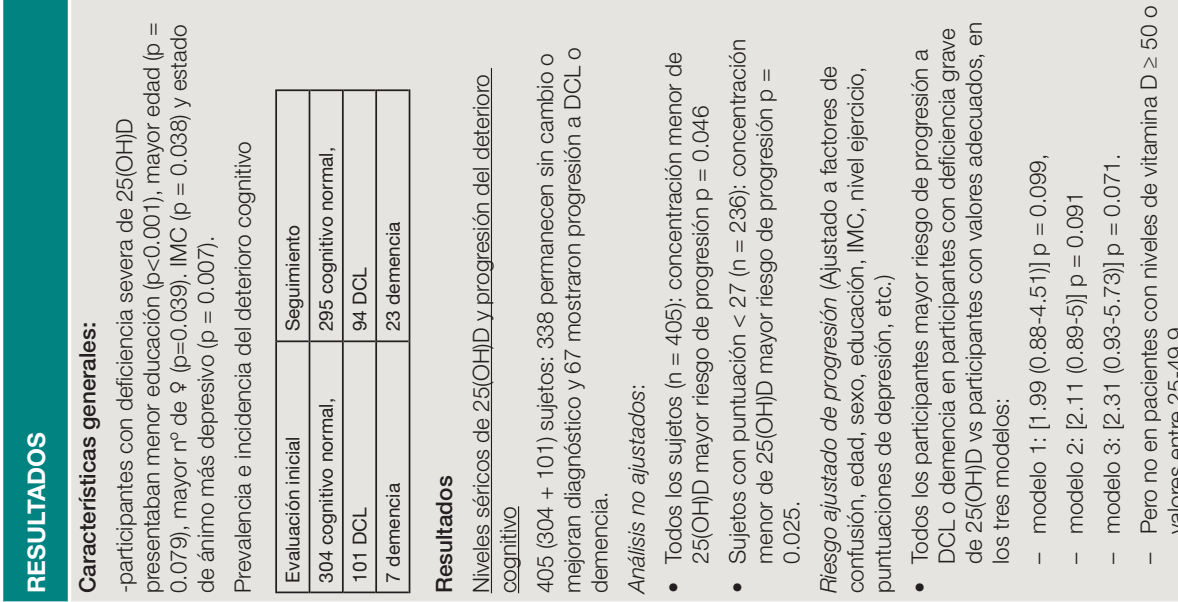

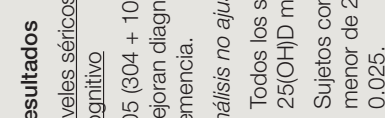

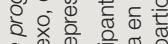

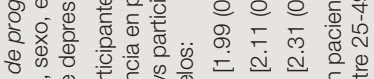

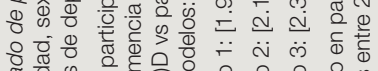

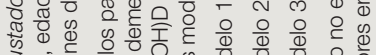

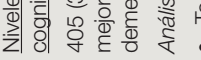

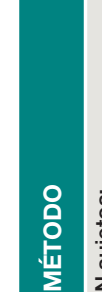

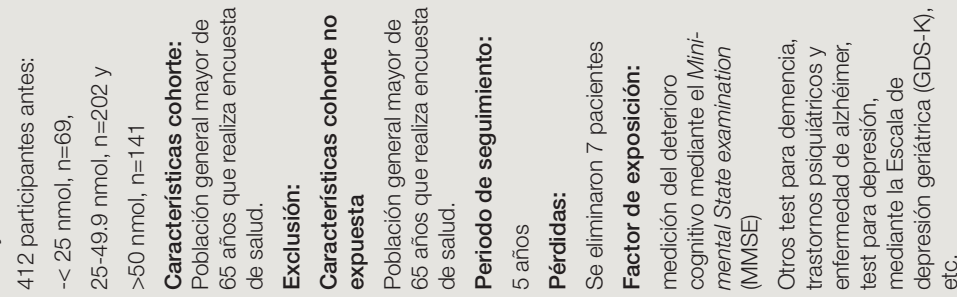

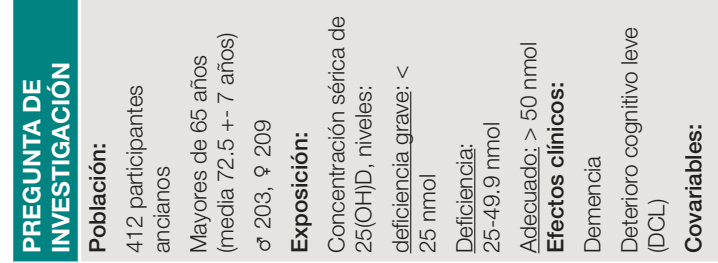

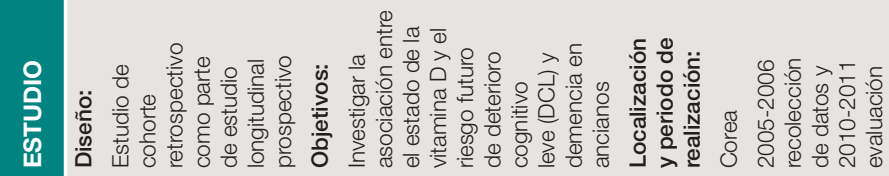

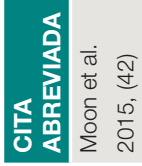



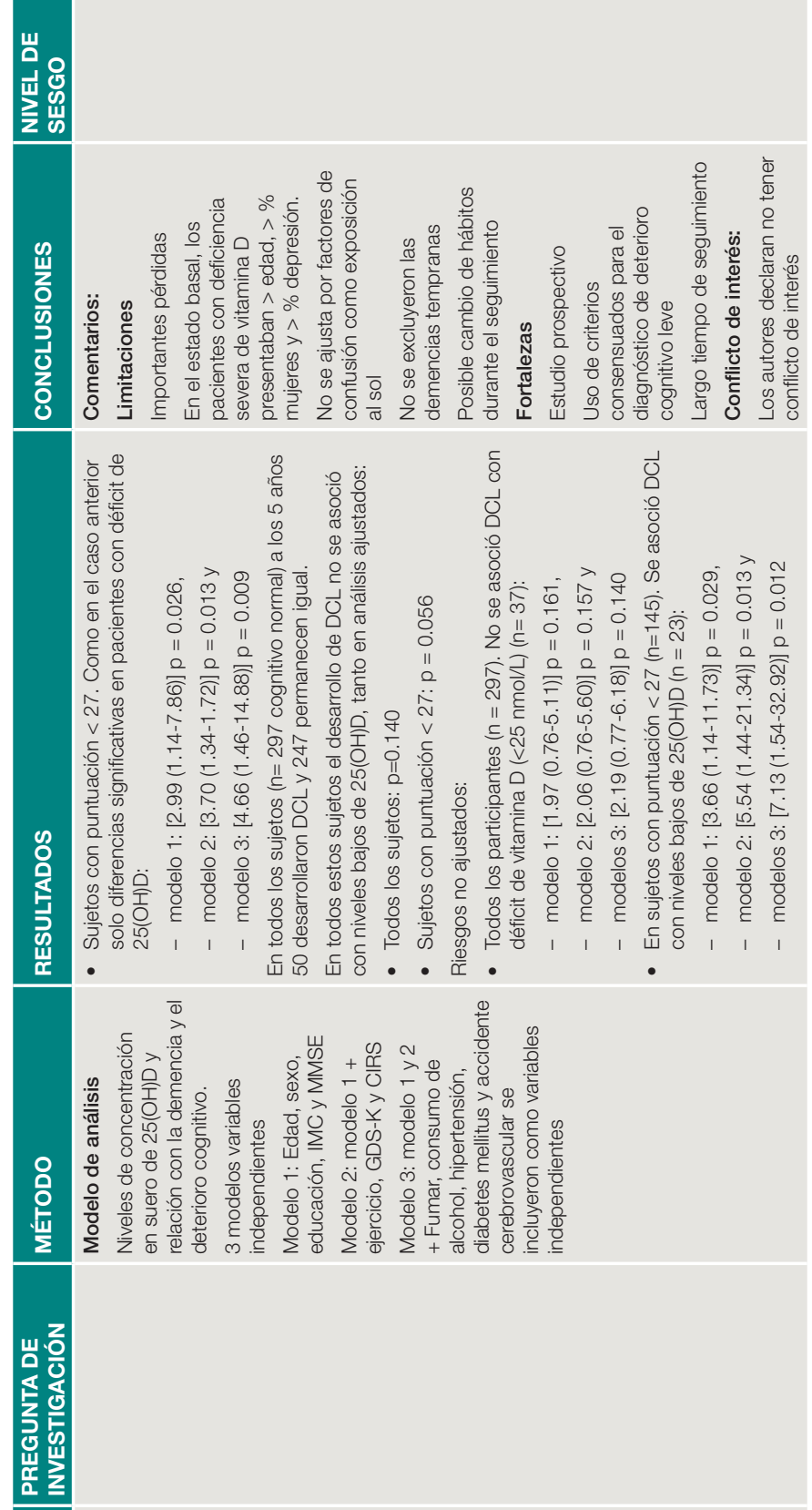


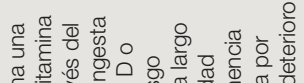

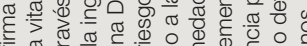

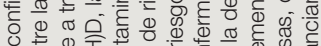

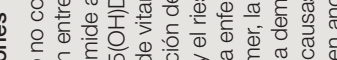

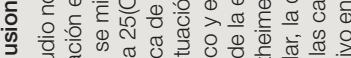

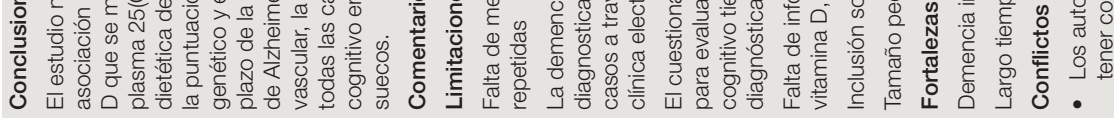

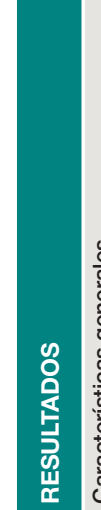

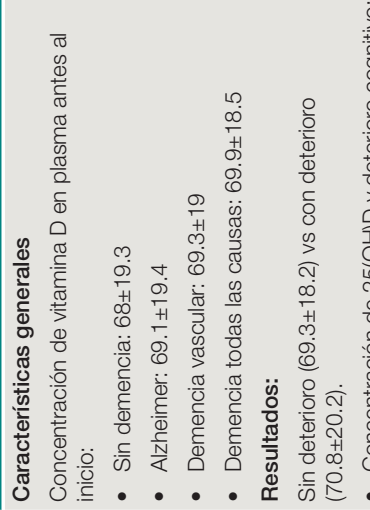

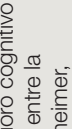

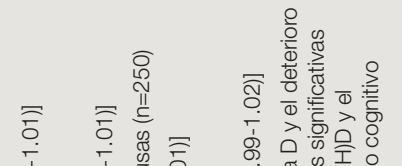

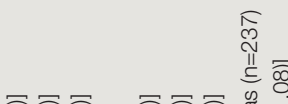

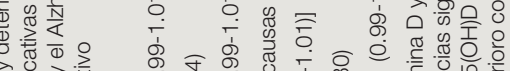

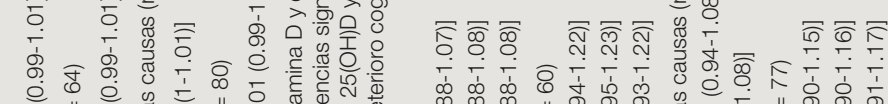

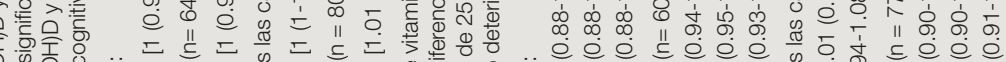

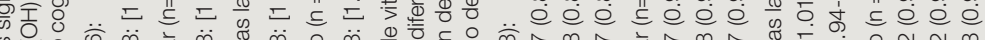

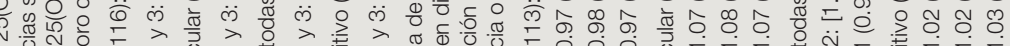
ه্

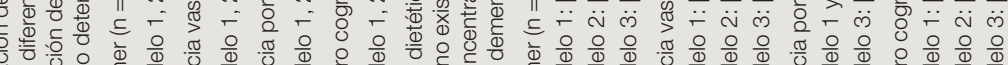

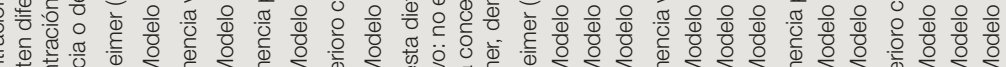

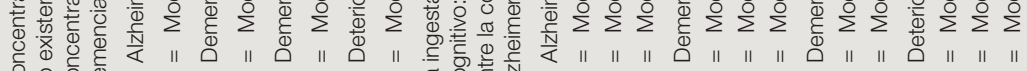
○े ๘

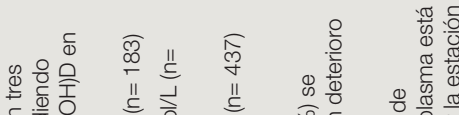

$$
\text { • }
$$

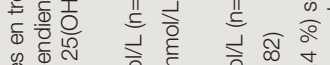

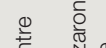

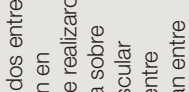

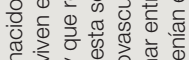

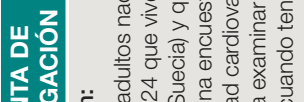

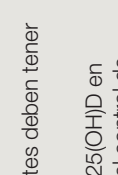

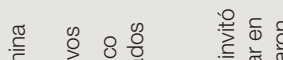

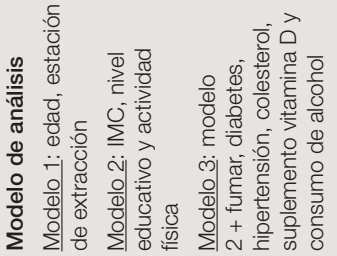

18.

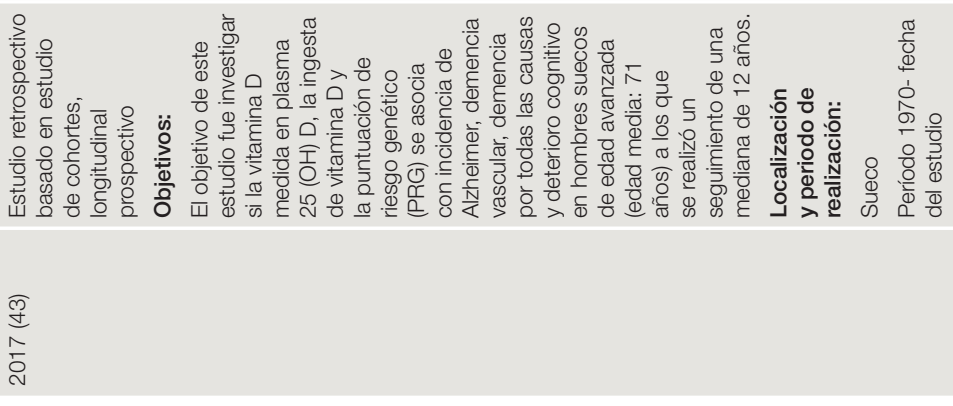



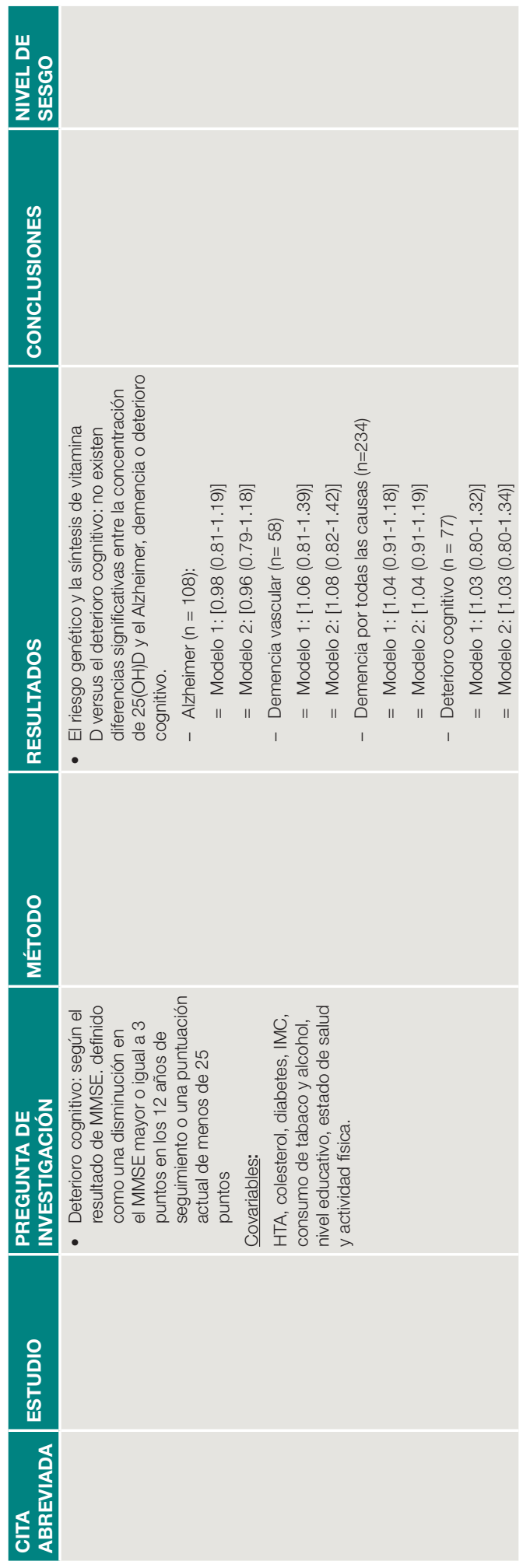


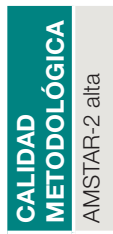

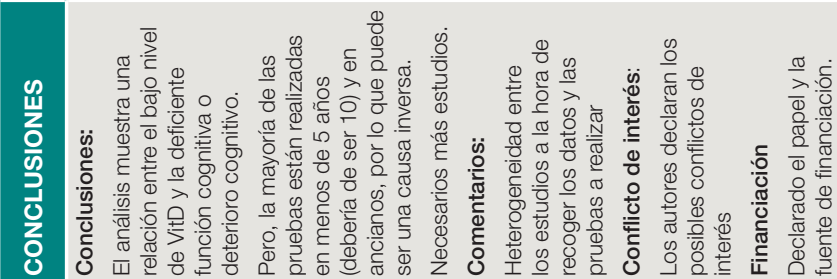

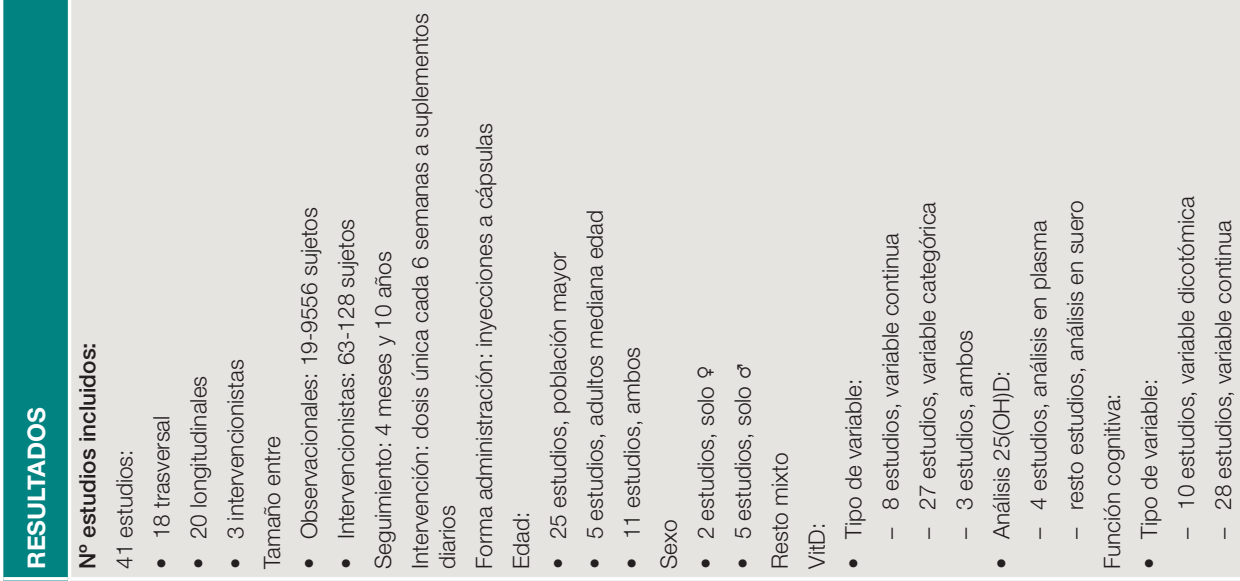

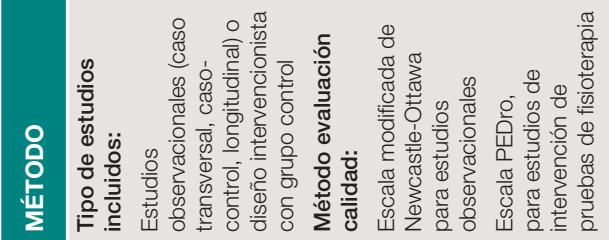

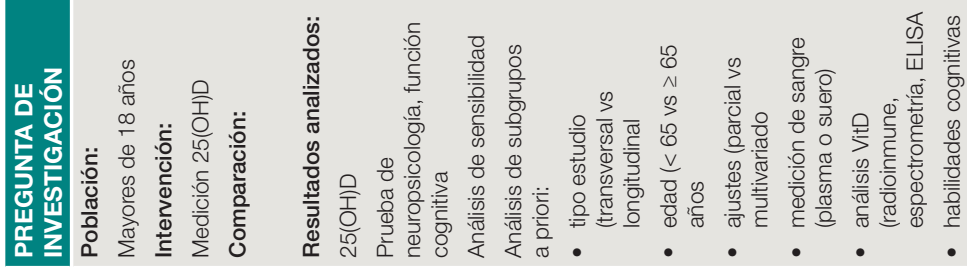

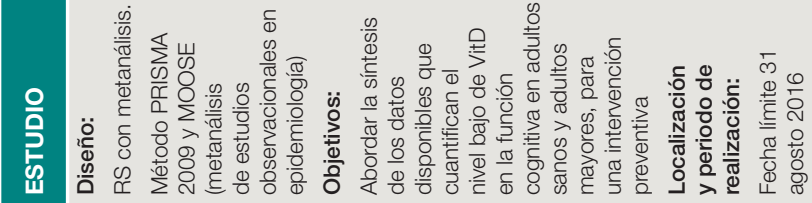

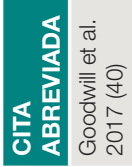



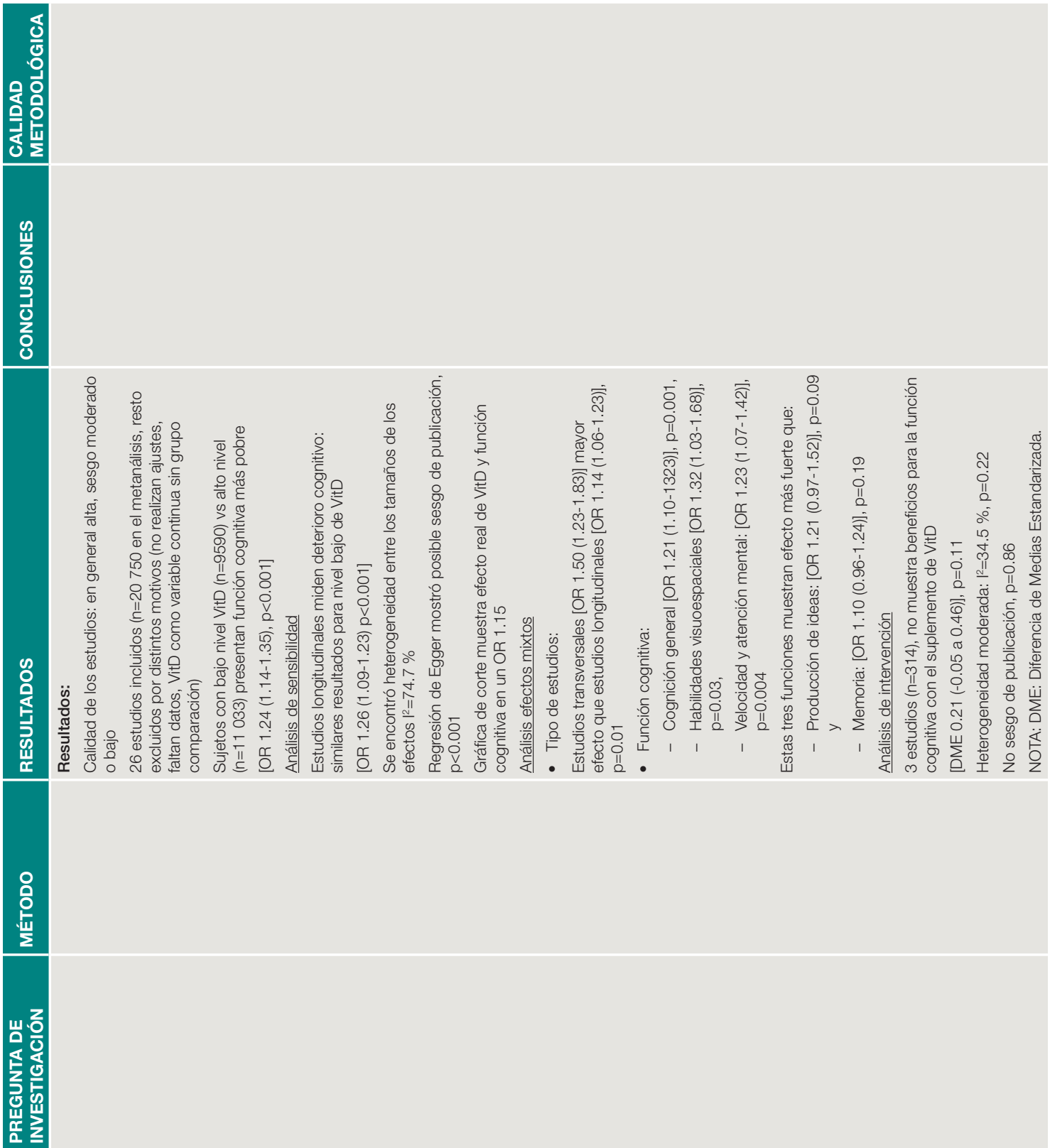

음 


\section{Wil}

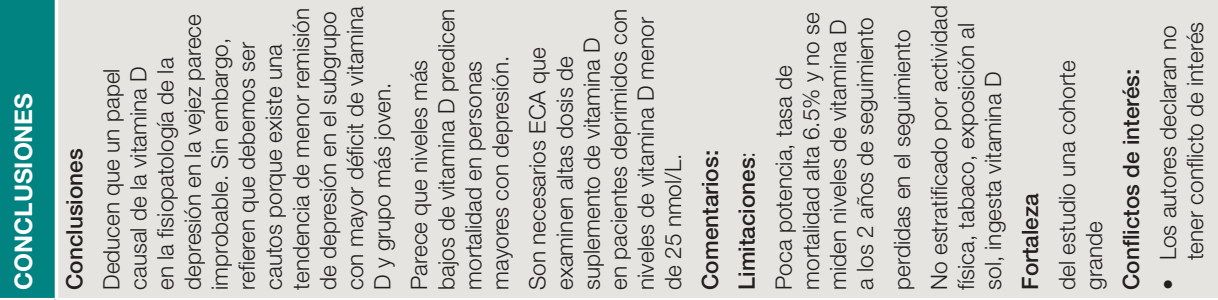

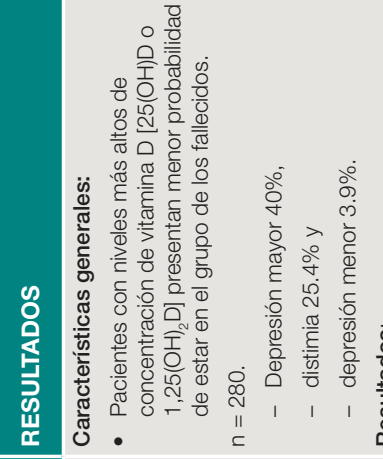

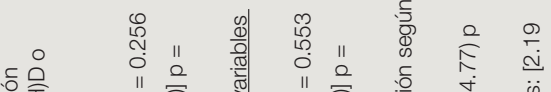

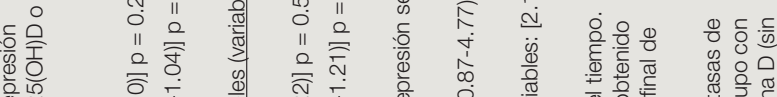

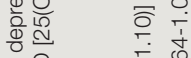

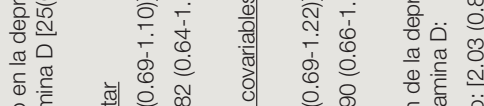

क ब

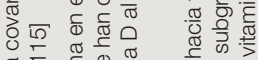

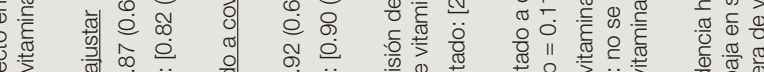

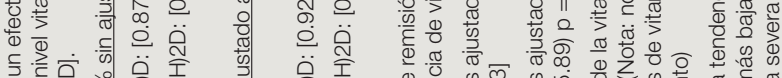

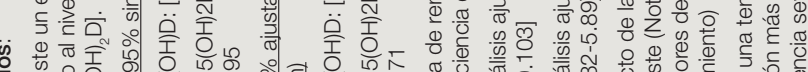

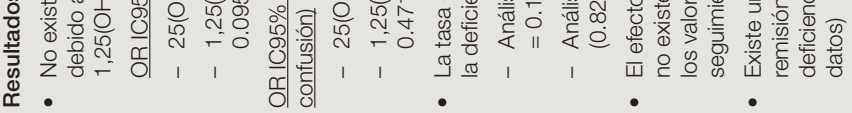

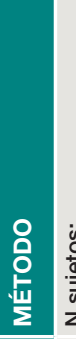

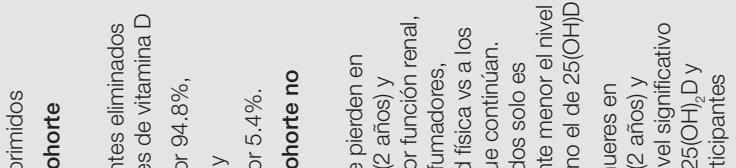

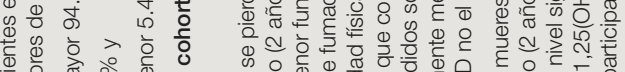

o 0 o

o

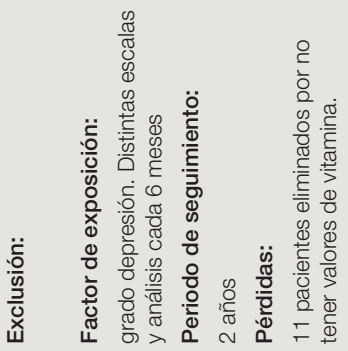

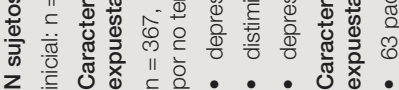

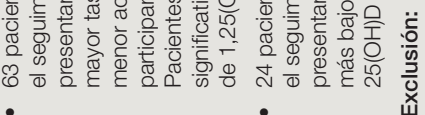

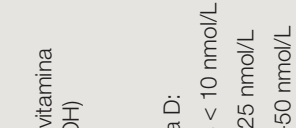

죵

드 $\bar{\Phi}$

के

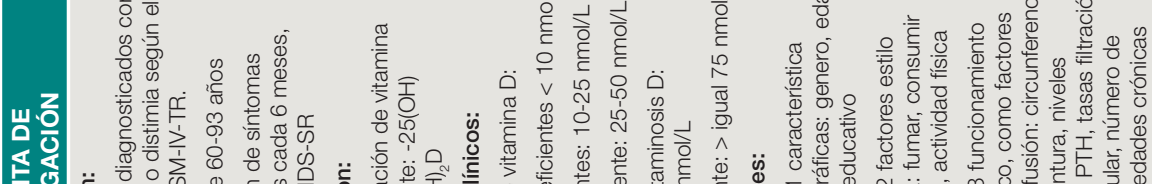

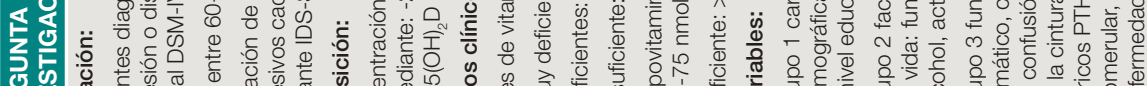

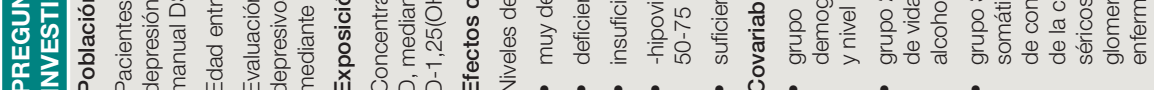

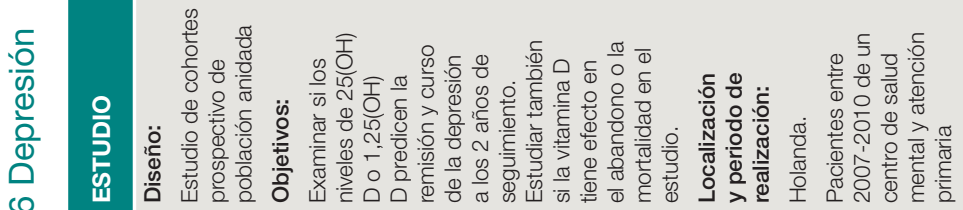

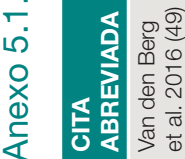




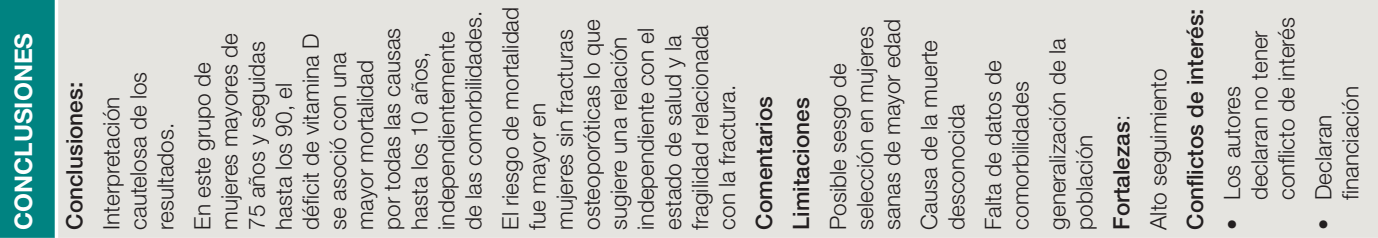

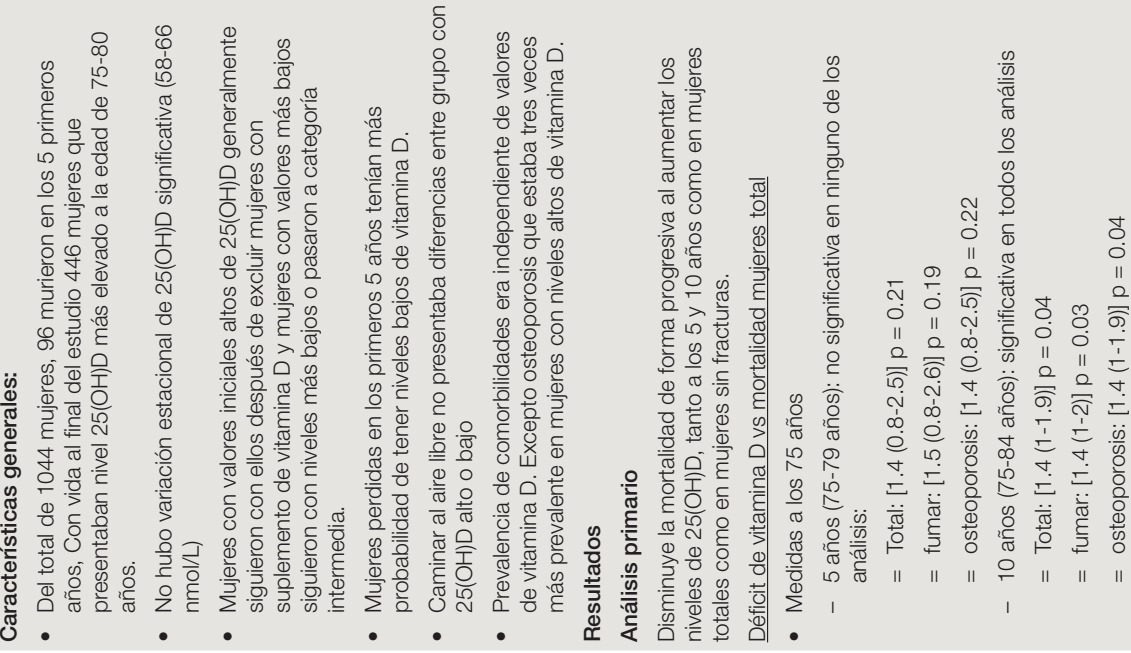

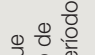<smiles>O=[SH]OC1CCCCC1=O</smiles>

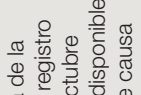

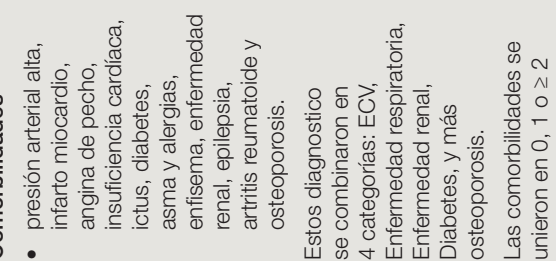

능 응

나을

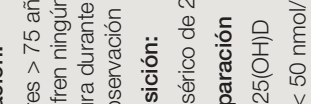

ठें है हे

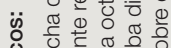

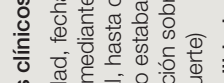




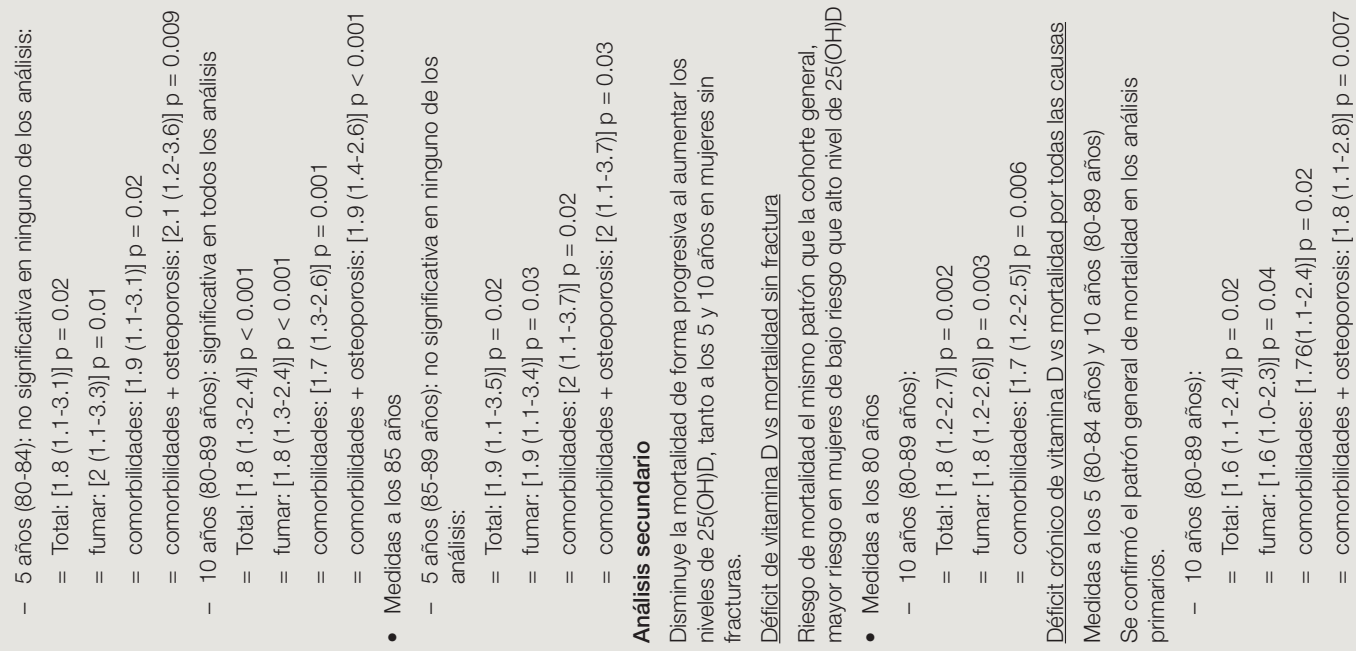

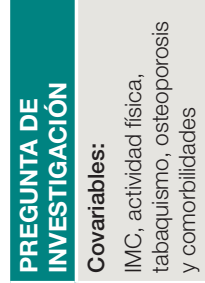




\section{II}

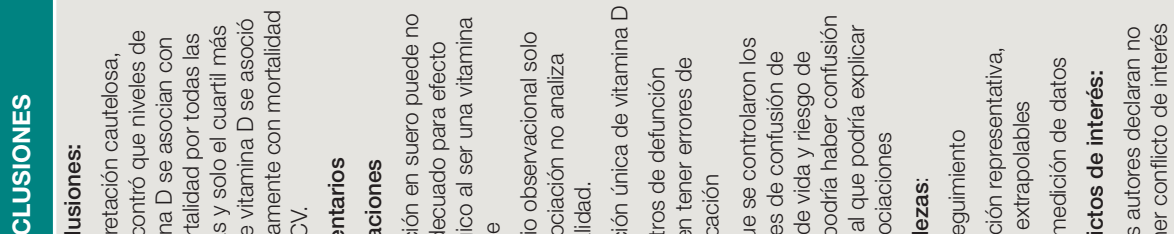

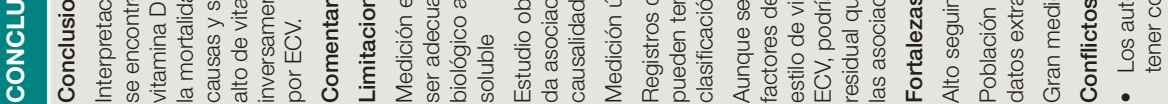

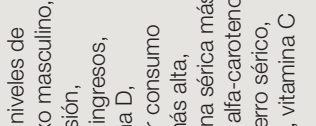

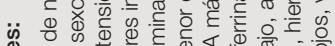

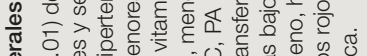

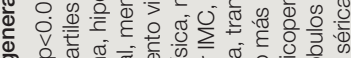

\&

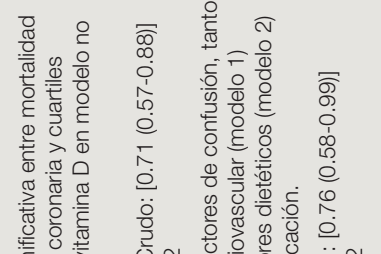

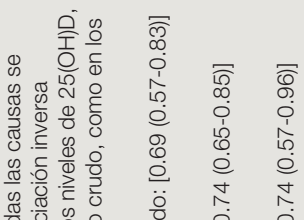

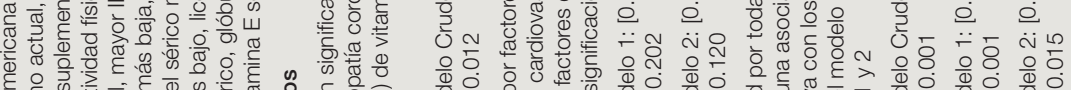

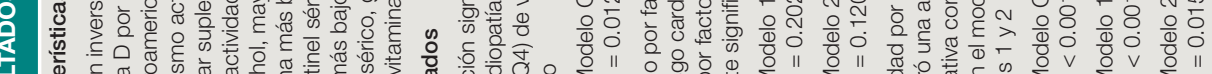

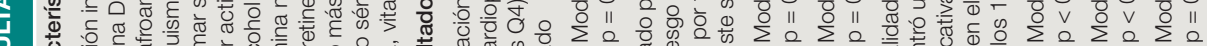
क

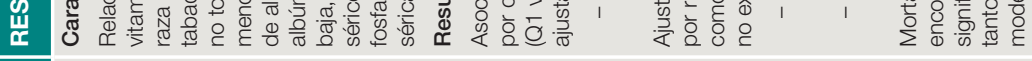

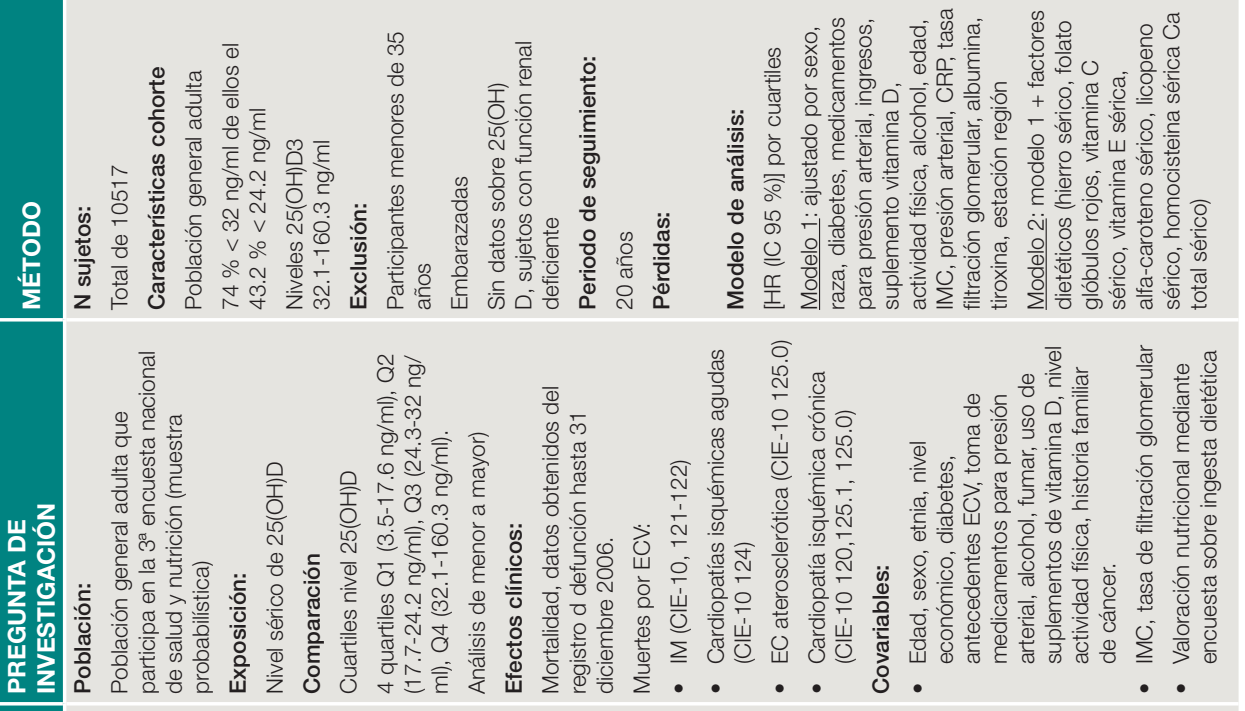

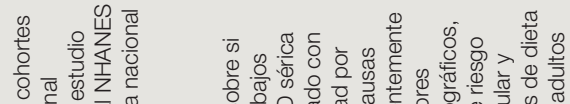

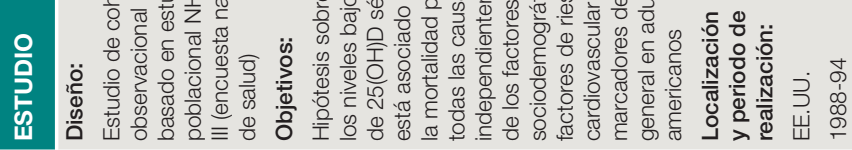

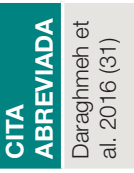




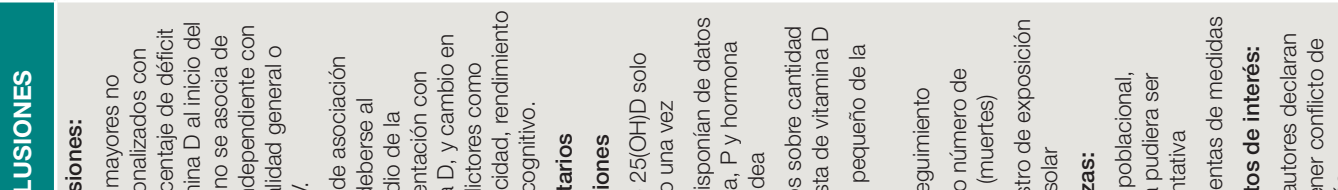

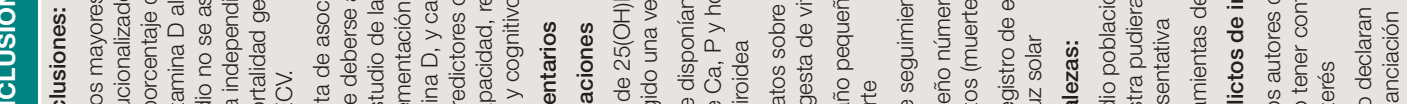

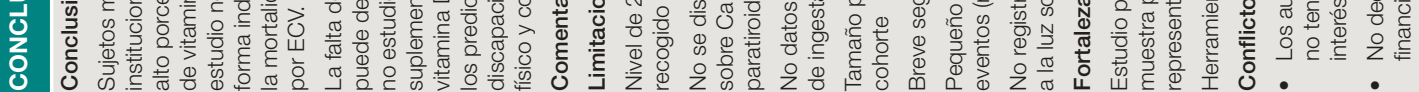

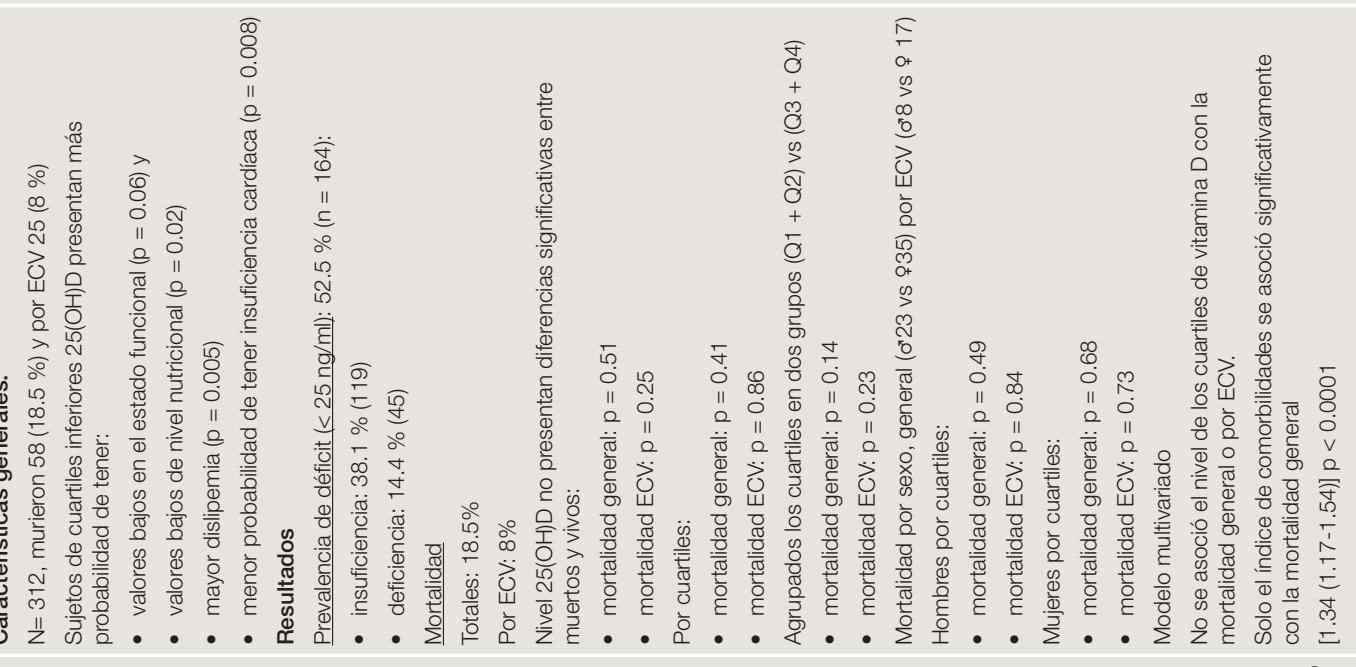

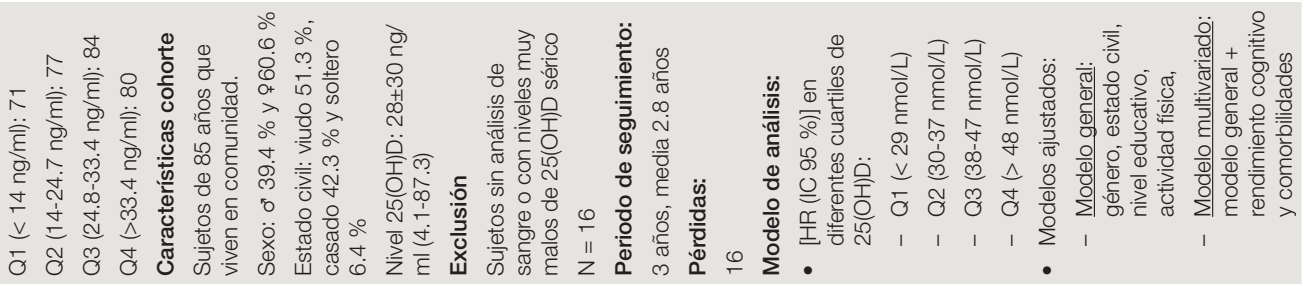

I IH $=$

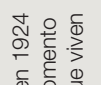

岩离

क्षे

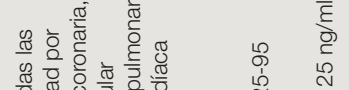

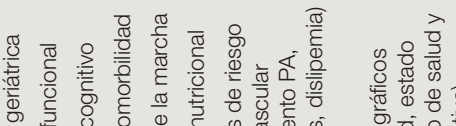

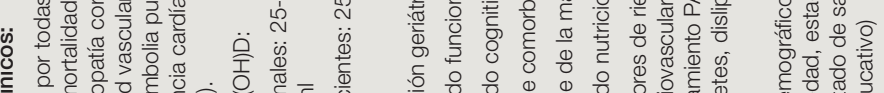

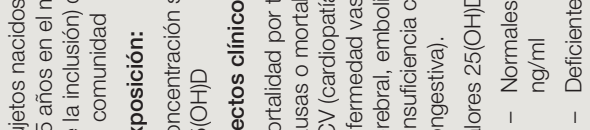

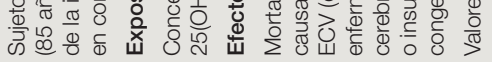

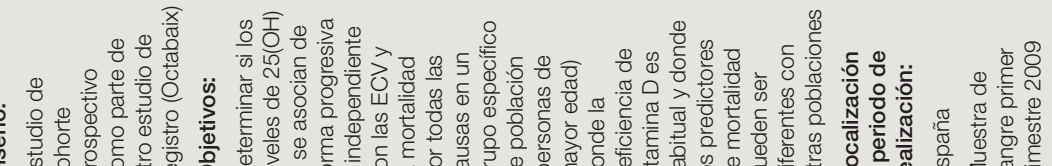

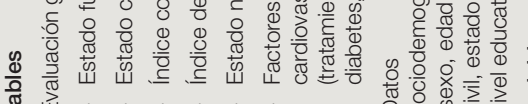

음

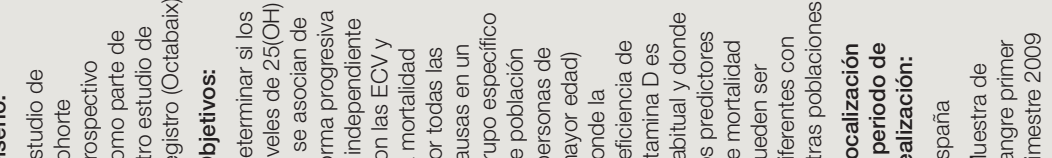

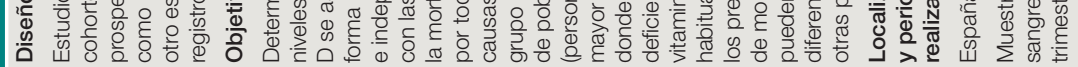

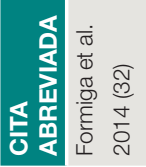




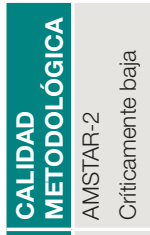

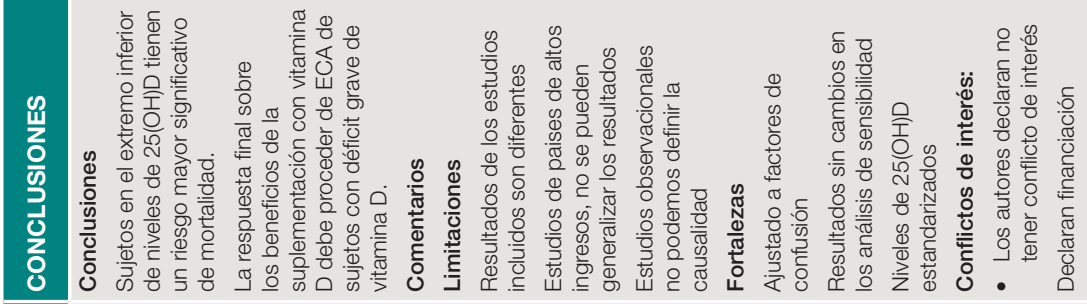
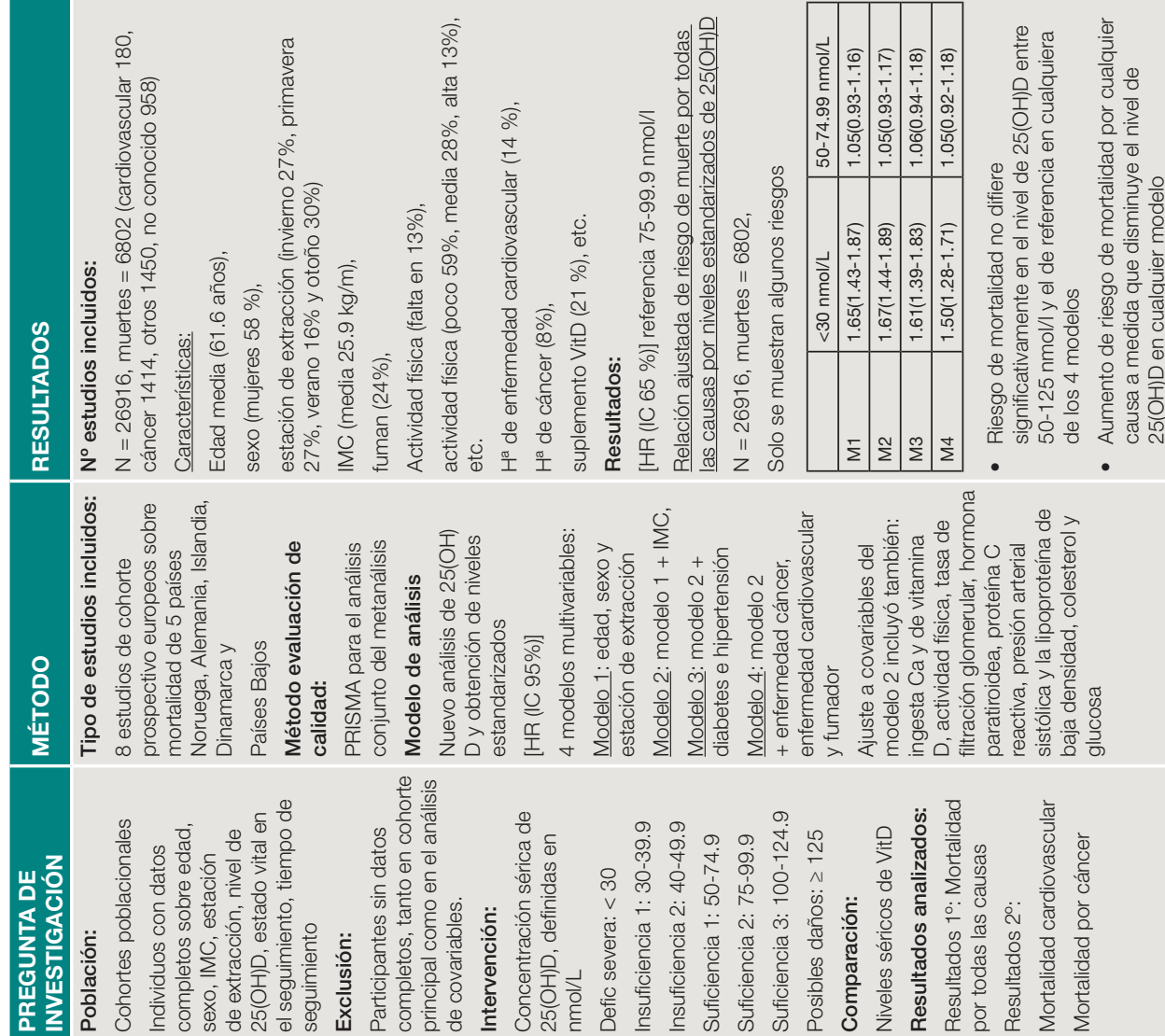

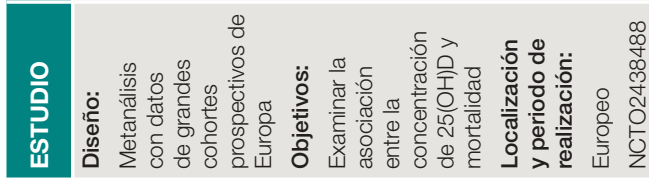

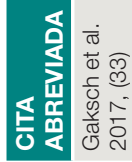




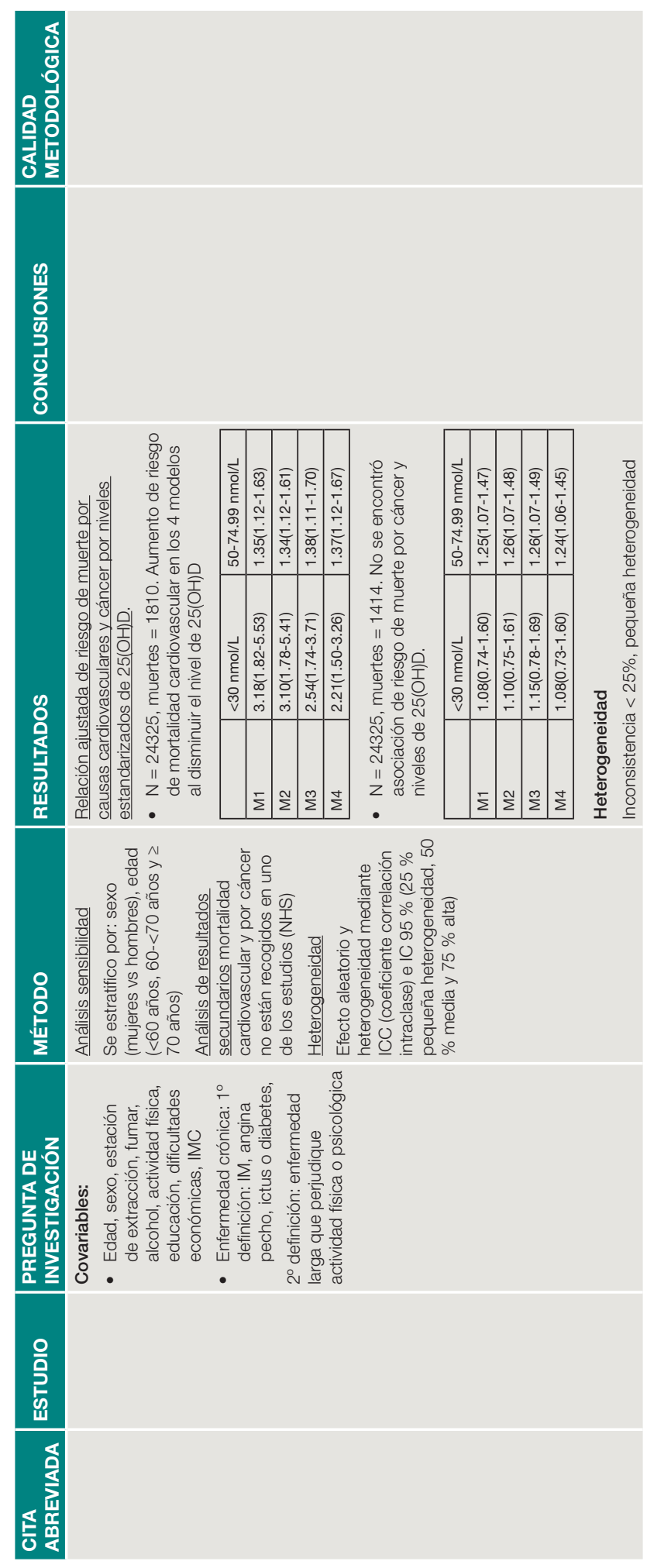




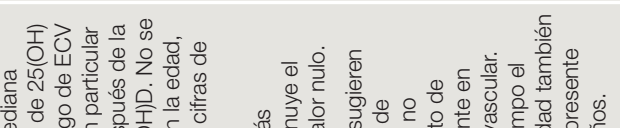

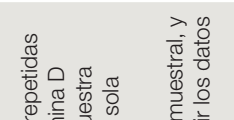

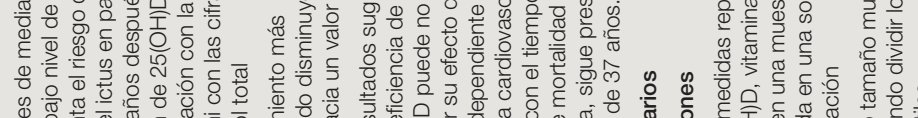

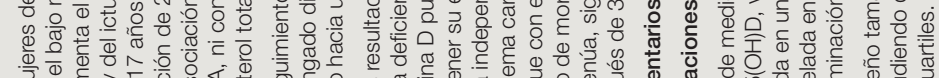

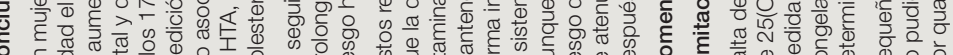
品

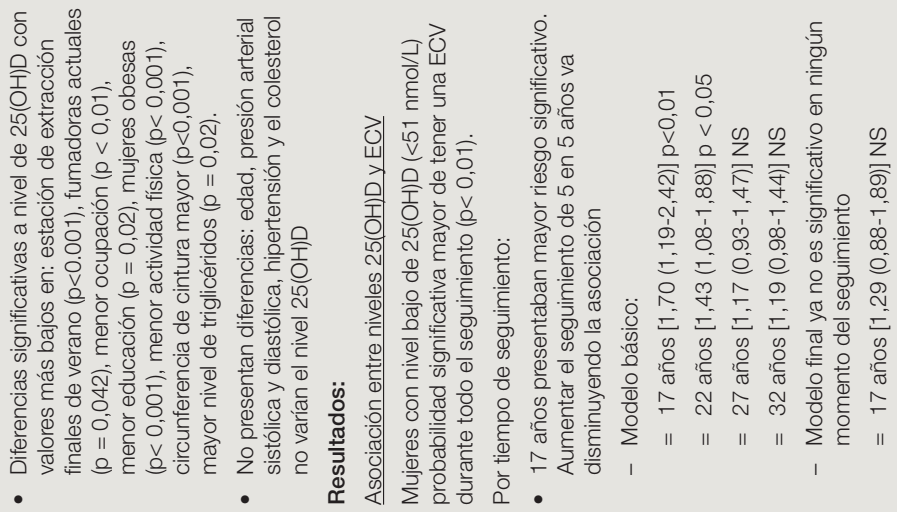

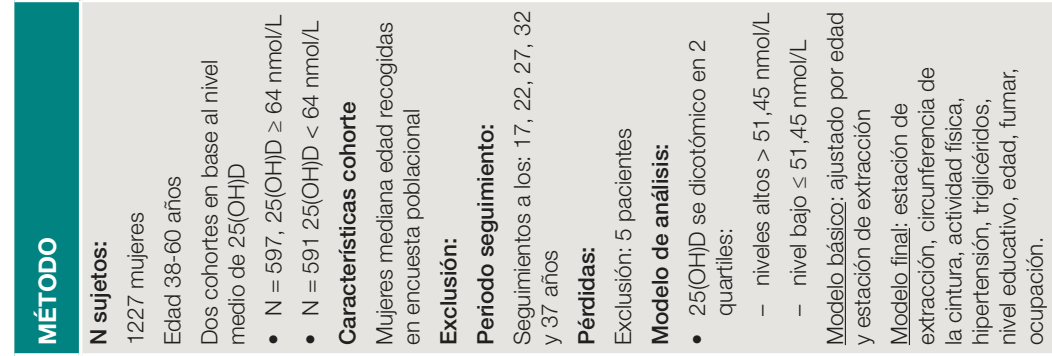

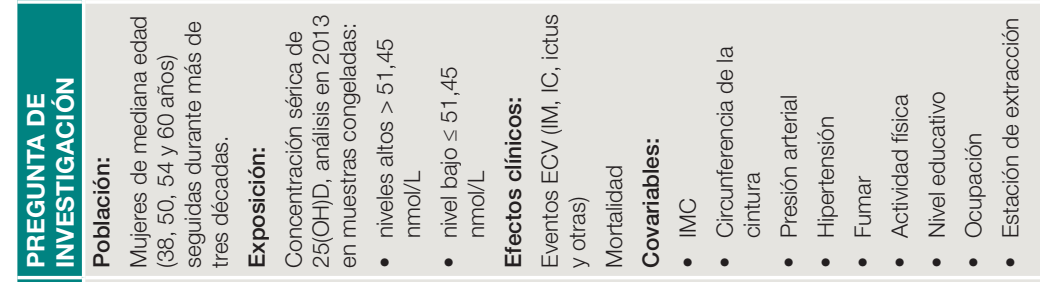

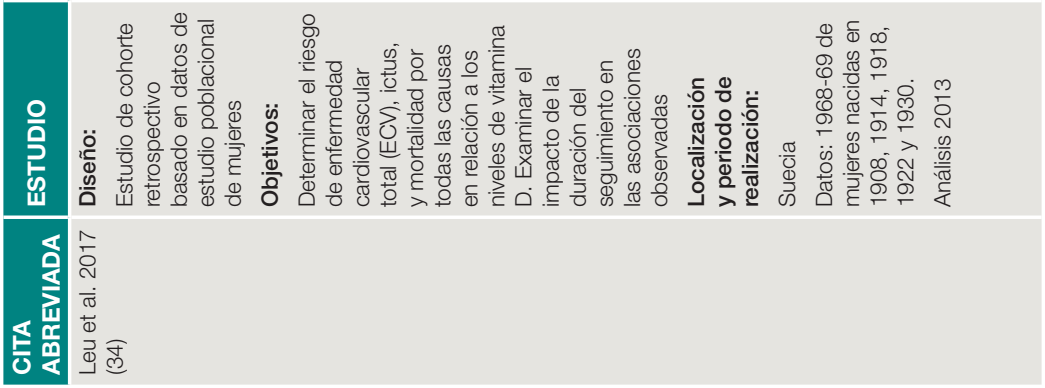




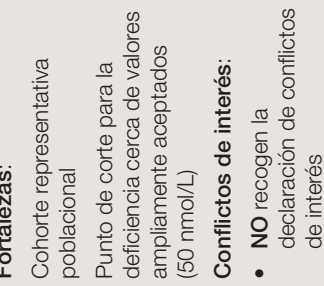
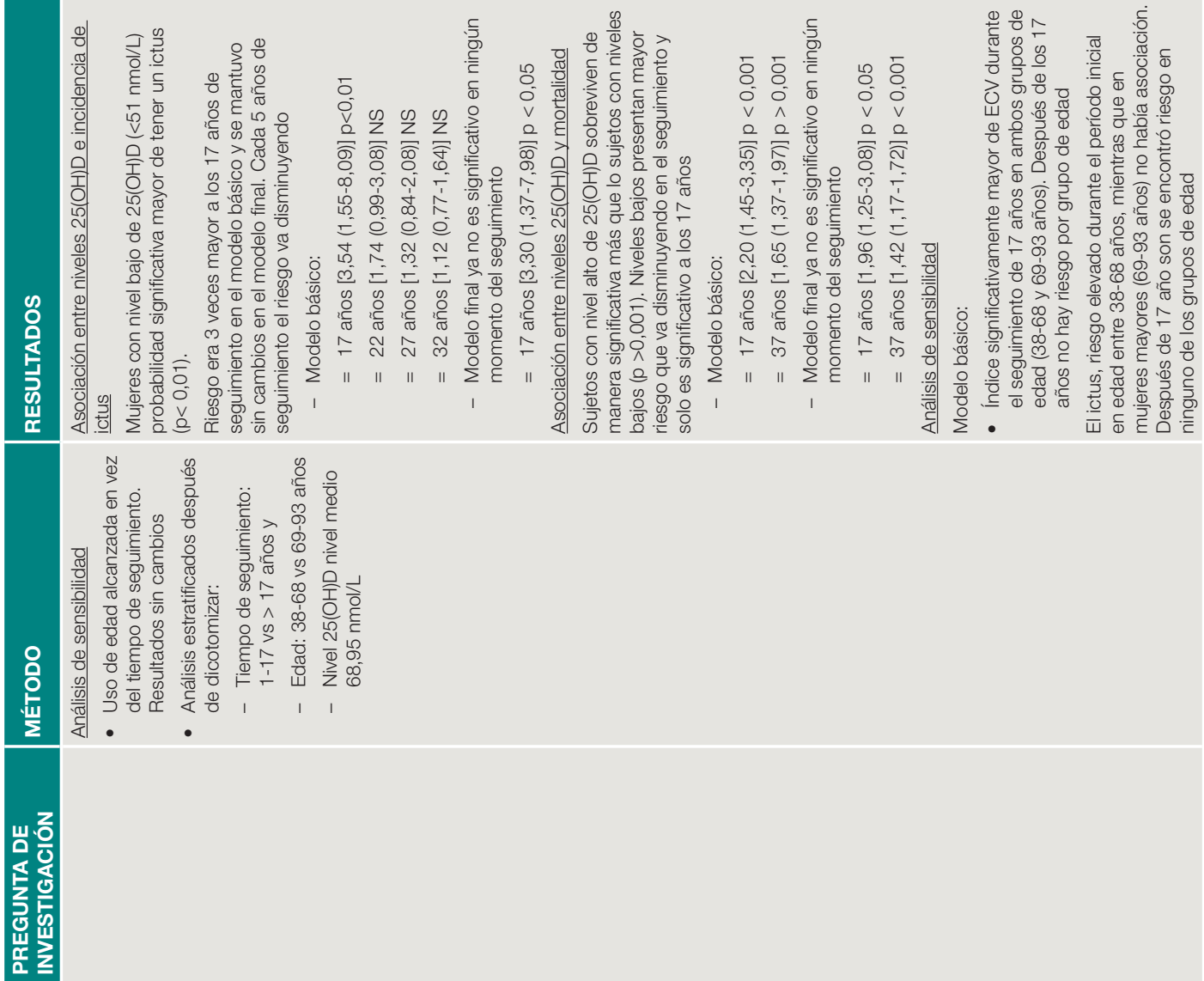

음 


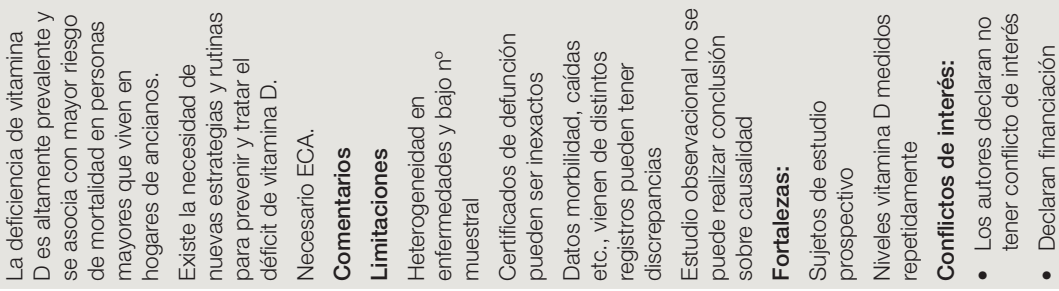

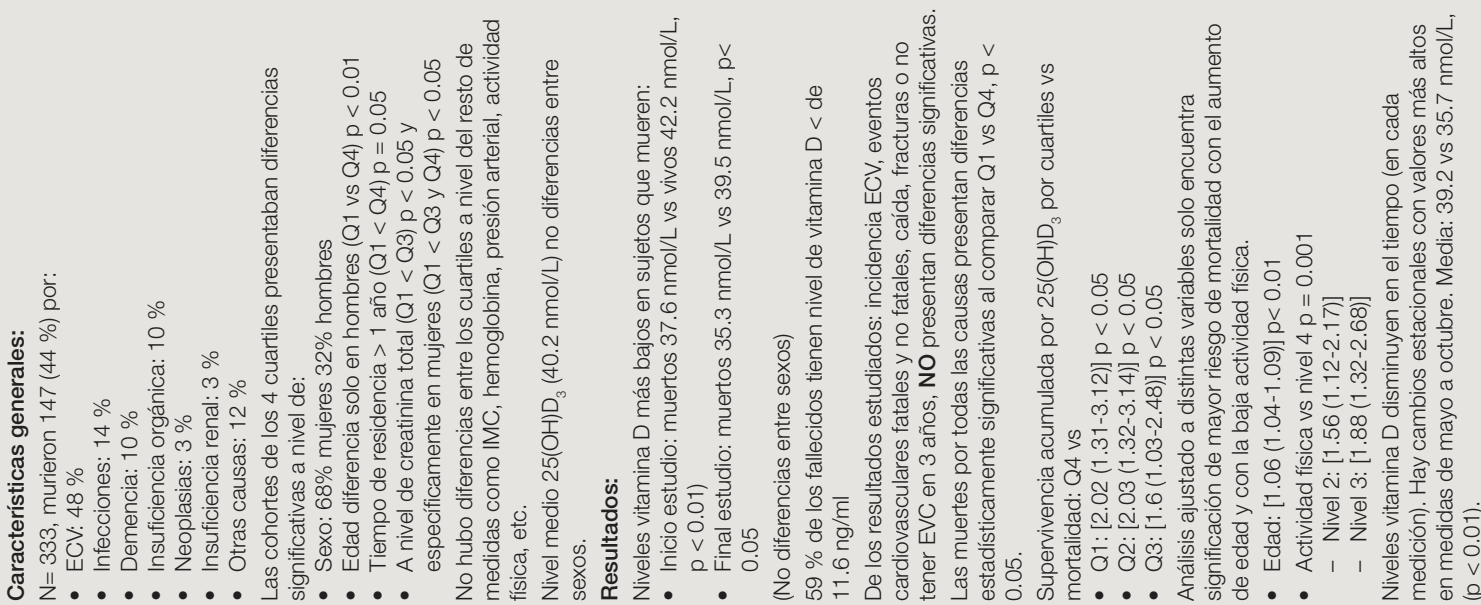
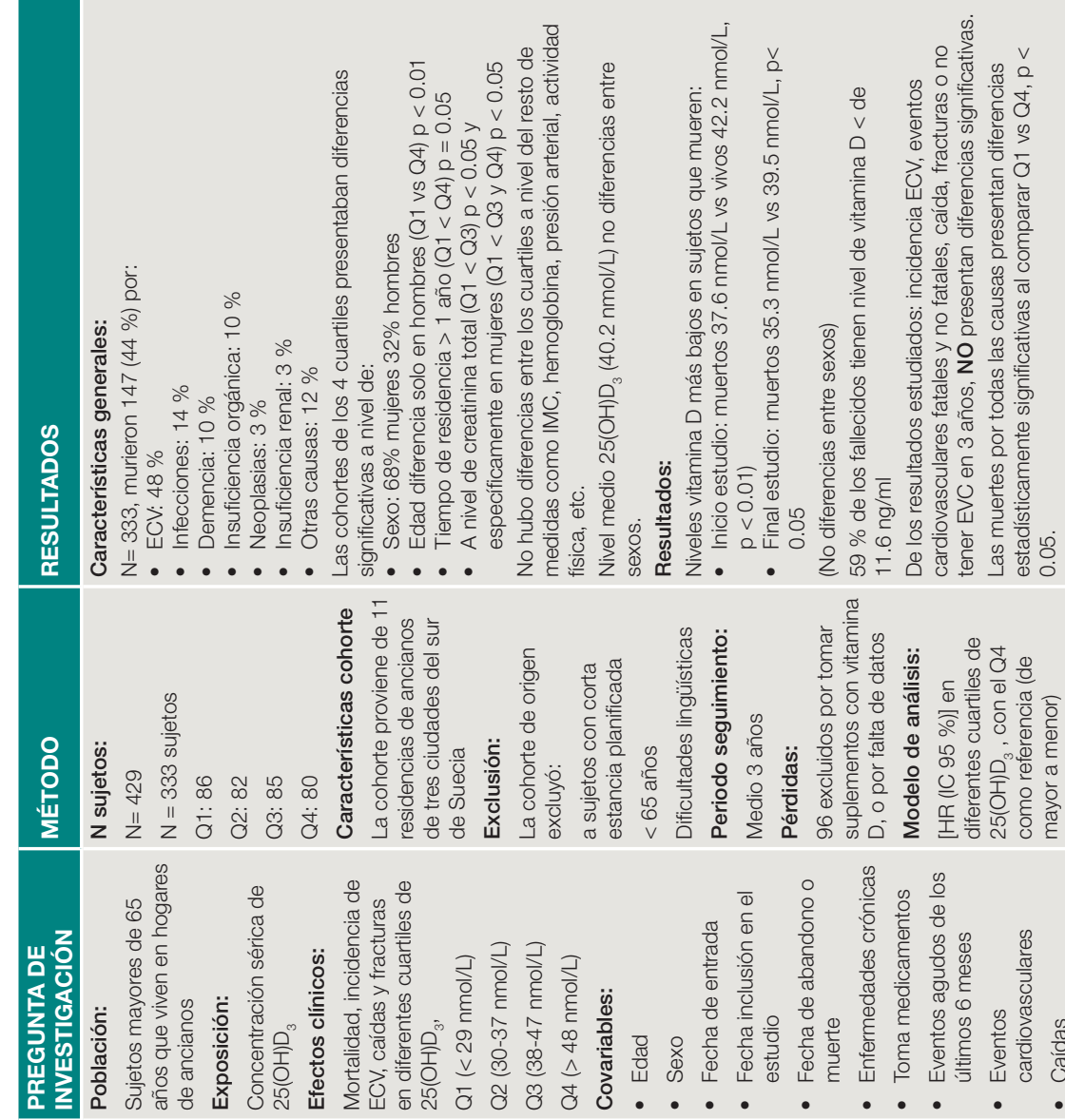

.
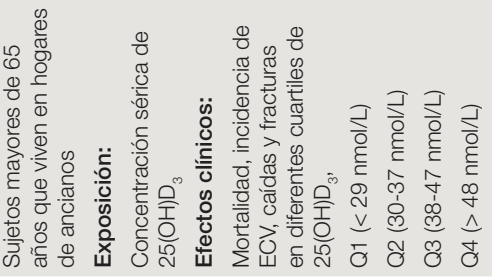

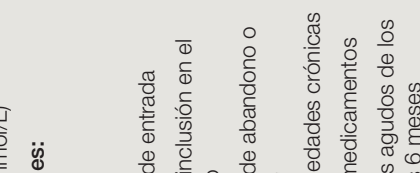

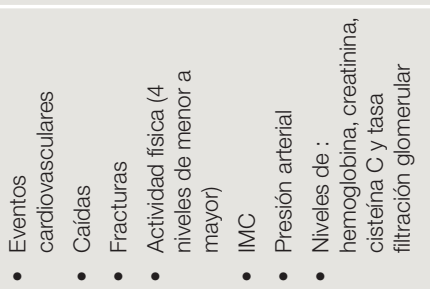

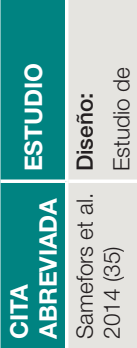




\section{III}

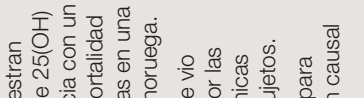

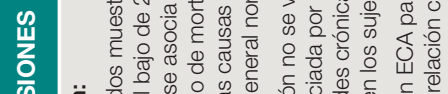

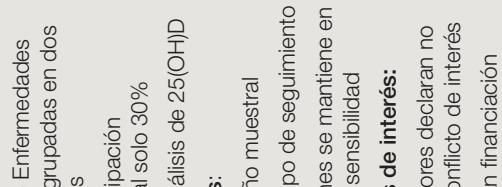

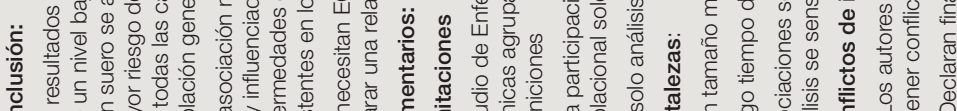

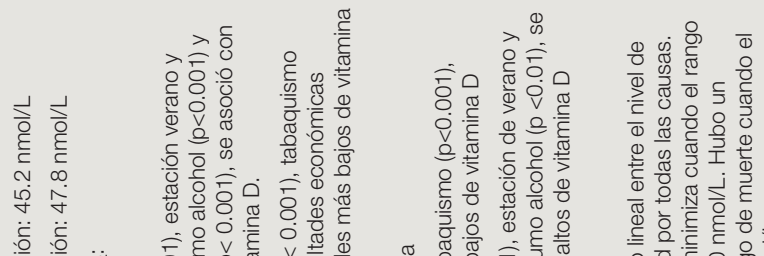

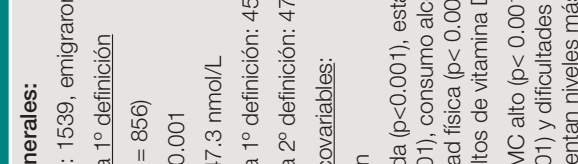

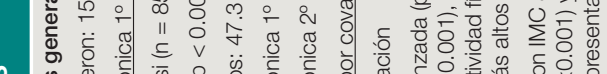

की

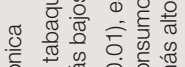

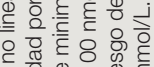

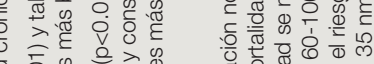

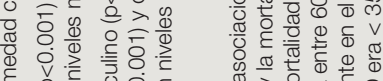

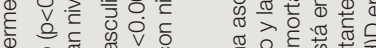

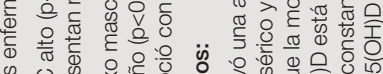

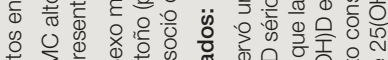

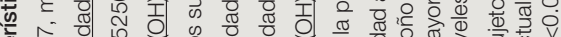

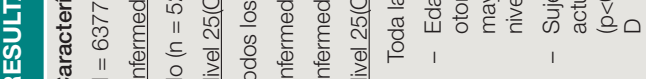

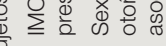

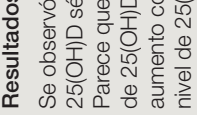

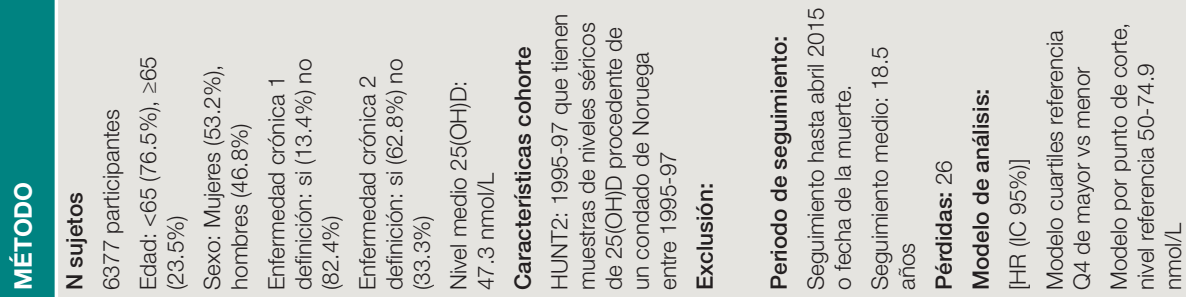

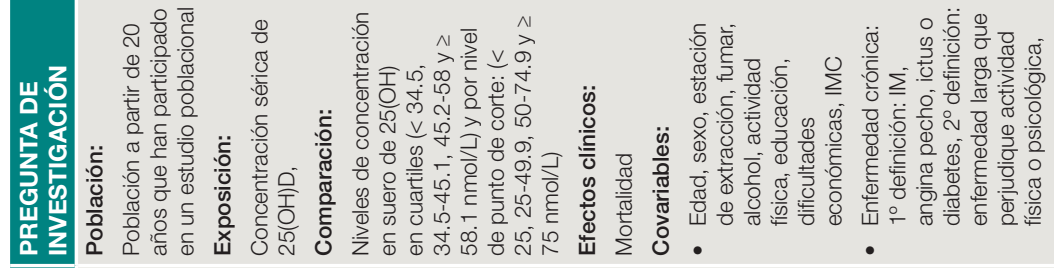

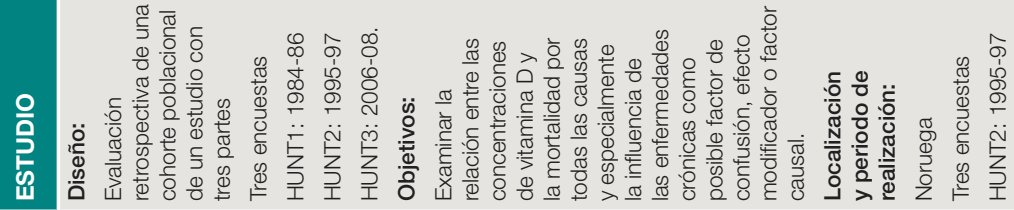

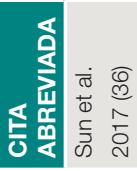




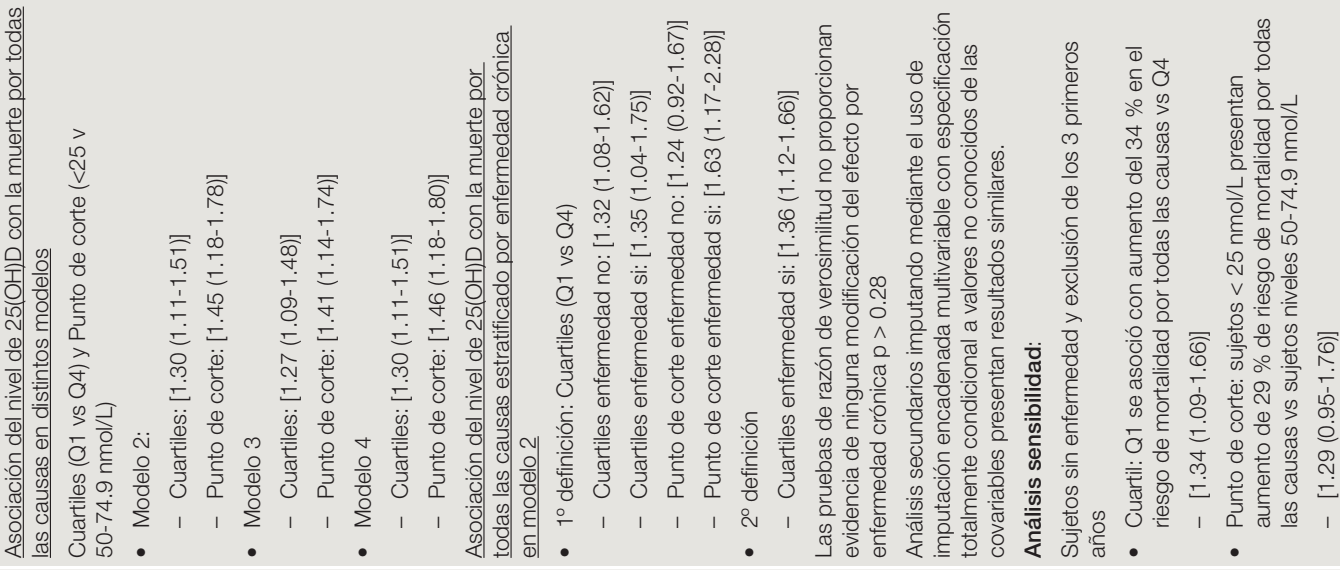

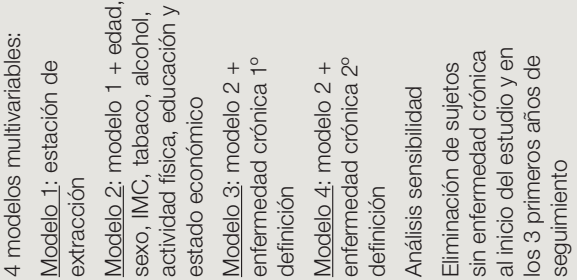




\section{III}

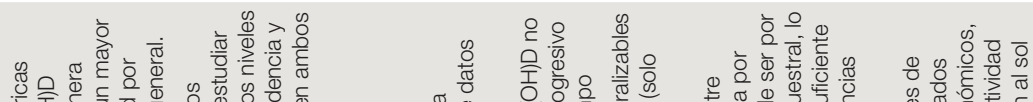

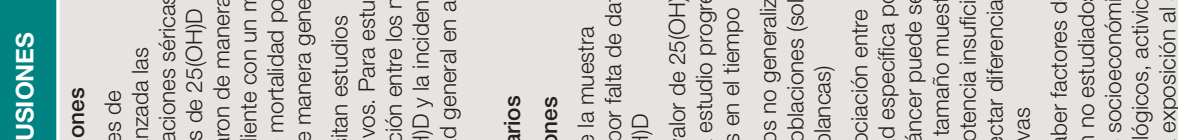

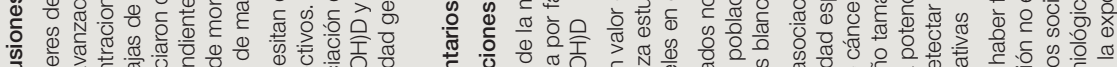

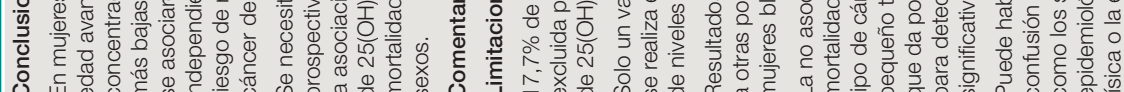

응

ㄱำ $\frac{1}{2}$.

응 응

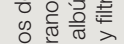

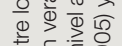

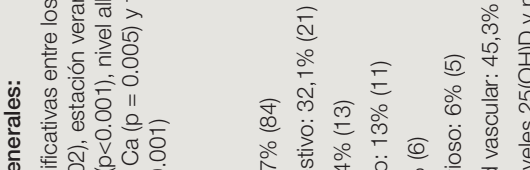

$\stackrel{\ddot{N}}{\stackrel{0}{\oplus}}$

\section{을}

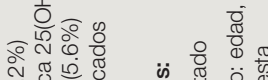

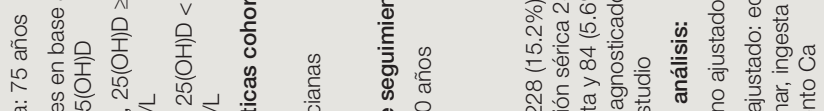

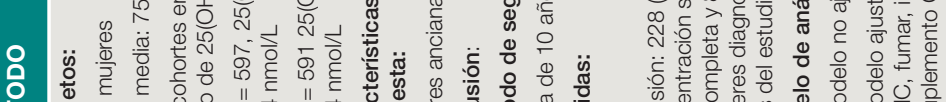

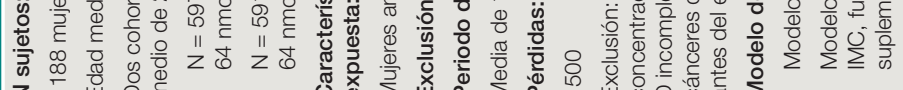

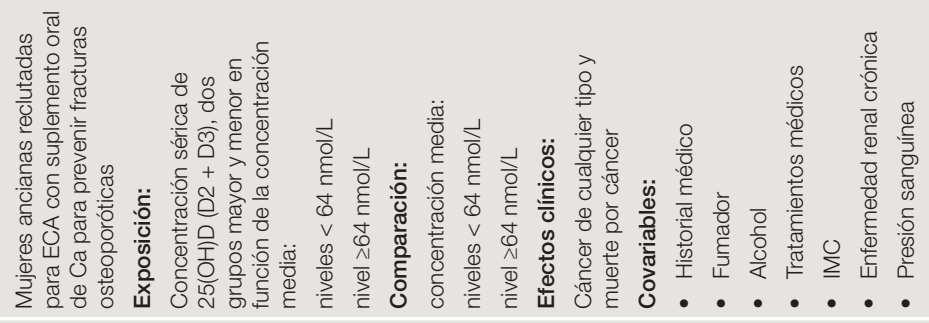

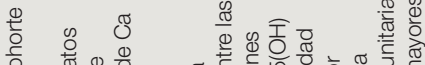

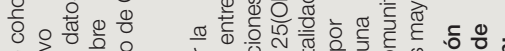

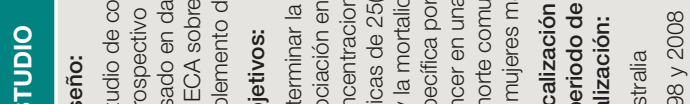

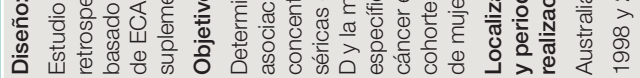

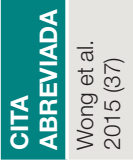




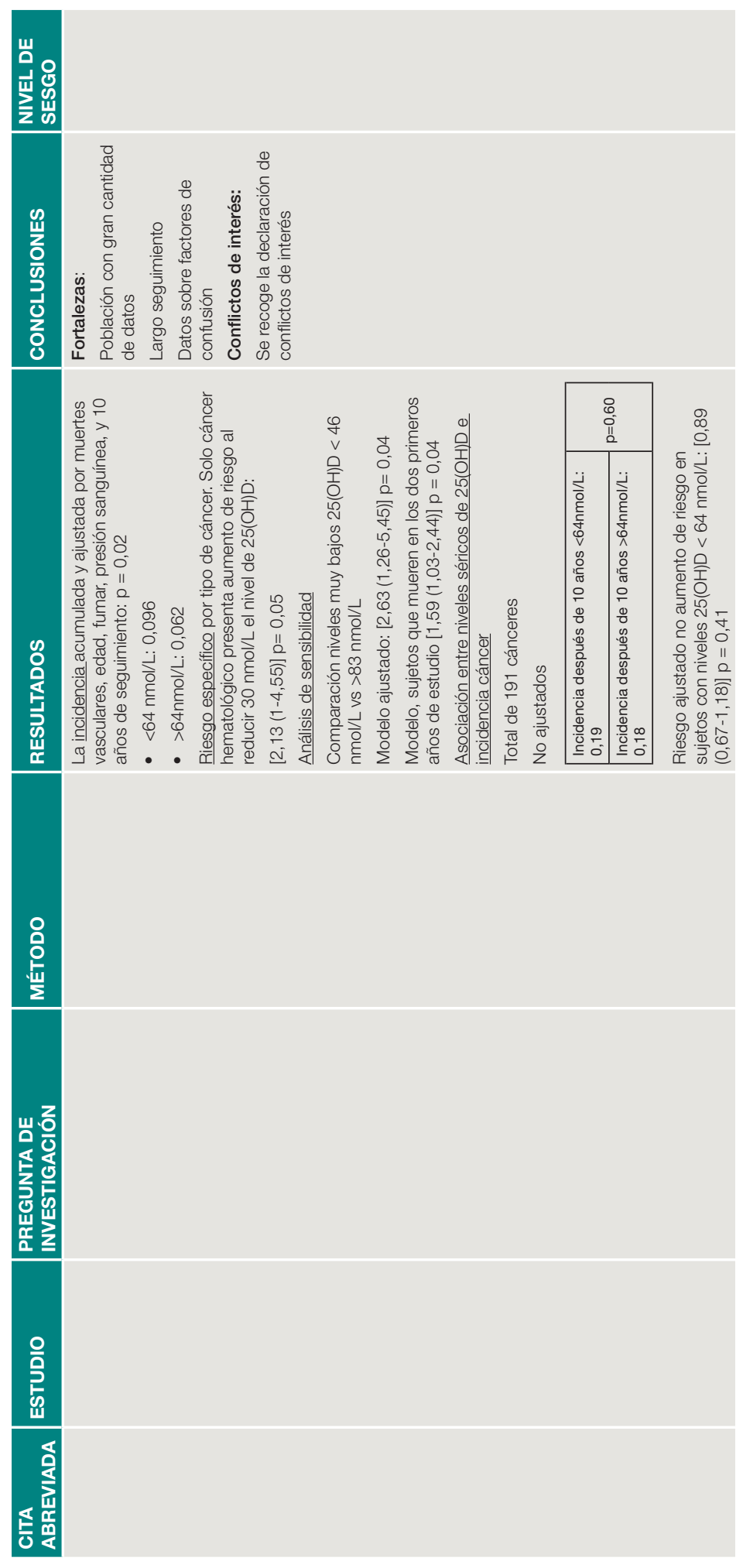




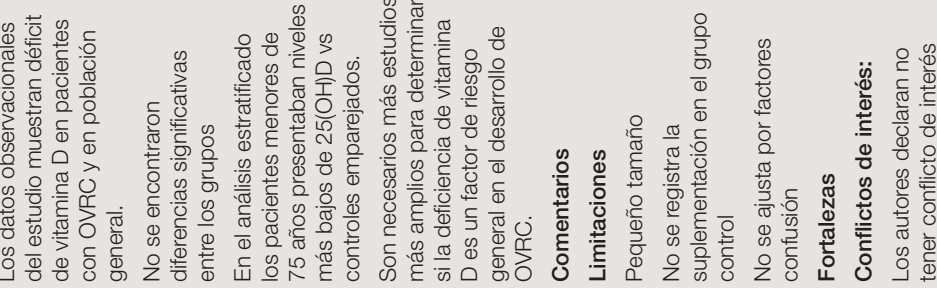

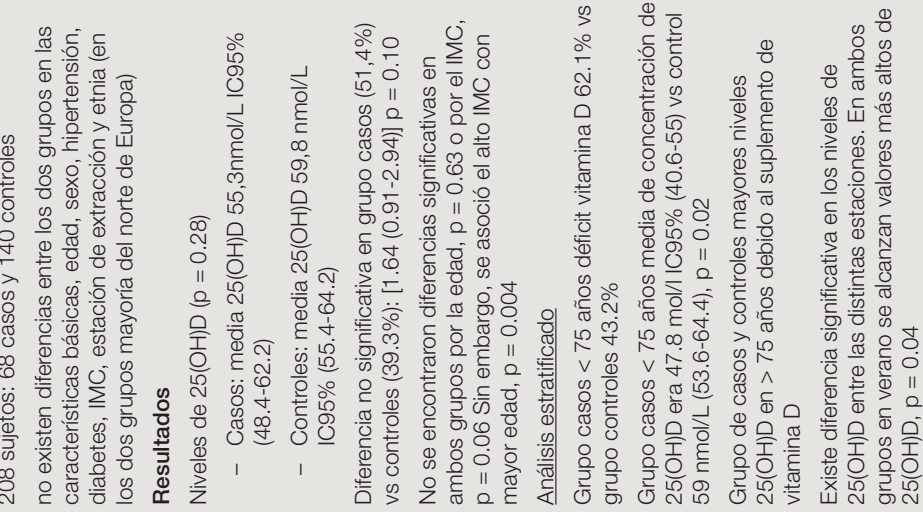

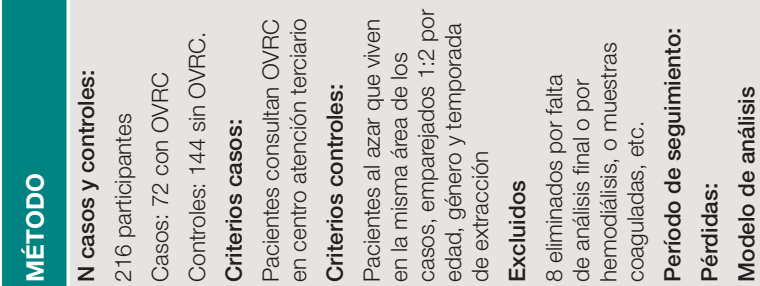

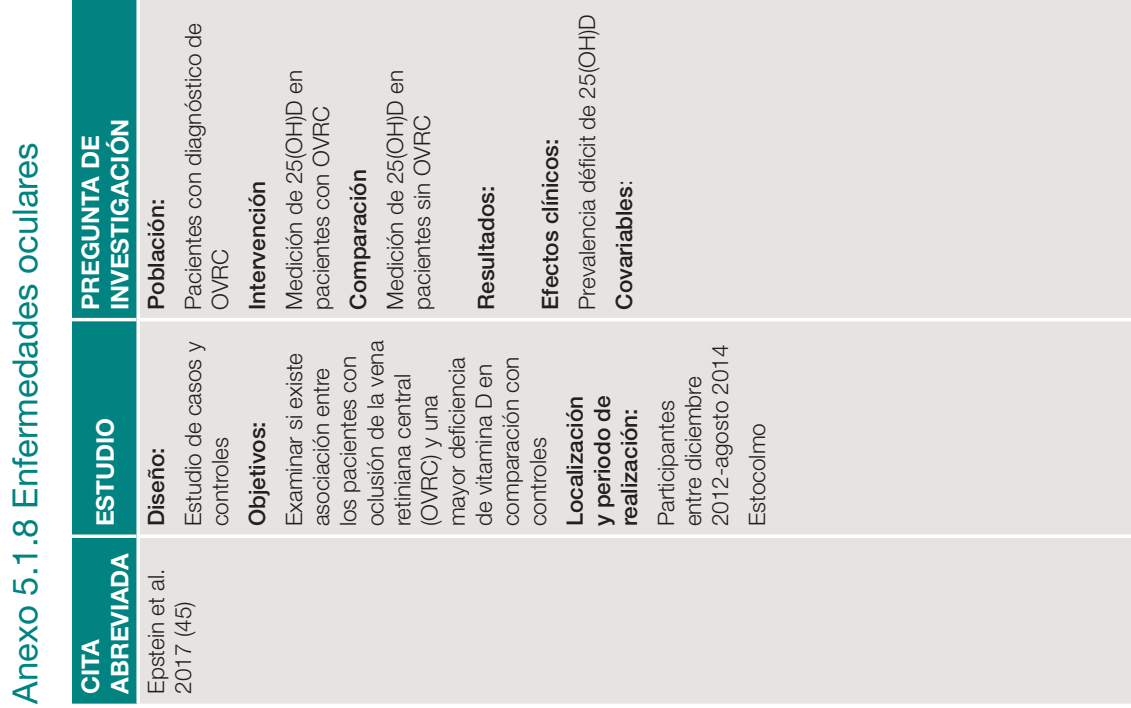




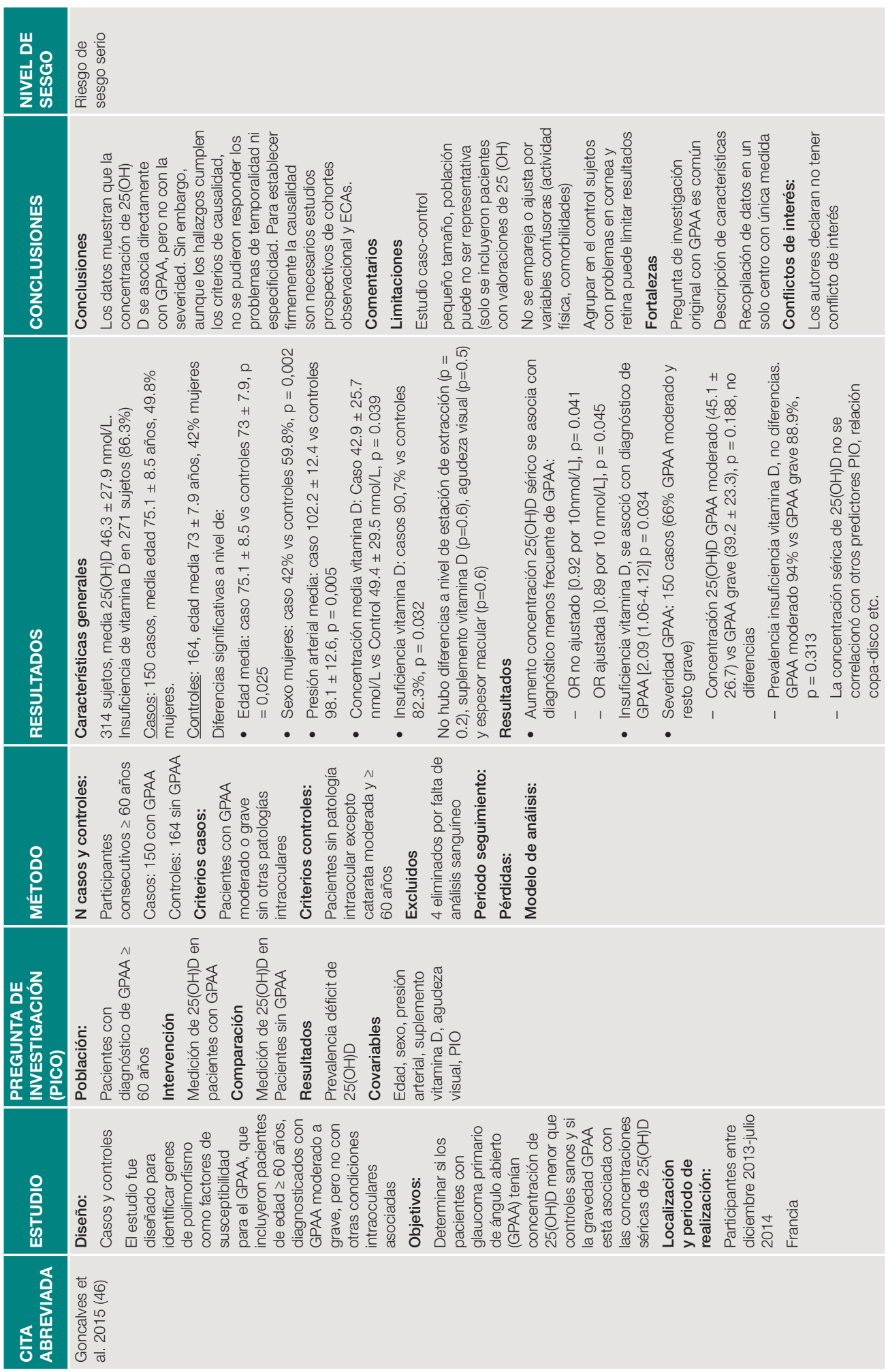




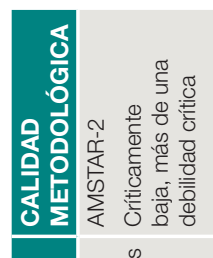

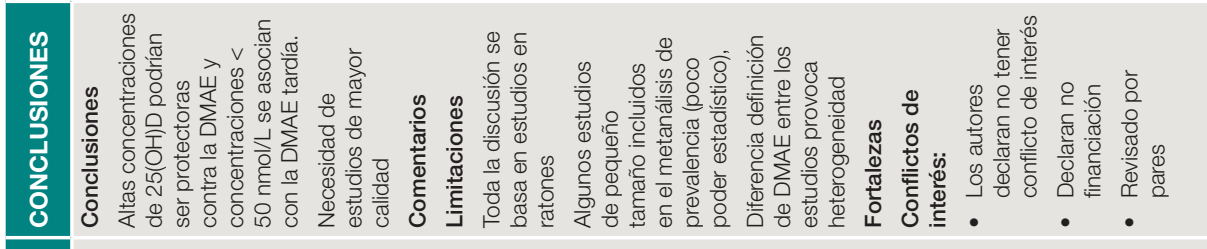

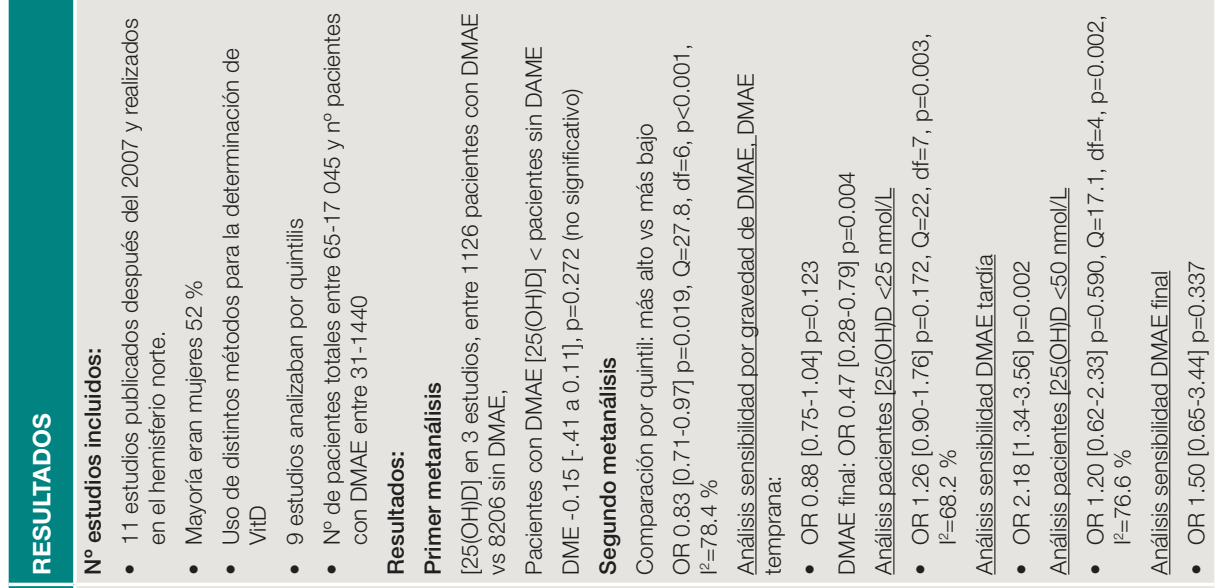

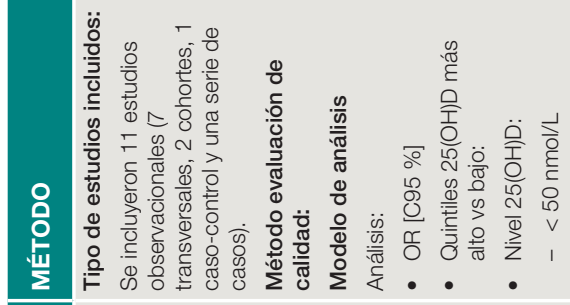

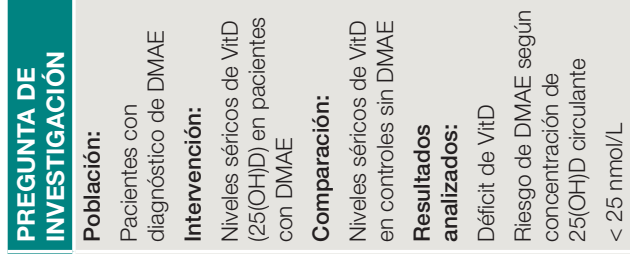

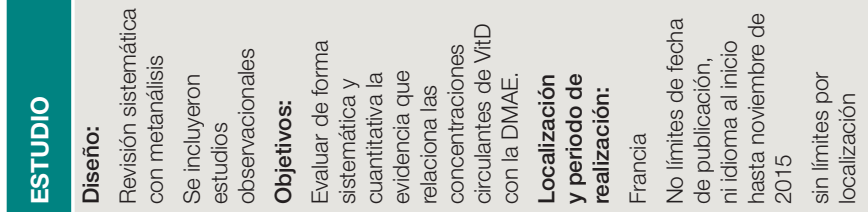

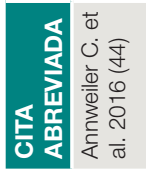




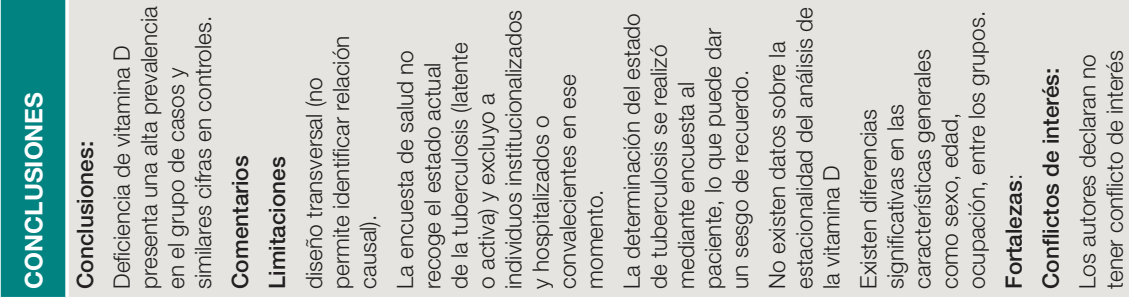

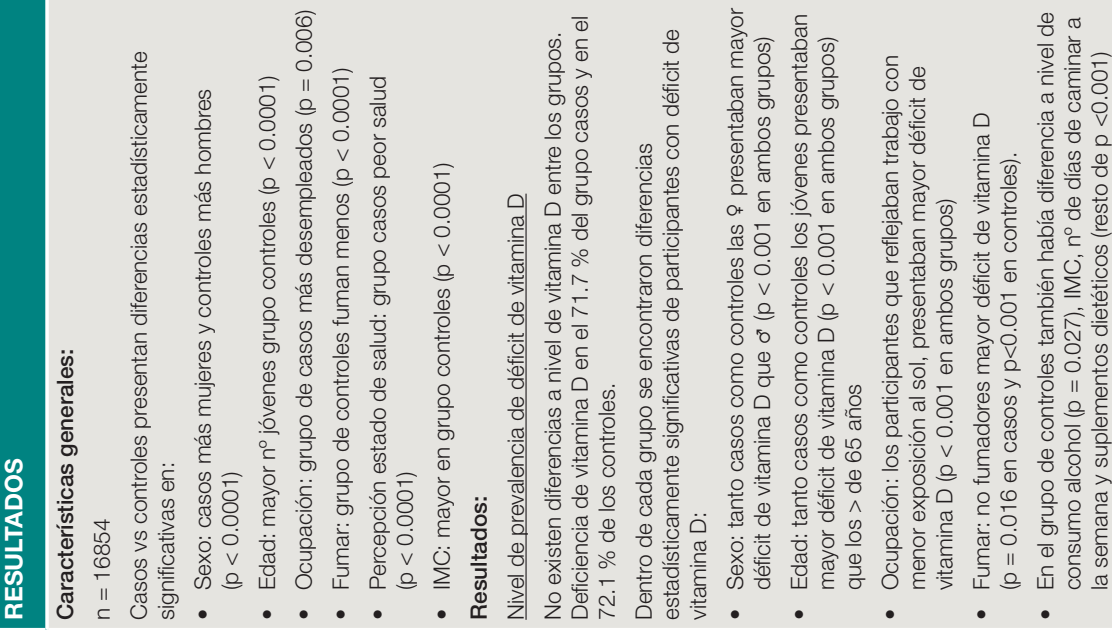

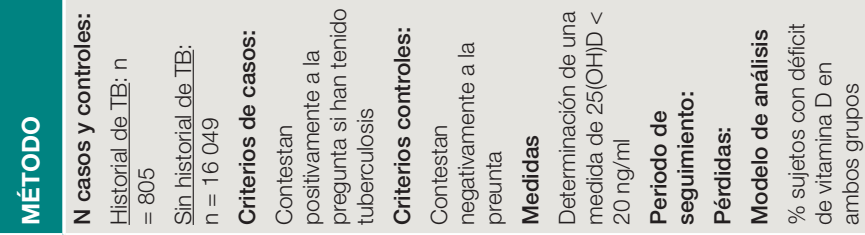
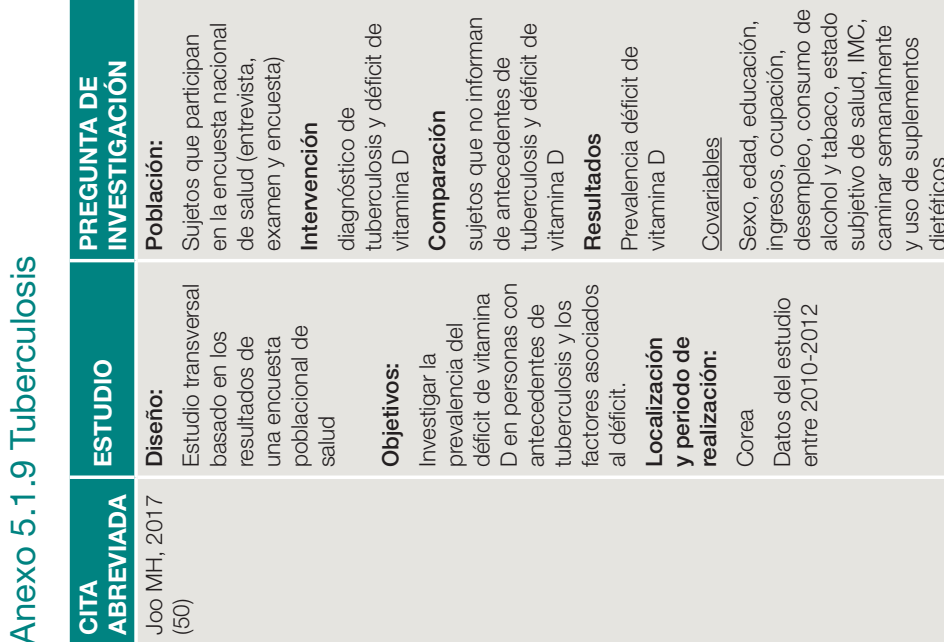
罚l

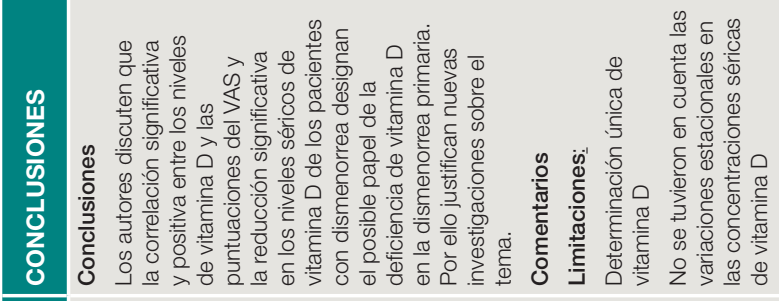

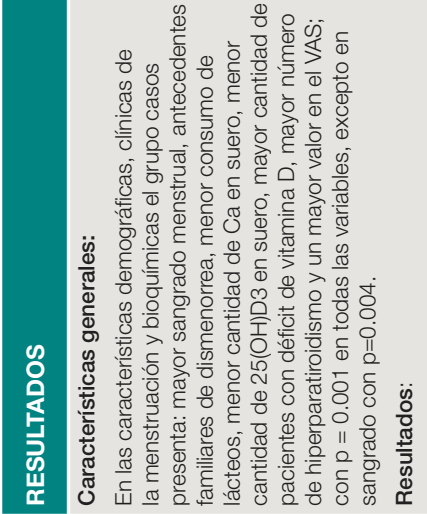

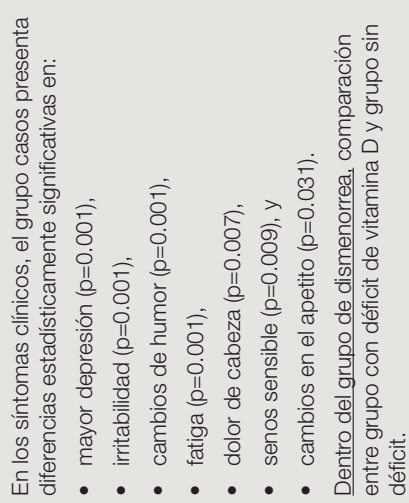

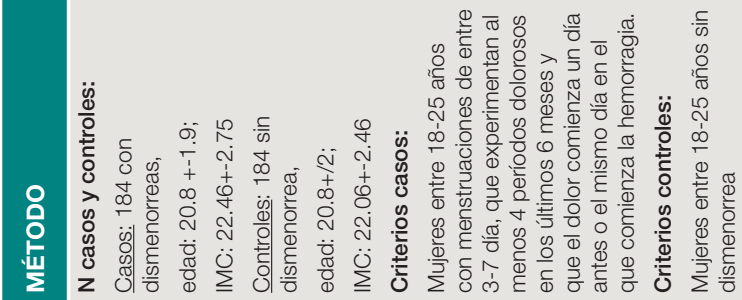

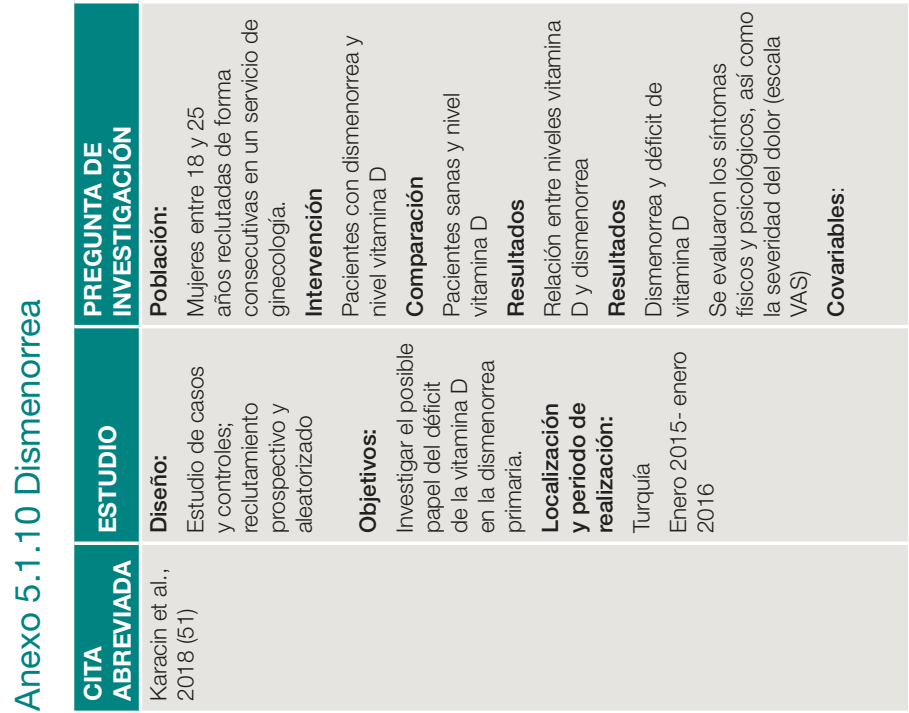




\section{蹅。}

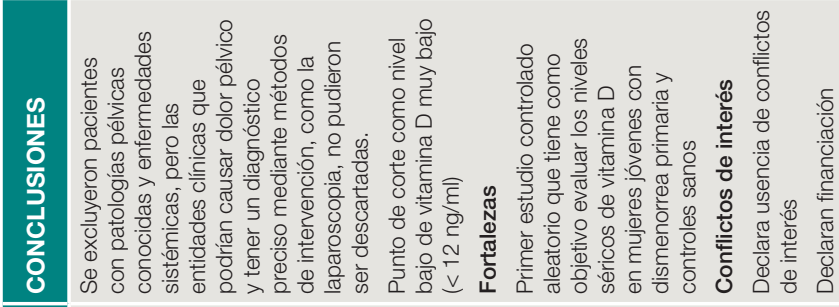
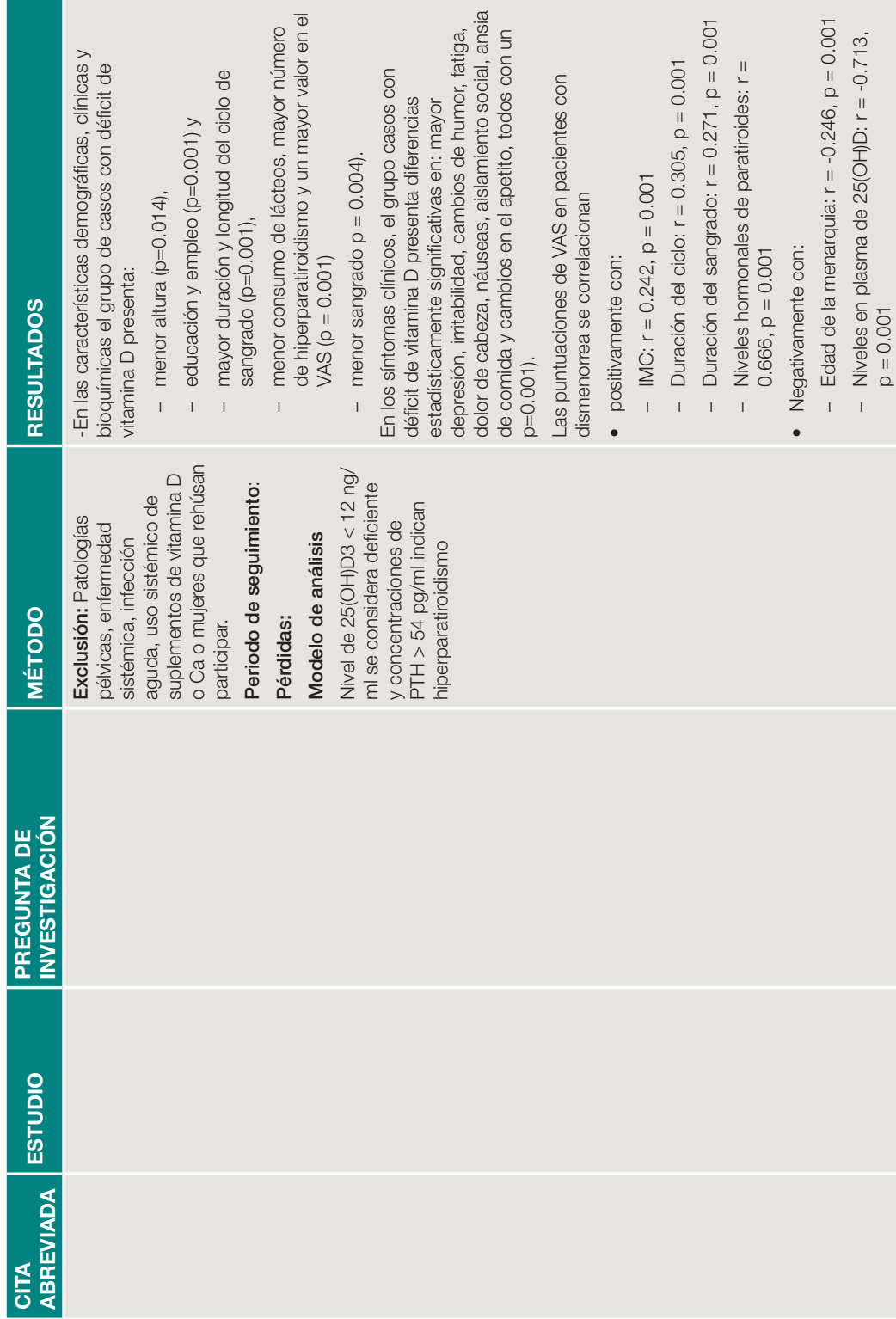


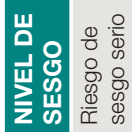

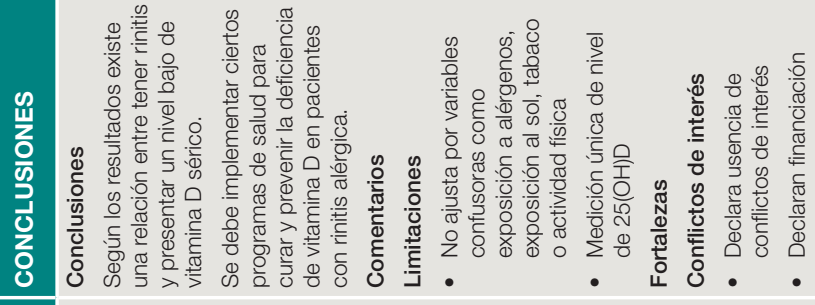

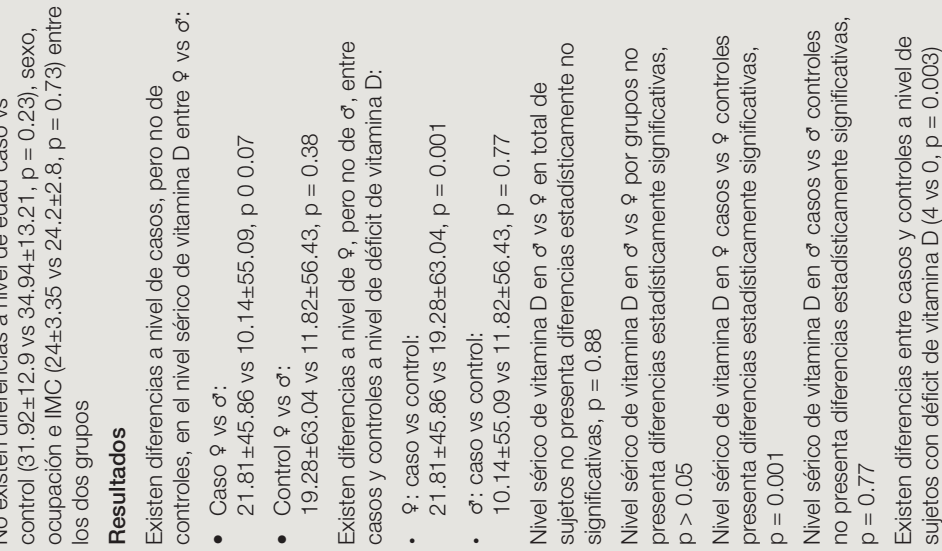

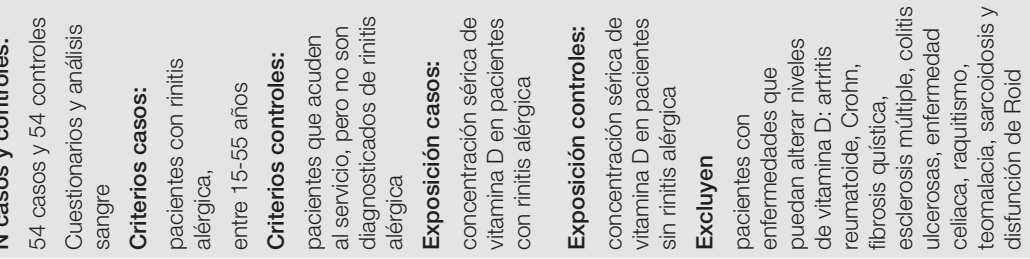
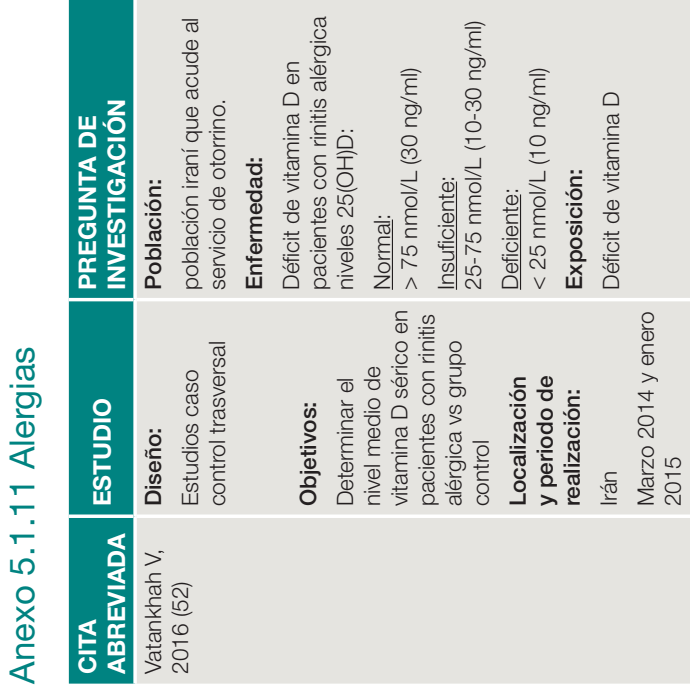
III

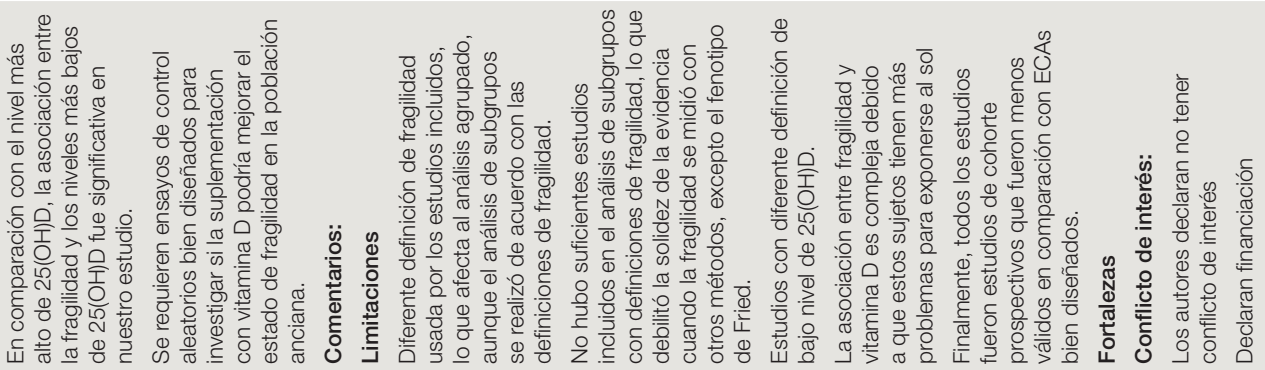

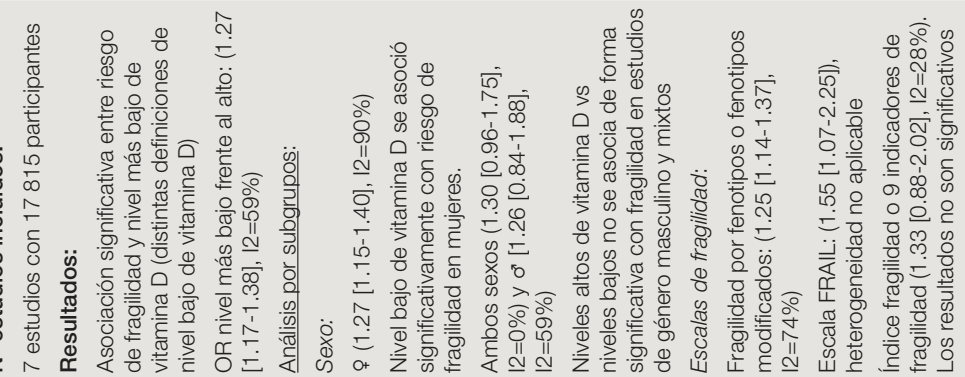

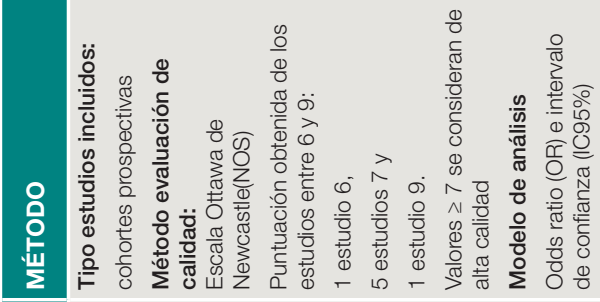




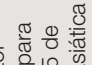

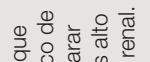

ब

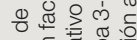

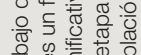

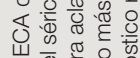

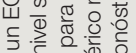

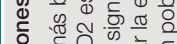

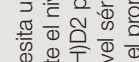

5

은

ํㅓㄷ 

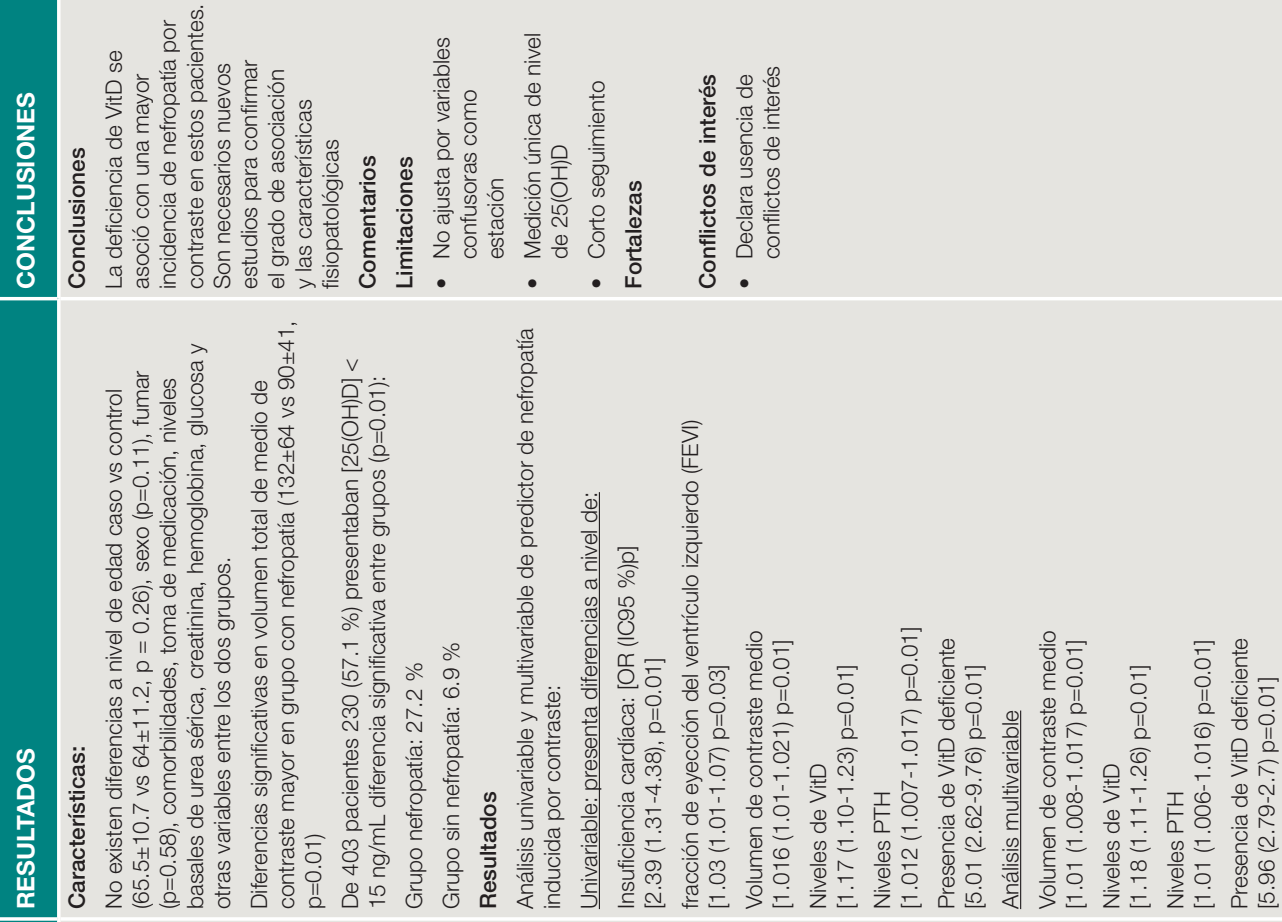

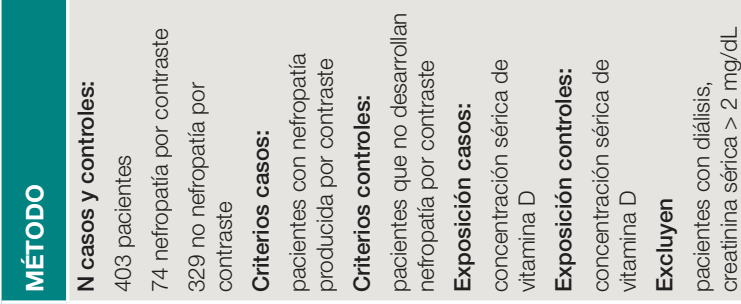

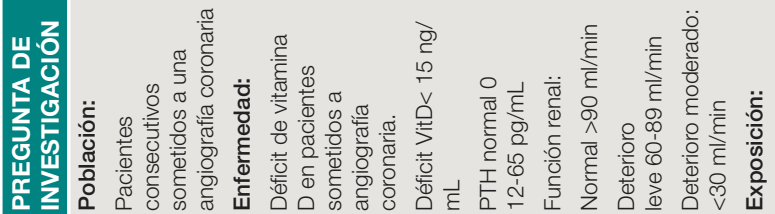

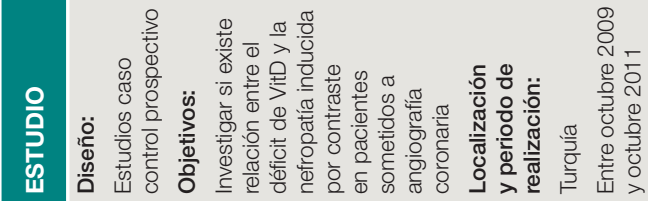




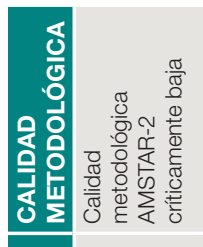

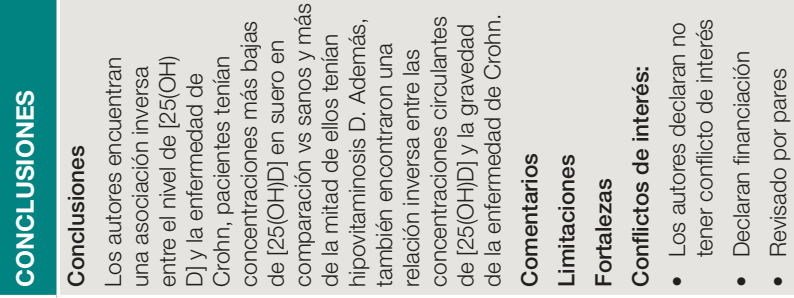
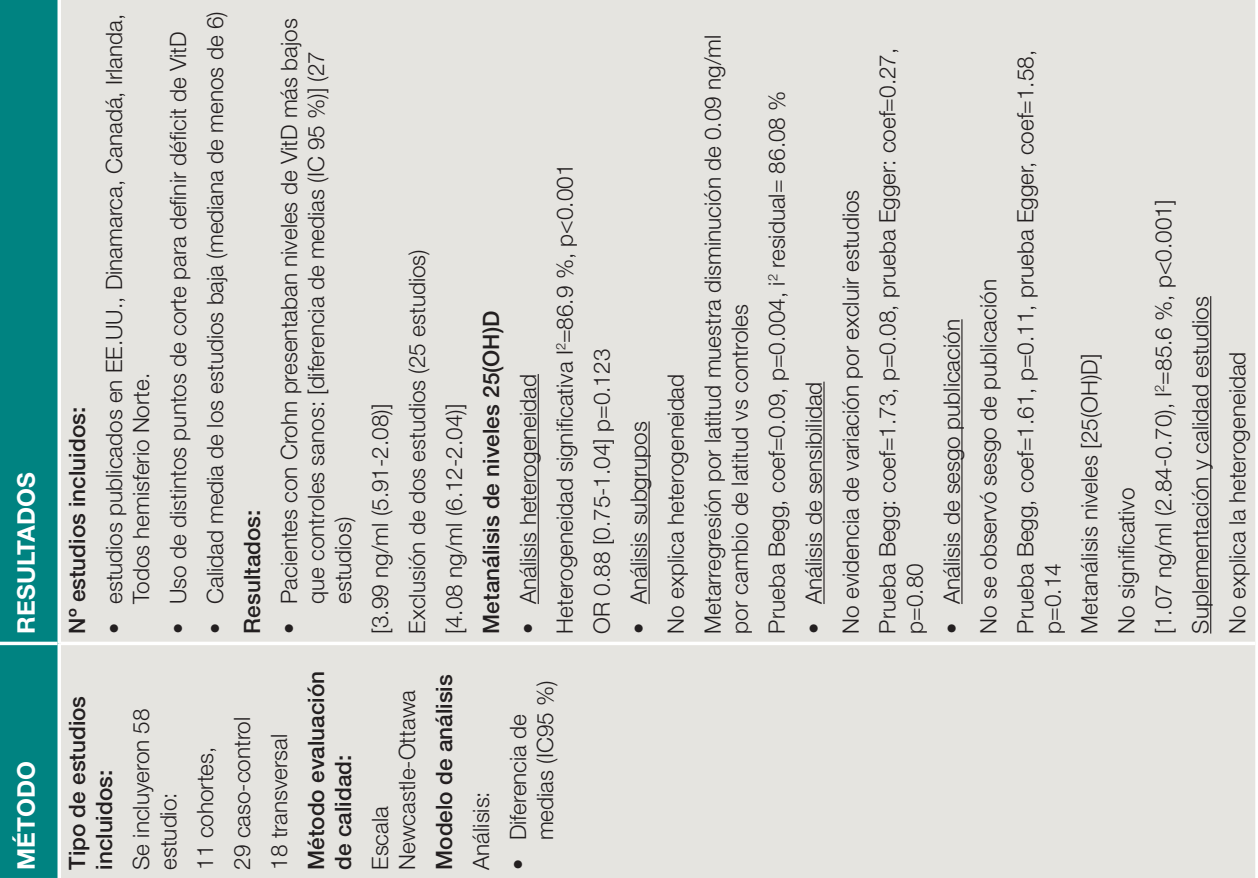

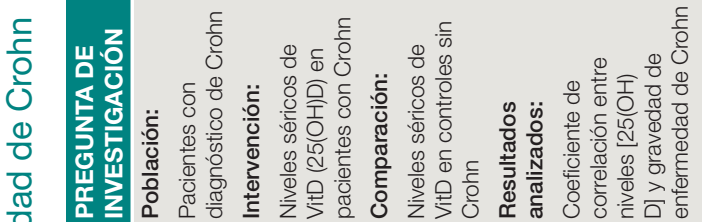

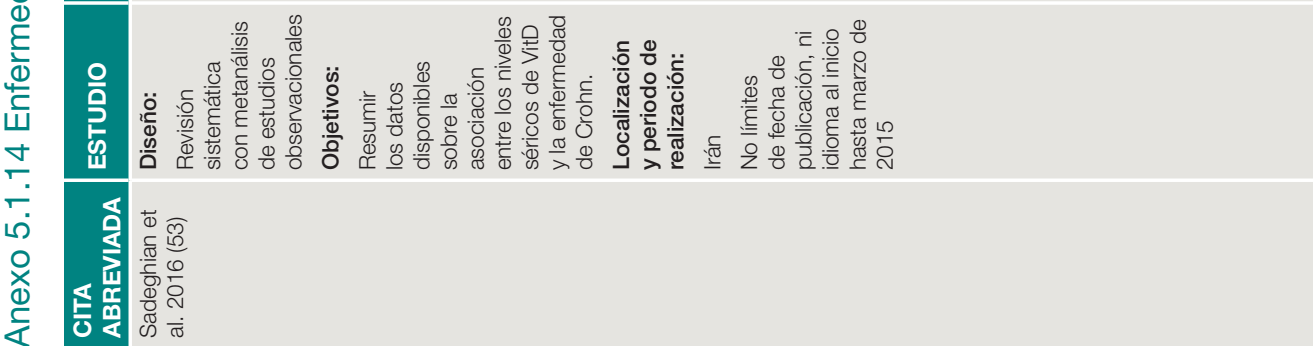




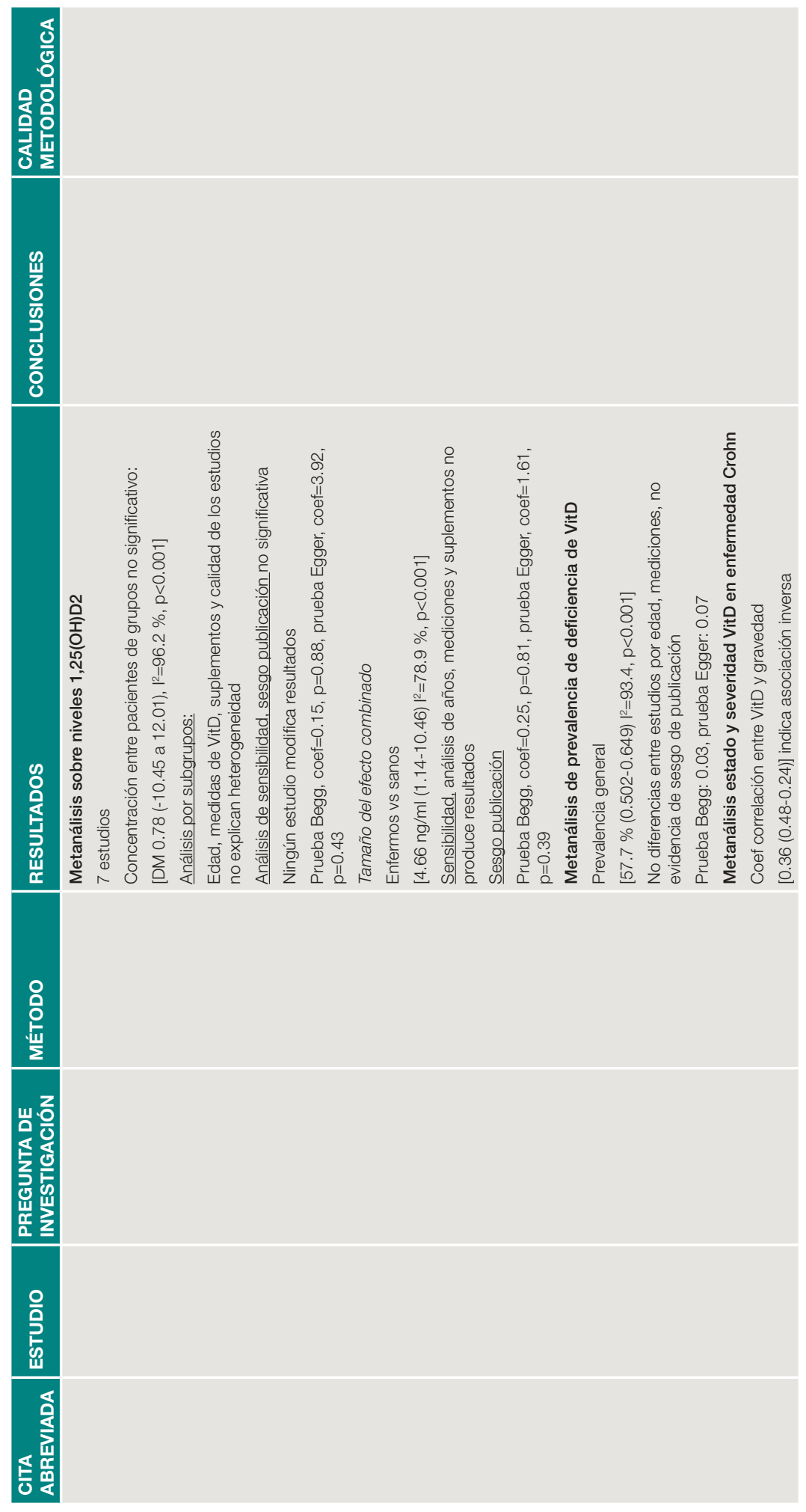




\section{III}

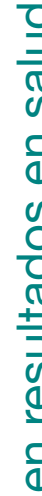

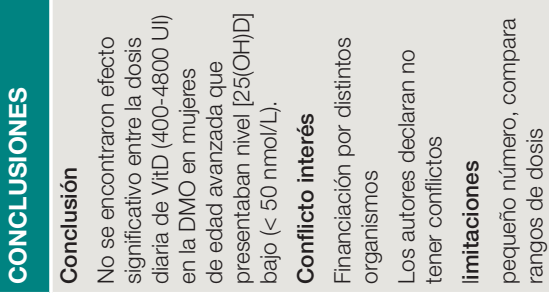

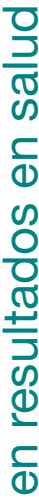

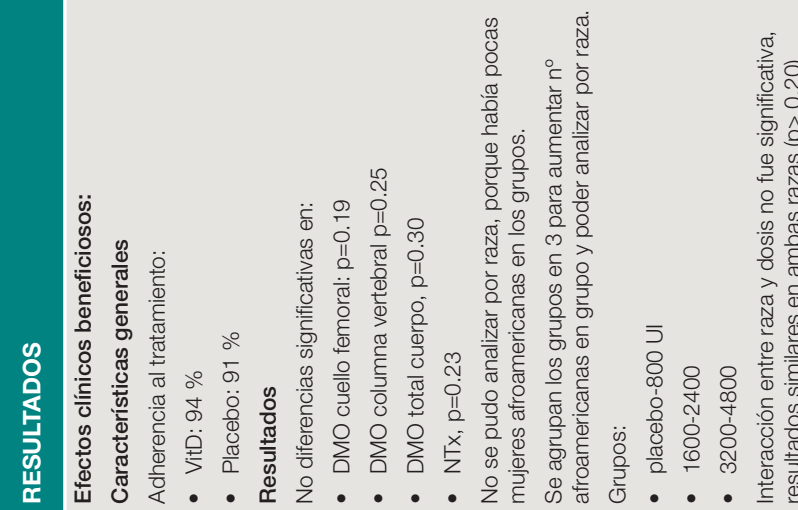

.

요

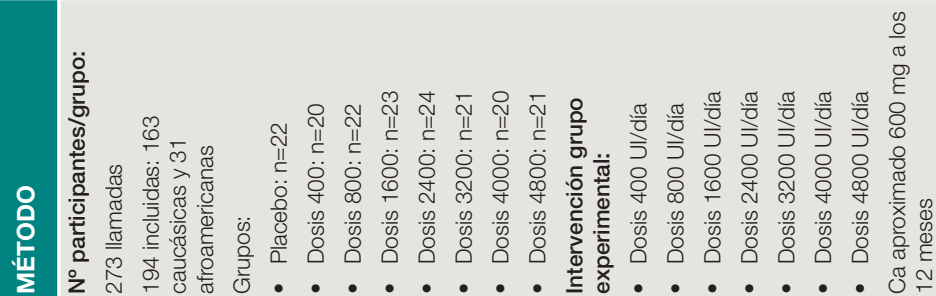

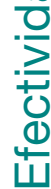

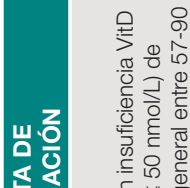

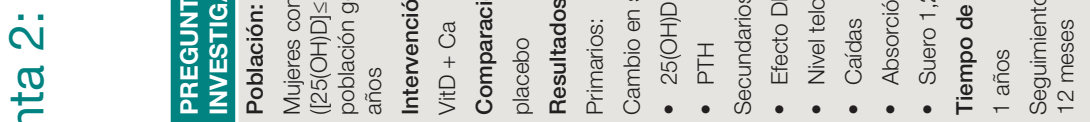

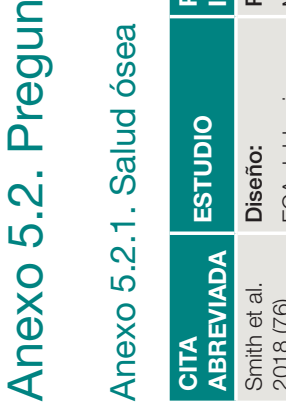




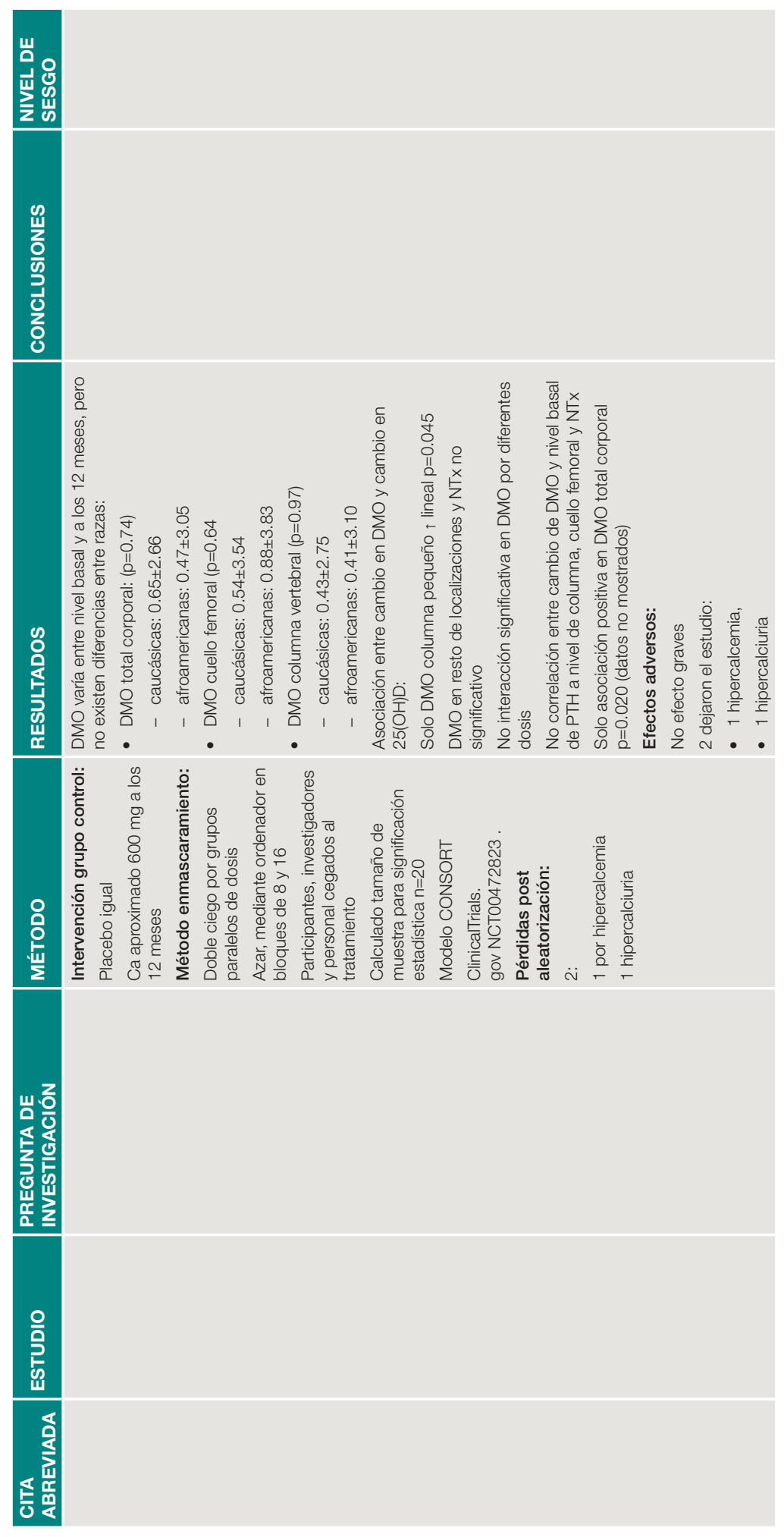




\section{.}

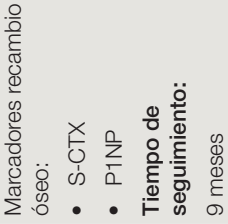

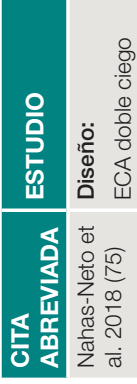



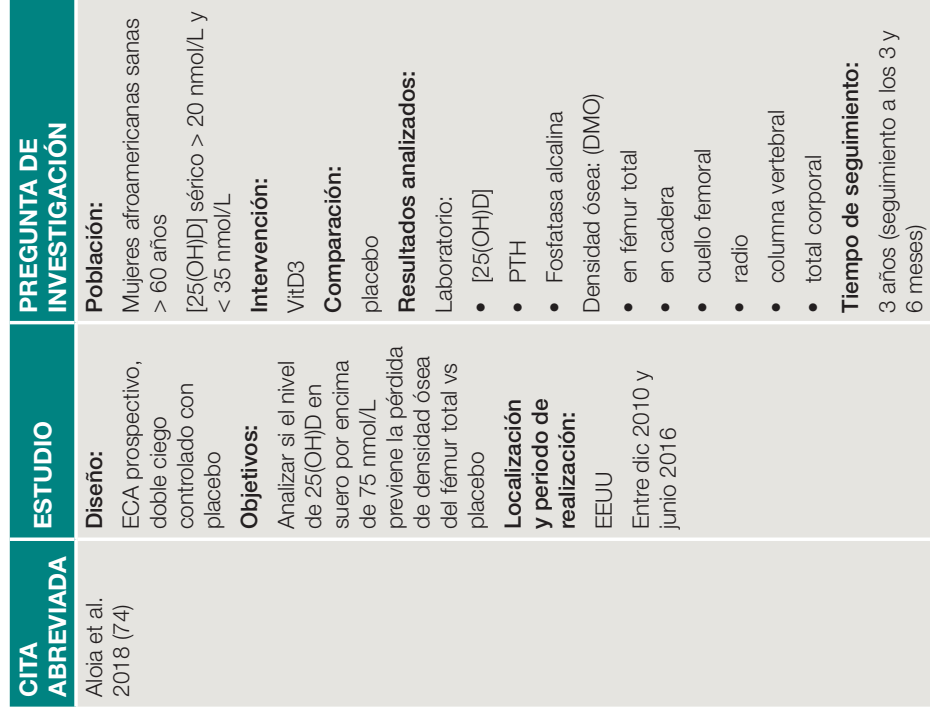
III
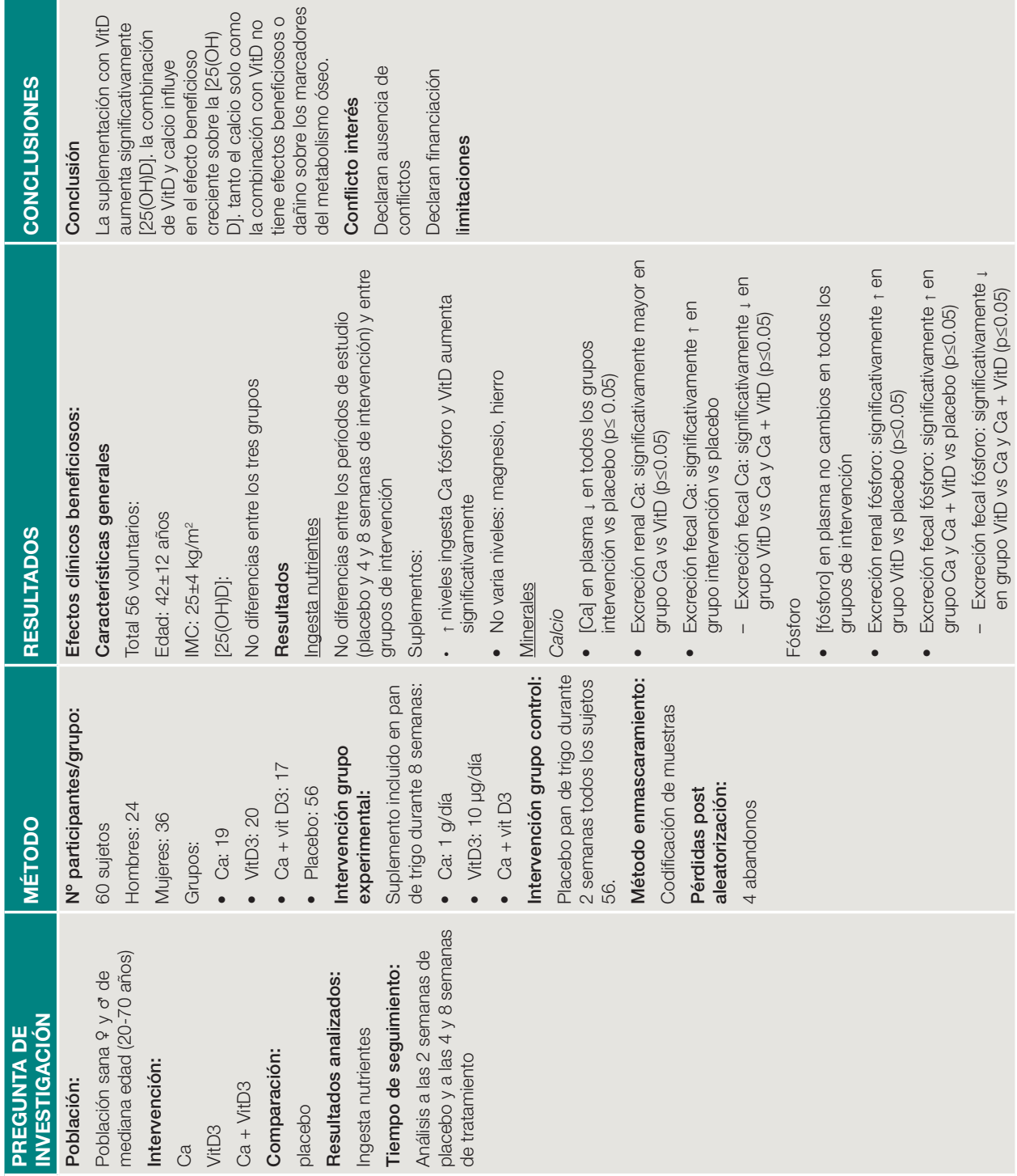

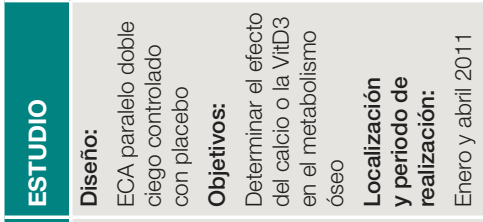

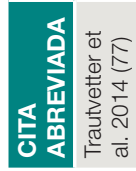



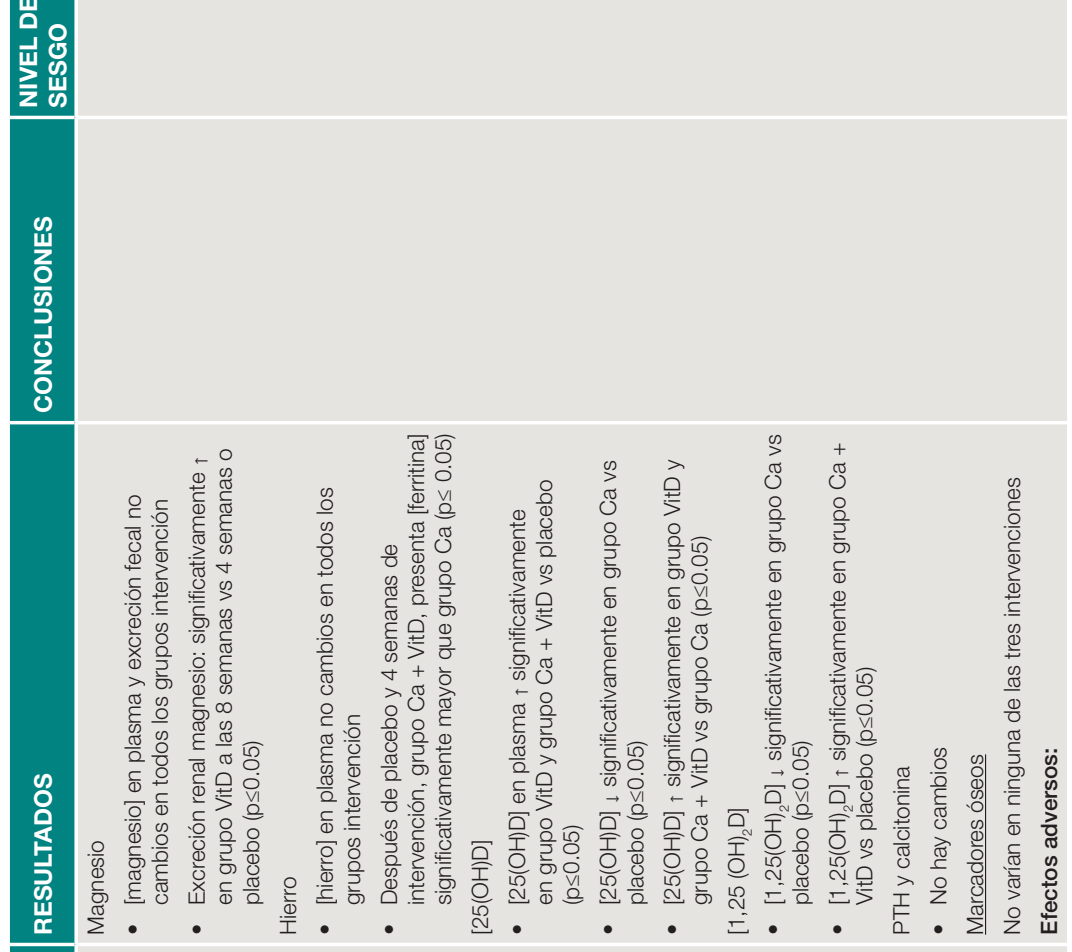

음
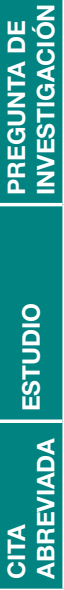

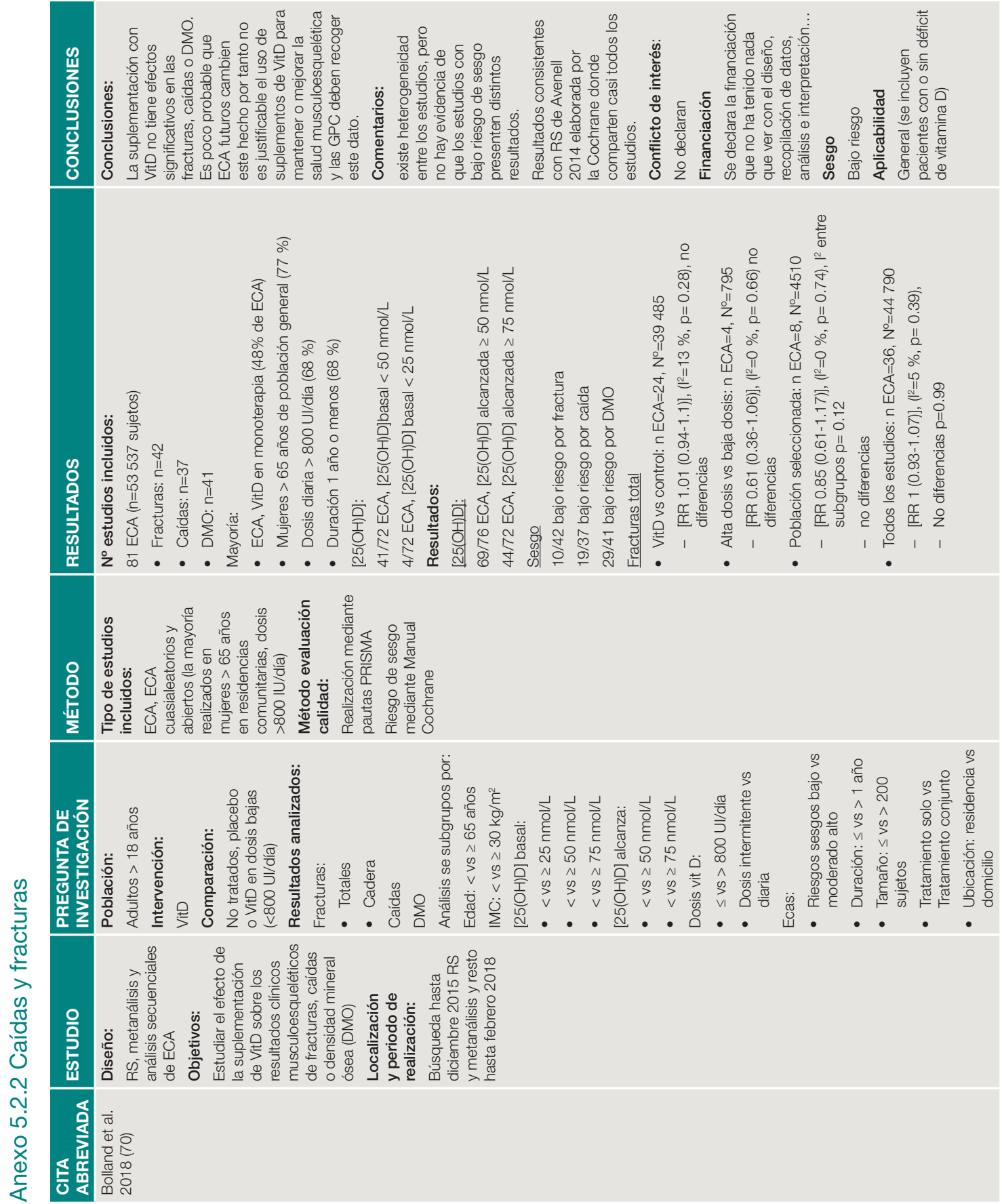


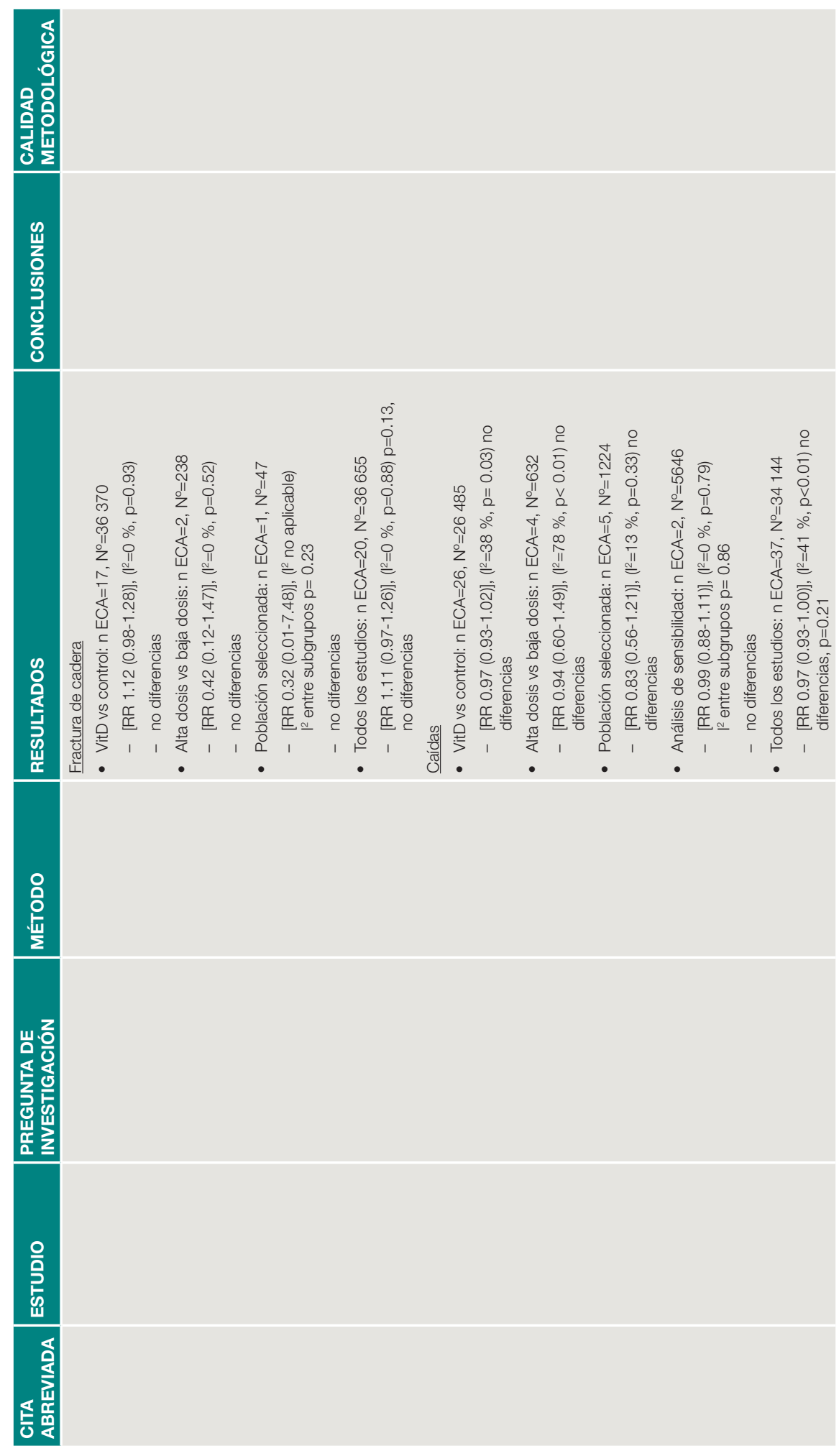




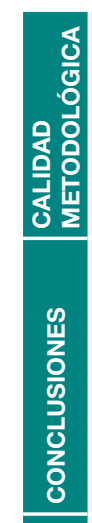

电

ब.

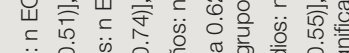

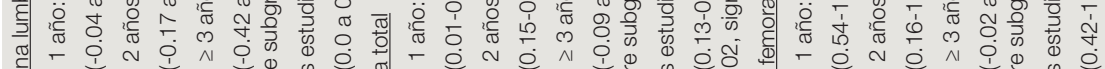

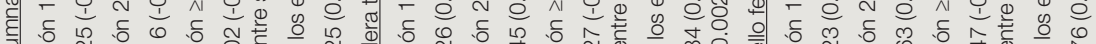

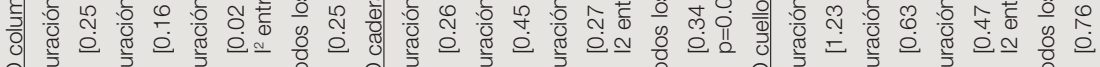

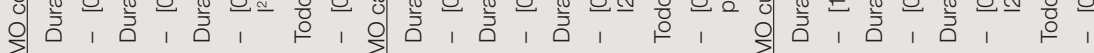
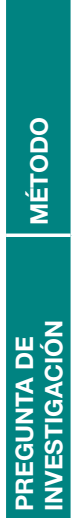

음 


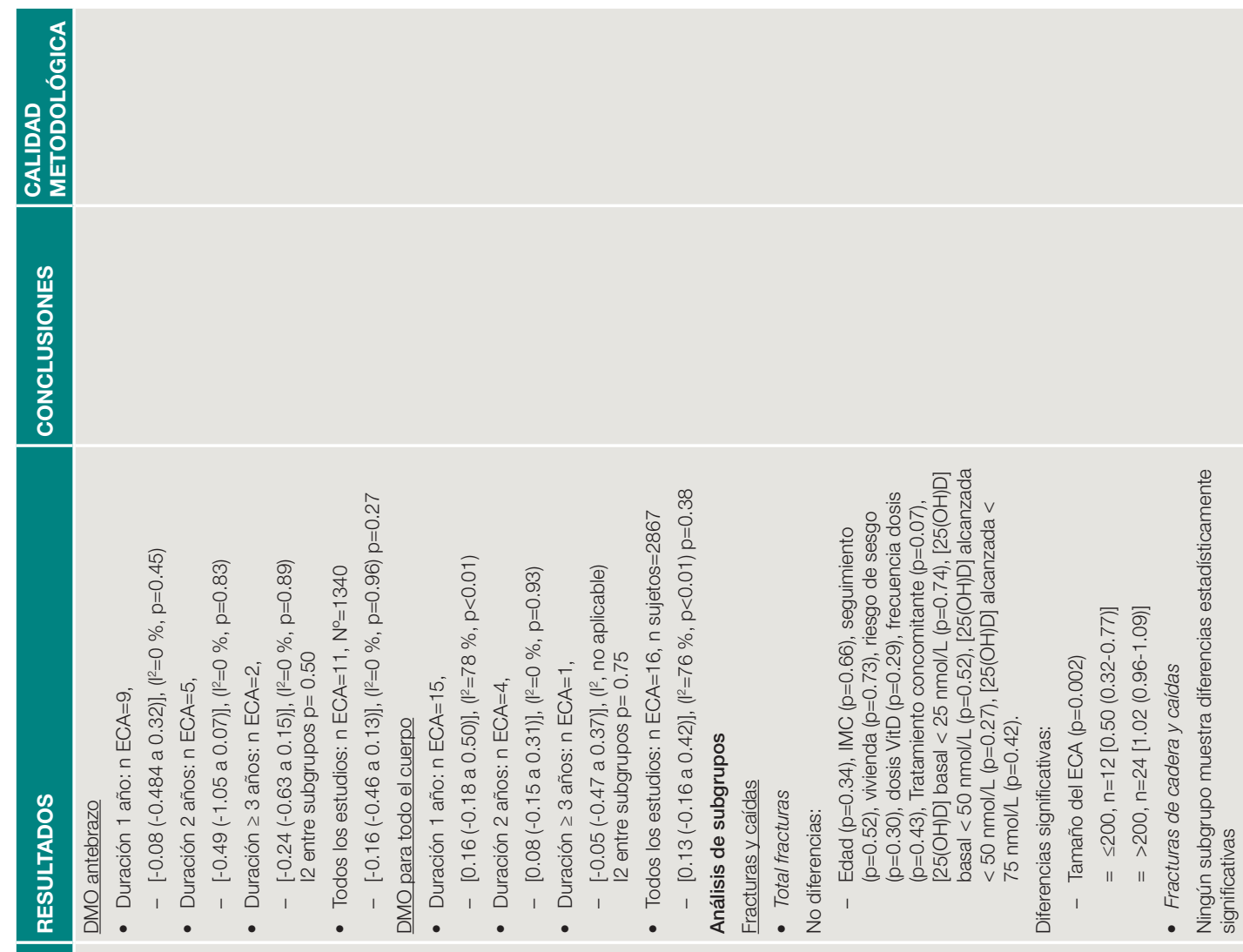

을

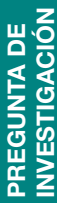

을 


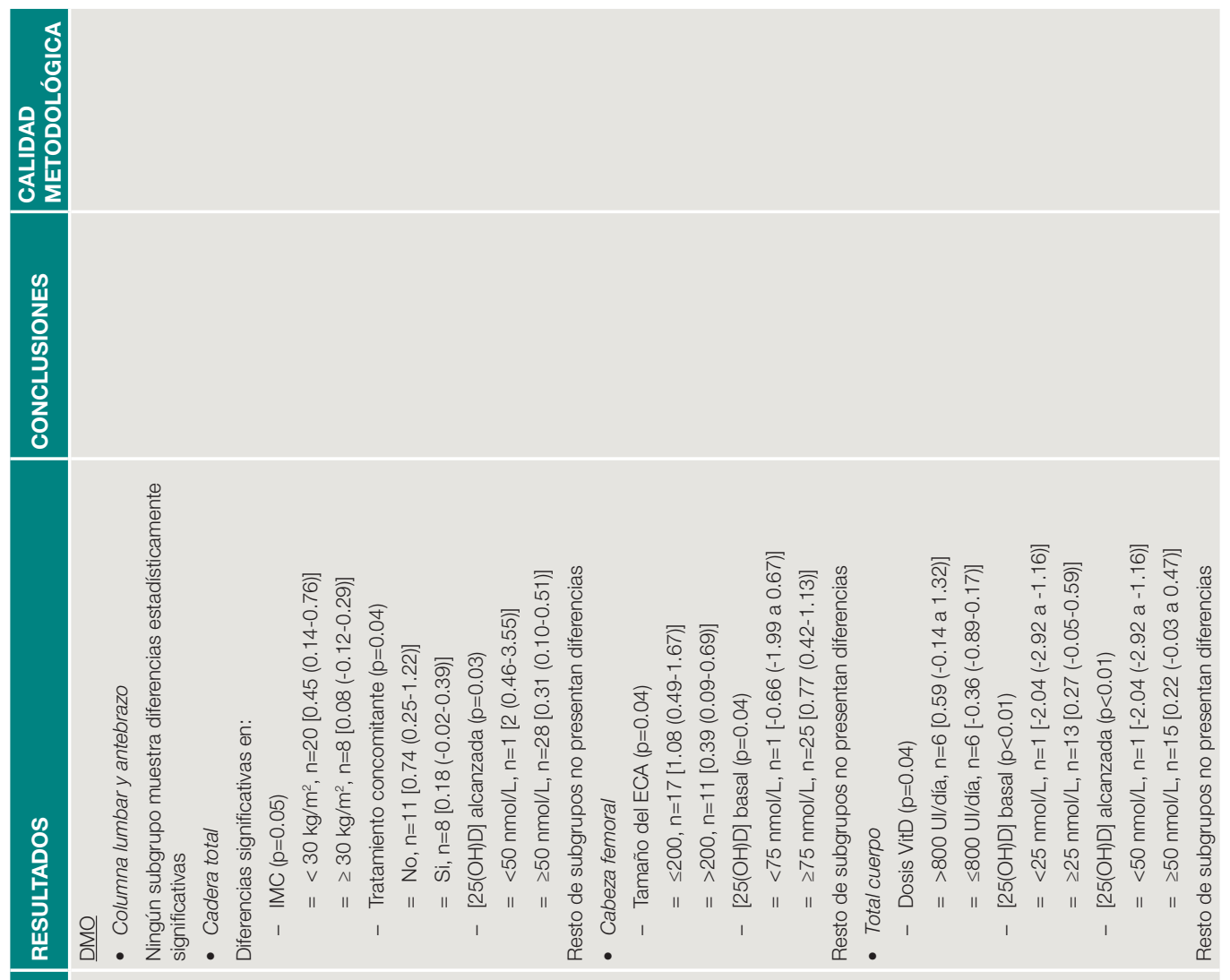

음

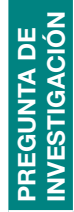

음

卢高 


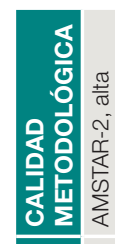

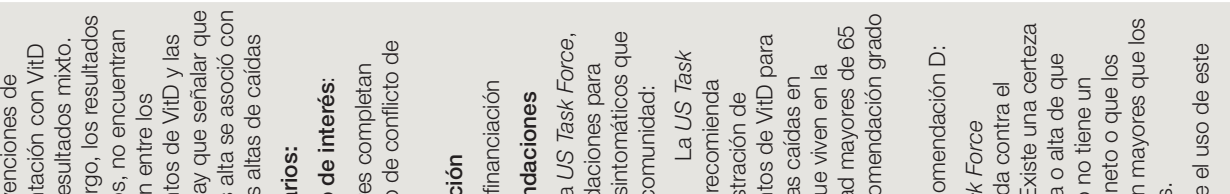

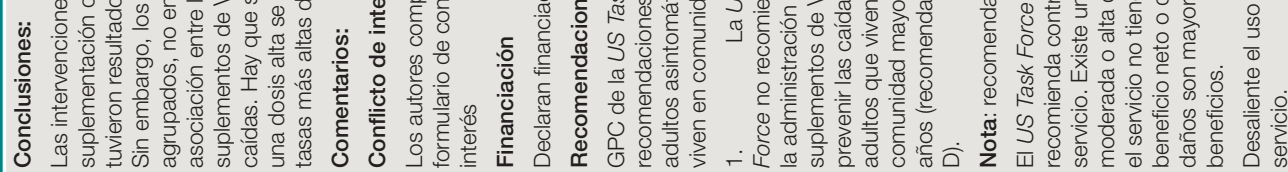

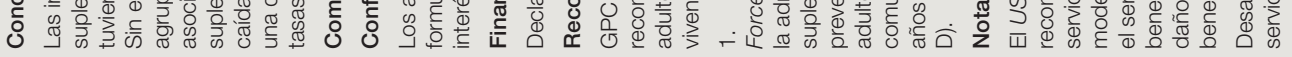
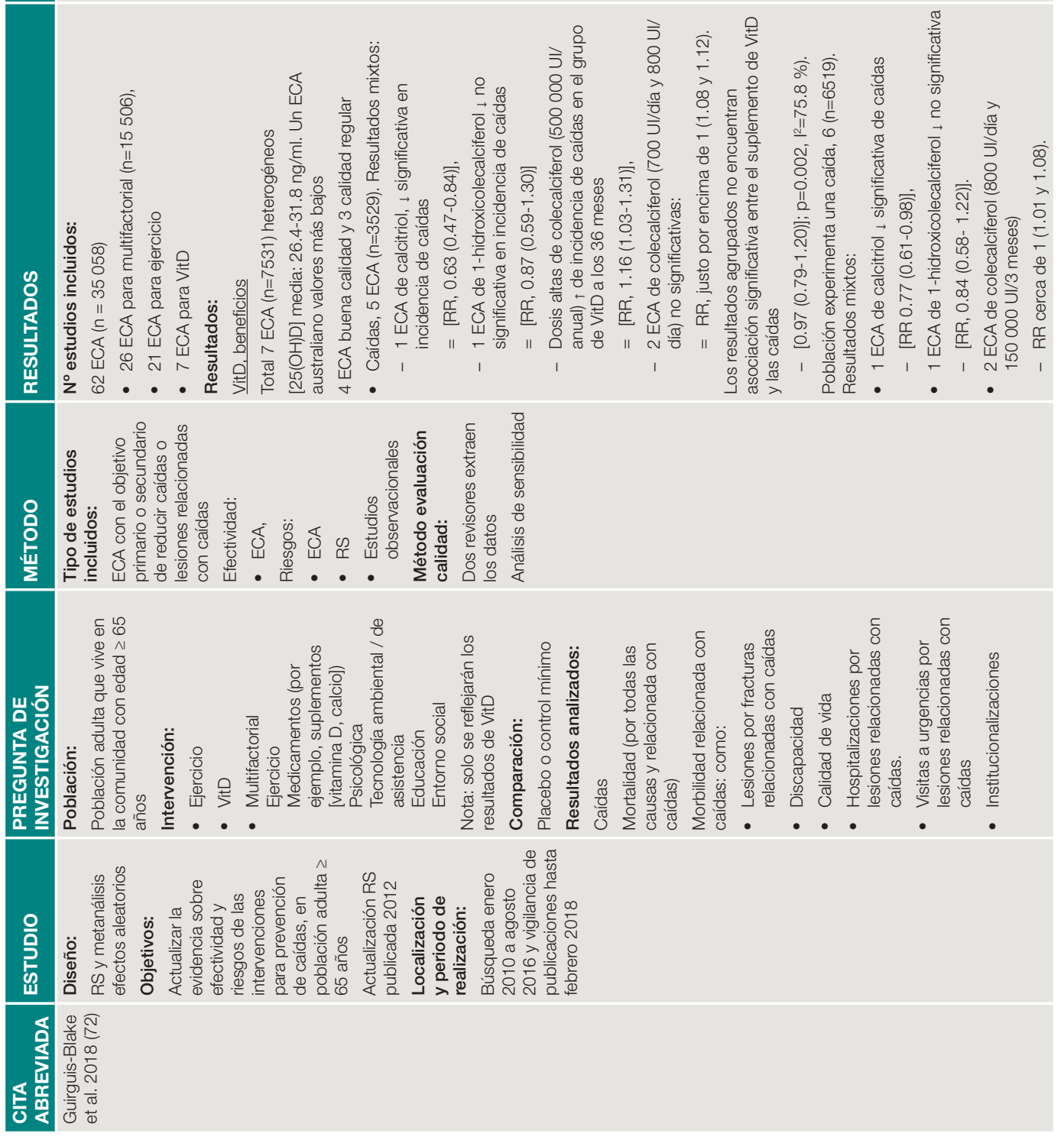

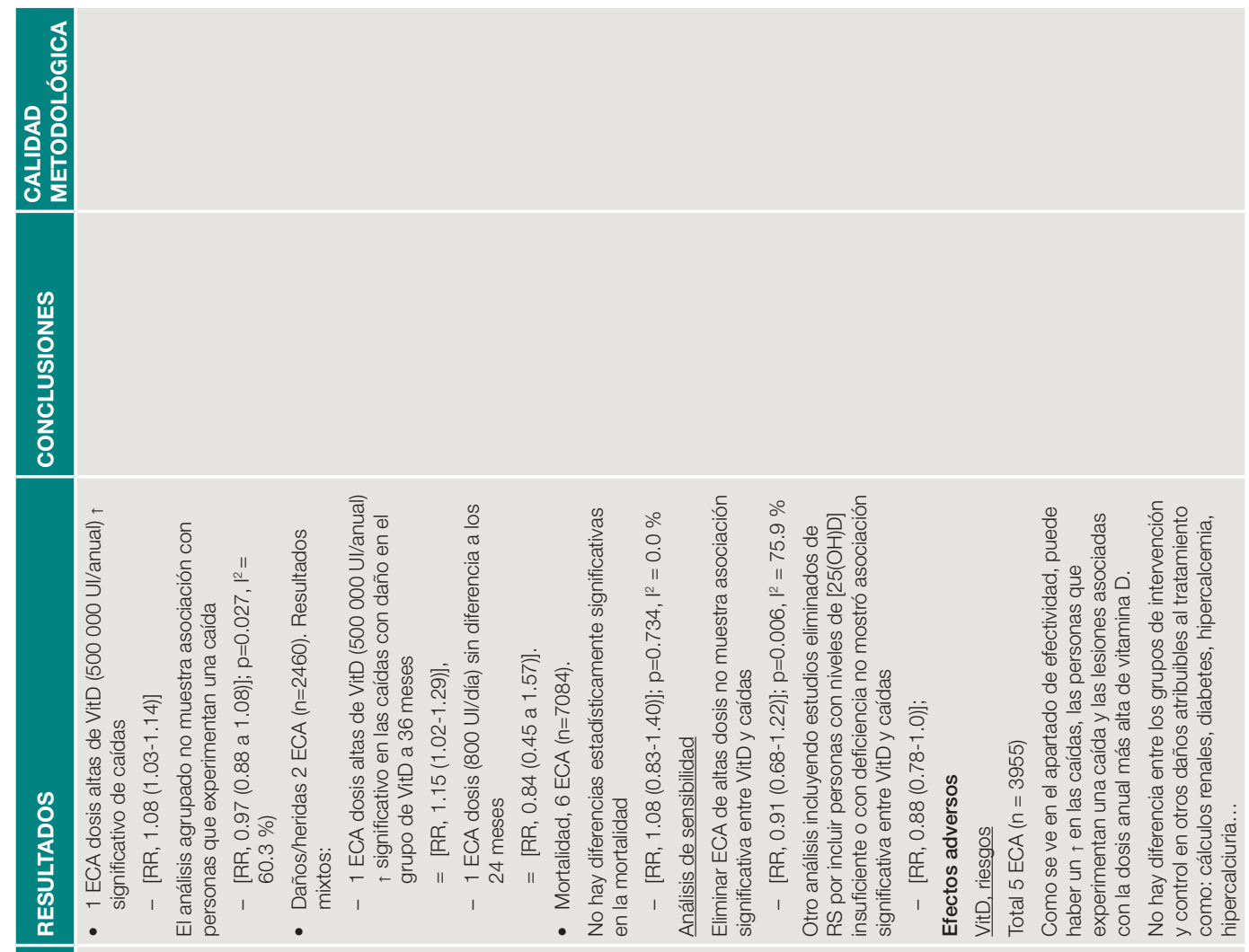

웅

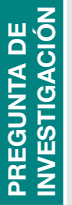

음

笔誉 


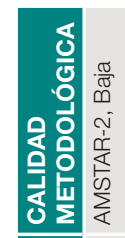

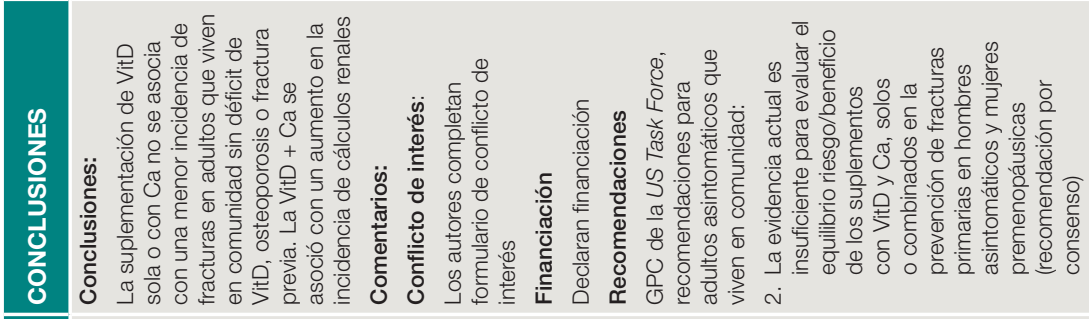

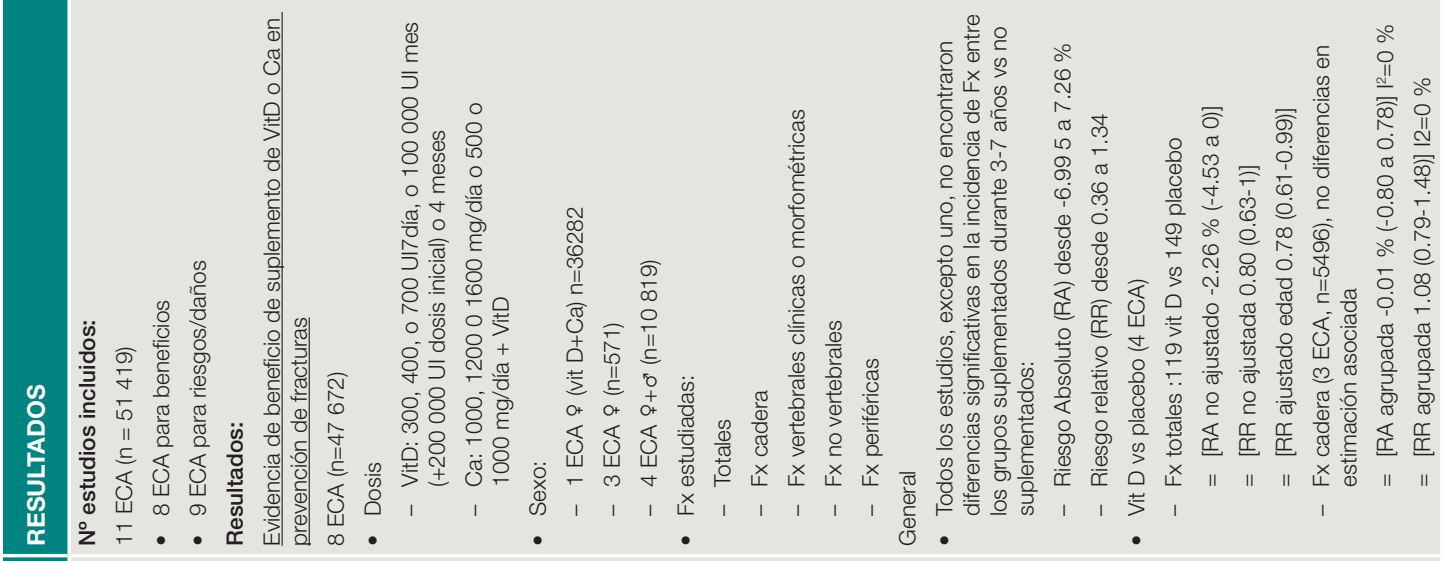

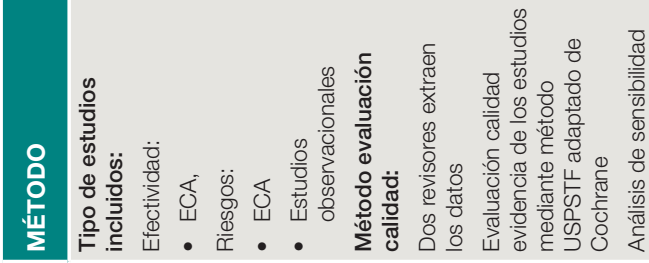

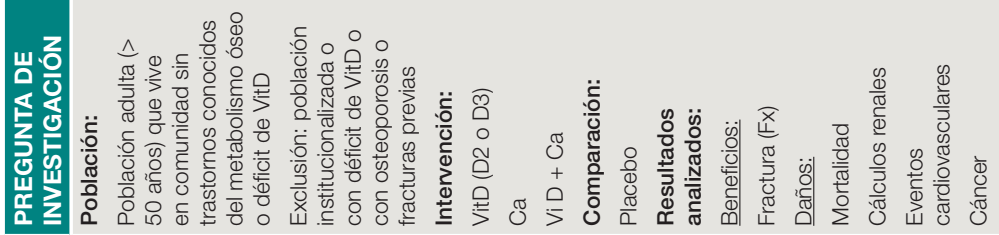

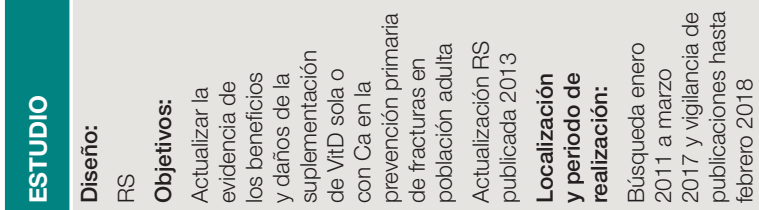

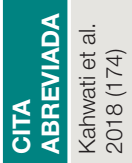




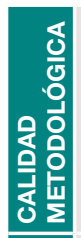

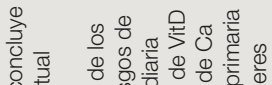

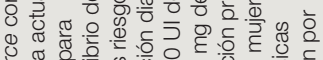

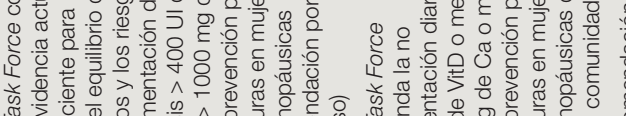

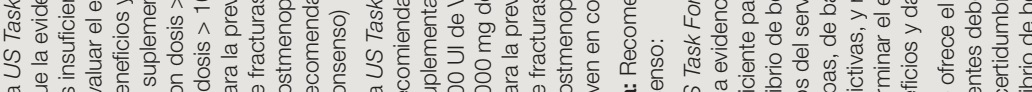

๙

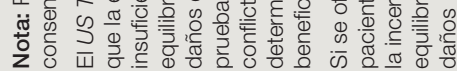

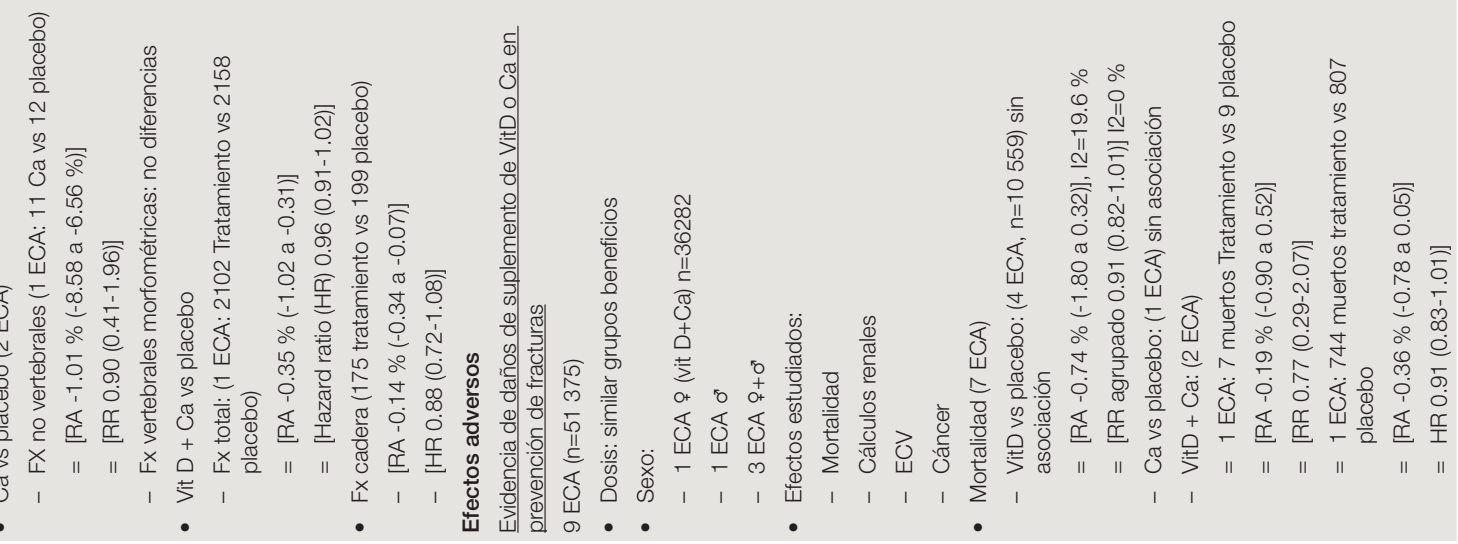

응

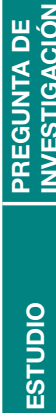

悹 

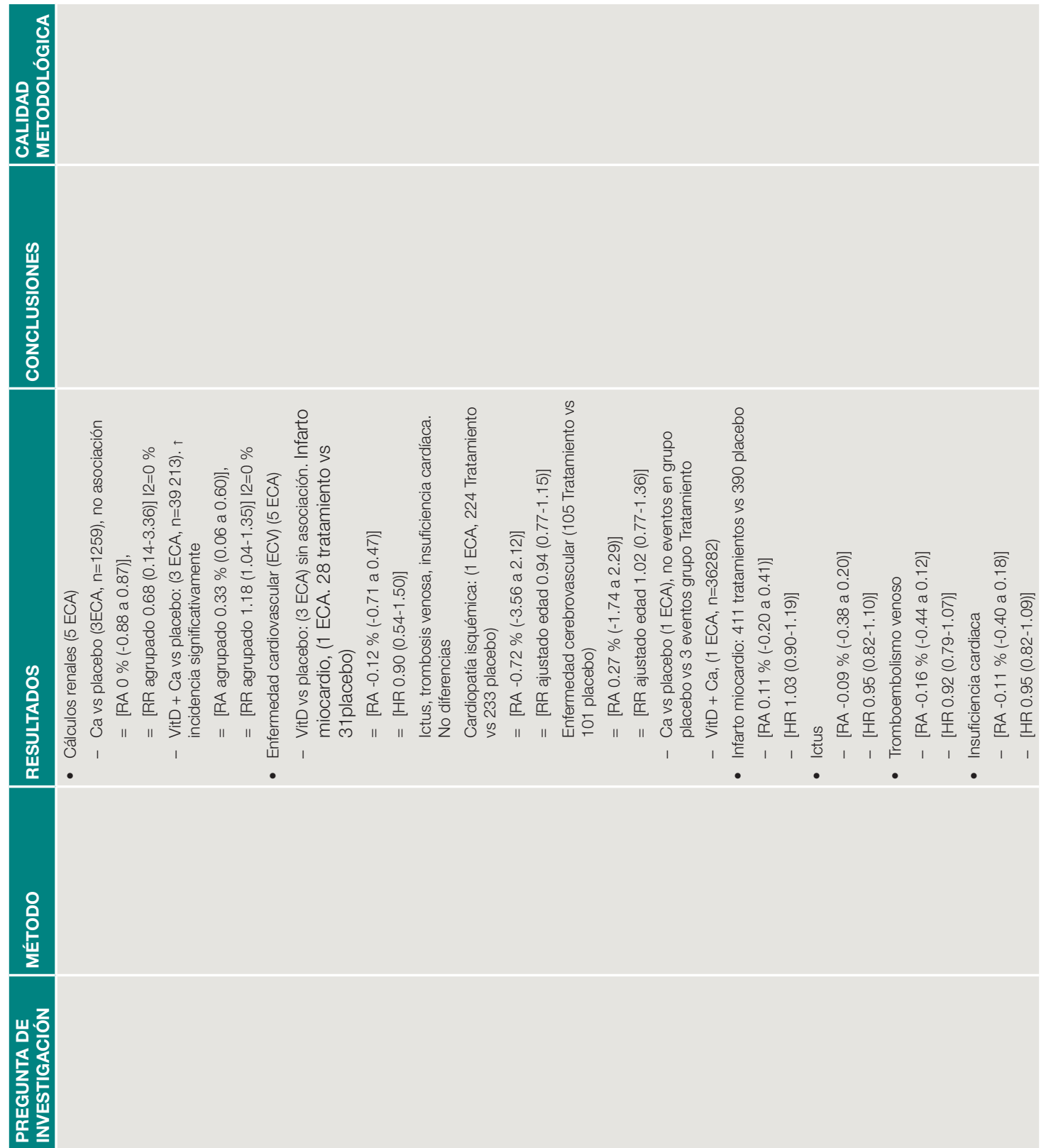


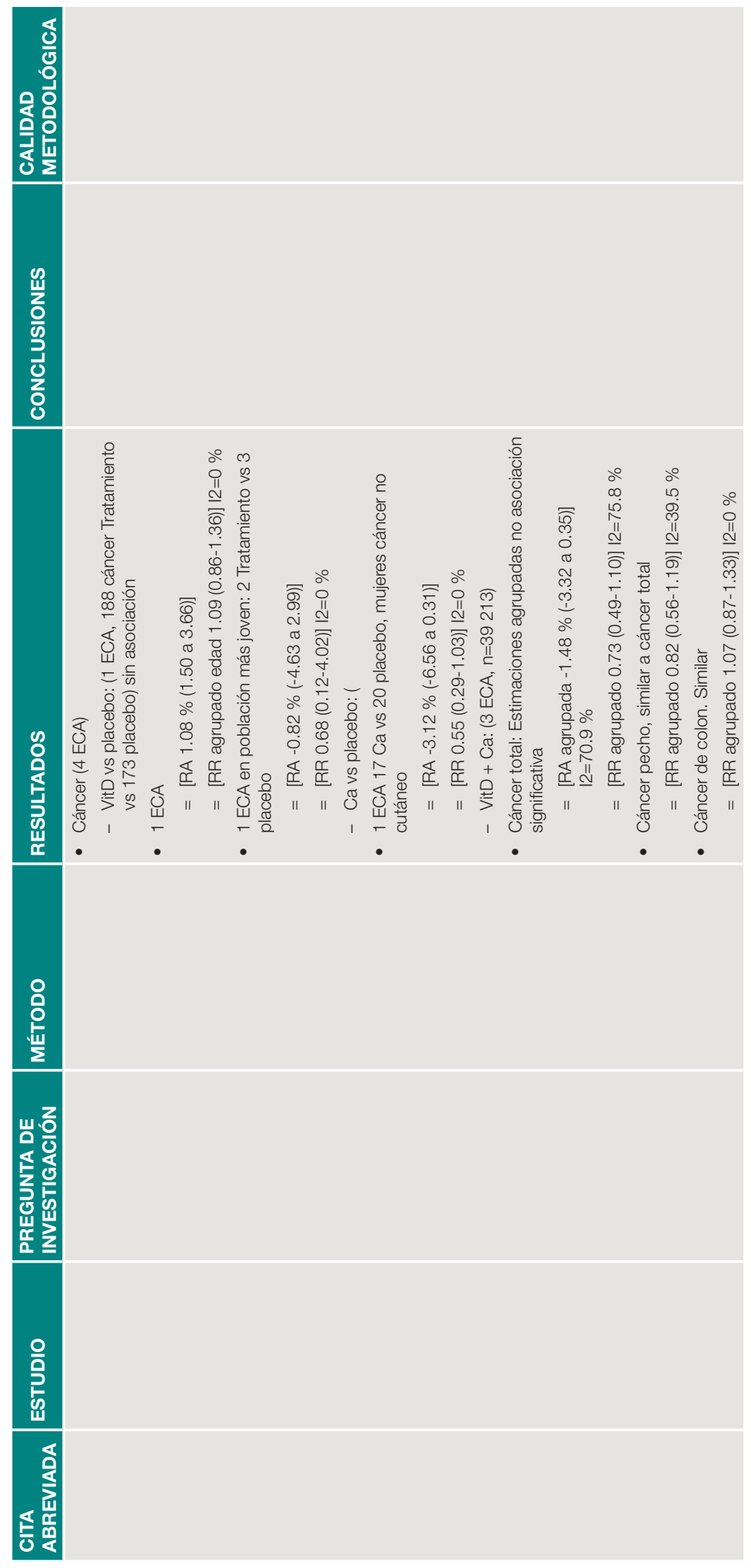




\section{耍

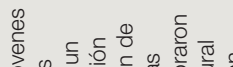 \\ $\frac{8}{0}$

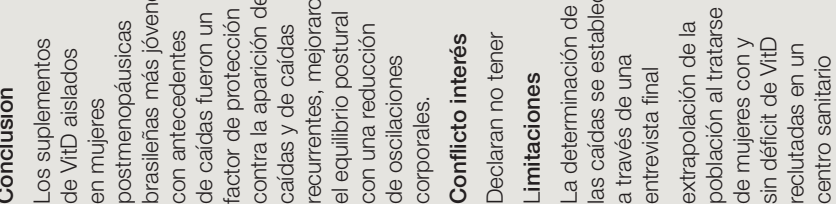

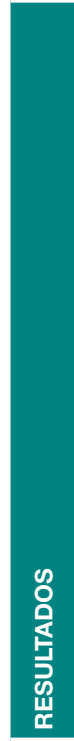

0
0
0
0
0
0
0
0
0
0
0
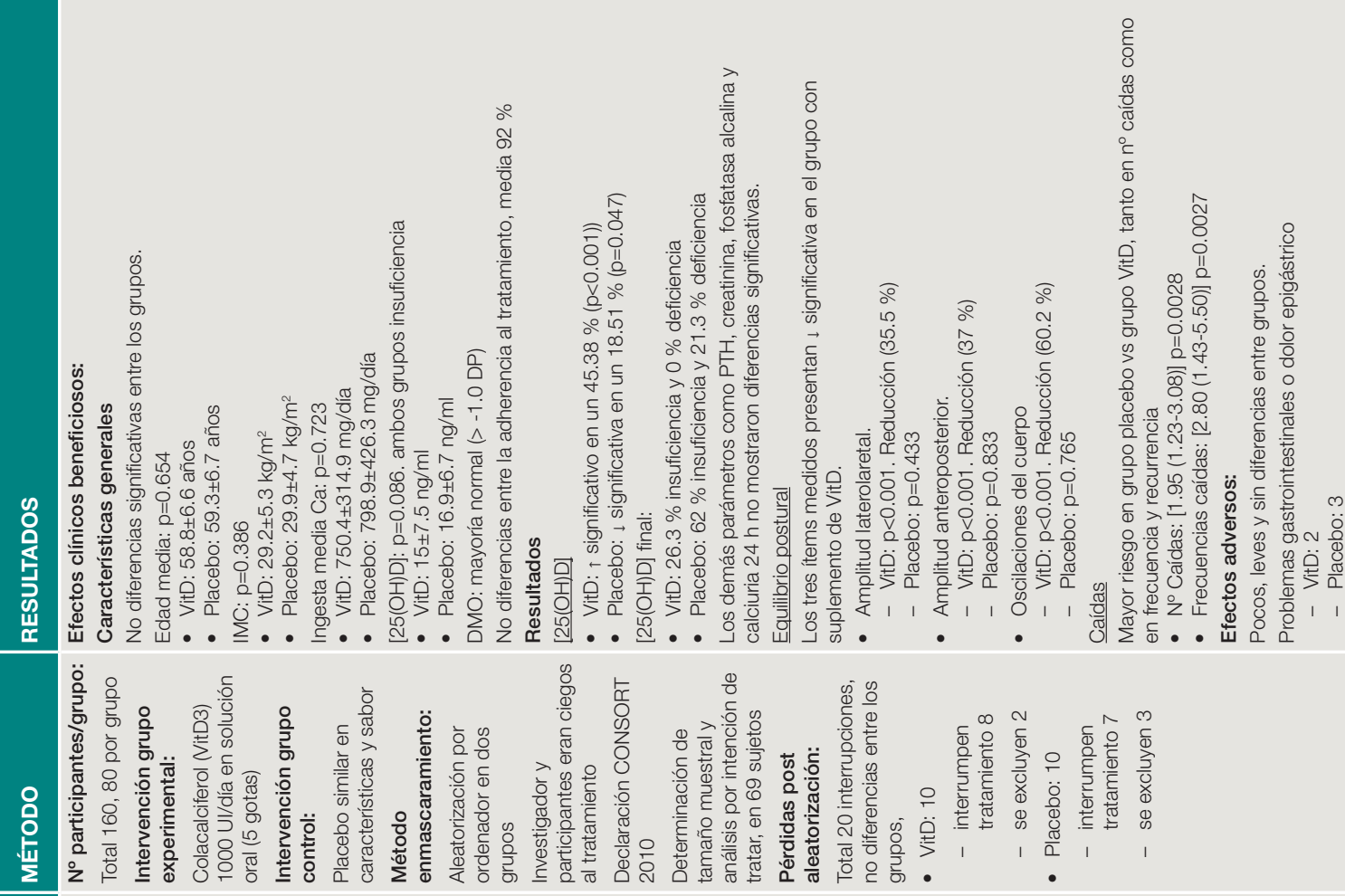

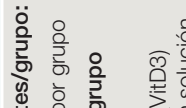

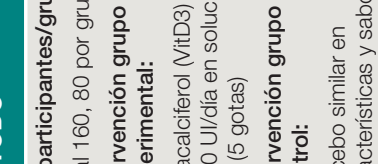

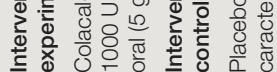
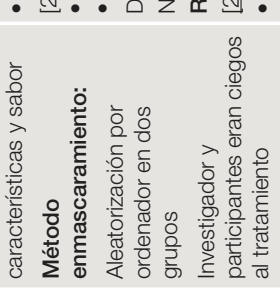

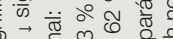

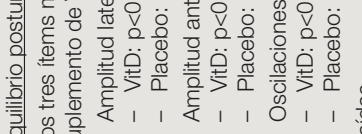

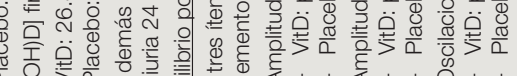

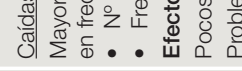

$\ddot{8}$

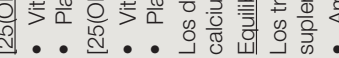

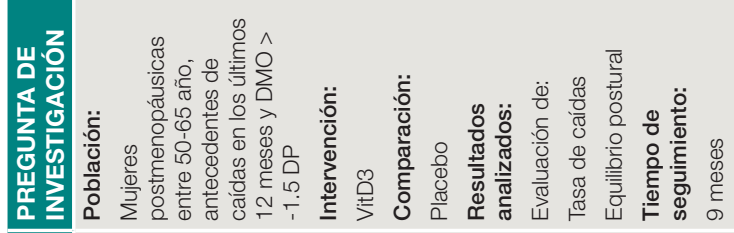

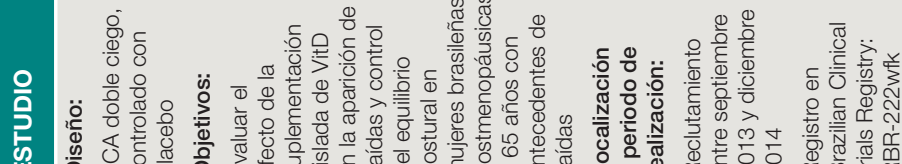
兽

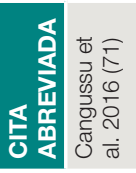




\section{I}

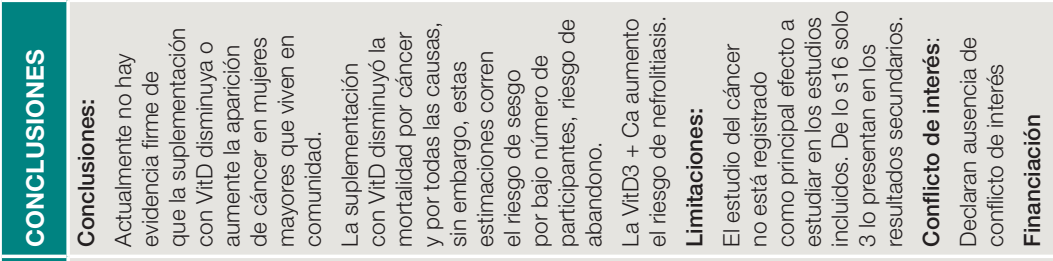
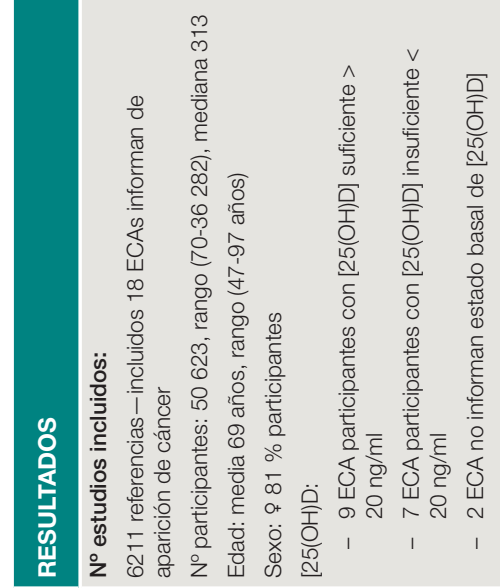

क्ष.

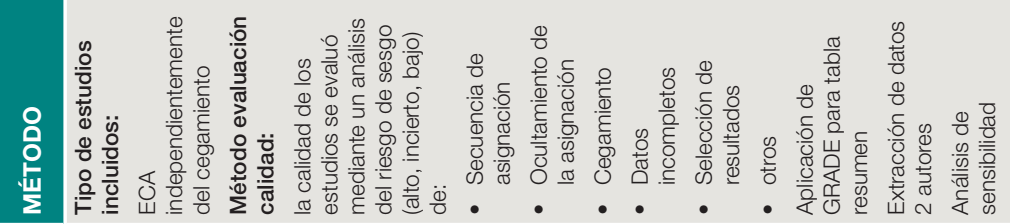
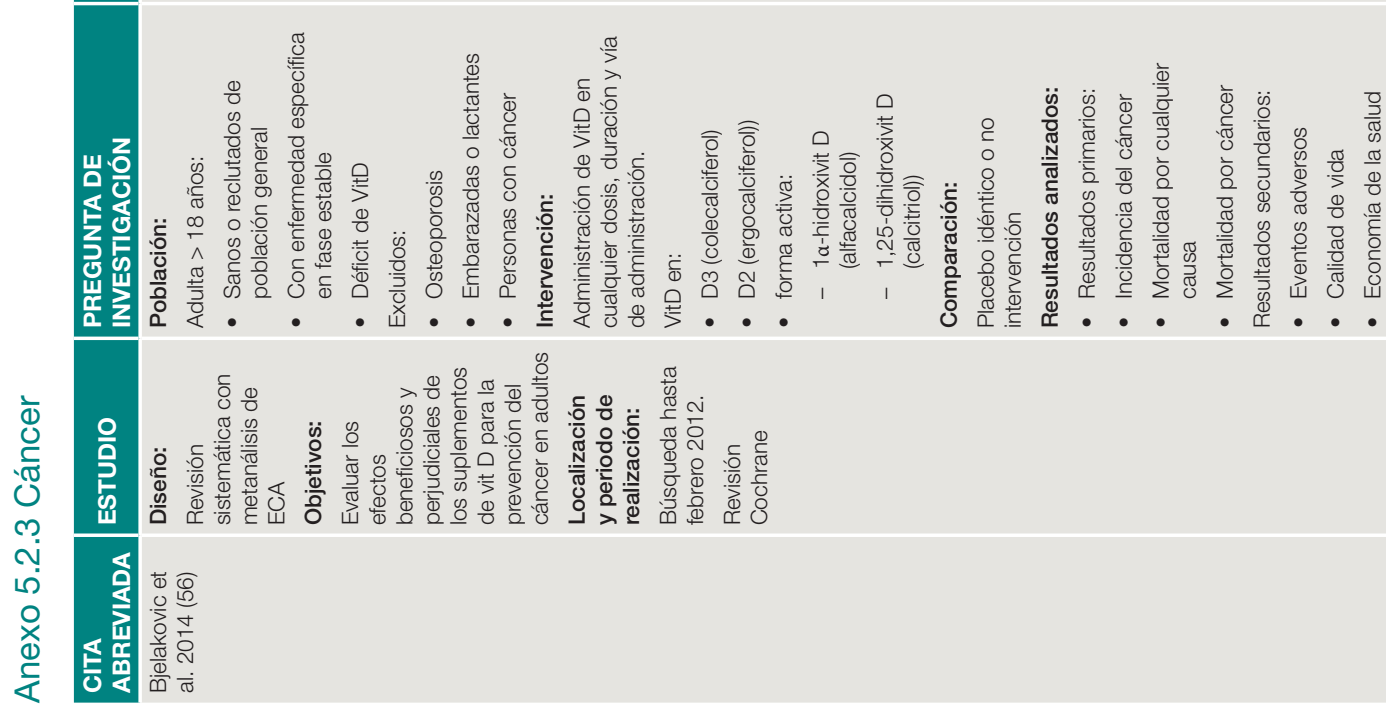

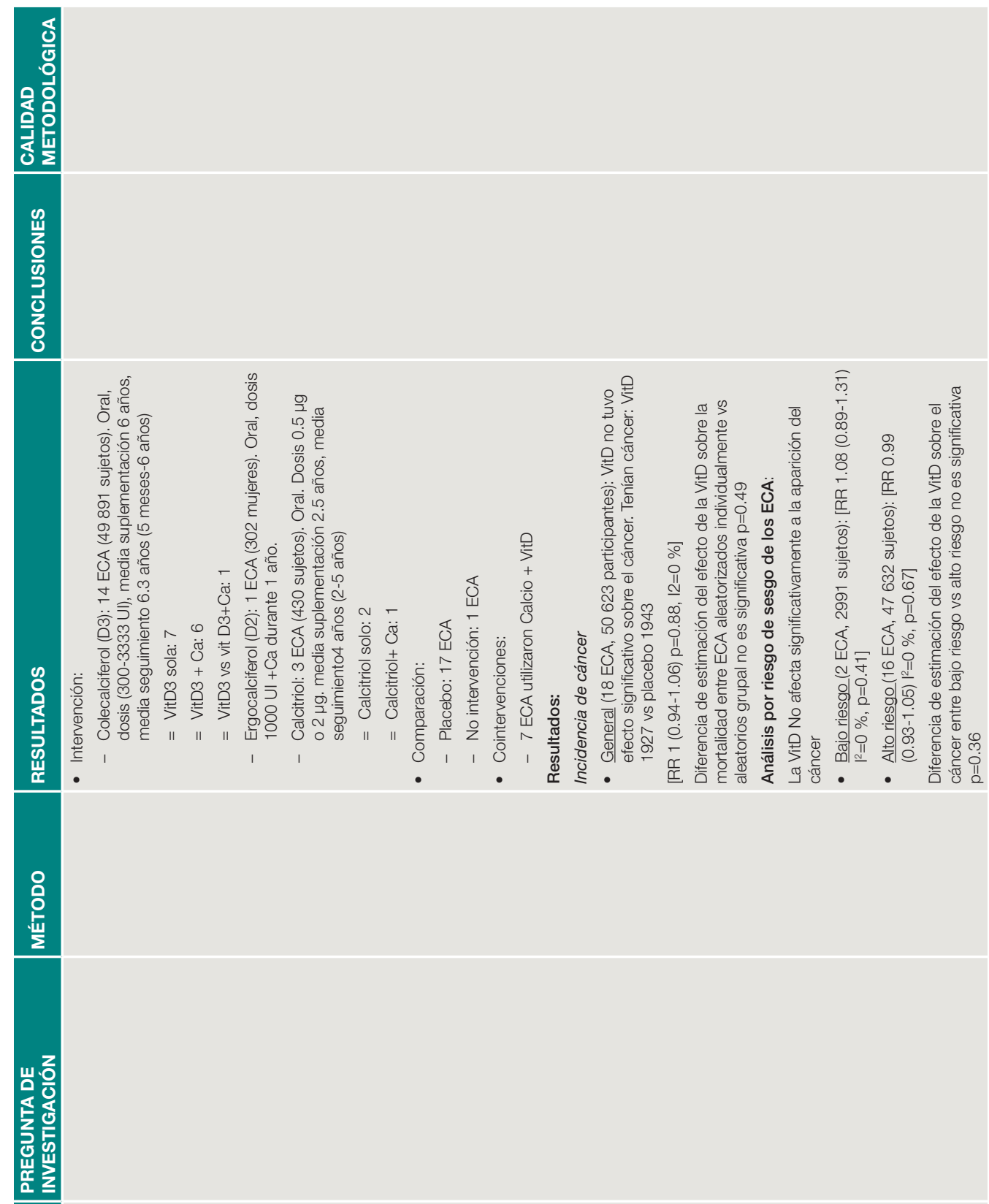

음 


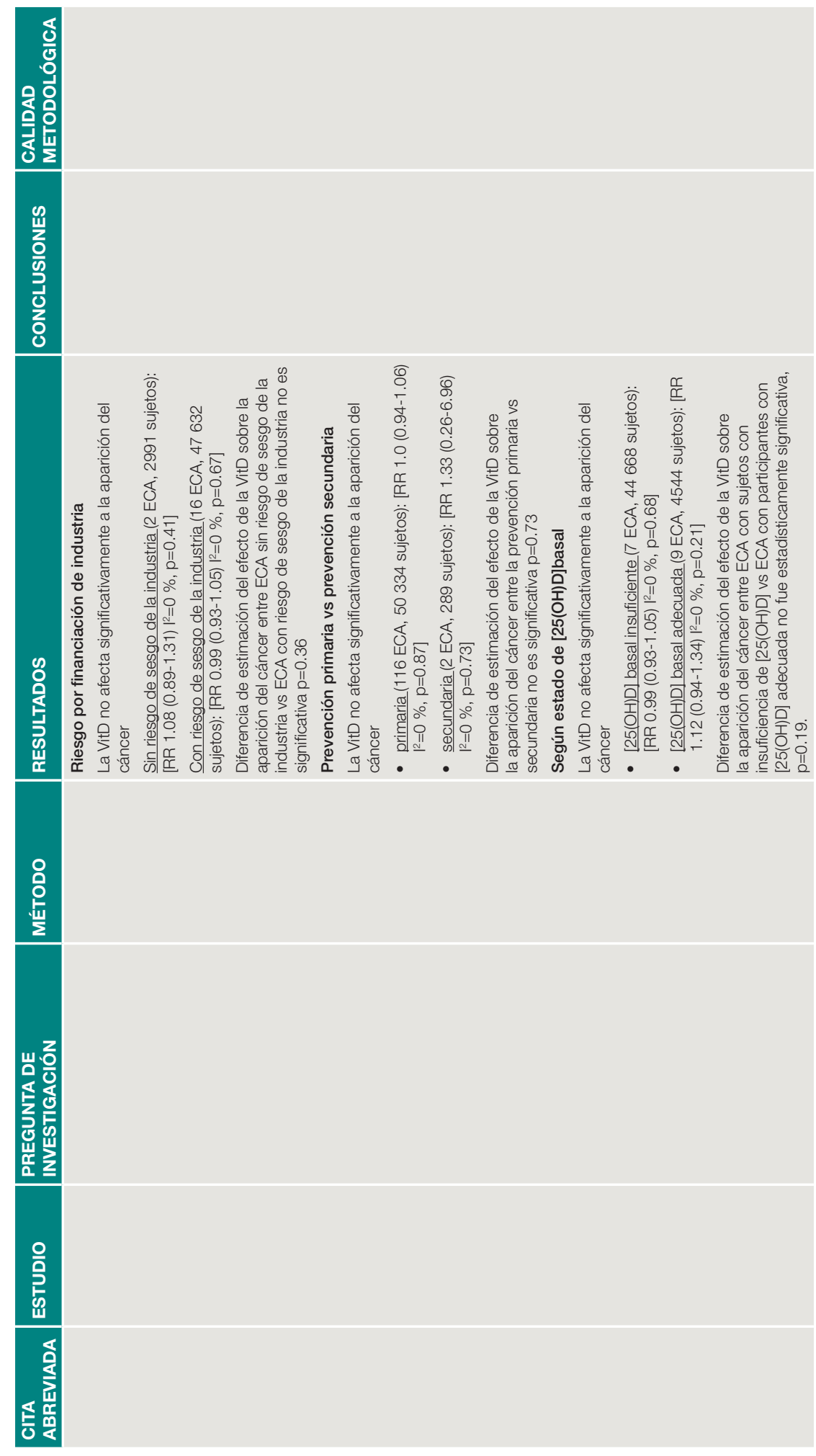




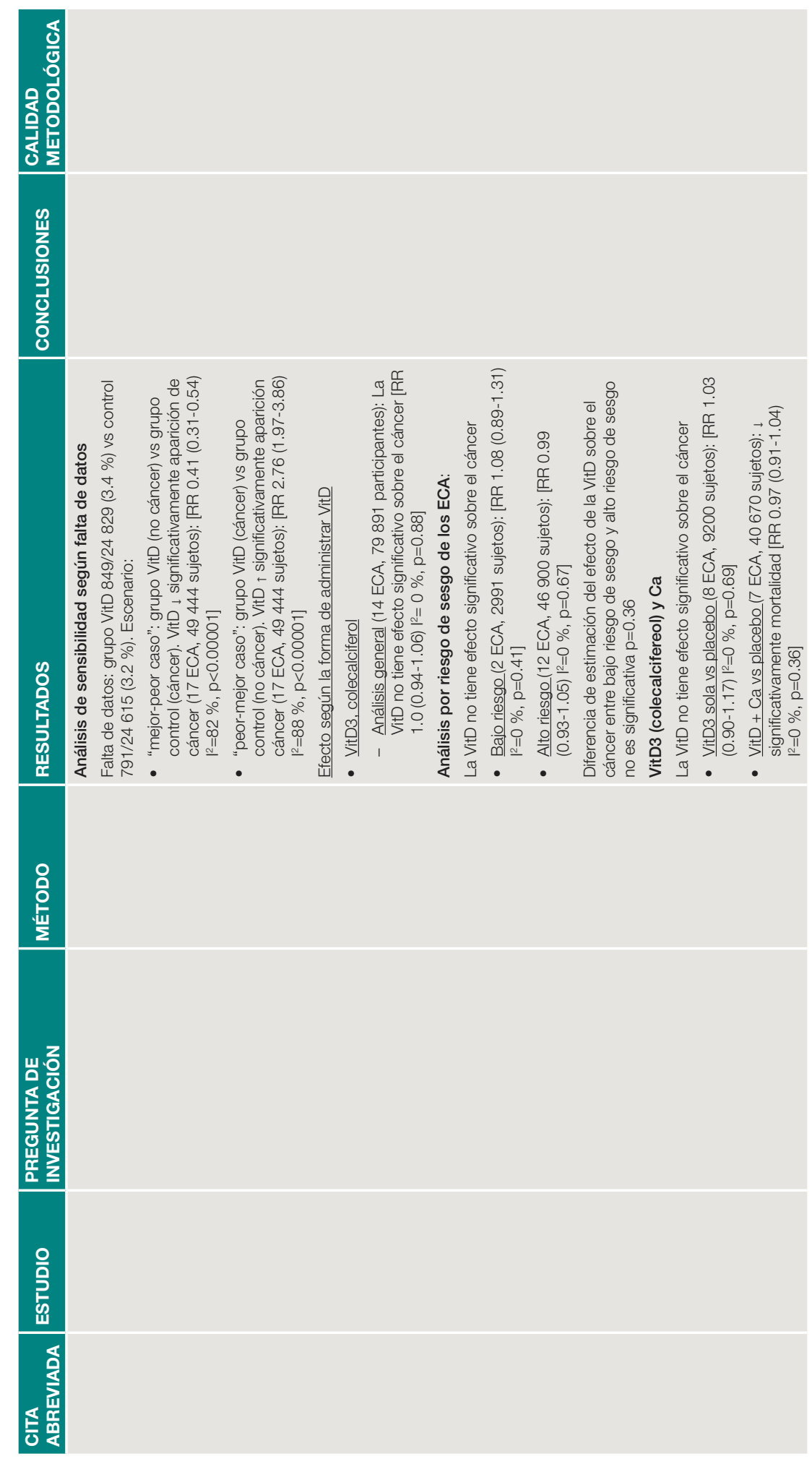




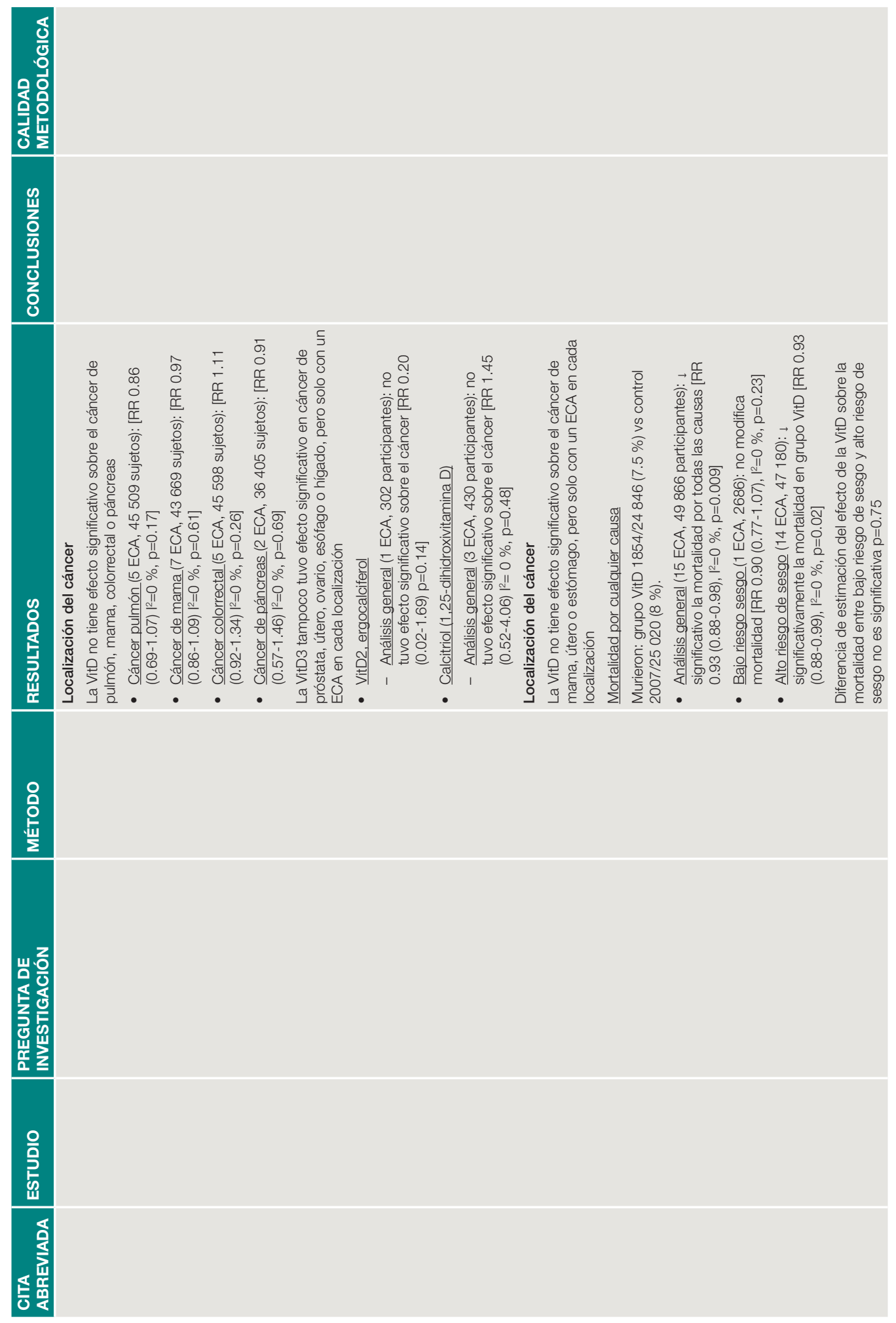



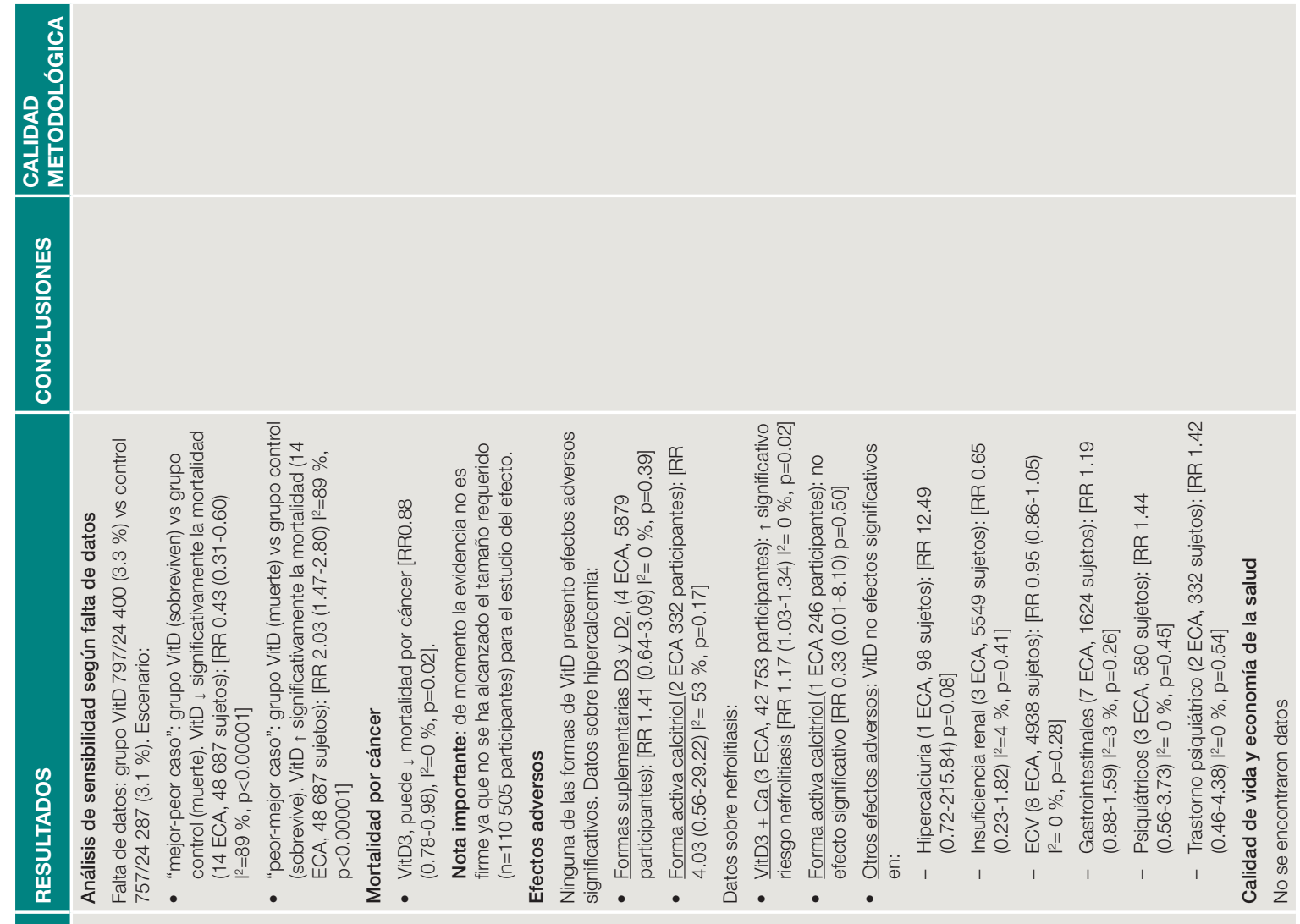

음

高 


\section{Ifli}

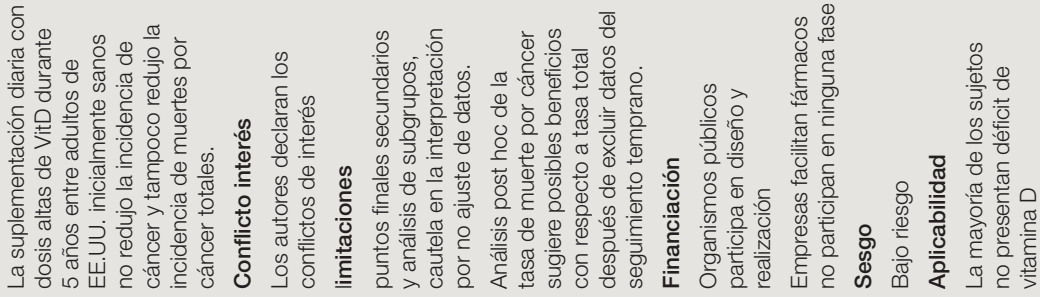

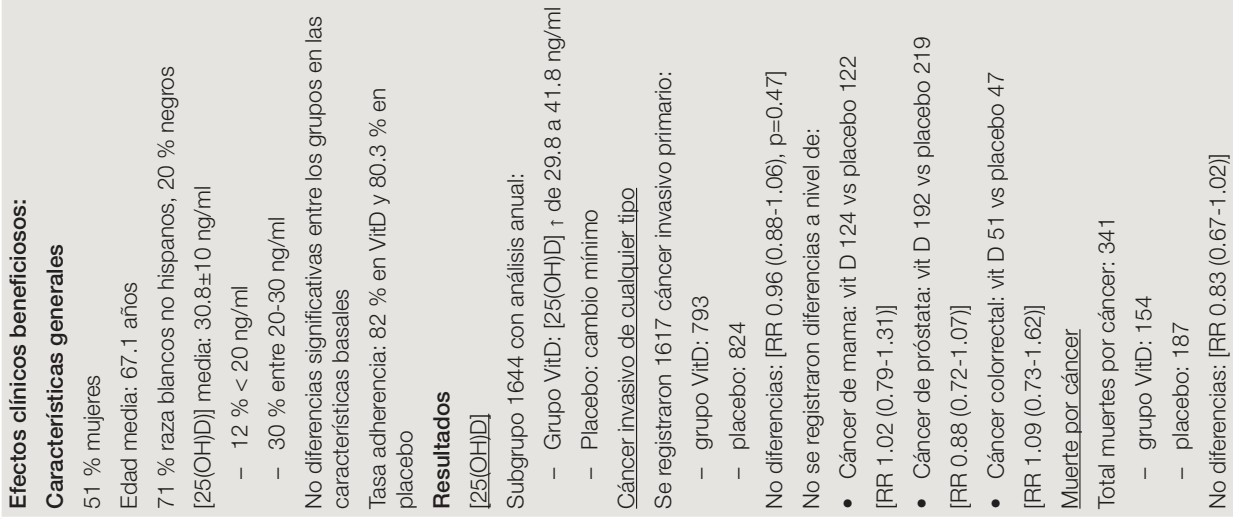

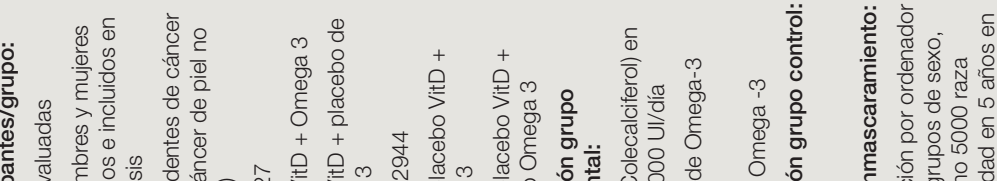

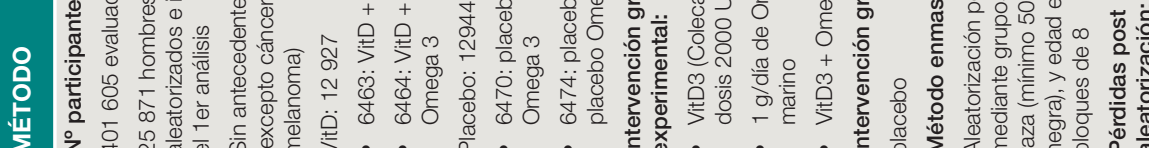

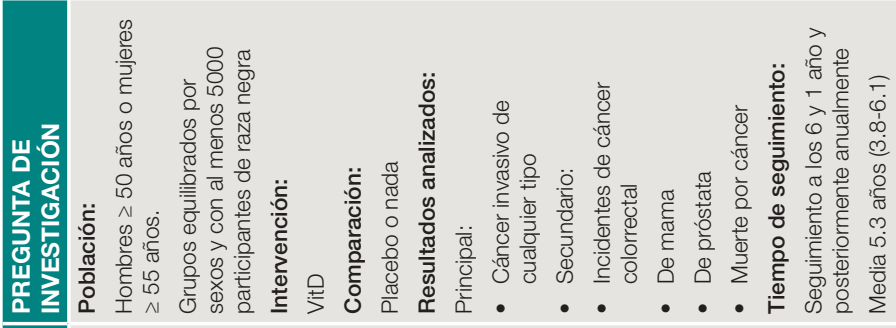

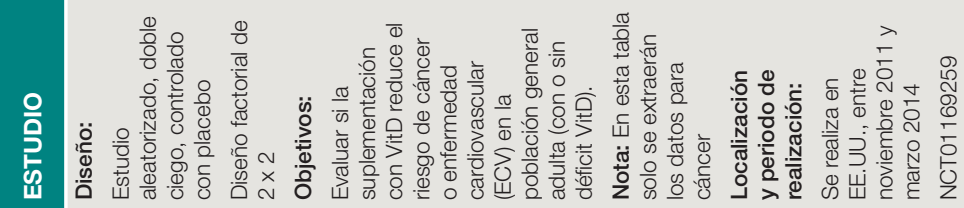




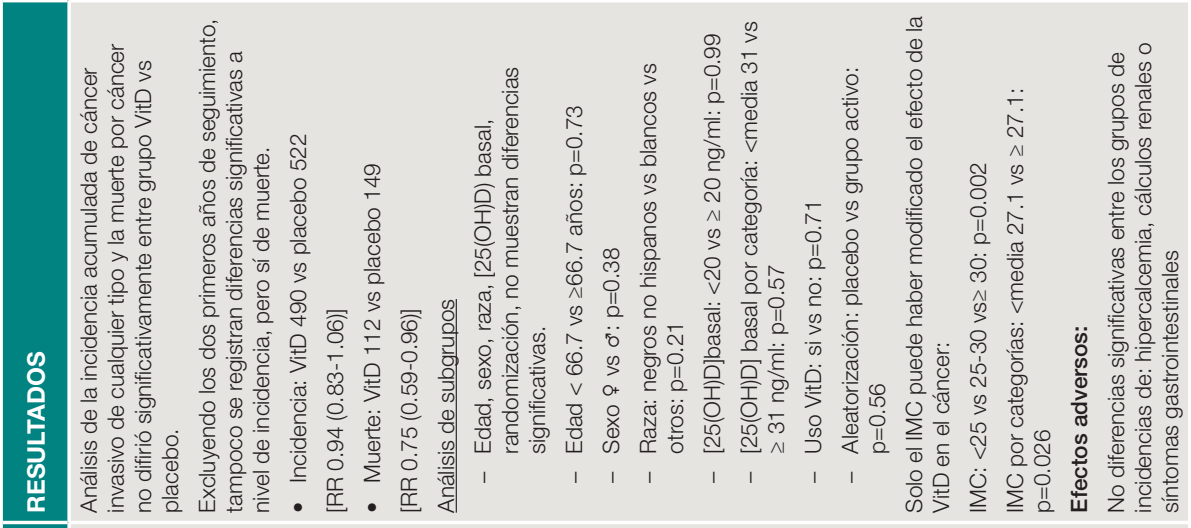

음

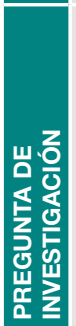

음 


\section{Hill}

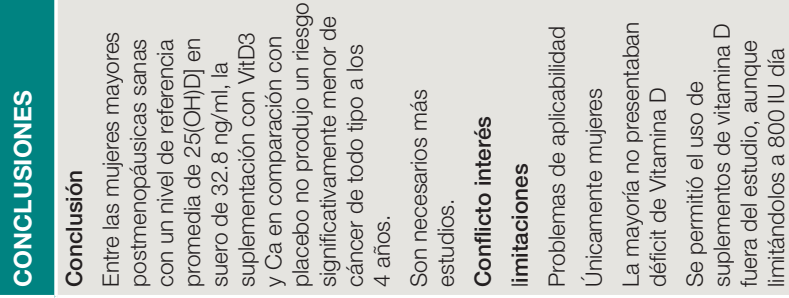

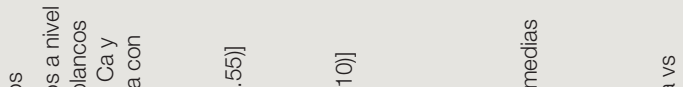

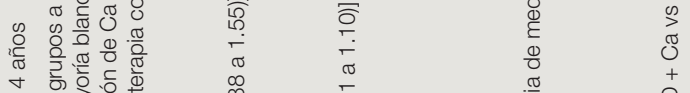

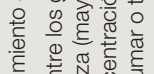

is

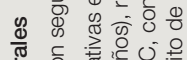

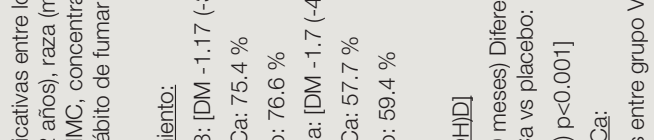

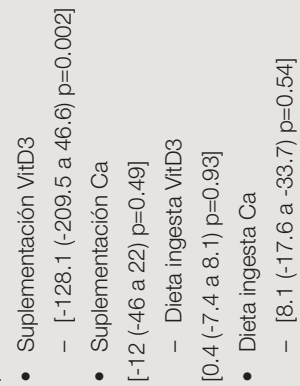

里

ब 흥 응

:

हैं

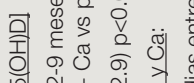

幽

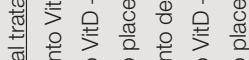

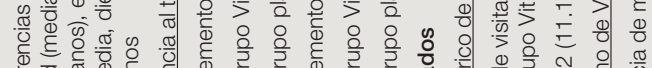

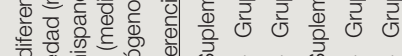

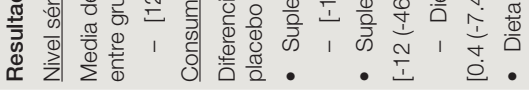

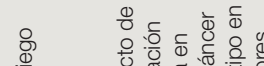

을

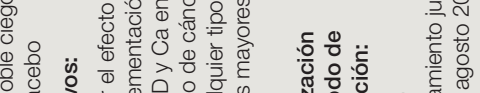

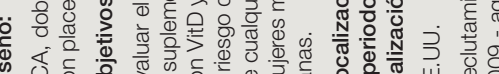

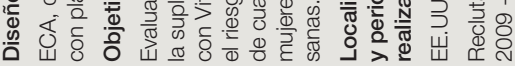

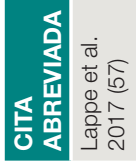




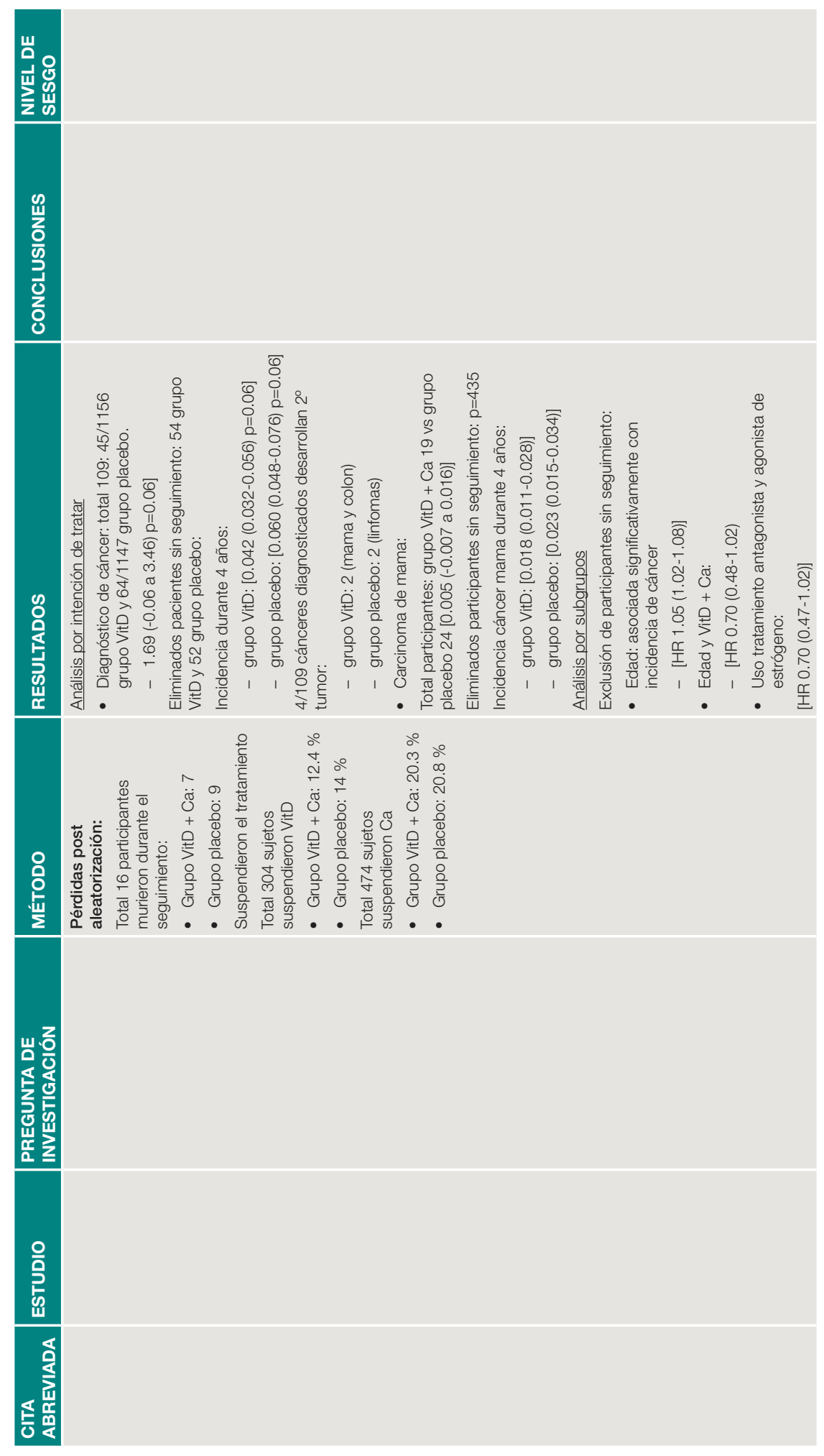




$$
1
$$




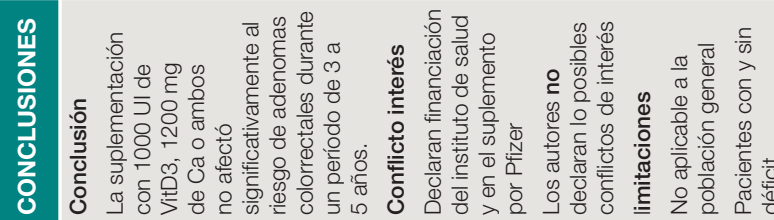

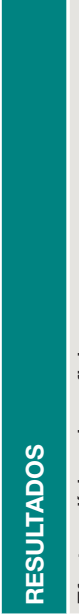

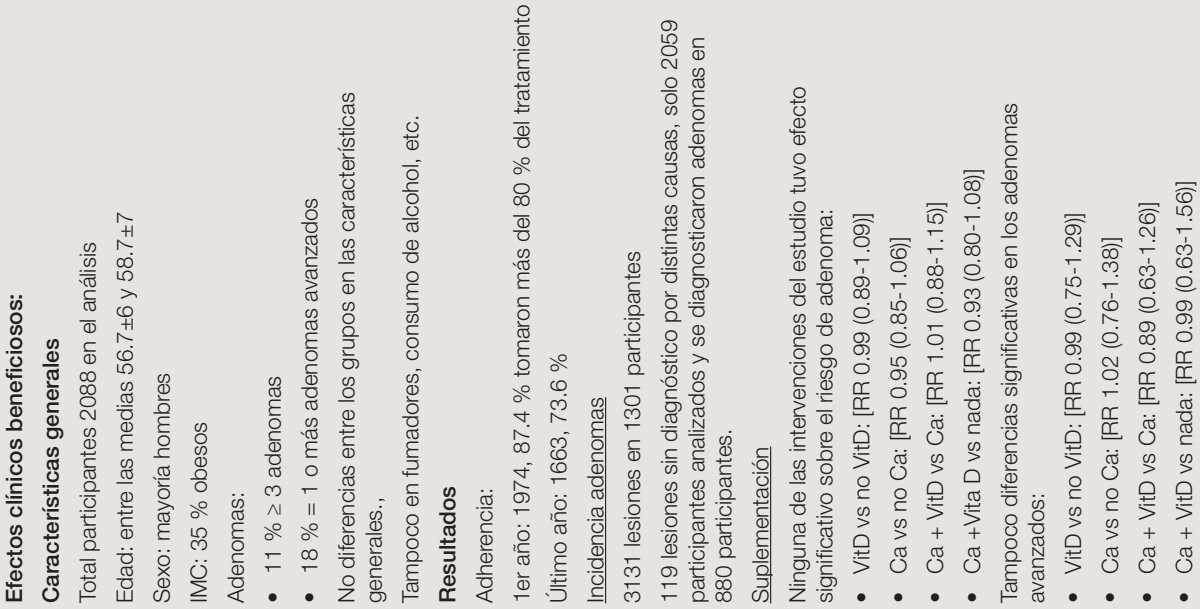

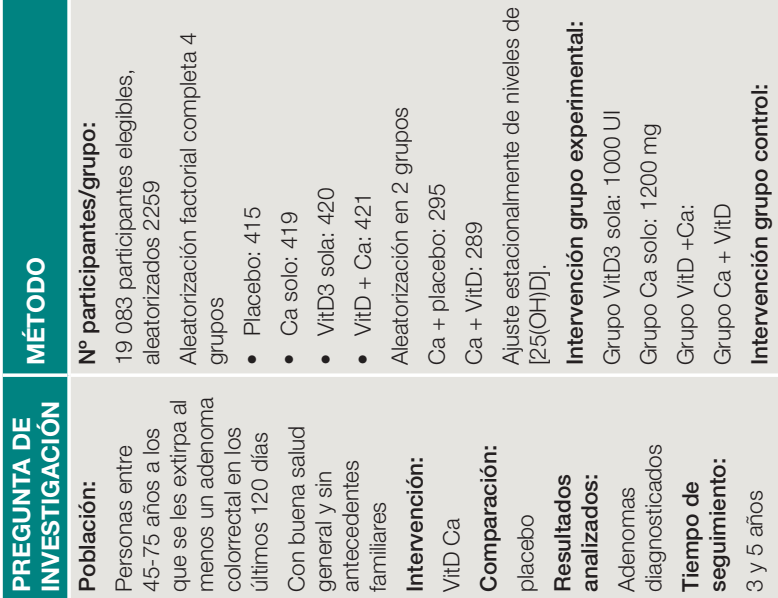

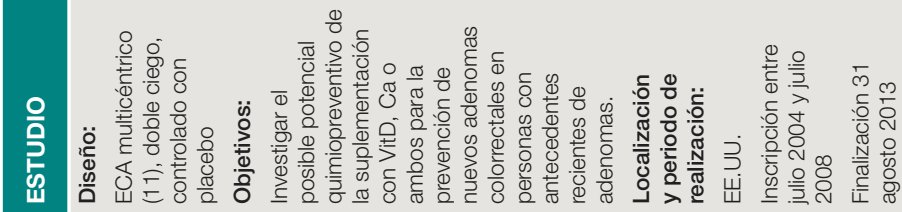

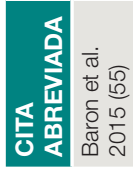




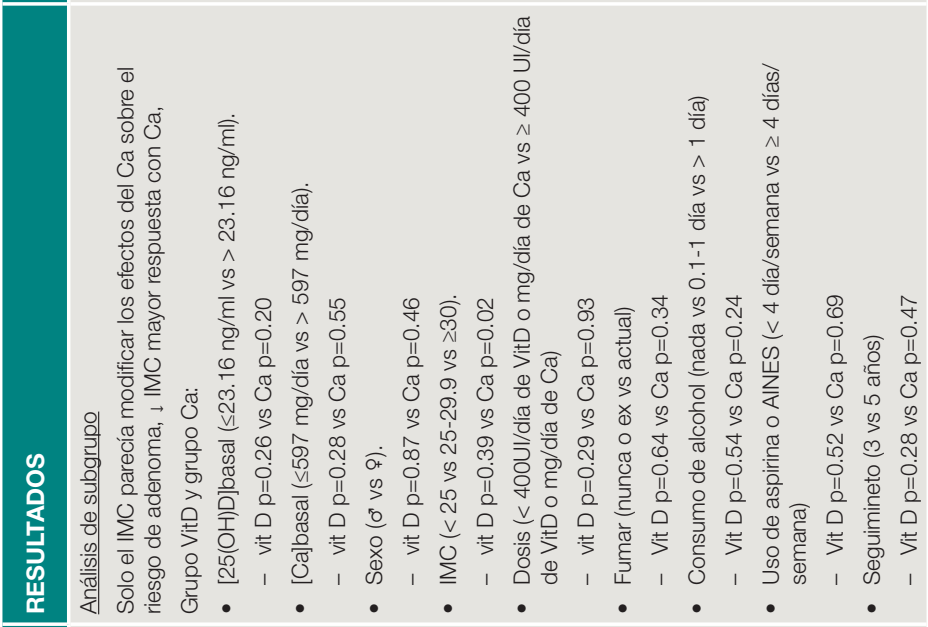

옹

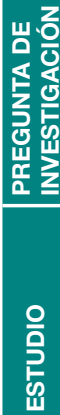

选夏 


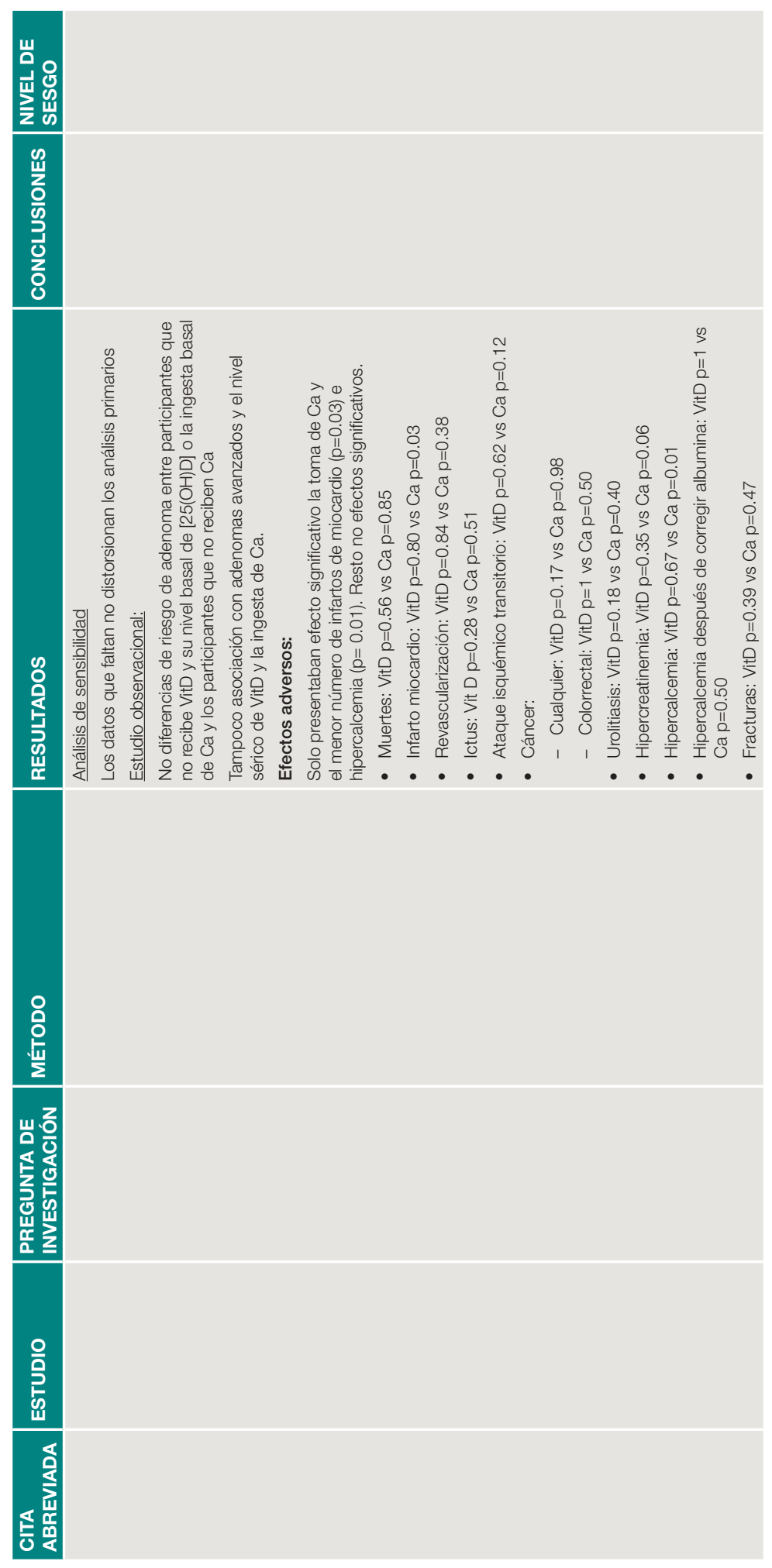




\section{II}

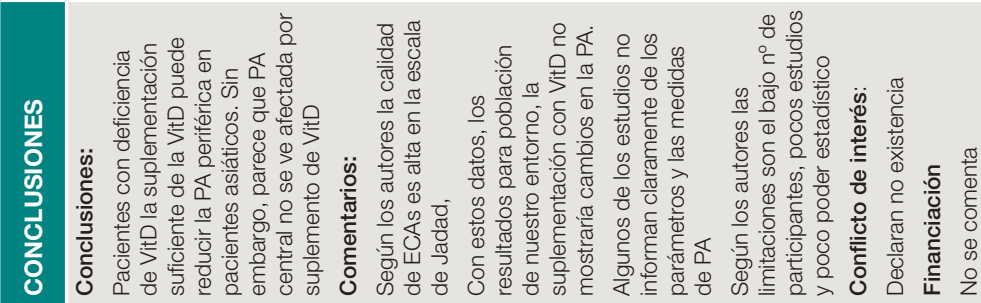

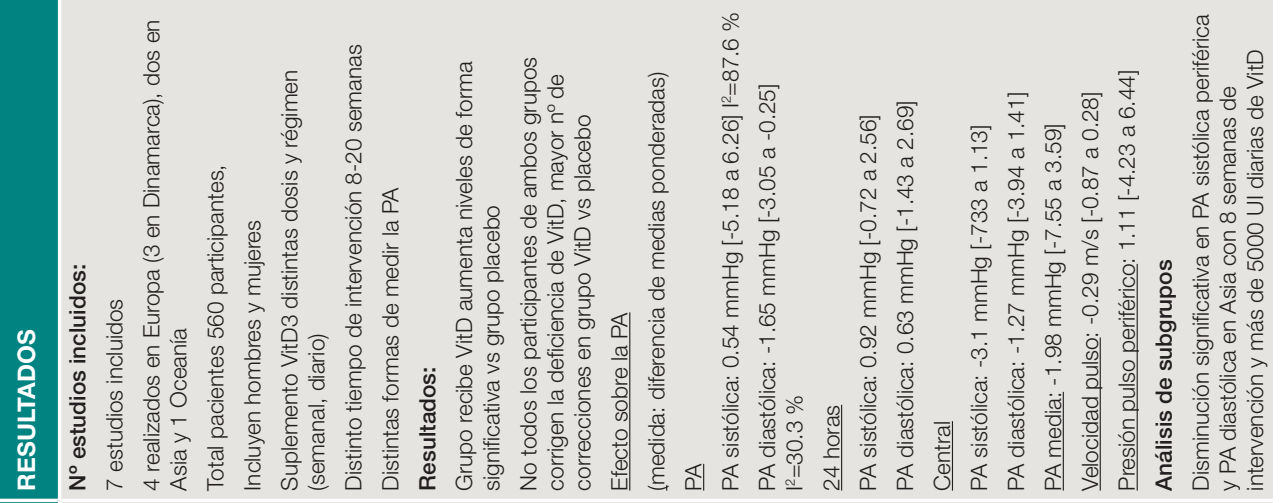

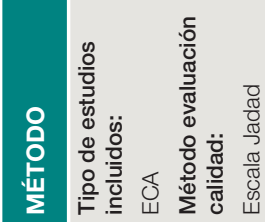

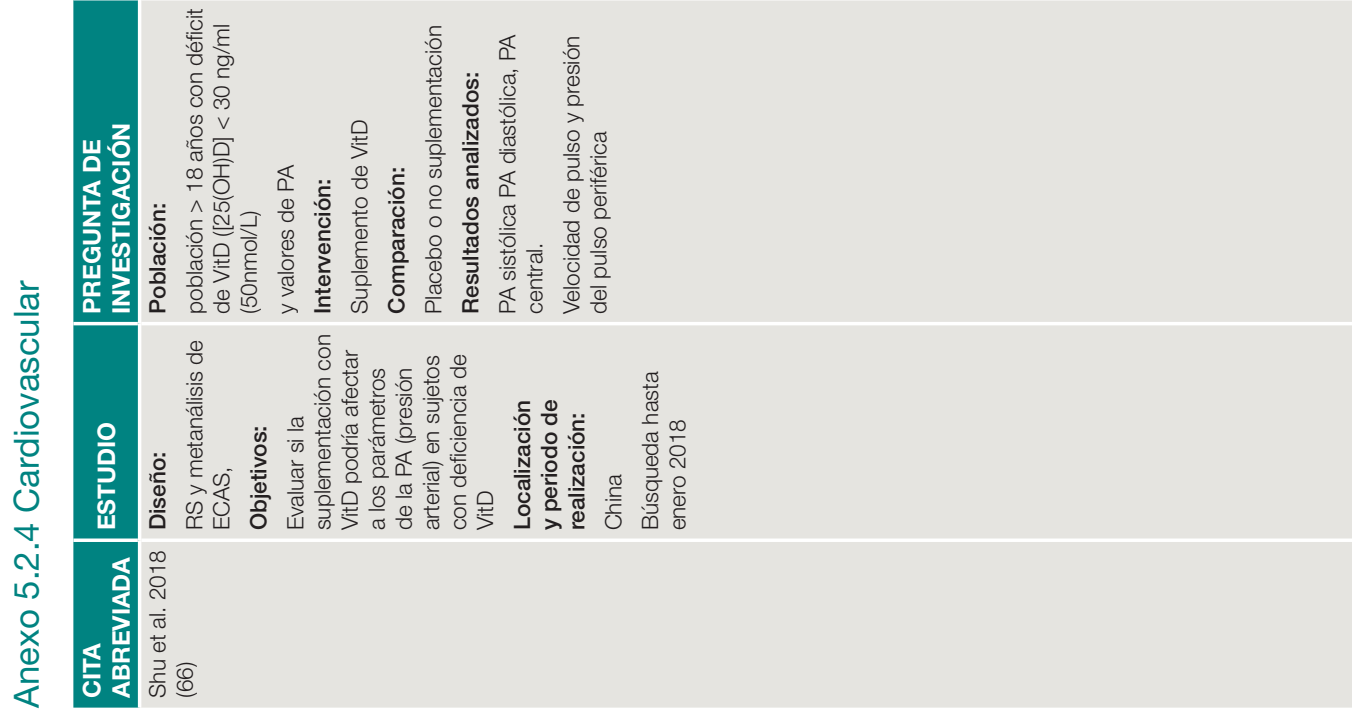




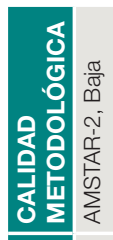

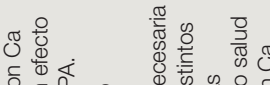

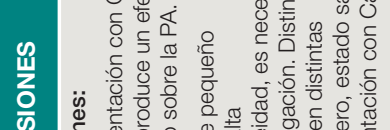

心

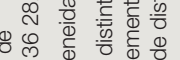

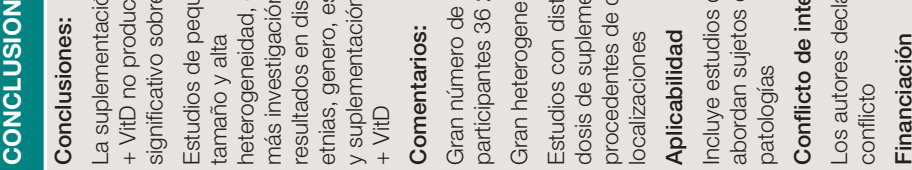

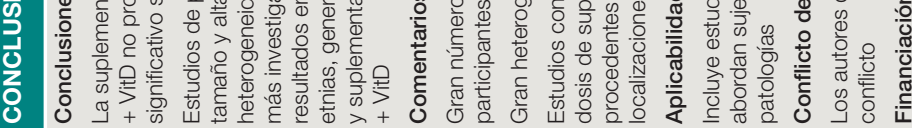

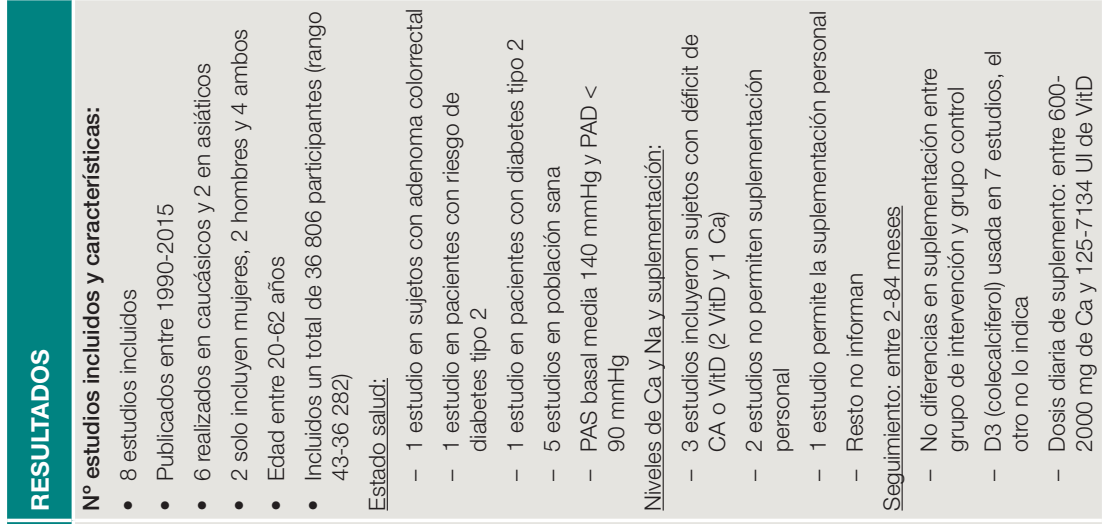

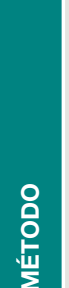

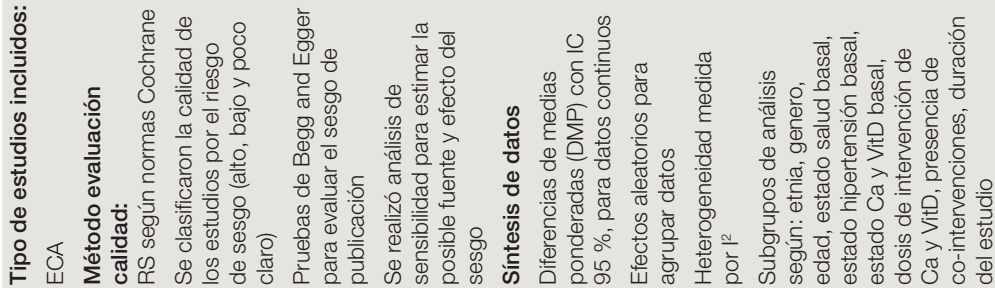

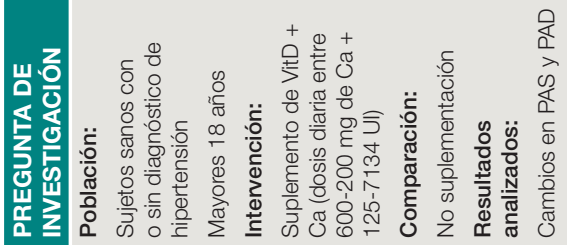

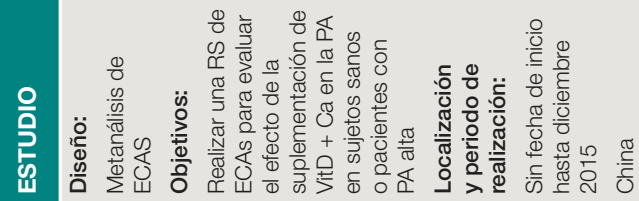

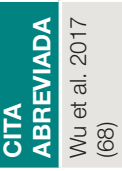



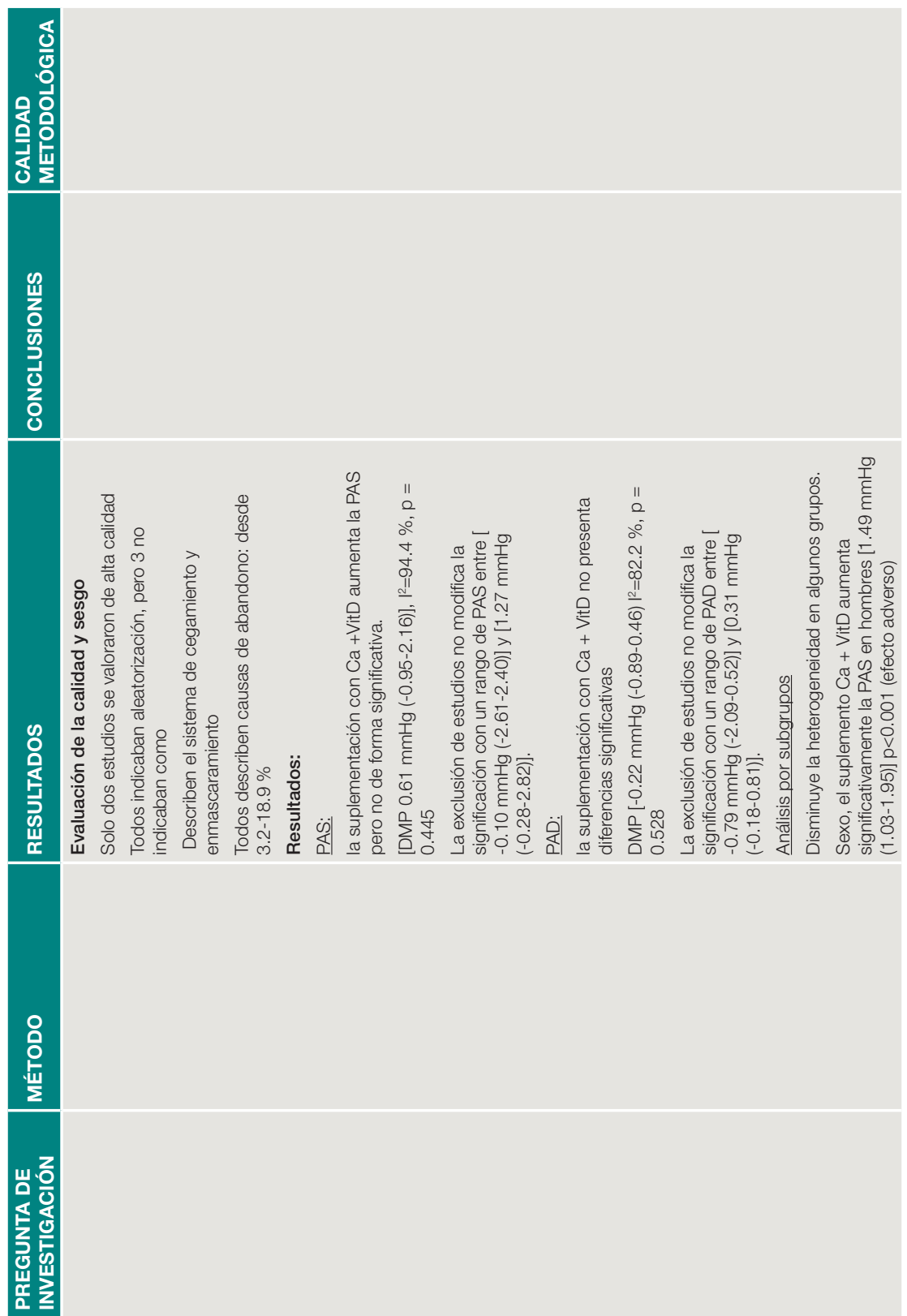

음

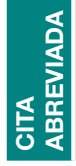




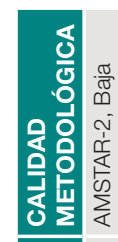

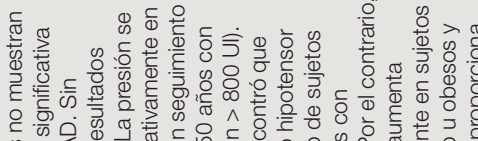

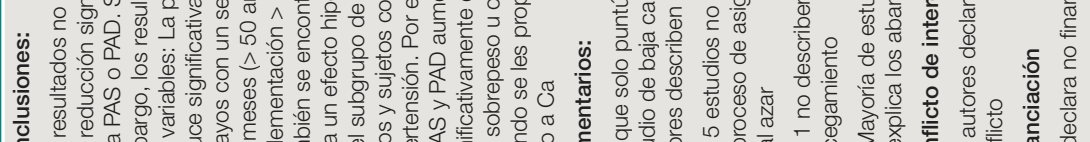

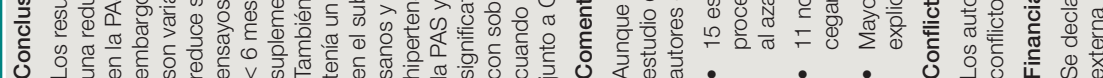
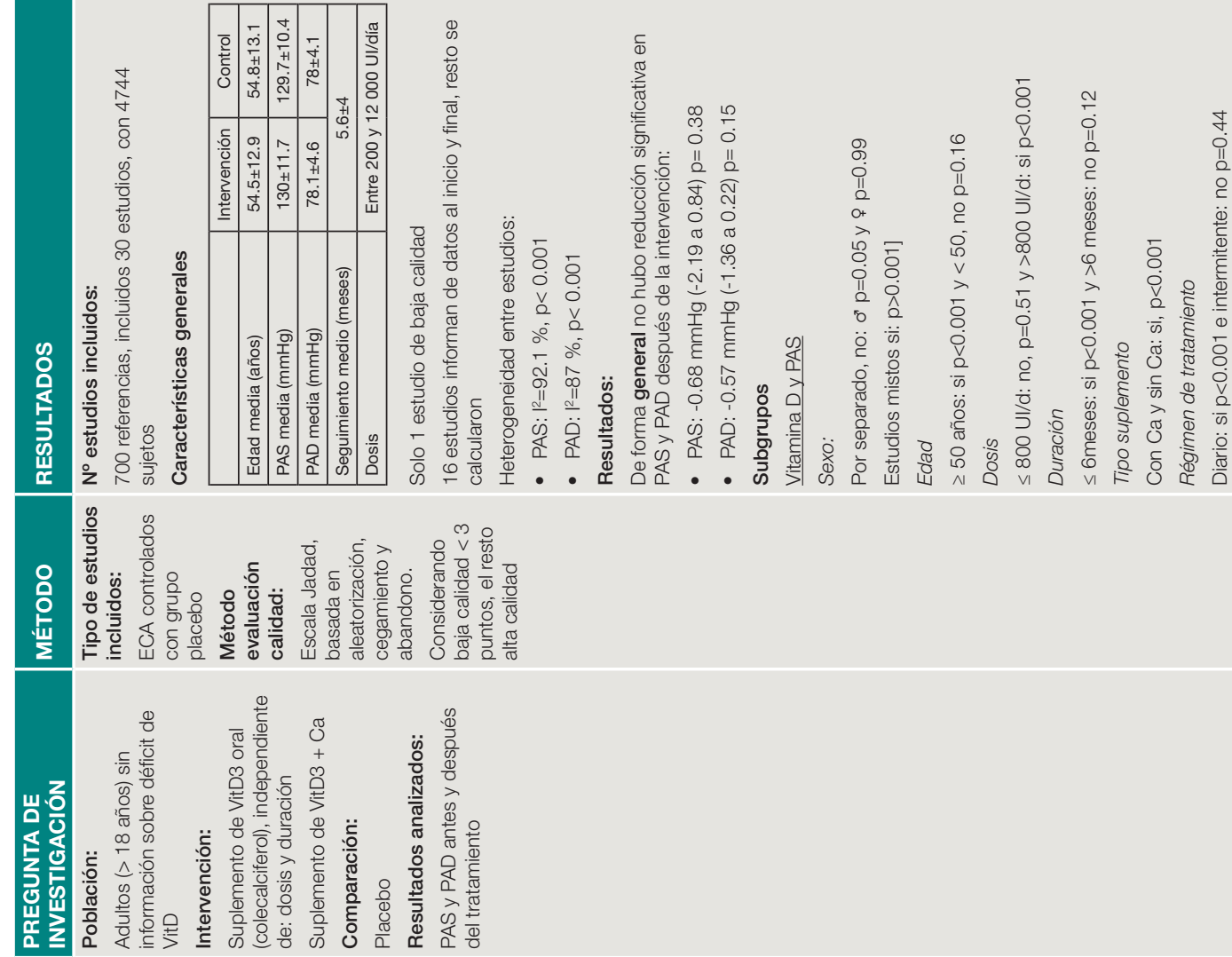

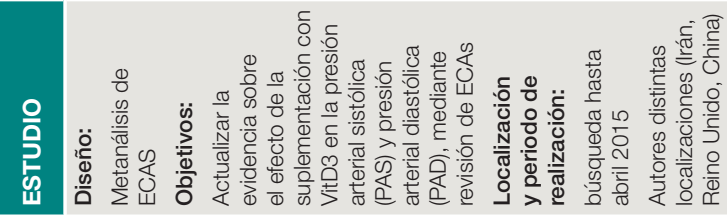

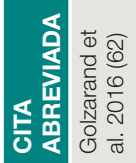




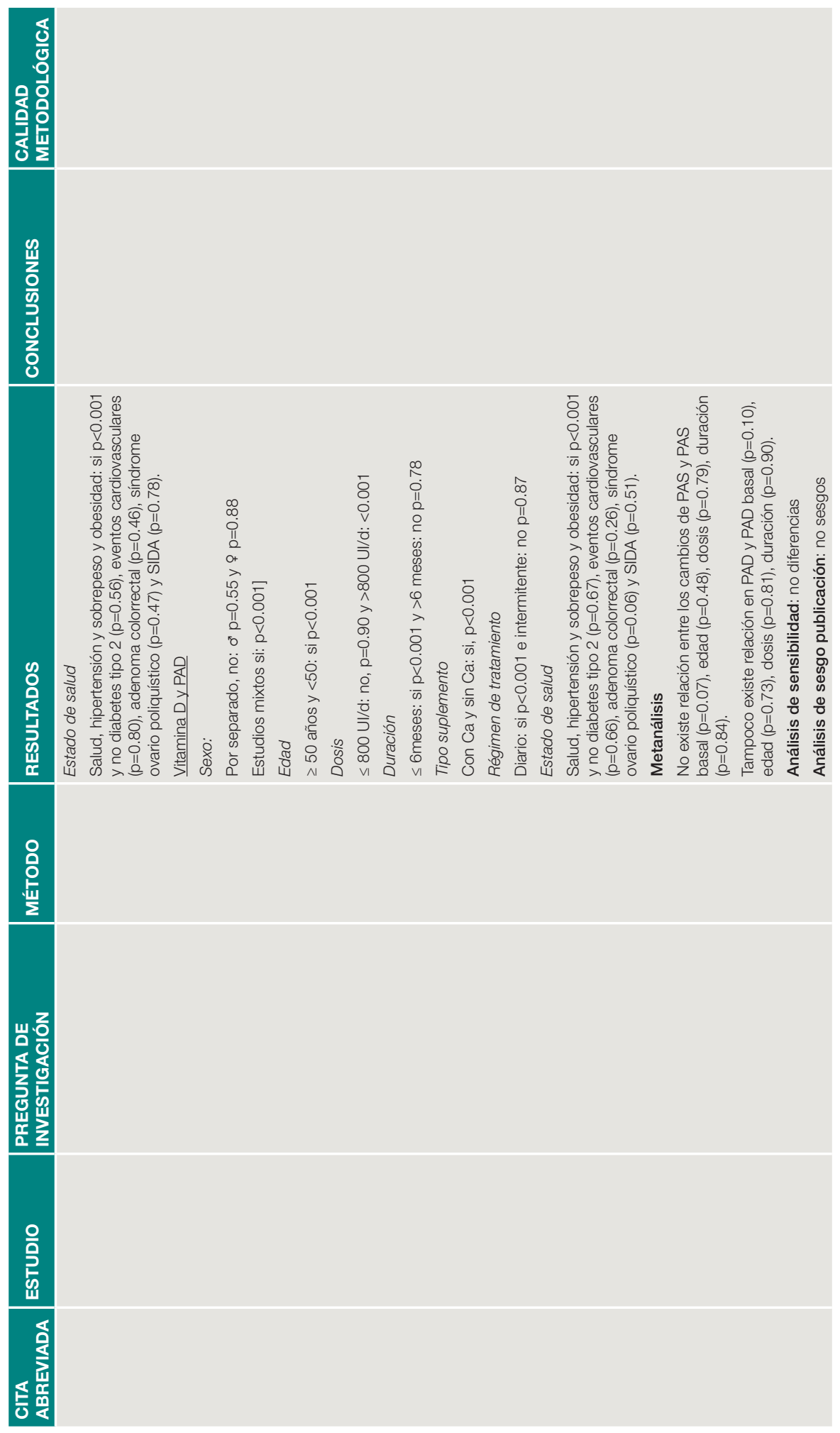


MIIIIIIIIIIIIII

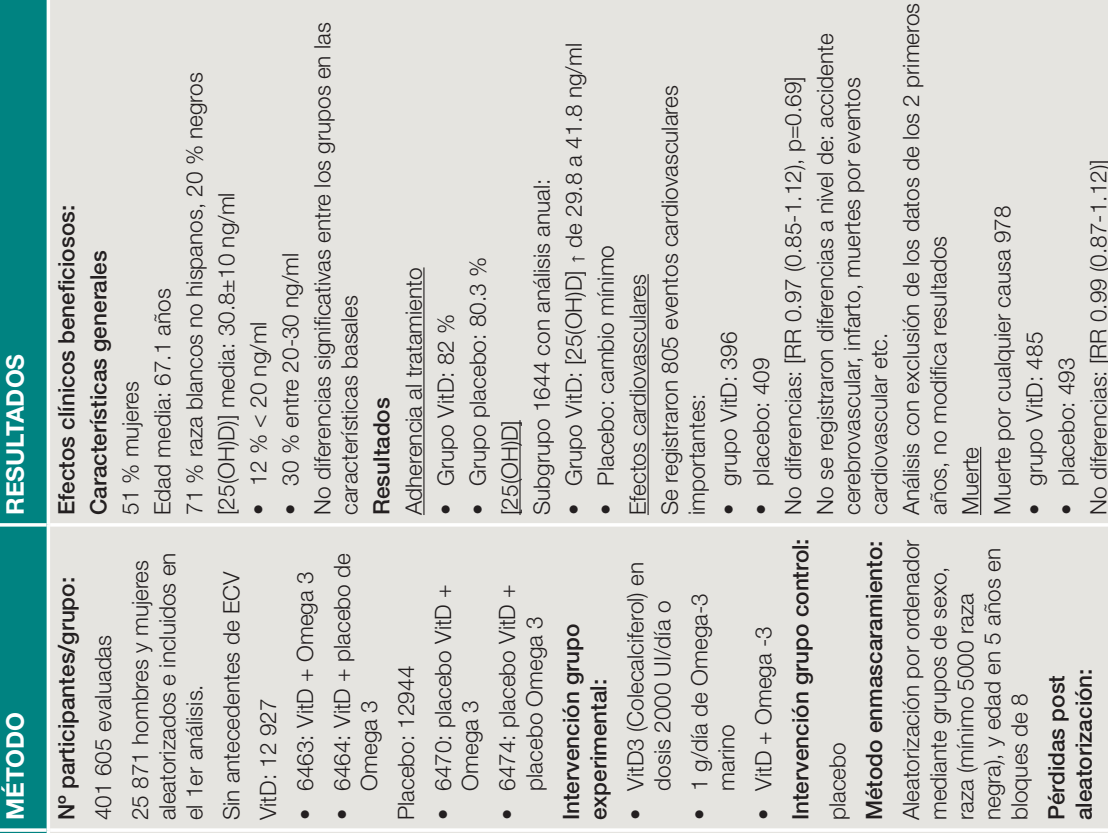

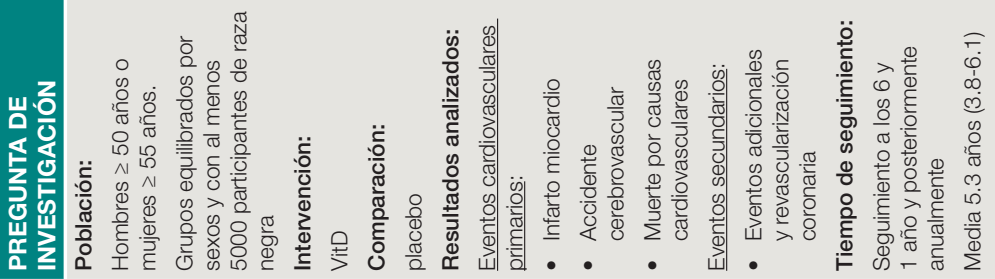

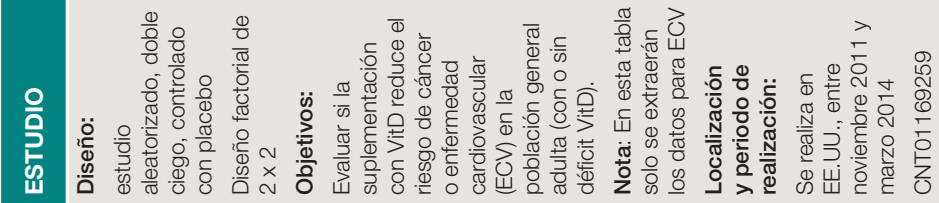

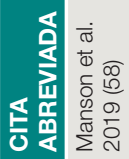




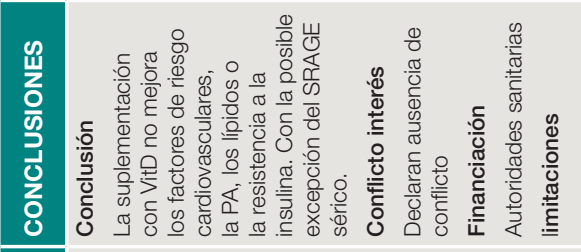

$\frac{0}{0} \frac{0}{0} \frac{0}{0}$ 


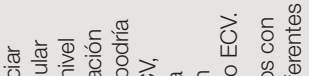
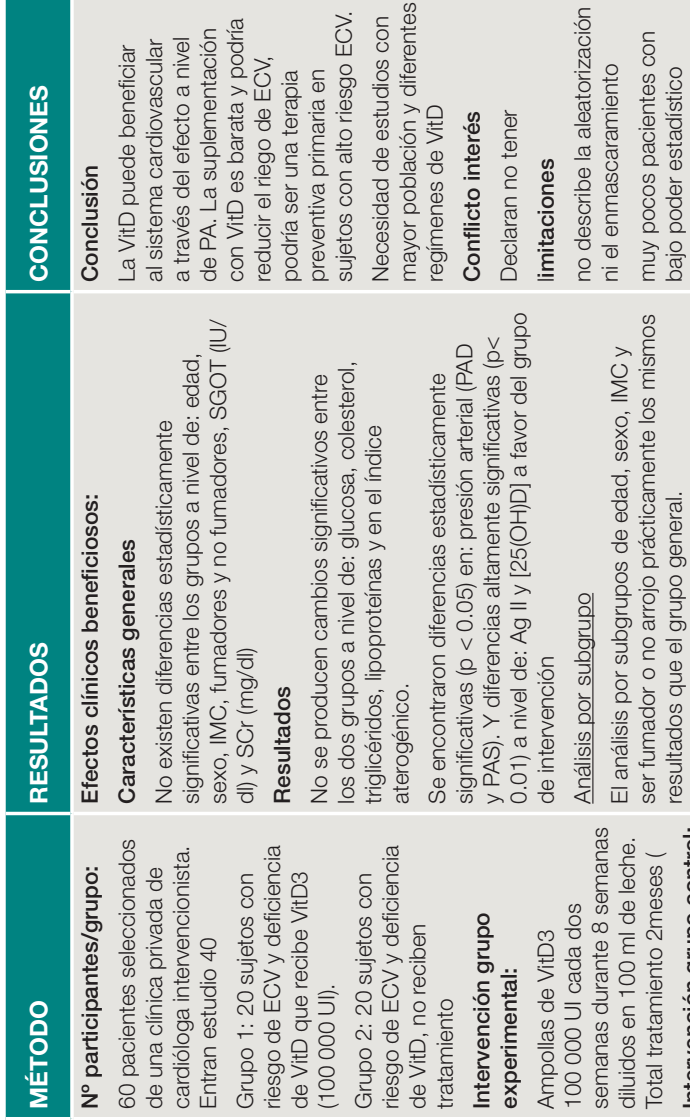

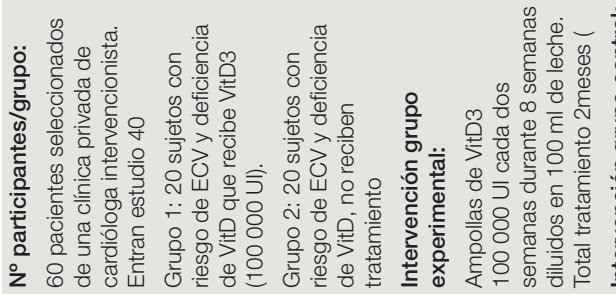

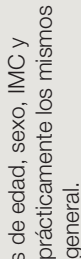

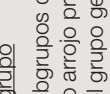

के

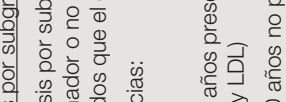

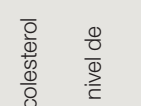

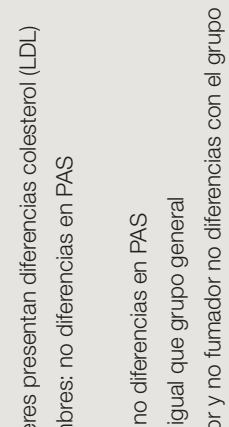

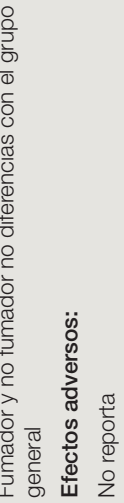

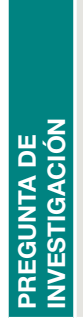

\section{$\circ$}

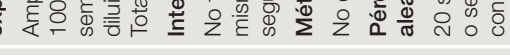

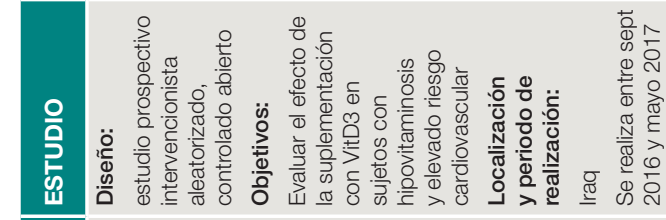

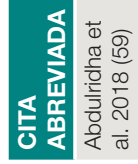


il

.

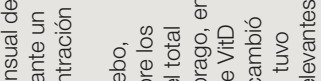

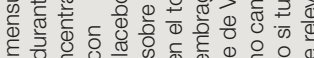

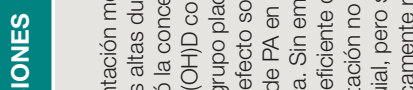

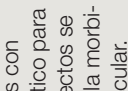

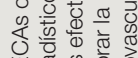

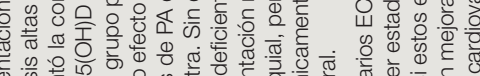

T)

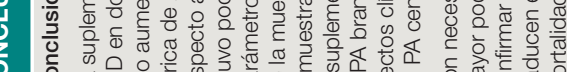

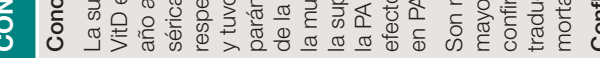

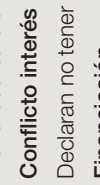

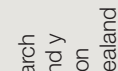

웡요

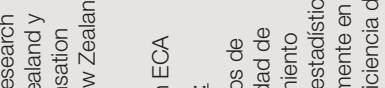

$\frac{5}{\text { क }}$

ฯ

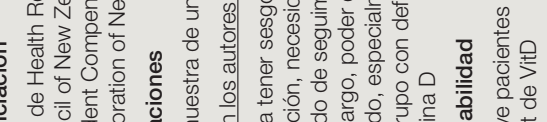

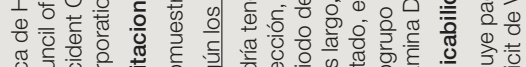

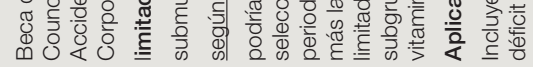

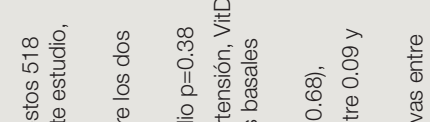

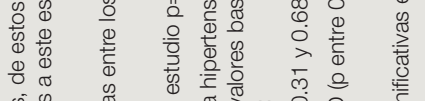

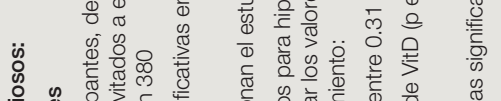

की

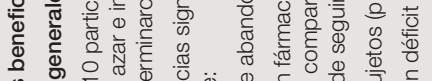

\& 80.000

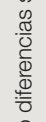

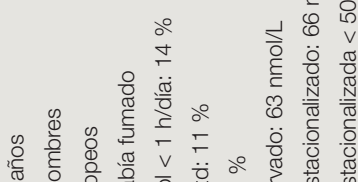

के वे

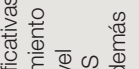

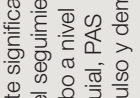

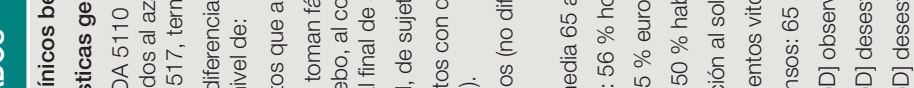

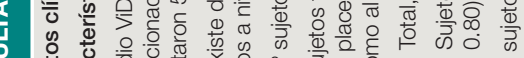

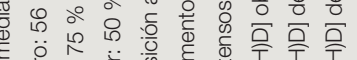
का

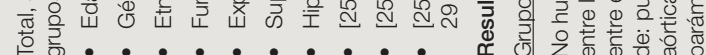

橤

\section{ᄃ。}

里

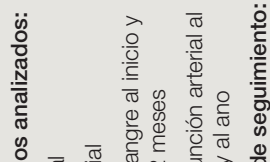

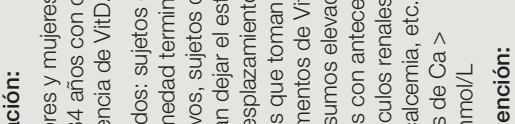

$\begin{array}{lll} & \\ 0 & \end{array}$

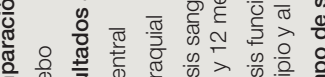

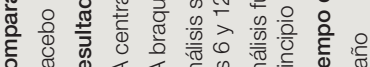

\begin{tabular}{ll}
\hline \\
\hline
\end{tabular} 

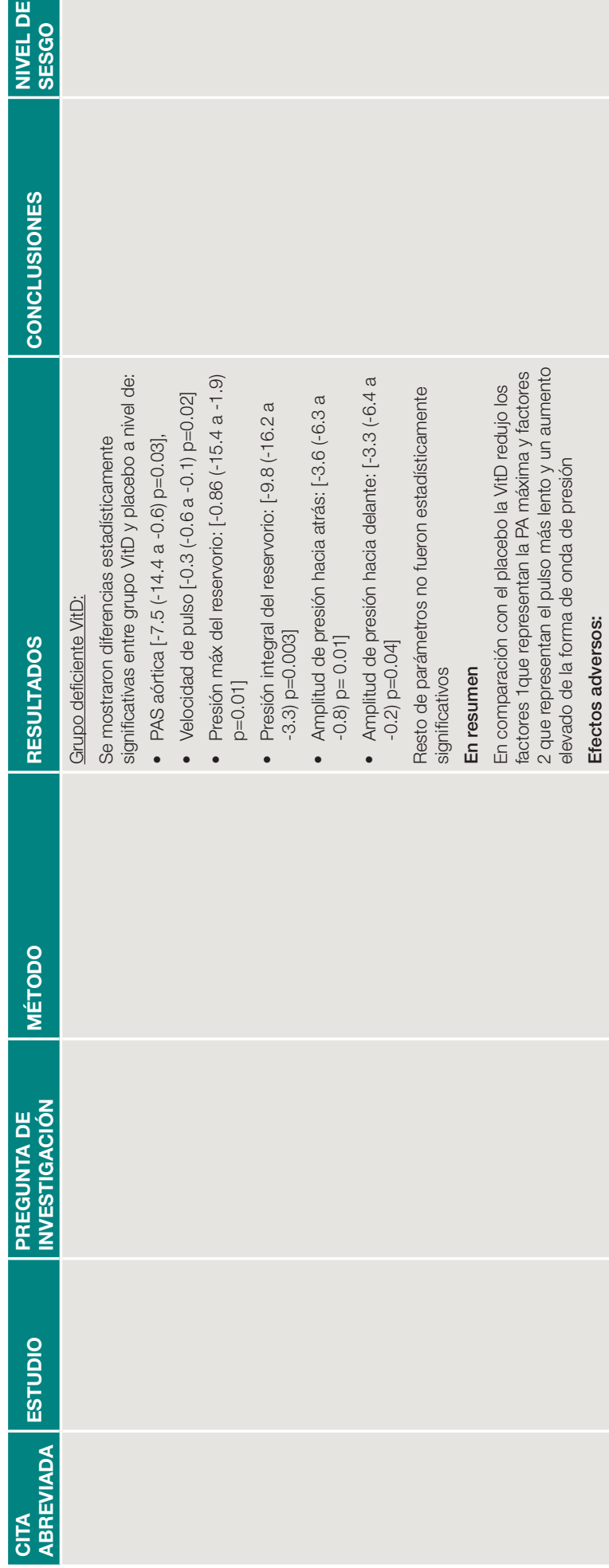


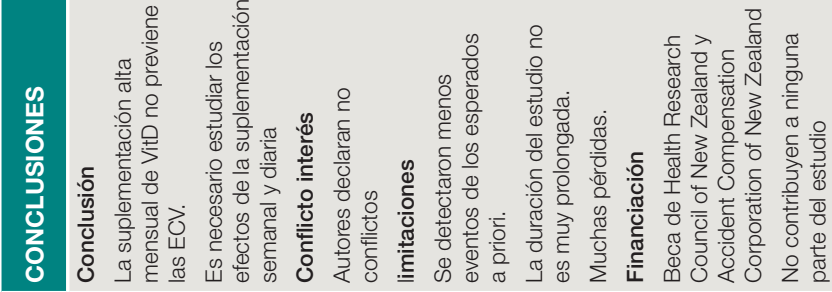

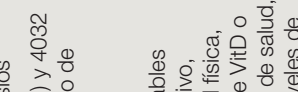

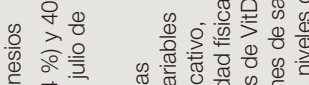

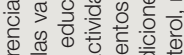

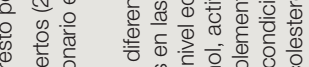

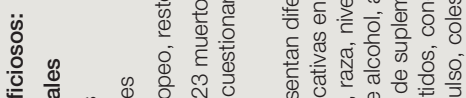

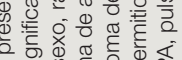

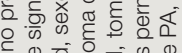

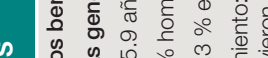

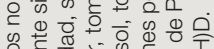

\section{융요

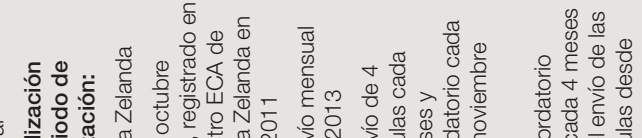

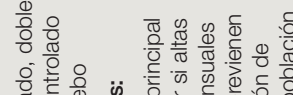

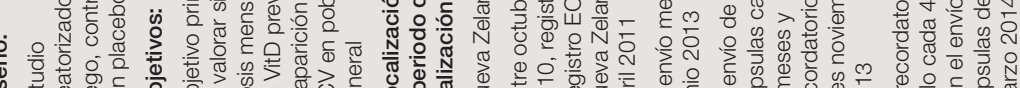

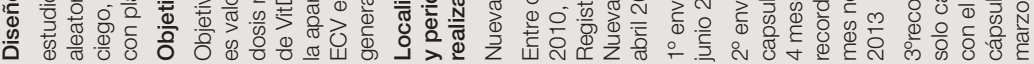

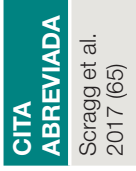




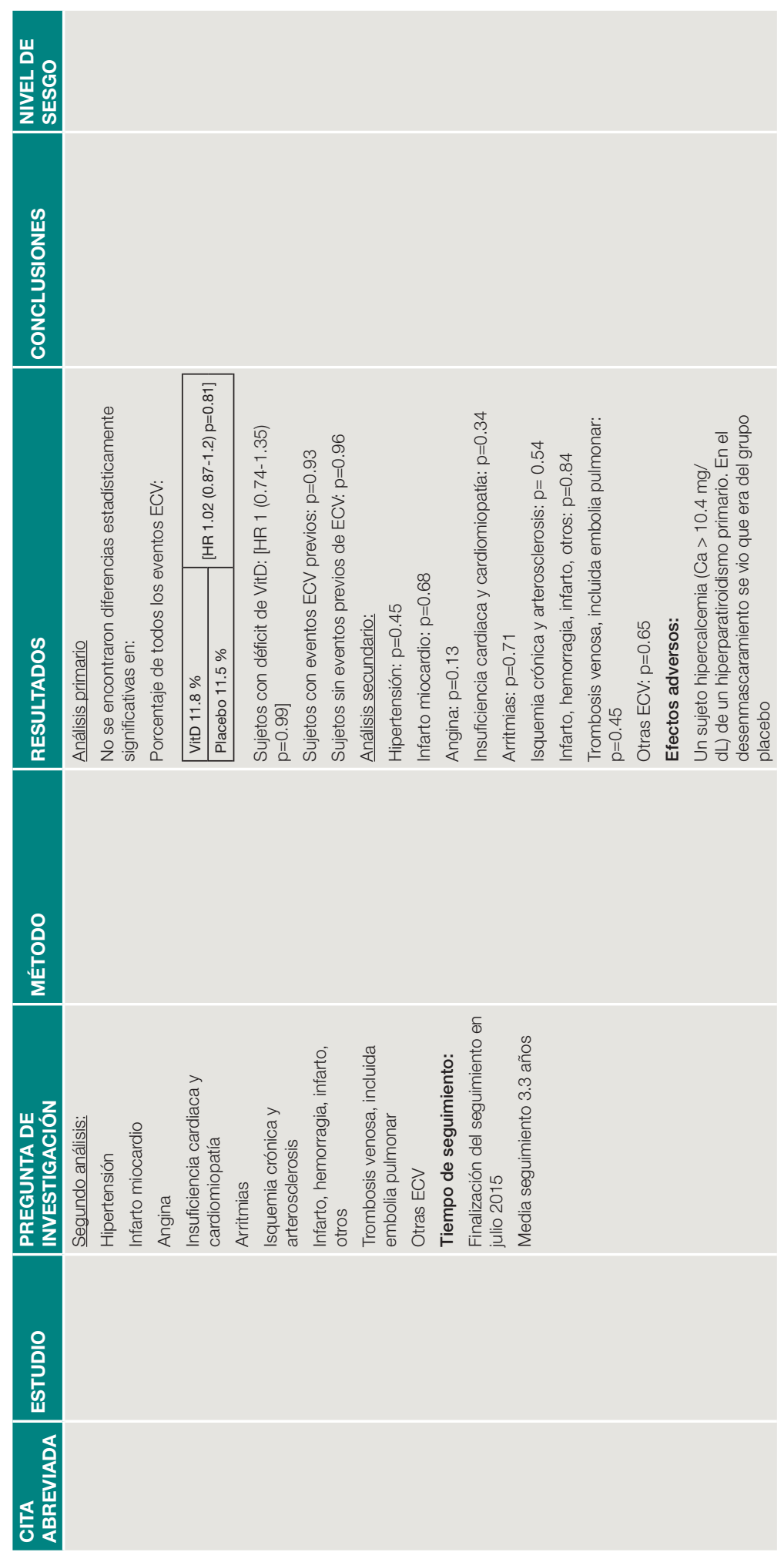




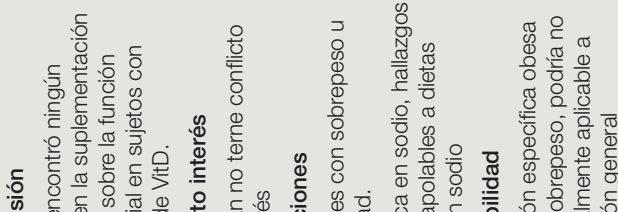

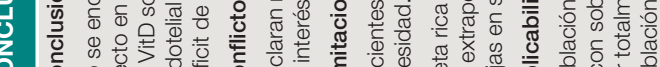

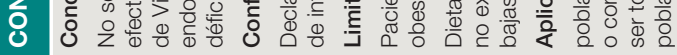

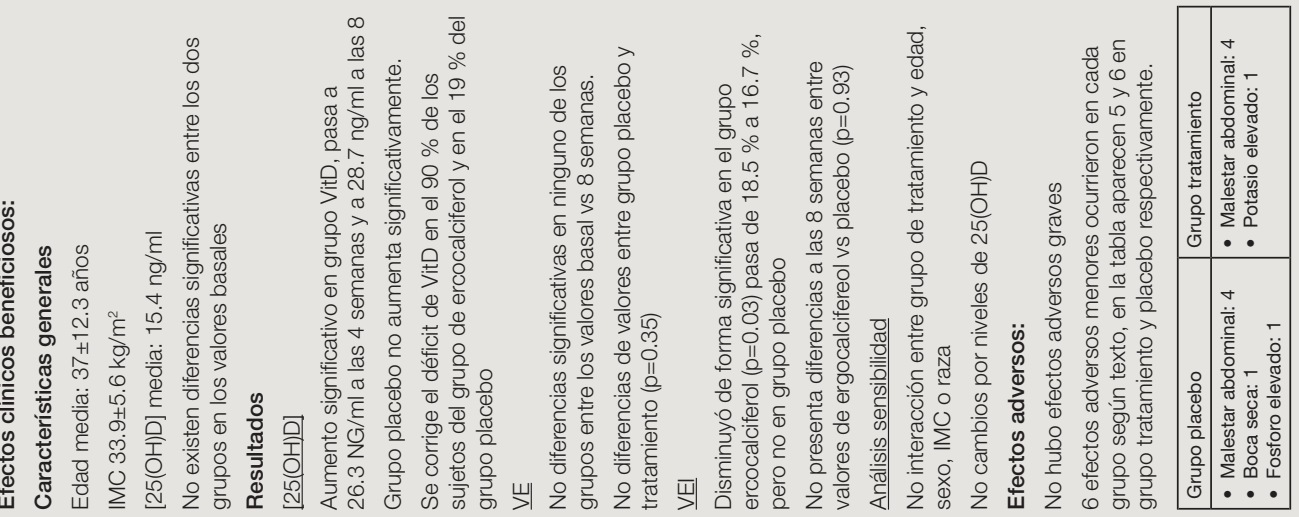

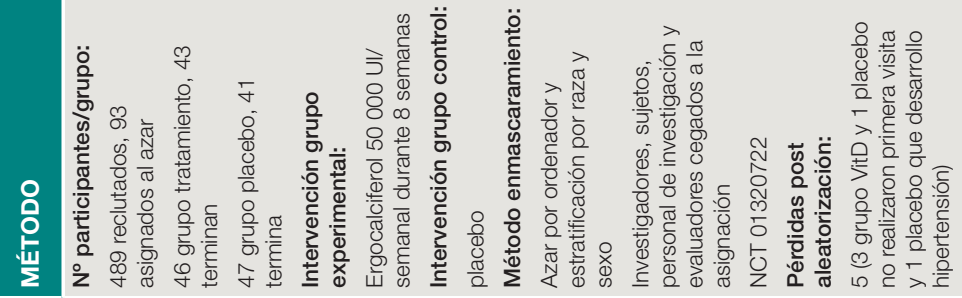

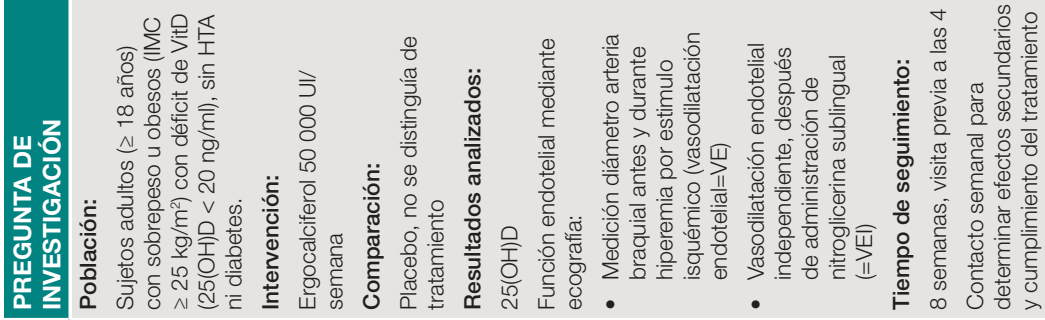

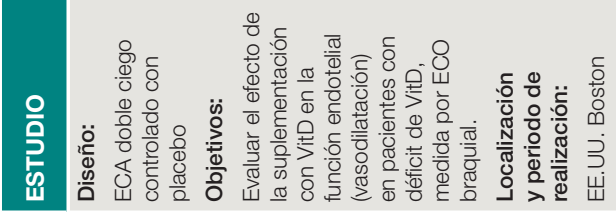

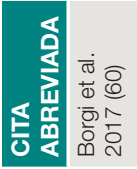




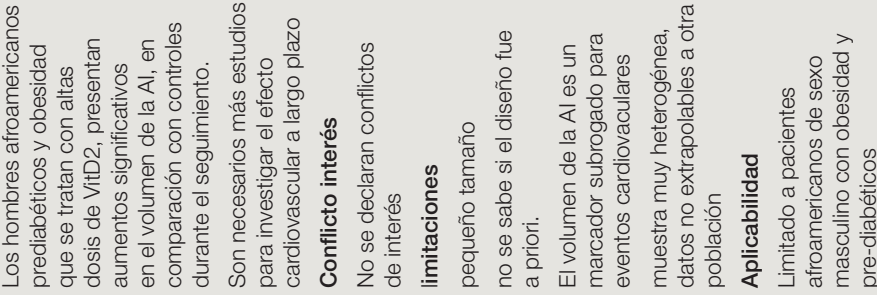
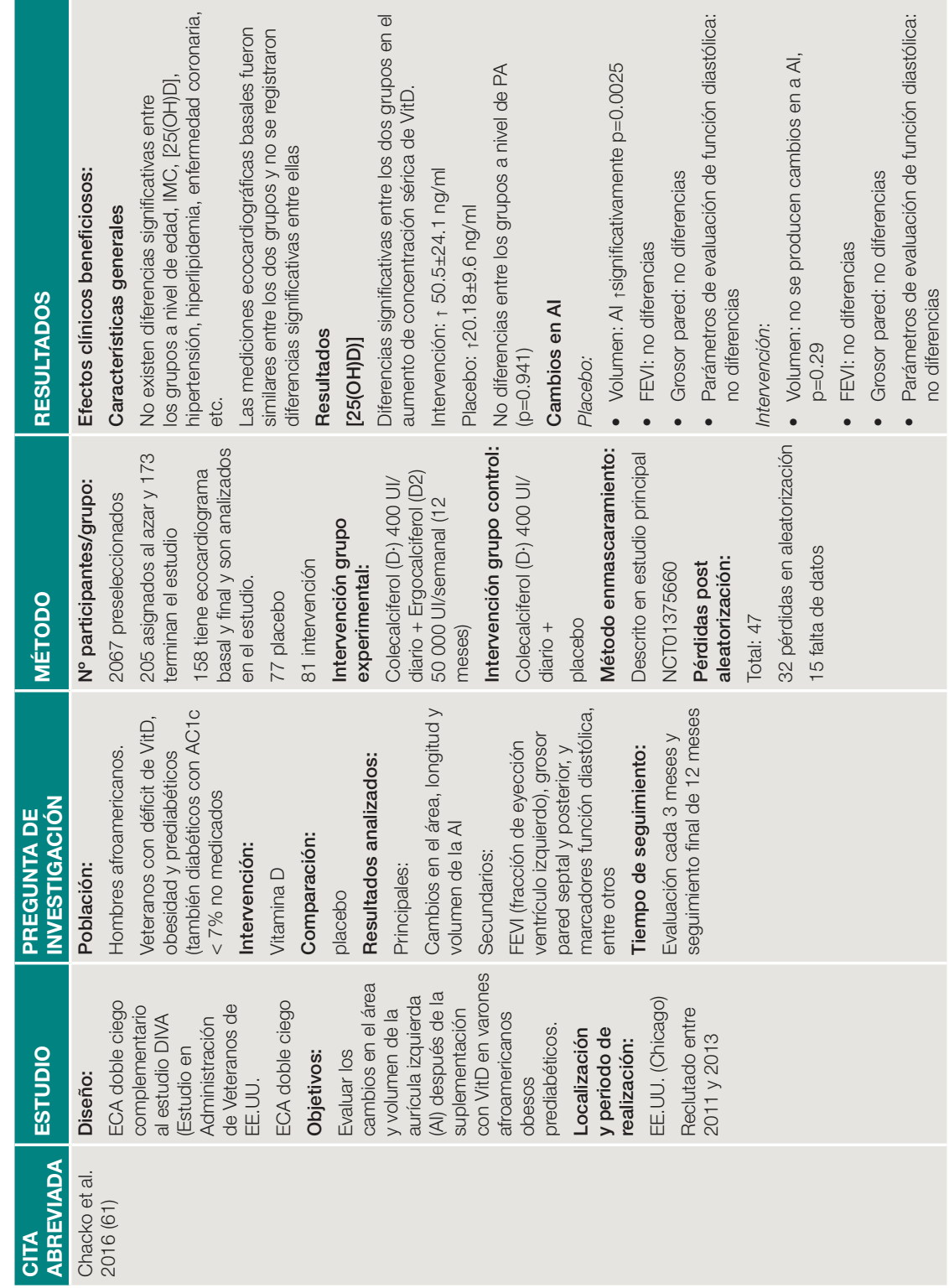


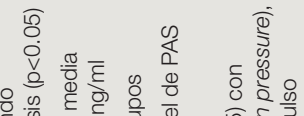

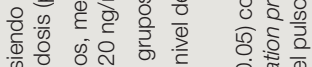

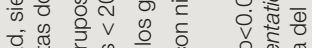

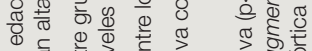

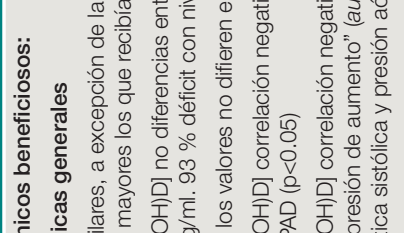

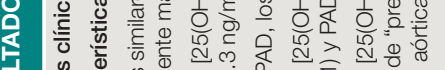

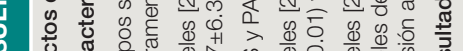

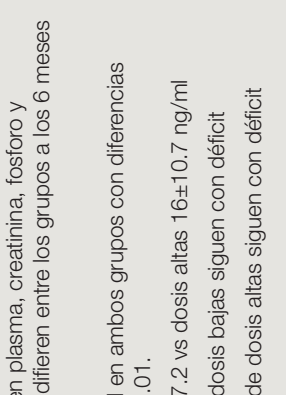

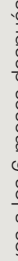

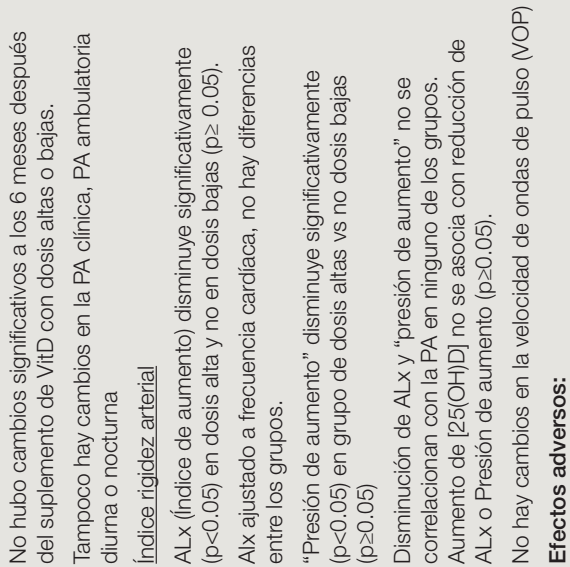

סु 0

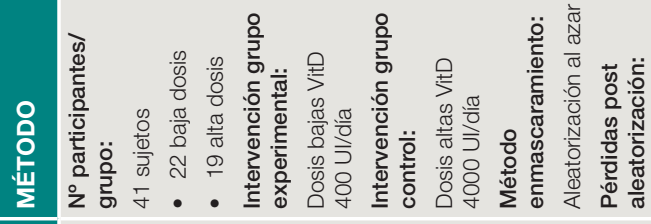

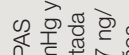

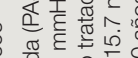

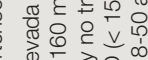

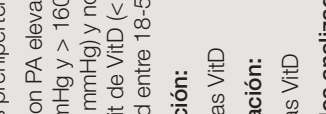

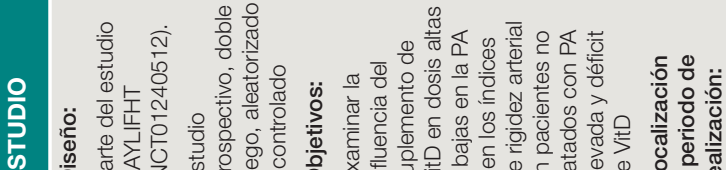

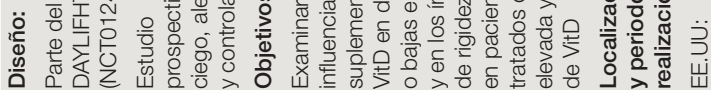

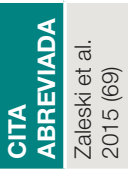




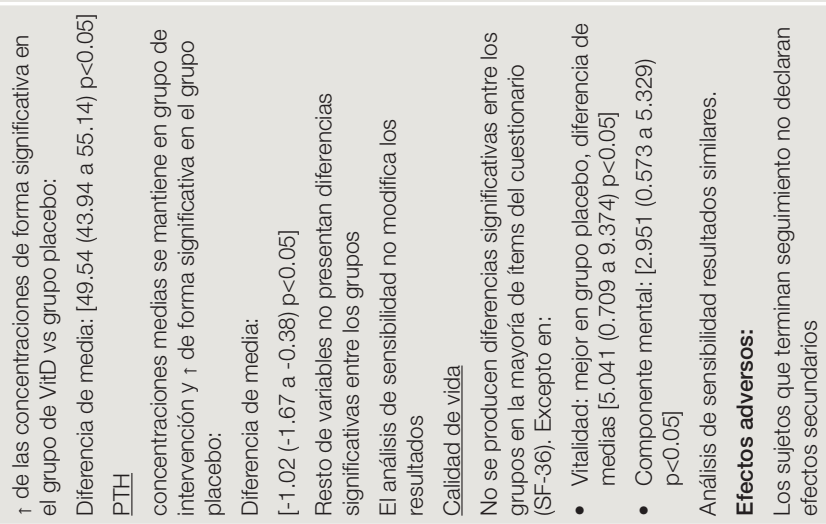

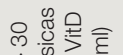

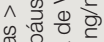

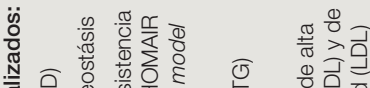

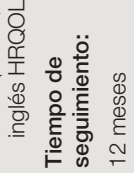

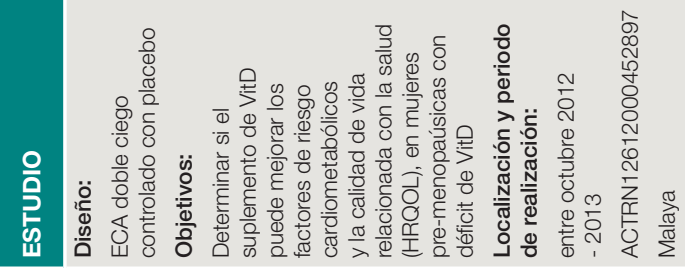

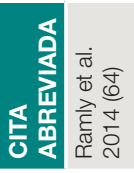




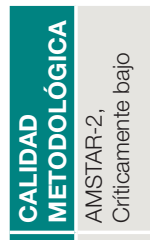

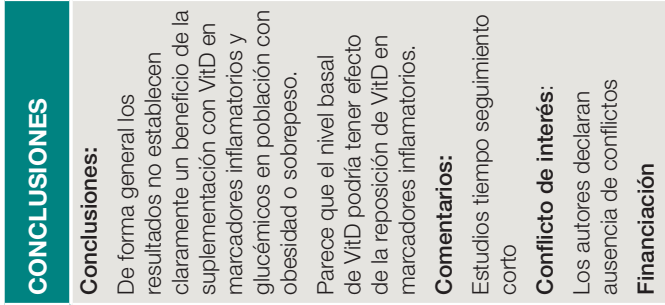

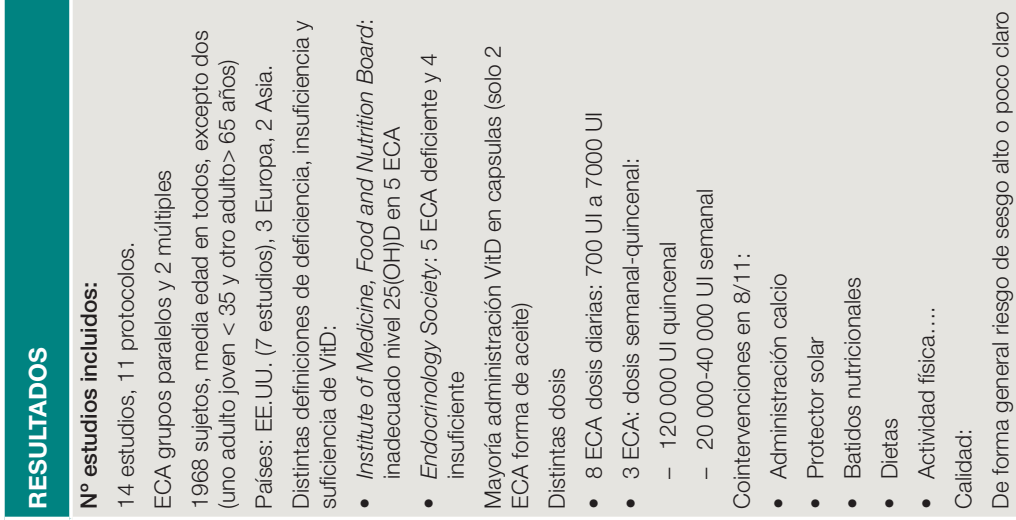

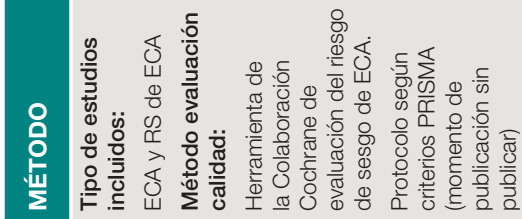
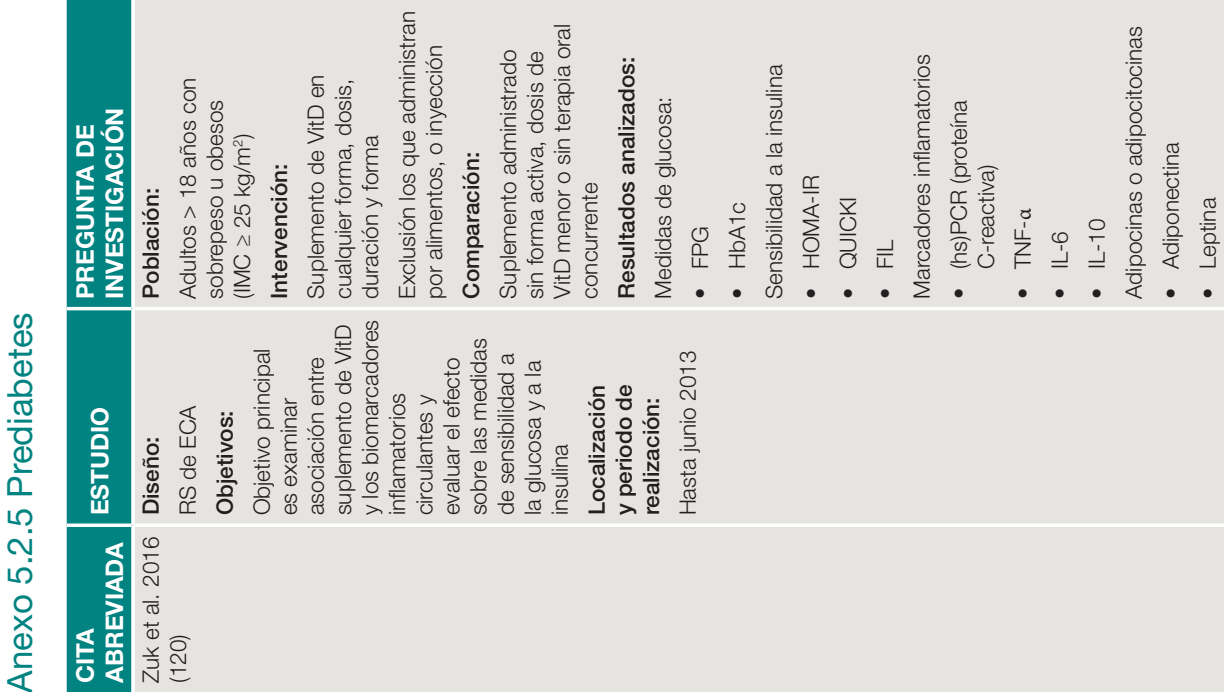

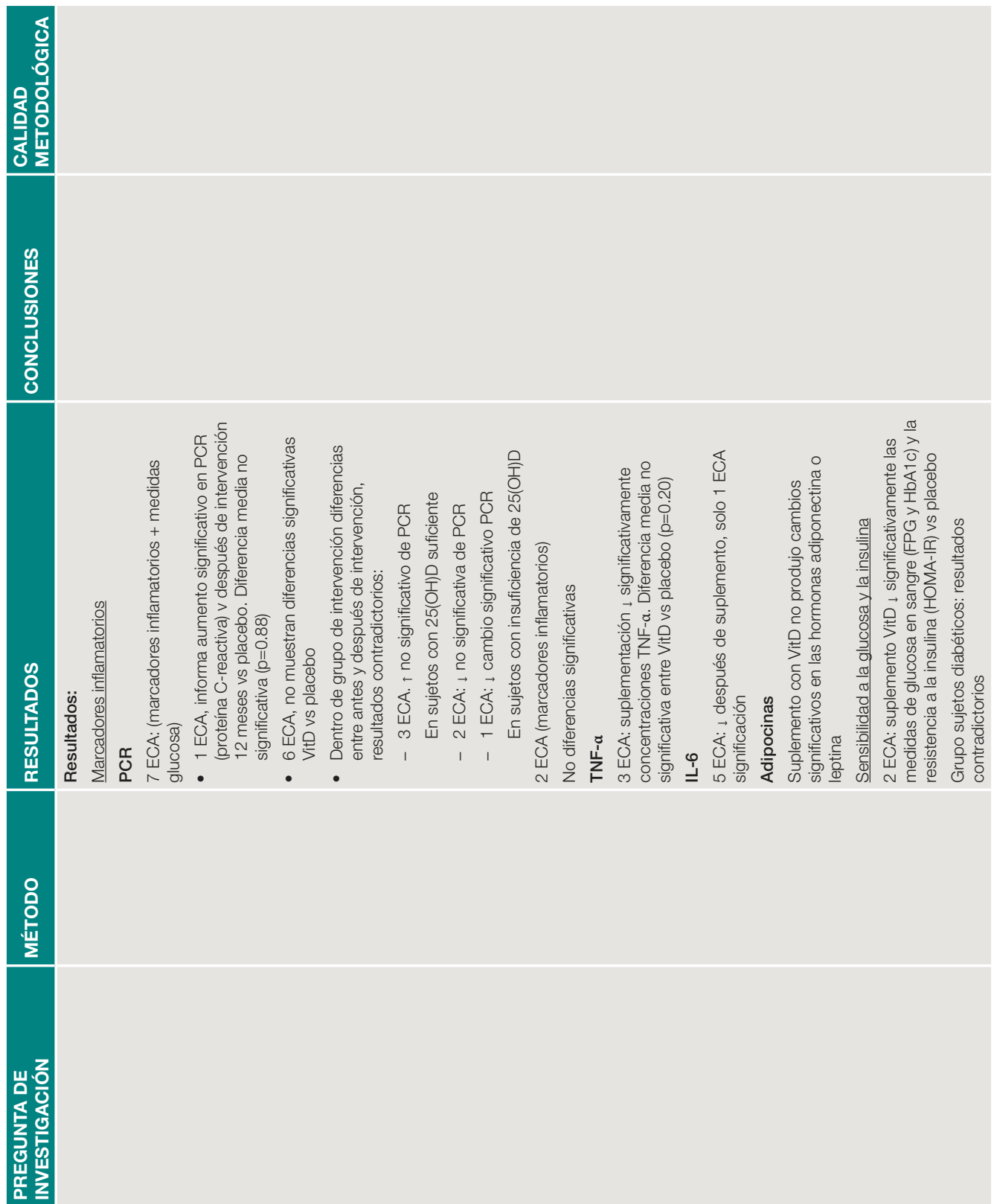

음 
ifil

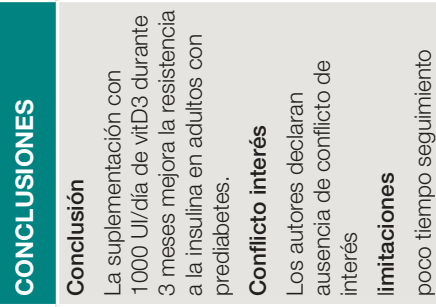

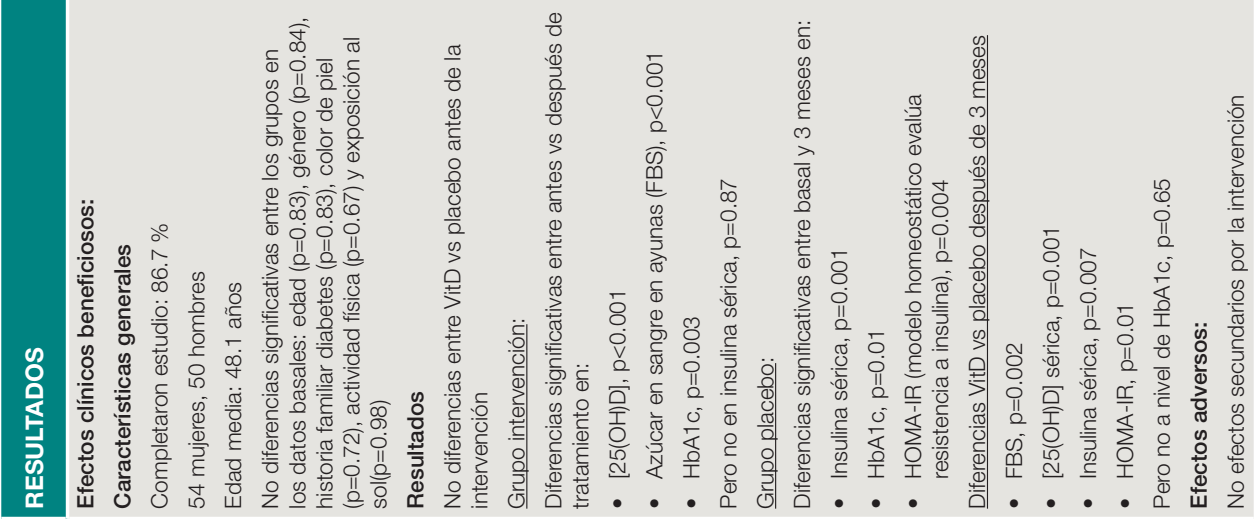

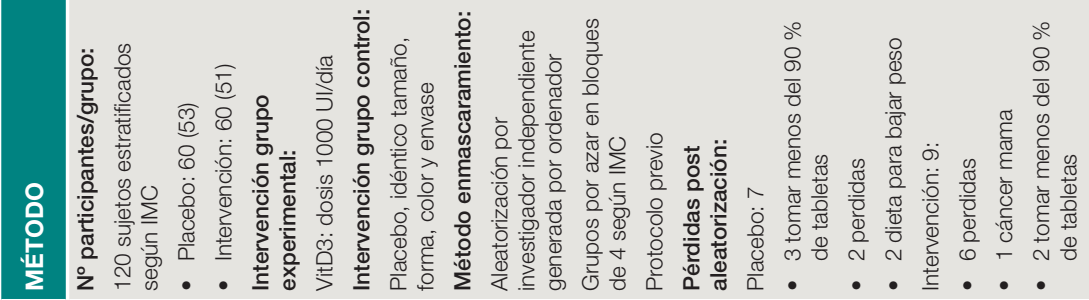

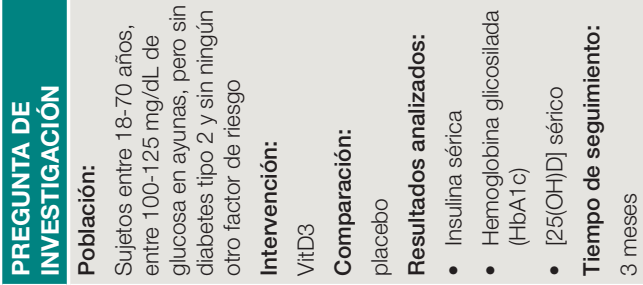

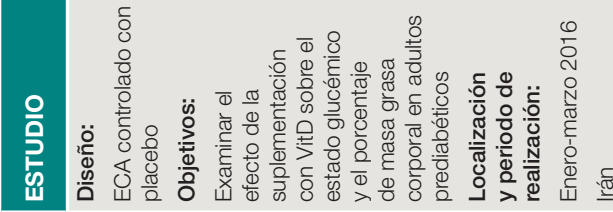

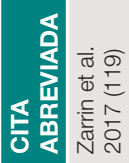




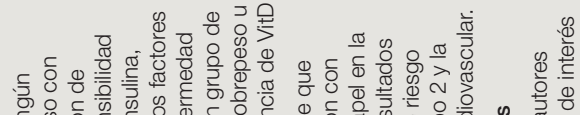

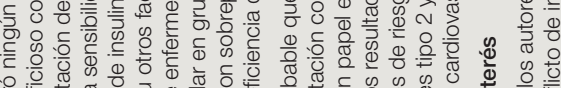

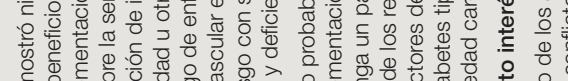

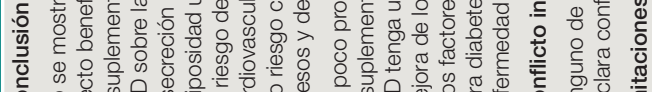
○

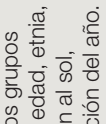

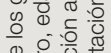

î

:

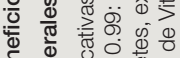

क्ष.

(1)

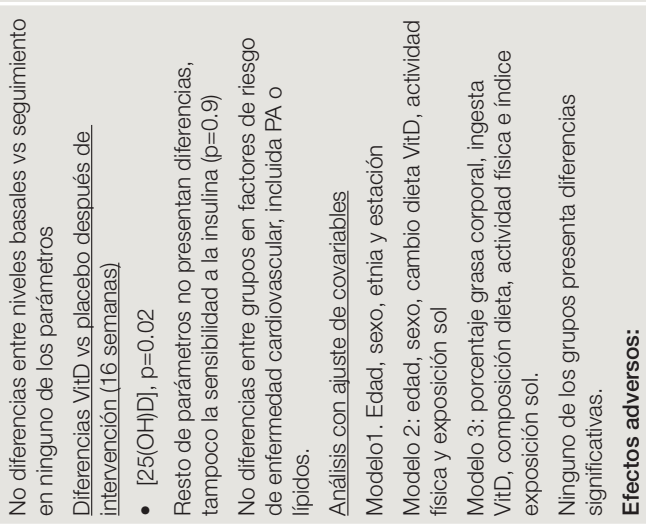

을 2 을

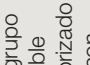

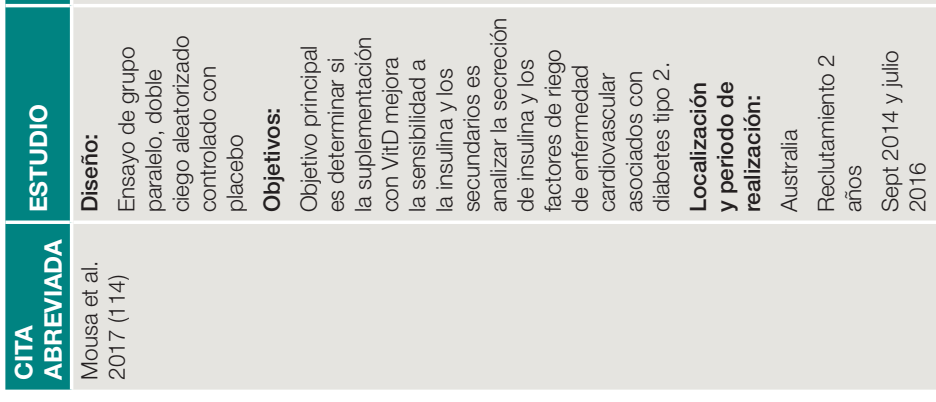




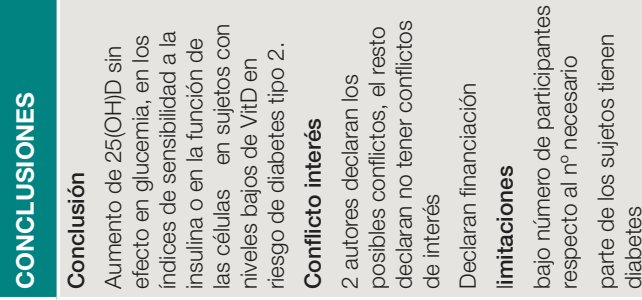

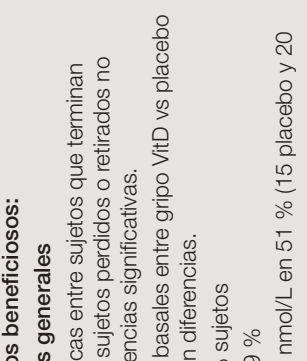

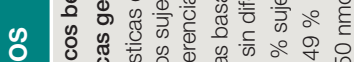

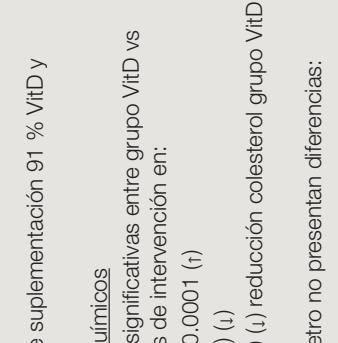
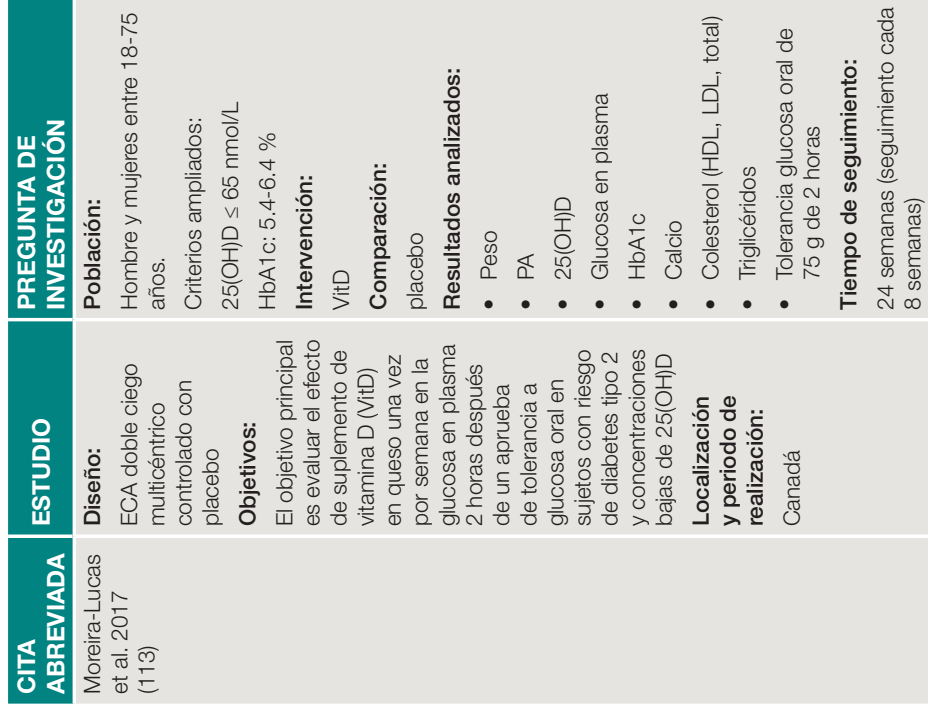

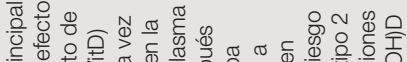

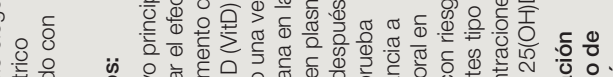

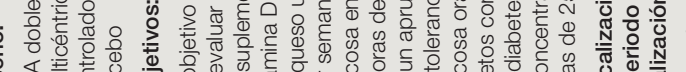

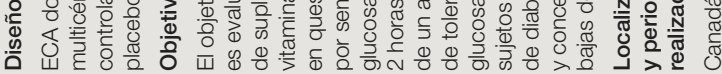

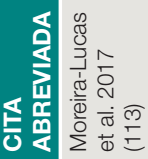




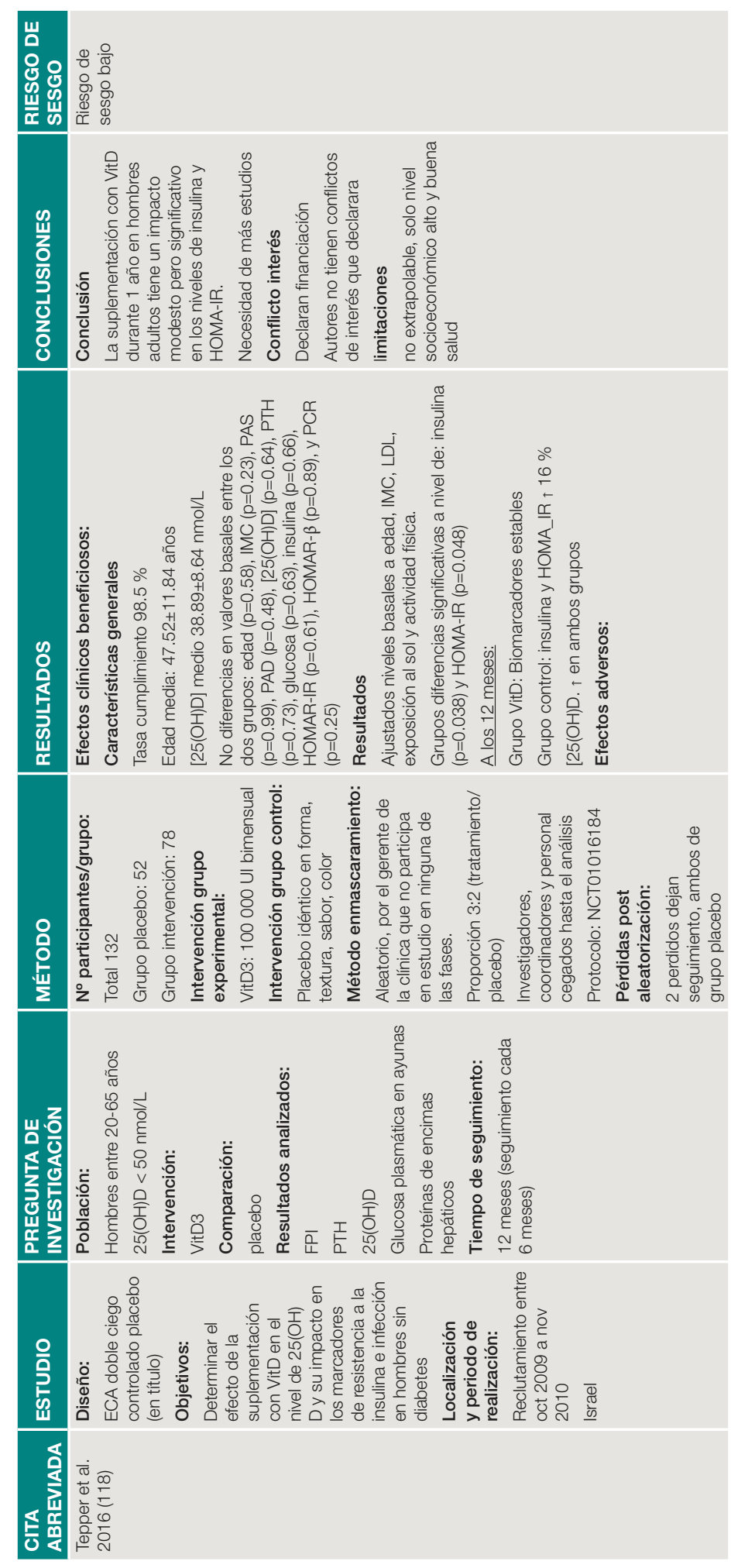


HIIIII)

융

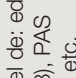

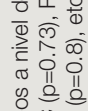

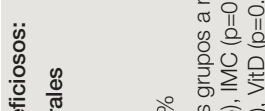

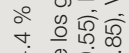

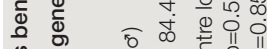

:

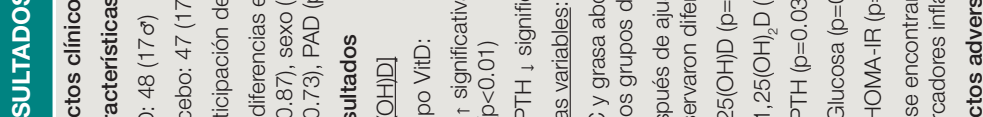

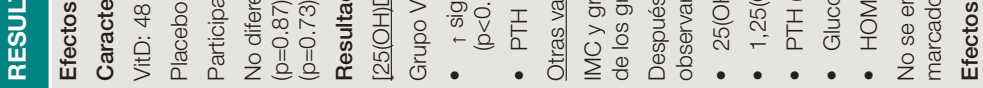

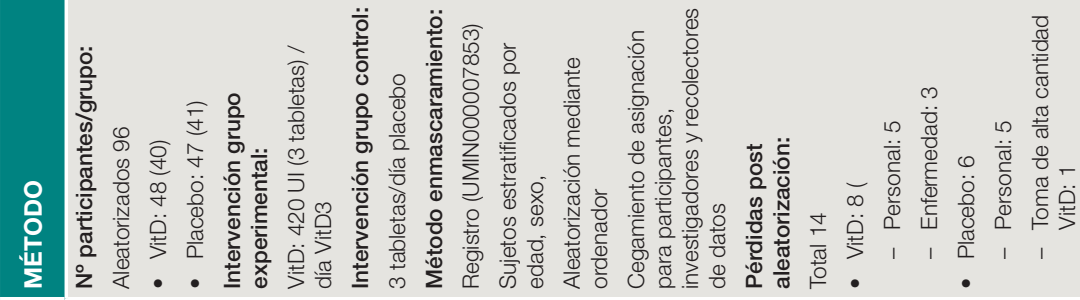

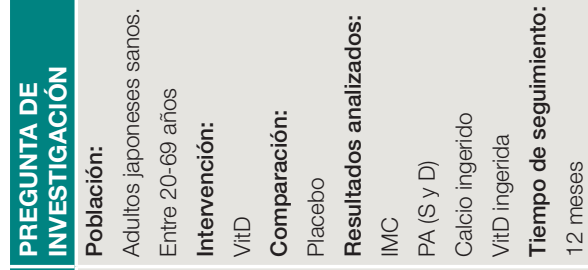

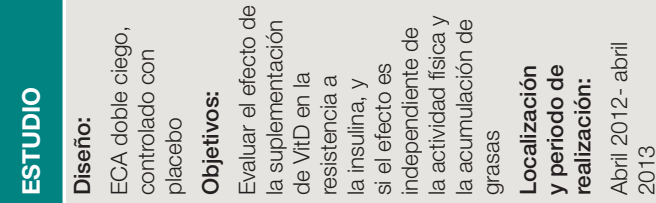

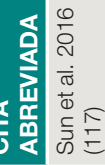




\section{罚}

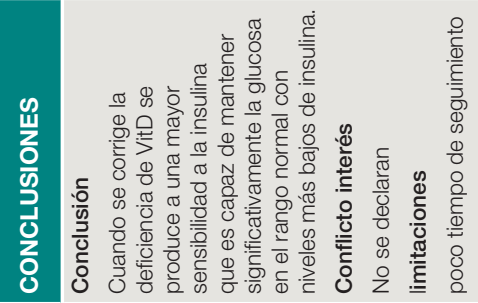
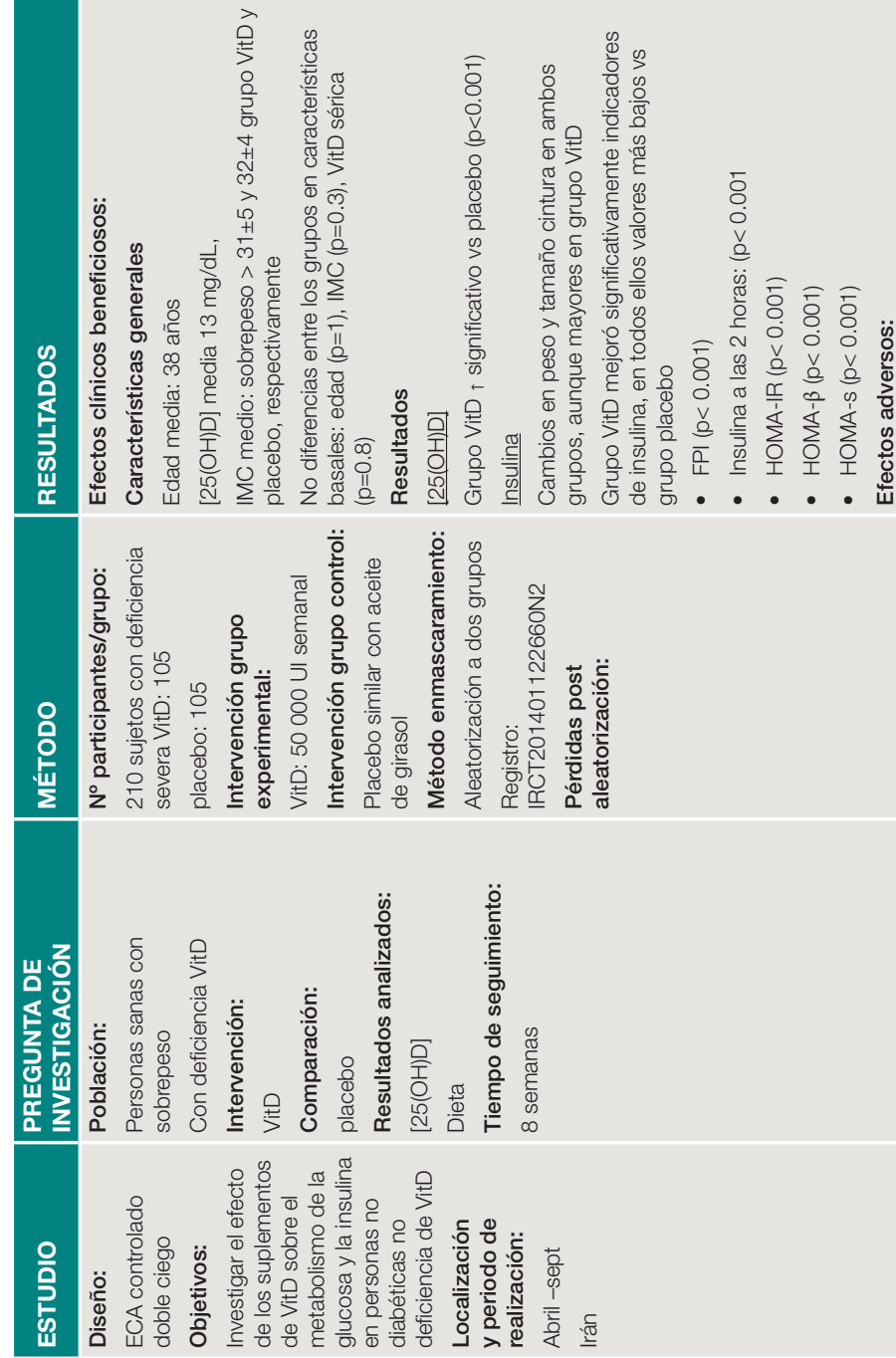

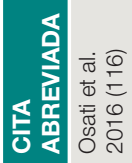


III

IIIIIIIII

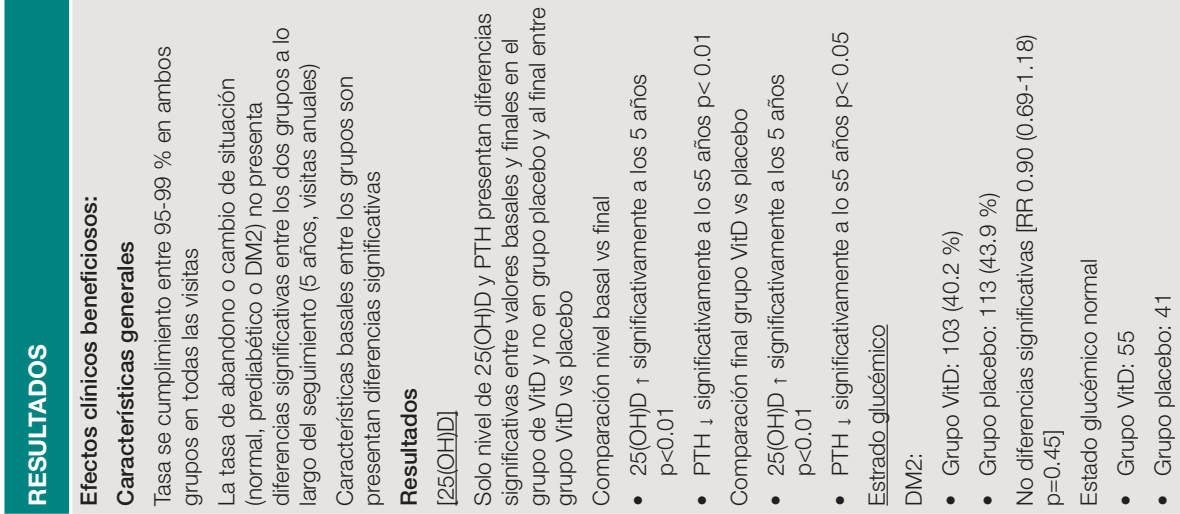

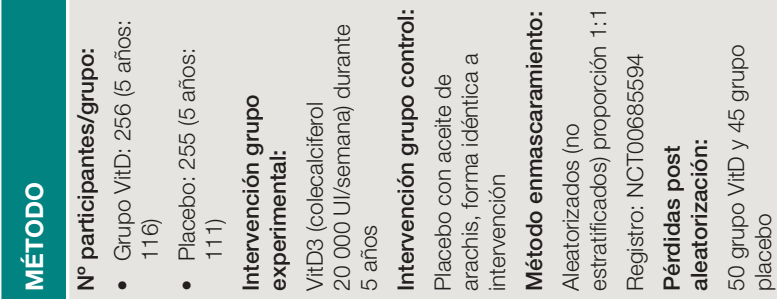

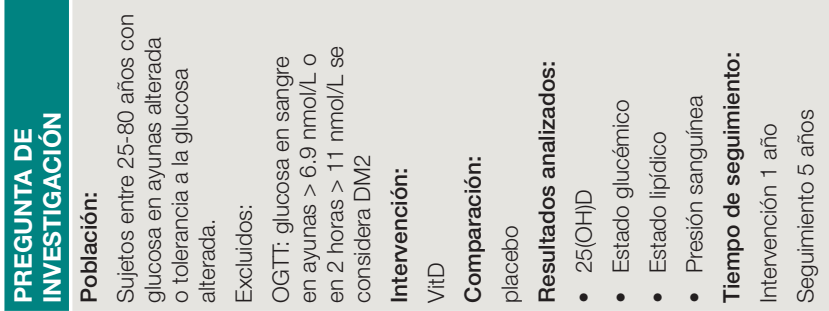

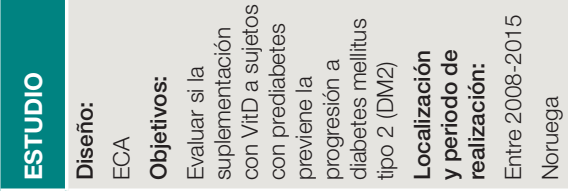

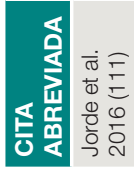




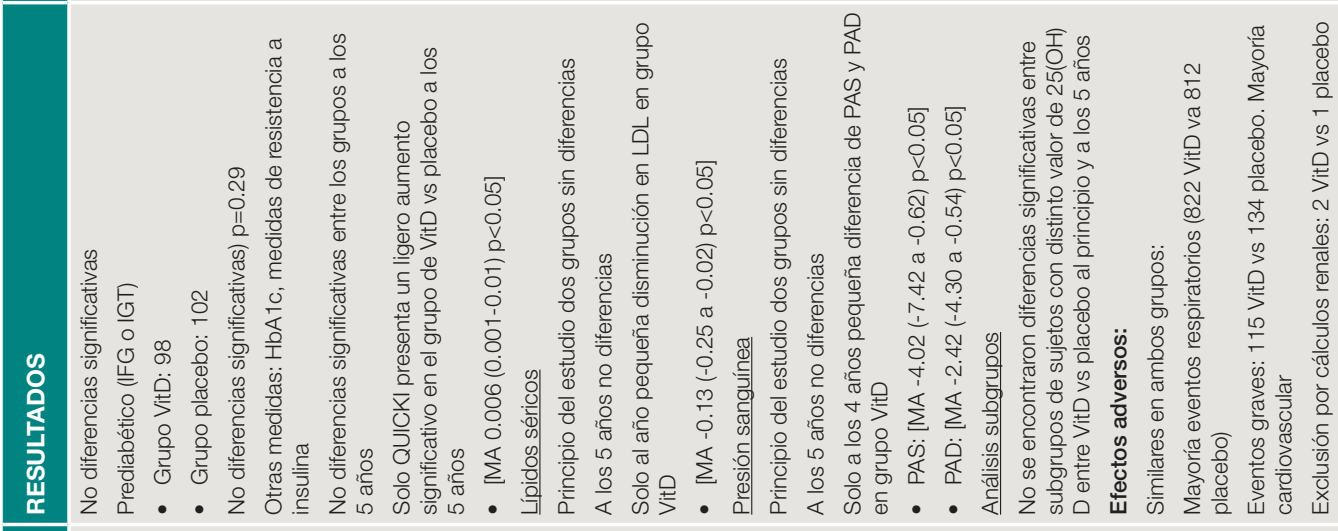




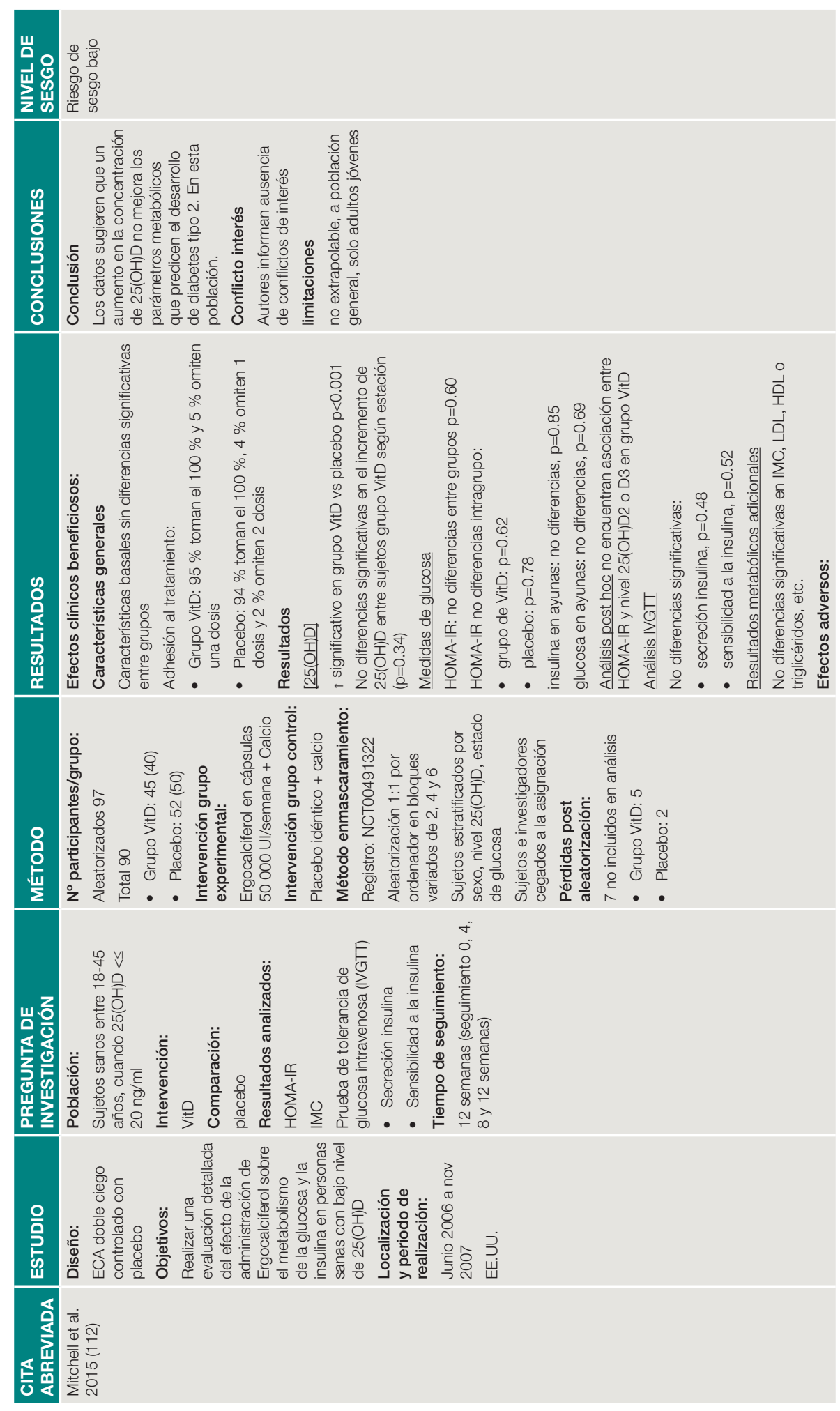



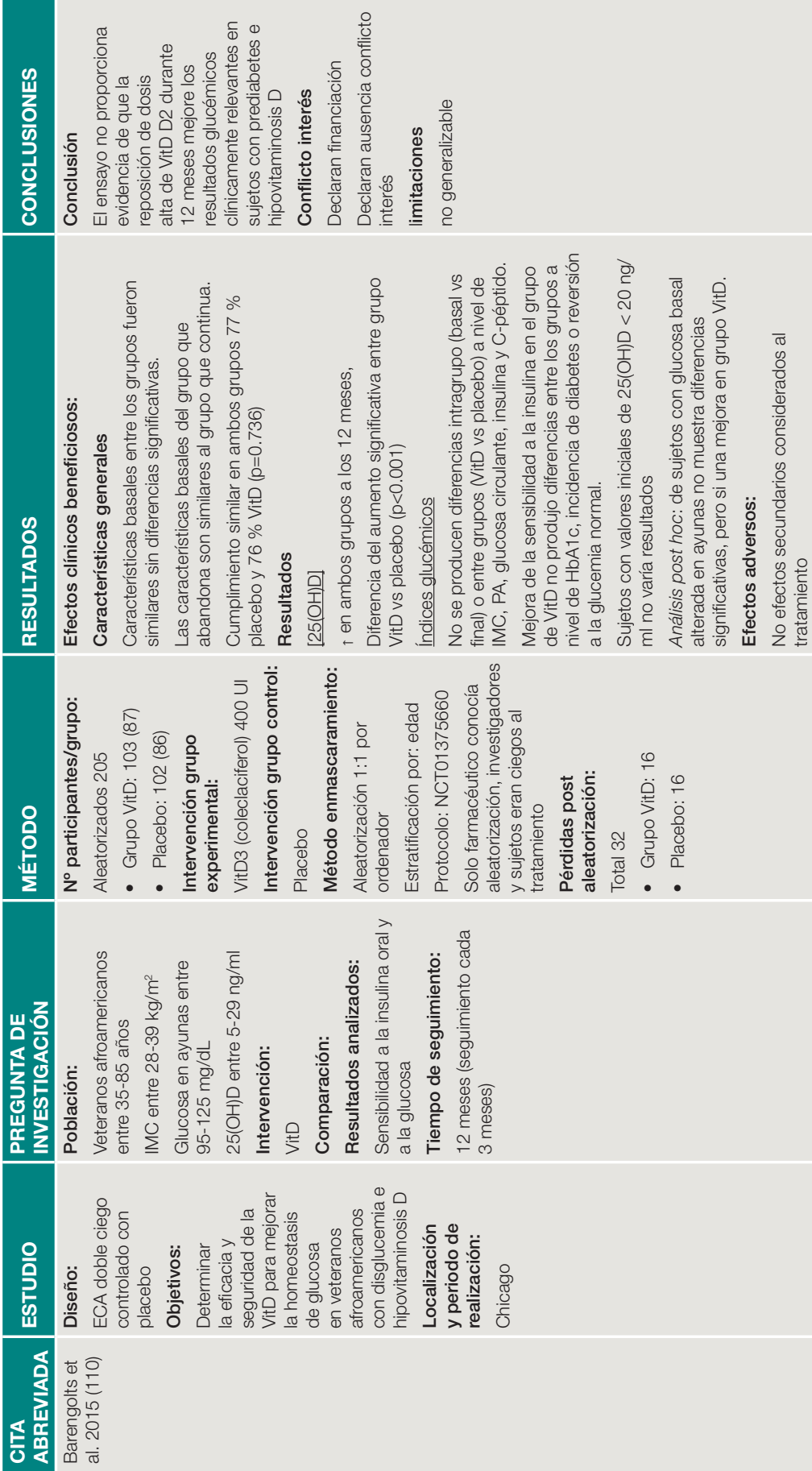


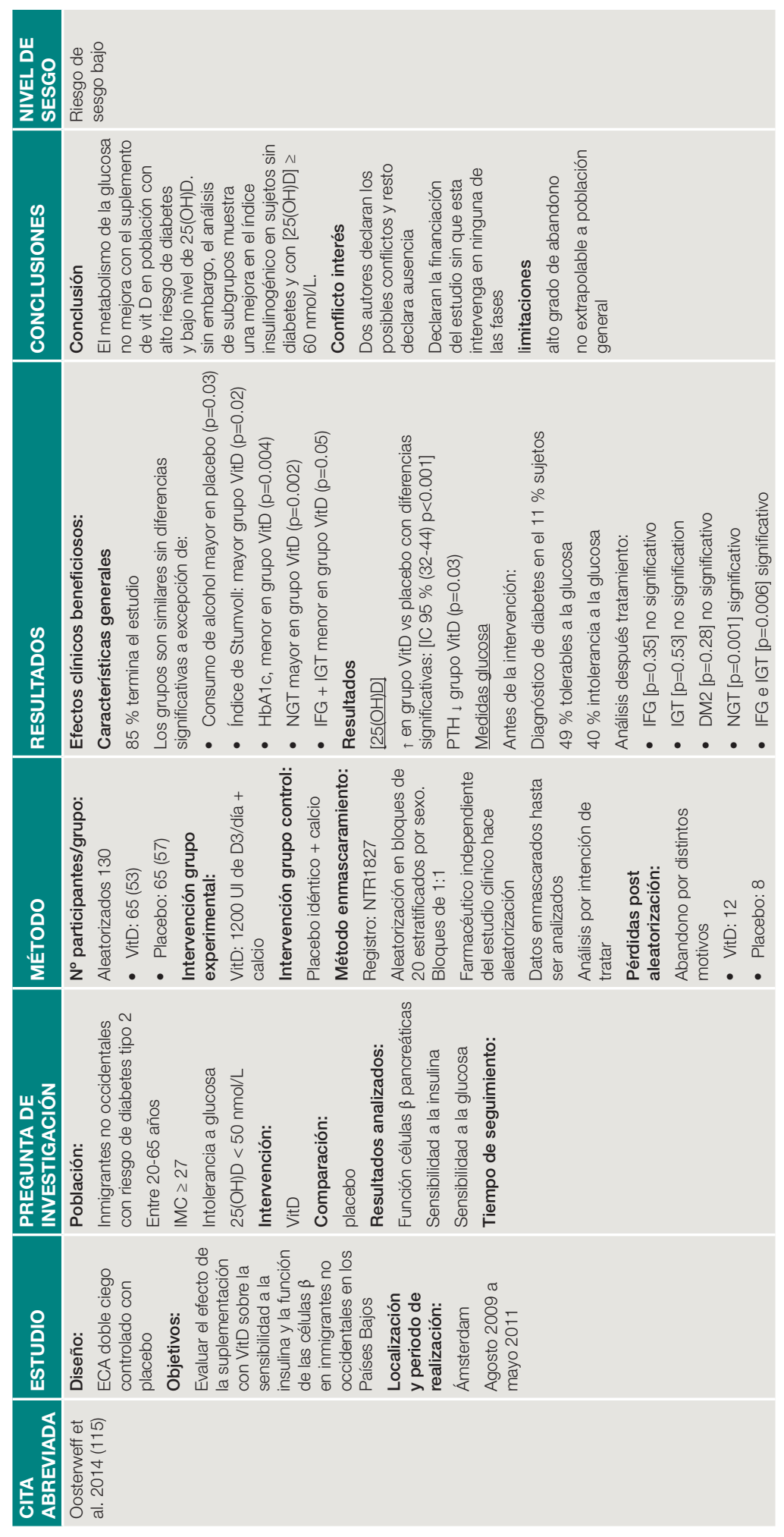



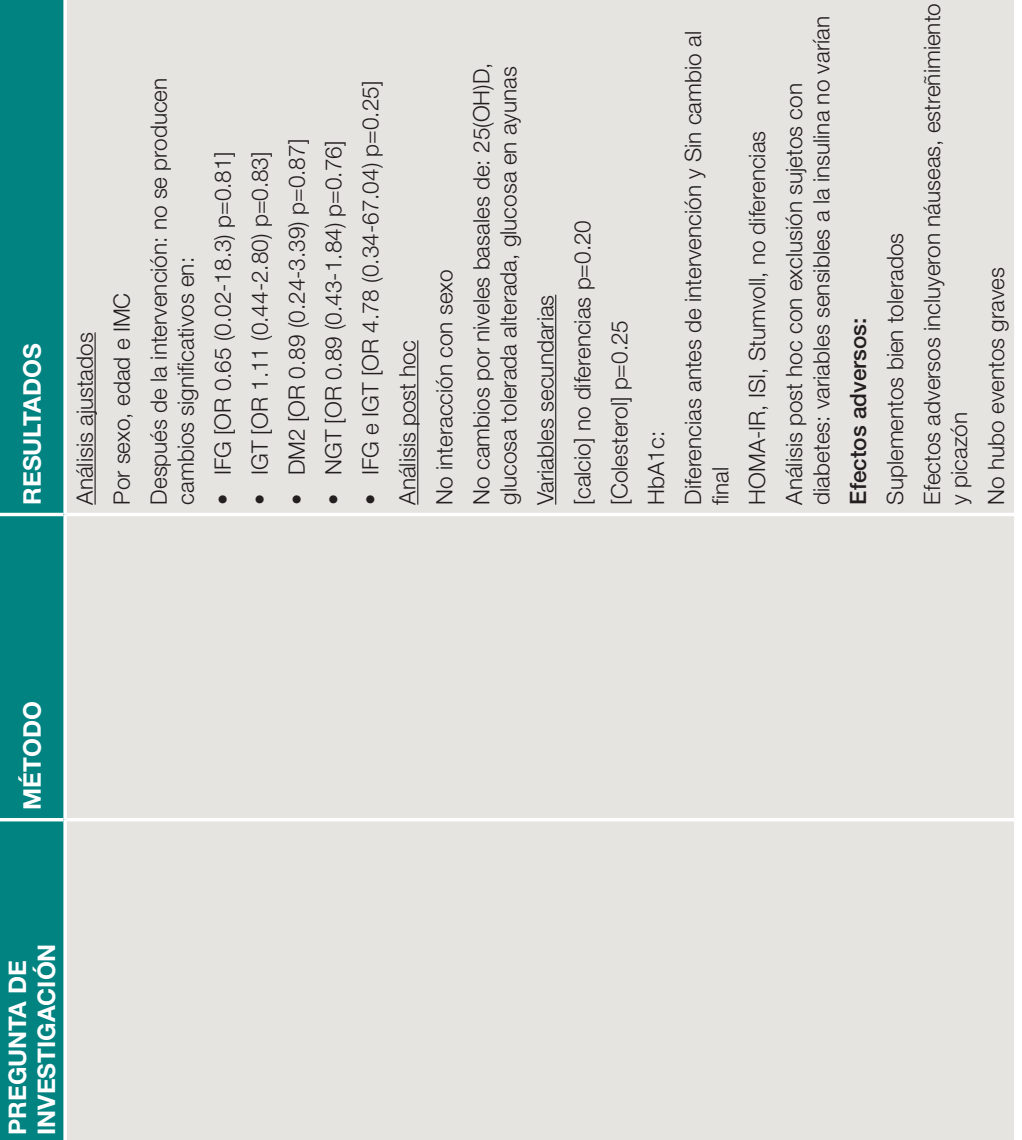

음

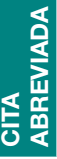




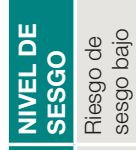

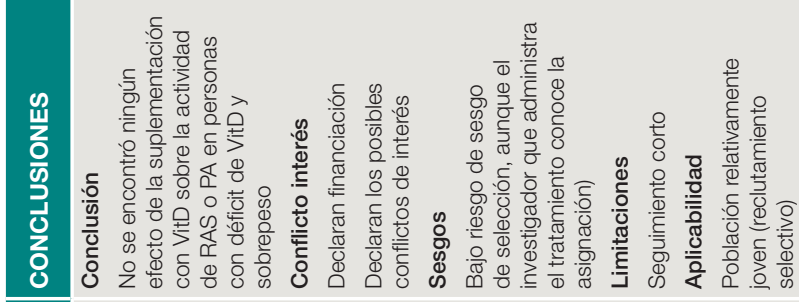

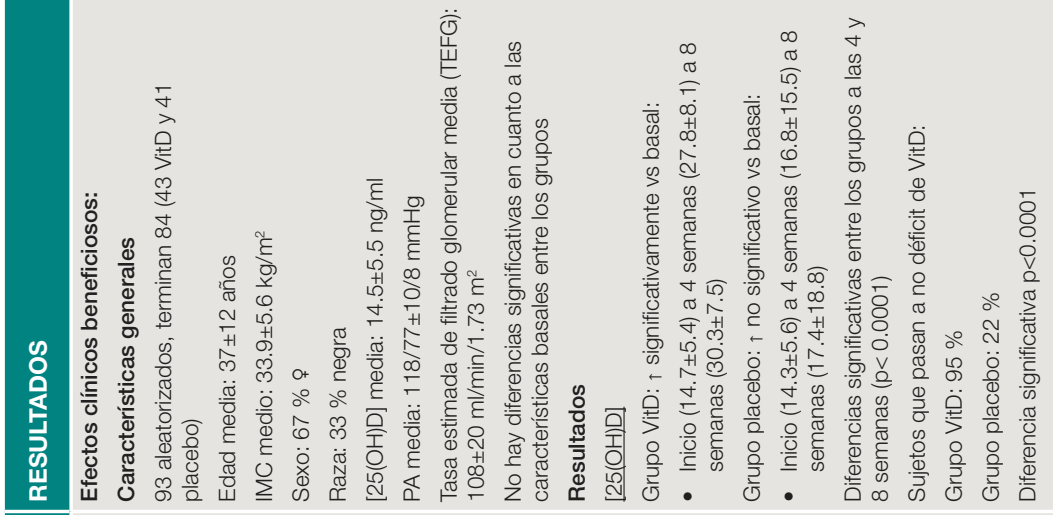

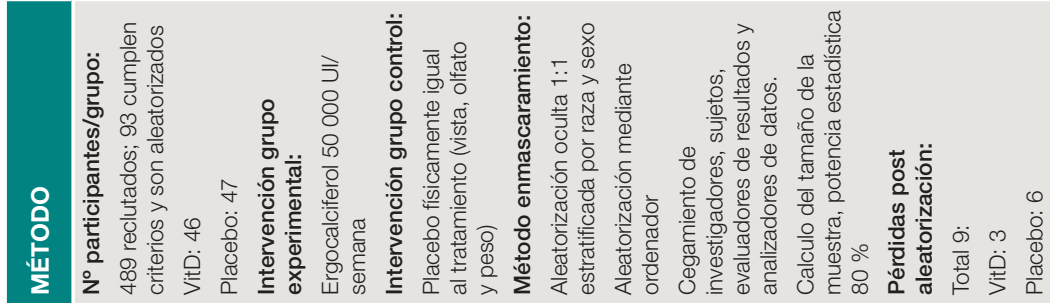

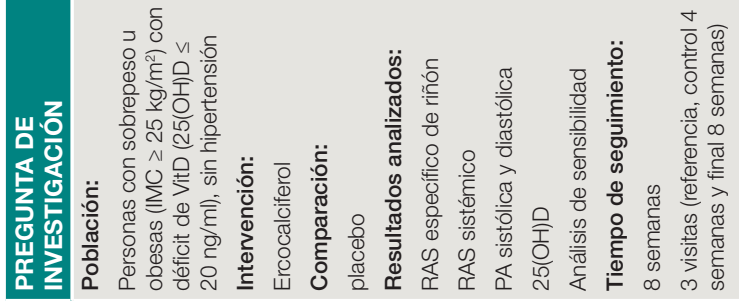

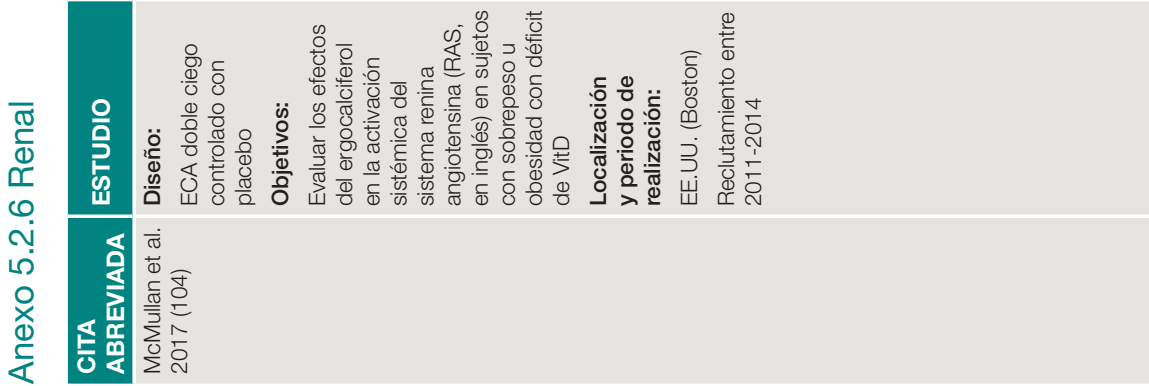




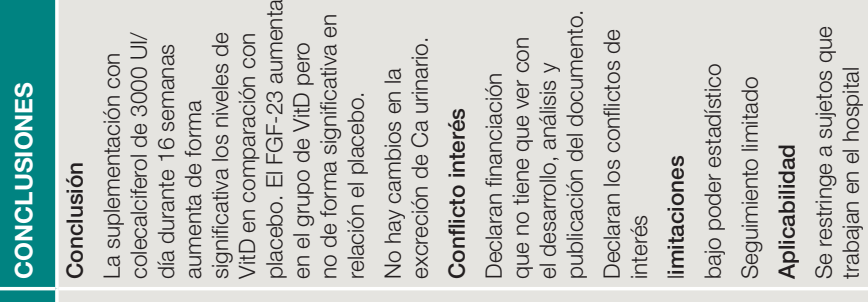
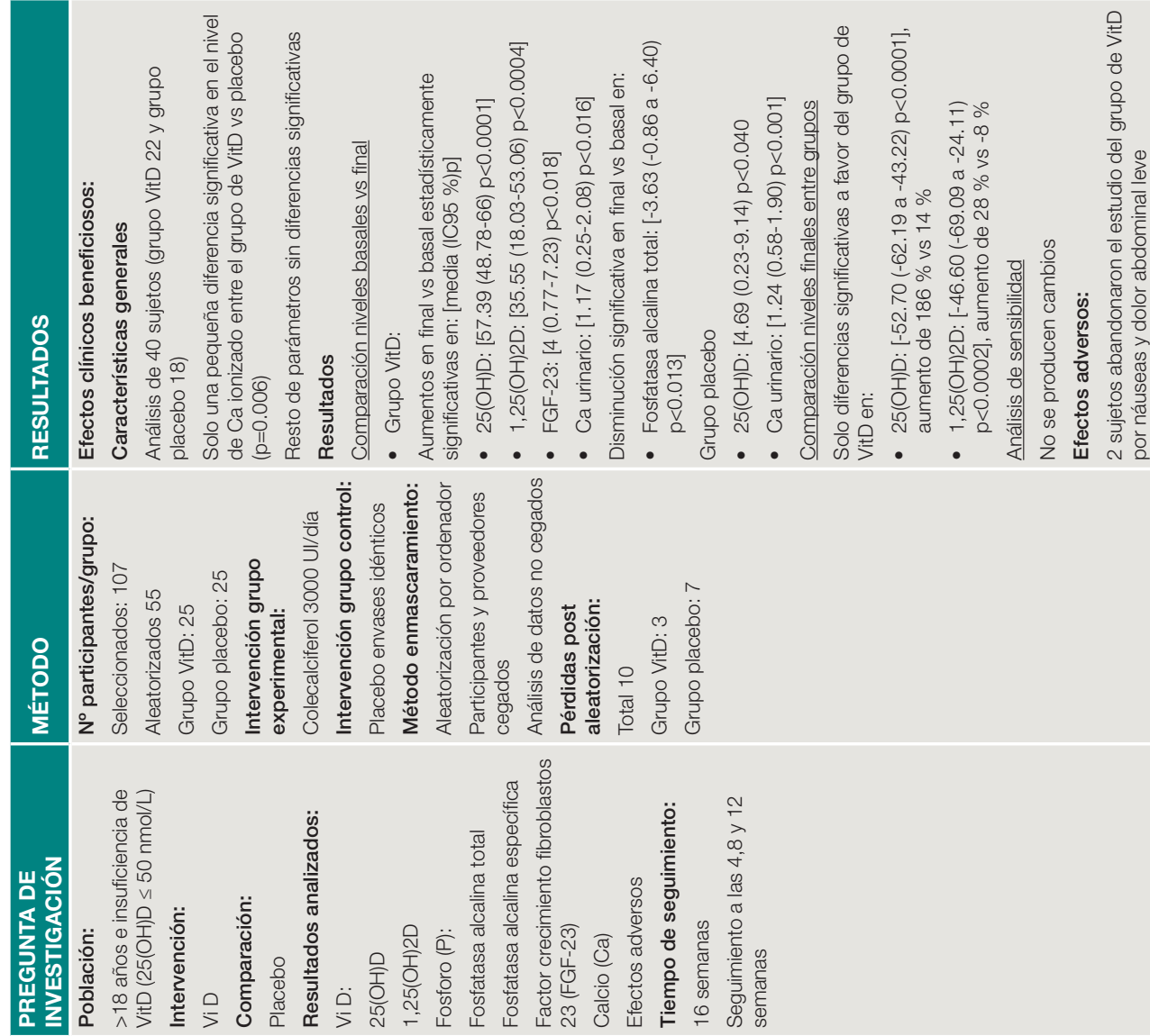

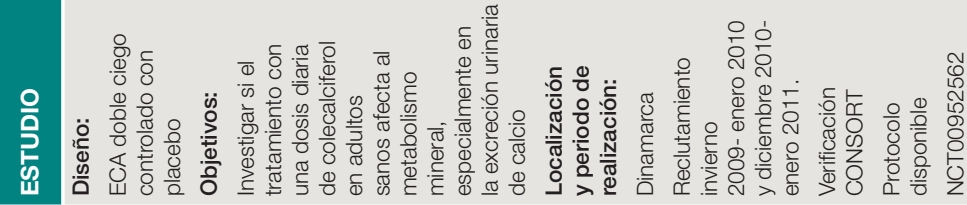

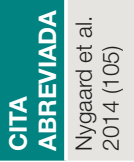




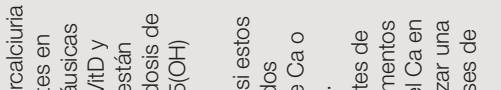

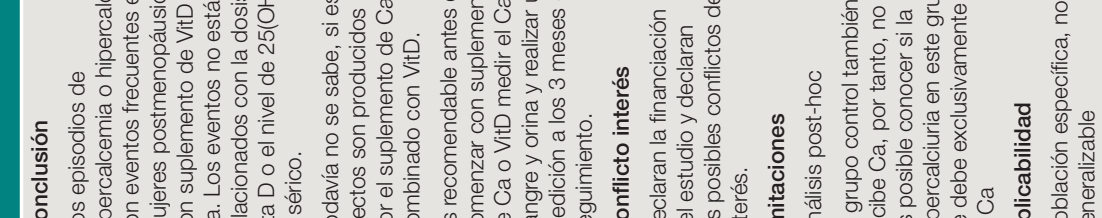

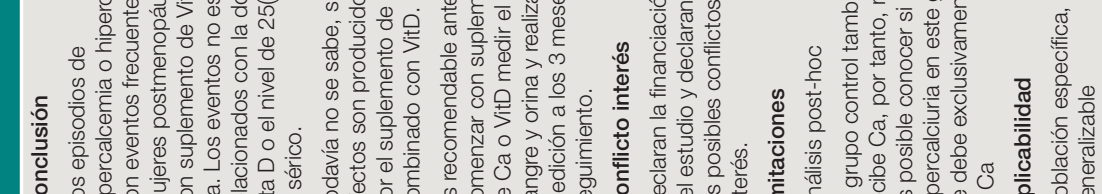

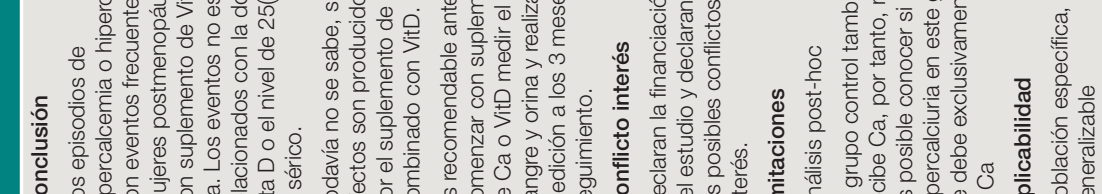
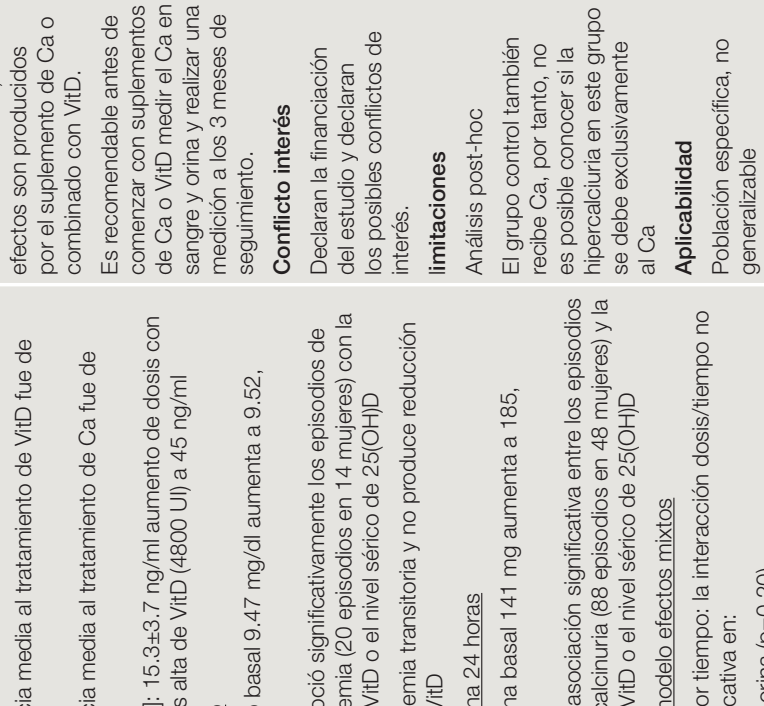

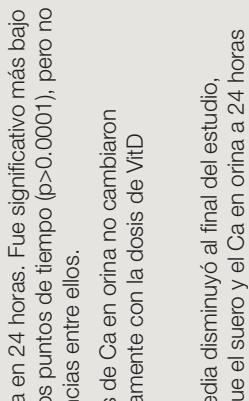

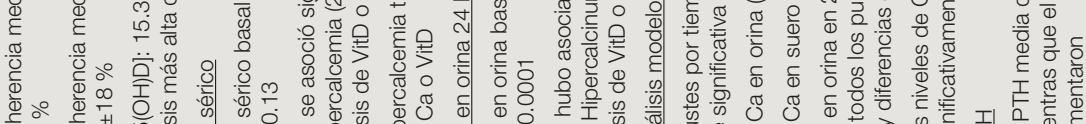

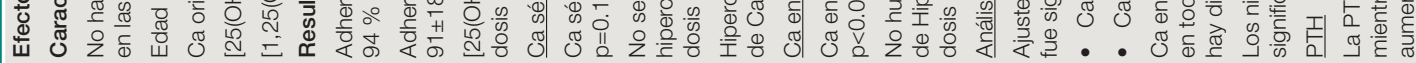



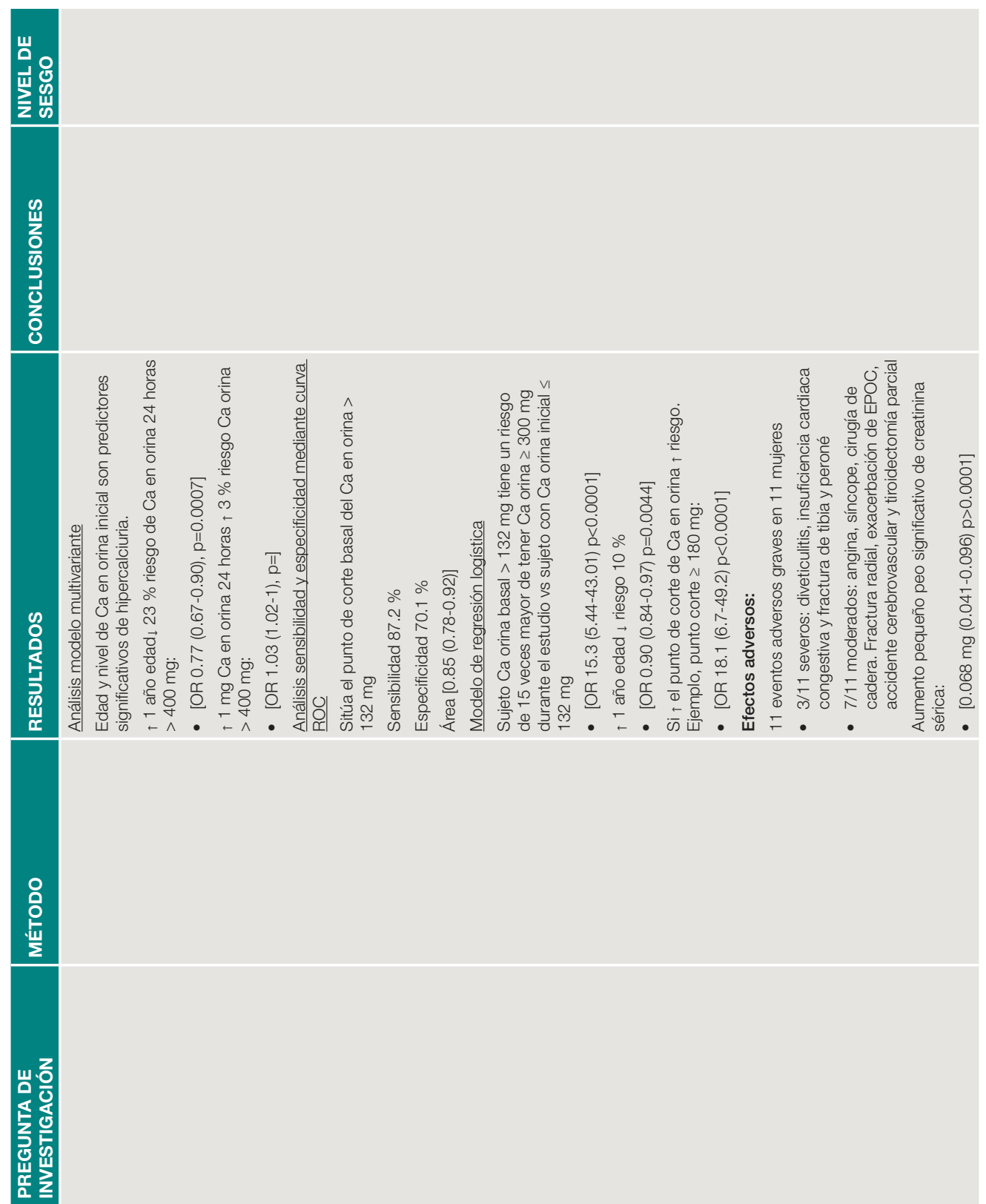

음 
III

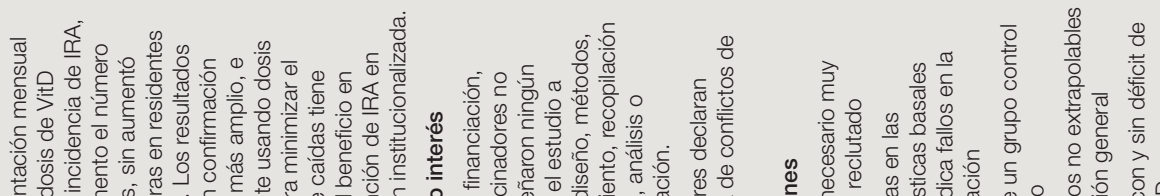

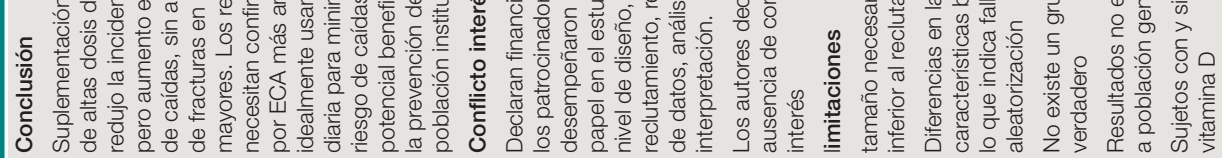

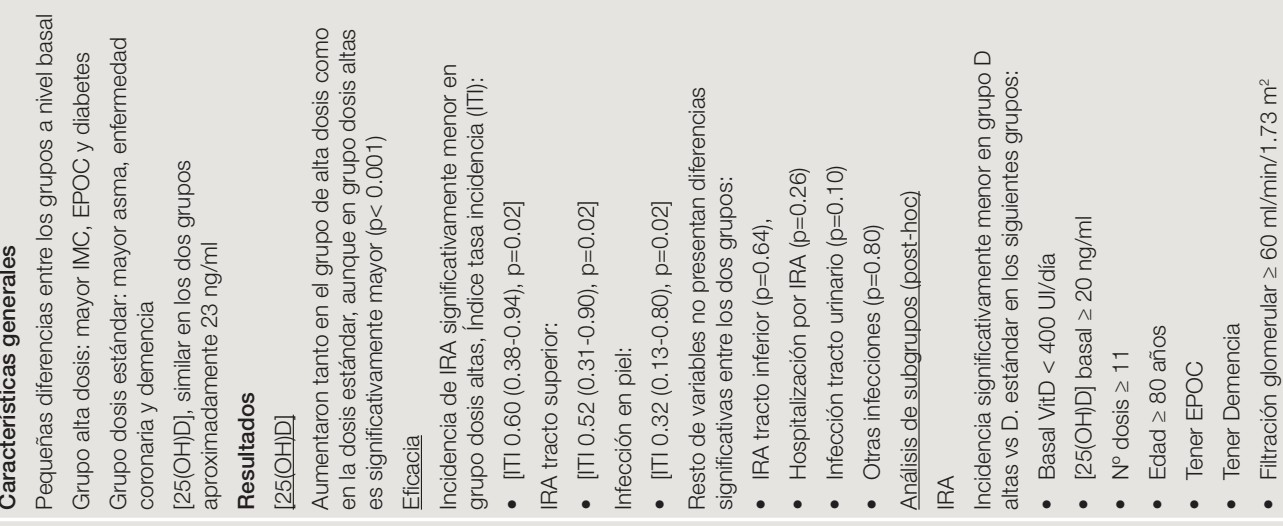

0

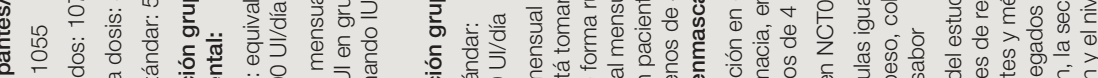

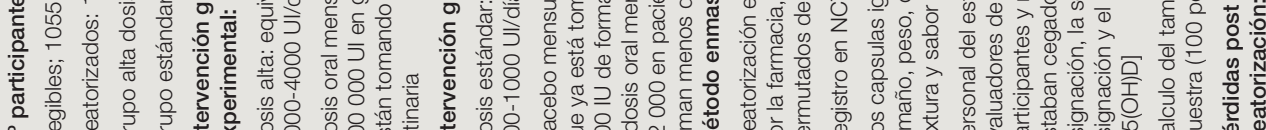

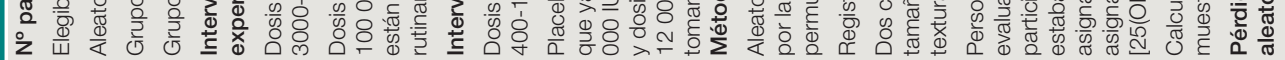

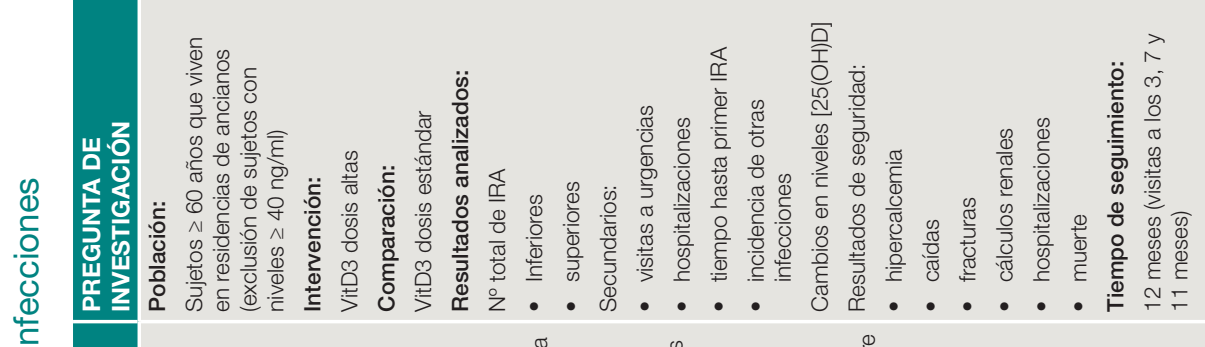

产

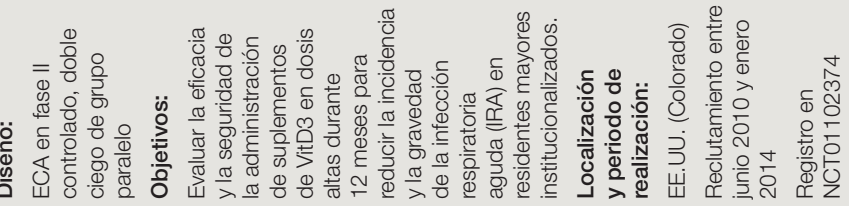

กั

用

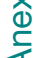

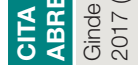




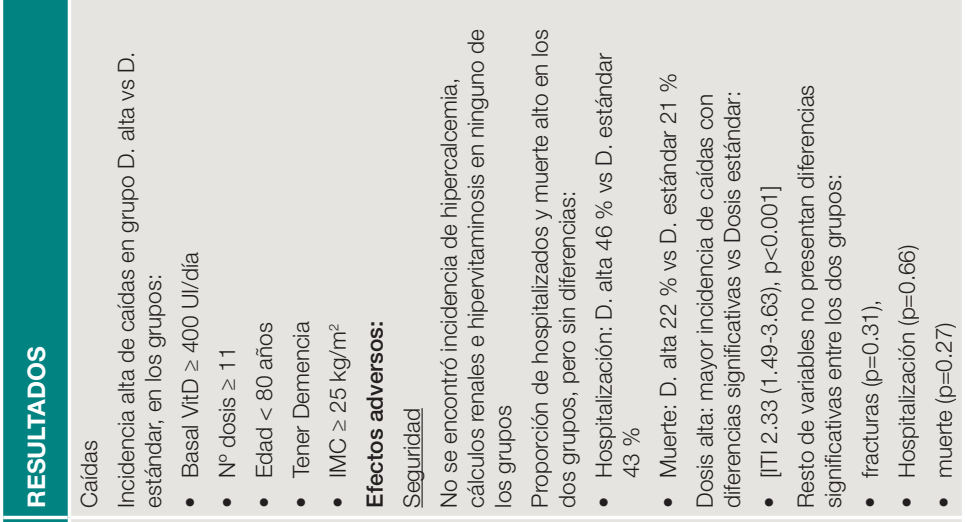

옹

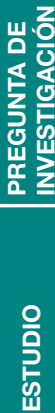

틍 


\section{III}

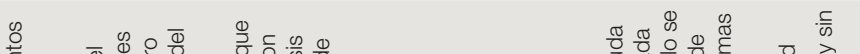

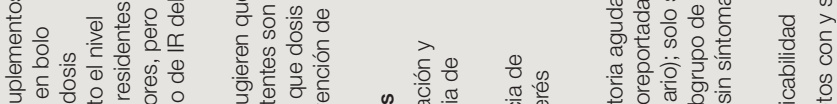

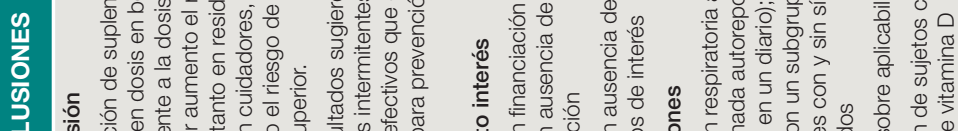

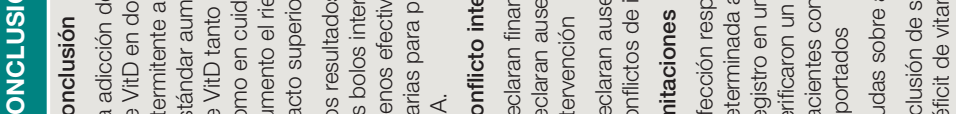
O

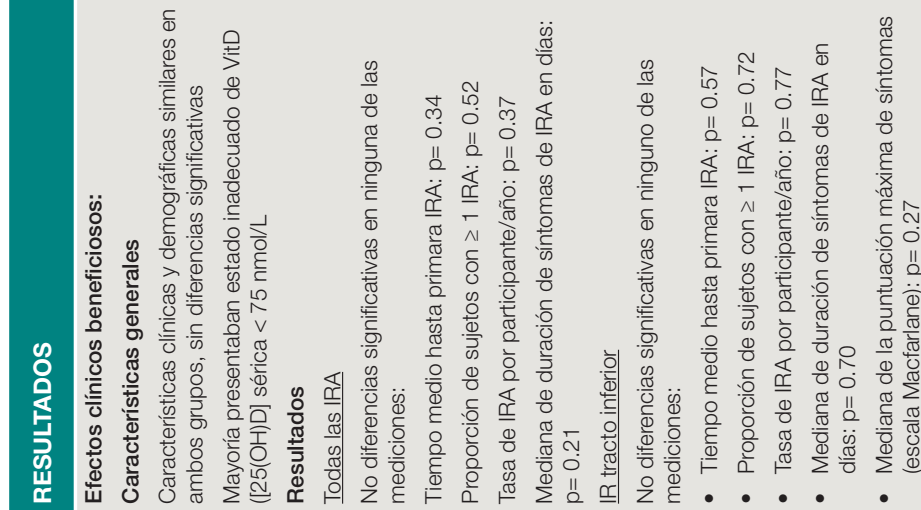

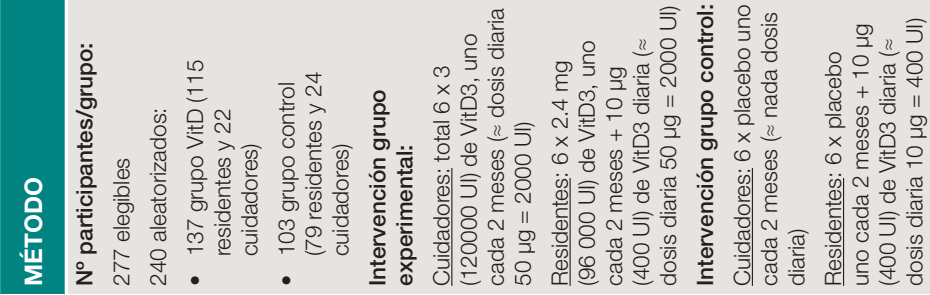

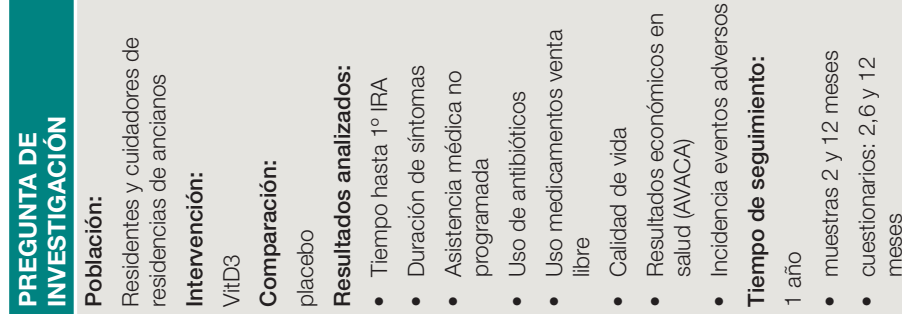

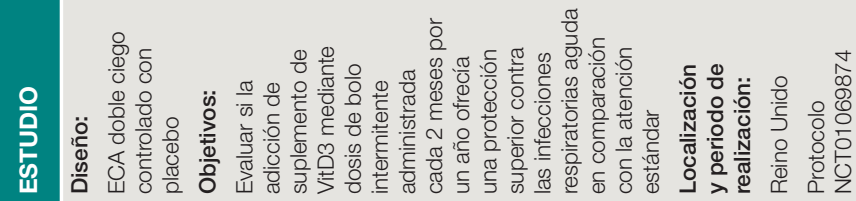

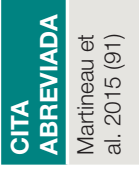




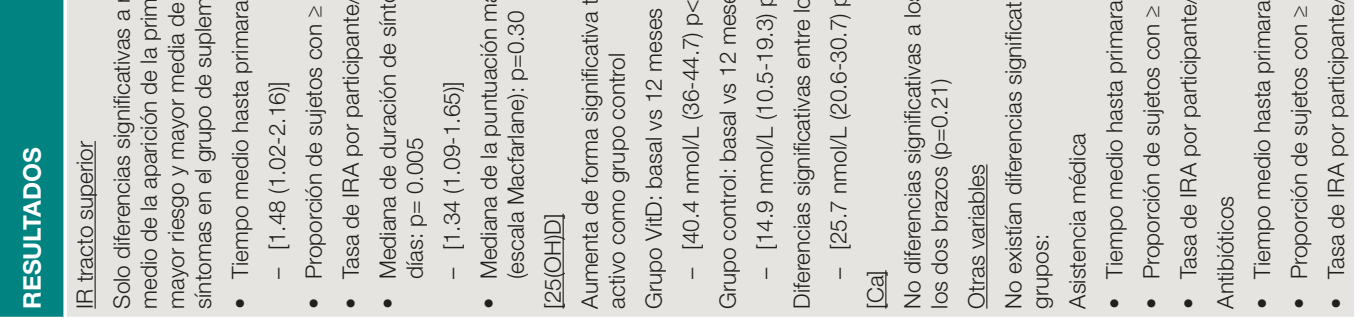

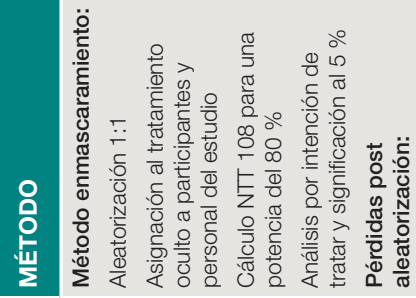

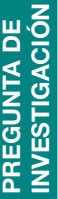

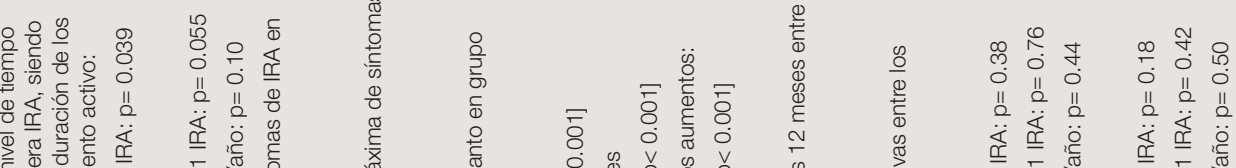
厄. 


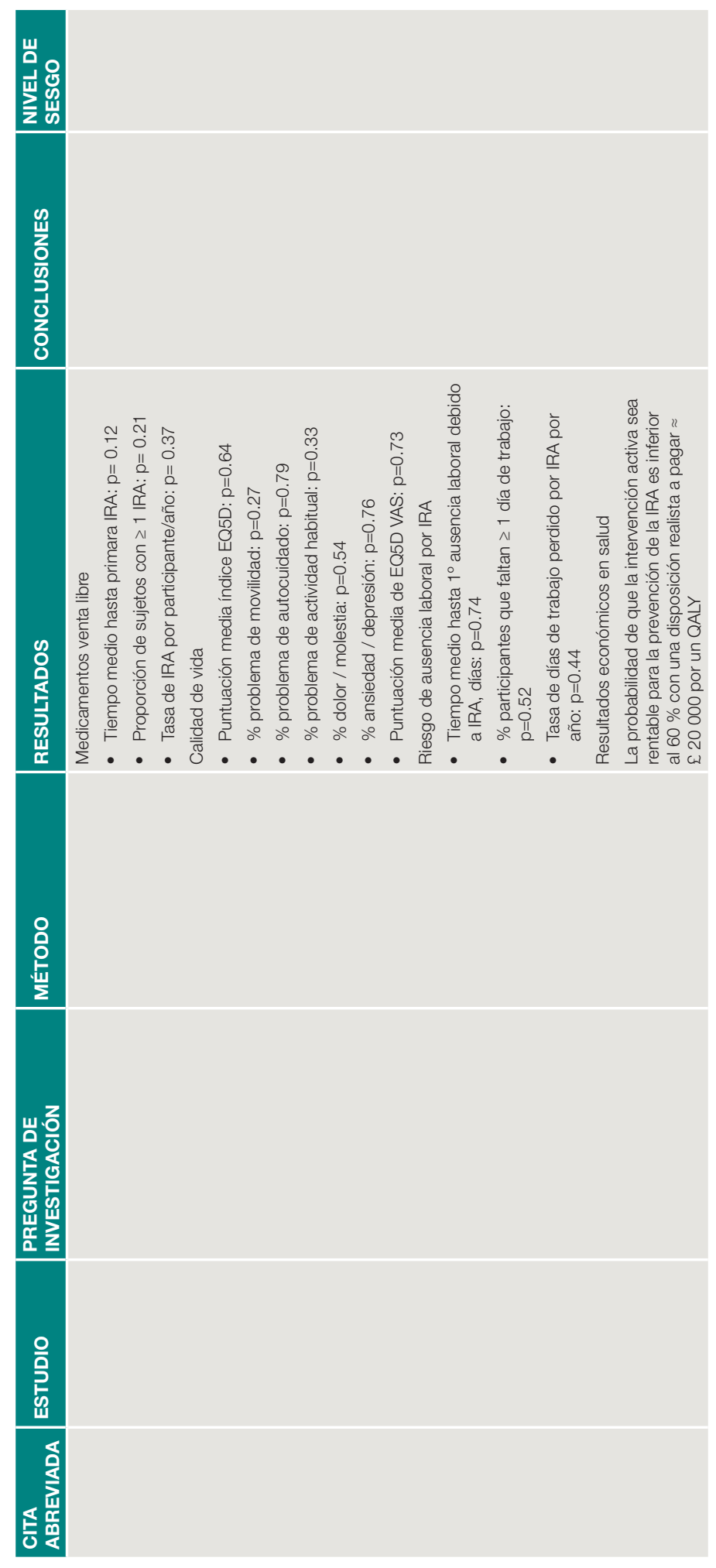




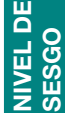

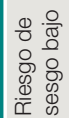

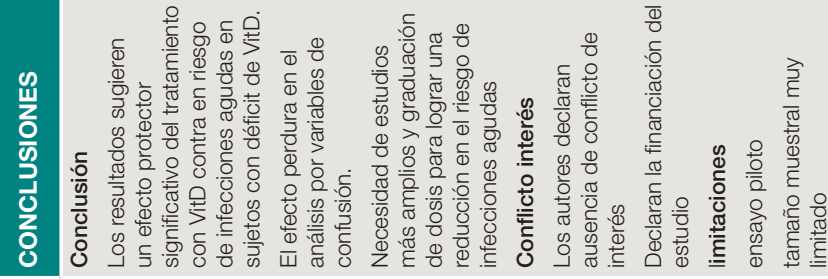

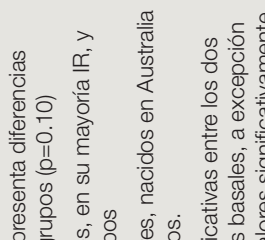

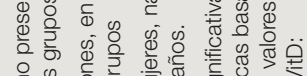

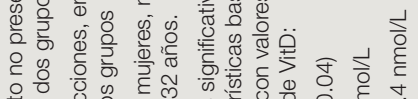

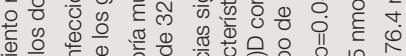

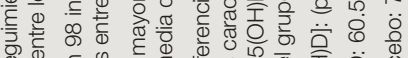

क

$\frac{\sqrt{0}}{0.0}$

을

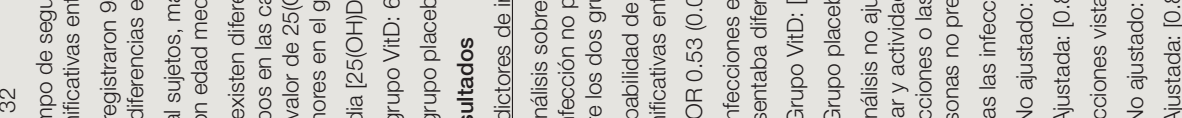

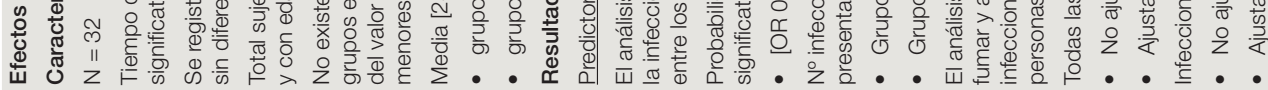

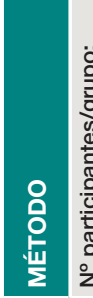

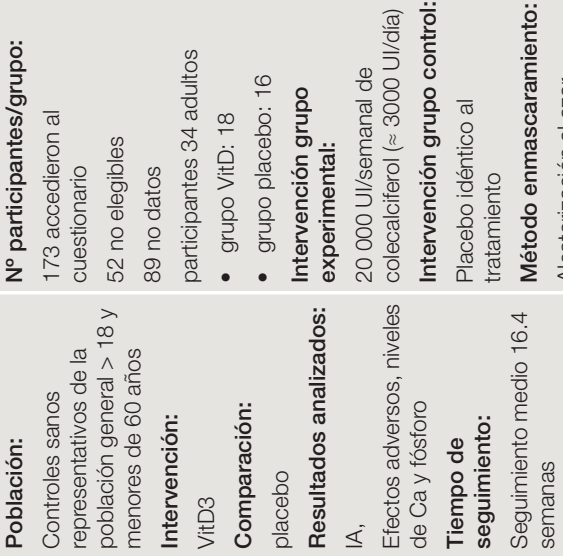

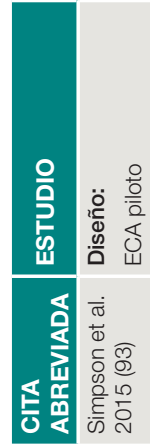




$$
1
$$




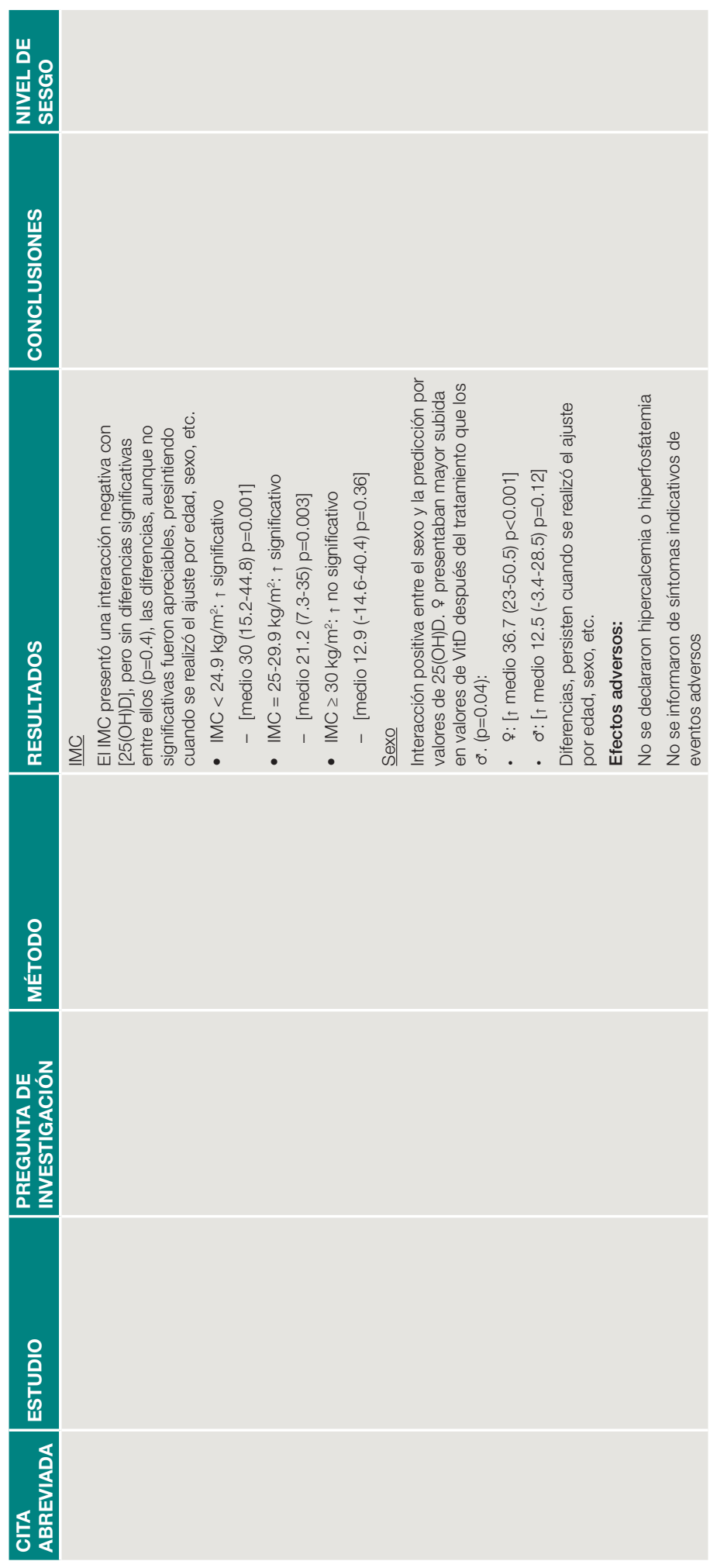




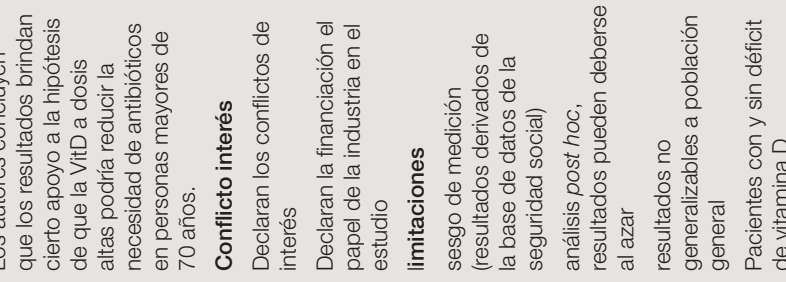

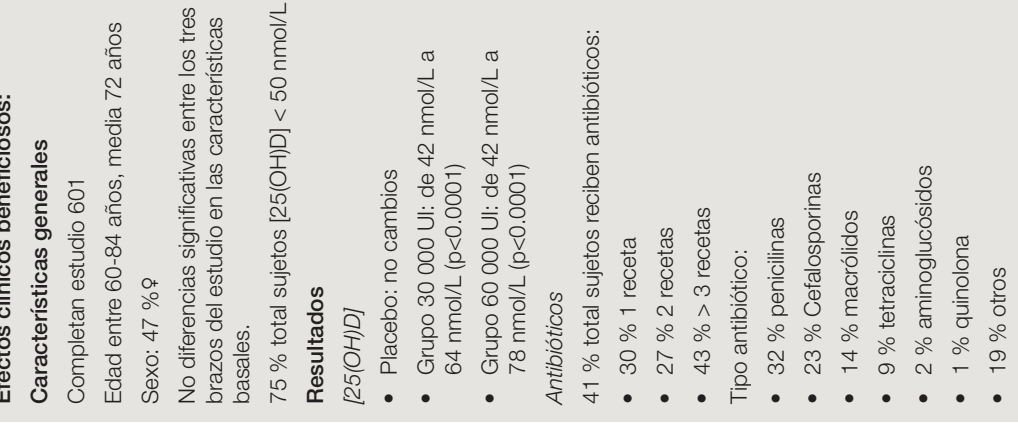

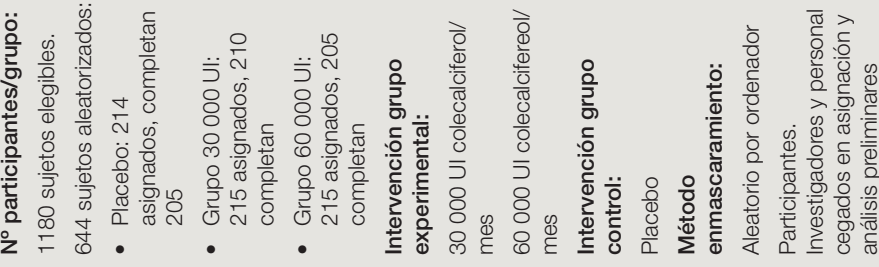

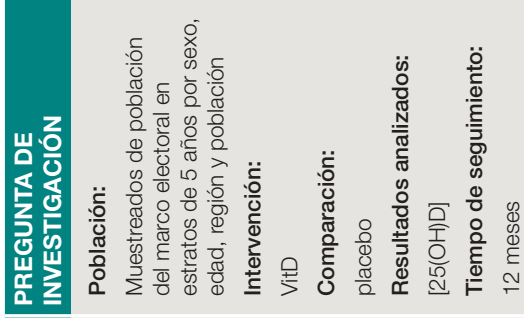

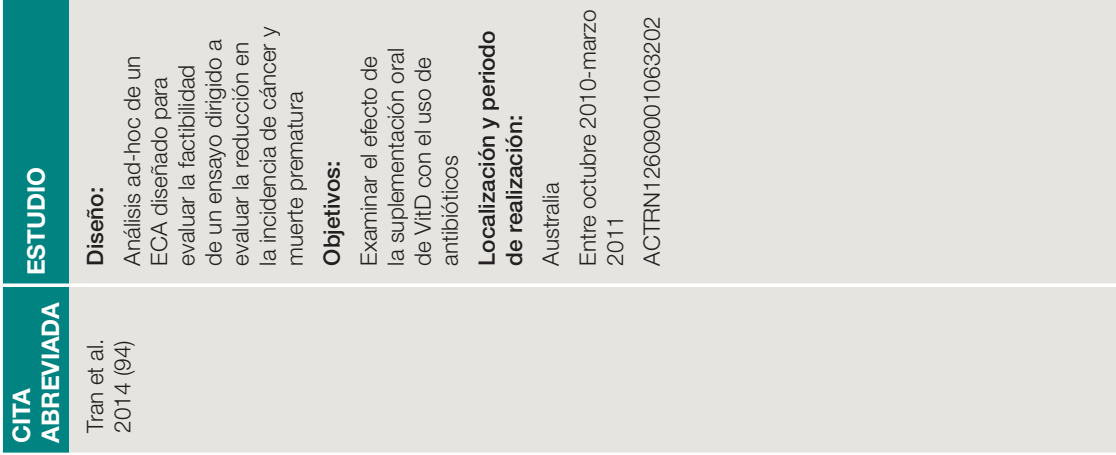




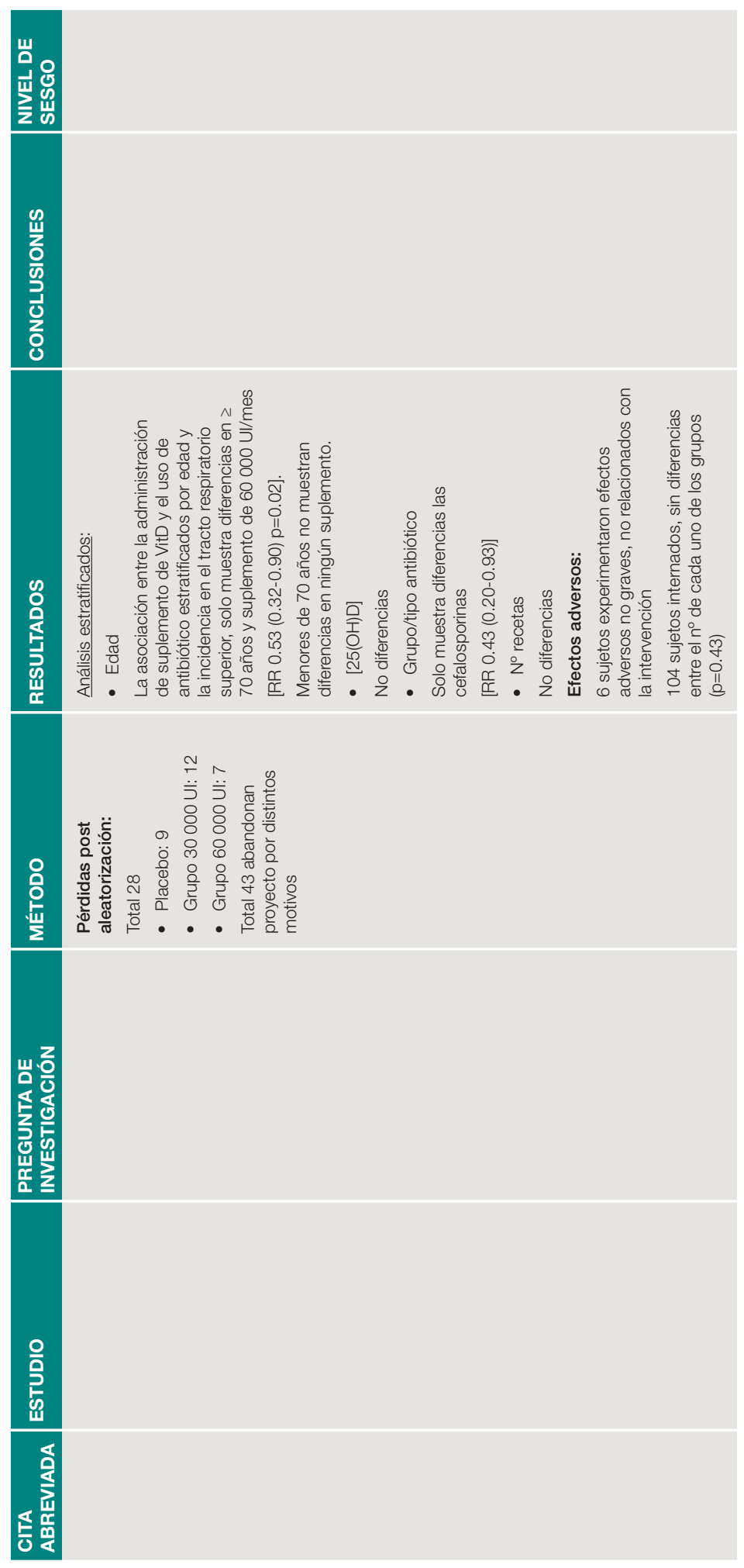




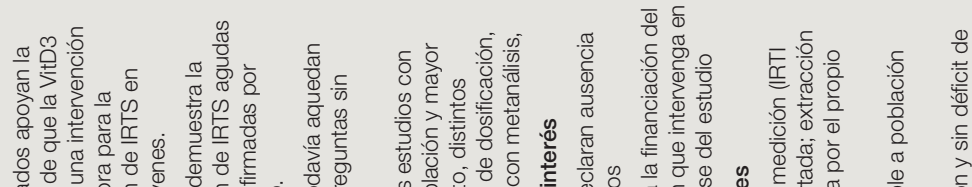

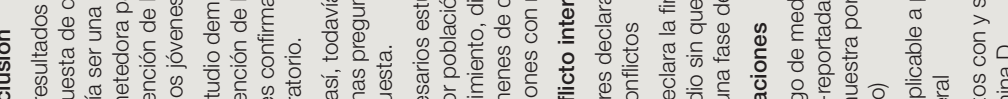

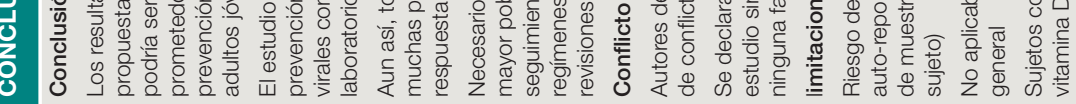

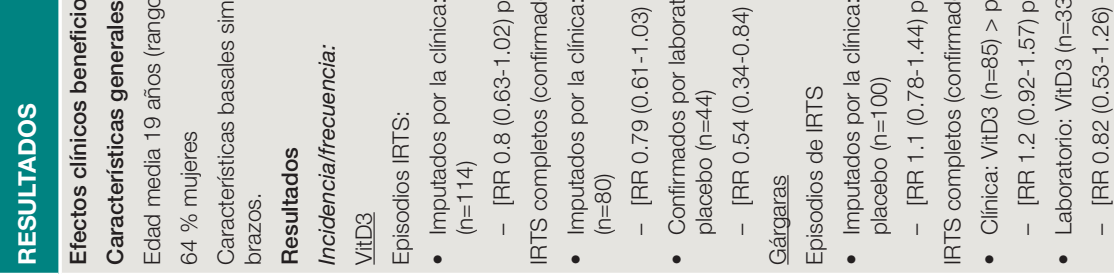

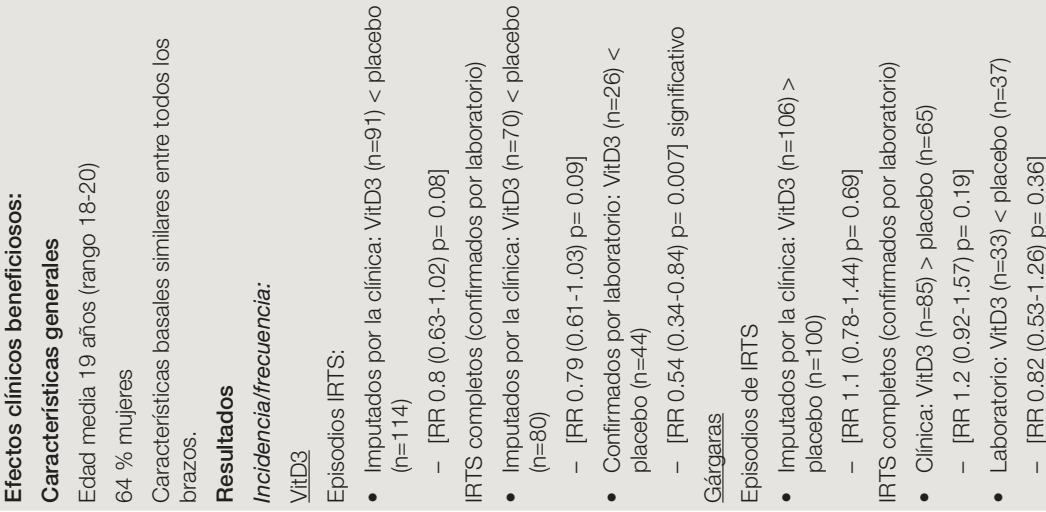

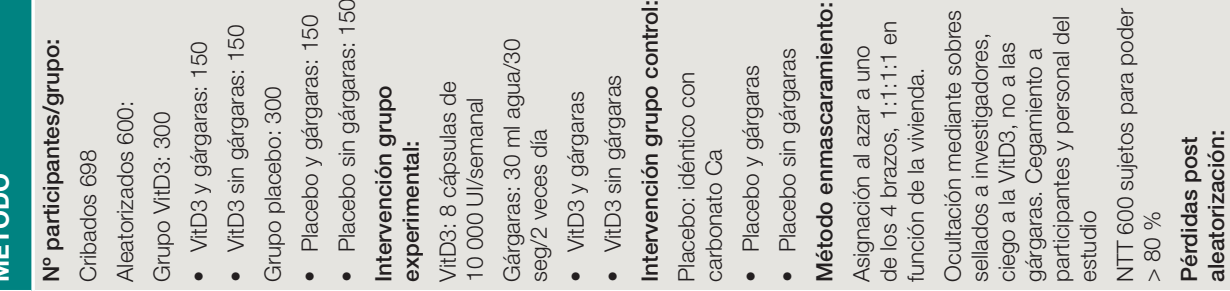

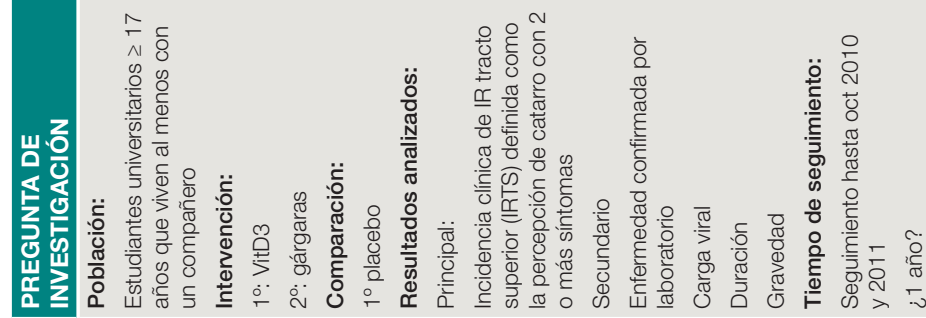

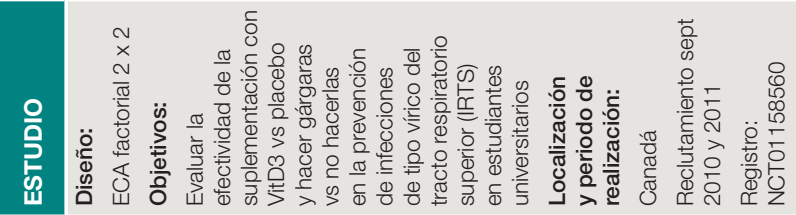



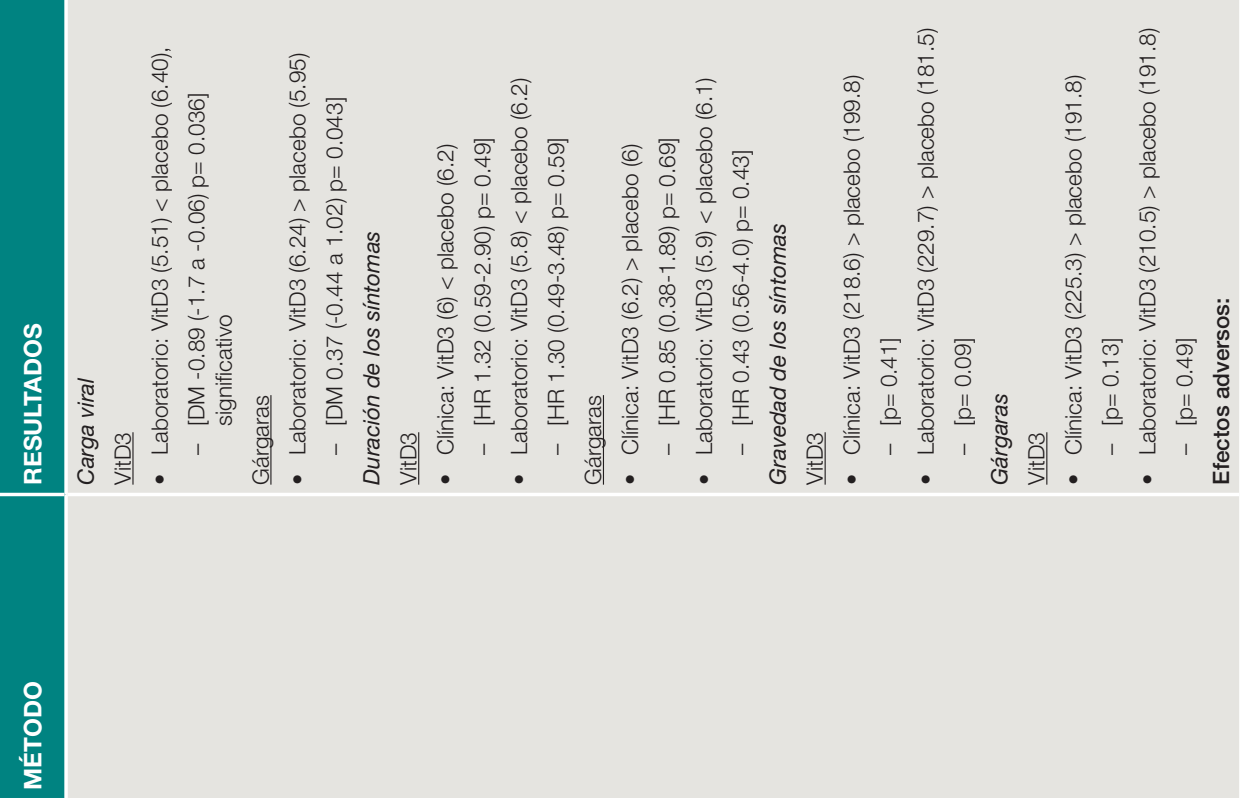

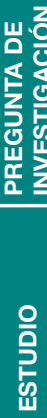




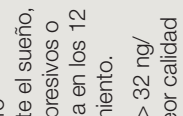

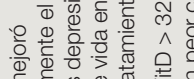

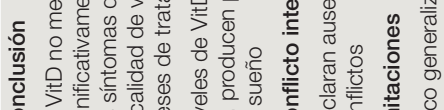

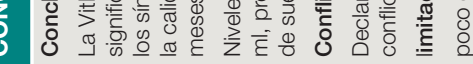

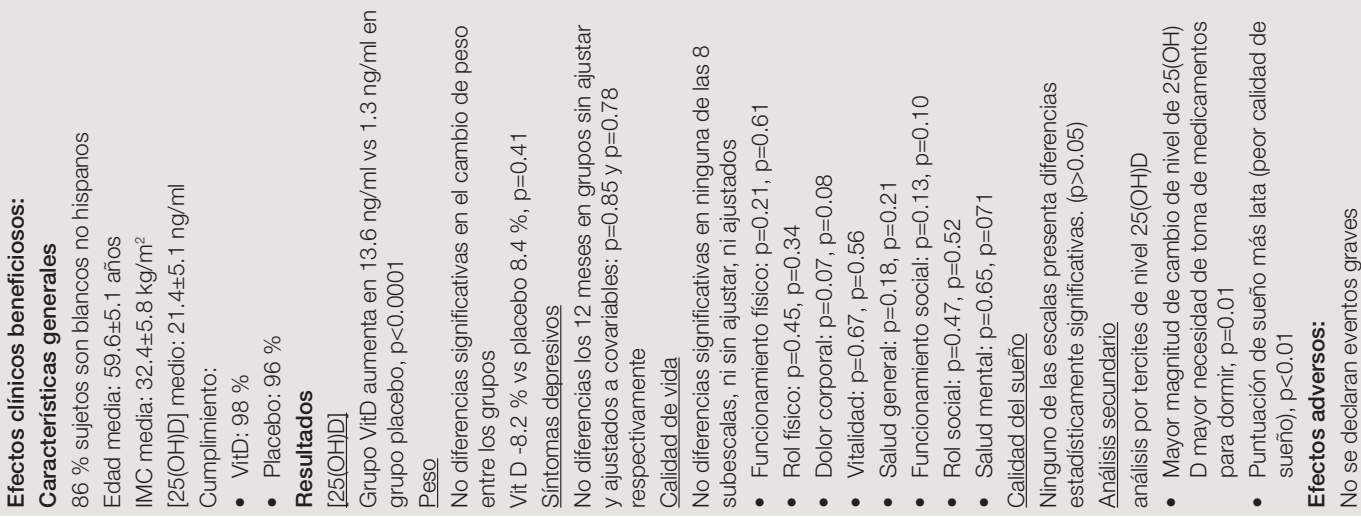

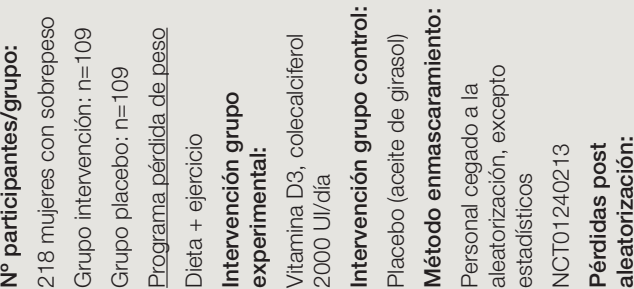

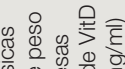

क⿻

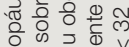

岁花

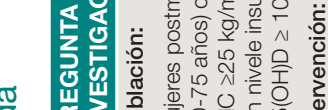

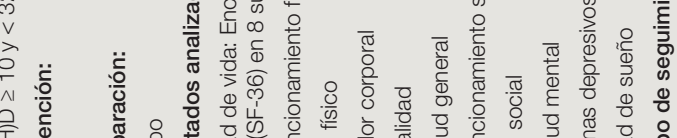

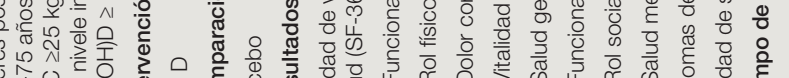

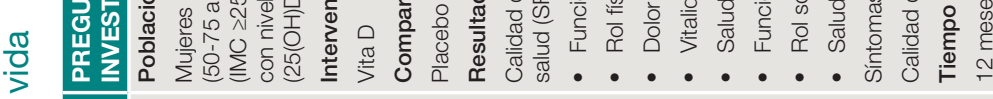

ก

เก

을

$\stackrel{\infty}{\stackrel{\infty}{4}}$

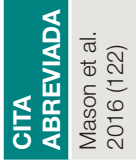




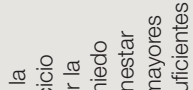

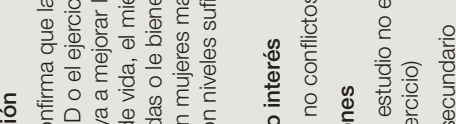

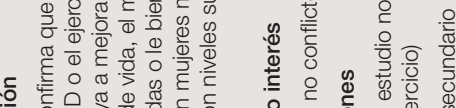

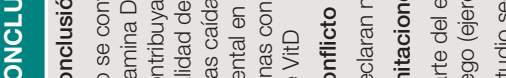

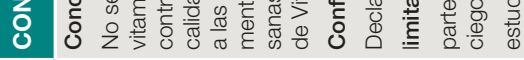

\section{)}

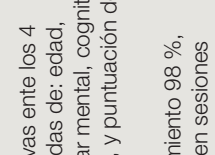

i.

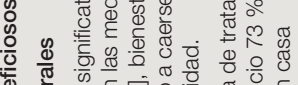

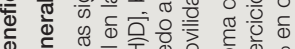

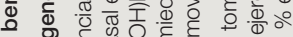

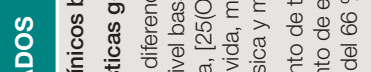

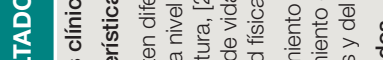

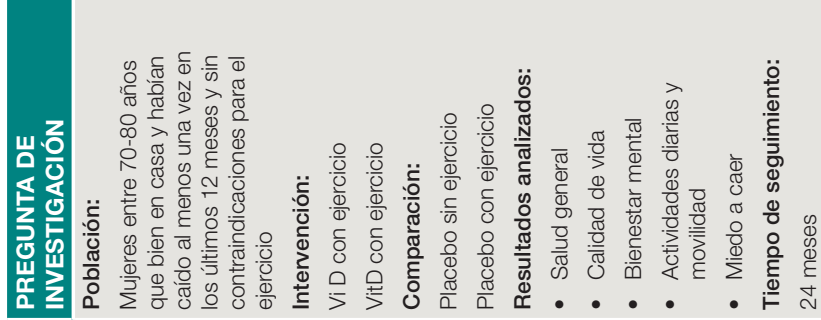

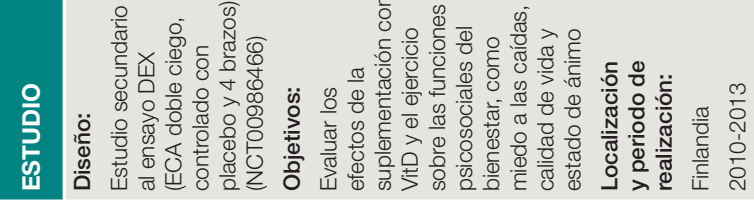




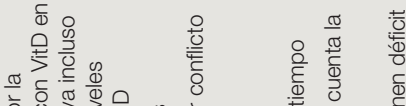

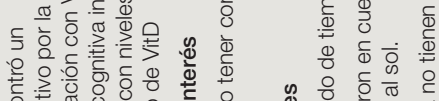

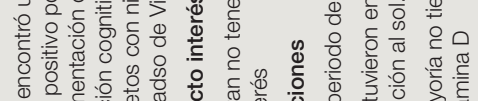

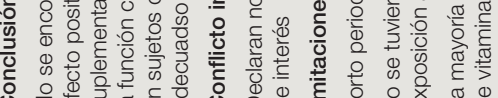

ن

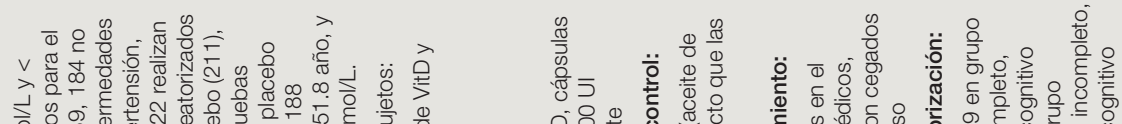

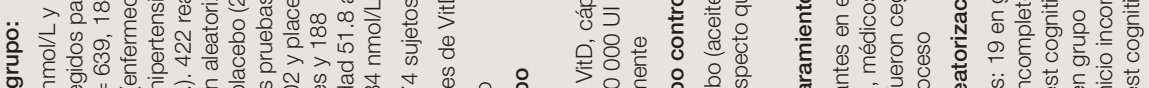

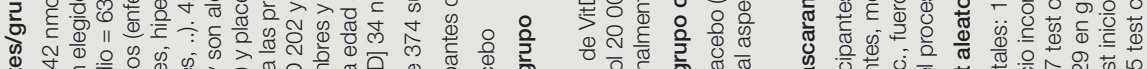

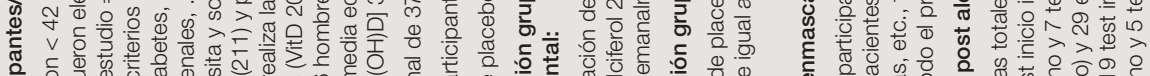

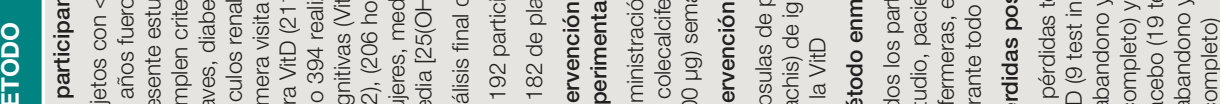

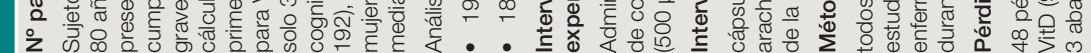

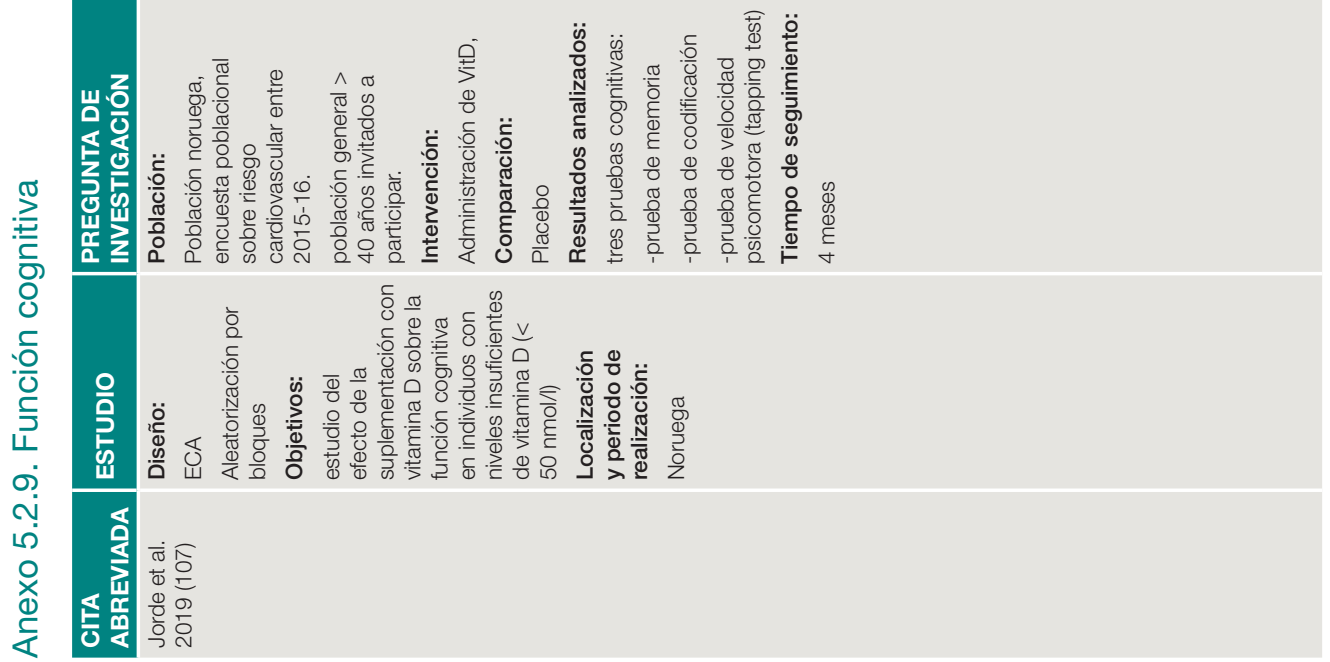




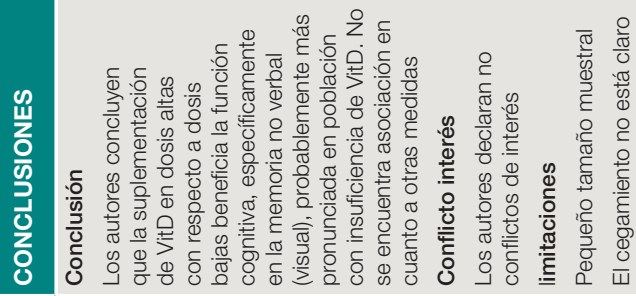
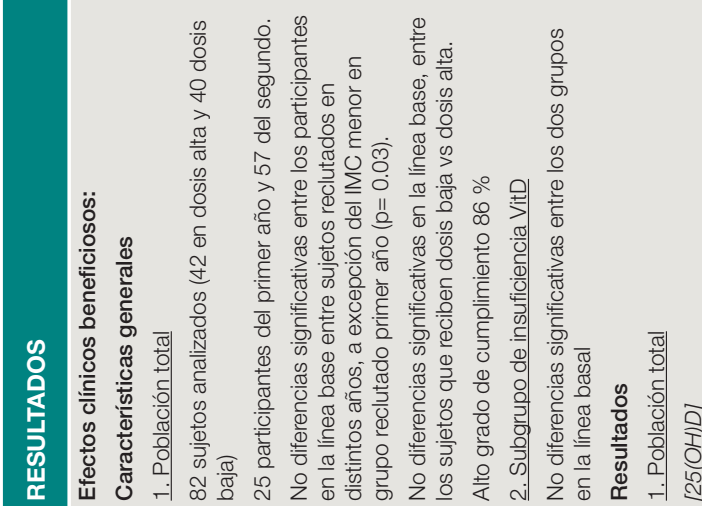

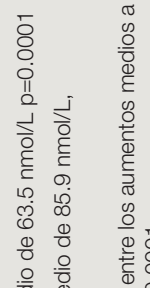

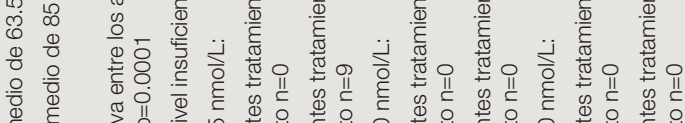

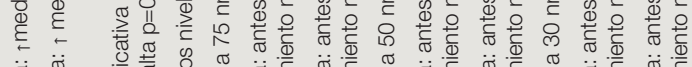

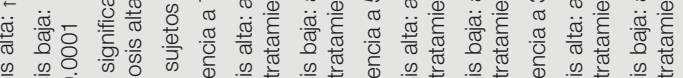

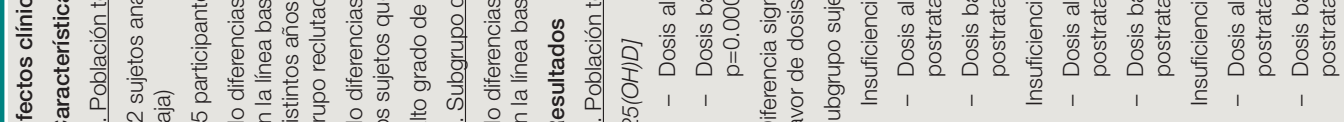
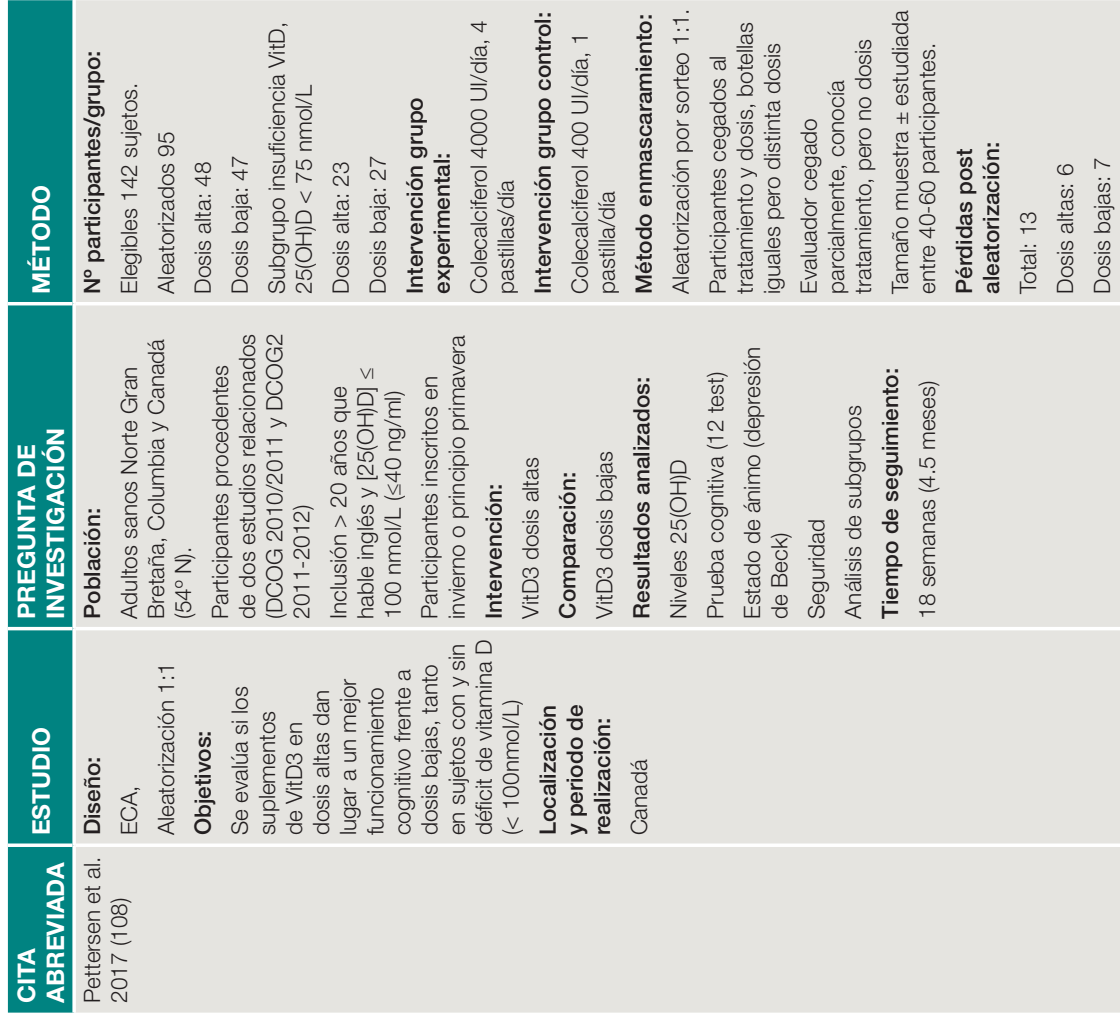

$$
\begin{aligned}
& \text { Г. } \quad \text { ब } \\
& \text { :응 }
\end{aligned}
$$

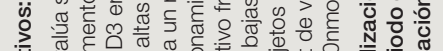

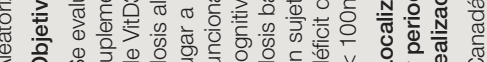

$$
\begin{aligned}
& \text { thos }
\end{aligned}
$$




$$
\mid
$$



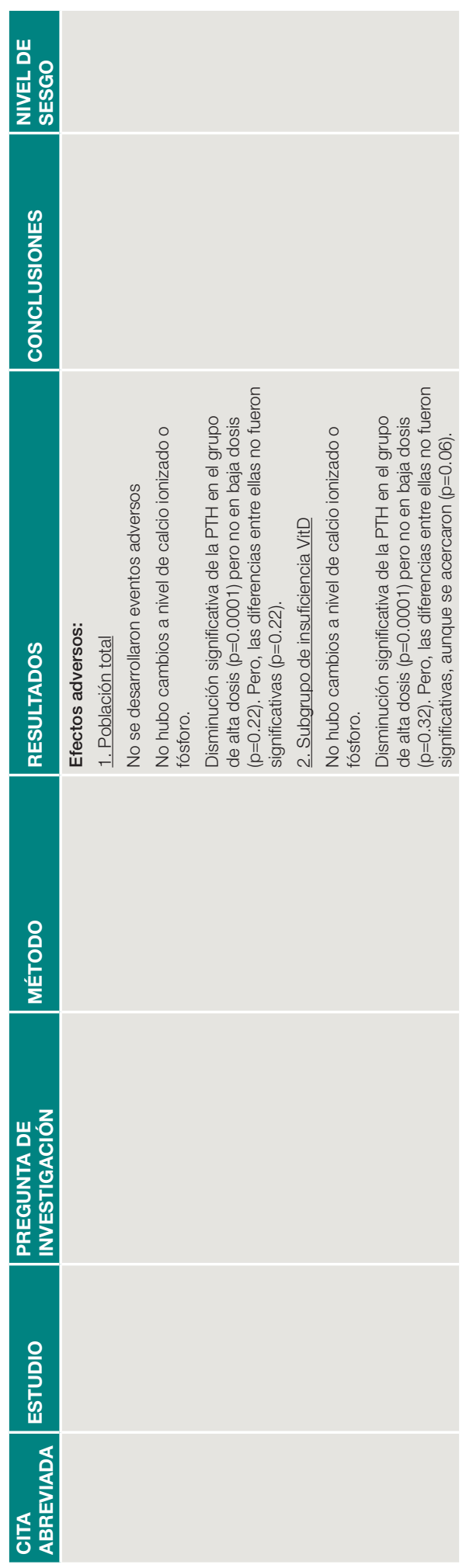


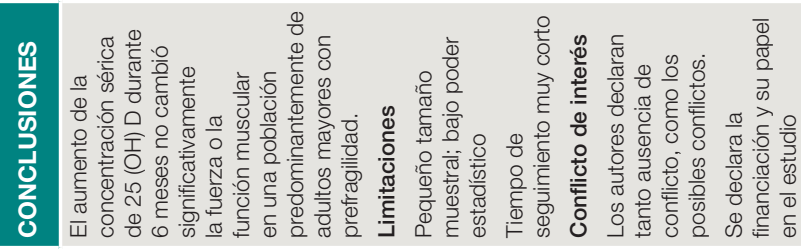

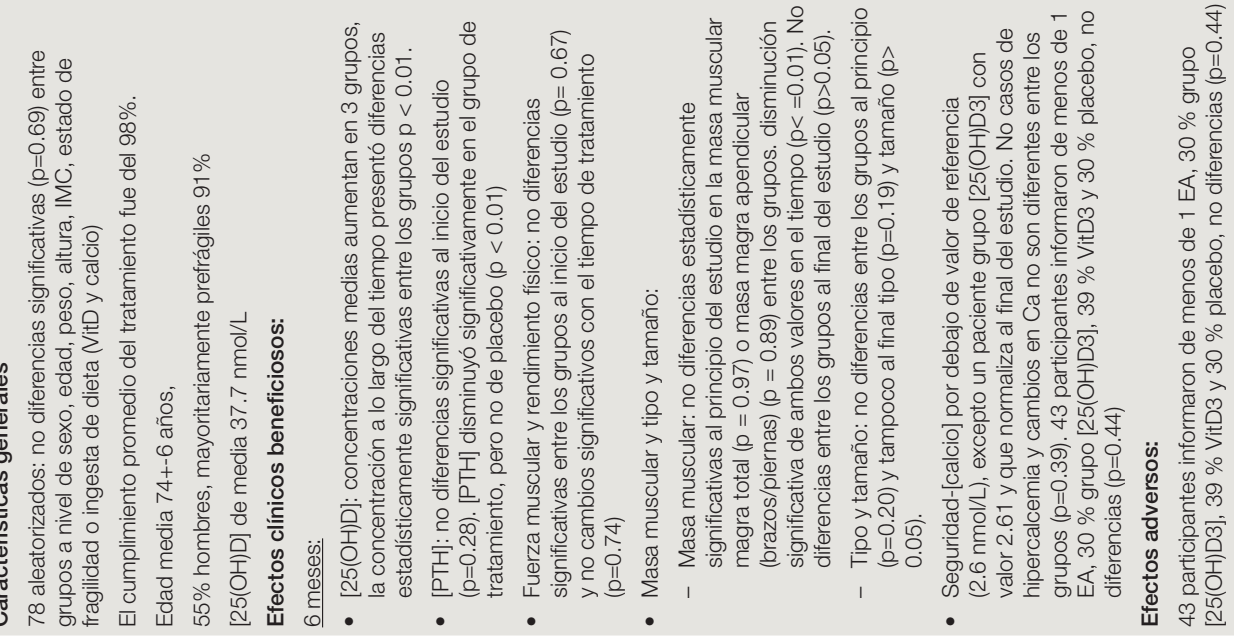

$\frac{\frac{8}{2}}{\frac{8}{2}}$

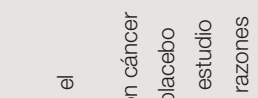

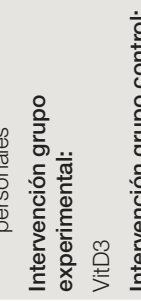

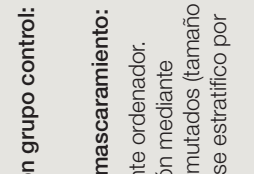

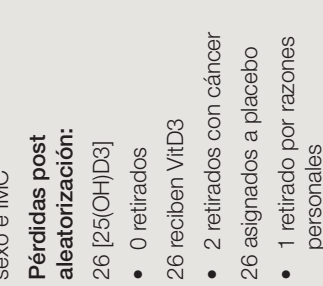

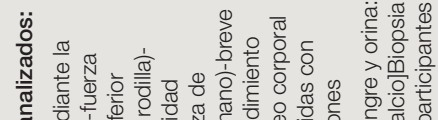

주은

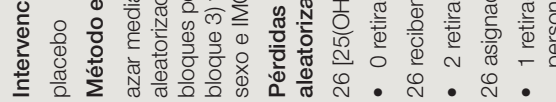

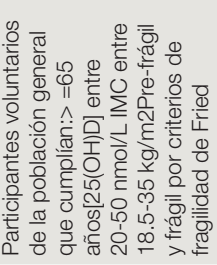

$\frac{\sqrt{1}}{\sqrt{d}}$

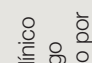

\section{siting}

$\frac{1}{2}$

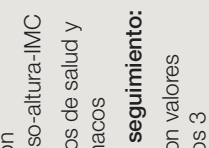

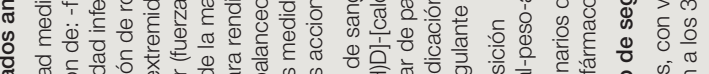

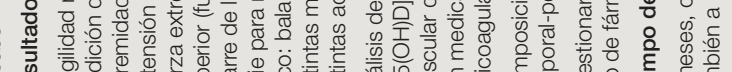

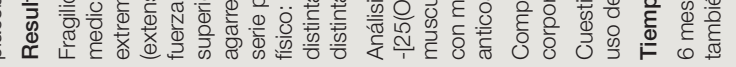

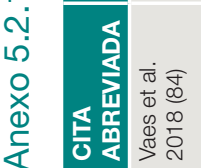



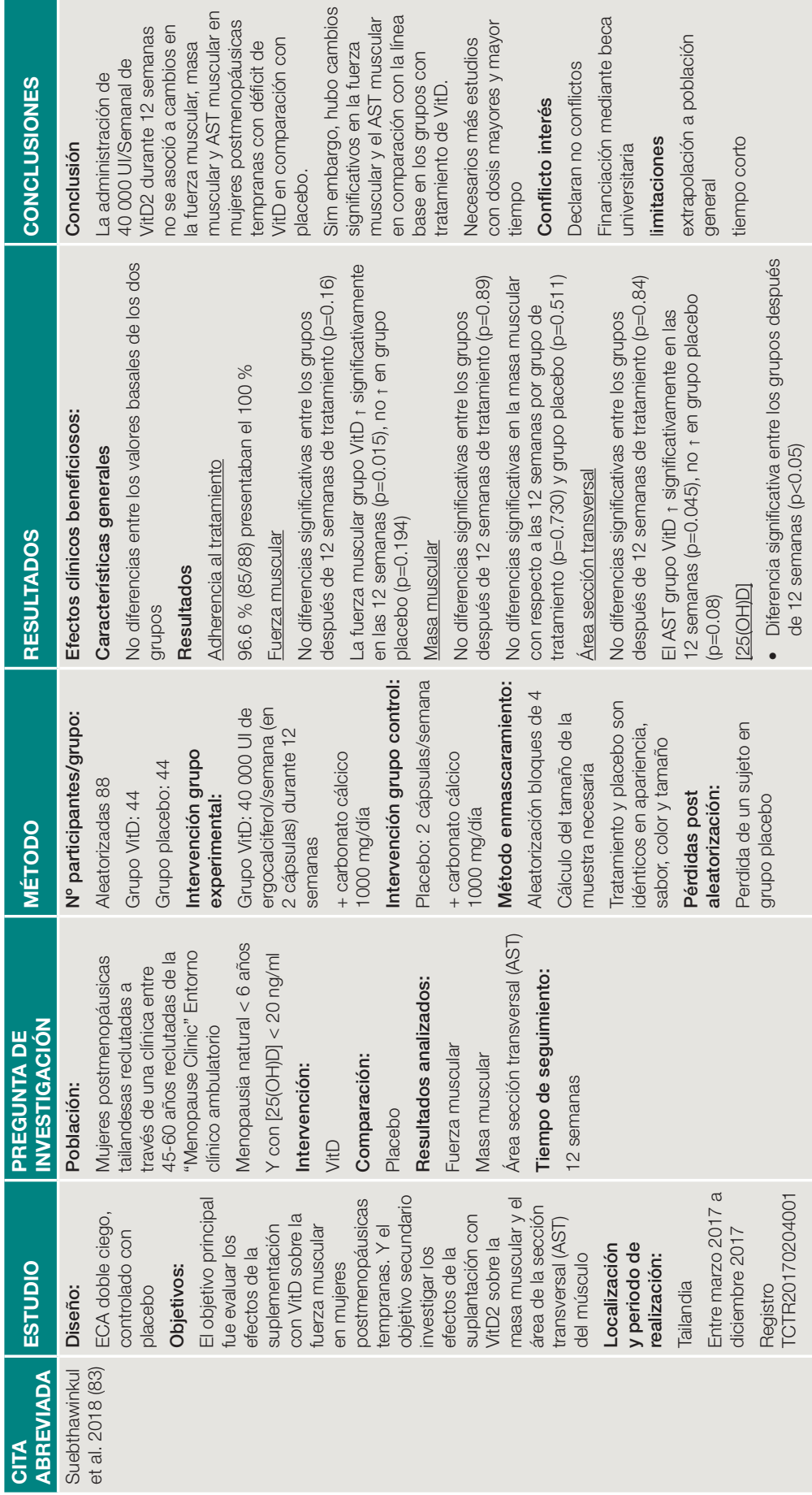


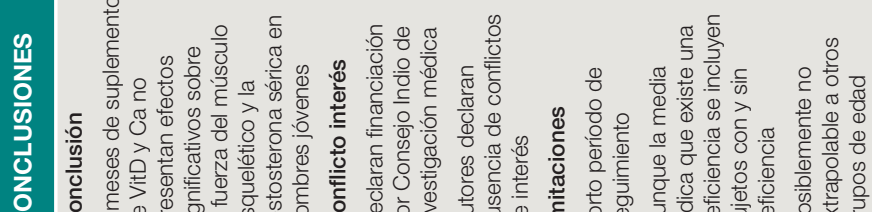 ठ}
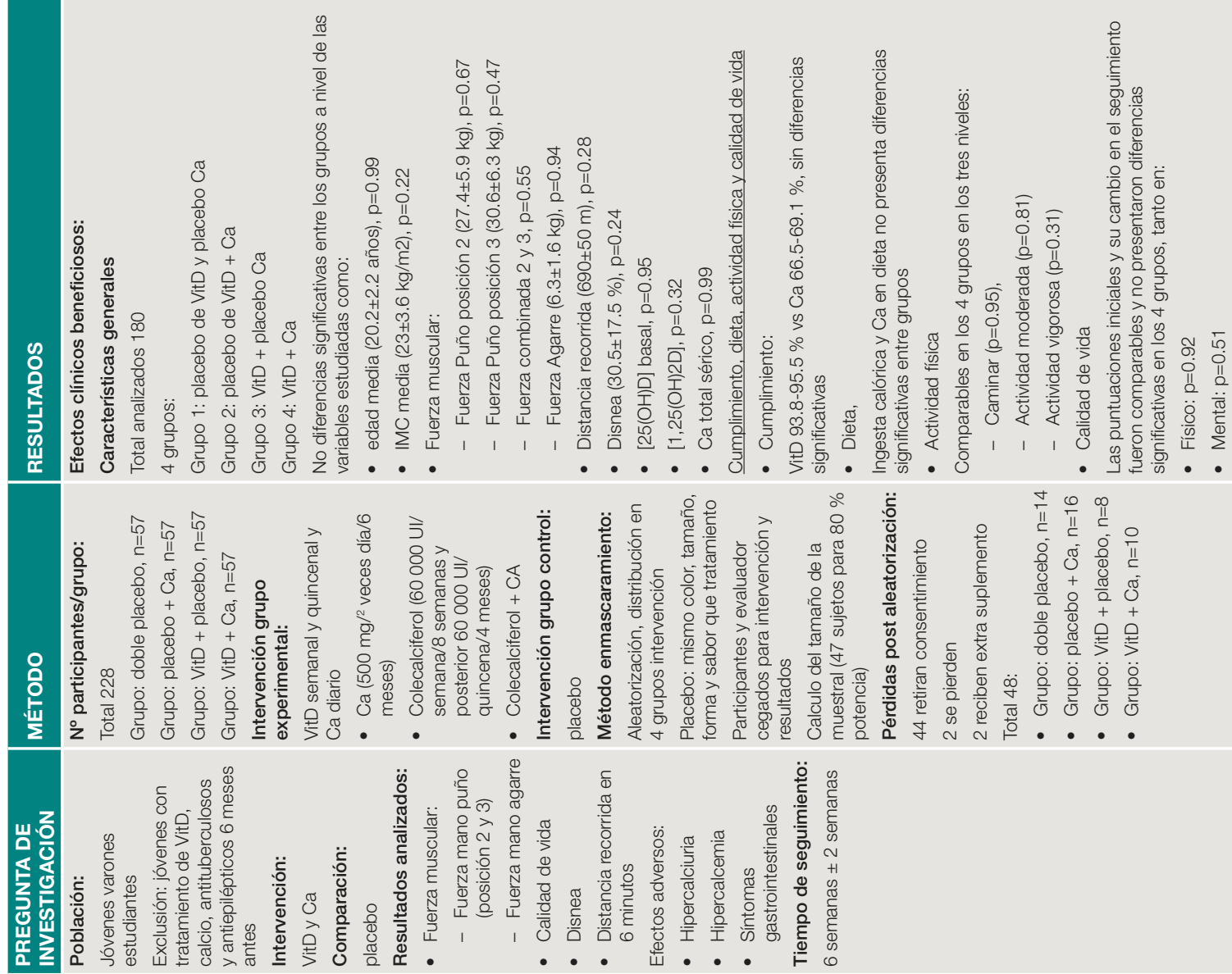

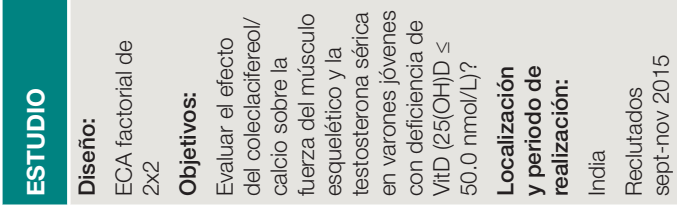

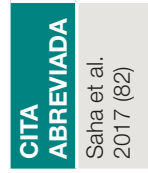



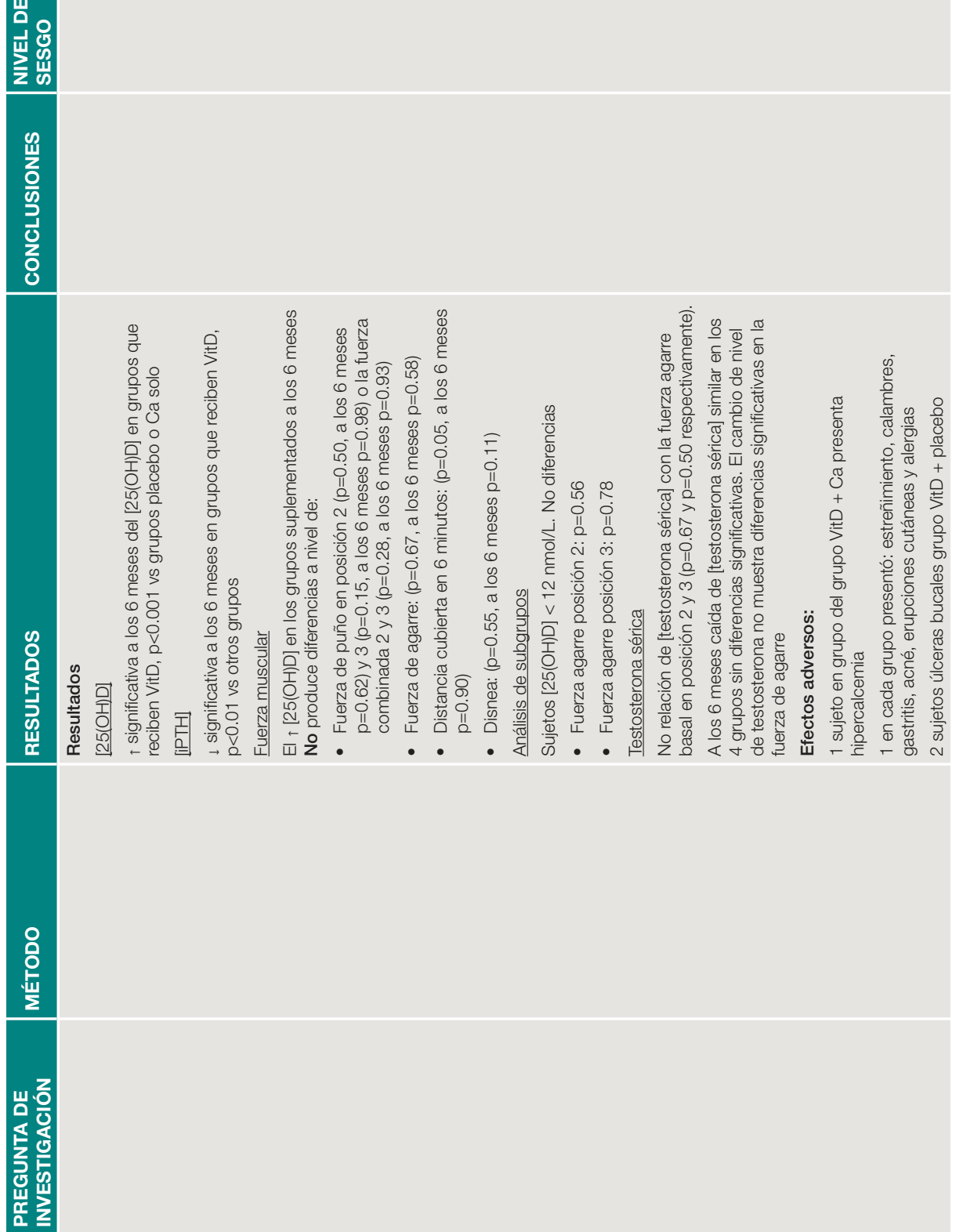

음 


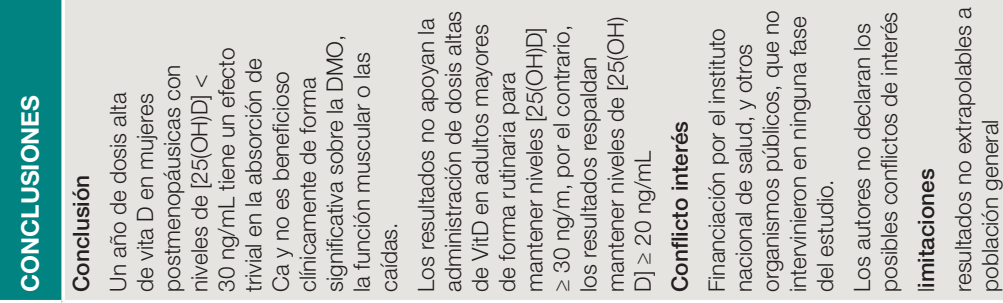

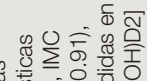

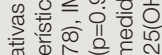

次

헤의 응 क

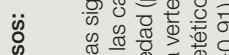

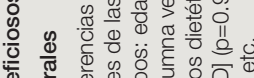

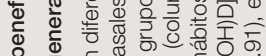

⿰彳

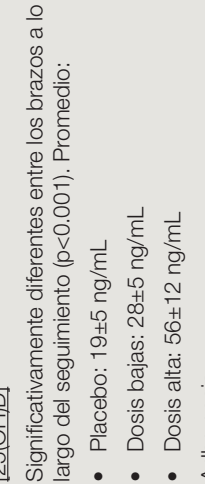

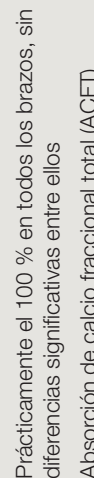

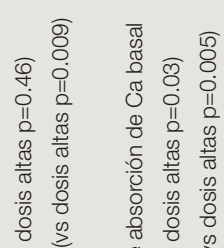

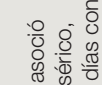

다요응

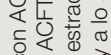

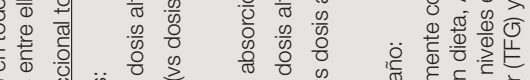

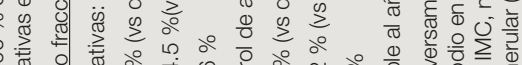

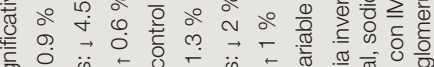

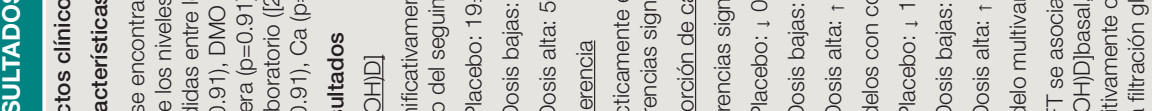

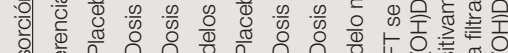

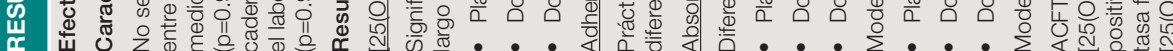

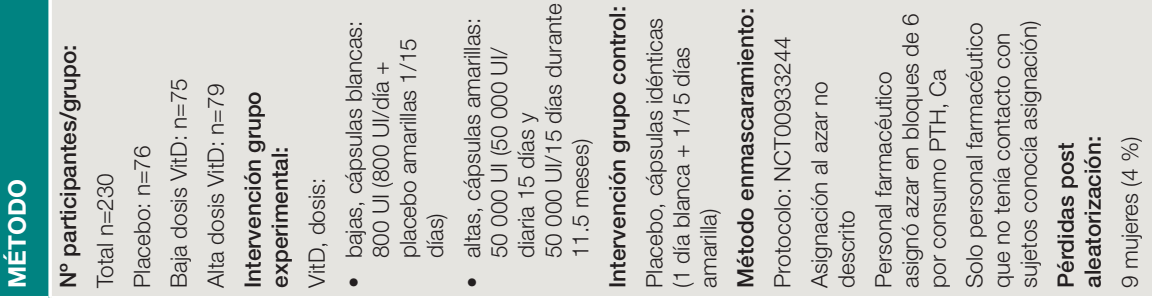

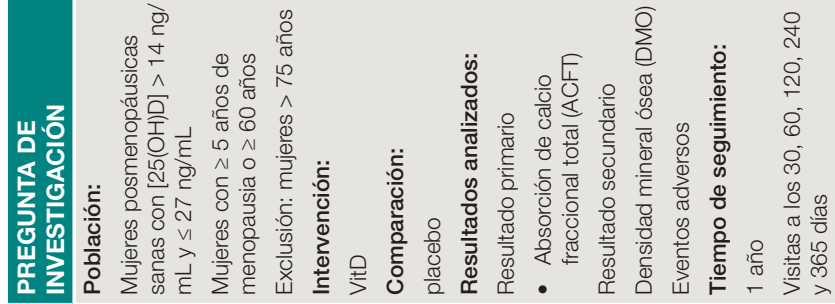

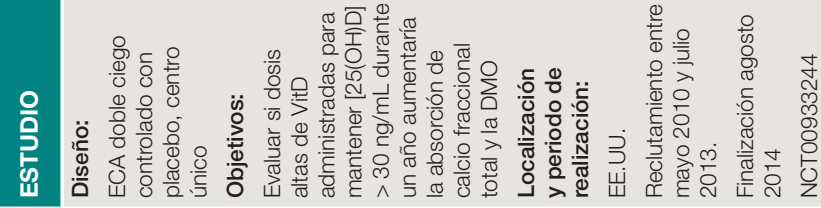

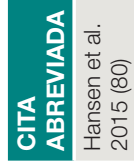




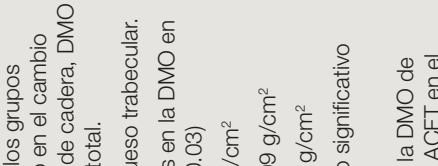

ब

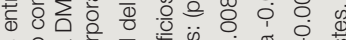

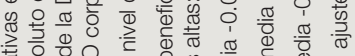

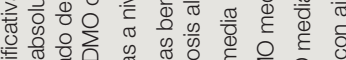

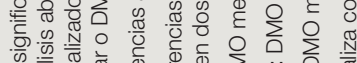

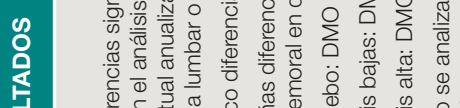

융.응

을.

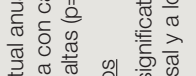

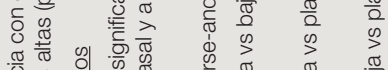

क्ष

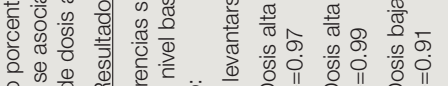

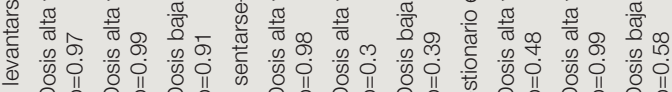

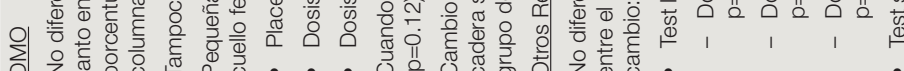




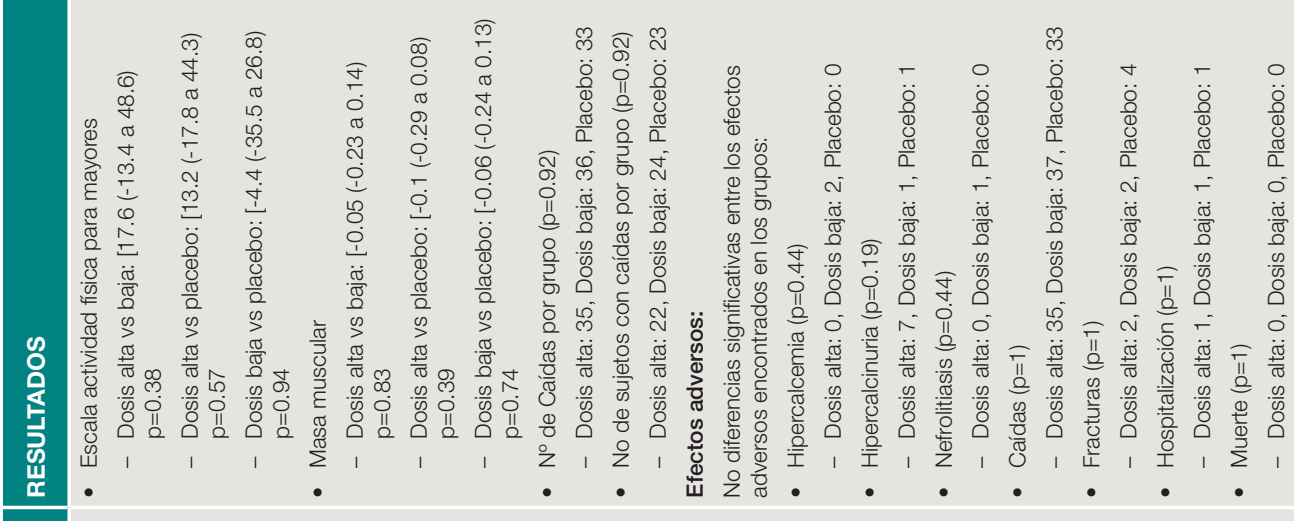



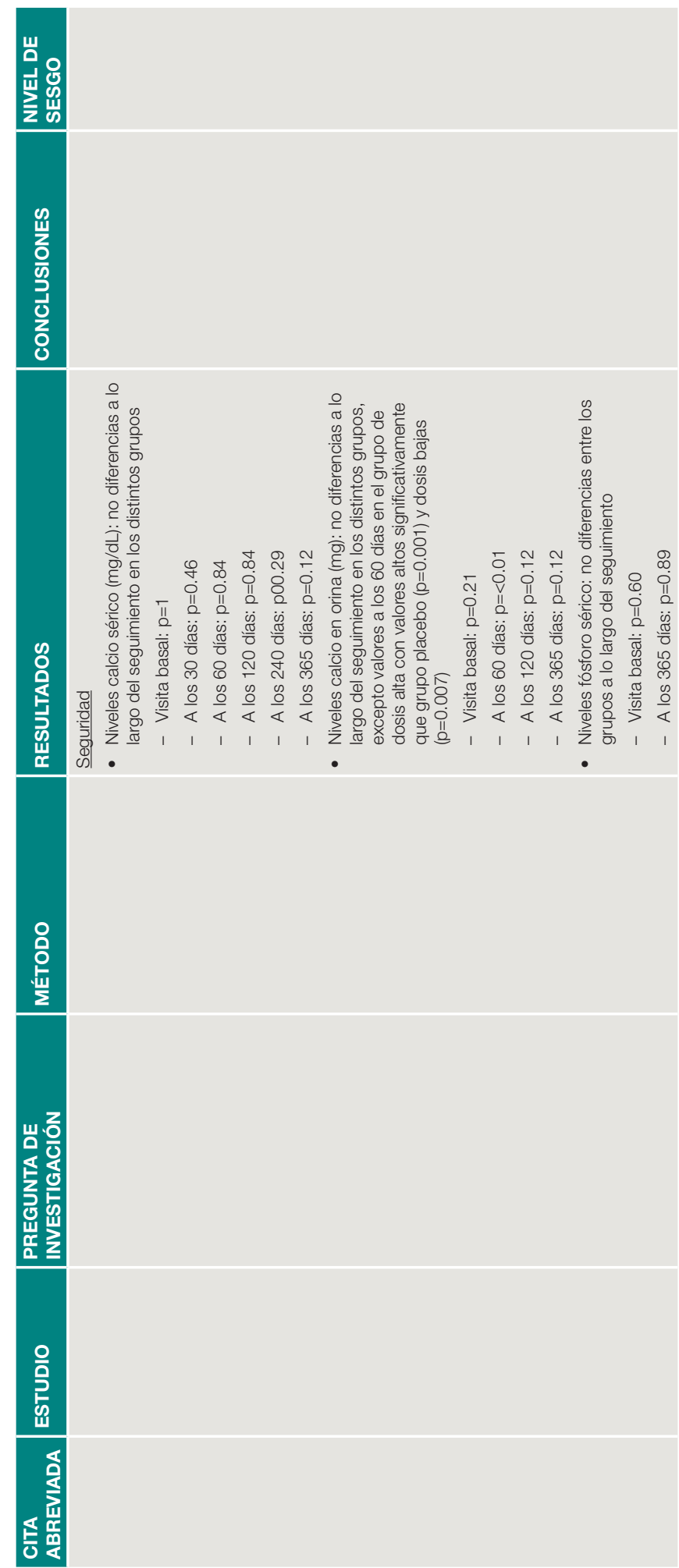


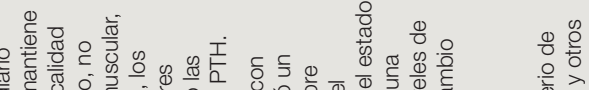

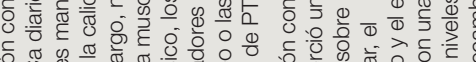

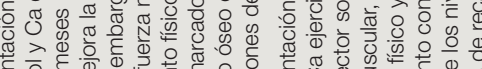

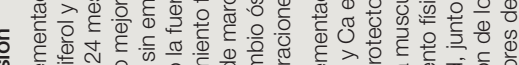

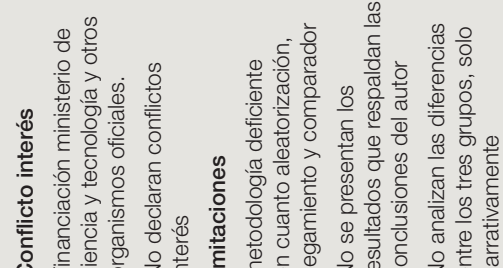

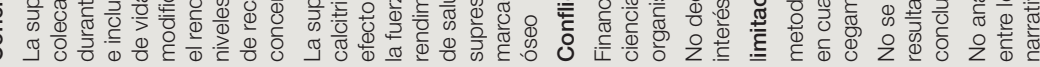

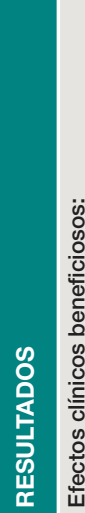

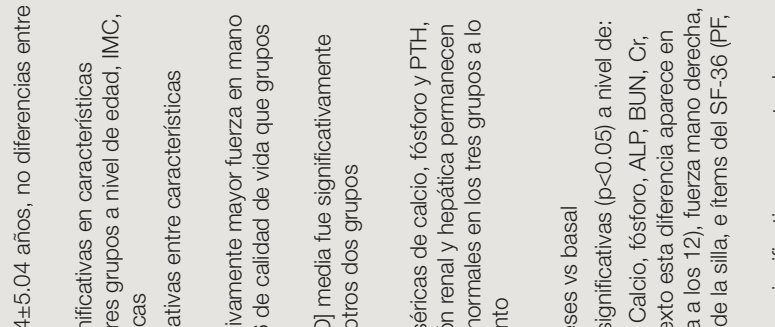

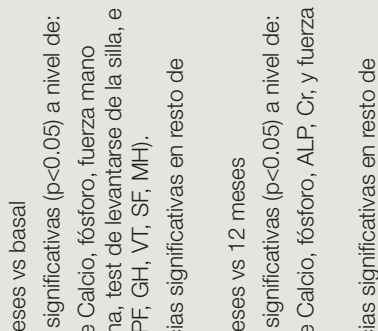

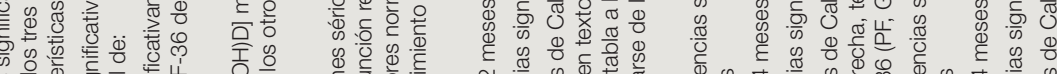

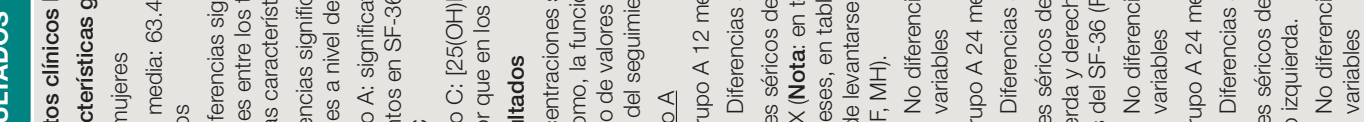

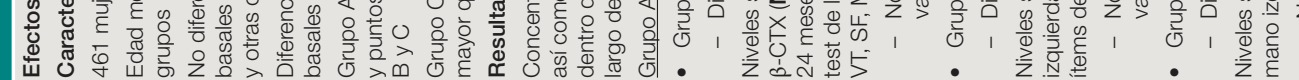

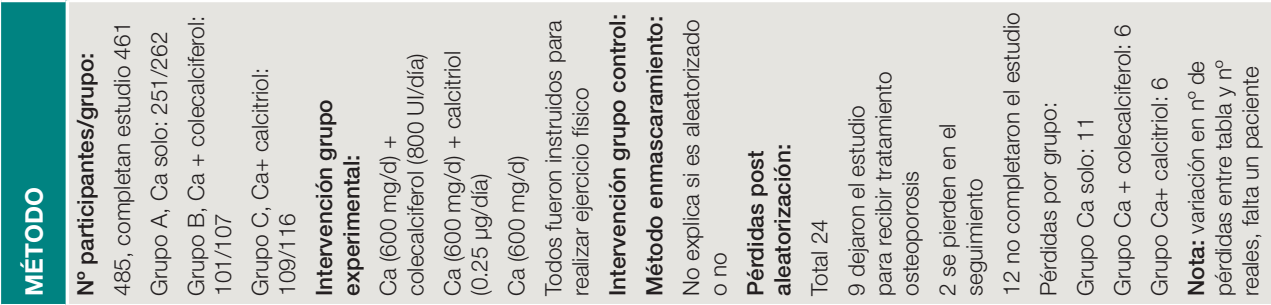

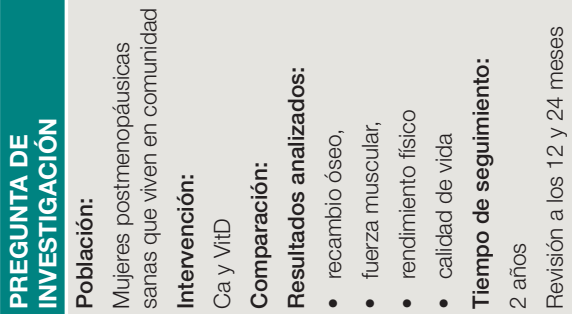

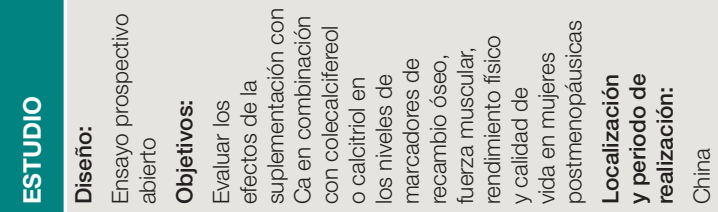

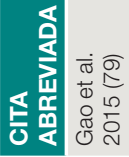


IIIIIIIIIIIIIIIIII!)

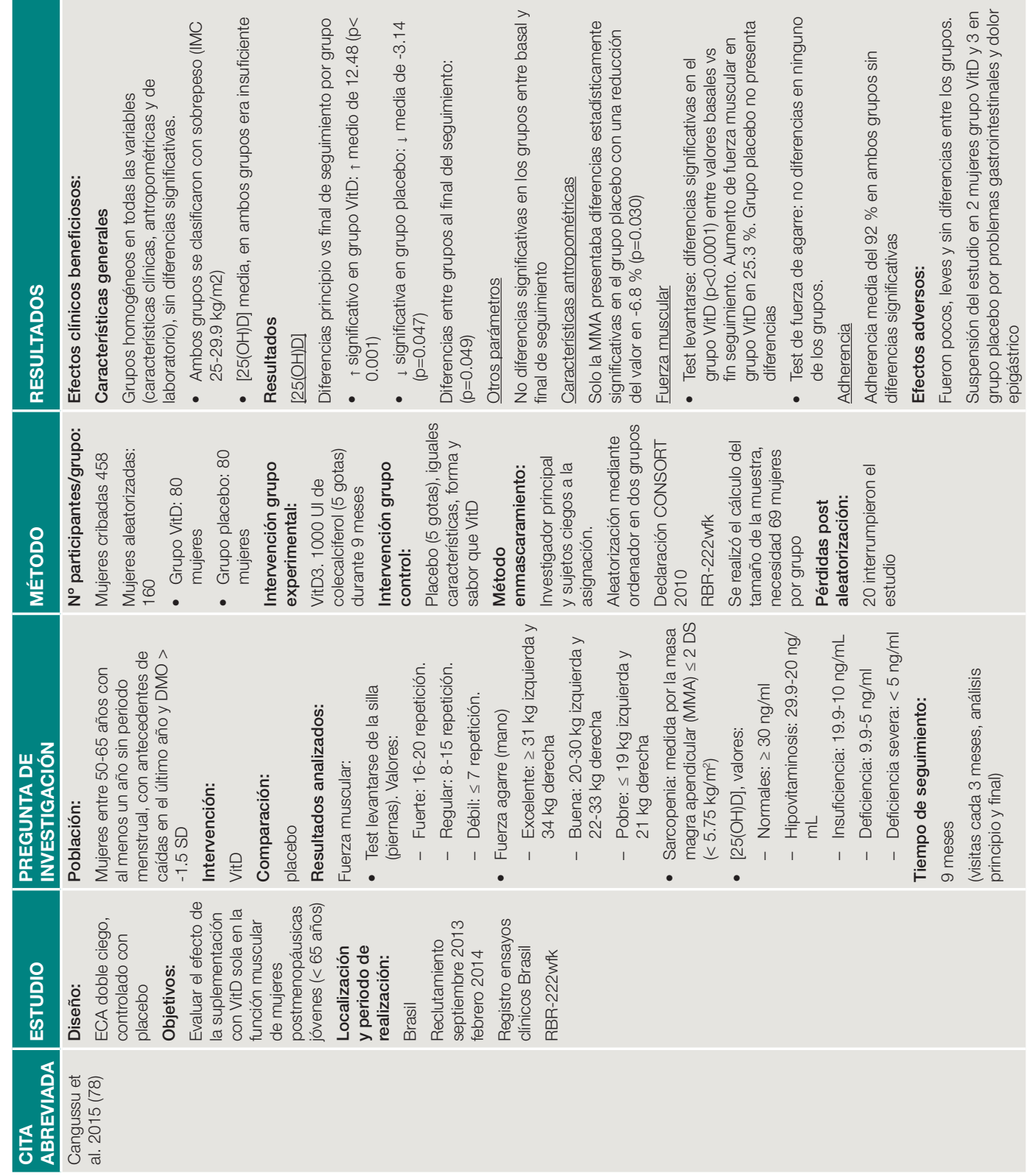




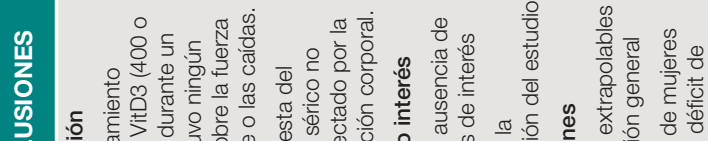

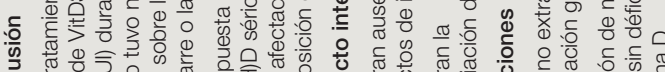

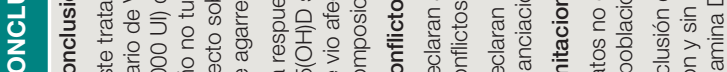 o}
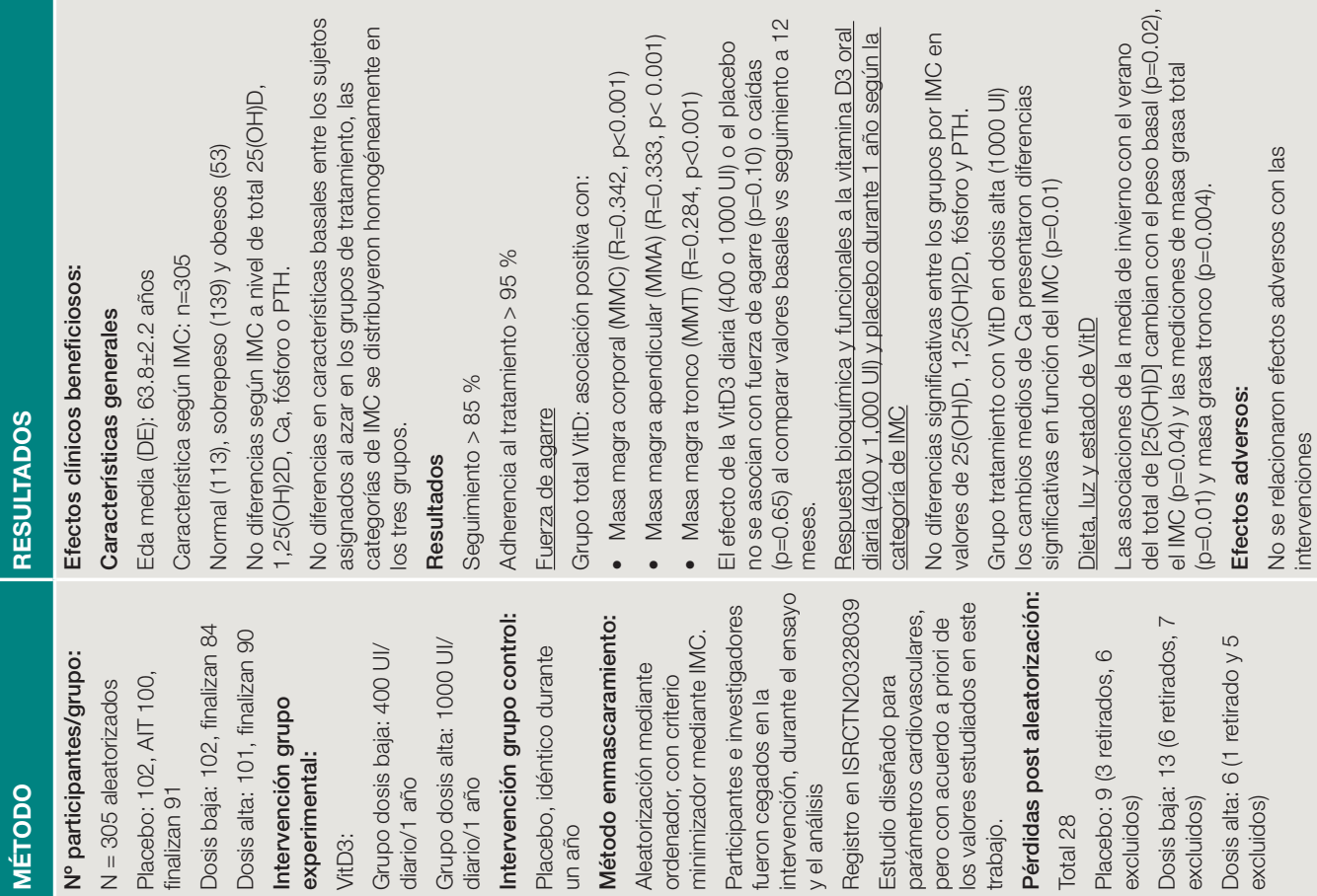

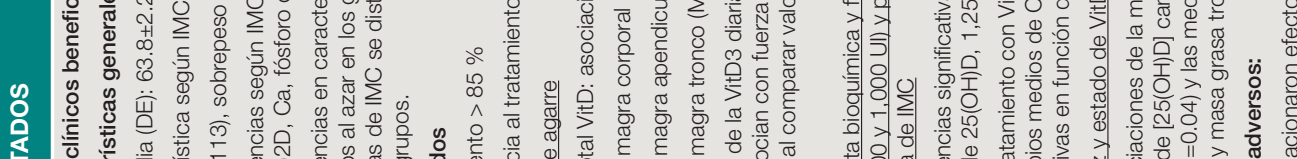

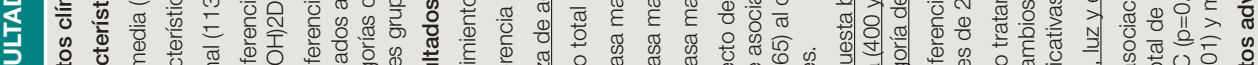

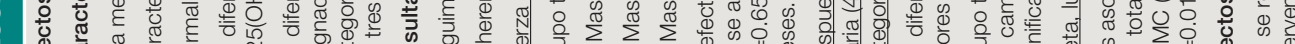
宸 崖

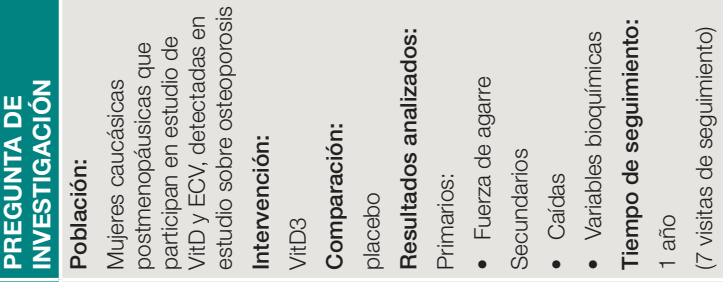

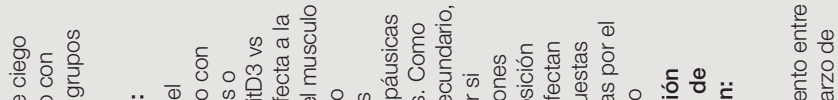

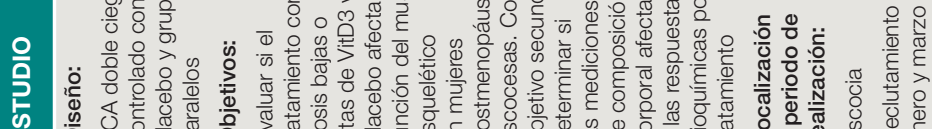

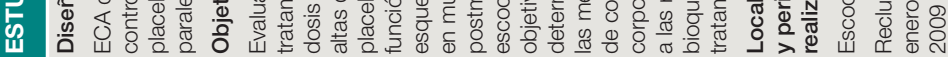

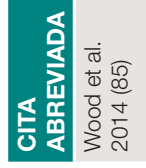




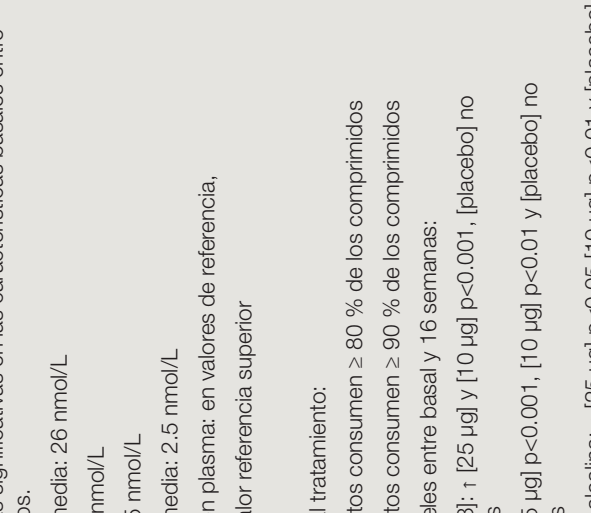

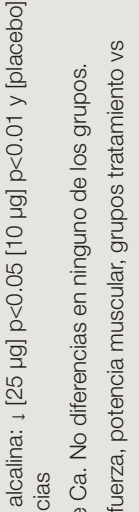

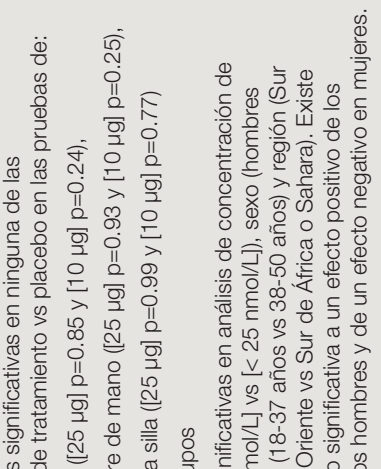

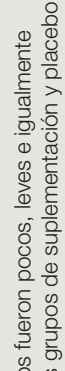
का

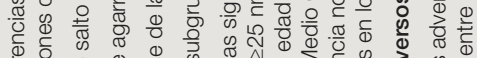

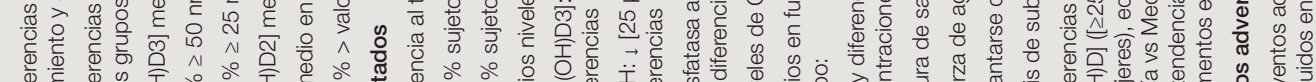

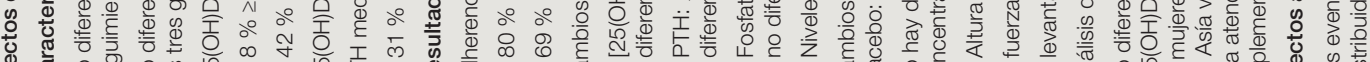

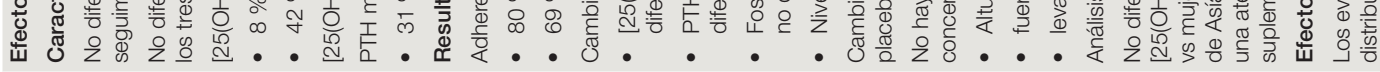

\section{ळ}

$\ddot{0} \quad \frac{\dot{\sigma}}{0} \frac{\dot{\sigma}}{\tilde{0}}$

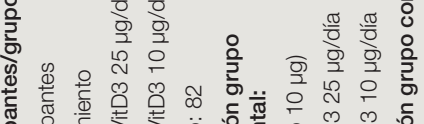

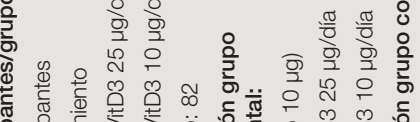

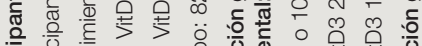




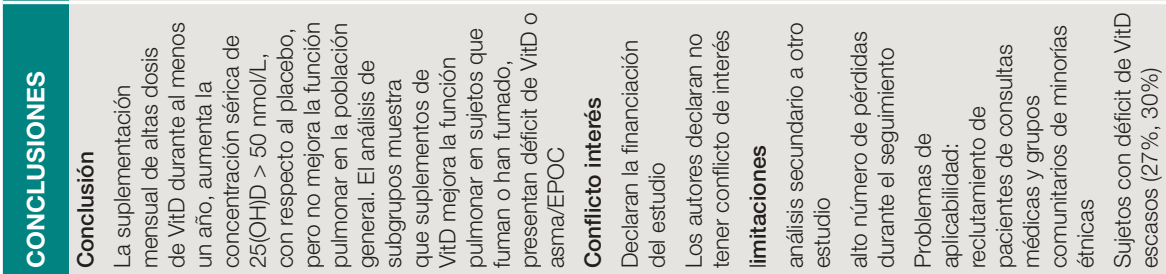
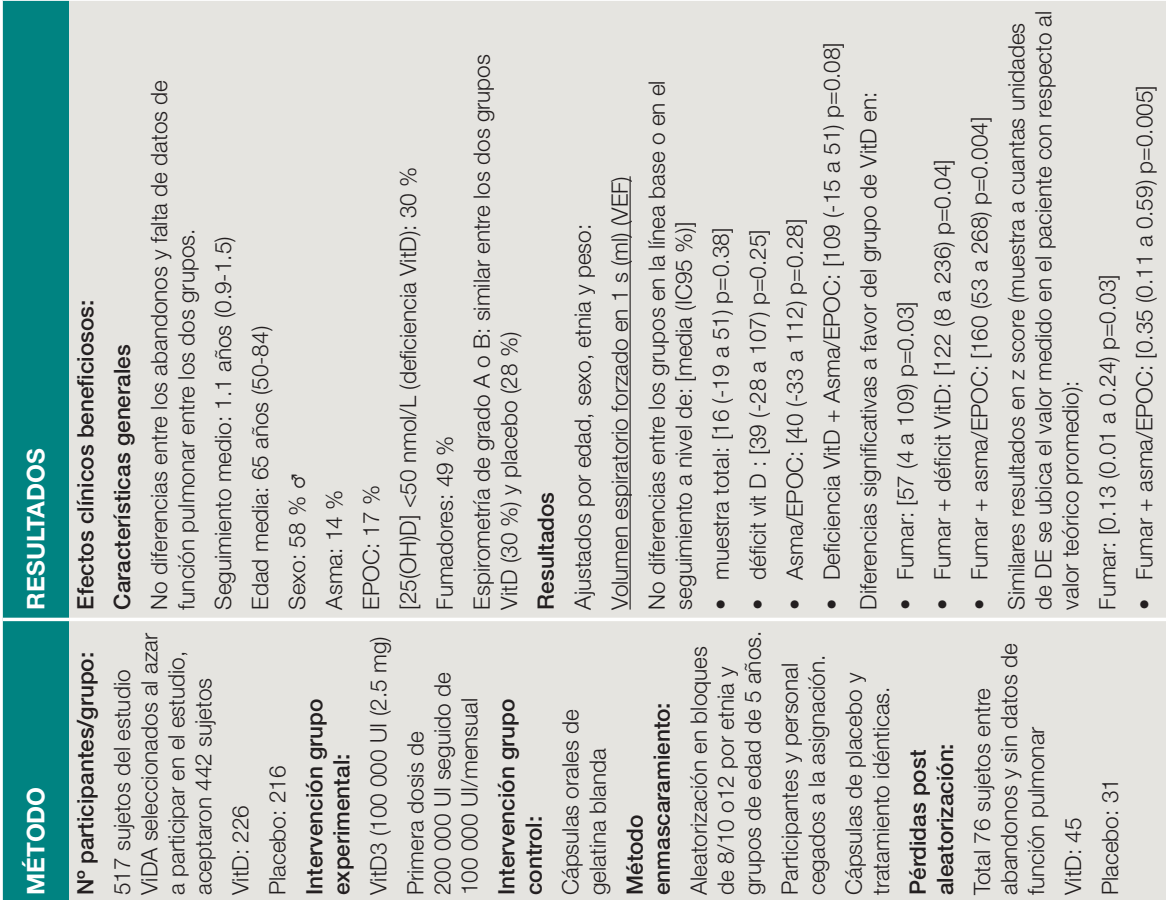

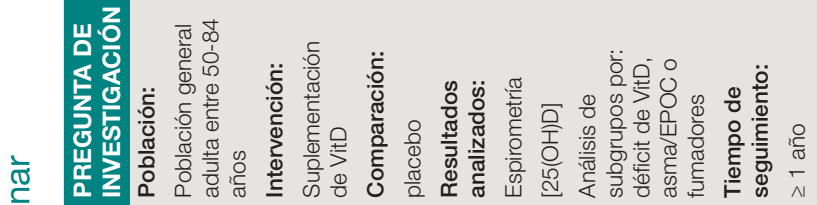

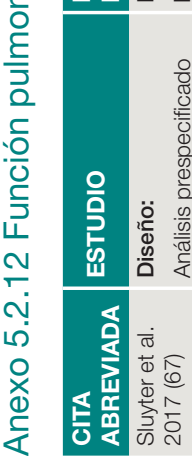




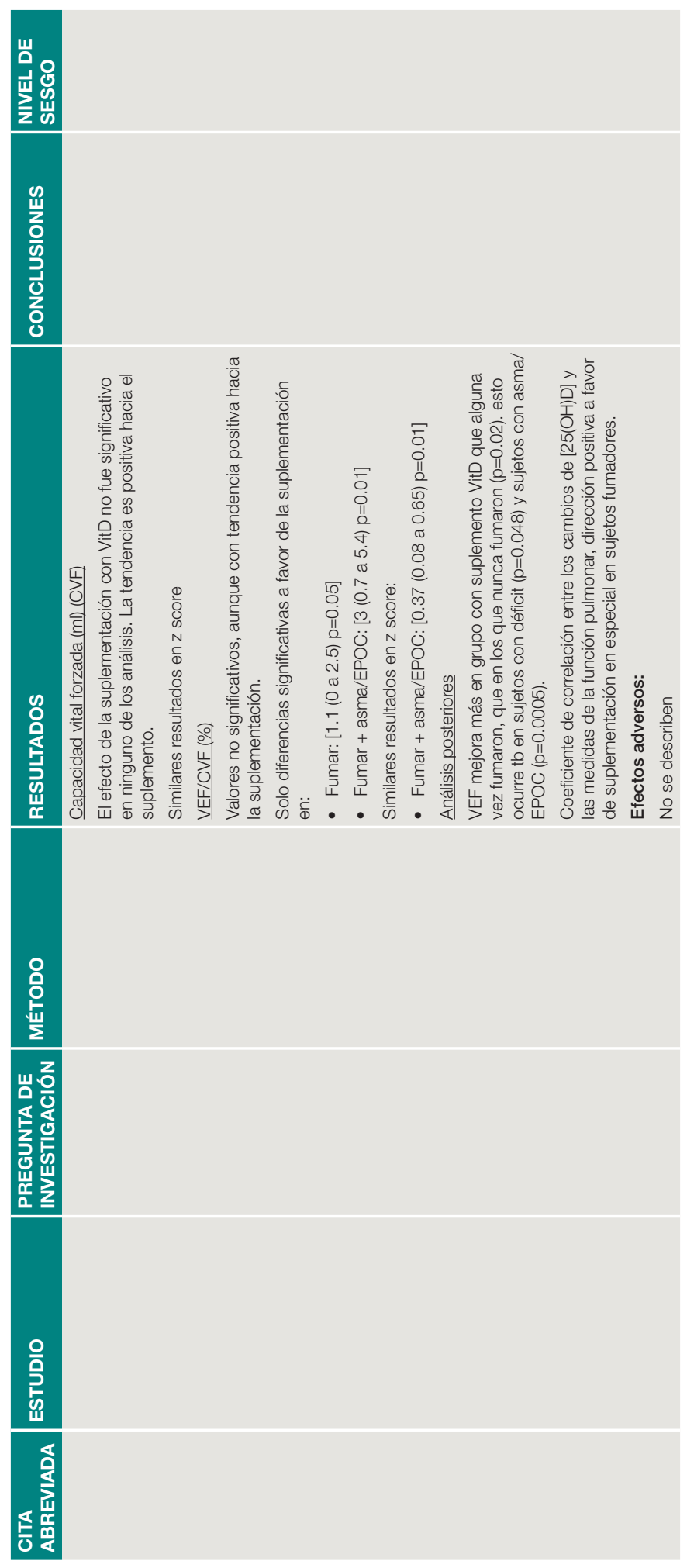




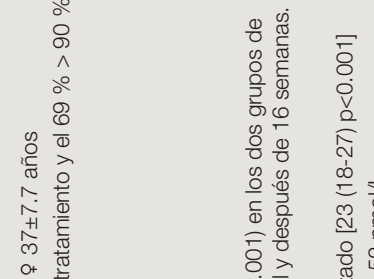

ï $\quad \stackrel{0+}{>}$

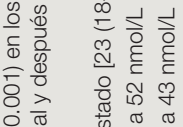

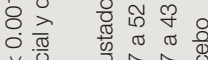

\&

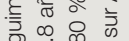

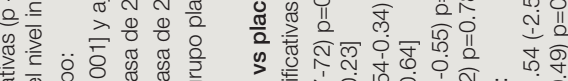

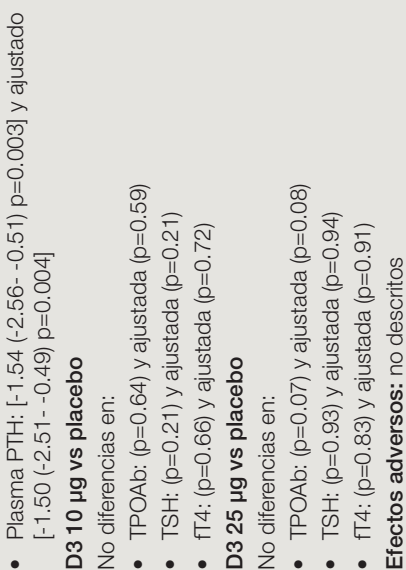

\section{$\stackrel{\infty}{-} \frac{0}{\overline{0}}$}

\section{$\overline{5}$}

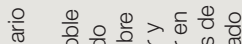

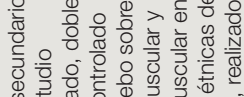

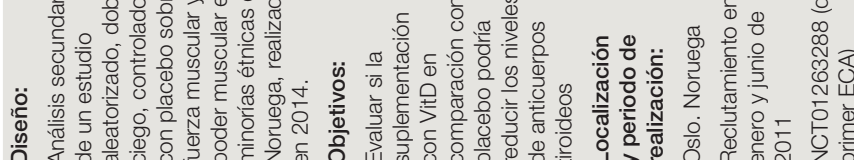

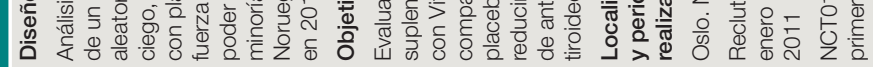

\section{ก \\ เก \\ เి

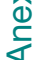

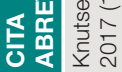




\section{WIIII}

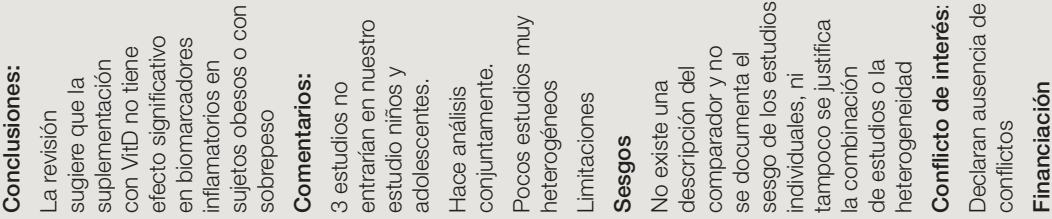

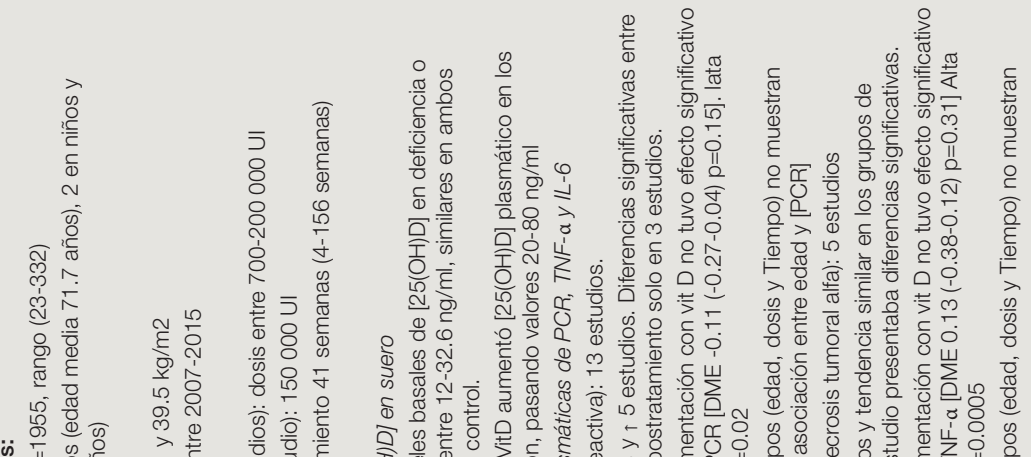

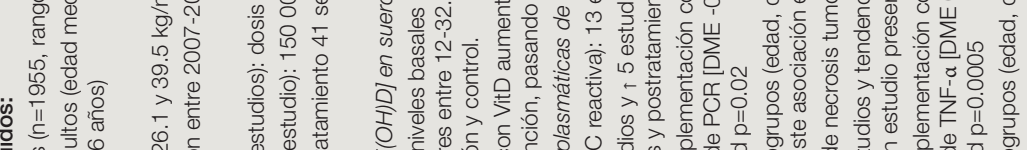

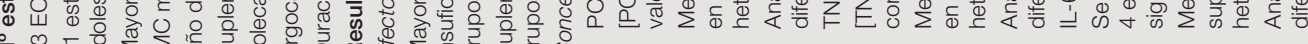

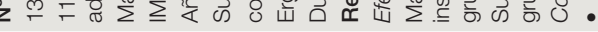



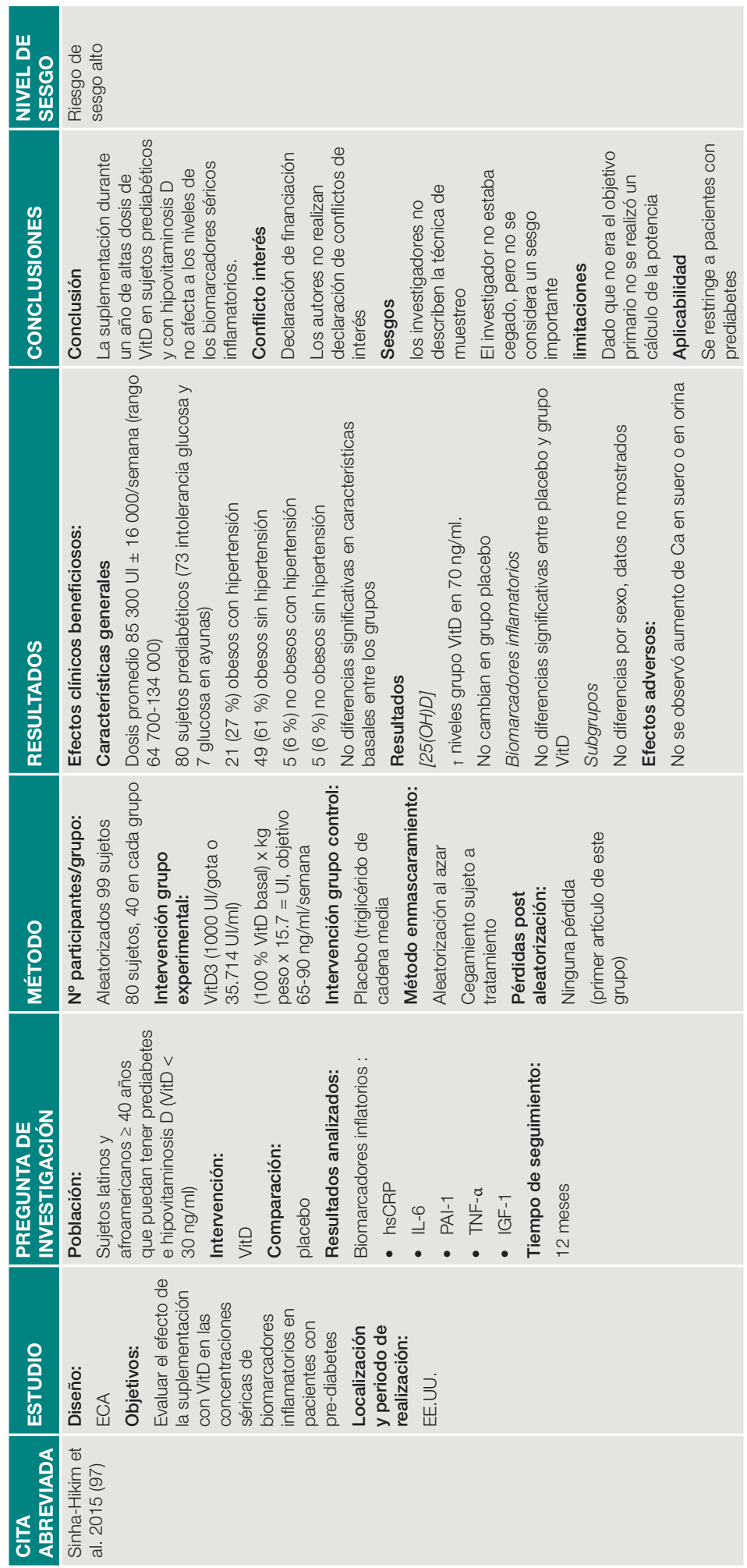


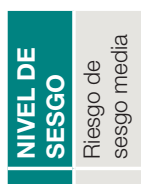

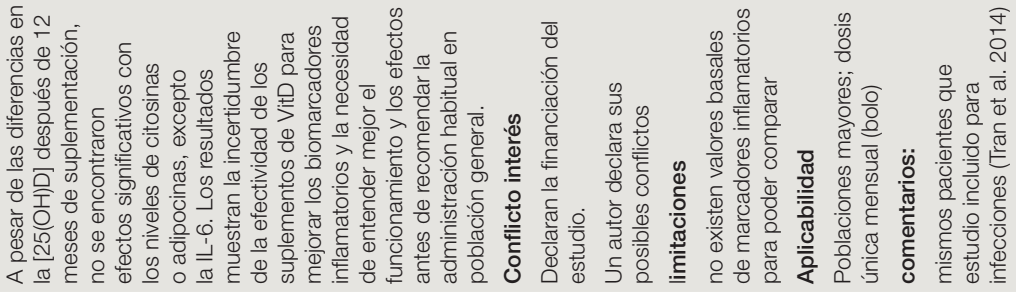
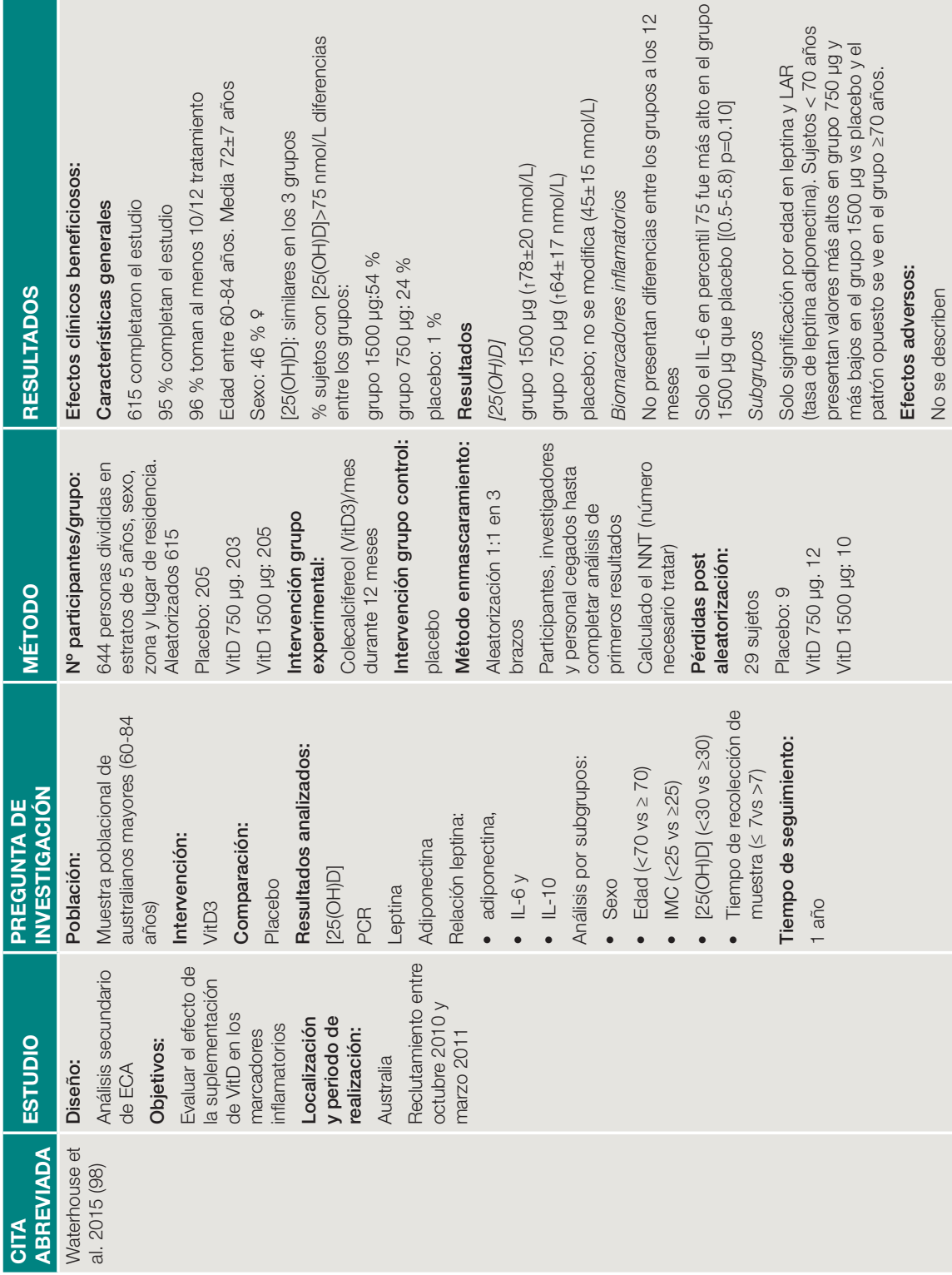


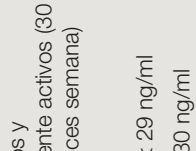

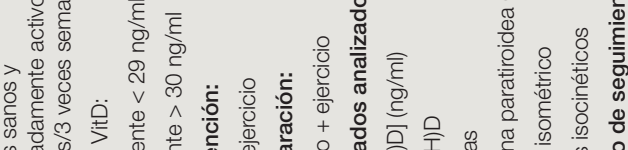

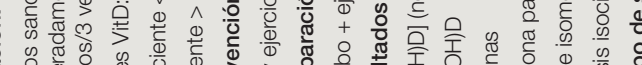




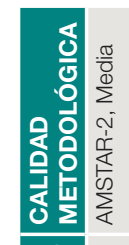

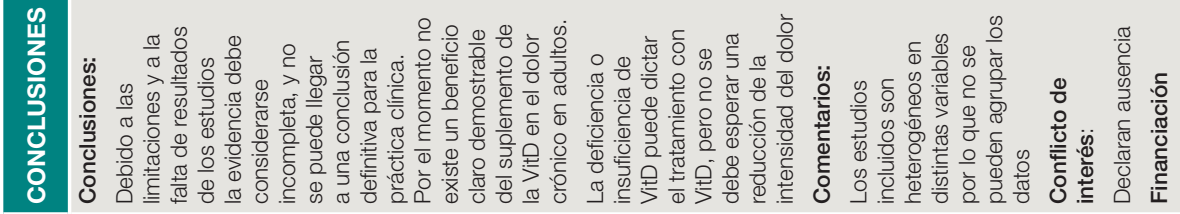

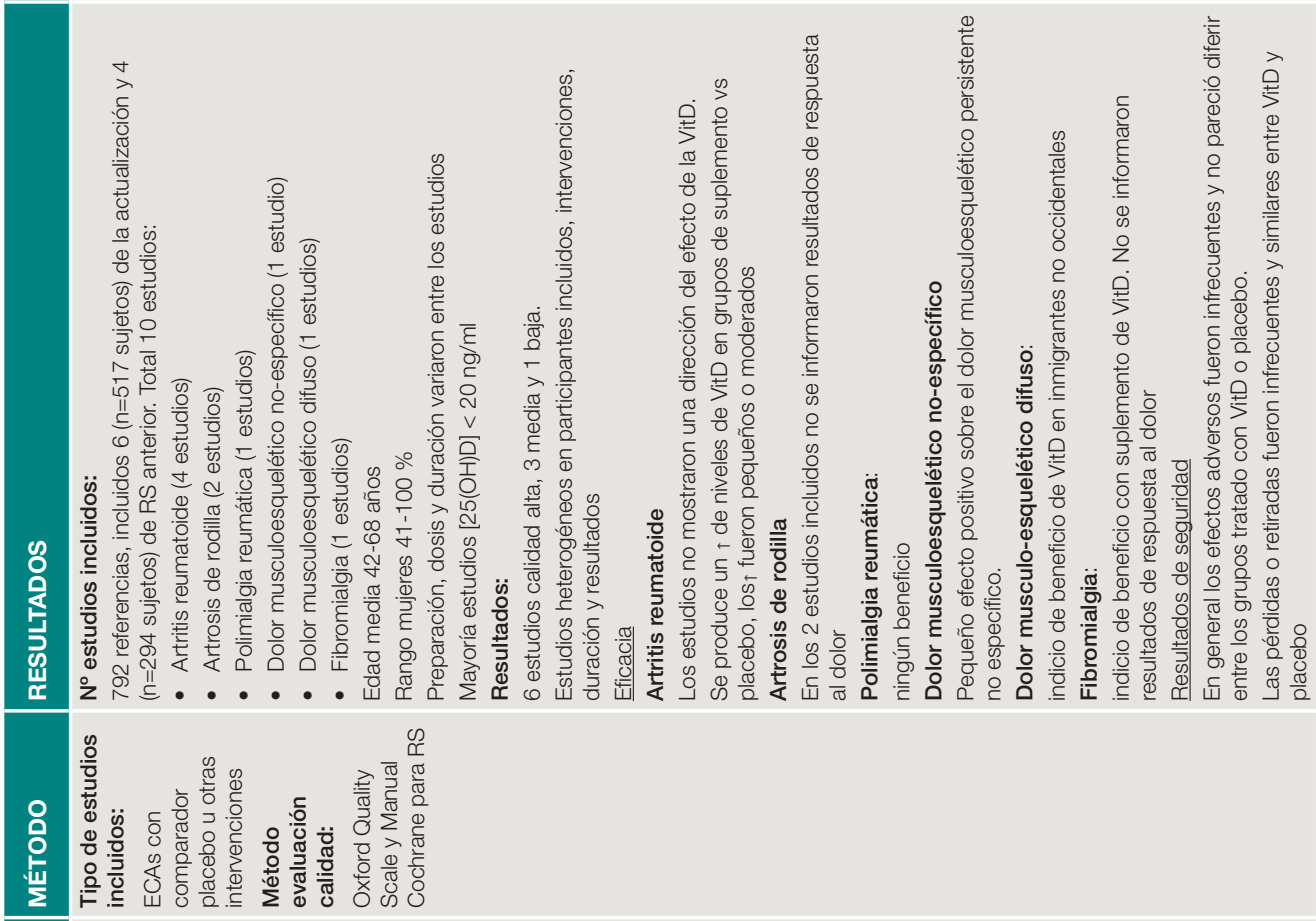

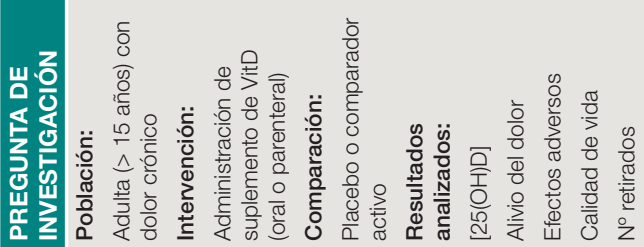

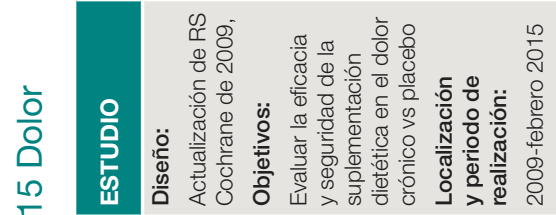

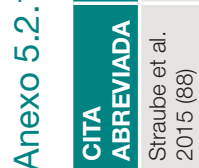




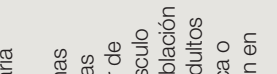

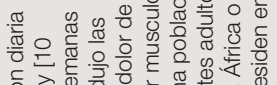

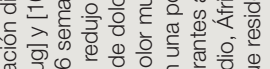

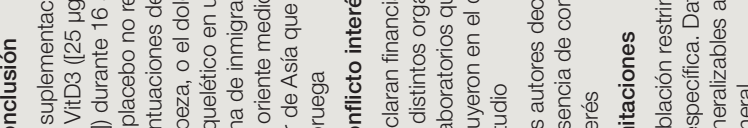

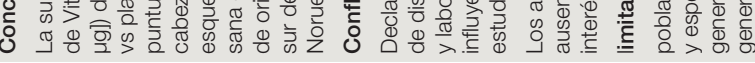

\section{\&ु

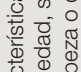 \\ 竞 $\frac{0}{0} \frac{0}{0}$ \\ ऽ。}

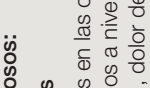

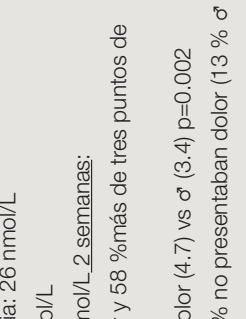

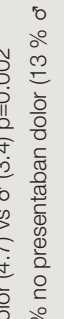

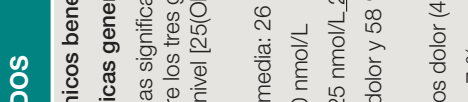

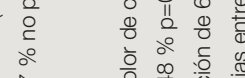

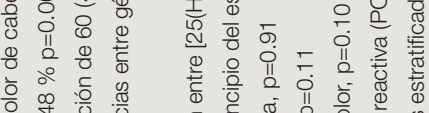

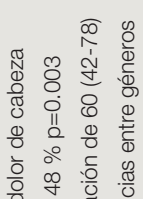

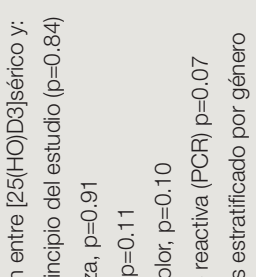

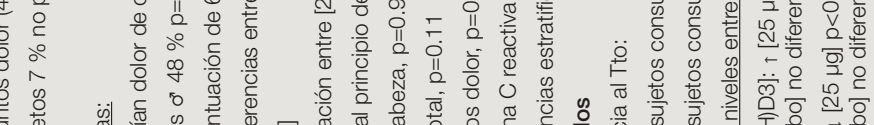

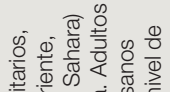

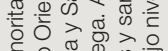

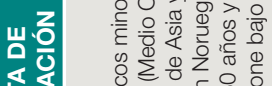

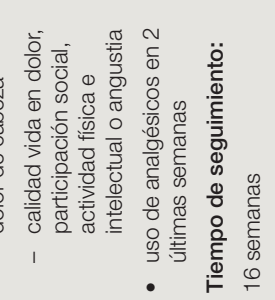

융

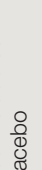

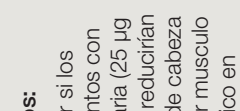

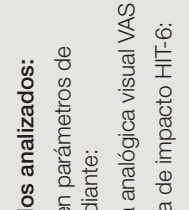

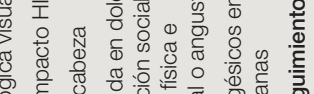




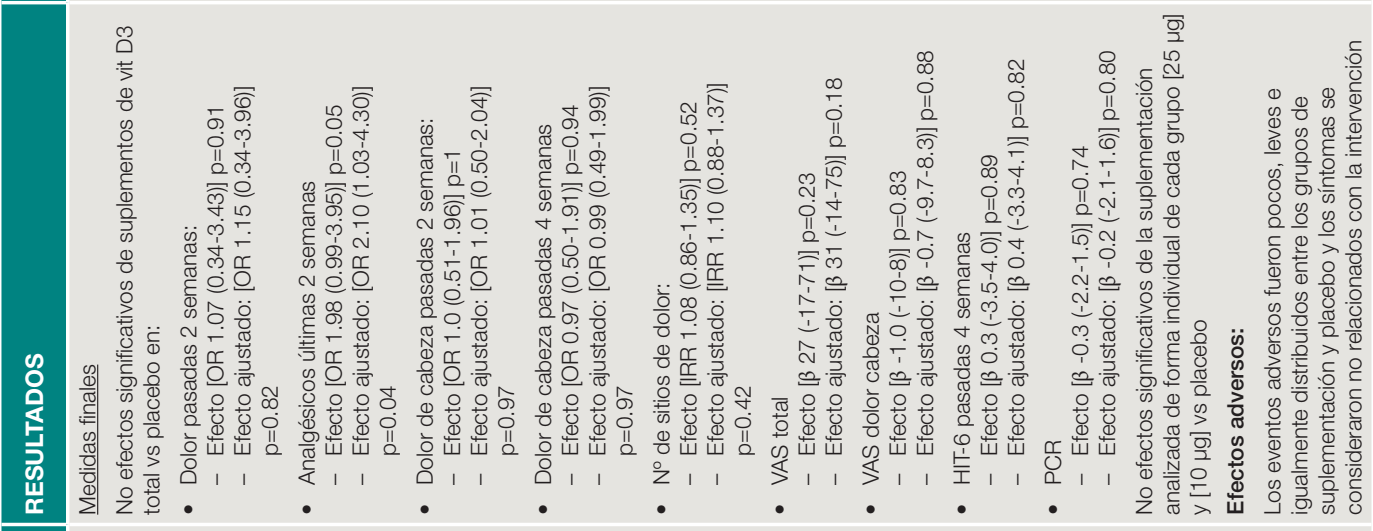




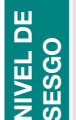

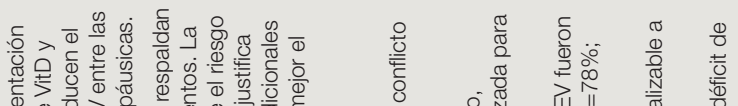

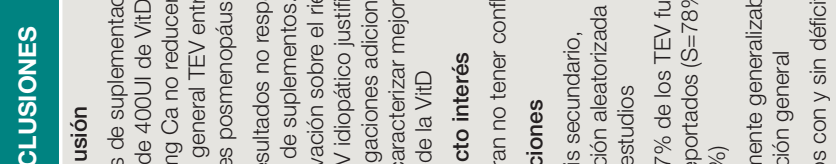

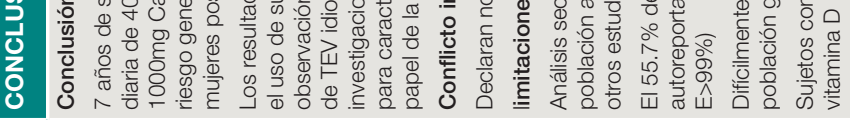
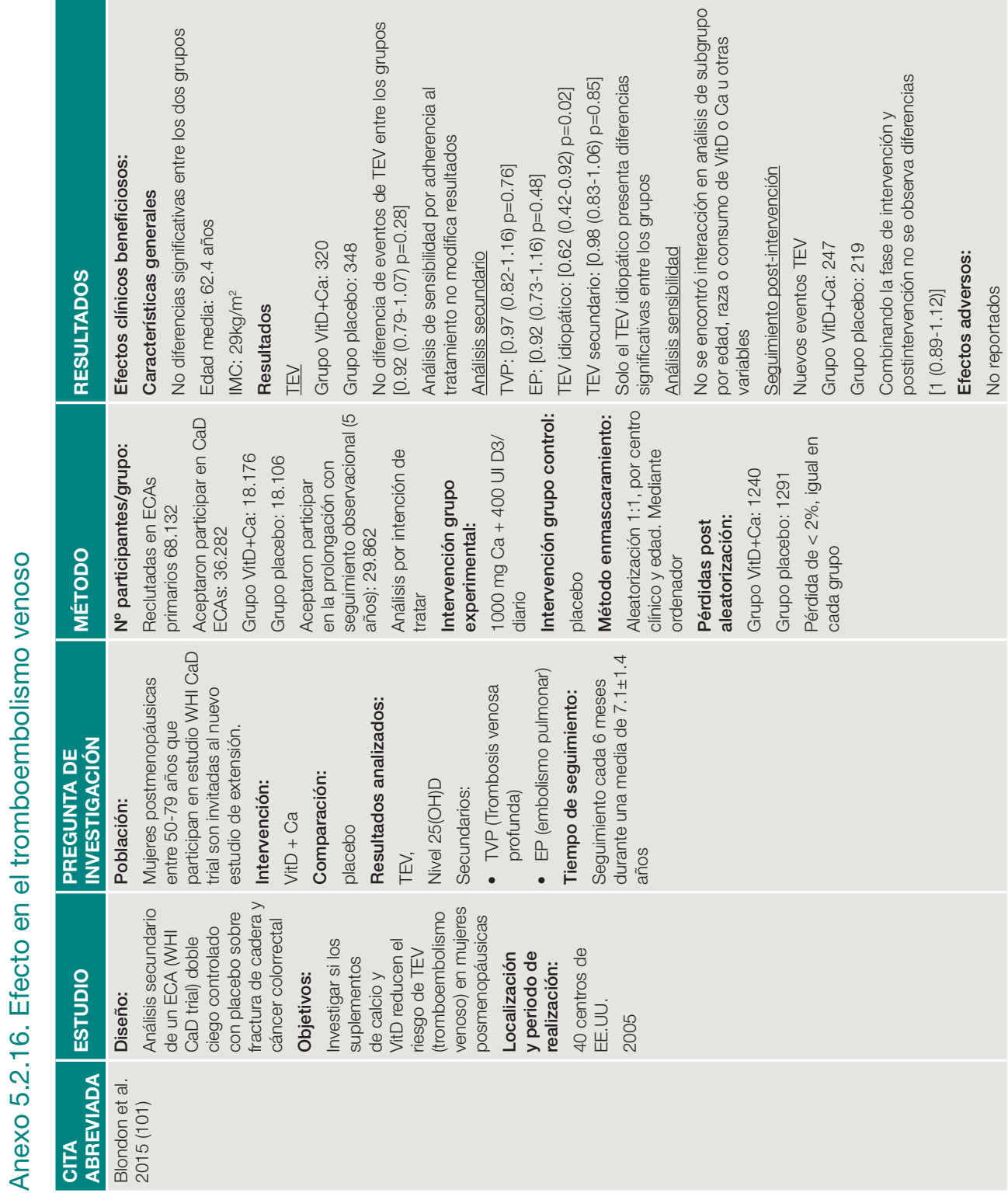


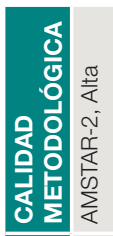

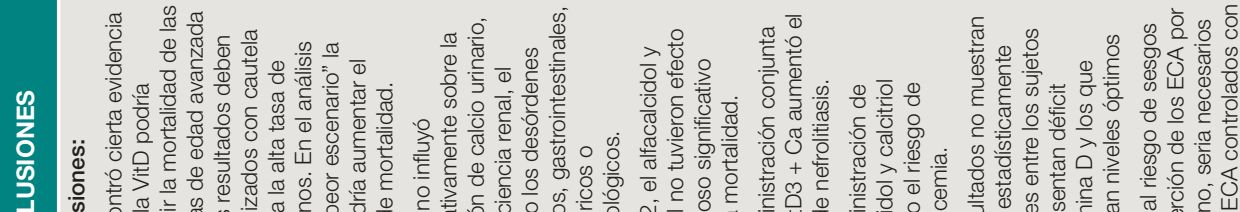

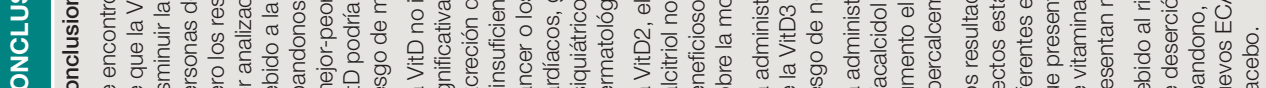

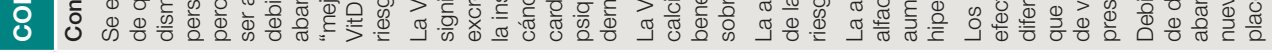
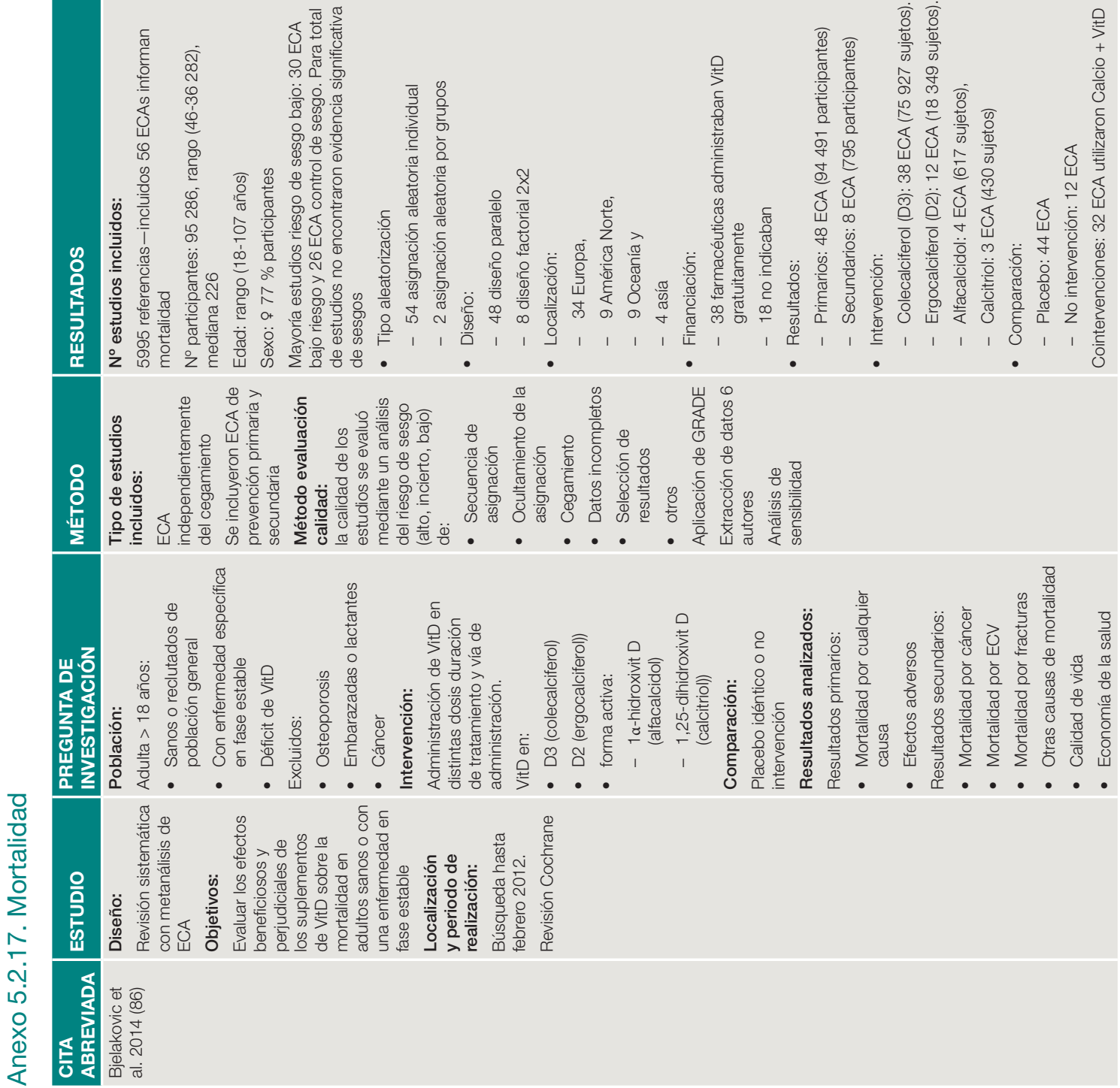


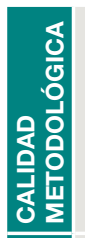

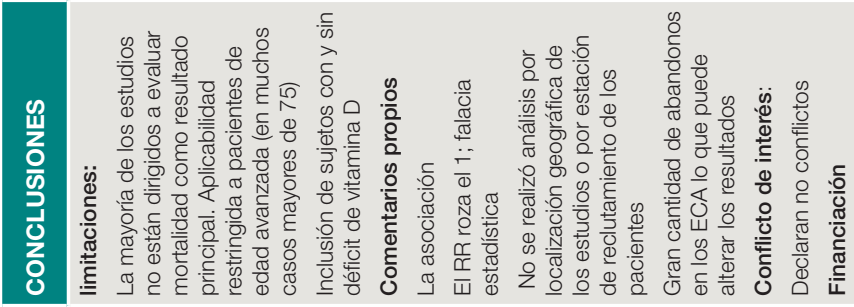

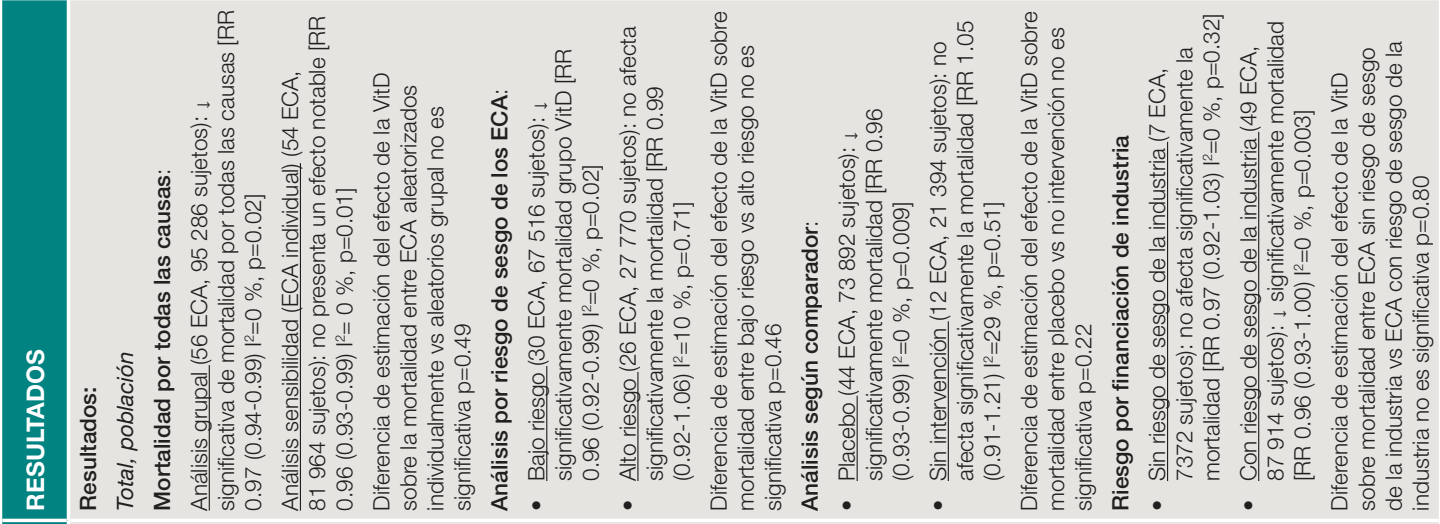

웅

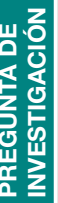

음 


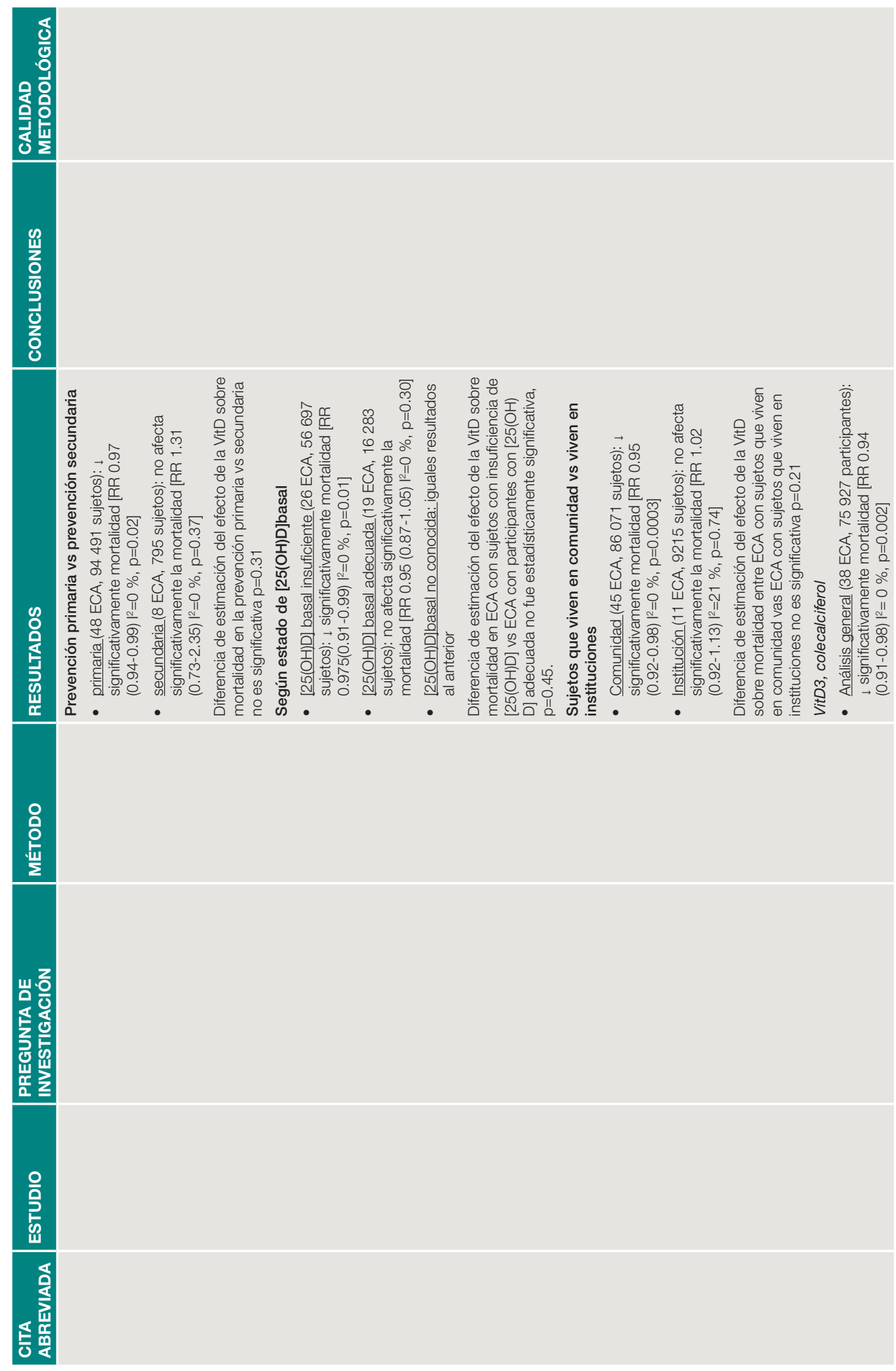




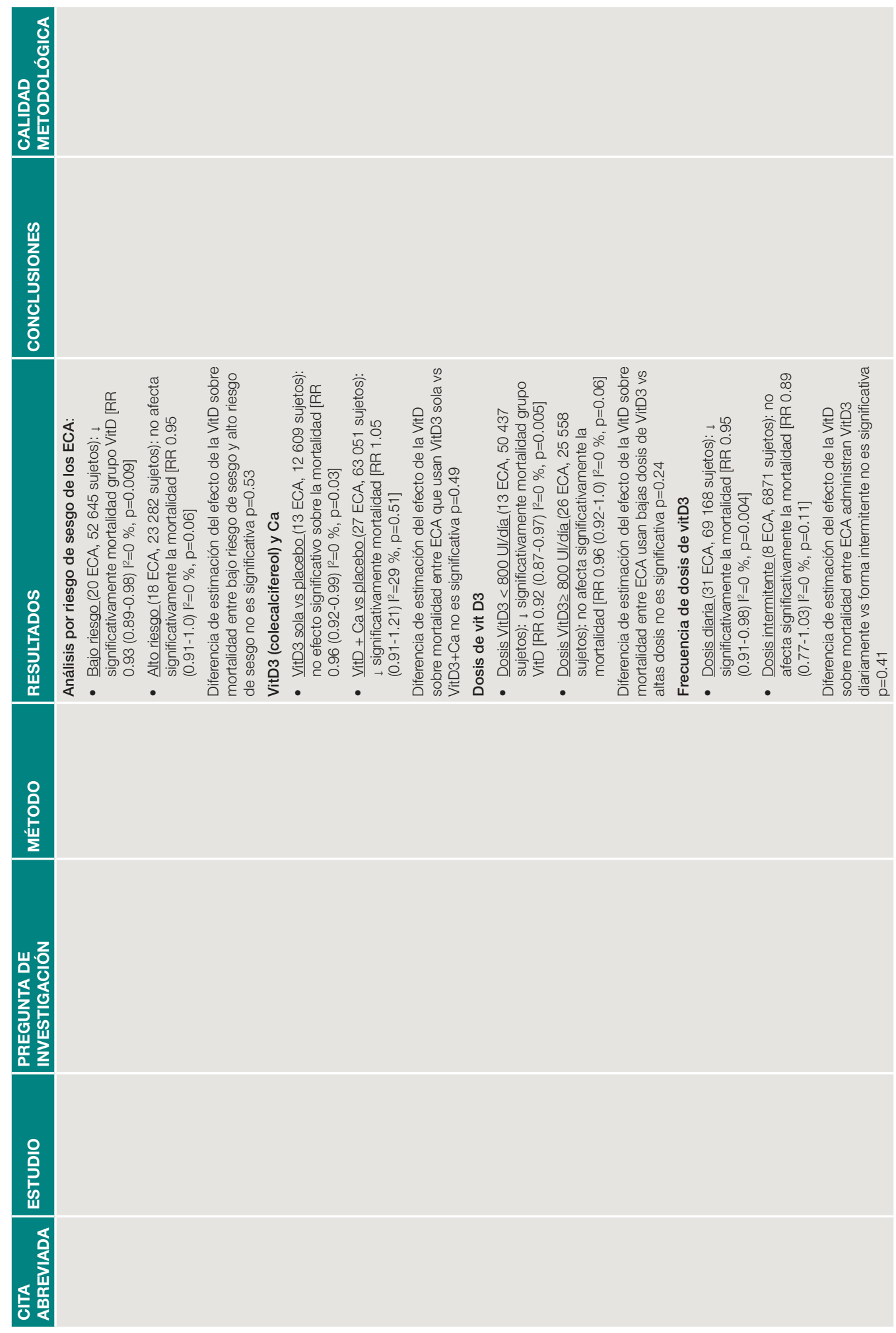




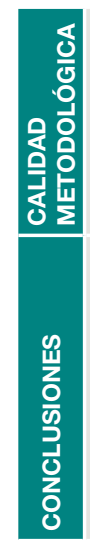

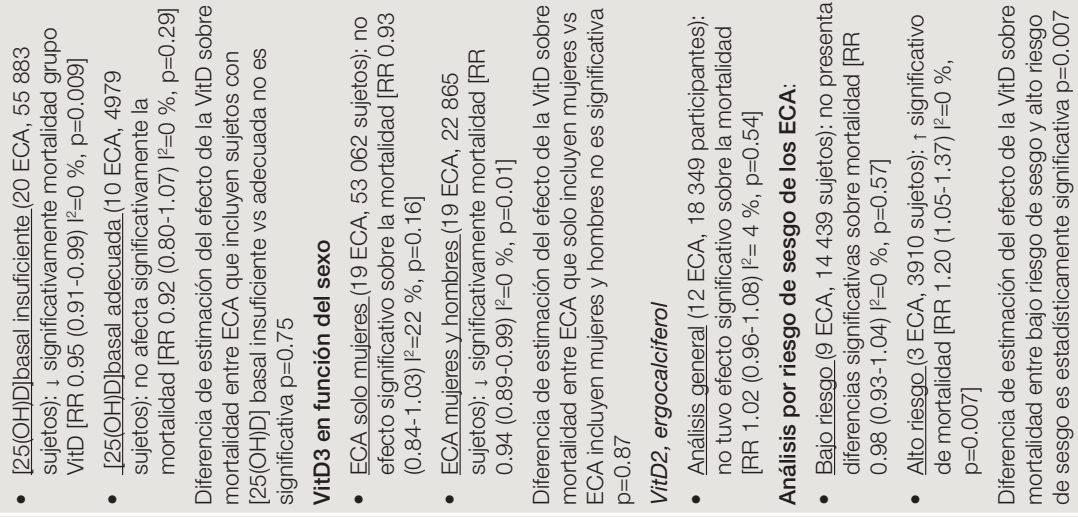

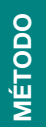

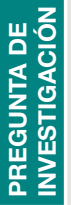

咅 


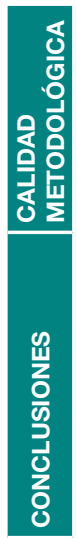

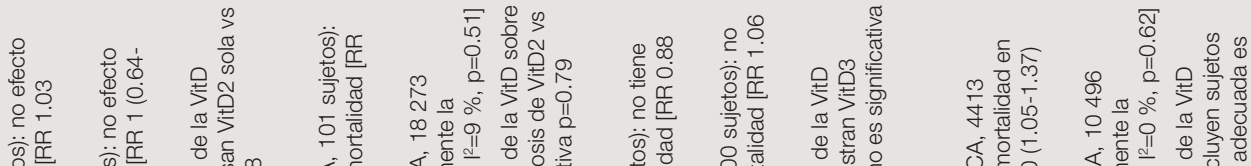

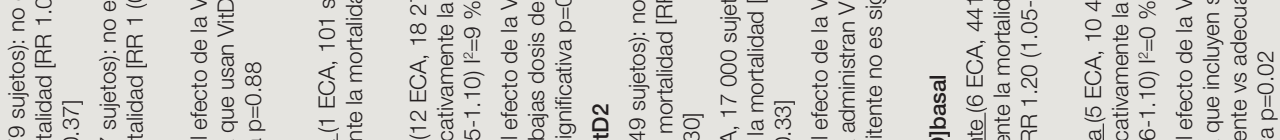

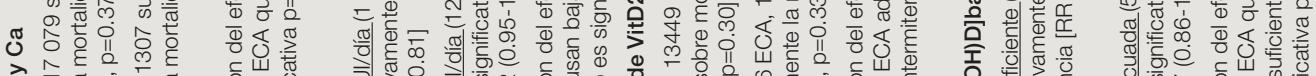
तิ

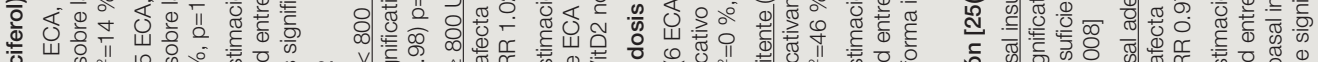

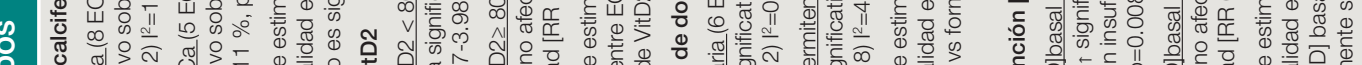

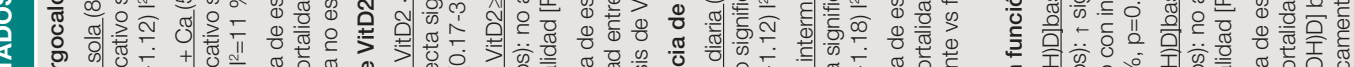

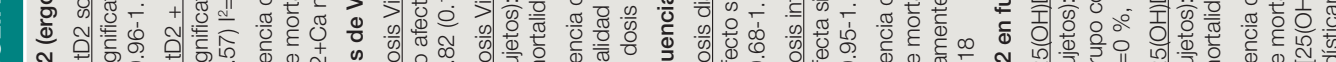
골

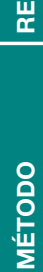

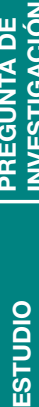




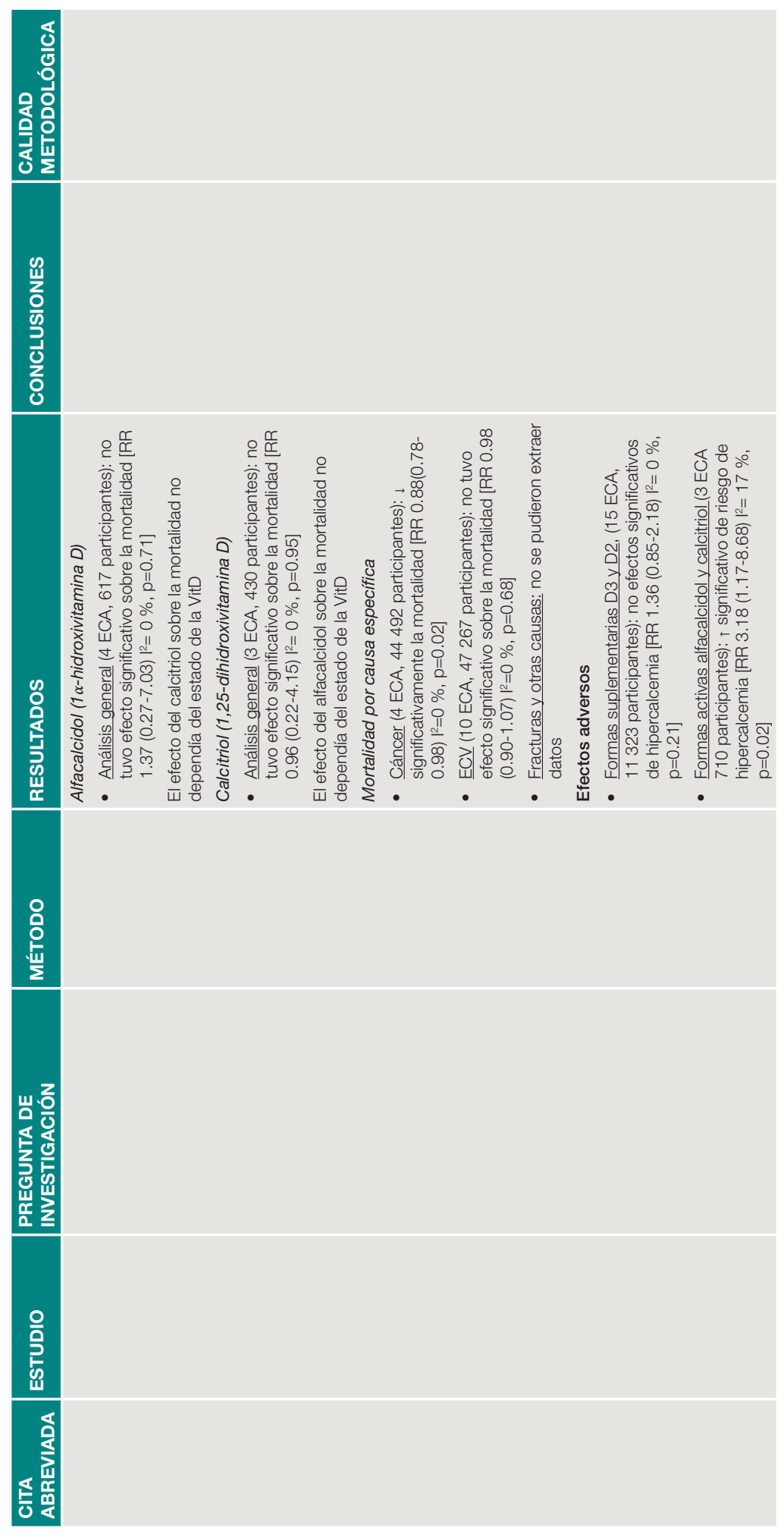



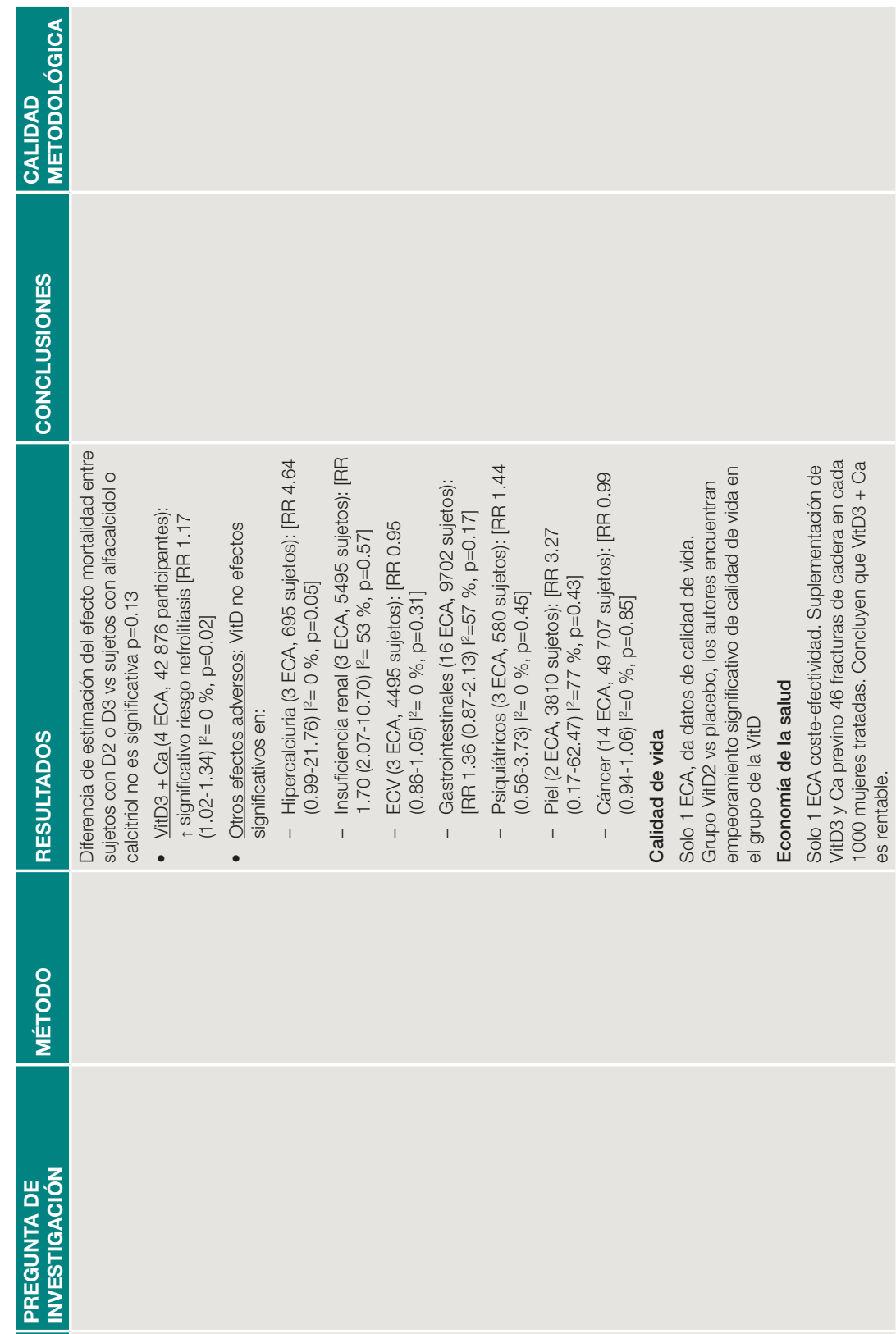


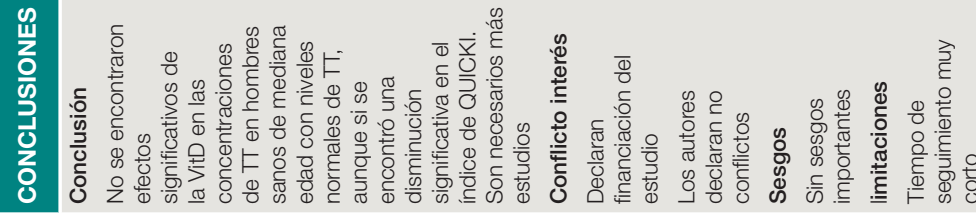

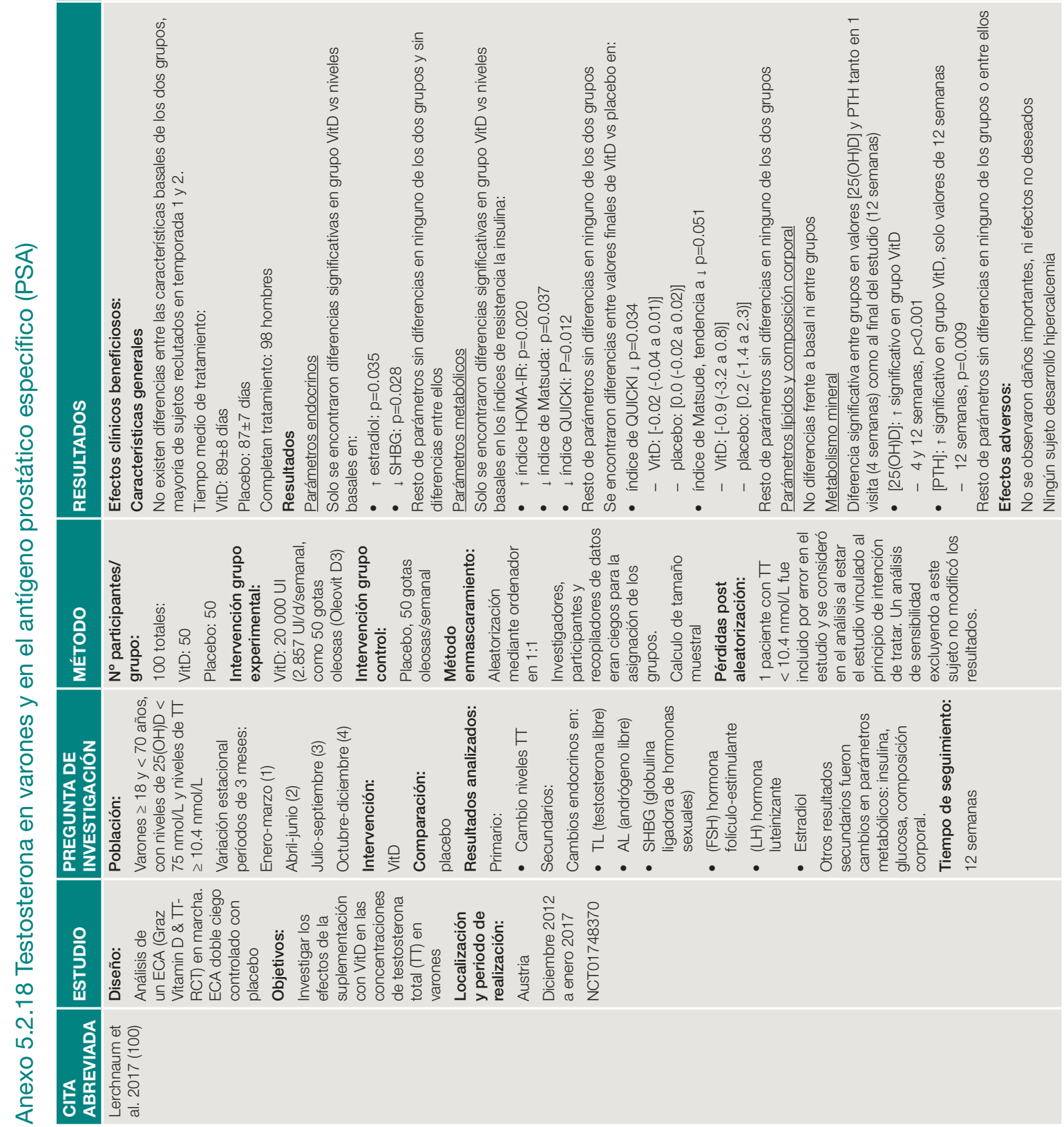




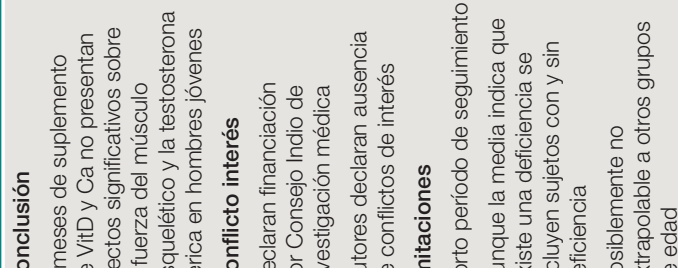

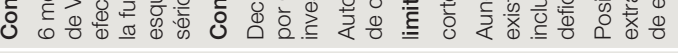

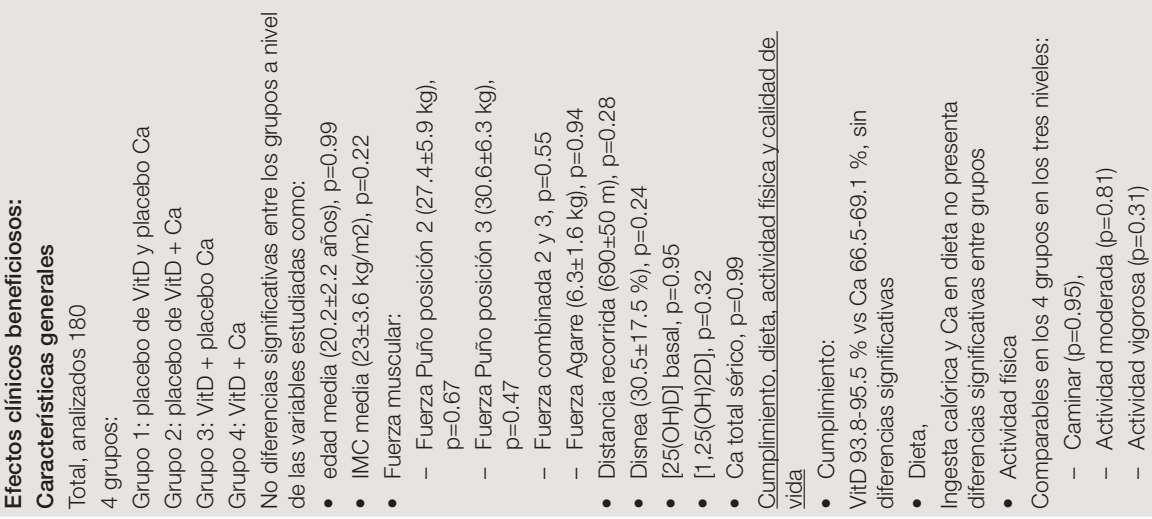

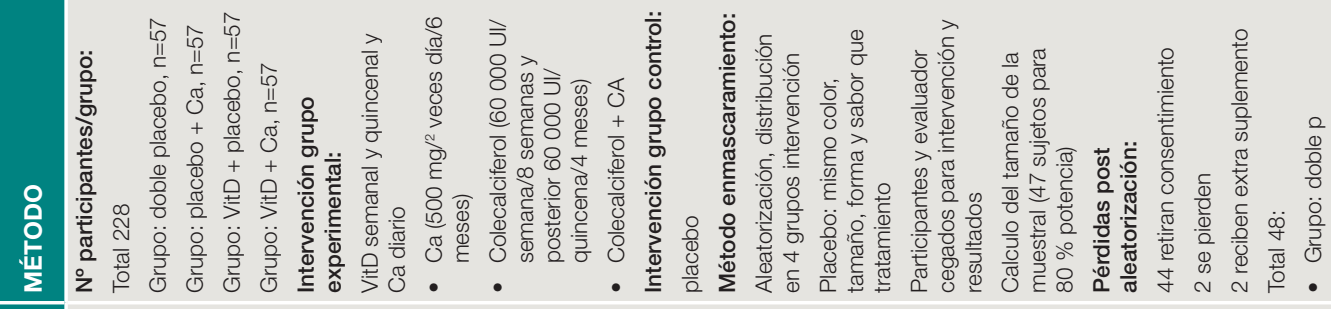
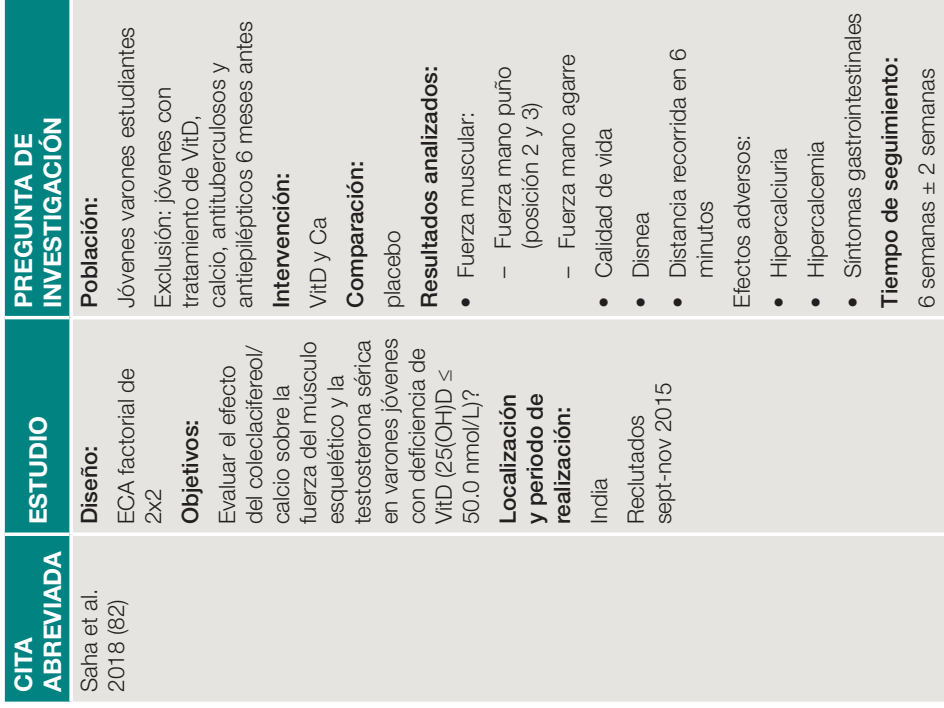


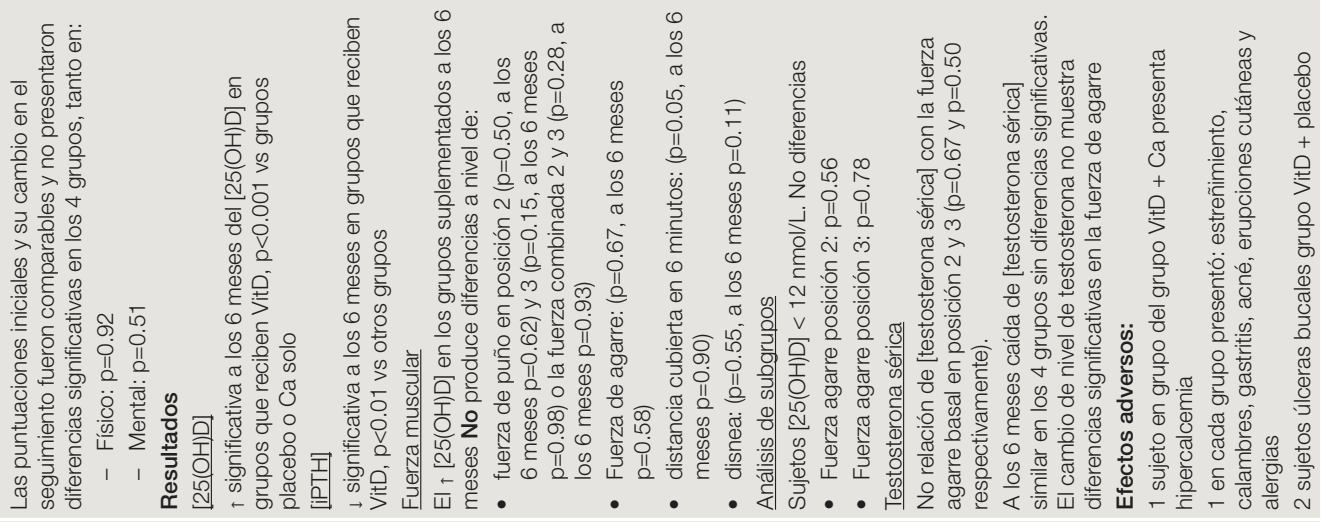

울
댈

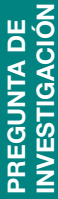

음 

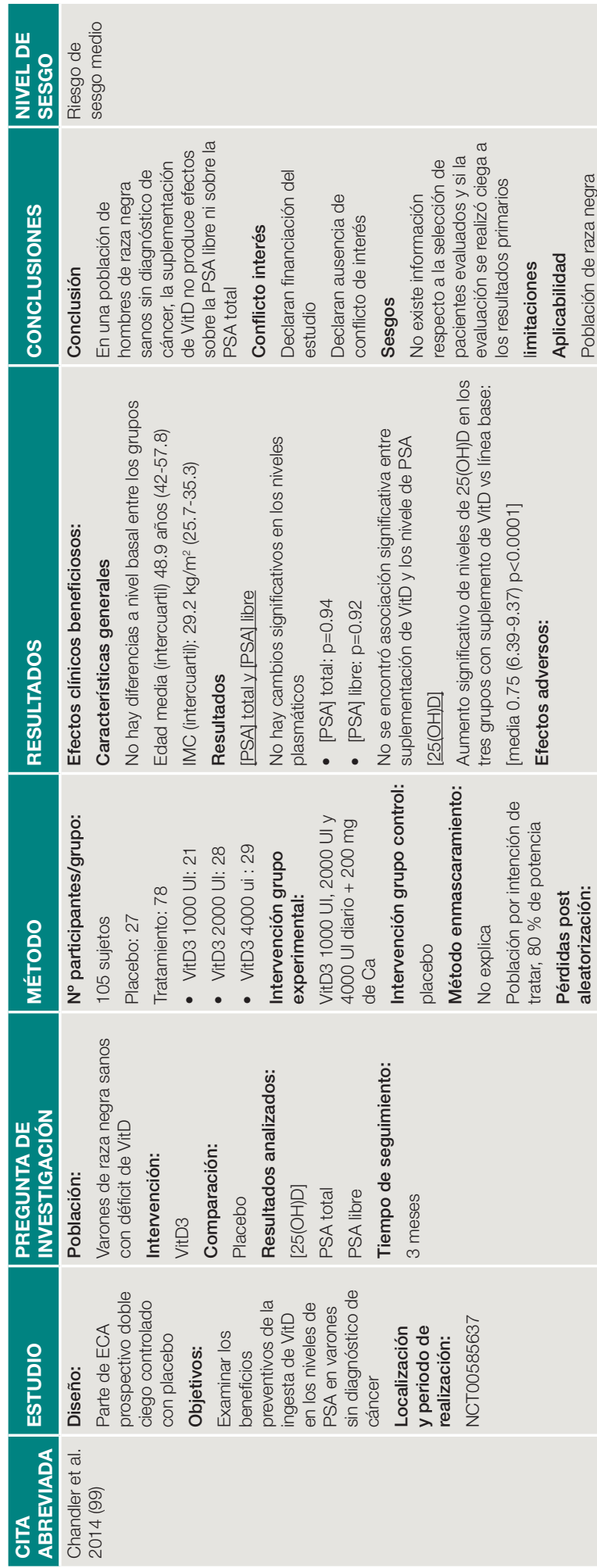



\section{Anexo 6. Fármacos comercializados en España que contienen vitamina D}

\section{https://cima.aemps.es/cima/publico/lista.html (AEMPS)}

\begin{tabular}{|c|c|c|}
\hline $\begin{array}{l}\text { Principio } \\
\text { activo }\end{array}$ & Medicamento & Laboratorio \\
\hline \multirow{20}{*}{ Colecalciferol } & $\begin{array}{l}\text { Deltius (10000 Ul/ml gotas orales } 1 \text { frasco solución } \\
10 \mathrm{ml} \text { ) }\end{array}$ & Italfarmaco \\
\hline & $\begin{array}{l}\text { Deltius ( } 25000 \text { UI } 1 \text { frasco unidosis, ampollas bebibles, } \\
\text { solución oral } 2.5 \mathrm{ml} \text { ) }\end{array}$ & Italfarmaco \\
\hline & $\begin{array}{l}\text { Deltius ( } 25000 \mathrm{UI} 4 \text { frascos unidosis, ampollas bebibles, } \\
\text { solución oral } 2.5 \mathrm{ml} \text { ) }\end{array}$ & Italfarmaco \\
\hline & Deltius (25000 UI 4 cápsulas) & Italfarmaco \\
\hline & Devik (25000 UI 4 sobres unidosis solución oral 2.5 ml) & Kern Pharma \\
\hline & Divisun (800 UI 30 comprimidos) & $\begin{array}{l}\text { Mylan } \\
\text { Pharmaceuticals } \\
\text { S.L. }\end{array}$ \\
\hline & Benferol (800 UI 28 cápsulas) & $\begin{array}{l}\text { Angelini } \\
\text { Farmacéutica S.A. }\end{array}$ \\
\hline & Benferol (5600 UI 4 cápsulas) & $\begin{array}{l}\text { Angelini } \\
\text { Farmacéutica S.A. }\end{array}$ \\
\hline & Benferol (25000Ul 4 cápsulas mensual) & $\begin{array}{l}\text { Angelini } \\
\text { Farmacéutica S.A. }\end{array}$ \\
\hline & Benferol (50000 UI 2 cápsulas choque) & $\begin{array}{l}\text { Angelini } \\
\text { Farmacéutica S.A. }\end{array}$ \\
\hline & Benferol (100000 UI 1 cápsula) & $\begin{array}{l}\text { Angelini } \\
\text { Farmacéutica S.A. }\end{array}$ \\
\hline & Colecalciferol Rovi (30000 UI, 1 comprimido) & Rovi \\
\hline & Colecalciferol Rovi (30000 UI, 4 comprimidos) & Rovi \\
\hline & Thorens (25000 UI 4 cápsulas) & Effik \\
\hline & $\begin{array}{l}\text { Thorens ( } 25000 \text { UI } 4 \text { frascos unidosis, ampollas } \\
\text { bebibles, solución oral } 2.5 \mathrm{ml} \text { ) }\end{array}$ & Effik \\
\hline & Videsil (100000 UI 1 ampolla bebible, solución oral) & Gebro Pharma \\
\hline & Videsil (25000 UI 4 ampollas bebibles, solución oral) & Gebro Pharma \\
\hline & Videsil (50000 UI 1 ampollas bebibles, solución oral) & Gebro Pharma \\
\hline & $\begin{array}{l}\text { Vitamina D3 Kern Pharma (2000 Ul/ml solución oral } \\
\text { oleosa } 10 \text { ml) }\end{array}$ & Kern Pharma \\
\hline & $\begin{array}{l}\text { Vitamina D3 Kern Pharma (2000 UI/ml solución oral } \\
\text { oleosa } 30 \text { ml) }\end{array}$ & Kern Pharma \\
\hline
\end{tabular}




\begin{tabular}{|c|c|c|}
\hline & $\begin{array}{l}\text { Ácido alendronico/colecalciferol Aristo EFG } \\
\text { (70 mg/2800 Ul } 4 \text { comprimidos) }\end{array}$ & $\begin{array}{l}\text { Aristo Pharma } \\
\text { Iberia S.L. }\end{array}$ \\
\hline & $\begin{array}{l}\text { Ácido alendronico/colecalciferol Aristo EFG } \\
\text { (70 mg/5600 UI } 4 \text { comprimidos) }\end{array}$ & $\begin{array}{l}\text { Aristo Pharma } \\
\text { Iberia S.L. }\end{array}$ \\
\hline & $\begin{array}{l}\text { Ácido alendronico/colecalciferol Aurovitas EFG } \\
\text { (70 mg/2800 UI } 4 \text { comprimidos) }\end{array}$ & $\begin{array}{l}\text { Aurovitas Spain } \\
\text { S.A.U. }\end{array}$ \\
\hline & $\begin{array}{l}\text { Ácido alendronico/colecalciferol Aurovitas EFG } \\
\text { (70 mg/5600 UI } 4 \text { comprimidos) }\end{array}$ & $\begin{array}{l}\text { Aurovitas Spain } \\
\text { S.A.U. }\end{array}$ \\
\hline & $\begin{array}{l}\text { Ácido alendronico/colecalciferol Kern Pharma EFG } \\
\text { (70 mg/2800 UI } 4 \text { comprimidos) }\end{array}$ & Kern Pharma \\
\hline & $\begin{array}{l}\text { Ácido alendronico/colecalciferol Kern Pharma EFG } \\
\text { (70 mg/5600 UI } 4 \text { comprimidos) }\end{array}$ & Kern Pharma \\
\hline & $\begin{array}{l}\text { Ácido alendronico/colecalciferol Lorien EFG } \\
\text { (70 mg/2800 Ul } 4 \text { comprimidos) }\end{array}$ & $\begin{array}{l}\text { Laboratorios } \\
\text { Lorien S.L. }\end{array}$ \\
\hline & $\begin{array}{l}\text { Ácido alendronico/colecalciferol Lorien EFG } \\
\text { ( } 70 \text { mg/5600 Ul } 4 \text { comprimidos) }\end{array}$ & $\begin{array}{l}\text { Laboratorios } \\
\text { Lorien S.L. }\end{array}$ \\
\hline & $\begin{array}{l}\text { Ácido alendronico/colecalciferol Qualigen EFG } \\
\text { (70 mg/2800 Ul } 4 \text { comprimidos) }\end{array}$ & $\begin{array}{l}\text { Neuraxpharm } \\
\text { Spain S.L. }\end{array}$ \\
\hline & $\begin{array}{l}\text { Ácido alendronico/colecalciferol Qualigen EFG } \\
\text { (70 mg/5600 UI } 4 \text { comprimidos) }\end{array}$ & $\begin{array}{l}\text { Neuraxpharm } \\
\text { Spain S.L. }\end{array}$ \\
\hline & $\begin{array}{l}\text { Ácido alendronico/colecalciferol Ratiopharm } \\
\text { (70 mg/2800 UI } 4 \text { comprimidos) }\end{array}$ & Ratiopharm \\
\hline & $\begin{array}{l}\text { Ácido alendronico/colecalciferol Ratiopharm } \\
\text { ( } 70 \text { mg/5600 UI } 4 \text { comprimidos) }\end{array}$ & Ratiopharm \\
\hline & $\begin{array}{l}\text { Ácido alendronico/colecalciferol Sandoz EFG } \\
\text { (70 mg/5600 Ul } 4 \text { comprimidos) }\end{array}$ & $\begin{array}{l}\text { Sandoz } \\
\text { Farmaceutica S.A. }\end{array}$ \\
\hline & $\begin{array}{l}\text { Acido alendronico/colecalciferol STADA EFG } 70 \\
\mathrm{mg} / 2800 \text { UI } 4 \text { comprimidos }\end{array}$ & STADA \\
\hline & $\begin{array}{l}\text { Acido alendronico/colecalciferol STADA EFG } 70 \\
\text { mg/5600 UI } 4 \text { comprimidos }\end{array}$ & STADA \\
\hline & $\begin{array}{l}\text { Ácido alendronico/colecalciferol Tevagen } \\
\text { ( } 70 \mathrm{mg} / 2800 \text { Ul } 4 \text { comprimidos) }\end{array}$ & $\begin{array}{l}\text { Teva Pharma } \\
\text { S.L.U. }\end{array}$ \\
\hline & $\begin{array}{l}\text { Ácido alendronico/colecalciferol Tevagen } \\
\text { ( } 70 \mathrm{mg} / 5600 \text { UI } 4 \text { comprimidos) }\end{array}$ & $\begin{array}{l}\text { Teva Pharma } \\
\text { S.L.U. }\end{array}$ \\
\hline & Adrovance (70 mg/2800 UI 4 comprimidos) & $\begin{array}{l}\text { Ferrer } \\
\text { Internacional }\end{array}$ \\
\hline & Adrovance (70 mg/5600 UI 4 comprimidos) & $\begin{array}{l}\text { Ferrer } \\
\text { Internacional }\end{array}$ \\
\hline & Fosavance (70 mg/2800 UI 4 comprimido ) & MSD \\
\hline & Fosavance (70 mg/5600 UI 4 comprimidos) & MSD \\
\hline & $\begin{array}{l}\text { Fosavance Mediwin Limited } 70 \text { mg/2800 UI } 4 \\
\text { comprimidos }\end{array}$ & DIFARMED \\
\hline & $\begin{array}{l}\text { Fosavance Mediwin Limited } 70 \text { mg/5600 UI } 4 \\
\text { comprimidos }\end{array}$ & DIFARMED \\
\hline
\end{tabular}




\begin{tabular}{|c|c|c|}
\hline & $\begin{array}{l}\text { Adiaval EFG ( } 2500 \mathrm{mg}(1000 \mathrm{ca}) / 880 \text { UI } 30 \\
\text { comprimidos masticicables (tiras)) }\end{array}$ & Lacer \\
\hline & $\begin{array}{l}\text { Bonesil D Flas ( } 1500 \mathrm{mg}(600 \mathrm{ca}) / 400 \text { UI } 60 \\
\text { comprimidos bucodispersables) }\end{array}$ & Italfarmaco \\
\hline & $\begin{array}{l}\text { Cadelius D ( } 1500 \mathrm{mg} \text { (600 ca)/1000 UI } 30 \text { comprimidos } \\
\text { bucodispersable) }\end{array}$ & $\begin{array}{l}\text { Especialidades } \\
\text { Farmaceuticas } \\
\text { Centrum }\end{array}$ \\
\hline & $\begin{array}{l}\text { Calcial D (1500 mg (600 mg ca)/400 UI } 60 \text { comprimidos } \\
\text { masticables) }\end{array}$ & Alacan \\
\hline & $\begin{array}{l}\text { Calcio D Isdin (1500 mg (600 mg ca)/400 UI } 60 \\
\text { comprimidos masticables) }\end{array}$ & Isdin \\
\hline & $\begin{array}{l}\text { Calcio/vitamina D3 Aristo EFG (1500 mg ( } 600 \text { mg } \\
\text { ca)/400 Uli } 60 \text { comprimidos masticables tubo) }\end{array}$ & $\begin{array}{l}\text { Aristo Pharma } \\
\text { lberia S.L. }\end{array}$ \\
\hline & $\begin{array}{l}\text { Calcio/vitamina D3 Kern Pharma EFG ( } 1500 \text { mg } \\
\text { (600 mg ca)/400 Ul } 60 \text { comprimidos masticables) }\end{array}$ & Kern Pharma \\
\hline & $\begin{array}{l}\text { Calcio/vitamina D3 Rovi (1250 mg (500 ca)/1000 UI } 30 \\
\text { comprimidos masticables (tubo)) }\end{array}$ & Rovi \\
\hline & $\begin{array}{l}\text { Calcio/vitamina D3 Rovi (2500 mg (1000 ca)/880 UI } 30 \\
\text { comprimidos masticables (tubo)) }\end{array}$ & Rovi \\
\hline & $\begin{array}{l}\text { Calcio/vitamina D3 Rovi ( } 2500 \mathrm{mg} \text { (1000 mg ca)/880 UI } \\
30 \text { comprimidos efervescente) }\end{array}$ & Rovi \\
\hline Colecalciferol/ & $\begin{array}{l}\text { Calcio/vitamina D3 Sandoz (2500 mg (1000 ca)/880 UI } \\
30 \text { comprimidos masticables (frasco)) }\end{array}$ & $\begin{array}{l}\text { Sandoz } \\
\text { Farmaceutica S.A. }\end{array}$ \\
\hline $\begin{array}{l}\text { carbonato, } \\
\text { calcio }\end{array}$ & $\begin{array}{l}\text { Calciumosteo D ( } 1200 \text { mg ca/800 UI } 30 \text { comprimidos } \\
\text { efervescentes) }\end{array}$ & $\begin{array}{l}\text { Glaxosmithkline } \\
\text { Consumer } \\
\text { Healthcare }\end{array}$ \\
\hline & $\begin{array}{l}\text { Calciumosteo D ( } 2500 \mathrm{mg}(1000 \mathrm{mg} \mathrm{ca}) / 880 \mathrm{UI} 30 \\
\text { comprimidos efervescentes) }\end{array}$ & $\begin{array}{l}\text { Glaxosmithkline } \\
\text { Consumer } \\
\text { Healthcare }\end{array}$ \\
\hline & $\begin{array}{l}\text { Calodis ( } 2500 \text { mg (1000 mg ca)/880 UI } 30 \text { comprimidos } \\
\text { efervescente) }\end{array}$ & $\begin{array}{l}\text { Esteve } \\
\text { Pharmaceuticals, } \\
\text { S.A. }\end{array}$ \\
\hline & $\begin{array}{l}\text { Carbocal D (1500 mg (600 mg ca)/400 UI } 60 \\
\text { comprimidos masticables) }\end{array}$ & $\begin{array}{l}\text { Farmasierra } \\
\text { Laboratorios S.L }\end{array}$ \\
\hline & $\begin{array}{l}\text { Carbocal D (1500 mg (600 mg ca)/400 UI } 60 \\
\text { comprimidos) }\end{array}$ & $\begin{array}{l}\text { Farmasierra } \\
\text { Laboratorios S.L }\end{array}$ \\
\hline & $\begin{array}{l}\text { Cimascal D Forte ( } 1500 \mathrm{mg} \text { ( } 600 \mathrm{mg} \mathrm{ca}) / 400 \mathrm{UI} 60 \\
\text { comprimidos masticables) }\end{array}$ & Italfarmaco \\
\hline & $\begin{array}{l}\text { Cimascal D Forte Flas ( } 1500 \mathrm{mg}(600 \mathrm{ca}) / 400 \mathrm{UI} 60 \\
\text { comprimidos bucodispersables) }\end{array}$ & Italfarmaco \\
\hline & $\begin{array}{l}\text { Demilos ( } 1500 \mathrm{mg} \text { ( } 600 \mathrm{ca}) / 1000 \mathrm{UI} 30 \text { comprimidos } \\
\text { bucodispersable) }\end{array}$ & Italfarmaco \\
\hline & $\begin{array}{l}\text { Disnal (1500 mg (600 mg ca)/400 UI } 60 \text { comprimidos } \\
\text { masticables) }\end{array}$ & Italfarmaco \\
\hline & $\begin{array}{l}\text { Ideos ( } 1250 \mathrm{mg} \text { ( } 500 \mathrm{mg} \text { ca)/400 UI } 30 \text { comprimidos } \\
\text { masticables) }\end{array}$ & $\begin{array}{l}\text { Theramex } \\
\text { Healthcare Spain } \\
\text { S.L. }\end{array}$ \\
\hline
\end{tabular}




\section{Colecalciferol/ carbonato, calcio}

\begin{tabular}{|c|c|}
\hline $\begin{array}{l}\text { Ideos (1250 mg (500 mg ca)/400 UI } 60 \text { comprimidos } \\
\text { masticables) }\end{array}$ & $\begin{array}{l}\text { Theramex } \\
\text { Healthcare Spain } \\
\text { S.L. }\end{array}$ \\
\hline $\begin{array}{l}\text { Ideos Forte (500mg ca/1000 UI } 30 \text { comprimidos } \\
\text { masticables) }\end{array}$ & $\begin{array}{l}\text { Theramex } \\
\text { Healthcare Spain } \\
\text { S.L. }\end{array}$ \\
\hline $\begin{array}{l}\text { Ideos Unidia ( } 2500 \text { mg (1000 ca)/880 UI } 30 \text { sobres } \\
\text { efervescentes) }\end{array}$ & $\begin{array}{l}\text { Theramex } \\
\text { Healthcare Spain } \\
\text { S.L. }\end{array}$ \\
\hline $\begin{array}{l}\text { Ideos Unidia Elam Pharma (2500 mg (1000 ca)/880 UI } \\
30 \text { sobres efervescentes) }\end{array}$ & Elam Pharma \\
\hline $\begin{array}{l}\text { Mastical D (1250 mg (500 ca)/1000 UI } 30 \text { comprimidos } \\
\text { masticables fresa) }\end{array}$ & $\begin{array}{l}\text { Takeda } \\
\text { Farmaceutica } \\
\text { España S.A. }\end{array}$ \\
\hline $\begin{array}{l}\text { Mastical D (1250 mg (500 ca)/400 UI } 60 \text { comprimidos } \\
\text { masticables fresa) }\end{array}$ & $\begin{array}{l}\text { Takeda } \\
\text { Farmaceutica } \\
\text { España S.A. }\end{array}$ \\
\hline $\begin{array}{l}\text { Mastical D (1250 mg (500 ca)/400 UI } 60 \text { comprimidos } \\
\text { masticables limón) }\end{array}$ & $\begin{array}{l}\text { Takeda } \\
\text { Farmaceutica } \\
\text { España S.A. }\end{array}$ \\
\hline $\begin{array}{l}\text { Mastical D (1250 mg (500 ca)/400 UI } 60 \text { comprimidos } \\
\text { masticables naranja) }\end{array}$ & $\begin{array}{l}\text { Takeda } \\
\text { Farmaceutica } \\
\text { España S.A. }\end{array}$ \\
\hline $\begin{array}{l}\text { Mastical D (1250 mg (500 ca)/800 UI } 30 \text { comprimidos } \\
\text { masticables limón) }\end{array}$ & $\begin{array}{l}\text { Takeda } \\
\text { Farmaceutica } \\
\text { España S.A. }\end{array}$ \\
\hline $\begin{array}{l}\text { Mastical D Unidia ( } 2500 \text { mg ( } 1000 \text { mg ca)/800 UI } 30 \\
\text { comprimidos masticables) }\end{array}$ & $\begin{array}{l}\text { Takeda } \\
\text { Farmaceutica } \\
\text { España S.A. }\end{array}$ \\
\hline $\begin{array}{l}\text { Micaldeos (1250 mg (500 ca)/1000 UI } 30 \text { comprimidos } \\
\text { masticables (tubo)) }\end{array}$ & $\begin{array}{l}\text { Procare Health } \\
\text { Iberia, S.L. }\end{array}$ \\
\hline $\begin{array}{l}\text { Natecal D (1500 mg (600 mg ca)/400 UI } 60 \\
\text { comprimidos masticables) }\end{array}$ & Italfarmaco \\
\hline $\begin{array}{l}\text { Natecal D Flas (1500 mg (600 ca)/400 UI } 60 \\
\text { comprimidos bucodispersables) }\end{array}$ & Italfarmaco \\
\hline $\begin{array}{l}\text { Osmille D (1500 mg (600 ca)/1000 UI } 30 \text { comprimidos } \\
\text { bucodispersable) }\end{array}$ & Effik \\
\hline $\begin{array}{l}\text { Ostine (1250 mg (500 mg ca)/400 UI } 30 \text { comprimidos } \\
\text { masticables) }\end{array}$ & $\begin{array}{l}\text { Angelini } \\
\text { Farmacéutica S.A. }\end{array}$ \\
\hline $\begin{array}{l}\text { Ostine (1250 mg (500 mg ca)/400 UI } 60 \text { comprimidos } \\
\text { masticables) }\end{array}$ & $\begin{array}{l}\text { Angelini } \\
\text { Farmacéutica S.A. }\end{array}$ \\
\hline $\begin{array}{l}\text { Veriscal D (1500 mg (600 mg ca)/400 UI } 60 \\
\text { comprimidos masticables) }\end{array}$ & Italfarmaco \\
\hline $\begin{array}{l}\text { Veriscal D Flas ( } 1500 \text { mg (600 ca)/400 UI } 60 \\
\text { comprimidos bucodispersables) }\end{array}$ & Italfarmaco \\
\hline
\end{tabular}




\begin{tabular}{|c|c|c|}
\hline \multirow{2}{*}{$\begin{array}{l}\text { Colecalciferol/ } \\
\text { carbonato, } \\
\text { calcio/ } \\
\text { lactogluconato, } \\
\text { calcio }\end{array}$} & $\begin{array}{l}\text { Calciumosteo D (1200 mg ca/800 UI } 30 \text { comprimidos } \\
\text { efervescentes) }\end{array}$ & $\begin{array}{l}\text { Glaxosmithkline } \\
\text { Consumer } \\
\text { Healthcare }\end{array}$ \\
\hline & $\begin{array}{l}\text { Calciumosteo D (600 mg ca/400 Ul } 60 \text { comprimidos } \\
\text { efervescentes) }\end{array}$ & $\begin{array}{l}\text { Glaxosmithkline } \\
\text { Consumer } \\
\text { Healthcare }\end{array}$ \\
\hline $\begin{array}{l}\text { Colecalciferol/ } \\
\text { pidolato, calcio }\end{array}$ & $\begin{array}{l}\text { Osvical D (4500 mg/400 UI } 60 \text { sobres polvo suspensión } \\
\text { oral) }\end{array}$ & Alter \\
\hline \multirow{6}{*}{ Calcifediol } & Hidroferol (0.1mg/ml gotas orales solución 10 ml) & Faes Farma \\
\hline & Hidroferol (0.1mg/ml gotas orales solución 20 ml) & Faes Farma \\
\hline & Hidroferol (0.266 mg 10 ampollas bebibles $1.5 \mathrm{~m}$ ) & Faes Farma \\
\hline & Hidroferol (0.266 mg 10 cápsulas blandas (pvc/pvdc-al)) & Faes Farma \\
\hline & Hidroferol (0.266 mg 5 cápsulas blandas (pvc/pvdc-al)) & Faes Farma \\
\hline & Hidroferol choque (3 mg ampollas bebibles $1.5 \mathrm{ml}$ ) & Faes Farma \\
\hline \multirow{2}{*}{ Calcitriol } & Rocaltrol (0.25 mcg 20 cápsulas) & Roche Farma \\
\hline & Rocaltrol (0.5 mcg 20 cápsulas) & Roche Farma \\
\hline \multirow{3}{*}{ Alfacalcidiol } & Etalpha (0.25 mcg 30 cápsulas) & Leo Pharma \\
\hline & Etalpha (0.5 mcg 30 cápsulas) & Leo Pharma \\
\hline & Etalpha (2 mcg/ml gotas orales solución 10 ml) & Leo Pharma \\
\hline
\end{tabular}


